


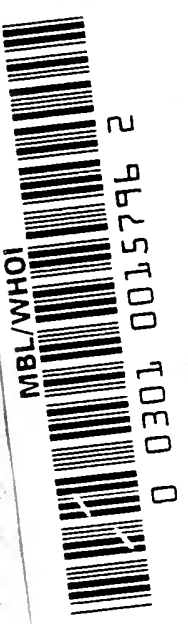


$25=$ 



\section{AN INTRODUCTION TO THE STUDY OF THE PRO'TOZOA}




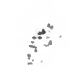




\title{
AN INTRODUCTION TO THE
}

\section{STUDY OF THE PROTOZOA}

\section{WITH SPECIAL REFERENCE TO THE PARASITIC FORMS}

\author{
BY \\ E. A. MINCHIN, M.A., Pн.D., F.R.S. \\ PROPESSOR OF PROTOZOOLOGY IN THE UNIVERSITY OF LONDON
}

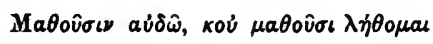

ILLUSTRATED

SECOND IMPRESSION

\author{
LONDON \\ EDWARD ARNOLD \\ 1922


$-3+2$ 


\section{PREFACE}

This book, as its name implies, is intended to serve as an introduction to the subject with which it deals, and not in any way as a complete treatise upon it. The science of "protozoology," as it is now generally termed, covers a vast field, and deals with an immense series of organisms infinitely varied in form, structure, and modes of life. In recent years the recognition of the importance of the Protozoa to mankind in various ways, and especially from the medical point of view, has focussed attention upon them, and has brought about a great increase of our knowledge concerning these forms of life. To set forth adequately and in full detail all that is now known about the Protozoa would be a task that could not be attempted in a volume of this size, but would require a work many times larger.

The aim of the present work is essentially didactic-that is to say, it is intended to furnish a guide to those who, having at least some general knowledge of biology, desire a closer acquaintance with the special probloms presented by the Protozoa. First and foremost, it attempts to define the position of these organisms in Nature, and to determine, as far as possible, in this way exactly what should be included under the term "Protozoa," and what should be excluded from the group. Secondly, its function is to guide the student through the maze of technicalities necessarily surrounding the study of objects unfamiliar in daily life, and requiring, consequently, a vocabulary more extensive than that of common language; and with this aim in view, care has been taken to define or explain fully all technical terms, since confusion of thought can be avoided only by a clear understanding of their exact significance and proper application. Thirdly, it aims at introducing the student to the vast series of forms comprised in the Protozoa and their systematio classification, based on their mutual affinities and inter-relationships, so far as these can be inferred from theif structural peculiarities and their life-histories. And, incidentally, attention has been drawn speoially to those parts of the subject where the Protozoa throw 
great light on some of the fundamental mysteries of living matteras, for example, sex-and a special chapter dealing with the physiology of the Protozoa has been added.

In so wide a field it is almost necessary to exercise some favouritism in the choice of objects, and greater stress has been laid in this work upon the parasitic forms, both on account of the many interesting biological problems which they present, and also because they come into closer relationship with the practical needs of human life than the non-parasitic species. The author wishes, however, to point out clearly that he is not a medical man, but one who approaches the study of the parasitic Protozoa solely from the standpoint of a naturalist who is more concerned, so to speak, with the interests of the parasite than with those of the host. Consequently, purely medical problems-such as, for example, the symptoms and treatment of diseases caused by trypanosomes and other Protozoa-are not dealt with in this book, since the author deems it no part of his task to attempt to instruct medical men concerning matters with which they are better acquainted by their training and experience than himself. The needs of medical men have, however, been specially kept in view, and the author hopes that the book will succeed in supplying them with useful information, at least from a general zoological or biological standpoint.

In a science, such as protozoology, which is growing actively and receiving continually new additions, and in which most of the data are based upon an elaborate and delicate technique, there are necessarily many controversial matters to be dealt with. In such cases the points at issue have been reviewed critically, and the author has, wherever possible, attempted to give a lead by indicating more or less decisively what is, in his opinion, the most probable solution of the problem under discussion. Such judgments, however, are not intended to be put forward in a dogmatic or polemical spirit, since the author recognizes fully that any conclusion now reached may be upset entircly by fresh evidence to the contrary.

The vast literature of the Protozoa would, if cited in full, easily fill by itself a volume of the size of the present one. It has been necessary, therefore, to restrict the limits of the bibliography as much as possible, both by selecting carefully the memoirs to be cited and by abbreviating their titles. The works selected for reference comprise, first, comprehensive treatises which deal with the subject, or with some part of it in a general way, and in which full references to older works will be found; secondly, classical memoirs on particular subjects. also containing, as a rule, full bibliographies; and, thirdly, such memoirs of recent date as have 
been deemed worthy of citation. In the many cases where the same authors have published several works on a given subject, only the last of them is cited-for example, the volume of researches published recently by Mathis and Léger (473) covers the ground of the earlier memoirs published by these authors, which are therefore not cited; similarly, the memoir upon amœbæ by Nägler (95) covers the earlier work of Hartmann and Nägler upon Amoba diploidea. Since it was quite impossible to make the bibliography in any way exhaustive, the aim has been to make it, like the rest of the book, introductory to the subject. It is hoped that any reader who, desirous of pursuing further some special subject, consults the references cited will find in them and in the further works quoted in them the means of acquiring complete information with regard to modern knowledge concerning all the points in question. The following classes of memoirs are not cited, however, in the bibliography, unless there was some special reason for doing so : faunistic works, papers describing new species, and writings of a pclemical character.

New memoirs on Protozoa are being published continually, so rapidly, and in so many different periodicals (some of them very difficult to obtain), that the author fears he may himself have overlooked many such, especially of those publications which have appeared very recently, while the book was in course of preparation. For such omissions, some of which have already come under his notice, he can but apologize, and at the same time promise that they shall be rectified in future editions, if the patronage of those interested in the subject enables further editions of this book to be published. The present edition does not, however, profess to deal with works published later than 1911 .

In order to further the object of making this book a guide to the technicalities of the subject, the plan has been adopted of printing in heavier black type in the index the numbers of those pages on which the term cited is fully explained, or, in the case of taxonomic names, is referred to its place in the systematic classification. In this way the index can be used as a glossary by anyone wishing to ascertain the significance of a technical term, or, though necessarily to a more limited extent, the systematic position of a genus, family, or order of the Protozoa. All that is necessary for this purpose is to look up the word in the index, and then to turn to the page or pages indicated by black type.

The author has, in a few cases, modified the technical terminology in current use, or has made additions to it. The adjective in general use relating to chromatin is "chromatic." with its various deriva- 
tives ("achromatic," ete.) ; since, however, these adjectives have a totally different meaning and use in optics, they have been altered to chromatinic, etc., in so far as they relate to chromatin. New terms used in this book are chromidiosome (p. 65, footnote), endosome (p. 73), as an equivalent to the German Binnenkörper, and gregarinula (p. 169).

In conclusion, it is the author's pleasant duty to return thanks to those of his colleagues who have kindly rendered him assistance in his task. $\mathrm{He}$ is especially indebted for much help and many valuable suggestions and criticisms to $\mathrm{Dr}$. H. M. Woodcoek, whose unrivalled knowledge of recent bibliography has been throughout of the utmost assistanee ; and to Dr. J. D. Thomson and Miss Muriel Robertson for many helpful diseussions upon matters of fact or theory. Dr. A. G. Bagshawe, Professor J. B. Farmer, F.R.S., Mr. W. F. Lanchester, Dr. C. J. Martin, F.R.S., and Dr. P. Chalmers Mitchell, F.R.S., have kindly read through some of the chapters, and have given valuable advice and critieism. In justice to these gentlemen, however, it should be stated that they are in no way responsible for any of the theoretical opinions put forward by the author. The majority of the figures have been specially drawn. from the original sources, or from actual preparations by Mr. R. Brook-Greaves and Miss Mabel Rhodes, to both of whom. the author's best thanks are due.

\section{Lister Institute of Preventive Medicine, Chelsea, S.W., July I, 1912.}




\section{CONTENTS}

CHAPTER

I. Ixtroddctory-The Distinctive Characters of the Protozoa aNd of therr Principal Subdivisions - - - $1-12$

1I. The Modes of Life of the Protozos - - - - 13-28

The Four Types of Nutrition, 13-15 ; Problems of Parasitism 15-28.

III. The Organization of the Protozon-External Form and Skgletal Structures $\quad$ - $\quad$ - $\quad$ - $\quad$ - $29-39$

IV. The Organization of the Protozoa (continued)-The ProtoPLASMIC BODY - $\quad$ - $\quad$ - $\quad$ - $\quad$ - $\quad$ - $40-44$

V. Tho Organization of the Protozos (continued)-Differentiations OF tHE ECTOPLASM AND ENDOPLASM - - - 45-64

A. Ectoplasmic Organs-(1) Protective, 45; (2) Kinetio and Locomotor, 46 ; (3) Excretory, 60 ; (4) Sensory, 61.

B. Endoplasmic Organs, 62.

VI. The Organization of the Protozoa (continued)-The Nuclear Apparatus-Chromatis, Nuclets, Chromidia, CentroSOMES, AND BLEPHAROPLASTS - - $\quad$ - $\quad$ - $\quad 65-99$

Vil. The Reproduction of the Protozos - - - - - $\quad$ - 100-124

Types of Fission, 100 ; Division of the Nucleus, 101 ; Division of the Cell-Body, 122.

VIII. Syneamy and Sex nN the Protozoa - - - - $\quad$ - 125-161

Nature of the Sexual Process, 125; Occurrence of Sexual Phenomena and their Importance in the Life of the Organism, 128 ; Maturation and Reduction, 142 ; Examples of Syngamy and Reduction in Protozoa, 147 ; Theories of the Origin and Significance of the Syngamic Process, 154. 
CHAPTER

IX. Polymorphism and Lifre-Cycles of the Protozoa -

A. Polymorphism, 162.176 ; B. Lifo-Cycles, 177.185.

X. The General Physiology of the Protozoa

(1) Nutrition and Assimilation, 187; (2) Respiration, 195;

(3) Exeretion and Secretion, 197 ; (4) Transmutation of Energy, 199; (5) Reactions to Stimuli and Environments, 201 ; (6) Degeneration and Regeneration, 208.

XI. Systematio Review of the Protozoa: The Sarcodiva

A. Rhizopoda-I. Amœbæa, 218; II. Foraminifera, 231 ; III. Xenophyophora, 237 : IV. Mycetozoa, 239.

B. Actinopoda-V. Heliozoa, 244 ; VI. Radiolaria, 249.

XiI. Systematio Review of the Protozoa: The Mastigophora 257-279

I. Flagellats, 257; II. Dinoflagellata seu Peridiniales, 276 ; III. Cystoflagellata seu Rhynchoflagellata, 278.

XIII. The Hemoflaghuntes and Aluied Forms

I. Trypanosoma, 283 ; II. Trypanoplasma, 300 ; III. Crithidia, 312 ; IV. Leptomonas, 313 ; V. Leishmania, 316 ; VI. Prowa. zekia, 319.

XIV. The Sporozon: I. The Gregarines and Coocidia - $323-355$

I. Gregarinoidea, 326 ; II. Coceidia, 341.

Comparison of the Life-Cycles of Gregarines and Coccidia, 354.

XV. The Sporozoa: II. The Hamosporidia - $356-397$

(1) Hæmamœbæ, 357 ; (2) Halteridia, 305; (3) Leueocytozoa, 369 ; (4) Hæmogregarines, 371 ; (5) Piroplasms, 378 ; Affinities of the Hæmosporidia, 388 ; of the Telosporidia, 395.

XVI. The Sporozoa: III. The Neosporidia - $398-429$

I. Myxosporidia, 399 ; II. Actinomyridia, 409 ; III. Microsporidia, 411 ; IV. Sarcosporidia, 419 ; V. Haplosporidia, 423.

Incertos Sedis, 425. 
XVIII. Afrinitiks axd Classipication of the Man Subdivisions-

Doubtrul Grovps

- $462-474$

General Phylogeny of the Protozoa, 463.

Spirochætes, 466 ; Chlamydozoa, 470.

BIBLIOQRA PHY .

INDEX
- $475-504$

- $505-517$ 



\section{AN INTRODUCTION TO THE STUDY OF THE PROTOZOA}

\section{CHAPTER I}

\section{INTRODUCTORY - THE DISTINCTIVE CHARACTERS OF THE PROTOZOA AND OF THEIR PRINCIPAL SUBDIVISIONS}

The Protozoa are a very large and important group of organisms, for the most part of minute size, which exhibit a wide range of variation in structural and developmental characters, correlated with the utmost diversity in their modes of life. Nevertheless, however grcatly adaptation to the conditions of life may have modified their form, structure, or physiological properties, a certain type of organization is common to all members of the group. The most salient feature of the Protozoa is their unicellular nature; that is to say, the individual in this subdivision of living beings is an organism of primitive character, in which the whole body has the morphological value of a single " cell," a mass of protoplasm containing nuclcar substance (chromatin) concentrated into one or more nuclei. However complex the structure and functions of the body, the organs that it possesses are parts of a cell (" organellæ "), and are never made up of distinct cells; and at least one nucleus is present, or only temporarily absent, as a constant integral part of the organism. The unicellular nature of the Protozoa, though a constant character, cannot, however, be used by itself to define the group, since it is also a peculiarity of many other distinct types of simple living things.

As an assemblage of organisms of primitive nature from which, in all probability, the ordinary plants and animals have originated in the remote past by divergent processes of evolution, the Protozoa have always possessed very great interest from the purely scientific and philosophical point of vietw. Of recent years, however, they have also acquired great practical importance from the relations that have been discovered to exist between Protozoa of parasitic habit and many diseases of man and animals. Hence the study of the Protozoa has received an immense impetus, and has been 
cultivated zealously even by many who are not professod biologists, with the result that our knowledge of these organisms has made very great strides in the last two decades, and is advancing so rapidly that it becomes increasingly difficult for any single person to keep pace with the vast amount of new knowledge that is published almost daily at the present time.

While the attention that is now focussed upon the Protozoa has led to a most gratifying increase of scientific and medical knowledge concerning particular forms, it tends frequently to a certain vagueness in the notions held with regard to the nature and extent of the group as a whole. This is owing largely to the fact that many are now attracted to the study of the Protozoa whose aims are purely practical, and who investigate only a limited number of species in minute detail, without having an adequate foundation of general knowledge concerning other forms. Hence $i v$ is important to attempt to frame a general definition of the Protozoa, or at least to characterize these organisms in such a way as to enable a distinction to be drawn between them and other primitive forms of life. This object may be attained logically in two ways-either by considering the distinctive characters of the group, or by cnumerating the types of organisms which constitute it; in more technical phraseology, by determining either the connotation or the denotation of the term Protozoa. To attempt this task will be the object of the present chapter.

The name Protozoa was first used in $1820^{*}$ as an equivalent of the German word Urthiere, meaning animals of a primitive or archaic type. This fitting designation superseded rapidly the older term Infusoria (Infusionsthierchen), used to denote the swarms of microscopic organisms which make their appearance in organic infusions exposed to the air. The word Infusoria is now employed in a restricted sense, as the name of one of the principal subdivisions of the Protozoa (pp. 12 and 430).

The first attempt at a scientific definition of the Protozoa was given by von Siebold, who defined them, from a strictly zoological standpoint, as unicellular animals. This definition, or a modification of it, is still the one given, as a rule, in zoological textbooks ; and from this time onwards the animal kingdom was subdivided universally into the Protozoa and the Metazoa. The Protozoa, as organisms in which the individual is a single cell, are regarded as those which come first ( $\pi \rho \hat{\omega} \tau$ ros) in the ascending scale of animal life, or in the course of organic evolution; the Metazoa, in which the individual is an organism composed of many cells, come after ( $\mu \in \tau a ́)$ the simpler forms of life in rank and time.

* For the detailed history of the growth of scientific knowledge of the Protozoa, see Bütschli (2), pp. i-rviii. 
Siebold's generalization was a great stop in advance, introducing clear and orderly ideas into the place of the chaotic notions previously held, and setting definite limits to the group Protozoa by excluding from it various types of organisms, such as Sponges, Rotifers, etc., which had hitherto been classed as Protozoa, but which were now referred definitely to the Metazoa. Nevertheless Siebold's definition presents many difficulties, especially when considered from a wider standpoint than that of the zoologist. This will be apparent if the two words of the definition given above, " unicellular animals," be considered critically.

1. "Unicellular."-Accepting the standpoint of the cell-theory, it has already been noted that many other organisms besides Protozoa must be regarded as single cells. Moreover, it is found that many organisms which must be classed as Protozoa appear constantly in a multicellular condition; such are the well-known genus Volvox and its allies, besides examples of other orders. In all cases of this kind, however, the constituent cells are morphologically equivalent, and are to be regarded as complete individuals more or less independent, showing as a rule no differentiation, or, if any, only into reproductive and vegetative individuals; and the multicellular organism as a whole is to be regarded as a colony of unicellular individuals primitively similar but secondarily differentiated, it may be, in relation to special functions. Such multicellular Protozoa present, in fact, a perfect analogy with the colonial forms seen in many groups of animals higher in the scale, especially the Colentera, where also the members of a colony, primitively equivalent and similar amongst themselves, may become differentiated secondarily for the performance of distinct functions by a process of division of labour among different individuals. It is not possible to confound the multicellular Protozoa with the Metazoa, in which the organism is not only composed of many cells, but exhibits also cell-differentiation based on mutual physiological dependence of the cells on one another, leading to the formation of distinct tissues; that is to say, aggregations or combinations of numerous cells, all specialized for the performance of a particular function, such as contraction, secretion, and so forth.

The essential feature of the Protozoa, as contrasted with the higher animals or plants, is to be sought in the independence and physiological completeness of the cell-individual. The Metazua are tissue-animals, in which the primitive individuality of the cell is subordinated to, or has a restraint imposed upon it by, tho corporate individuality of the cell-aggregate. In the Protozoa the cells are complete individuals, morphologically and physiologically of equal value. If, however, as few will doubt, the Metazoa have been evolved from simple unicellular ancestors, similar to the 
Protozoa, then there must have existed an unbroken scries of transitions between these two types of living beings. Hence, as in all attempts to classify living beings, sharp verbal distinctions between Protozoa and Metazoa are rendered possible only through the extinction of intermediate forms, or by ignoring such forms if known to exist. It is expedient rather to recognize distinct types of organization characteristic of the Protozoa and the Metazoa respectively, and to compare and contrast them, than to attempt to limit these groups by precise definitions.

2. "Animals."-This part of the definition raises more difficulties than their cellular nature. In the higher forms of life the distinction between animals and plants is an obvious and natural one; it is by no means so in the lower organisms. In the ranks of the simplest living creatures, those of animal nature are not marked off by any sharply defined structural or other features from those of vegetable nature, and cannot be separated from them in any scheme of classification which claims to be founded upon, or to express, the true natural affinities and relationships of the objects dealt with. As will be explained more fully in the next and subsequent chaptr rs, the distinction between animal and vegetable is, at its first appearance, nothing but a difference in the mode in which the organisms obtain their living. Forms that are obviously closely allied in all their characters may differ in this respect, and in some cases even one and the same species may nourish itself at one time as a plant, at another as an animal, according to circumstances. In short, the difference between plant and animal is primarily a distinction based upon habits and modes of life, and, like all such distinctions, does not furnish characters that can be utilized for systematic classification until the mode of life has continued so long, and the habit has become so engrained, as to leave an impress upon the entire structural characteristics of the organism.

The Protozoa cannot therefore be defined strictly and consistently as organisms of animal nature, for, though the vast majority of them certainly exhibit animal characteristics, it is impossible to exclude from the group many which live temporarily or permanently after the manner distinctive of the vegetable kingdom, and which are plants, to all intents and purposes, leading on in an unbroken series to the simplest algæ.

For this reason it has been proposed to unite all the simplest and most primitive forms of life in one "kingdom" under the title Protista (Protistenreich, Haeckel), irrespective of their habit of life and metabolism, whether animal or vegetable. The kingdom Protista is then to be considered as equivalent in systematic value to the animal and vegetable kingdoms, which in their turn are restricted in their application to true animals and plants as ordinarily 
understood. The term Protista thus unites under a single systematic category the vast assemblage of simple and primitive living beings from which the animal and vegetable kingdoms have taken origin, and have developed, by a continuous process of natural evolution, in different directions in adaptation to divergent modes of life.

The conception of a Protistan kingdom separate from the animal and vegetable kingdoms is open to the objection that it contains organisms which are indubitably of animal or vegetable nature respectively. The relations of the Protista to other living things may be represented graphically by the accompanying diagram (Fig. 1), where the circle represents the Protista, the two triangles the animal and vegetable kingdoms respectively. It is seen that the separation of the Protista as a systematic unity cuts across the ascending series of evolution ; to express it figuratively, it is a transverse cleavage of the phylogenetic " tree." A truly natural classification of living things, however, is one which expresses their genetic affinities and follows their pedigrees and lines of descent; it should represent a vertical cleavage of the ancestral tree. Judged by this standard, the kingdom of the Protista can only be regarded as a convenient makeshift or compromise, rather than as a solution of a difficult problem-that, namely, of giving a natural classification of the most primitive forms of life.

Whether the kingdom Protista be accepted or not as a natural and valid division of living beings, it is imperative to subdivide it further, not only on account of its vast extent and unwieldy

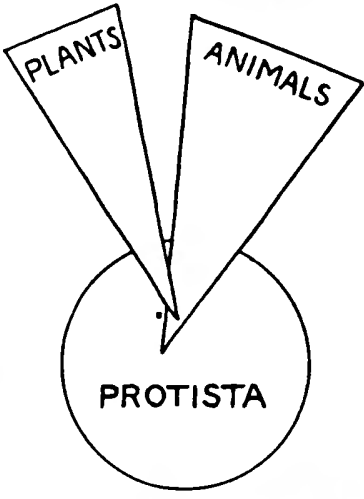

Fig. 1.-Graphic representation of the relation of the animal and regetable kingdoms to the kingdom of the Protista (Protistenreich). The Protozoa are represented by the portion of the triangle representing the animal kingdom which lies within the circle representing the Protista. size, but also because it comprises organisms very diverse in nature, requiring for their study the application of methods of technique and investigation often entirely different in kind. Hence in actual practice the Protista are partitioned among at least three different classes of scientific workers-zoologists, botanists, and bacteriologists-each studying them by special methods and to some extent from different points of view.

It is necessary, therefore, to consider from a general standpoint the principal types of organization comprised in the kingdom Protista, and we can recognize at the outset two chief grades of structure, bearing in mind always that transitional forms between them must exist, or at least must have existed.

In the first grade, which is represented by the Bacteria and allied groups of organisms, a type of organization is found which is probably the more primitive, though by many regarded as the 
result of degencration and specialization. These organisms do not conform to the type of structure of the cell, as this word is usually understood, since they do not exhibit, speaking generally, a division of the living body substance into a nucleus distinct from the cytoplasm;* but the chromatin is distributed through the protoplasmic body in the condition of scattered lumps or granules (" chromidia"), and in many cases it constitutes, apparently, the whole or a very large proportion of the substance of the body.

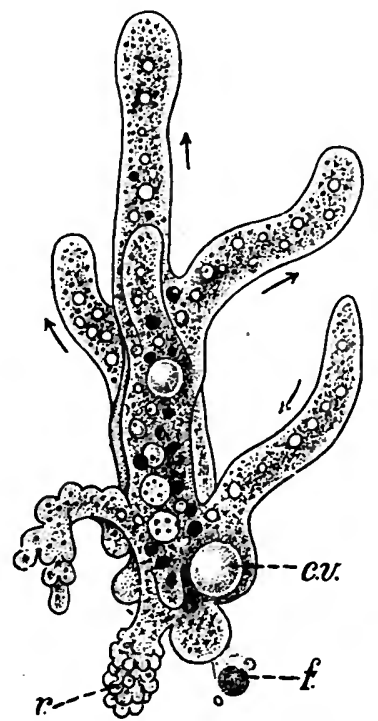

A

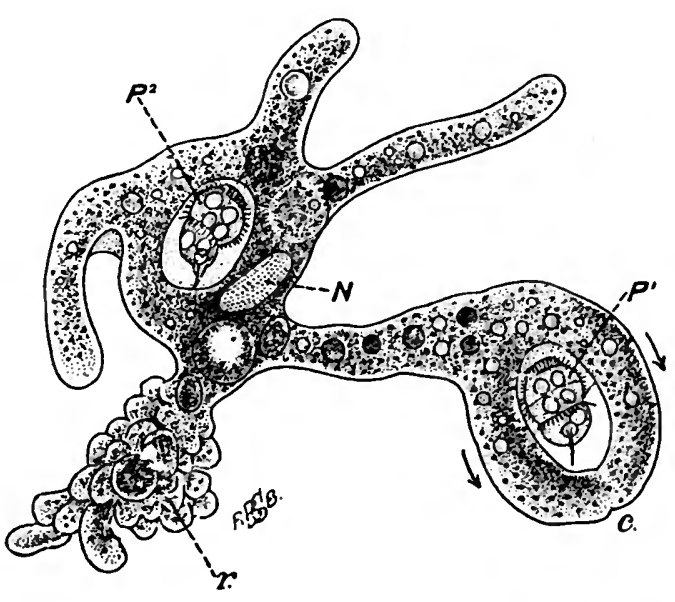

B

Frg. 2.-Amaba proteus. A, An individual in active movement; the arrows indicate the direction of the currents in the protoplasm; at $r$ is soen a pseudopodium which is nearly completely retracted and has assumed a mulberrylike appearance ; $c . v$., contractile vacuole; $f$., fæcal matter extruded at the cnd of the body posterior in movement; the nucleus is obscured by the opacity of the protoplasm. $B$, An individual in the act of capturing its prey $\left(P^{1}\right)$, an Infusorian (Urocentrum); two pscudopodia have flowed round it, as shown by the arrows, and met at the point $c$, enclosing tho prey ; another Infusorian $\left(P^{2}\right)$ is seen in a food vacuole in the body; $N$., nucleus; other letters as in $A$. After Leidy (226), magnified 200 diameters.

Further, the body in organisms of the bacterial type is of definite form, limited in many cases by a rigid envelope or cuticle, and special organs of locomotion are either absent or present in the form of so-called "flagella," structures perhaps different in nature from the flagella of truly cellular organisms. But the most remarkable and significant feature of organisms of the bacterial type is seen in the many different modes of metabolism and assimilation

* The significance of tho terms " nucleus," "cytoplasm," " chromidia," etc., $k$ ill be oxplained more fully in subsequent chapters. 
seen to occur amongst them. Although their metabolism is in general distinctly of a vegetative or saprophytic type, it ofter exhibits peculiarities not found in any true plant.*

In the second grade of the Protista, the organism possesses the characteristics of a true cell, in that the body shows a differentiation of the living subetance into two quite distinct parts - the cytoplasm, or general body-protoplasm, in which is lodged at least one nucleus, a body representing a concentration and organization of the chromatin-substance. In some cases the nuclear substance or chromatin may be in the scattered, chromidial condition

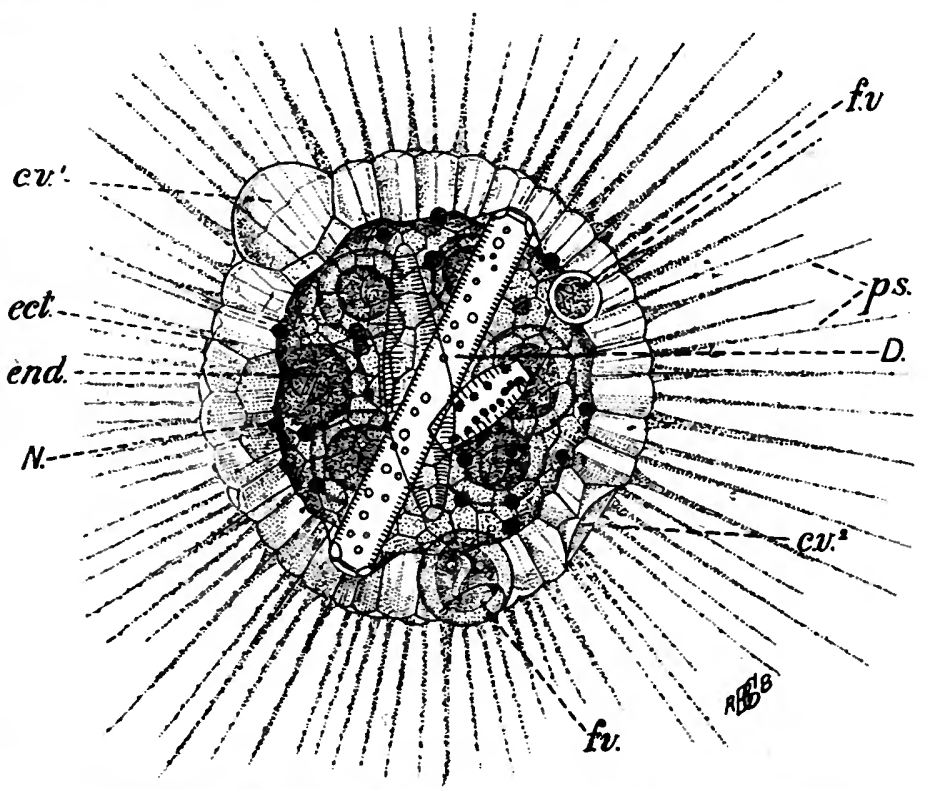

Frg. 3.-Actinaspharium eichhornii. ect., Ectoplasm; end., endoplasm ; c.v. ${ }^{1}$, a contractile vacuole at its full size; $c . v^{2}$, a contractile vacuole which has just burst ; $f v$., $f . v$., food vacuoles ; $D$., a large diatom engulfed in the protoplasm ; ps., peeupopodia ; $N$., one of the numerous nuclei. After Leidy (226), magnified 250 diameters.

during certain phases of the life-history, but such a condition is comparatively rare and probably always temporary. The bodyprotoplasm may be limited by a firm envelope, or may, on the other hand, be naked, in which case the body-form may be quite indefinite. Organs of locomotion, when present, are of various kinds ; and these organs may serve also for the capture and ingestion of food. And, finally, the metabolism is always one of the four types

- For a eummary and review of different modes of metsholism among bacterial organisms, article "Fermentation" in Thorpe's "Dictionary- of Applied Chemistry " (Longmans). 
described in more detail in the next chapter-numely, animal-like (holozoic), plant-like (holophytic), fungus-likc (saprophytic), or at the expense of some other living organism (parasitic).

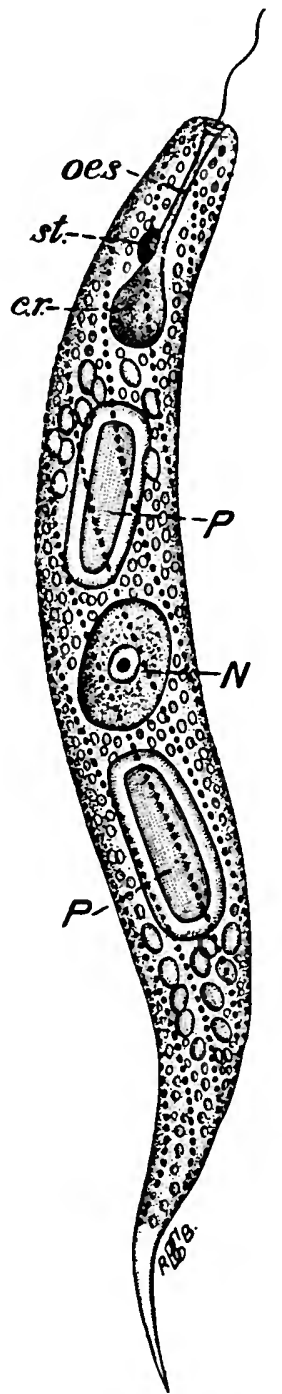

F1G. 4.-Euglena spirogyra. ox., Esophagus; st., stigma ; c.r., reservoir of the contractilo vacuole; $P, P$, paramylum - bodies ; $N$., nucleus. After Stein.
The cellular organisms that constitute the second or higher grade of the Protista are commonly partitioned between botanists and zoologists as Protophyta (unicellular algæ and fungi) and Protozoa respectively. It has been pointed out already, however, that this

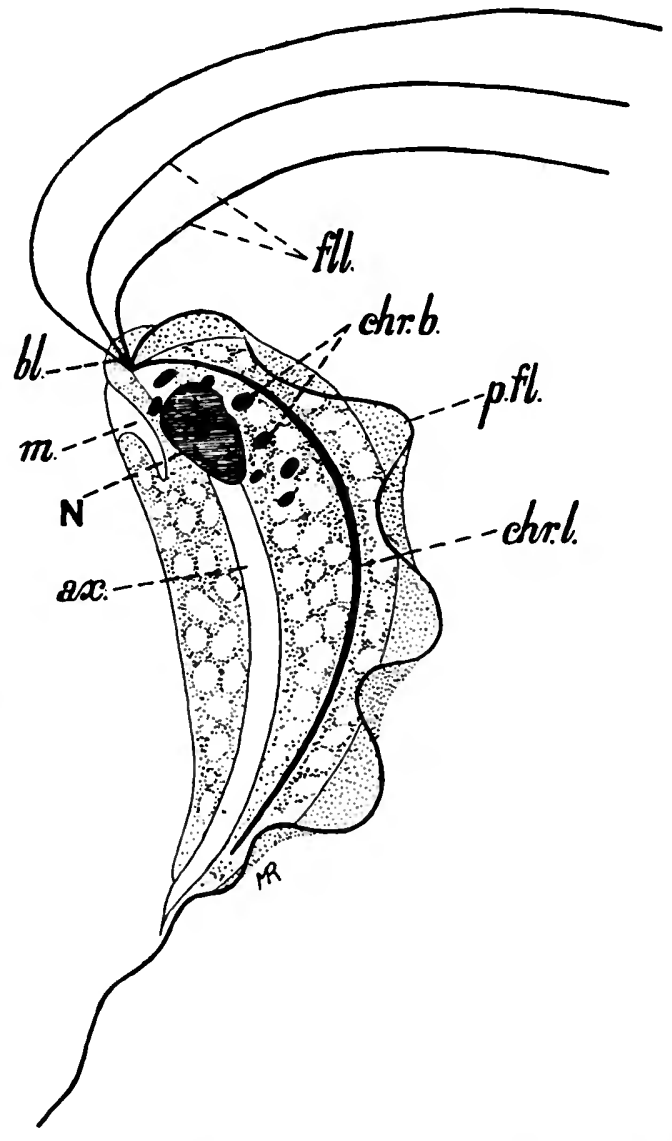

Fra. 5.-Trichomonas eberthi, from the intestine of the common fowl. $f l$., Anterior flagella, three in number; $p$. $f$., posterior flagellum, forming the edge of the undulating membrano; chr.l., "chromatinic line," forming tho base of the undulating membrane; chr.b., "chromatinic blocks"; $u l$., blepha roplast from which all four flagella arise; $m$., mouth-opening; $N$., nucleus $a x$., axostyle. After Martin and Robertson. 
method of subdividing them is purely arbitrary and artificial ; it leads to the result that many forms are claimed by both sides, and are always to be found described in both botanical and zoological treatises. It is nevertheless convenient for many reasons to retain the group Protozo3, even though we are obliged to include in it some forms which are plants in every sense of the word. The systematist who desires to give a rigidly logical definition of the Protozoa is, then, confronted with a dilemma : either to exclude from it forms with plant-like metabolism which naturally belong to it, or, by admitting such forms, to impair the universal applicability of the definition given. Such difficulties arise in every attempt to apply rigid verbal definitions to natural groups of living things; they are the direct outcome of the fact that all organisms have undergone and are undergoing a process of evolution, whereby they adapt themselves to new conditions of life and acquire new characters, as a result of which any two forms now distinct are or have been, connected by intermediate forms.

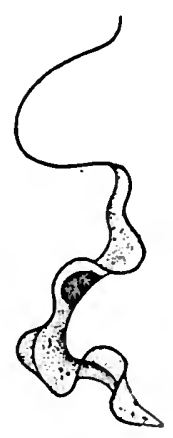

A

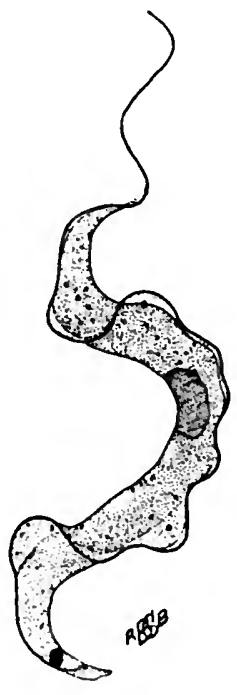

B

Fig. 6.-Trypanosoma remaki of the pike. A, Slender form ("var. parva"). $B$, Stout form ("var. magna"). After Minchin, $\times 2,000$.

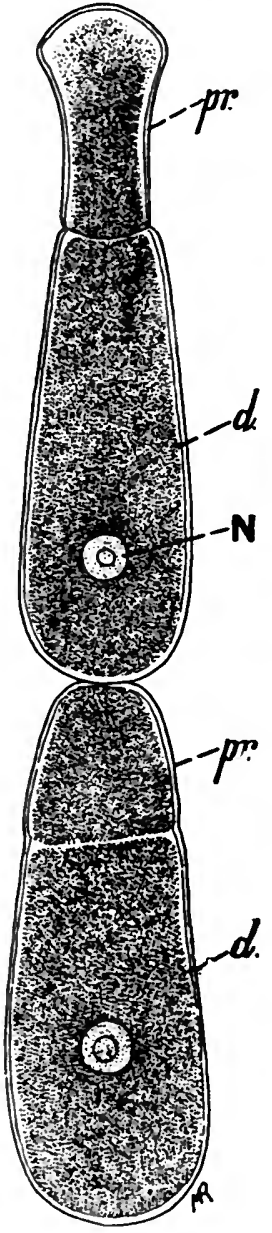

Fuc. 7. - Gregarina polymorpha, parasite of the digestive tract of the mealworm; "syzygy" of two individuals attaehed to one another. In each individual, $N$., nucleus; $p r .$, protomerite, or anterior segment of the body; $d$., deutomerite, or posterior segment. After Schneider. 
The attempt, therefore, made in the following paragraph to give a diagnosis of the Protozoa must not be regarded as a definition of the group in the rigidly logical sense, but merely as the construction of general type, the characters of which are liable to a certain amount of variation in special cases-a compromise between the claims of logic and the versatility of Nature.

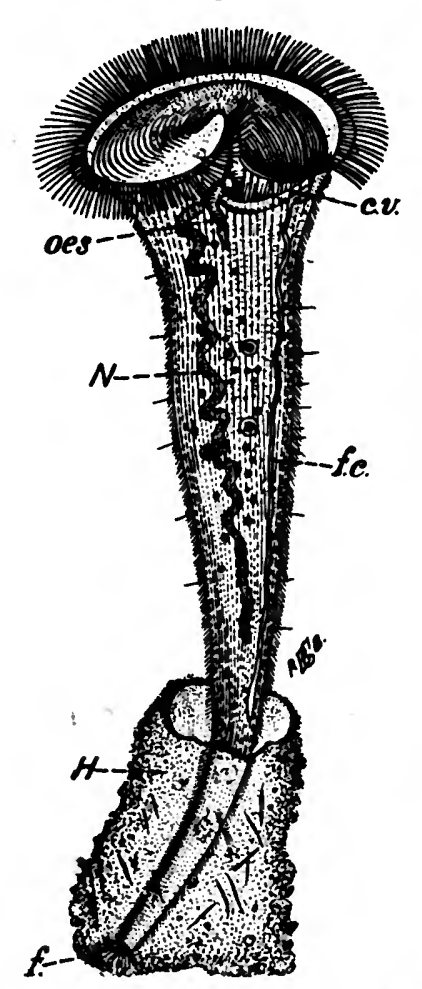

Fı. 8. - Slentor roeselii, fully expanded. ces., Esophagus; $N$, band-like macro. nucleus c.v., contractile vacuole, connectod with a long feeding-canal (f.c.) strotching down the body ; $H$, gelatinous house into which the animal can retmct itsolf completely; $f$., fibres attaching the extremity to the stalk to the bisuse. Aftor Stein.

The Protozoa, then, are Protista in which the organization is of the cellular type, with nucleus distinct from the cytoplasm. They are unicellular, in the sense that the cell constitutes an entire individual, which may exist singly and independently or in the form of cellcolonies; but in the latter case the cells are not subordinated to the individuality of the entire cellaggregate by the formation of

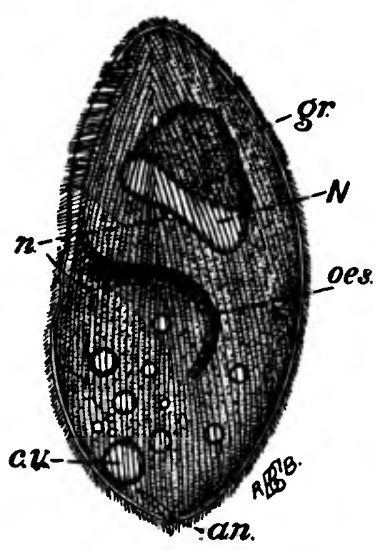

FiG. 9. - Nyctotherus cordiformis, parasite of the rectum of the frog. $N$, Macronucleus ; $n$, inicronucleus; gr., mass of granules in front of the macronucleus ; oes., cesophagus; c.v., contractile vacuole; an., anus (oytopyge). After Stein.

tissues. The body protoplasm is naked or clothed with a firm envelopo, which is usually not of the nature of cellulose. Protoplasmic organs are usually present for purposes of locomotion and for the capture and ingestion of food Chlorophyll is usually absent as a cell-constituent, and the metabolism is usually of the animal type. To these characters it may be added, though not as special peculiarities of Protozoa, that reproduotion takes place 
always by some form of fission-that is to say, division of the body into smaller parts ; that the plenomena known as "syngamy" and "sex" occur, perhaps universally, throughout the group; and that it is very characteristic of Protozoa, as compared with other Protista, to exhibit in their life-history a developmental cycle, more or less complicated, in the course of which the organism may appear under very different forms at different stages in its development.

The Protozoa, as thus understood, are commonly divided into four main subdivisions, termed "classes." Other methods of classifying the Protozoa have been suggested, whioh will be considered later; for the present the oldestablished subdivisions are sufficient for our purpose.

Class I., Sarcodina.*Protozoa in which the protoplasmic body is naked or noncorticate - that is to say, without a limiting envelope in the form of a cuticle, membrane, or stiff cortical layer; consequently the body tends to be either more or less spherical in floating forms, or to have an irregular, continually changing shape in creeping forms. Organs serving for locomotion and capture of food are furnished by tem. porary extensions of the living protoplasm, termed pseudopodia. A skeleton or shell may be present. Examples

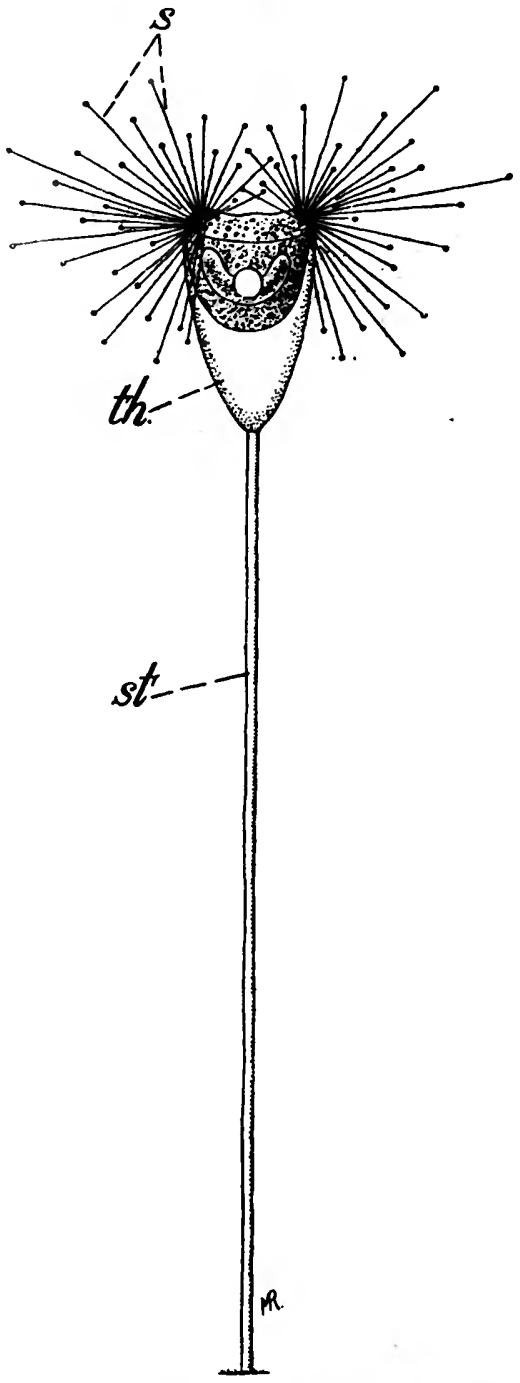

Fig. 10.-Acineta grandis. st., Stalk; th., theca ; 8., suctorial tentacles. After Saville Kent.

* The namo is derived from sarcode, the term coined by Dujardin to denote the living substance, subsequently named by von Mohl protoplasm, the term now
universally employed. 
are Amoba (Fig. 2), Diffugia (Fig. 16), Actinospharium (Fig. 3), etc.

Class II., Mastigophora.*-Protozoa in which the organs of locomotion and food-capture in the adult are flagella, slender filaments which are capable of performing active whip-like, lashing movements. The body-protoplasm may be naked or corticate. Examples are Euglena (Fig. 4), Trichomonas (Fig. 5), Trypanasoma (Fig. 6), etc.

Class III., Sporozon.-Protozoa occurring always as parasites of other organisms, and without definite organs for locomotion or ingestion of food in the adult condition. The reproduction takes place, typically, by formation of resistant seed-like bodies, termed spores, containing one or more minute germs, termed sporozoites. Examples are Gregarina (Fig. 7), Coccidium (Fig. 152), the malarial parasites (Fig. 156), etc.

Class IV., Infusoria.-Protozoa in which the organs of locomotion and food-capture are cilia, small vibratile filaments distinguished from flagella by their smaller size, by differences in their mode of movement, and by being present usually, in primitive forms at least, in great numbers like a fine fur over the whole or a part of the surface of the body. The cilia may be present throughout life (subclass Ciliata), or only in the early stages of the lifehistory (subclass Acinetaria). The body-protoplasm is always corticate. Examples are Stentor (Fig. 8), Nyctotherus (Fig. 9), Acineta (Fig. 10), etc.

Bibliography.-For a list of general works on Protozoa, see p. 476.

* Derived from the Greek $\mu d \sigma \tau \iota \xi$. a whip, equivalent to the Latin flagellum. 


\section{CHAPTER II}

\section{THE MODES OF LIFE OF THE PROTOZOA}

ProtozoA, as simple protoplasmic organisms, can only exist in an active state in a fluid medium. Hence the free-living, non-parasitic forms are aquatic, either marine or fresh-water in habitat. A certain number of species, however, are semi-terrestrial in their mode of life, creeping on damp surfaces or living in a minimum of moisture. Examples of such forms are the Amœbæ, etc., found in the soil, or Mycetozoa, which in the plasmodial phase (p. 239) creep on tree-trunks, logs, and so forth. None of these forms however, can remain active in perfectly dry surroundings, but pass into a resting state when desiccated.

It has been stated already that the methods by which Protozoa gain their livelihood vary greatly in different cases. Considered generally, these methods may be classified under four types :

I. The majority of Protozoa nourish themselves after the manner of animals - that is to say, they are entirely dependent for food and sustenance on other organisms which they capture, devour, and digest. Such forms are said technically to be holozoic, a word signifying " completely animal-like"; they are unable to utilize simpler chemical substances in order to build up the protein constituents of the living body, but require proteins ready-made for their sustenance.

II. A certain number of Protozoa-all, with rare exceptions, belonging to the class Mastigophora-possess in their body-substance peculiar colour-bearing corpuscles, so-called chromatophores or chromoplasts, containing chlorophyll or a pigment of allied nature, by means of which they are able to decompose carbon dioxide in the sunlight, liberating the oxygen and making use of the carbon in order to build up the protein and other constituents of the living body. Such organisms are entirely similar in their metabolism to the ordinary green plants, and are hence termed holophytic, or " completely plant-like."

The holophytic condition, in which the chlorophyll-bodies form an integral part of the structure of the body, and are to be regarded simply as protoplasmio organs, must be distinguished carefully from a state of things often 
found in holozoic Protozoa of all classes-namely, the presence in the body substance of symbiotic independent organisms of vegetable nature, as described below.

III. A certain number of Protozoa that have no chlorophyll or similar pigment in their bodies are, nevertheless free from the necessity of preying upon other organisms in order to obtain their sustenance, since they are able to live upon organic substances in solution, the products of the metabolism or decay of other living organisms. Such forms are termed saprophytic (or saprozoic), since their mode of life is similar to that of a saprophyte, such as a fungus. It is not necessary that they should be supplied with ready-made proteins in their food, since they are able to build up their protein constituents from substances of simpler chemical nature. Many examples of saprophytic forms are found amongst the free-living Flagellata.

Lauterborn (17) has coined the useful term sapropelic (from the Greek $\pi \eta \lambda \sigma s$, mud) to denote a mode of life which must be regarded as a special type of the saprophytic method, partly also of the holozoic-namely, the mode of life of those fresh-water organisms that live in a mud or ooze composed almost entirely of the decaying remains of dead plants and other débris of a similar nature. A very characteristic fauna occurs under these conditions.

IV. Finally, many Protozoa of all classes live as parasites-that is to say, at the expense of some other living being, which is termed the host.

These four modes of life can be used only to a very limited extent for classificatory purposes; it is only possible to do so in those cases where a particular habit of life, long continued, has resulted in definite structural characteristics, and more especially in the loss of organs requisite for other modes of life-as, for example, in the case of the subdivision Phytoflagellata, of the order Flagellata, where the holophytic habit has become so ingrained that only structural features proper to vegetable life are retained.

In other cases it is clear that a given habit of life in different organisms does not necessarily indicate close affinity between them. In the first place, we find closely allied forms living in different ways. Examples of all the four methodg of metabolism described above are to be found in the single order Flagellata, and throughout the Protozoa there are commonly to be found parasitic forms closely allied to free-living forms. In the second place, different types of metabolism may be found as transitory phases in the life of one and the same individual or species. Thus the common Euglena (Fig. 4), a flagellate possessing chromatophores and living normally in a holophytic manner, is able to maintain itself as a saprophyte if deprived of the sunlight necessary for a holophytic mode of life (p. 188) Striking examples of variability in the mode of nutrition are seen also in the section Chrysomonadina of 
the Flagellata, where a given species may be either holozoic or holophytic,* according to circumstances.

The bionomics of Protozoa - that is to say, their relations to their environment and to other organisms-constitute a very important branch of knowledge, both practical and theoretical, especially in the case of parasitic forms. Considering the subject from the point of view of the four modes of life already described, it is clear, in the first place, that the holophytic forms are entirely independent of all other living organisms, and tequire for their continued existence only sunlight and a suitable environment, containing the necessary inorganic substances, at a temperature which permits the continuance of vital processes and activities.

Saprophytic organisms, however, in so far as they require for their sustenance materials produced by living bodies, are dependent directly or indirectly upon other organisms for their existence. Purely holozoic forms, also, cannot exist without other forms of life upon which, or upon the products of which, they can feed. But neither holozoic nor saprophytic organisms are dependent, as a rule, upon any other particular form of life, but only upon living things generally; though in some cases such forms may be specialized in their nutrition to such an extent as to be unable to exist without some particular food.

A parasitic form, on the other hand, is entirely dependent, as a rule, for its existence on some particular organism or limited group of organisms which constitute its host or hosts. It must, however, be understood clearly that an organism living in or upon the body of another organism is not necessarily a parasite by any means. In the first place, a distinction must be drawn between parasitism and symbiosis, by which is meant an association of two organisms for mutual benefit. $\dagger$ Good examples of symbiosis are seen in some of the Sarcodina, Radiolaria, and Foraminifera, the protoplasm of which contains constantly intrusive organisms, known as zoochlorelloe or zooxanthella, according as they contain a green or a yellow pigment. Zoochlorellæ are green algæ of the order Protococcacece; zooxanthellæ are holophytic flagellates of the suborder Cryptomonadina-e.g., Cryptomonas schaudinni, symbiotic in the foraminifer Peneroplis (Winter, 28). These organisms penetrate

* For example, the species Chromulina flavicans. See Bütschli (2), vol. ii., p. 865.

† The term "symbiosis" is often much misused, especially by medical writers, by whom it is commonly applied to any association of two distinct organisms; for instance, "pure mixed cultures" of amobr with some species of bacillus, where the amabso are simply feeding on the bacteria, are often spoken of as "symbiosis," although the advantage is clearly only on one side in such an association. It should be understood that the term "symbiosis" is a tochnical term of long standing in biology, and is used not merely in its strict etymological significance of "living together," but in the special and restricted sense of "living together for the mutual benefit of the two organisms concerned." 
into the body of their host, lose their flagella, and nourish themselves by means of their pigment, which has the nature and properties of plant-chlorophyll ; that is to say, it decomposes carbon dioxide in the sunlight and liberates oxygen. The carbon dioxide is obtained from the respiratory processes of the host, which in its turn utilizes the oxygen produced by the symbionts (p. 197), and thus each organism supplies the needs of the other. When the host enters upon its reproductive processes and breaks up into a vast number of swarm-spores, the symbionts develop flagella and swim off, doubtless to seek for lodging elsewhere.

It is a matter of convenience to distinguish as epizoic those organisms which live upon, or are attached to, and as entozoic those which live within, the body or substance of the particular form of life with which they are associated. Epizoic forms may, be entirely harmless to the creature upon which they occur; they may simply utilize its body as a coign of vantage where they readily obtain their food, which may consist in some cases of nutritious substances dropped or rejected by the animal that carries them; or they may obtain the benefits of shelter or transport, especially when the epizoic form in question is itself of sedentary habit. Every naturalist is acquainted with the sea-anemones that live habitually upon hermit-crabs, probably the advantage of both animals-at all events, to the detriment of neither. There are many similar cases among Protozoa. The appendages of Crustacea, especially of the Cladocera and Copepoda, are often thickly beset with sessile Vorticellids and Acinetaria, which obtain a convenient lodging, but provide their own board. Other forms occur similarly on the stems of hydroids, as, for example, Acineta papillifera on Cordylophora lacustris. Amœbæ are found creeping on the exterior of Calcareous Sponges, nourishing themselves on diatoms and other organisms. Similar instances could be multiplied indefinitely.

On the other hand, epizoic forms may be dangerous' parasites, nourishing themselves at the expense of the animal they infest, and sometimes inflicting much damage upon it. It can be easily understood that an epizoic form which at first lived harmlessly upon some animal, drawing its supplies of food from the surrounding medium, might acquire the habit ultimately of obtaining its nourishment from the living substratum upon which it has planted itself. Examples of epizoic parasites are the flagellate Costia necatrix (p. 272) and the ciliate Ichthyophthirius multifiliis (p. 450), both of which are epizoic parasites of fishes, attaching themselves to the skin and destroying the epidermis ; as a result, the way is left open for fungi and bacteria to penetrate the skin, and so produce ulceration and suppuration, which may be fatal.

All certain instances of Protozoa acting as external parasites aro 
found amongst aquatic animals, and it can be readily understood that a delicate protoplasmic organism could only pass from one host to another in a fluid medium, or by tho help of special mechanisms adapted to aerial transport or transmission by contact. It should be mentioned, however, that some human contagious skindiseases are suspected to be due to the agency of parasites of the nature of Protozoa.*

Like the epizoic forms, there are many entozoic Protozoa which inhabit the bodies, and especially the intestines, of other animals, but which are in no way to be regarded as parasites; they feed merely on various substances to be found there, such as waste particles of food, excreted or fæcal matter, or on other organisms, such as bacteria, yeasts, and the like-in short, on substances which from the point of view of the host are superfluous, or even harmful. Many examples of such organisms could be cited; a good one is the common Chlamydophrys stercorea, found in the fæces and digestive tract of man and many animals. The common intestinal flagellates belonging to the genus Trichomonas (Fig. 5) and other genera are, similarly, not to be regarded as true parasites in any sense of tho word. The common Lophomonas blattarum (Fig. 45) from the intestine of the cockroach feeds chiefly upon bacteria and yeasts. Many of these intestinal Protozoa are perhaps useful, rather than harmful, to their hosts.

On the other hand, the vast majority of organisms, Protozoa or otherwise, that live in the interior of other living creatures arc there for no good or useful purpose ; their habitat is alone suffieient to render them suspect. Two modes of parasitism may be distinguished from a general point of view. On the one hand, the para sito may merely intercept the food of the host and rob it of its sustenance. On the other hand, the parasite may nourish itself upon the living substance or vital fluids of its host.

Organisms which rob the host of its food may do so in one of two ways. They may appropriate the raw food-material, which they then ingest and devour after the strictly holozoic method of feeding ; examples of this mode oflife are possibly to be found in the extensive infusorian fauna to be found in the stomachs of ruminants. Or they may absorb the fluid products of the digestion of the host by diffusion through the surface of the body of the parasite; examples of this mode of parasitism are to be seen, probably, in the case of the Gregarines so common in the guts of insects. Parasites of the

* For example, the so-called Coccidioides immitis, a name given to bodies found in certain South American skin diseases; sce Blanchard (633), p. 168. Molluscum contagiosum has also beon attributod to parasites referred by some to the Protozos. In both these instances, however, the exact nature of the parasitic bodies is far from clear; the parasite of molluscum contagiosum should probably be reforred to the Chlamydozoa (p. 470). 
type, that may be denoted as food-robbers are in general vory harmless to their hosts.

Those parasites, however, that nourish themselves on the substance of the host may produce the most dangerous effects on its health and well-being. As in the case of the food-robbers, parasites of this kind may absorb their food in one of two ways. They may devour solid portions of the host's body in a holozoic manner ; an example of this is seen in Entamoeba histolytica (Fig. 90), the parasite of amœbic dysentery, which devours portions of the host's tissue, such as epithelial cells, or blood-corpuscles. But more usually the parasites absorb their nourishment in a fluid form through the surface of their body, doubtless by the help of enzymes secreted by them. Hence it is typical of true parasites to have lost all trace of special organs for the capture, ingestion, or digestion, of solid food.

Just as in the epizoic mode of life a harmless or even beneficial commensalism may degenerate by insensible gradations into dangerous parasitism, so the same is true of the entozoic habit. An organism which begins by being a scavenger readily becomes a food-robber. Lophomonas, for instance, may be seen to contain starch-grains and other substances which probably belong to the food of its host. A further but easy gradation leads to the entozoio organism devouring portions of its host. A good example of this is seen in two of the entozoic amobæ of the human intestine : the common Entamoeba coli (Fig. 89) appears to be chiefly a scavenger, harmless to its host, and not deserving the reproach of parasitism ; on the other hand, $E$. histolytica is a dangerous parasite. So also an entozoic organism, which begins by merely absorbing the products of digestion, may end by absorbing the substance of its host. It is highly probable that in many entozoic organisms the mode of feeding may vary according to circumstances, and that an organism which may be a harmless commensal under some conditions may become a more or less dangerous parasite under others.

The entozoic Protozoa which are truly parasitic may inhabit a variety of situations in the bodies of their hosts. In some cases the host is another species of Protozoon, into the body of which the intruder penetrates, living either in the cytoplasm or the nucleus. Amœbæ are very subject to the attacks of intranuclear parasites, and the young stages of many Acinetans are parasitic upon other Infusoria. When the host is one of the Metazoa, the invading organism may be in like manner intracellular or intranuclear in habitat; or it may penetrate into the tissues, living amongst and betwcen the constituent cells; or it may inhabit, finally, one of the internal cavities of the body, such as the digcstive tract, $g$ meral body-cavity, spaces containing blood or lymph, cavities of the renal 
or urinary organs, etc., either living free in the cavity it inhabits, or attached to the lining epithelium.

As diverse as the modes of parasitism among Protozos are the effects they produce on their hosts. Some parasites produce no perceptible disturbance in the well-being of their host; even when they destroy cells and portions of tissues, the damage may be slight, and is quickly made good without appreciable permanent injury being done. From this condition of more or less perfect harmlessness there is a continuous gradation in the ascending capacity for harmfulness, culminating in species which bring about the death of their hosts with greater or less rapidity. Hence parasitic Protozoa are commonly distinguished as pathogenic or non-pathogenic; but since there is no precise limit to the degree of sickness or indisposition which justifies the application of the term "pathogenic," it is perhaps more convenient to distinguish them as lethal or nonlethal. It is not possible, however, to lay down hard-and-fast distinctions in these matters, since a parasite which is not lethal under some ciroumstances may become so under othars ; for instance, an animal living a free and natural life may be quite well able to resist the attacks of parasites to whioh it succumbs in captivity. Moreover, it must be borne in mind that such terms as "lethal" or "pathogenic" can only be applied to a parasite in its relation to a particular host, since, as will be shown below, a parasite which is harmful to one host may be harmless to another.

It is far from clear in what way the pathogenic effects of parasitic Protozoa are produced. If the action and reaction of host and parasite were relations dependent simply on the number or relative total bulk of parasites present in a given host, the problems of parasitism would be comparatively simple; but in many cases this is obviously very far from being the case. The effect produced by a given species of parasite upon a given species of host is a specific reaction, which differs markedly when one of the two dramatis personce is changed. It is not uncommon to find insects with their digestive tract or body-cavity crammed with parasitic Gregarines of relatively large size, but apparently none the worse for it. On the other hand, large mammals may succumb to the effects of minute parasites in relatively scanty numbers-in the sense, that is, that the aggregate bulk of the parasites may be infinitesimal compared to the bulk of the host. A better comparison is furnished by considering closely-allied species of parasites and hosts respectively. A rat may have its blood swarming with Trypanosoma lewisi, without apparently being any the worse for it. On the other hand, in a man dying of sleeping sickness, caused by $T$. gambiense, or in a ruminant dying of nagana (tsetse-fly disease), caused by $T$ : brucii, the trypanosomes may be so scanty as to be exceedingly 
difficult to detect.* These facts suggest strongly that the parasites produce specific toxins ; but the " sarcocystine" produced by parasites of the genus Sarcocystis (Sarcosporidia) $\dagger$ is almost the only case up to the present, in which a toxin has been isolated from a Protozoan parasite. Laveran and Pettit (19), however, claim to have obtained " trypanotoxins" from trypanosomes.

Considering the facts of parasitism generally, as a problem of natural history, two guiding principles must be borne in mind clearly : the first is that any organism, parasitic or otherwise, tends to be adapted in the best possible manner to the circumstances of its natural environment; the second is that, so long as a parasite is entirely dependent on its host for its existence, it is to its utmost disadvantage to bring about the death of its host. When, therefore, a given parasite is constantly lethal to a particular host or hosts, one of two explanations must be sought for : either the case is one of a disharmony - that is to say, of imperfectly-adjusted relations between the host and parasite; or the parasite must obtain from the death of the host advantages in the matter of the continuance of the species sufficient to compensate for the temporary loss through destruction of individuals.

The conditions to which a parasite requires to be adapted are different in many ways from those that influence the life of a freeliving organism. When once a parasite has obtained a footing in its proper host, the problem of food-supply is solved for it, since it finds itself lodged in the midst of abundant nutriment so long as its host lives. On the other hand, if the species is to be maintained, it is essential that the parasite should be able to infect new hosts, a difficult undertaking, and one in which the chances are all against the parasite in most cases. To insure dissemination of the species a large number of offspring must be produced, and special mechanisms and adaptations may be necessary to insure their reaching their destination. Hence, the more parasites become specialized and adapted to their peculiar mode of life, the more the organs and functions of nutrition tend to become simplified, and the greater the tendency to elaboration and extreme fertility of the reproductive function.

Considered gonerally, a parasitic Protozoon reproduces itself within a given host with one of two results : in the first place, with that of overrunning the host and establishing itself there; in the second place, with that of producing forms destined to infect new hosts. Forms produced in the first manner may be termed the " multiplicative phases"; their function is to produce a stock of the parasite. From the stock are given off what may be termed

* Compare Laveran and Mesnil (391), pp. 146-150.

$\dagger$ Laveran and Mesnil (18); Teichmann (25); Teichmann and Braun (26). 
the " propagative phases," which as a rule do not multiply further in the host in which they are produced, but await their chance of being transferred to a new host; and if such a chance be not given to them, they die off and are replaced by fresh propagative forms from the stock (see further below, Chapter IX., p. 166).

So long as the nutritive or multiplicative function is the most important one in the life of a parasite, and until it has matured its propagative phases, the death of the host is the greatest disaster that can befall it. The ideal host, from the point of view of a parasite, is one that is "tolerant" to it-that is to say, one that can support the presence of the parasite and koep it supplied with the nutriment it requires, without suffering in health or vigour to any marked extent. When once, however, the parasite has made the necessary provision for propagating the species, the life or death of the host may become a matter of indifference to the parasite, or may even in some cases be necessary for the dissemination of the offspring. This will be apparent from a consideration of the methods by which parasitic Protozoa infect new hosts.

The passage of a parasite from one host to another includes two mancuvres : the passing out from the first host, and the passing into the second. Primitively it may be supposed that this migration was effected simply by the unaided efforts of the parasite itselfthat is to say, that the active motile parasite would force its way out of one host, move freely in the surrounding medium, and sooner or later attack and penetrate a fresh host. This primitive method of transference doubtless occurs in many cases, especially amongst epizoic forms (e.g., Ichthyophthirius, p. 450). In the case of entozoic parasites its occurrence is less common, but it is found in a certain number of cases. The young stages of many Acinetaria, parasitic in Ciliata, probably seek out their hosts and penetrate into them; after a period of juvenile parasitism they leave the host's body and become free-living, non-parasitic organisms. Active migration of this kind, however, is very raro amongst entozoic parasites. In the first place, the conditions of life within a living body, in the midst of organic fluids, are so different from those in the open water, whether salt or fresh, that it is hardly to be expected that a delicate unicellular organism adapted to the one mode of life could stand the sudden change to the other. In the second place, it is clear that active migration of parasitic Protozoa could only be effected when the host is an aquatic animal, and not when it leads a terrestrial life. The only instances of active migration known with certainty to occur in the case of Protozoa parasitic on terrestrial animals are those in which the parasite can penetrate a mucous membrane, and is thus ablc to pass from one host to another when two such surfaces are in contact. In this way the trypanosome of dourine in horses 
( $T$. equiperdum) passes from one host to another during coitus, and the transmission of the parasite of syphilis is another instance.

Speaking generally, and excluding for the moment those cases in which the transmission is brought about by means of an intermediary host, the propagative phases of the parasitic Protozoa take the form of inactive, resting stages in which the body of the parasite is protected against adverse external conditions by tough protective membranes. In the form of resistant cysts or spores, the parasites in a dormant state offer a passive and inert resistance to the elements; they are disseminated like seeds, and they germinate when they reach a suitable environment, but not till then.

Many, perhaps the majority of parasitic Protozoa, occupy positions in the body of the host whence the propagative phases can pass without difficulty to the exterior. This is the case when the parasite is lodged in organs which have ducts or passages leading directly or indirectly to the exterior-such as, for instance, the digestive tract and its dependencies, or the urinary organs and ducts. In all such cases the propagative stages of the parasite pass harmlessly to the exterior. The host may in this manner get rid entirely of its parasites, without, however, necessarily acquiring immunity to fresh infections ; or, on the other hand, the parasite may keep up its numbers in the host by continual multiplication to produce a stock from which are sent forth incessant relays of the propagative phases destined to infect new hosts. In the majority of parasitic Protozoa the relations to the host are of this type, and the parasites are neither lethal nor pathogenic tc any great extent.

On the other hand, there are many instances in which parasitic Protozoa occupy a position in the body of the host whence escape by anatomical channels is not possible. This is the case when the parasite inhabits some closed space in the body, such as the colome or general body-cavity, or the blood-system; or when it attacks deeply-situated cells or tissues of the body. In some cases where natural means of exit from the body occur, they may be unsuitable for the dissemination of the parasite, as in the case of those forms parasitic in the genital organs of one sex of the host. In cases of this kind there are at least six known methods whereby parasitic Protozoa are disseminated and transferred to fresh hosts.

1. The resistant stages of the parasite may be set free by the death and decay of its host. This appears to be the manner in which some of the tissue-infecting parasites of the order Myxosporidia, especially the family Myxobolidoe, are disseminated ; they are for the most part parasites of fishes, and are often very deadly in their effects.

2. The parasite may cause tumours and ulcers, which suppurate, and so set free the cysts or spores of the parasite. This, again, is 
an effect often produced by tissue-parasites, such as the Myxobolida, or by species of Microsporidia. In such cases also the parssite is pathogenic to its host, and frequently lethal.

3. The parasite remains in the host until the latter is eaten by some animal which preys upon it. The propagative phases of the parasite are able, however, to resist digestion by the animal that has devoured their former host, and pass unaltered through its intestine, to be finally cast out with the dejecta. This is almost certainly the method by which the common Monocystis of the earthworm infects its host. The parasite produces resistant spores in the worm; the worm is eaten by a bird, mole, frog, or some other animal, through the digestive tract of which the spores pass unaltered; they are scattered abroad with the fæces, and may then be swallowed by another earthworm, in which they germinate and produce an infection.

4. As in the last case, the hast, together with its parasites, is devoured by some animal, in which, however, the parasite is not merely carried passively, but again becomes actively parasitic. Hence in this case there is an alternation of hosts, one of the two hosts becoming infected by devouring the other. This mode of infoction, which is well known to occur commonly among parasitic worms, such as Cestodes, is probably also frequent among Protozoa ; but at present only two cases of it are known with certainty. One is that of the species of the genus Aggregata (vide infra, p. 353), parasites of crabs and cephalopods, such as the cuttle-fish and the octopus. In the cephalopod the parasite forms resistant spores which pass out with the fæces, and may then be devoured by crabs. In the crab the spores germinate and give rise to a second form of the parasite, which lives and multiplies in its new host. If, as frequently happens, the crab is eaten by a cephalopod, the parasite completes its life-cycle by becoming once more a parasite of the cephalopod. Another case is that of Homogregarina muris in the rat-mite (p. 376, infra).

5. The Protozoa parasitic in the blood of vertebrates are disseminated by blood-sucking invertebrates, such as leeches, ticks, or insects, which take up the parasites by sucking the blood of an infected animal. Later on the parasite may be inoculated into a second vertebrate host by the invertebrate when it sucks blood at a later feed. In some cases the transference of the blood-parasite may be effected in a purely direct and mechanical manner by the invertebrate, but in most cases the invertebrate plays the part of a true host, in which the parasite multiplies and goes through a cycle of development. Hence in such cases also there is an alternation of hosts and a complicated life-cycle, of which the life-history of the malarial parasite is a good example (vide infra, p. 359). It 
need only be noted here that in such cases resistant spores or cysts become unnecessary and superfluous for the propagation of the parasite, and tend to disappear from its developmental cycle.

6. In some cases the parasite may penetrate the ovary of its host, . pass into the ova, and thus infect the embryo and the next generation. Transmission of this kind is known in a certain number of eases; it is never the sole method of transmission, but is always supplementary to other methods. For instance, in "pébrine" of silkworms, caused by Nosema bombycis, the spores of the parasite are liberated in the ordinary way from the caterpillar either with the fæces or by its death, and are then eaten accidentally on the leaves by other silkworms; but a certain number of the parasites penetrate into the ovary and form spores, which pass through the pupal and imaginal stages of the host into the next generation of silkworms, which are born infected. In this way the parasite is able to tide over the winter season, when the ordinary method of infection would be impossible. The blood-parasites of the genus Piroplasma (p. 384, infra) afford another example of germinative infection in the ticks which transmit them.

To turn now to the methods by which parasitic Protozoa penetrate into new hosts; there are four known methods, which, after what has been said, can be summarized very briefly. The commonest is the method of casual or contaminative infection, where the host infects itself accidentally by taking up the propagative phases of the parasite from its surroundings-most usually by way of the mouth, with the food, but it may be by way of the respiratory organs. Other modes of infection are the contagious, as in dourine, already mentioned; the inoculative, as in malaria and other diseases caused by blood-parasites ; and the so-called "hereditary" or "germinative" method, as in Nosema bombycis and other cases.

From the foregoing summary of the methods by which parasitic Protozoa are propagated from one host to another, it is clear that there are very fow cases in which it is of direct advantage to the parasite to cause the death of its host. Even where it is necessary, for the propagation of the parasite, that the host should be destroyed by some other animal, as in the case of the Monocystis of the earthworm, the interests of the parasite are not furthered, and may, indeed, be damaged, if it cause disease or death to the host. In the case of blood-parasites, transmitted by the inoculative method, it may be necessary for the propagation of the parasite that the required phases should be sufficiently abundant in the blood of the vertebrate host to insure the invertebrate host becoming infected when it sucks the blond then large numbers of the parasite may be detrimental to the well-being of the host to a greater or less extent, 
and one interest of the parasite may, so to speak, clash with another. But in all cases alike it is perfectly clear that the death of the host before the parasite has matured its propagative phases leads simply to the extirpation of the parasite, and is a suicidal policy on its part, a glaring disharmony in Nature. This conclusion is borne out by a general survey of the facts of parasitism in the Protozoa, since the vast majority of these parasites are quite harmless to their hosts, and lethal parasites, greatly in the minority when compared with harmless forms, must be considered as exceptional and aberrant types of parasites, from a general point of view.

The parasitic Protozoa of lethal properties present a problem which can be best attacked by considering and comparing two cases of closely allied parasites, the one harmless, the other lethal, to their hosts. Very instructive cases of this kind are furnished by trypanosomes (vide infra, p. 285). The common parasite of the rat, Trypanosoma lewisi, is perfectly harmless as a rule to its host, and the infection runs a very definite course. When the parasite is introduced into the blood of a healthy and susceptible rat, it enters at once upon a period of rapid multiplication, which lasts about twelve days. At the end of that time the parasite swarms in the rat's blood, without perceptibly affecting its general health. After about twelve or thirteen days the multiplication of the parasite ceases entirely ; the swarming period lasts generally about a month, and after that the parasites begin steadily to diminish and disappear, until after a variable length of time, usually three to five months, the blood is quite free from them, and the rat, cured from the attack, is now quite immune to the parasite, and cannot be infected by it a second time.

The behaviour and effects of a pathogenic trypanosome, such as $T$. brucii, when introduced into a rat's blood, contrast sharply with that just described. Not only do the trypanosomes begin to multiply at once, but they never cease to do so while the host remains alive. By the fifth or sixth day there are practically more trypanosomes in ihe blood than blood-corpuscles, and the death of the host soon follows when this stage has been reached.

Trypanosoma lewisi is a type of a well-marked group of trypanosomes, which may be conveniently denoted the lewisi-group (Fig. 11). Such are T. cuniculi of the rabbit; T. duttoni of the mouse; $T$. rabinowitschi of the hamster ; $T$. blanchardi of the dormouse; T. microti of Microtus arvalis; and T. elyomis of the lérot (Eliomys quercinus). All these species of trypanosomes are exceedingly similar in their appearance and structure; each species, however, appears to be perfectly specific to its particular species of host. The trypanosome of the rat, for instance, will not flourish in any other host not even in a mouse, under normal circumstances. 
Roudsky suggests that all this group of trypanosomes constitutes in reality a single species ; in any case, it is reasonable to regard them as forms recently evolved from a common ancestor, incipient species which have not advanced beyond the stage of physiological differentiation.

In like manner, $T$. brucii is a type of a group of trypanosomes which may be termed the brucii-group (Fig. 12); other members of it are $T$. gambiense, the parasite of human sleeping sickness; $T$. evansi, causing surra in horses; $T$. equiperdum, of dourine in horses ; and several other species. These forms also are exceedingly similar in appearance and structure, though easily distinguishable from members of the lewisi-group. They aro all of them very lethal, as a rule, to their hosts ; and they differ further from the try-
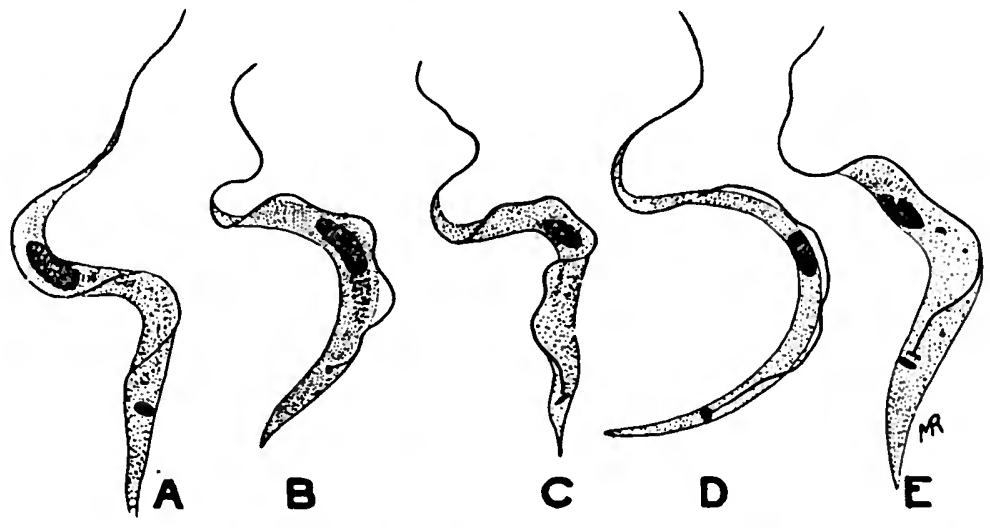

Fro. 11.-Trypanosomes of the lewisi-group. A, T. lewisi (rat); B, T. duttoni (mouse); C, T. cuniculi (rabbit); D, T. microti (Microtus arvalis); E, T. elyomis (Eliomys quercinus); A and C, from preparations ; B, after Thiroux ; D, after Laveran and Pettit; E, after França. All figures magnified 2,000 diameters.

panosomes of the lewisi-group in the fact that a given member of the brucii-group is not specific to a particular host, but can flourish and exert its lethal powers in a great variety of vertebrate hostsa fact which, coupled with their very similar morphology, renders the exact determination of the species of this group very difficult, and often a matter of controversy. All these facts point to the brucii-group being also desoended from a common ancestral form; they may be regarded as incipient species in which the process of evolution has not yet the degree of physiological specialization reached in the lewisi-group. This view receives support from, the fact that a now race or apecies of the brucii-group has been made known this year (1911)-namely, $T$. rhodesiense, a trypanosome pathogenic to human beings which appears to have come into existence as a species very recently. 
A further point of great interest in this connection is that $T$. brucii in Africa appears, from the observations of Bruce, to occur as a natural parasite of wild game, and to be as harmless to these its natural hosts as $T$. lewisi is to rats. The physiological difference between these two species is that $T$. lewisi is perfectly specific to its natural host, whereas $T$ brucii is capable of flourishing in others, with most deadly effects. Hence the pathogenic properties of $T$. brucii would appear to be exerted on hosts to which

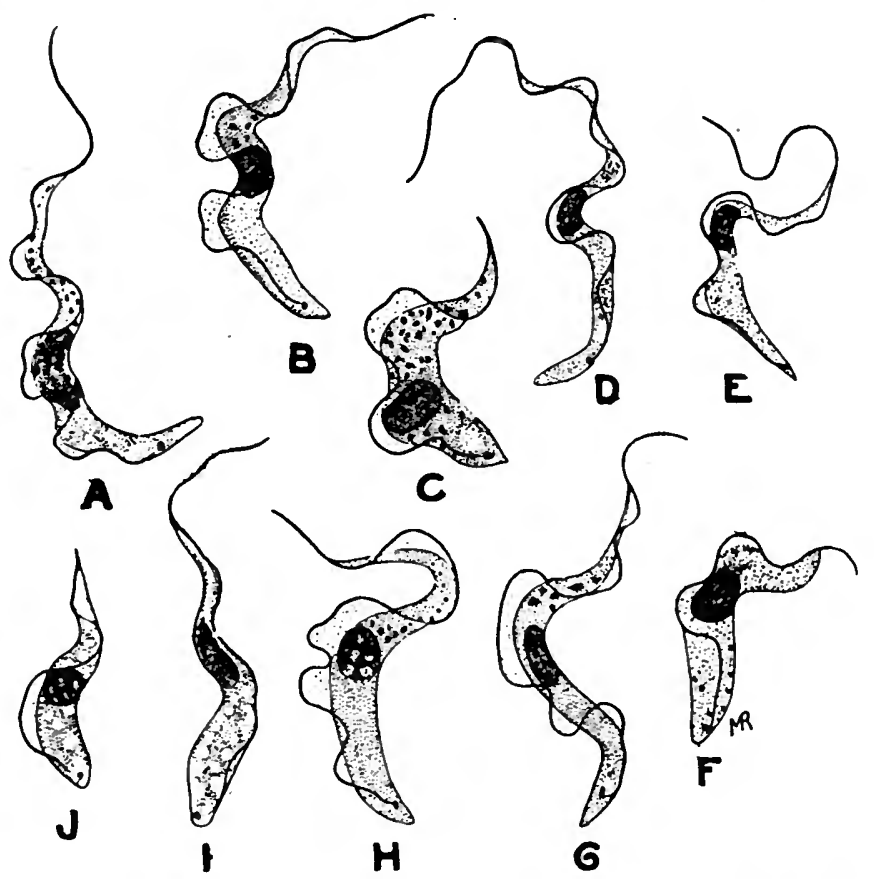

Fra. 12.-Trypanosomes of the brucii-group. A, B, C, T. brucii of "nagana," three forms-slender, intermediate, and stumpy ; D, E, F, T. gambiense of sleeping sicknoss, the three corresponding forms ; G, H, T. evansi of "surra," two forms; I, T. vivax; J, T. nanum. A to C, I, and J, after Bruce, Hamerton, Bateman, and Mackio (411); $G$ and $H$, after Bruce (404); D to F, from preparations. All figures magnified 2,000 diameters.

it is a new parasite, and not on those to which, like $T$. lewisi, it has established harmonic relations in the course of evolution. The pathogenic properties of $T$. brucii, and doubtless of other similar forms, may from this point of view be characterized as a disharmony associated with the first steps in the origin of species.

The problem of the origin of diseases caused by parasites is essentially a problem of the same nature as the origin of species. The first step in the formation of new species is a process of variation in an established species. Similarly, in the process of forma- 
tion of new species of parasites, the first step would be the acquisition by the parasite of the power of living in hosts other than that to which it is specific. How such a variation might arise in Nature is impossible to cenjecture in the present state of knowledge ; but some experiments that have been carried out upon $T$. lewisi show that conditions can modify the apparent fixity of its characters. Roudsky $(22,23)$ found that after prolonged culture on artificial media, and subsequent rapid passages through rats, it was possible to infect mice with $T$. lewisi. Wendelstadt and Fellmer (27) have shown that $T$. lewisi, if inoculated into cold-blooded vertebrates, can persist there for a time, and then becomes virulent to rats.* In both aases it is evident that the normal specific properties of the parasite have been induced to vary by changes in the conditions of life, with the result that they become similar to those characteristic of the pathogenic trypanosomes.

If it be true that a parasite attacking a new host is at first pathogenic to it, but tends in the course of evolution to establish more harmonic relations with the host, the question arises as to how such relations are brought about. There are two organisms concerned, and the problem affects them both. In the case of the host the adaptation to the effects of the parasite may bo both individual and racial, in the latter case to be perhaps largely explained by the elimination of individuals less fitted by their constitution to resist the parasite. In the case of the parasite, also, the problem may be considered from both points of view ; deadly strains of the parasite contribute to their own destruction. Interesting observations bearing on the individual adaptability of strains of Schizotrypanum cruzi have been made by Chagas (425). This parasite, when inoculated into guinea-pigs, was found to kill them in about six days; this is its initial virulence to this host. After repeated passages through guinea-pigs, it was found that the virulence diminished, until guinea-pigs inoculated with strains of attenuated virulence lived as much as six weeks beforo they succumbed to the effects of the parasite. If, when this result had been attained, the parasite was given a singlo passage through a marmoset, it was then found to have regained its primary virulence to guinea-pigs.

The study of the exact mechanism of the physiological relations between parasites and their hosts is the task of the investigations upon immunity and kindred problems which now engross so large a share of the attention of scientific workers, but which cannot bo considered here in detail.

Bibliography.-For references, sec p. 476.

* See also Sleeping Sickness Bulletin, No. 22, p. 412, and No. 24, p. 81. 


\section{CHAPTER III}

\section{THE ORGANIZATION OF THE PBOTOZOA-EXTERNAL FORM AND SKELETAL STRUCTURES}

A UNICELLULAR organism of any kind is a more or less minute mass or corpuscle of the living substance, protoplasm, containing usually other substances, fluid, solid, or even in some rare instances gaseous, in greater or less amount-substances which are either the product of its own vital activity or have been taken up into the body from without. As will be shown in more detail in the next chapter, protoplasm is a substance or complex of substances which, considered in the aggregate, exhibits the physical properties. of a viscid fluid. Some samples of protoplasm may be less, others more fluid, but the essentially fluid nature of the whole mass of protoplasm composing the cell-body is very obvious, as a rule, in the case of Protozoa.

A drop of a fluid substance, when suspended in another fluid with which it is not miscible, tends immediately, under the action of the physical laws of surface-tension, to assume the geometrical form in which the surface is least in proportion to the mass ; that is to say, it tends to become a perfect sphere, except in so far as this tendency may be altared or modified by the contact or pressure of other bodies, or by the operation of other forces or conditions which oppose the action of surface-tension.

The sphere may therefore be regarded as the primary form of the living cell-the form, that is to say, which the organism tends to assume under the influence of physical forces when not checked or inhibited in their operation by other factors. A great many Protozoa exhibit the spherical form in a striking manner, especially those species which float more or less freely in the water, such as the Heliozoa (Fig. 3) and Radiolaria (Fig. 13). But the majority of Protozoa depart more or less widely from the primitive spherical form, for reasons which must be considered in detail.

In the first place, departure from a spherical form may be merely temporary, the result of vital activity producing altered conditions of surface-tension. In order that a drop of fluid may assume a spherical form as the result of surface-tension, its surface must be 
homogeneous-that is to say, of similar nature in all parts; if, however, its surface be heterogeneous, and differs in different parts, local inequalities of surface-tension may be the result, and then a perfectly spherical form cannot be maintained so long as the surface remains heterogeneous. Thus an organism, such as an amoba, in which the protoplasm is quite naked and exposed at the surface of the body, tends always to have a spherical form in the resting state ; but when it enters npon a phase of vital activity, it may assume various forms which can be explained by supposing that the surfacetension is altered at one or more regions of the surface as the result

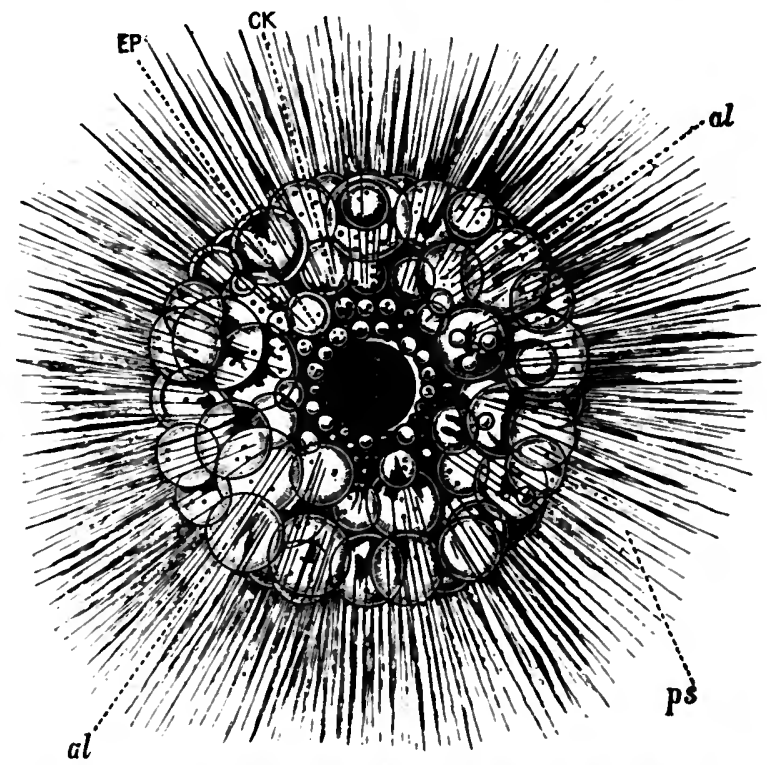

FIa. 13.-Thalassicolla (Thalassophysa) pelagica, Haeckel, an example of a species of floating habit combined with radiate symmetry and spherical body-form. $C K$, Central capsule; $E P$, extracapsular protoplasm; al, vacuoles in the calymma (see p. 251); ps., pseudopodia. The small dots in the calymma represent " yellow cells" (p. 252). After Lankester, magnified 25 diameters.

of local changes in chemical constitution, brought about by the vital activity of the protoplasm (Rhumbler, 34, and p. 200 infra). In consequence, the spherical form characteristic of the resting state undergoes modification in various ways when the organism becomes active. In floating forms the sphere throws out radiating processes, so-called "pseudopodia," in all directions (Figs. 3, 13). In creeping species the body assumes the indefinite and constantly changing form, with pseudopodia extruded in every direction, which is characteristic of the amoba (Fig. 2), and hence commonly termed " amcboid." In all such cascs, when the animal passes into a 
resting, inactive condition, or when the vital activity is temporarily inhibited by some shock or stimulus, such as an electric current suddenly turned on, physical forces reassert their sway, and under the influence of surface-tension the pseudopodia are retracted, and the body rounds itself off and returns to the spherical form.

Apart, however, from temporary and variable departures from the primary and.fundamental spherical form, many unicellular organisms exhibit a constant body-form which is often widely different from the sphere, and which is characteristic of particular species, or for the corresponding stages in the life-history of a given species, and varies only within the narrowest limits, if at all.

The problem of form-production in Protozoa, like all other biological problems, may be considered from two points of view. In the first place, there is the question why a particular species has such and such a form. The answer to this question must be sought in the habits and mode of life of the species and its relation to the environment. In general it may be said that each species possesses, or tends to possess, the body-form best adapted to its particular mode of life, though it is not always easy to trace the correlation of form and habit in special cases. A broad distinction may, be drawn, however, between species which move freely in their environment and those which are fixed and sessile in habit. In freely-moving species, again, a further distinction can be drawn between those which float or swim in the medium, and those which creep on a firm substratum. Free-swimming species tend to the form of an ovoid, more or less elongated, with the longitudinal axis lying in the direction of forward movement (Fig. 14). Creeping forms tend to be more or less flattened, and spread, as it were, upon the substratum, leading in extreme cases to the differentiation of a ventral surface, in contact with the substratum, from a dorsal surface on the opposite side. Sedentary forms tend to be more or less vasiform, often with the point of attachment drawn out into a stalk or peduncle of greater or less length. A frequent peculiarity of the body-form in Protozoa, whether fixed or free, is the tendency to a more or less pronounced spiral twist. Bilateral symmetry, on the other hand, is a comparatively rare phenomenon in these organisms ; examples are found among the Flagellata-e.g., Lamblia intestinalis (Fig. 117).

The second question which arises is, Given a particular specific form, how is the form developed and maintained, on physiological or mechanical principles? To this question the answer must be sought in the structure of the individual, and more especially in the formation and possession of special structural elements, more or less rigid in nature, which determine the form and support the soft body. Such structures may be external to the body, in the 
form of cuticular productions or envelopes of various kinds, or internal, in the form of an axis or framework. Both these types of form-determining or skeletal elements, as they may be termed broadly, may be present together in a given organism.

1. Cuticular and Exoskeletal Structures.-In the Sarcodina generally, and in a few examples of the Mastigophora and Sporozoa, the body-protoplasm is quite naked at the surface, as already stated, and not covered by any cuticle or firm covering. With these exceptions, the bodies of Protozoa are clothed by a firm cortical

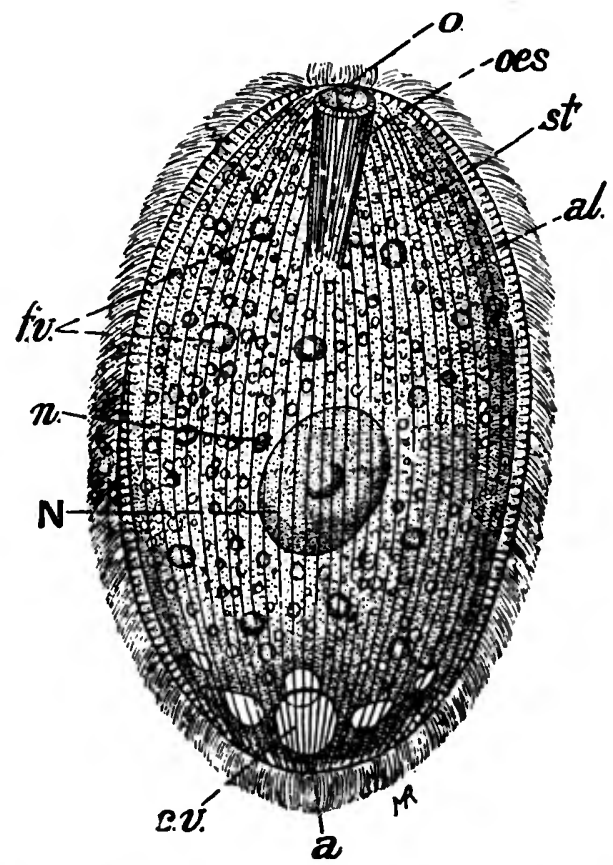

Fia. 14.-Prorodon teres $N$, Macronucleus ; $n_{1}$ micronucleus ; 0 , mouth ; aes., cesophagus surrounded by rod-apparatus (p. 433); f.v., food vacuoles; c.v., contractile vaeuole surrounded by feeding-vacuoles; al., alveolar layer; st, meridional rows of cilia ; $a$., anal pore. After Schewiakoff, magnified 660 diameters.

layer, which is produced either as a differentiation of, or secretion by, the most superficial layer of the protoplasmic body, and which receives various names in different cases.

The very first beginnings of a cortical layer are seen in some species of amœbæ, such as Amoba verrucosa-species in which the protoplasm, extremely viscid and slow-flowing, forms a delicate investing pellicle at the surface. In these cases the pellicle is so thin that it does not hinder the amoboid movement appreciably (Fig. 23). A further advance is seen in some of the Flagellata, 
where a thin cuticle is present which permits changes in shape, caused by the contractility of the enclosed protoplasmic body. Such forms are not amoboid, but exhibit rhythmical changes of form produced by contractions of the superficial body-layer in a manner somewhat recalling peristaltic movement, and are commonly said to be metabolic (Fig. 15) ; and since such movements are characteristic of some species of the genus Euglena, they are sometimes called euglenoid.

In most cases, however, in which a cuticle or firm cortex is present, a definite and characteristic body-form is maintained, subject only to such changes as may result from curvatures of the body, or temporary shortening of its axis in a particular direction, brought about by the contractility of the living body. An envelope of this kind, which may vary in consistence from a thin, flexible cuticle to a rigid inflexible cuirass, or "lorica," inhibits eompletely the natural tendency of the fluid protoplasmic body to round itself off-a tendency, however, which frequently reasserts itself during resting phases of the organism, when the cortex may be softened or absorbed. Hence it is very common to find that the resting phases of Protozoa revert to the primitive spherical form, whatever the shape characteristic of the organism in an active state.

A close-fitting cortex or cuticle which is essentially a part of the body itself must be distinguished clearly from structures built up by the organism externally to the body ta afford shelter or support. Such a structure is termed variously a "shell," " test," or " house." The formation of protective shells, into which the body can be completely retraeted,

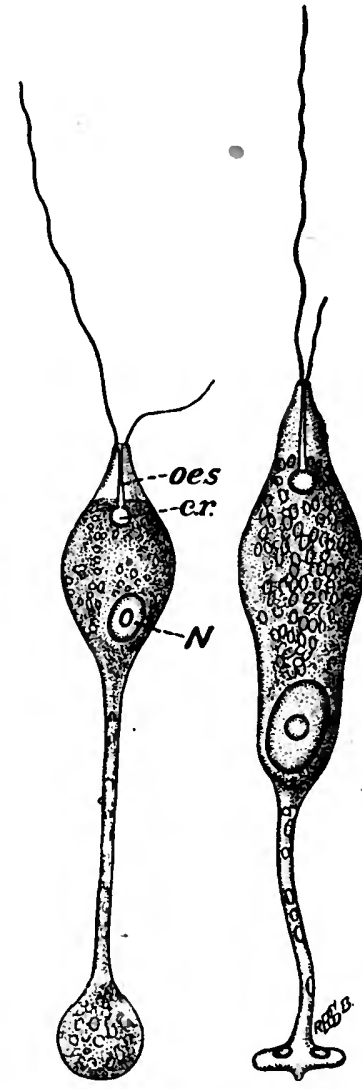

Fig. 15.-Astasia tenax, two individuals showing the changes of form duo to metabolic movemont. œes., Esophagus ; c.r., reservoir of the contractile vacuole; $N .$, nuclcus. After Stein. and from which it can emerge to a greater or less extent, is of extremely common occurrence amongst the naked-bodic d Sarcodina. The forms of these shells, their structure and mode of formation, exhibit an almost infinite variety, and can oilly be described here in a quite general manner. 
As regards material, the shells may bc composed of elements secreted by the organism (" autophya," Haeckel), as in Hyalosphenia (Fig. 16, B), or of foreign particles taken up by the animal from its surroundings ("xenophya"), as in Diffugia (Fig. 16, A). Skeletal elements secreted by the organism may be of organio or inorganic nature. In the former case they are probably chitinous in most cases, or composed of a substance allied to chitin; in the latter they are either calcareous or siliceous. A good example of the formation of a shell is seen in Euglypha (Fig. 59), where the chitinous plates composing it are formed first of all in the interior of the protoplasmic body, and pass to the surface to build up the shell. When the shell is built up of foreign particles, the material employed may vary greatly, and consists generally of particles of sand, grit, etc.,

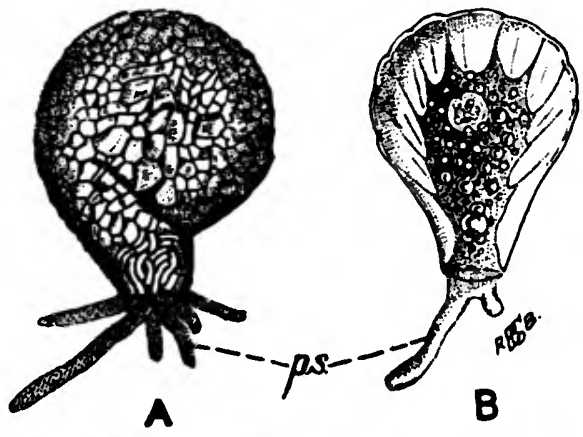

Fro. 16.-Examples of shells or houses formed by Protozon. A, Diffugia spiralis, which forms a house built up of foreign bodies; B, Hyalosphenia cuneata, in which the house is built up of plates secreted by the animal itself (compare also Euglypha, Fig. 59). Both these species belong to the order Amœbæa ; the pseudopodia (ps.) are seen stresming out of the mouth of the shell. After Leidy ; A magnified 250, B 500 dismeters.

taken up at hazard from the environment. Such shells are described technically as "arenaceous." In the case of Difflugia, Verworn (36) was able to cause it to build up its test of various materials, such as particles of coloured glass or other substances, when these were supplied to it exclusively. Many species of Foraminifera, however, form their tests exclusively of particular materials under natural conditions. Thus, in the genus Haliphysema (Fig. 17) the test is formed of sponge-spicules; in Technitella thompsoni the calcareous plates of echinoderms are selected; and other instances could be cited in which the organism selects habitually for its sholl certain materials from a varied environment in which the particular materials required may be far from common in occurrence relatively to other particles apparently equally suitable (see especially Heron-Allen and Earland). Verworn (36) found that 
in the case of Difflugio the foreign particles used are taken up by the pseudopodia during the process of being retracted; the surface of the pseudopodium then becomes wrinkled, and particles of debris are caught in these wrinkles, and so drawn into the interior of the protoplasmic body, in which they are stored up in the fundus of the shell, like the plates in Euglypha, and are utilized in the growth of the shell, or in repairing damages to it, or in building a new shell when the animal reproduces itself by division.

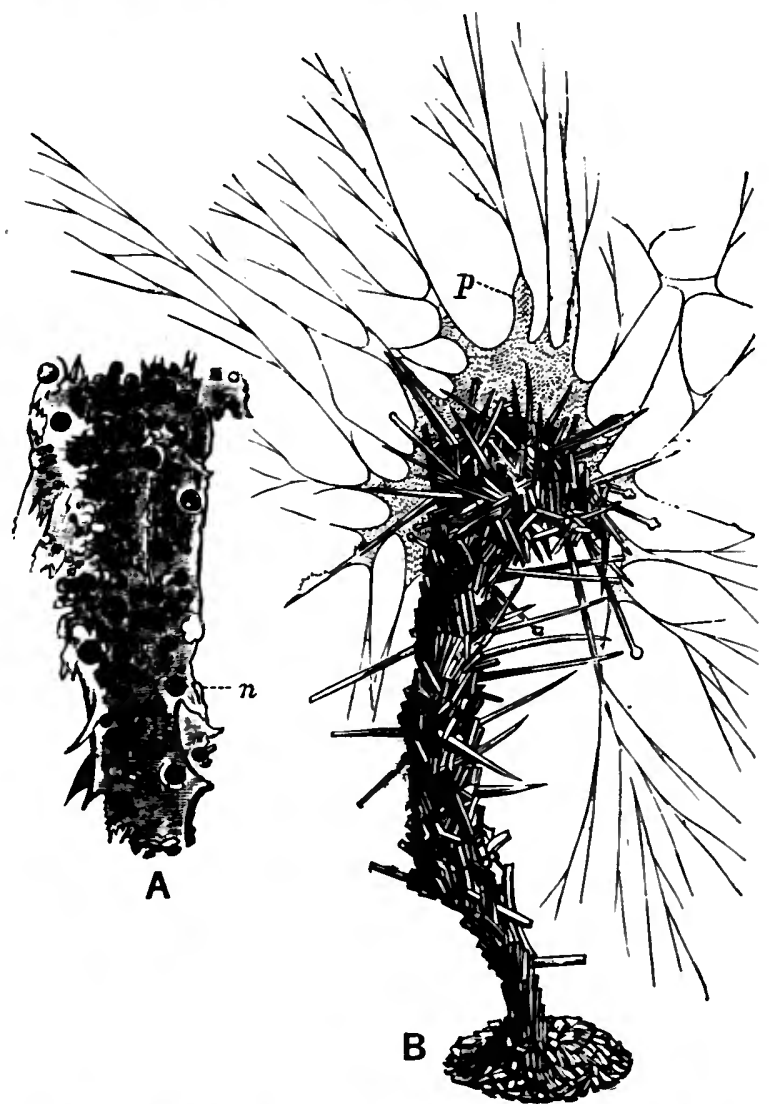

Fro. 17.-Haliphysema tumanowiczii, a foraminifer which builds up its house out of sponge-spicules. A, part of the protoplasm stained to show the nuclei $(n$.$) ;$ B, living specimen with expanded pseudopodia ( $p$.). After Lankester (11).

The simplest architectural type of shell or test is a simple spherical or oval capsule, usually with a large aperture at one pole through which the protoplasm is able to creep out in order to capture food or perform the function of locomotion (Fig. 16). The wall of the test may be imperforate, or may have fine pores through which also the protoplasm can stream out. With oontinued growth of the organism, the original shell may become too small for its requirements. Then the organism may reproduce itself by fission. 
and the daughter-individual forms a new shell for itself. In many cases the shell formed by the daughter is larger than that of the parent; for instance, in Centropyxis aculeata and other species, in which the young individuals multiply by fission, and each time they do so, the new shell formed is largor than the old one, until the full size of the adult individual is reached (Schaudinn, 131), after which point the new shell formed after the process of fission is of the same size in both the parent and the daughter-individual. In such cases the shell is always a single chamber, and is described technically as " monothalamous."

In other cases, however, the organism does not multiply by fission whon it has outgrown its first shell, but forms a new shell of larger size which is in continuity with its first shell; the protoplasmic body now occupies both the chambers of the shell formed in this way. With further growth more chambers are formed, giving rise to a complex "polythalamous" shell composed of many chambers all occupied by the protoplasmic body (p. 232, infra). For a detailed study of the developmental mechanics of shell-formation, see Rhumbler (35).

2. Internal Skelelal Structures.-In many cases in which the protoplasmic body is naked at the surface, or bears only an extremely thin cuticle, a definite body-form may be maintained by means of internal supporting fibrils or other similar structures (Koltzoff, 30,31 ). In some cases such structures may be of temporary nature. A beautiful example of this is seen in the delicate organic axes formed in the pseudopodia of Heliozoa (Fig. 22), in the form of slender needle-like rods secreted by the protoplasm to stiffen the pseudopodia, and absorbed again when the pseudopodia are retracted. In other cases, supporting structures of organic nature may be permanent constituents of the protoplasmic body; such are the axial rods, or " axostyles," found in many flagellates, such as Trichomonas (Fig. 5, ax.), Lophomonas (Fig. 45), etc., slender flexible rods of organic substance which form a supporting axis for the body. Previous to division the axostyle is absorbed, and new axostyles are formed in the daughter-individuals. The axostyles are stated to arise from a centrodesmose (p. 103, infra) formed in the process of division of the blepharoplast (Dobell, 236) or of the centriole of the nucleus (Hartmann and Chagas, 62); the centrodesmose persists after division is complete, and its two halves become the axostyles of the two daughter-individuals. In Trichomonas eberthi, however, Martin and Robertson (348) find that the axostyles arise after division quite independently of the centrodesmoses or other nuclear structures. In Octomitus (Fig. 116) two axostyles are present.

From supporting structures of organic nature, such as the axostyles or the organic axes of the pseudopodia mentioned above, it is not difficult to derive the more rigid and permanent elements known as "spicules," in which the organic basis becomes indurated by depasits of dforganic mineral substance. In some cases spicules may perhaps consist entirely of mineral substance deposited directly within the living substance without any organic basis. In 
either case the spicules grow by accretion-that is to say, by deposition of fresh layers of inorganic substance upon that already laid down-and if such accretion takes place at one end of a rod-shaped spicule, it may have the result that the opposite extremity of the spicule is pushed outwards by the continued growth, with the result that the oldest portion of the spicule projects freely far beyond the limits of the body.

As regards material, spicules are usually either calcareous or siliceous-in the first case generally carbonate of lime, in the second

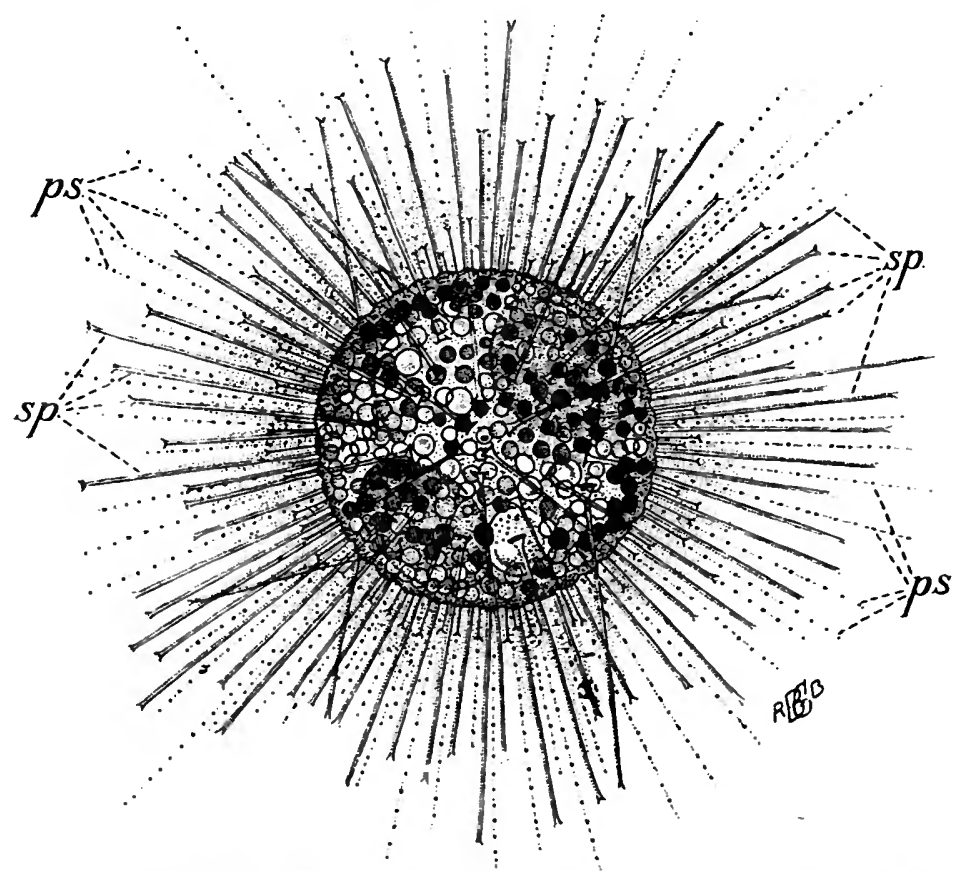

Fig. 18.-Acanthocystis chatophora, a Heliozoon with a skeleton of slender radiating siliceous spicules, each forked at the distal end. In the interior of the body are seen numerous symbiotic algæ (dark) and non-contractile vacuoles (clear); one vacuole of larger size is seen, probably the contractile vacuole. $8 p$., $8 p$., Spioules ; ps., ps., pseudopodia. After Leidy, magnified 750 diameters.

case amorphous silica In the family Acanthometrida among the Radiolaria the spicules are formed of a substance which was thought to be of organic nature, and was named " acanthin," but which has been found to consist of strontium sulphate.

As regards their form and relation to the body, the spicules in the simplest cases are rod-shaped or needle-like elements disposed radially or tangentially. A simple type of spicular skeleton is seen in Acanthocystis (Fig. 18), in which elongated siliceous rods, fre- 


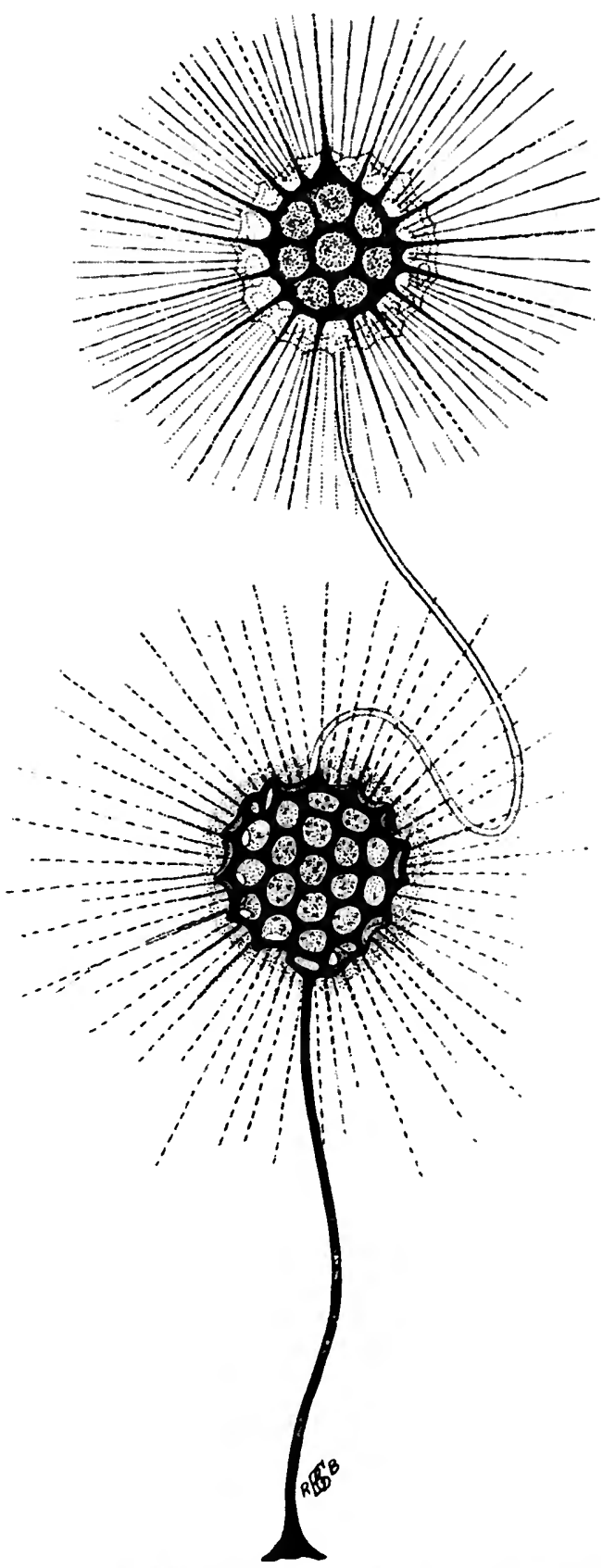

Fic. 19.-Clathrulina elegans, a Hcliozoon with a lattice-liko skcleton, attachod by a stalk. Two individuals are seen, the younger with its stalk attached to tho head of the older ; in the younger the lattice-work is still very delicate. Both individuals are sending out numemus radiating pseudopodia, very delicate and s!ender. After Leidy, magnified 750 diametcrs. 
quently branched at their distal ends, are arranged like radii of the spherical body, projecting freely for some distance from the surface. In other cases the spicules may be disposed tangentially to the body, as in the family Collides amongst the Radiolaria, and in other forms belonging to this order. From a simple type of skeleton composed of separate spicules, more complicated types of skeletons are derived by fusion of the spicules to form a connected framework. The commonest, type of this is a fusion of tangentially-disposed spicules to form a lattice-work; an example of this is seen in Clathrulina (Fig. 19), in which a lattice-like skeleton is formed at the surface of the body, standing off from it like a shell. Skeletons of this type are especially characteristic of the Radiolaria, a group in which the architecture of the skeleton may reach a very high degree of complication and exhibits endless variety. The latticelike framework, made up of tangentially-arranged spicules united together, may be further strengthened by radially-disposed beams. As the animal grows, it may outgrow the framework first laid down, and another lattice-work is formed concentric with the first, and connected with it by radial beams; later on a third and a fourth such framework is formed, as the organism continues to grow in size. Skeletons formed in this way may be " homaxon "- -that is to say, built up on the axes of a sphere; or " monaxon," with ono principal axis ; or may follow various plans of symmetry, or may be asymmetrical (p. 250, infra).

Bibliography.-For references, see p. 477. 


\section{CHAPTER IV}

\section{THE ORGANIZATION OF THE PROTOZOA (Continued)- THE PROTOPLABMIC BODY}

THE substance composing the bodies of Protozoa was termed originally sarcode by Dujardin; but after it had been shown to be identical in nature with the living substance of the cells of animals and plants, the same term was employed universally for both, and the word protoplasm, coined by von Mohl to designate the living substance of plant-cells, supplanted the older term sarcode, which has now quite dropped out of current use.

It would be impossible within the limits of the present work to discuss in detail the various theories that have been put forward with regard to the nature and constitution of protoplasm; they can only be summarized in brief outline here. Protoplasm, when seen under the microscope with powers of moderate strength, presents itself as a viscid, semi-fluid substance, sometimes clear and hyaline in special regions, but always showing, throughout at least the greater part of its substance, numerous granulations, which vary greatly in size, from relatively coarse grains to those of the minutest size visible with the power of the microscope used. The most important of these granulations are the so-called "chromatingrains," which are discussed fully in Chapter VI. ; in this chapter only non-chromatinic granules are dealt with. The coarser protoplasmic grains may be present in greater or less quantity, or may be entirely absent; they are to be regarded for the most part as so-called inetaplastic bodies-that is to say, as stages in, or byproducts of, the upward or downward metabolism of the organism. On the other hand, the minute, ultimate granules, or " microsomes," are never absent, except over limited areas, in any sample of protoplasm. It is on the constant presence of granules that the so-called granular theory of protoplasm, especially connected with the name of Altmann, has been founded. On this view, each minute granule is regarded as an elementary organism, or "bioblast," capable in itself of all vital functions, and equivalent to a single free-living bacterium, just as a single cell of a Metazoan body may be compared with a single Protozoan organism. Protoplasm, on this view, is re- 
garded as a colony of bioblasts, imbedded in a fluid matrix, comparable in a'general way to a zooglœa-colony of bacteria.

A special and important class of motaplastic granules are the so-called "deutoplasmic" bodies, consisting of reserve food-materials stored up in the protoplasmic substance. Examples of such are the yolk-granules of ova, the paraglycogen-grains of gregarines, the plastinoid bodies of coccidia, starch-grains in holophytic forms, etc.

Amongst the granulations of the protoplasm, special mention must be made of the bodies known generally as chondriosomes and mitochondria, but also by a variety of other names (cytomicrosomes, bioblasts, spherules or sphero. plasts, and, collectively, ergastoplasm). The chondriosomes aro not to be classed with the temporary, metaplastic inclusions, but are permanent elcments of the cell-protoplasm. The chondriosomes of Protozoa have recontly been the subject of detailed study by Fauré-Fremiet (38.5). In the living condition they are small transparent bodies, feebly refractile, and of a pale grey tint. In shape they are generally spherical, and vary from $0.5 \mu$ to $1.5 \mu$ in diameter. In some cases the chondriosome appears homogeneous in structure ; in others it presents the appearance of a vacuole with fluid contents and a denser peripheral layer. In contact with water or with weak alkalis they swell up immediately. When the nucleus (in Infusoria the micronucleus) divides, the chondriosomes alse divide simultaneously, and the daughter-chondriosomes are sorted out between the two daughter-cells; they have, however, no direct relation with the nucloar apparatus. In the process of division each chondriosome becomes first rod-like, then dumb-bellshaped, and is finally constricted directly into two halves.

A purely chemical definition of the chondriosomes, according to FaurćFremict, cannot be given. They exhibit the reactions of a fatty acid, and can be considered as combinations of fatty acids or of phosphates of albumin. The physiological function of the chondriosomes is not clear, but FauréFremiet considers that they "play an important part in the life and evolution of the sexual cell," in Protozoa or Metazoa, and are active in the elobora. tion of deutoplasmic substances of fatty nature, into which they may be transformed directly.

It has been shown, however, that the minute granules of protoplasm do not lie isolated from one another, suspended freely in a matrix, but are seen in the microscopic image to be connected with one another by fine lines or darker streaks, the whole forming a delicate network, at the nodes of which the granules are lodged. In some eases the granule itself is perhaps only an optical effect produced by a node of the network. On these appearances has been founded the so-called reticular theory of protoplasm, connected especially with the names of Heitzmann, Schäfer, and others. On this view protoplasm has been regarded as composed of an exceedingly fine reticulum, a network or feltwork ramifying in all planes, bearing the granulations at its nodal points, and bathed throughout by a fluid, more or less watery sap, or enchylema. The fibrillar theory of Flemming may be regarded as a modification of the reticular theory.

Against the reticular theory of protoplasm, it may be urged that it leads to physical diffieulties, in view of the generally fluid. nature of protoplasm. For the reticulum must itself be either of a fluid or a solid nature; if fluid, it presents the condition of one fluid 
suspended in the form of a network in another fluid with which it does not mix -a condition which could not exist for more than an instant of time, since the fluid reticulum must break up immediately into minute droplets. If, on the other hand, the reticulum is of rigid consistence, the protoplasm as a whole could not be fluid, any more than a sponge soaked in water could behave as a fluid mass in the aggregate. The difficulty can, however, be overcome by supposing the apparent reticulum to be the optical expression, not of a fine network of fibtils, but of delicate lamellæ limiting minute closed chambers, or alveoli. Then the fine line seen with the microscope joining any two adjacent nodal points would be the optical section of the wall or lamella separating two contiguous alveoli, and protoplasm as a. whole would possess a honeycombed structure comparable to that of a fine foam or lather-the fluid lamellæ of the foam represented by the apparent reticulum of the protoplasm, and the air-contents of the individual bubbles represented by the enchylema. Or, to express the state of things in a different manner, protoplasm could be regarded as an emalsion of very fine structure, composed of two fluids not miscible with one another-namely, the more fluid enchylema, which is suspended in the form of minute droplets in the more viscid substance forming the alveolar framework. This is the so-called alveolar theory, especially connected with the name of Bütschli ; by this conception of protoplasmic structure, not only are the necessary physical conditions satisfied, but an explanation is given for many peculiarities of protoplasmic bodies, such as the radiate arrangement of the meshes of the reticulum commonly observed either at the surface of the body or around solid or fluid bodies contained in the protoplasm, and so forth.

The various theories that have been mentioned all assume tacitly that protoplasm is monomorphic - that is to say, that it possesses one fundamental type of minute structure. Fischer, on the other hand, seeks to unite all the different theories by supposing that protoplasm is a polymorphic substance-that is to say, one that may exhibit a diversity of structure at different times and under different conditions, as the result of changes produced by its inherent vital activity. Thus, he supposes that a given mass of protoplasm may be at one time homogeneous, and at another time granular, reticular, fibrillar, or alveolar, as the result of a process of "vital precipitation," and that by reabsorption of the structural elements it may return to a homogeneous condition. Fauré-Fremiet (38 and $38.5)$ also regards protoplasm as a homogeneous fluid, which is pre. cipitated by reagents, and which normally contains, in suspension, a certain number of granulations, some temporary, others permanent in nature ; compare also Degen (154). 
Those investigators of the Protozoa who have expressed an opinion on the subject have been for the most part in favour of the alveolar theory of protoplasm, since it was first propounded by its author, Bütschli (see especially Rhumbler). Protozoa as a rule are very favourable objects in which to study the foam-like structure of the protoplasm (compare Schaudinn, 130, p. 188). But whatever view be held as to the ultimate structure of protoplasm, its essentially fluid nature is very apparent in these organisms, and is a point upon which it is very important to be clear. The fluid condition of the living substance is manifested directly by the streaming movements to be observed in it, and indirectly by a number of phenomena, such as the tendency, already mentioned, of the body to round itself off when at rest, and the tendency of all vacuoles to assume a spherical form. A vacuole is a drop of fluid suspended in the protoplasmic body, and may be regarded as formed by the bursting and running together of many minute alveoli, just as a large bubble in a foam may arise by the union of many smaller ones; or by the gradual enlargement of a single alveolus by diffusion of fluid into it from neighbouring alveoli, until it attains proportions relatively gigantic. Vacuoles assume uniformly spherical contours, except when they are deformed by mutual pressure from crowding together or from other causes. In some cases the protoplasm may be so full of coarse vacuoles that it exhibits an obvious frothy structure, which must by no means be confounded with the ultimate alveolar structure of the protoplasm, a structure which is exceedingly delicate, only to be observed with high powers of the microscope and with careful attention to all details of microscopic technique. Examples of vacuolated bodies are seen especially in Heliozoa-e.g., Actinosphorium (Fig. 3).

The statement, however, that protoplasm generally is of fluid nature admits of its exhibiting many degrees of fluidity, and some samples of protoplasm are far more viscid than others. This is true both of different species of organisms, of the same species at different phases of its development, and of different parts of the same organism. In some cases portions of the protoplasm may be stiffened to a degree that perhaps oversteps the ill-defined boundary between the liquid and solid states of matter. In a great many Protozoa, perhaps the majority of them, the protoplasm of the body is divisible, more or less distinctly, into two regionsnamely :

1. An external or cortical zone, termed ectoplasm or ectosarc ; in appearance and consistence typically clear, hyaline, more refringent, finely granular or without visible granulations, and of more viscid nature ; in function protective, kinetic, excretory, and sensory.

2. An internal or medullary region, the endoplasm or endosarc; 
opaque, less refringent and coarsely granular ; the seat of trophic and reproductive functions.

These two zones of the protoplasmic body are, in the more primitive forms, differentiations of the protoplasm more or less temporary and transient in nature. For instance, in an amœba which is in active movement, fluid endoplasm is constantly flowing along the axes of the pseudopodia towards their tips, where it comes into contact with the surrounding medium, the water or other fluid in which the amœba lives. Under the influence of the medium the endoplasm is converted into ectoplasm, becomes of stiffer, less fluid consistence, and loses its coarse granulations. At the samc time, at the hinder end of the amoba, ectoplasm is continually passing into the interior of the body, where it becomes liquefied and granular in structure, and is converted into endoplasm (Rhumbler 34).

In Protozoa, however, which do not exhibit amœboid movement, the ectoplasm and endoplasm may be two independent layers, well defined and perfectly separate the one from the other. The ectoplasm is the seat of those functions which are connected with the relation of the organism to the outer world, to the environment in which it lives; the endoplasm, on the other hand, is concerned specially with the internal affairs, so to speak, of the protoplasmic body. In the following two chapters the various organs of the Protozoa will be considered under the headings of the layer from which they are formed, and according to the functions they perform

Bibliography.-For references, see p. 477. 


\section{CHAPTER V}

\section{THE ORGANIZATION OF THE PROTOZOA (Continued)- DIFFERENTLATIONS OF THE ECTOPLASM AND ENDOPLASM}

\section{A. Ectoplasmic Organs.}

THE various structures and organs produced from the ectoplasm are best classified by the functions they subserve, under the headings of protective, kinetic and locomotor, excretory, and sensory mechanisms.

1. The protective function of the ectoplasm is often seen in organisms in which no cuticle or envelope is present. It has been observed, for instance, that the species of Myxosporidia that inhabit the gall-bladders or urinary bladders of their hosts resist the effects of the medium in which they live so long as their ectoplasm is intact, but succumb if it be injured.

In most Protozoa other than those belonging to the class Sarcodina, however, a special protective envelope or cortex is present at the surface of the body, and such forms are commonly said to be corticate. A cuticle may be formed in various ways, distinguished by the use of different terms. It may represent the entire ectoplasm, modified in its entirety to form an envelope, as in the periplast of the Flagellata ; it may represent a transformation or modification of only the most superficial layer of the ectoplasm, as in the pellicle of the Infusoria and of some amœbæ-for instance, Amoeba verrucosa, the epicyte of the gregarines, etc. ; or it may arise as a secreted layer deposited at the surface of the ectoplasm, and not derived from a modification of the substance of. the ectoplasm itself, in which case it is termed a " cell-membrane."

Whatever its mode of origin, the cuticle may be developed to a very variable degree, from the thinnest possible membrane, sometimes very difficult to discover, to a thick and tough investment which may be termed a "cuirass" or "lorica" ("Panzer "), when it is formed by thickening of a pellicle ; or a " house" or "shell," when it is a greatly thickened cell-membrane standing off from the body. In many cases the cuticle undergoes local thickenings to form spikes or hooks, which may serve as organs of attachment, as in the epimerite of gregarines (Fig. 142). 
In addition to the passive protection afforded by a cuticle, organs of active defence may be present in the ectoplasm in the form of bodies known as trichocysts, found commonly in many ciliate Infusoria (p. 447, Fig. 187) ; they are little oval or spindle-shaped bodies which on suitable stimulation are converted explosively into a stiff thread which is shot out from the surface of the body. (For the nematocyst-like organs known as "polar capsules," in Myxosporidia and allied organisms, see p. 399, infra.)

2. The ectoplasm is shown to be the seat of movement both by the fact that motile organs arise from it and by the frequent presence in it of special contractile mechanisms. The motile organs which are found in the Protozoa are pseudopodia, flagella, cilia with their various modifications, and undulating membranes; any of these structures may subserve the function of food capture in addition to, or instead of, that of locomotion. These organs will now be described in order, after which contractile mechanisms will be dealt with.

(1) Pseudopodia are organs of temporary nature, extruded from the protoplasm when required, and retracted when no longer needed. They can be formed, probably, in all cases in which the body protoplasm is naked, or limited only by a cuticle not of sufficient thickness to inhibit the movements of the underlying protoplasm. They arise simply as an eruption of the protoplasm at some point at the surface of the body, forming an outgrowth or process which varies greatly in different cases as regards size, lcngth, width, composition, and activity.

Pseudopodia always arise in the first instance from the ectoplasm, and may consist throughout of this layer alone, in which case they are relatively stiffer and more rigid; or a core of endoplasm may flow into the pseudopodium when it has grown to a certain length, in which case the pseudopodium is more fluid and flexible. The formation of a pseudopodium is best studied in a common amoba, such as Amoeba proteus (Fig. 2) or A. limax (Fig. 20); it is then seen to arise as a protrusion of the ectoplasm, forming a shallow prominence at the surface of the body. The prominence continues to grow out from the body, and is at first hyaline, transparent, and free from granulations, since it consists of ectoplasm alone. In some cases the pseudopodium may grow to a relatively very large size, and still consist of clear ectoplasm alone, as in Entamoba histolytica (Fig. 90), a form rather exceptional in this respect; more usually, so soon as the budding pseudopodium has reached a certain not very great size, a core of granular endoplasm flows into it and forms the axial part of the pseudopodium. It is then easier to study the formation of the pseudopodium, since the granules in the endoplasm permit the characteristic flowing movements and currents to 
be followed. In the growing pseudopodium a strong current can be observed flowing down the axis to the tip, and there spreading out and breaking up into weaker currents which turn round and flow backwards along the surface of the psendopodium. In amobæ with a very viscid surface layer the back-currents are very feeble, ceasing a short way from the tip of the pseudopodium, and often scarcely discernible, or even absent altogether; in species with a fluid ectoplasm, however, the back - currents are distinctly seen, and muy even pass back and bend round again to join the forward axial current, as described by Rhumbler (34) in Amoba blatto.

While the extrusion of the pseudopodium is an active process, the retraction requires nothing but the action of purely physical forces of surface-tension to explain it. The protoplasm then flows back into the body of the animal, and may present some characteristic appearances in doing so. If one surface is in contact with the substratum on which the animal is creeping, the adhesion of the pseudopodium often causes the tip to be drawn out into slender processes like spikes or hairs. At the same time the surface of the

Fra. 20.-Diagram to show the protoplasmic currents in a limaxamcoba which is moving forward in the direction indicated by the large arrow on the left. The smaller arrows indicate the direction, and their length the intensity, of the currents in different parts of the body. A forwardly-directed "fountain current "starts from near the hinder end, and passes along the axis of the body to the extremity anterior in movement; there it turns ontwards and passes back along the sides of the body, diminishing rapidly in intensity, and finally dying out in the regions where the two dots are placed. After Rhumbler (34).

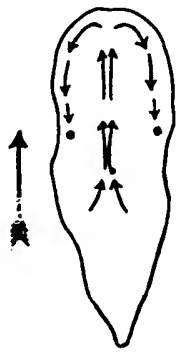

pseudopodium may present a wrinkled appearance, as the viscid ectoplasm shrinks in consequence of the rapid withdrawal of the fluid endoplasm.

The pseudopodia of different species of organisms, or even of the same species at different periods of the life-cycle, vary greatly in form, appearance, and structural characters, and the more important variations require a special terminology. In the first place, the pseudopodia may be broad and thick relatively to their length, as in Amaba proteus (Fig. 2) ; they are then termed "lobose" ("Jobopodia"), and usually have a core of endoplasm. A typical lobose pseudopodium is, in fact, nothing more than an outgrowth of the body-protoplasm as a whole. In the most extreme cases of this type, the whole body flows forward in one direction, forming, as it were, a single pseudopodium. Such a mode of progression is characteristic of Amoeba limax (Fig. 20) and other similar forms, in which the body glides forward like a slug as the animal creeps over substratum ; the end which is anterior in movement is rounded, 
while the posterior end commonly becomes drawn out into processes similar to those seen in a pseudopodium in process of retraction. In other forms, such as $A$. proteus (Fig. 2), the pseudopodia are sent out on all sides and balance each other, in which case there is very little translation of the body as a whole, and the pseudopodia serve chiefly for food-capture. If, however, the outflow of the pseudopodia is strongest on one side of the body, the organism moves in that direction as a whole, and the larger, more strongly developed pseudopodia counteract and overcome the pull exerted by those that are weaker. It will be readily understood, therefore, that the most rapid powers of progression are possessed by the slug-like amœbæ, in which a single pseudopodium drags the whole body along without opposition from others.

Rhumbler (34) has drawn attention to the existence of two modes of progression exerted by amœbæ of the lobose type. In the more fluid species which creep upon a substratum to which they adhere more or less firmly, like Amoba proteus, the animal progresses by a flowing movement, such as has been described; this is the commonest type of amoboid locomotion. On the other hand, in species of the type of $A$. verrucosa and $A$. terricola the very slightly fluid body is limited by a thin pellicle, and does not adhere to the substratum; then progression is effected by "rolling" movement. The animal throws out a number of pseudopodia on one side, which cause it ultimately to overbalance and roll over to that side ; by continued repetition of this procedure, a slow progression in a particular direction is effected. At other times, however, $A$. verrucosa may flow along like other amœba.

Contrasting with the lobose pseudopodia are the slender, threadlike, so-called "filose" pseudopodia, formed entirely of ectoplasm. Pseudopodia of this type can effect a slow creeping movement, but are not very effective for locomotion, and serve for food-capture principally, or even entirely, as in the radiate floating forms (Heliozoa and Radiolaria) ; food is entangled by them and drawn into the body. The filose pseudopodia may radiate from the body in all directions, remaining separate from one another, or they may anastomose to form networks, and are then termed "reticulose." Pseudopodia of the reticulose type are specially characteristic of the Foraminifera (Fig. 21). Radiatn pseudopodia which du not form anastomoses, on the other" hand, characterize the groups of the Heliozoa and Radiolaria, organisms of floating habit. As noted above, pseudopodia of the radiate type are generally supported by an axial rod, a secreted structure of firm, elastic nature, and are hence known as axopodia. The actual rod reaches some way into the endoplasm, often to the centre of the body, as in Acanthocystis (Fig. 18), Wagnerella (Fig. 48), etc.; it 


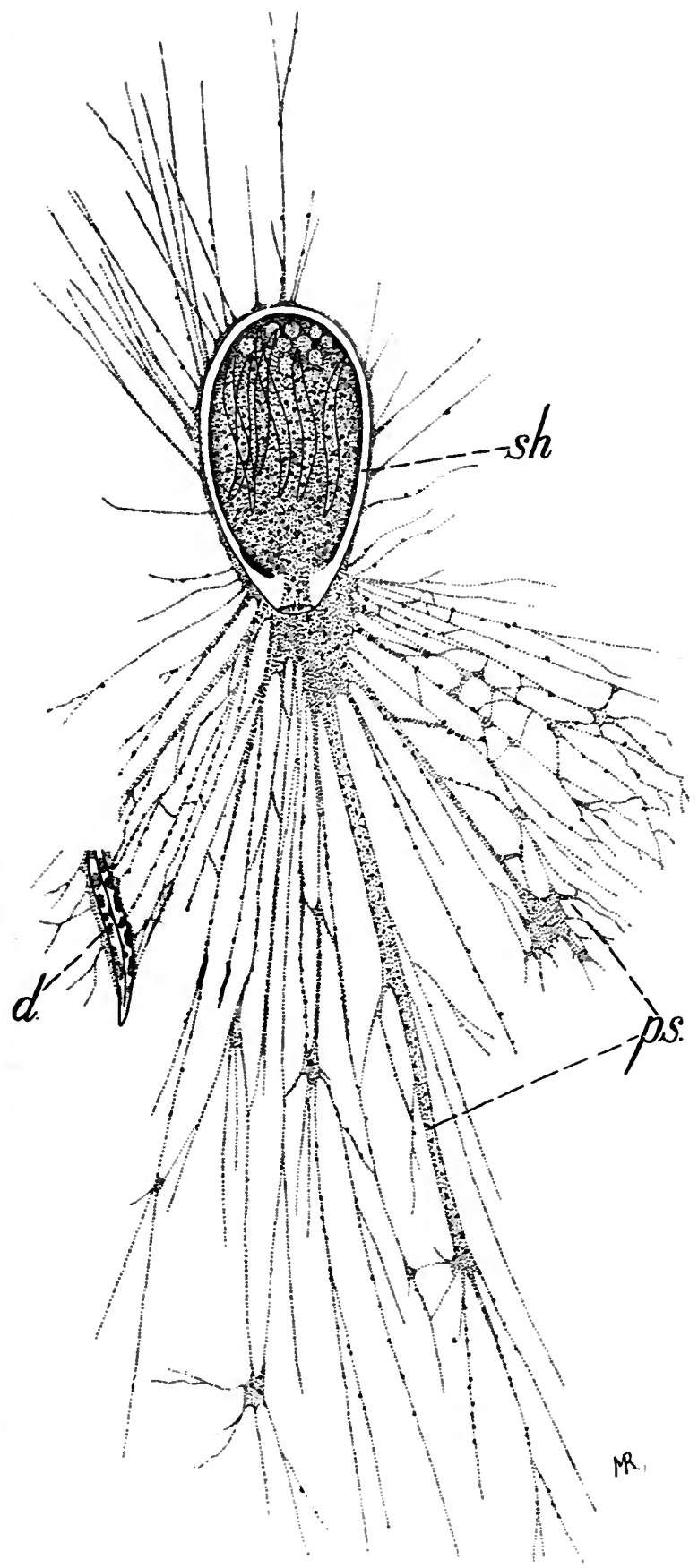

Fra. 21.-Gromia oviformis, M. Schultze (=G. ovoidea, Rhumbler), living specimen with outstretched pseudopodial network (ps.), in which a diatom (d.), Navicula sp., is entangled and will be drawn into the shell (sh.). Other diatoms are seen inside the shell, and at its fundus several nuclei aro seen asclear spherical bodies in the protoplasm. The pseudopodial network is drawn at a magnification of about 200 lincar, but for want of space is represented extending over about onethird of the area over which it commonly spreads. A part of the pseudopodial network is reflected back over the shell, and streams out backwards from the pole opposite to the shell-mouth. After M. Schultze. 
is probably of endoplasmic origin, and is pushed out from it in a centrifugal direction. As it grows out, the ectoplasm forms a sheath over it, and extends usually some way beyond it. When the pseudopodium is retracted, the axial rod is liquefied and absorbed by the protoplasm.

Food-capture is effected by the pseudopodia in various ways (see p. 189). In forms with lobose pseudopodia they flow round the body

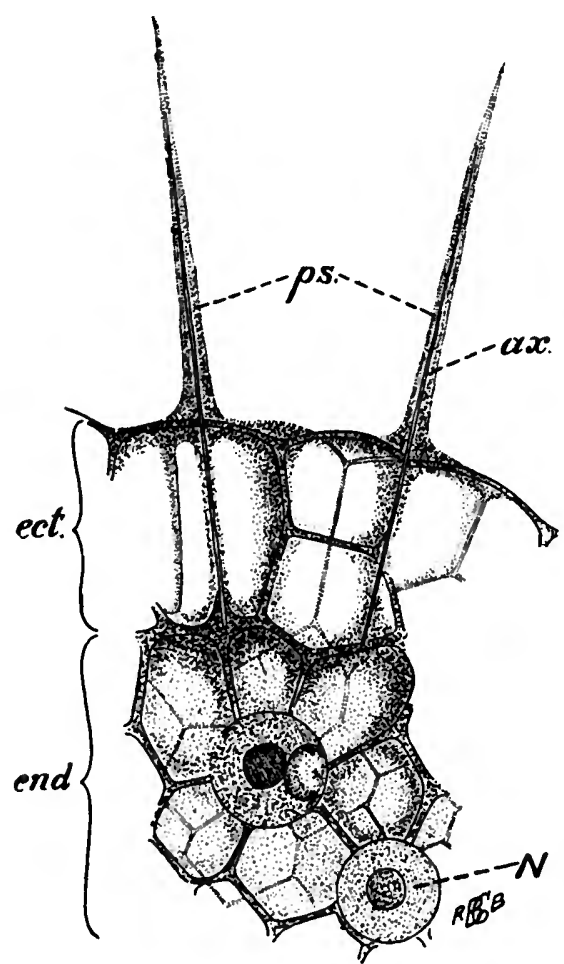

FIG. 22.-Portion of an Actinaspharium, magnified about 660 linear. ect., Ectoplasm with larger vacuoles; end., endoplasm with smaller vacuoles; $N$., nucleus ; $p s .$, pseudopodia ; ax., delicate axial rod in the pseudopodia. After Leidy.

gested, but draw it into their interior, as if by suction. In this manner $A$. verrucosa absorbs and devours filamentous algæ (Fig. 23), which are "imported" into the interior of the body and there coiled up and digested. Rhumbler has shown that this process can be imitated by drops of fluid ; for instance, a drop of chloroform in water will draw in a thread of shellac and coil it up in its interior in a manner similar to the ingestion of an algal filament by an amœba. on all sides, and finally imprisoning the prey in a closed chamber of the living substance, together with a drop of water which forms the food - vacuole (Fig. 2, $P^{1}, P^{2}$ ) in which the prey is digested (p. 192, infra). A very noticeable feature of pseudopodia of all kinds is their adhesiveness, due to the secretion of a slimy substance at the surface of the ectoplasm. In Diffugia, if the pseudopodia be touched gently with a glass rod, the slime can be drawn out into threads, like the muous of a snail (Rhumbler, 34). The adhesive power of the pseudopodia is of service both in adhering to the surface upon which they creep and in the capture of their food.

The slow - flowing amœbæ, such as $A$. verru$\cos a$, do not as a rule flow round the body to be in-

to be ingested, enclosing it 
The pseudopodia of the filose type adhere firmly to organisms suitable for food with which they come in contact, and it can be observed that the prey is both held fast and killed by them, indicating that the pseudopodia secrete some toxic substance in addition to that of an adhesive nature. In the reticulose type, diatoms and organisms of various kinds are entangled in the pseudopodial network (Fig. 21), and are generally digested there also.

In a few cases pseudopodia exhibit a peculiar form of movement known as nutation. An example of this is seen in the remarkable Heliozoon described by Schaudinn (43) under the name Camptonema nutans (Fig. 47), which possesses slender axopodia in which the axial

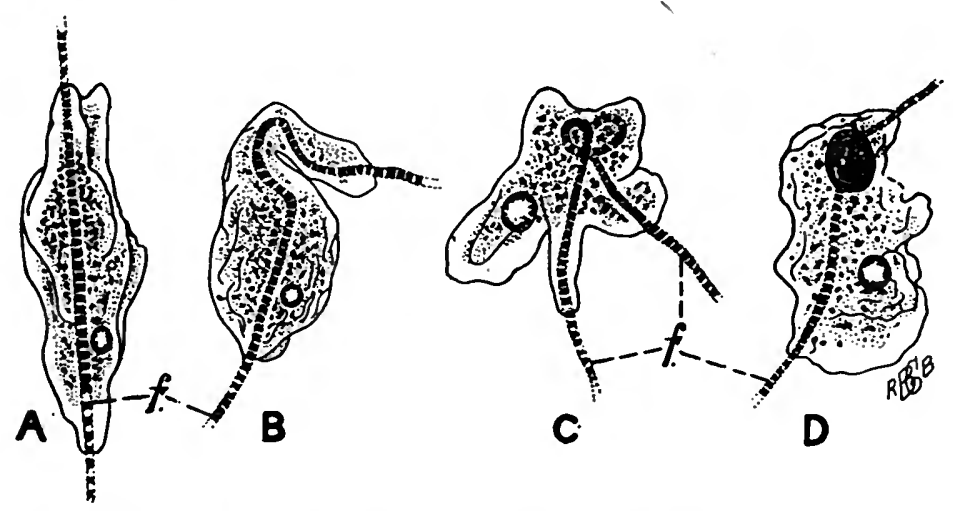

Frc. 23.-Four stages in the ingestion of an Oscillarian filament $(f$.$) by A m a b a$ verrucosa. In $A$ the amceba has crept along the filament; in $B$ one end of the amooba is bending up, and is about to fuse with the rest of the body, producing a twist in the filament ; in $C$ two have been produced; in $D$ a considerable length of the filament has been drawn into the amœba, and is twisted up into a stout coil. $A, B$, and $C$, are drawn at intervals of quarter of an hour, $D$ seversl hours later. After Rhumbler (34).

filament does not extend the whole length of the pseudopodium. The pseudopodia perform a slow rotating movement, and "describe the mantle of a cone, sometimes acute, sometimes obtuse, remaining stretched out straight for their entire length, and bending only at their base." Similar movements are performed by the pseudopodia of Trichosphørium (p. 229) and Wagnerella (p. 246). In Camptonema the pseudopodia also have the power of bending suddenly when brought in contact with prey, which they capture like the tentacles of a polyp. The bending takes place beyond the point at which the axial filament ceases. Movements of this kind are transitional to those seen in flagella.

(2) Flagella are vibratile thread-like extensions of the protoplasm, capable of performing very complicated lashing movements in 
every direction. A flagellum consists of an elastic axial core enclosed in a contractile sheath or envelope (Fig. 24), from the extremity of which the core protrudes freely in some cases, forming is so-called "end-piece." The flagellum takes origin from a more or less deeply-seated granule, the blepharoplast, or basal granule, which will be described in dealing with the nuclear apparatus (p. 82, infra). The elastic axis, arising from the blepharoplast, can be regarded as a form-determining element of endoplasmic origin, the sheath as an ectoplasmic motor substance. A flagellum

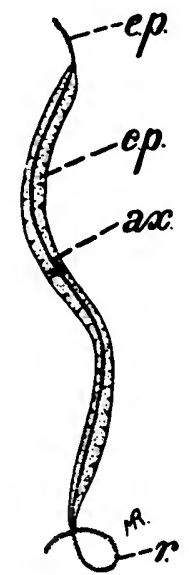

Fro. 24. - Structure of the flagellum of Euglena. ax., Axial filament; c.p., contractile protoplasm onveloping the axisl filament; e.p., ond - piece of the flagellum, consisting of the axial filament exposed ; $r$, root of the flagellum passing into the body (compare Fig. 84). Aftor Bütsohli (3). is usually cylindrical in form, with the axial filament central in cross-section, but may be band-like, with the axial filament at or near one edge; it is usually of even thickness throughout its whole length, but when the axial filament is exposed to form a terminal end-piece the flagellum tapers to a fine point.

Like pseudopodia, flagella serve primarily for locomotion, and secondarily for food-capture, which is effected by causing food-particles to impinge on some point or aperture at the surface of the body, where they are ingested. In their relation to locomotion two types of flagella can be distinguished, termed by Lankester pulsella and tractella respectively. A pulsellum is situated at the end of the body which is posterior in movement-that is to say, it is a flagellum which by its activity propels the body forwards. Flagella of this type occur in Oxyrrhis (p. 278) and in the Choanoflagellata (p. 271), but are comparatively rare in the Protozoa. In the majority of cases the flagella are tractellathat is to say, their action is such as to drag the body after them-hence they are situated at the end which is anterior in progression. Considered generally, the movements performed by tractella are of two types. In some cases the entire flagellum is thrown into even, sinuous undulations, and the body of the flagellate progresses with a smooth, gliding movement, which may be extremely rapid, and is then well expressed by the French phrase "mouvement en flèche"; this type of movement is well seen in the trypanosomes and allied genera, such as Leptomonas, etc. In most free-living flagellates, however, the flagellum is held out stiff and straight for the proximal two-thirds or so of its length, while the distal third performs peculiar whirling or pulsating 
movements, ${ }^{*}$ which drag the body along in a succession of more or less distinct jerks.

In many flagellated organisms, forwardly-directed flagella may be combined with so-called " trailing flagella" ("Schlepp-geissel "), which are directed backwards, running along the side of the body, either quite free (Fig. 25) or united to the body by an undulating membrane (Fig. 5). In such cases the trailing flagellum is perhaps the chief organ of propulsion, acting as a pulsellum, while the forwardly-directed flagellum or flagella may function more as tactile organs or feelers than as locomotor organs. The flagellum may also serve as an organ of temporary attachment in some cases, especially in parasitic flagellates; it then often exhibits at its distal extremity a distinct bead-like swelling or enlargement, doubtless of adhesive nature. Such terminal enlargements are sometimes seen, however, in free-swimming forms.

There are many grounds for assuming the existence of a gradual transition from flagella to pseudopodia and especially to the slender axopodia seen in Heliozoa, otc. In organs of each kind tho typo of structure is essontially similar, an axis of firm elastic nature, which is pushed out from the ondoplasm, in many cases from a basal granule of centrosomic naturo (p. 82), and is covered over by a sheath of contractile fluid ectoplasm. The difference between them is one of degree, the axopodia being relatively shorter in proportion to their thickness, and consequontly less flexiblo, but tho nutating and bending movements seen in axopodia aro cssentially similar in typo to those manifostod by flagella. The Heliozoa aro connected with the Flagellata by transitional forms which indicato that their psoudopodia have arisen as modifications of flagolla (p. 248). Goldschmidt, who discusses tho wholo question (41, pp. 116-122), de scribes in a Cercomonas-liko flagcllato tho shortening of the flagollum, and its transformation into a psoudopodium which swings to and fro. A flagellum may bo considered as having arisen by modification and spocialization of an axopodium, and as capable in many instances of reverting to that typo of organ. (Compare also p. 465, infra.)

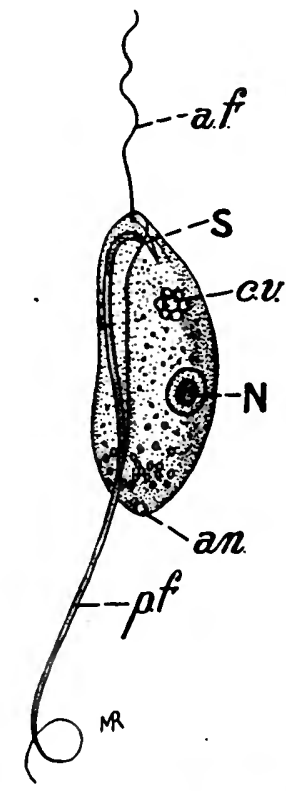

Fro. 25. - Anisonema grande, ventral view, showing the " heteromastigote " a rrangement of the flagella. a.f., Anterior flagellum ; p.f., posterior trailing flagellum; $S$, osophagus; c.v., contractile vacuole surrounded by a number of feeding vacuolcs ; $N$., nucleus; an., anus (cytopyge). After Stein.

(3) Cilia are slender, thread-like extensions of the ectoplasm which diffel from flagella mainly in three points: they are as a rule much shorter relatively to the size of the body; they are present usually in much greater numbers, and in their most primitive typc

* For a detailed description and analysis of these movements, see Delage and Hérouard (6), pp. 305-312. 
of arrangement form, as it were, a furry covering to the body ; and their movements are different from those of flagella. A cilium performs simple regular movements of alternate contraction and relaxation, whereby it is first bent like a bow, with a slight spiral twist (Schuberg, 44), and then becomes straightened out. again; from this it may be inferred that the contractile substance is developed mainly on one side of the elastio axis - on that side, namely, which becomes. concave during contraction-instead of ensheathing the axis completely, as in most flagella. Then the bending of the cilium would be the result of active contractility, acting against the elasticity of the axis, which is operative in causing the cilium to straighten out again when the contractile substance is relaxed.

Cilia are usually implanted in rows on the surface of the body, and their movements are co-ordinated in such a way that the contraction-or, as it may be better termed, the pulsation-of a given cilium takes place slightly after the one in front of it, and before the one behind it (Fig. 26). On the other hand, the neighbouring cilia of adjacent rows pulsate in unison; consequently, when a ciliated

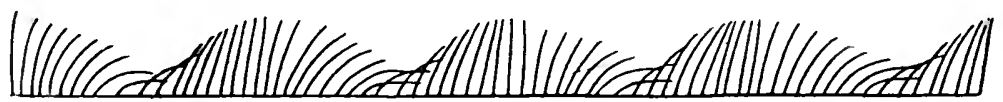

Fra. 26.-Diagram of oiliary movement, representing the successive phases of contraction and expsnsion in a row of cilia. After Verworn.

surface is seen from above with sufficient magnification, the movements of the cilia produce an optical effect similar to that seen in a cornfield when the wind blowing across it gives rise to an appearance of waves following each other in a continuous succession. When, however, a row of cilia is seen in sidc-view, the successive beats of the cilia may produce the illusion of a rotating wheel; hence the origin of such names as Rotifer, Trochophore, etc., applied to Metazoan organisms bearing rings or girdles of stout cilia.

In spite of the apparent differences between cilia and flagella, there is no diffioulty in regarding cilia as derived ancestrally from flagella by a process of modification and specialization in structure, movement, number, arrangement, and co-ordination. Like pseudopodia and flagella, cilia may serve both for locomotion and foodcapture. In many cases the cilia specialized for these two functions may be sharply distinct ; the food-capturing cilia, found in connection with the mouth and the peristomial region, are commonly much longer than the locomotor cilia, and show the tendency to form fusions presently to be described. In sedentary forms loco motor cilia may be absent in the ordinary state of the animal, and only developed temporarily during motile phases. On the other 
hand, in a purely parasitic form such as Opalina (p. 439), in which a mouth is entirely absent, only locomotor cilia are present.

The chief modifications of cilia, apart from variations in size and function, are the result of a tendency to adhere or fuse together ; thus arise various types of organs, of which the most common are the cirri, membranello, and undulating membranes. Cirri are organs resembling bristles, formed by fusion of a tuft of cilia, just as the hairs of an ordinary camcl's-hair paint-brush adhere when moistened so as to form a flexible pencil. In many cases the cirri have frayedout ends, in which the component cilia are distinct from one another; and reagents often cause a cirrus to break up into separate cilia. Cirri have a locomotor function, and are especially characteristic of the ciliate Infusoria which are of creeping habit (order Hypotricha, p. 440, infra). The cirri occur on the ventral surface of the body - that is to say, on the side of the body turned towards the substratum on which the organism creeps, using the cirri practically as legs.

Membranellæ are flapping or swinging membranes formed by fusion of two or more transverse rows of cilia implanted side by side, and adhering to form a flat membrane, the free edge of which often has a fringed or frayed border, representing the free ends of the component cilia. Membranellæ occur usually in the region of the peristome in spiral rows, implanted one behind the other, and each membranella performs simple movements of alternate flexion and expansion, comparable to those of a single cilium. Both in structure, origin, and movements, the membranellæ must be distinguished clearly from the undulating membranes presently to be desoribed.

Undulating membranes are sheet-like extensions of the ectoplasm, which perform rippling movements, comparable to those of a sail placed edgewise to the wind; or, better still, to the undulating movements performed by the dorsal fin of a sea-horse (Hippocampus) or a pipe-fish (Syngnathus) when swimming. The undulating membranes of Ciliata consist simply of a single row of cilia fused together. Such membranes are found commonly in the cesophagus of Infusoria; in the vestibule of Vorticellids there are two membranes of this kind. In some genera, such as Pleuronema (Fig. 27), they represent the principal food-capturing organ, and reach a great development. Pleuronema swims about by means of its cilia, and comes to rest sooner or later in a characteristic attitude, with the cilia projecting stiffly from the body ; the large undulating membrane is then protruded from the mouth, and serves by its movements to wait food-particles down the cesophagus.

Undulating membranes are also of common occurrence in the Flagellata, where they are of a different type from those of Ciliata. The undulating membrane in this class is always found in connec- 
tion with a flagellum, and is to be regarded as a web of the ectoplasm (periplast) connecting the flagellum to the surface of the body. Such a condition may arise either by attachment of a backwardly-directed trailing flagellum to the side of the body, as in Trichomonas (Fig. 5) and Trypanoplasma (Fig. 36), or by the shifting backwards of the point of origin of an anterior flagellum, as is well seen in the transition from crithidial to trypaniform phases in the development of trypanosomes (Fig. 131). As a rule, only the proximal portion of the flagellum is involved in the formation of

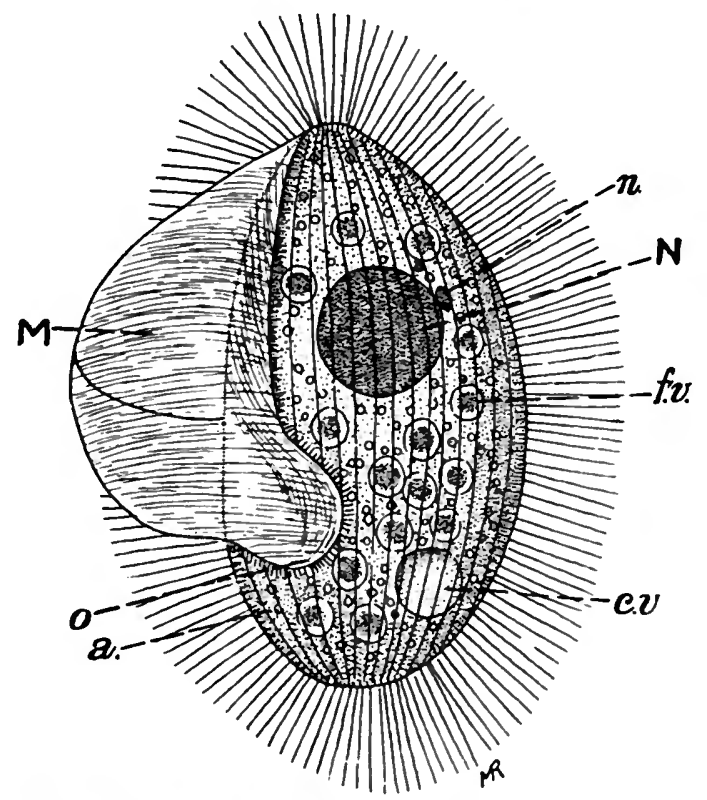

Fro. 27.-Pleuronema chrysalis. $M$, The undulating membrane; 0 , mouth; $N$, macronuclous; $n$, micronucleus ; c.v., contractile vacuole ; f.v., food vacuole; a., anal pore. After Schewiakoff, magnified 660 diameters.

the undulating membrane, and the distal portion projects freely beyond it; but in some cases a distal free portion of the flagellum may be quite absent, and then flagellum and undulating membrane are co-extensive (Fig. 12, J). Undulating membranes in Flagellata appear to be specially related to the endoparasitic mode of life, and in free-living species they are found rarely, if ever; they may be regarded as an adaptation to life in a broth-like medium, such as the intestinal contents, or the blood of a vertebrate, containing many suspended particles or corpuscles. In such cases the membrane may assist the organism to force its way between the solid bodies suspended in the fluid medium. Undulating membranes may, how- 
ever, serve for other functions than that of locomotion, in flagellates as well as in ciliates. In large, stout forms of trypanosomes, for example, the animal may remain perfectly still while its membrane is rippling actively, and in that case the function of the membrane is probably to cause currents in the fluid surrounding the body, and to change and renew the liquid bathing the body-surface. In such a case it has been noted that the undulating membrane may from time to time reverse the direction of its movements, the waves running for a time from the hinder end forwards, and then for a time in the opposite direction (Minchin and Woodcock, 42, p. 150). It is probable that the undulating membranes which pass down the vestibule of Vorticellids can reverse their movements in a similar manner, since this passage serves both for passage of foodparticles to the mouth and for the ejection of excreta from the anal pore and the contractile vacuoles.

The only structures found in free-living Flagellata which can be compared at all with undulating membranes are the peculiar " collars" found in the Choanoflagellata (Fig. 110), and also in tne collar-cells of sponges. Each collar is an extension of the ectoplasn which grows up from the edge of a circular area round the insertion of the flagellum, forming a membrane like a cuff or sleeve surrounding the basal portion of the flagellum, but quite distinct from the flagellum itself, and not formed in actual connection with it like the undulating membrane of a trypanosome. The collar differs further from a true undulating membrane in not being energetically motilc, but only slowly protrusible and retractile. It has been stated, both for Choanoflagellates and for the collar-cells of sponges, that the collar is formed by a spirally-folded membrane. Their function appears to be that of assisting in food-capture by a sessile, flagellated organism.

(4) Contractile mechanisms in Protozoa, when they are visible, take the form of so-called myonemes, minute contractile fibrils running in various directions in the ectoplasm, like an excessively minute system of muscle-fibres. Such elements are not found in Sarcodina or in the non-corticate forms of the other classes; in naked forms with amoboid movement the ectoplasm, as has been pointed out above, is only a temporary differentiation of the protoplasmic body, which can arise by conversion of the endoplasm, and which can be changed back again into endoplasm. Myonemes occur commonly, however, in those. Flagellata, Sparozoa, or Infusoria, which owe a definite body-form to the presence of a firm cuticle or cortex, representing a stable ectoplasm. The myonemes are oftell, however, extremely fine, and sometimes escape detection in cases in which we can infer their presence with certainty from the movements or contractions of the organism or of its ectoplasm. As a 
general rule they are visible more or less clearly in the larger, but not in the more minute, species. Thus, in trypanosomes, myonemes can be made out in large forms as delicate lines running parallel to the undulating membrane (Fig. 28), but in small species of trypanosomes it may be impossible to discover them, although the nature of their movements may leave no doubt as to the existence of contractile mechanisms in the ectoplasm. In other cases, both motile species possessing myonemes and non-motile species lacking them may occur within the limits of a single group, as in Gregarines, whare the motile species show a very distinct layer of myonemes (Fig. 29); while the non-motile

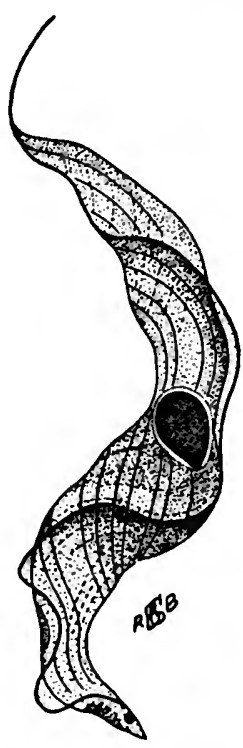

Fio. 28.-Trypanosoma pe ca, stout form stained with iron-hæmatoxylin to show myonemes. After Minchin, $\times 2,000$. forms have a much thinner ectoplasm, represented practically by the cuticle alone, with no trace of myonemes. In the non-motile trophozoites of the Coccidia myo-

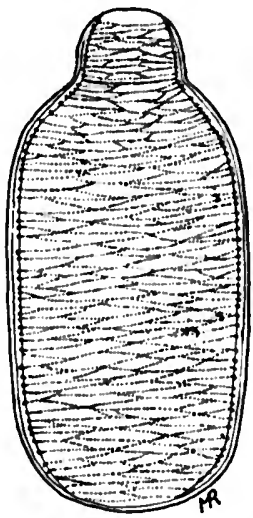

FIa. 29.-Gregarina munieri, showing the layer of myonemes at the surface of the body, sligbtly diagrammatic. After Schneider.

nemes are similarly absent. In the ciliate Infusoria the myonemes run parallel to, and beneath, the rows of cilia, and in species of large size and great powers of contractility, such as Stentor, the myonemes are lodged in canals and show a transverse striation (Fig. 186, $I$ ).

According to Schaudinn, these motile mechanisms, both flagella and myonemes, are derived from the achromatic spindle of a dividing nucleus. In the development of a trypanosome from a non-flagellated condition, he describes the entire kinetic apparatus as arising from a nuclear spindle consisting of two polar centrosomes connected by a centrodesnose (p: 103, infra), and by mantle 
fibres, but with chromosomes apparently rudimentary or absent. Such a spindle is stated to persist and to grow greatly in length, one pole of it finally projecting beyond the anterior end of the body. The centrosome at the proximal pole of the spindle becomes the blepharoplast or basal granule of the flagellum; the centrodesmose itself becomes the flagellum, or at least its axial elastic filament;

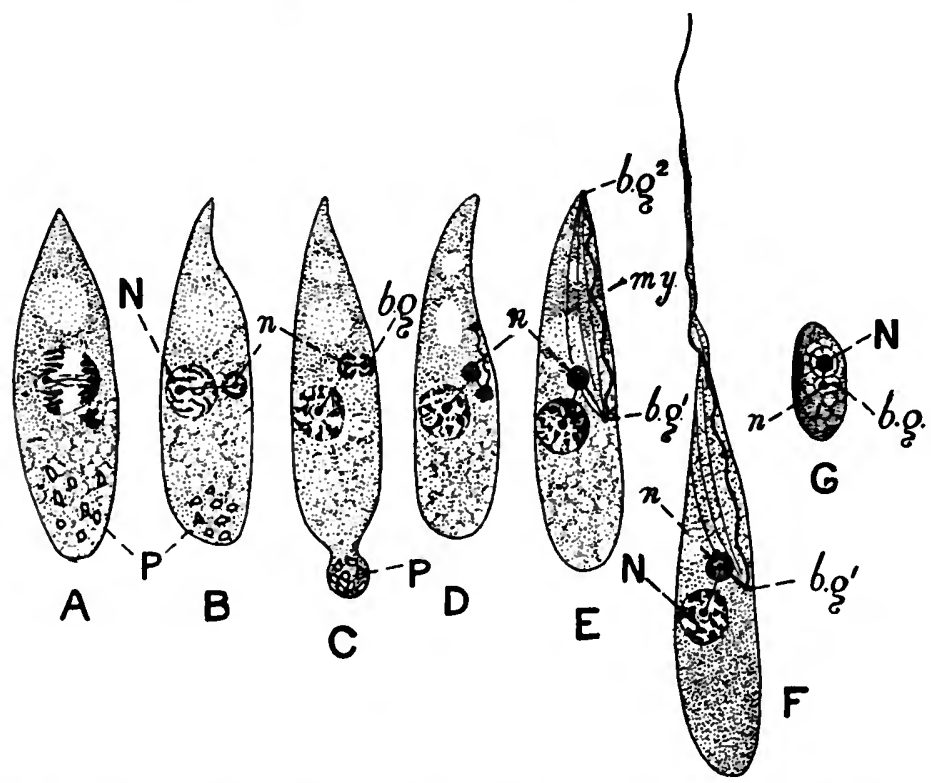

Fra. 30.-Development of the locomotor apparatus of trypanosomes. $A-F, \mathrm{De}$ velopment of Trypandsoma noctuce: $A$, the single nucleus of the "ookinete" is dividing into two unequal halves; in each half a centriole is seen, connected with its twin by a controdesmoso; $B$, the division of the nucleus complete; tho two sister-nuclei still connected by a centrodesmose uniting the centrioles; $C$, the smaller nuclous $(n$.) is dividing unequally to furnish a third nucleus (h.g.); $D, E$, the third nacleus is dividing to furnish a proximal (b.g. $\left.{ }^{1}\right)$ and a distal $\left(b . g .{ }^{2}\right)$ centriole, while the fibrils of tho achtomatio spindle become the myonemes $(m y) ;$.$F , development of the trypanosome -N$, trophonucleus; $n$, kinetonucleus; b.7.1, basal granule (true blepharoplast) of the flagellum. In $C$ the pigment $(P)$ present in the earlicr stages is being thrown off. After Schandinn (132).

$G$, stage in tho development of the merozoite of Tryfanosoma rotatorium into tho trypanosome-form; $N$, trophonucleus, still connected by a centrodesmose with $n$, the kinetonucleus, which has budded off $b . g$. , the basal granule of the flagellum. After Machado (469).

the distal centrosome is carried out on the tip of the flagellum; and the mantle fibres form the myonemes, stated in this case to be eight in number, of the body, which are continued on into the contractile sheath of the flagellum (Fig. 30). However fascinating the views put forward by Schaudinn, with regard to these points, may be, it must be stated that the greatest doubt attaches to the correctness 
of the obscrvations upon which they are founded, and that they lack confirmation entirely.*

3. Organs apparently of excretory function are present in many Protozoa as the so-called "contractile vacuoles," one or moredroplets of clear liquid which make their appearance in the ectoplasm, grow to a certain size, and then burst, emptying their contents to the exterior. When the contractile vacuole reaches its full size, it often bulges inwards far beyond the limits of the ectoplasm, and hence may appear to lie in the endoplasm; but its first appearance is always in the ectoplasm, to which it strictly belongs.

In non-corticate amœboid forms the contractile vacuoles simply empty themselves to the exterior, and the changing form of the body does not prrmit of determining whether the position of the vacuole is a constant one. It is common in amœbæ for the vacuole to be lodged in the region of the body which is hindmost in progression; but this may be simply the mechanical consequence of the streaming movements in the protoplasm, whereby the vacuole is carried along to the hinder end of the body. In corticate forms, on the other hand, the contractile vacuoles are constant both in number and position, and void their contents through a definite pore in the cuticle, directly or indirectly; in many Flagellata and Infusoria, for instance, the vacuoles do not discharge directly to the exterior, but into the osophagus or into a reservoir-vacuole communicating with the esophagus.

The growth of the contractile vacuole is caused by fluid draining into it from the body-protoplasm. In amœbæ and forms of simple structure no channels supplying the contractile vacuole are visible, and it must be supposed to be fed by a process of diffusion through the protoplasm from all parts of the body. In the highly-organized ciliate Infusoria, however, the deepest layer of the ectoplasm has a loose, spongy texture, and forms a definite excretory layer full of spaces containing fluid, which drains into one or more mair canals

* It must be added further that, to judge from the figures left by Schaudinn and published on Plate xxix. of his collected works ("Fritz Schsudinn's Arbeiten," Hamburg and Leipzig, 1911), the statements cited above appear to be founded on preparations made by a method of technique which is recognized generally as giving unsound cytological results-namely, the method of dried films stained by the Romanowsky stain. Schaudinn's statements are nevertheless cited above on account of the numerous theoretical discussions and speculations in modern protozoological and cytological literature of which they have been the foundation. For my part, I disbelieve entirely in the theory that the flagellum represents a centrodesmose between two centrosomes,' regard it as a simple ontgrowth from a blepharoplast of a nature essentially simuiar to the axopodium of a Heliozoon. It is curious that no one has as yet extended Schaudinn's theory to the axopodia, the axial filament of which should also represent a centrodesmose, if that view is correct for the axial filament of the flagellum, a view that seems to me quite unthinkable from a phylogenetio standpoint. Is it to be supposed that the formation of each psendopodium by a Heliozoon represents a rudimentary mitosis ? 
supplying the contractile vacuole or vacuoles. Thus, in Stentor (Fig. 8) the single vacuolc is fed by a canal running the length of the body, and in Paramecium (Fig. 185) the two vacuoles are each surrounded by a number of canals forming a star-shaped figure.

As regards the function of the contractile vacuoles, it should be noted in the first place that their contents are always fluid and watery, and never contain solid particles of any kind. The fluid which a contractile vacuole drains from the body is doubtless replaced by water absorbed from the surrounding medium by diffusion through the superficial layer of the protoplasm, or it may be through the mouth in some cases. The contractile vacuole is generally regarded as the organ of nitrogenous excretion, comparable functionally to the urinary organs of the Metazoa, but it is highly probable that the liquid discharged from it contains also the carbon dioxide produced by the respiratory process. Hence the contractile vacuole thay be regarded as both excretory and respiratory in function (see also p. 197, infra).

4. In the majority of Protozoa there are no organs for which a definite sensory or nervous function can be claimed, although these organisms show by their reactions to the environment or to stimuli that they possess sensory and psychical functions. In some cases, however, certain organs can be asserted to have a sensory function, exhibited in sensitiveness either to impressions

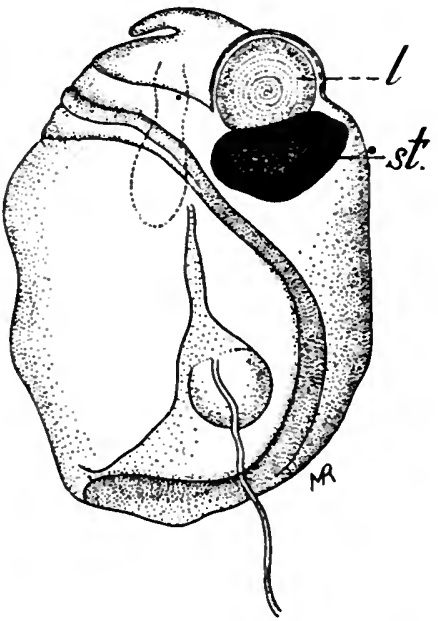

Fig. 31.-Pouchetia cornuta, one of the Dinoflagellata, to show the large stigma (st.), in front of which is a lens $(l$.$) . After Schütt$ (386). of touch or light. Thus, in many Flagellates the flagella appear to be tactile as well as locomotor in function, and in Cilinta tactile cilia occur, especially in the creeping hypotrichous forms.

Sensitiveness to light is a marked feature of many Protozoa, even of quite undifferentiated forms, such as amœbæ. Rhumbler (34) has shown that many amœbæ cease feeding in a strong light, and even disgorge food that they have taken in when suddenly subjested to the intense illumination necessary for microscopic study. This characteristic is, however, most marked in the holophytic species, to which light is a necessity for their plant-like metabolism. In the holophytic Flagellates a red pigment-spot, or stigma, is found constantly, situated close to the anterior end of the 
body (Fig. 4, st.). The belief that the stigma is the seat of lightperception receives support from the fact that in some cases it is found associated with lens-likc structures, which evidently serve to concentrate light upon it and act as dioptric elements, as in Pouchetia (Fig. 31).

\section{B. Endoplasmic Organs.}

The bulk of the endoplasm in proportion to that of the whole body varies greatly in different Protozon. In Flagellata, for example, the protoplasmic body must be considered as consisting almost entirely of endoplasm, the ectoplasm furnishing only the delicate periplast and myonemes. Similarly, in motionless parasitic forms, such as the Coccidia or the "colomic" Gregarines (p. 326, infra), the body within the cuticle is entirely endoplasm. On the other hand, in Ciliata, in which the ectoplasm may give rise to a number of different structures, the endoplasm is often a relatively restricted region of the body. In these examples that have been oited, the ectoplasm and endoplasm are probably stable layers, and their relative proportions are consequently more or less constant for a given phase of the life-history ; but in amooboid forms, as already pointed out, ectoplasm and endoplasm are interchangeable, and the amount of each layer present in an organism varies with the extent of its body-surface ; that is to say, the proportion of ectoplasm to endoplasm is greatest when the amoba is moving actively and throwing out many pseudopodia, and least when it is in a resting condition and has assumed the spherical form.

As stated above, the endoplasm is a fluid, granular substance, which contains various enclosures connected with the nutritive function, and also the nucleus or nuclei. Hence it may be regarded as the seat of trophic and reproductive functions. The nuclear apparatus will be dealt with in a separate chapter, since it belongs, strictly speaking, neither to the ectoplasm nor the endoplasm, though commonly lodged in the latter. In this chapter only the structural elements connected with the function of food ingestion and assimilation will be described.

The contents of the endoplasm vary greatly, according to the mode of life of the organism. In saprophytic and most parasitic forms no special organs are found in connection with the nutritive function, the food being simply absorbed in a soluble condition at the surface of the body, probably by the aid of enzymes secreted by the organism, but not by any recognizable organs. In holozoic and holophytic forms, however, special organs, differing widely in each case, are present for the assimilation or elaboration of food.

1. In holozoic Protozoa the organs of assimilation take the form of fond-vacuoles, minute droplets of fluid in which the solid particles 
ingested as food are suspended and gradually digested. In some cases, however, and especially when the prey is relatively large, no distinct fluid vacuole can be made out surrounding it, but the food appears to be simply lodged in the endoplasm itself; the vacuole is "virtual." When the digestion is completed, the insoluble fæcal residues are cast out of the body.

In Protozoa in which the body consists of naked, non-corticate protoplasm, the food is ingested, and the fæcal remains are expelled, at any point on the surface of the body. In corticate Protozoa, on the other hand, in which the body is limited by a resistant envelope or cuticle of a certain strength and thickness, food cannot be ingested at any point, but is taken in through a special aperture, a cell-mouth or cytostome. In such cases the organs of food-capture are either flagella or cilia, and by their action the food is wafted into the mouth. Primitively the mouth is a superficial aperture in the cuticle, opening into the endoplasm by means of a longer or shorter tube, the csophagus or cytopharynx. In the Peritricha (p. 433), however, the mouth and osophagus are, as it were, carried into the body at the end of an in-sinking of the ectoplasm, which forms a long tube or vestibule, comparable in its mode of formation to the stomodæum of the Metazoa. In any case the food-vacuoles are formed at the bottom of the esophagus, in the endoplasm. The mode in which the vacuoles arise, and the processes of digestion and defæcation, are discussed in a subsequent chapter (p. 189, infra).

2. In holophytic forms assimilation is carried on by cell-organs of the same nature as those found in the green cells of ordinary plants. Of primary importance are the chromatophores, or chromoplasts, bodies containing chlorophyll or allied pigments by means of which the organism is enabled to decompose carbon dioxide in the sunlight, setting free the oxygen and utilizing the carbon for building up the living substance. The chromatophores vary greatly as regards size, form, and number present in the cell-body. Other bodies of constant occurrence are pyrenoids, small glistening corpuscles which appear to serve as centres for the formation or storage of starch or similar substances of amyloid nature produced in the process of anabolism (see infra, p. 188).

In any Protozoa, whatever their mode of nutrition, the endoplasm contains usually various enclosures, which can be classed generally as metaplastic - that is to say, as products of the upward (anabolic) or downward (catabolic) metabolism of the living substance. Instances of anabolic products are the grains of starch or of the allied substance, paramylum, found in the holophytic forms, and the reserve food-materials-fat, "paraglycogen," and other substances-often stored up in considerable quantity in prepara- 
tion for developmental changes, especially in the female gamete, in a manner analogous to yolk-grains in an ovum. Instances of bodies resulting from catabolic activity are waste-products of various kinds in the form of granules, crystals, pigment-grains, etc., often present in great numbers, and giving the endoplasm an opaque and coarsely-granular appearance. A familiar instance of such wasteproducts is seen in the grains of melanin-pigment formed in the bodies of the malarial parasites (Fig. 156) as a result of the absorption and decomposition of the hæmoglobin of the red blood-corpuscle.

Many bodies present in the protoplasm of Protozoa may be considered as originally of metaplastic nature and origin, but as utilized secondarily for various functions. Such are the oil-drops in the intracapsular protoplasm of Radiolaria (p. 251), which appear to have a hydrostatic function, and also to serve as reserve foodmaterial in the development. It is also highly probable that both internal and external skeletons originated simply as excretions in the first instance-that is to say, as waste-products of the metabolism which have been utilized for the function of support, and subsequently adapted and modified in accordance with the special requirements of the organism.

Finally, as bodies of hydrostatic function, though not to be included necessarily under metaplastic products, are the peculiar gas-vacuoles of Arcella, bubbles of gas which can be secreted, absorbed, and formed agein, as circumstances may require, in and by the living protoplasm.

Bibliography.-For references see p. 477. 


\section{CHAPTER VI}

\section{THE ORGANIZATION OF THE PROTOZOA (Continued)-THE NUCLEAR APPARATUS-CHROMATIN, NUCLEUS, CHROMIDIA, CENTROSOMES, AND BLEPHAROPLASTS}

$\mathrm{OF}_{\mathrm{F}}$ all the parts or organs of the cell-body $y_{2}$ there is none of greater importance for the life and activities of the organism than the so-called nucleus, a term which, understood literally, means simply a kernel or central portion of the body, and conveys no idea of the true nature of the structure in question or of its significance for the life of the organism.

The cell-nucleus, in all its various modifications of form and structuro, is essentially and primarily a collection of grains and particles of a peculiar substance which has received the name chromatin, on account of its characteristic tendency to combine with ccrtain colouring matters and dyes. A nucleus may consist, perhaps, in some cases of little more than a single mass of chromatin, or of several such masses clumped together. In most cases, however, the chromatin is combined with other substances which may be termed comprehensively achromatin, and which are built up with the chromatin in such a way. as to produce a complicated nuclear structure, as will be described in detail presently.

The chromatin-substance is not necessarily, however, concentrated entirely in the nuclcus in all cases. In many Protozoa, especially amongst the Sarcodina, as, for example, Arcella (Fig. 32), Difflugia, and many other genera, the cell-body contains, in addition to one or more nuclei, extranuclear granules of chromatiı, termed chromidia,* which may be scattered in the cytoplasm

* The term " ehromidia," in tho German form "Chromidien," was coined by Hertwig (66) to denote the extranuclear grains of chromatin, and the whole mass of them in the cell-body was spoken of as a "Chromidialnetz." Subsequent authors, however, have used the word in its singular form, "chromidium," in a collective sense, to denote the entire mass of chromidia present in a cellular organ. ism, and not, as might have been expected, to mean the individual grains or particles of ehromatin which constitute the ehromidial mass. In order to avoid confusion, it is proposed in this work to use the term chromidiosome to denote the smallest ehromatin-particles of which the chromidial mass is made up, and which grow and nultiply by division like other elementary living bodies. It is clear, however, that the ehromidiosomes of which tho ehromidial mass scatterod in tho eytoplasm is built up aro in no way different in kind from the minutest granules of ehroniatin contained in the nucleus. The term "chromidiosome" must therefore be applied to the ultimate, individual grain or particle of ehromatin, alike whether it be lodged inside or outside a nuelcus. 
throughout the cell, or may be aggregated in certain regions of the body to form " chromidial masses" or "chromidial nets." It is even found that in some species a true nucleus may be absent temporarily during some phases of the life-cycle, all the chromatin being then in the form of chromidia, from which nuclei arise by a process of condensation and organization of the chromatin in combination with achromatinic elements. Such a condition may be regarded as a temporary reversion to a more archaic and ancestral condition, since, as has been pointed out already (Chapter I.), the Protista of the lower or bacterial grade of organization do not possess, speaking generally, a true nucleus, but only scattered grains of chromatin. Hence the chromidial condition of the chromatin may be ranked as an earlier and more primitive state, from which the strictly cellular grade of organization has been evolved by concentration of some or all of the chromatin to form a nucleus. In the tissue-cells of Metazoa, as a general rule, and in many Protozoa, the chromatin is concentrated entirely in the nucleus or nuclei, and chromidia do not occur.

Whatever view be taken as to the primitive or secondary nature of the chromidial condition (a question upon which individual opinions may differ considerably), the following facts can be stated definitely with regard to the chromidia. In some cases the chromidia can be observed to arise as extrusions of chromatin from the nucleus, which either casts off a certain amount of chromatin into the cytoplasm, while preserving its individuality, or may undergo complete fragmentation, becoming resolved entirely into chromidia, and ceasing to exist as a definite nucleus. In other cases, chromidia arise from pre-existing chromidia, by growth and multiplication of the chromidiosomes, thus keeping up a chromidial mass or stock which is propagated from cell to cell through many generations, independently of the nuclei present in addition to them in the cell.

The chromidial mass itself may vary considerably in structure in different cases or at different seasons ; the chromidiosomes may be arranged in clumps, strands, or trabeculæ, on a protoplasmic framework, and the mass is often vacuolated and contains substances other than chromatin. In Difflugia, Zuelzer (85) has shown that in the autumn the chromidial mass assumes a vacuolated or alveolar structure, and in each alveolus grains are formed of a carbohydrate substance allied to glycogen, which functions as reserve foodmaterial for the organism during the reproductive processes initiated at that season.

On the other hand, as chromidia arise from nuclei, so nuclei may arise from chromidia. In many Protozoa, as, for example, Arcella (Fig. 32), the formation of so-called " secondary" nuclei (which, however, do not differ from other nuclei except in their mode of 
origin), by concentration of chromidia into a clump or mass which acquires gradually the structure and organization of a true nucleus, is a frequent and normal occurrence in the life-cycle, as will be seen in subsequent chapters. Those who regard the chromidial condition as the more primitive will see in the formation of secondary nuclei from chromidia the ontogenetic recapitulation of the phylogenetic origin of the nucleus as a structural element of the cell-body.

From the foregoing it is seen that nuclei, in the Protozoa, do not necessarily arise from pre-existing nuclei ; the generalization "Omnis nucleus e nucleo," though it probably holds good universally for the cells of Metazoa, cannot be maintained for Protozoa if the term " nucleus" be taken in its strict sense. On the other hand, there

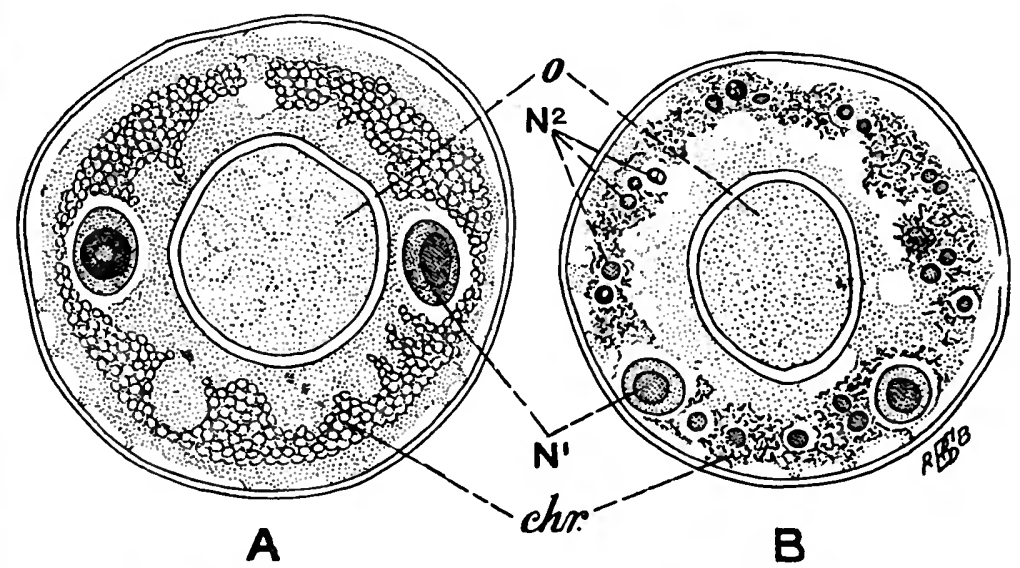

Fig. 32.-Arcella vulgaris, to show formation of secondary nuclei from the chromidia. $A$, Ordinary typo of individual, with two nuclei and a ring of chromidia; $B$, example in which secondary nuclei aro being formed in the chromidial ring. $N^{1}$, Primary nucleus ; $N^{2}$, secondary nucleus in process of formation; chr., chromidial ring; $o$. aperture of the shell. After R. Hertwig (65).

is no evidence that chromatin, within or without the nucleus, can ever arise de novo or in any way except from pre-existing chromatin, the particles of which grow and multiply as the result of processes of assimilation such as constitute the most essential characteristic of the living substance generally.

There is no doubt, however, that chromatin may itself give rise to other substances of achromatinic nature, and probably of simpler constitution, by a process of breaking down of its complex substance; and also that there may be present in the cell various substances very similar to chromatin in their properties and characteristics, representing stages in the building-up of the complex material of the chromatin-substance. In one or the other of these two ways it is possible to account for bodies in the cell known by various names, such as "metachromatinic grains," " chromatoid 
grains," and so forth-bodies which are often mistaken for true cliromatin, but which must be carefully distinguished from it, just as metaplastic bodies are to be distinguished from protoplasm. Among such bodies must be mentioned more especially the so-called "volutin-grains,"* which have attracted much attention of recent years, and which occur in various bacterial or unicellular organisms. The volutin-grains resemble chromatin in showing affinities for so-called " nuclear stains," which they hold more firmly than the ehromatin itself, when treated with reagents that extract the stain. According to Reichenow (78), volutin is a nucleic acid combination which is to be regarded as a special reserve-material for the formation of the nucleo-proteins of the chromatin-substance; during phases of the life-cycle in which the chromatin in the nucleus increases in quantity, the volutin in the cytoplasm diminishes, and, conversely, when the quantity of chromatin is stationary, the volutin-grains increase in number. Volutin-grains are thus seen to be bodies of totally different nature from chromidia, with which they are often confused on account of their similar appearance and staining reactions; chromidia are formed, typically, as extrusions from the nucleus into the cytoplasm; volutin-grains, on the other hand, are formed in the cytoplasm, and represent, as it were, a food-substance which is absorbed by the nucleus in the growth and formation of the chromatin. In some cases, however, the metachromatinie grains may represent chromidial extrusions from the nucleus which are breaking down or being modified into other substances; compare, for example, the extrusion of vegetative chromidia, which degenerate into pigment, from the nucleus of Actinospharium during a depression-period (p. 209).

The occurrence in the cell-body of volutin and other substances which resemble chromatin very elosely may often render extremely difficult the task of identifying and distinguishing the true chromatin, especially when it is not eoncentrated into a definite nucleus, but is scattered in the eytoplasm in the form of chromidial grains. 'The test upon which reliance is most usually placed for the identification of ehromatin is its staining propertles, and especially its readiness for combining with basic aniline dyes and certain other colouring matters. But this test is extremely inadequate and unreliable; on the one hand, as has been stated above, there are substances, such as volutin, which are coloured by "nuelear" stains more intenscly than the true chromatin itself; on the other liand, in cellular organisms which possess true nuclei eontaining undoubted chromatin, the staining reactions of the nuclei may be strikingly different in different cases. A good example of each of

* The namo " volutiu" was coincd by A. Moyer in 1904, and is derived from the fact that the substance was first studicd by him in Spirillum valutuns. 
these statements is furnished by the trypanosomes parasitic in vertebrate blood: on the one hand, these parasites often contain in their cytoplasm so-called " chromatoid grains," probably of the nature of volutin (Swellengrebel, 514), which stain in a similar manner to the nucleus; on the other hand, the nuclei of the parasites react to stains in a manner very different from the nuclei of the blood-cells amongst which they live. In short, it is not possible to name any stain or class of stains which can be relied upon either to combine with chromatin alone, or to stain chromatin in the same manner and to the same degree, at all times and in all cases* (compare Fig. 33). When, therefore, the adjectives "chromatinic" and "achromatinic" are used in the course of this work, it must be clearly understood that these terms signify that the bodies or substances to which they are applied consist or do not consist, as the case may be, of chromatin, and not that they stain or do not stain with certain dyes.

As regards the chemical nature of chromatin, it is characterized by containing protein-substances more complex in composition than any other part of the cell; it is not possible to say definitely, however, whether it is to be regarded as a single chemical substance or as a combination or mixture of several. Its most salient feature is its variability; judged by microchemical tests, no two samples of chromatin can be considered identical in composition, whether from different cells or even from the same cell at different times. Certain substances, especially phosphorus-compounds, are especially characteristic of nucleo-proteins, but it is not possible at the present time to define or identify chromatin by its chemical

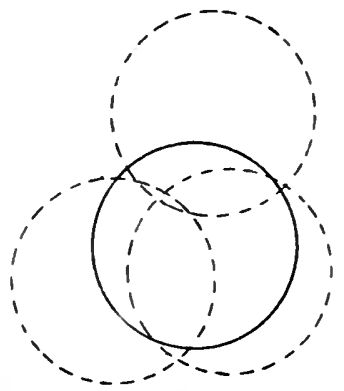

FIG. 33.-Diagram to represent in a graphic manner the action of colouring matters that stain chromatin. The eircle drawn with an uninterrupted line is supposed to represent a theoretically perfect chromatin-stain, which would stain chromatin always, and nothing else but chromatin; the circles drawn with interrupted lines ropresent the action of chromatin stains actually; they will stain chromatin as a general rule. though not invariably, but they will also stain other things which a re not chromatin. properties or composition.

All experience at the present time tends to show that the final test for the identification of chromatin in the cell is its relation to the vital activities and life-history of the organism. The term "chromatin" is thus to be regarded as denoting a biological or physiological, but not a chemico-physical, unity. A given body

* Methyl-green, acidulated with acetie acid, has sometimes been indicated as a most distinctively nuclear stain; but Hertwig (64) has shown that in the nuclei of Actinospharium this stain colours tho plastin-framework, and not the chromatin, and this author easts doubt on the alleged value of this stain as a reagent for demonstrating chromatin in the nucleus. 
or grain in the cell cannot be definitely identified as chromatin, in all cases, by any chemical or physical test, but only by its relation to the life and development of the organism as a whole, and more especially to the function of reproduction and the phenomena of sex, as will be shown more fully by means of concrete instances in subsequent chapters. The sum of modern knowledge with regard to the vital activities of living bodies and the life-histories of organisms, whether plants or animals, Protozoa or Metazoa, indicates that the chromatin exercises a regulative and determinative influence over the functions and properties of the cell-body. Direct experimental proof of the all-importance of the nucleus for the life of the cell is obtained by cutting Protozoa into pieces, some containing portions of the nucleus, others consisting of cytoplasm alone (p. 210, infra). Those pieces that contain nuclear substance are able to regenerate the lost parts of the body and to perform all the functions of life, and in particular those of assimilation, growth, and reproduction; those, on the contrary, that contain no portion of the nucleus rapidly lose the power of assimilation, and are unable to regenerate the body, to grow or to reproduce; and though they remain for a time irritable and capable of movement, they soon lose these properties. There are a number of facts which

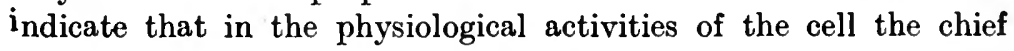
function of the nucleus is the formation of ferments; it is therefore all-important in regulating the assimilative processes of the living substance (p. 194).

The conception of chromatin as the directive and regulative centre of the cell-body renders intelligible a number of phenomena connected with it, such as the elaborate mechanisms which, as will be described in the next chapter, are gradually evolved and perfected for the exact partition of the chromatin in the reproduction of the cell by division, and the relation of chromatin to the sexual process. Further, the extremely variable nature of the chromatin-substance becomes at once intelligible on this view of its relation to the speeific charaeters and properties of the organism; for since every speeies of living being-perhaps, even, every individual of the same speeies-differs to a greater or less extent from every other: then, if such differences are determined by the chromatin, it follows that the chromatin must also differ to a corresponding degree in each case, and that consequently uniformity of character in different samples of chromatin cannot be expected to occur.

Hertwig $(67,92)$ considers that a certain quantitative relation of nucleus and cytoplasm is necessary in any cell for the normal continuance of the vital functions. This nucleo-cytoplasmic ratio ("Kernplasma-Relation") is subject to variations at different 
periods of life-history, but is the-same, normally, for corresponding phases of the life of the cell; it can be influenced by external conditions, such as food and temperature, and also by internal factors, undergoing changes in a regular manner, in harmony with changing functional conditions of the cell. In cultures of a given species at a lower temperature, multiplication is slower and the organisms grow larger and possess larger nuclei ; with increase of temperature the reverse takes place (compare p. 206, infra). It has also been observed that, in long-continued cultures of Protozoa, periods of active assimilation and multiplication are followed by periods of depression, during which assimilation and reproduction are at a standstill, even in the midst of abundant nutriment (see especially Calkins, 5). The depression-periods are characterized by an increase of the nuclear substance relatively to the cytoplasm, a "hyperchromasy" of the cell, which may lead to the death of the individual unless compensated by the elimination and absorption of part of the nuclear substance (p. 209, infra); when the balance has been thus restored, the organism becomes normal and feeds and multiplies again. From this conception of a definite relation between the mass of the nucleus, or rather of the chromatin, and that of the cytoplasm, Hertwig has deduced a number of important consequences to which reference will be made in subsequent chapters.

The influence exerted by the chromatin upon the life of the organism may be manifested in two ways, which may be termed, for convenience, actual and prospective, respectively. In the first case it regulates the metabolism and functions, both trophic and kinetic, of the cell in which it is contained, and is then commonly termed vegetative chromatin, or trophochromatin. In the second case it may be dormant and inactive in the cell that contains it, remaining latent, as it were, until carried on to future generations in. the course of cell-reproduction; at a later period the whole or a part of this latent chromatin may become active, determining the nature and properties of the offspring, and thus serving as the vehicle for hereditary transmission of the characters of antecedent generations. Such temporarily dormant chromatin is commonly termed generative chromatin, or idiochromatin. It is probable that in all Protozoa the cell-body contains chromatin both in the active and inactive state, the one regulating the vital functions of the living body, the other remaining dormant, in reserve for future generations.

The validity of this conception, according to which the chromatin present in an organism is regarded as being either vegetative or generative in function, must be tested by its capacity to account for the facts of the development and life-cycle which will be considered more fully in subsequent chapters. There are no means of recognizing and distinguishing vegetative and generative chro- 
matin except by their respective relations to the life-cycle, at certain periods of which, as will be seen, the nuclear apparatus is entirely reconstituted, effete vegetative chromatin being eliminated from the organism, either cast out or absorbed, and its place taken by reserve generative chromatin. It is only necessary to remark that some authorities speak of vegetative and generative chromatin as if they were two distinct kinds of substance, whereas they are probably to be considered rather as two phases or states of one and the same chromatin. Vegetative chromatin is that which is in a state of functional activity, and which thereby tends to become exhausted and effete in its vital powcrs, exhibiting in consequence the phenomena of "senility." Generative chromatin, on the contrary, by remaining inactive, conserves its " youth" unimpaired, and constitutes a reserve from which the worn-out vegetative chromatin can be replaced. Generative chromatin of one generation may become vegetative chromatin in the next.

As regards their distribution in the cell-body, in some cases vegetative and generative chromatin cannot be distinguished by the observer as separate structural elements, but are mixed up together in the same nucleus ; in other cases, however, they occupy distinet situations in the body. Thus, in Sarcodina it is common for the vegetative chromatin to be lodged in the principal nucleus or nuclei, while the generative chromatin occurs in the form of chromidia, as in Arcella (Fig. 32), or vice versa. In the Infusoria there are two kinds of nuclei, which are shown by their behaviour to consist, the one of vegetative, the other of generative chromatin. Chromidia, when present in the cell, may also differ in kind, being in some cases extrusions from the nucleus of purely vegetative chromatin, in process of climination, while in other cases, as already mentioned, the chromidia, or a part of them, represent the generative chromatin (see p. 150, infra).

The nuclei of Protozoa exhibit great variety of structure and form as compared with the relatively uniform structure of the nuclei of Metazoa. As stated already, the constituent substances or structural elements in any nucleus may be distinguished broadly as chromatinic and achromatinic: the former consisting of the chromatin, the primary and essential element never absent in any nucleus; the latter comprising, various accessory structures, ancillary to the chromatin, and not all of them invariably present in any given nucleus. Amongst the principal achromatinic constituents of nuclei in general must be mentioned the following: (1) linin, occurring in the form of a framework, which stains feebly or not at all by chromatin-stains, and which presents the appearance of a delicate reticulum or network, the optical expression of an alveolar structure; (2) a fluid enchylema or nuclear sap, filling 
the interstices of the linin-framework; (3) plastin, a substance which has staining reactions different to those of chromatin, and which occurs in lumps or masses forming the ground-substance of the nucleoli or karyosomes presently to be described. The whole nucleus is commonly enclosed in a membrune, but this structure is probably formed in different ways in different cases, and may be absent. In addition to these various constituents, there are commonly present also in connection with nuclei bodies of kinetic nature: Such are the centrosomes or centrioles, which appear to control, or at least to act as centres for, the movements which the various parts of the nucleus perform during the process of reproduction by division.

The structure and appearance of nuclei depend chiefly on the manner in which the chromatin is distributed. Two principal types of structure may be distinguished : in the first the chromatin is concentrated into a single mass or grain, or, if other grains are present in the nucleus, they are smaller and relatively insignificant in size; in the second a number of grains are present which are more or less equal in size. In the condition with a single, or one greatly preponderating, mass of chromatin, the nuclear space is not as a rule filled by it, but presents the appear-

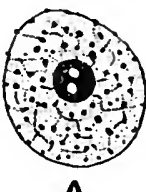

A
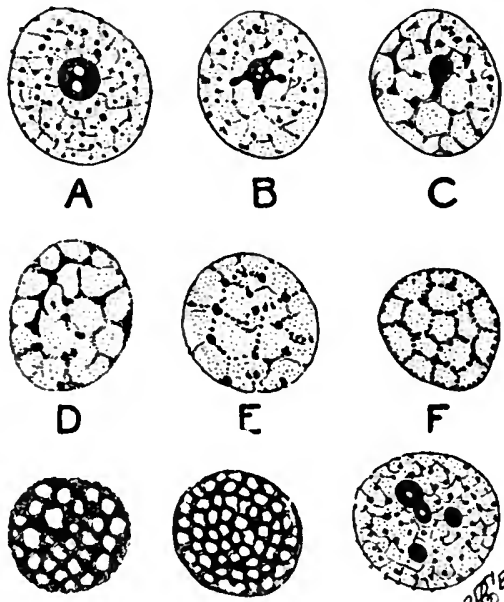

G
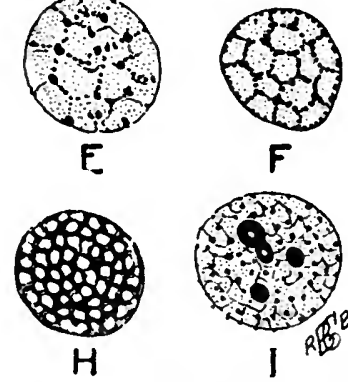

Fra. 34.-Cyclical vegetative changes in the resting nuclei of Trichospharium sieboldi. $A$, Stage with finely-meshed chromatic network and large karyosome (see p. 76); $B$, the meshes of the notwork widening, the karyosome budding off blocks of chromatin into it; $C$, the same prócess carried farther; $D$, coarse network containing scanty chromatin at thic nodes, karyosome wanting; $E$ to $G$, the chromatin increases greatly in quantity. covering the linin-framework-in $G$ the meshes of the network are becoming finer; $H$, the network has beenmo finemeshed again; $I$, a karyosome is being formed by condensation of thr chromatin at certain points, leading to the condition of $A$ again. After Schnudinn, $\times 2,250$. ance of a vesicle containing the chromatin-mass at or near its centre; consequently such nuclei are commonly termed "vesicular" in type, and the chromatinic mass may be termed generally, and without further determination of its precise nature, an endosome ("Binnenkörper"). When, on the other hand, the chromatin is in the form of numerous grains, they are generally distributed more or less evenly throughout the nuclear cavity ; such nuclei are termed "granular." 
Every transition from the one type of structure to the other may be found in the nuclei of Protozoa ; in a vesicular nucleus the principal mass of chromatin may break up into smaller grains which become distributed throughout the nuclear cavity; in a granular nucleus some or all of the grains of chromatin may be clumped together, and become fused to form a principal or single mass of chromatin. Such changes may take place during successive periods of activity of one and the same nucleus (Fig. 34). It is usual to speak of the condition of the nucleus as " resting" when it is not actually undergoing the process of reproduction by division; but it must be borne in mind that, so long as the cell is in a state of physiological activity of any kind, the nucleus also shares in this activity, and, strictly speaking, cannot be said to be resting. The activity of the nucleus is expressed in continual changes in its structure and rearrangements of its chromatin-substance and other constituents. In the gregarine Porospora gigantea, Léger and Duboscq (72) have observed changes taking place rhythmically in
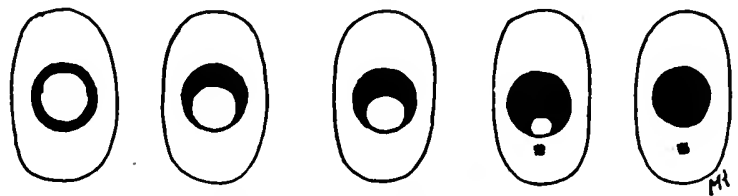

Fra. 35.-Successive stages of the karyosome (sec p. 76) of Porospora gigantea, showing the transformation of a hollow into a homogeneous karyosome by expulsion of a vacuole of clear viscous fluid into the nuclear cavity, where it forms a little mass of chromatin in front of the micropyle. After Léger and Duboseq (72).

the living condition (Fig. 35) ; compare also Chagas (48.5). Hertwig (64) has shown that the structure of the nucleus of Actinosphorium can be correlated with the functional activities of the cell. Thus a condition with the chromatin all concentrated to form a central endosome is found prior to division of the nucleus, and is also found when the animal is being starved; on the other hand, when it is supplied with abundant nutriment and is feeding actively, the chromatin-grains spread over the whole nuclear space. Since, however, abundant food also leads to frequent nuclear division, the condition with the chromatin concentrated at the centre also occurs during active cell-metabolism, as well as during hunger-periods.

In the simplest condition of the nucleus the grain or grains of chromatin are lodged in a space or vacuole, containing a clear fluid or nuclear sap, but not enclosed by a definite membrane. Nuclei of this simple type of structure are seen in some of the primitive forms, such as the small amœbæ of the limax-type, in which the 
nucleus consists of a large mass of chromatin suspended in the nuclear sap. In some cases no other structural elements can be made out ; in others the nuclear sap contains granules of peripheral chromatin varying in size from the most minute and scarcely visible particles to distinct grains. For a simple nucleus of this type the term "protokaryon" has been proposed; it is just such a nucleus as may be imagined to have arisen by a concentration of chromidiosomes at one spot in the cell-body, and in many cases such nuclei can be seen to be formed actually in this manner. The kinetonucleus of trypanosomes may be considered as a nucleus of this type in which the single mass of dense chromatin fills almost or quite completely the space in which it lies. In other cases there may be a clump of chromatin-grains more or less equal in size, filling the nuclear cavity, as in the nucleus of hæmogregarines. When there are numerous grains of chromatin, those placed superficially may be united to form a limiting layer which may be termed a "false" or "chromatinic" membrane, in distinction to a true nuclear membrane, which is an achromatinic structure. Even in nuclei of the most simple type, however, substances or structures accessory to the chromatin are probably always present.

In the first place, it is very probable that the grain or grains of chromatin do not lie loosely and freely in the nuclear vacuole, but are suspended in it, in all cases, by a delioate achromatinic framework, presenting the appearance of a fine network or reticulum, at the nodes of which the chromatin-grains are lodged. It is true that in many of the minute and primitive forms no such framework has been made out, and is believed by many observers to be absent; but on that view it is difficult to account for the definite position of the chromatin, its changes of position during division, and the frequent appearance, during this prccess of an achromatinic spindle, phenomena that may be noted even in the simplest cases. The achromatinic framework is often very fine and delicate, and its substance stains feebly or not at all with the colouring matters commonly employed in microscopical technique; hence it is very probable that it has often been overlooked in cases where it is really present. When there is but a single mass of chromatin, or one grain very much larger than all the others, the achromatinic reticulum presents the appearance of very delicate threads of linin radiating from the principal mass of chromatin to the periphery. When, on the other hand, there are numerous grains more or less equal in size, the reticulum is seen as fine lines passing from each grain of chromatin to each of the grains adjacent to it. In all probability the apparent "threads" of the reticulum are but the optical expression of the walls or partitions separating alveoli, and there is no reason for considering the achromatinic reticulum or 
linin framework as different in any essential point from the alveolar framework of the general protoplasm, with which, in nuelei that lack a true membrane, it is perfectly eontinuous. Hertwig (66) regards the eytoplasmic framework as achromatinic substance in intimate combination with chromatin; the nuclear framework, on the other hand, as pure achromatinic substance (linin) from which the ehromatin has beeome separated out and organized into special struetures independent of the framework in which they are lodged. Similarly, the nuclear sap filling the nuclear space and the interstices of the reticulum must be identified with the enchylema of the body-protoplasm. As compared with the alveolar structure of the general protoplasm, that of the achromatinic nuclear framework is eharacterized chiefly by the larger size of the alveoli, and, consequently, the greater distinctness of the apparent reticular strueture.

A true nuclear membrane, when present, is probably formed in all cases from the aehromatinic framework. In the nuelei of Actinosphorium, according to Hertwig (64), the membrane is a superfieial condensation of the achromatinic retieulum. The membrane may attain to a considerable thiekness and appear doubly-contoured in optical section, separating the nuelear framework completely from the extranuclear protoplasn; ; but it is always a strueture very readily absorbed and re-formed, and it appears to present no nbstacle to the passage rif substance from the nueleus into the eytoplasm, or vice versa. Awerinzew (47), on the other hand, regards the nuclear meinbrane as a product of the eytoplasm.

In addition to the achromatinie framework, plastin is eommonly, if not invariably, present in the form of masses or bodies which reecive different names, aceording as they eonsist of pure plastin or of plastin impregnated to a greater or less extent with ehromatin. In the vesienlar type of nucleus, the endosome may perhaps consist, in some cases, of pure chromatin, but in most eases, if not always, it is composed of a matrix or ground-substanee of plastin in whieh the chromatin is lodged. An endosome of this kind is termed a karyosome, or ehromatin-nucleolus; as a rule it has the form of a rounded mass, occupying the centre of the nucleus, sometimes of more than one such mass, but in a few cases it may have the form of a eresecnt or eap (" calotte ") closely applied to the nuclear membrane. In the gramular type of nucleus, on the other hand, there may be one or more masses of pure plastin eontaining no chromatin ; such a body is terned a nucleolus simply, or a " plastin-nueleolus." In the nuclei of the tissue-cells of Metazoa, true nucleoli oceur almost invariably ; in the nuelei of Protozoa, however, pure plastinnucleoli are not of eommon oeeurrence, but have been deseribed in a few instances-for example, in the hæmogregarine-nucleus 
(Reichenow, 78). As a general rule in the Protozoa, the plastin-substance is found as the matrix of karyosomes, but also as that of other masses of chromatin, such as the chromosomes of the dividing nucleus (see next chapter). Goldschinidt (41) observed that the formation of generative chronidia in Mastigella (p. 265) was preceded by the extrusion of plastin from the nucleus into the cytoplasm, to serve as a matrix for the chromatin which passed out from the nucleus subsequently. In Actinosphcerium, Hertwig has shown that a karyosome or chromatin-nucleolus, present during certain states or phases of nuclear activity, may give off its chro. matin-substance into the nuclear framework (reticulum), leaving the plastin-matrix as a body which is then seen to consist of a reticular framework similar in structure to the achromatinic reticulum of the nuclear framework, but distinguished from it by smaller meshes (alveoli) enclosed by thicker walls, as well as by its different staining properties. Certain phases of the development of Actinosphorium are further characterized by the formation in the nucleus of numerous small plastin-nucleoli, each consisting of a single vesicle (alveolus) of plastin containing nuclear sap.

Thus, a nucleus in its full complication of structure, and apart from the centrosomic elements, to be discussed presently, consists of the following parts : (1) An achromatinic framework or nuclear reticulum; (2) a true membrane, formed from the achromatinic framework, and separating the nuclear contents from the surrounding cytoplasm ; (3) nuclear sap, pervading the entire nuclear cavity ; (4) plastin, in the form of one or more bodies or masses which may consist either of pure plastin (nucleolif or of plastin impregnated with chromatin (karyosomes); and (5) the chromatin, which may be present either in the form of granules lodged at the nodal points of the reticulum, and scattered evenly or unevenly throughout the nuclear framework, or may be concentrated in a karyosome, or may combine both these two modes of distribution in various ways. Achromatinic framework and nuclear sap may be considered as a part of the general body-protoplasm, enclosed within the nuclear space, and set apart from the cytoplasm as a special nucleoplasm; plastin, on the other hand, is probably to be regarded as a product derived from the chromatin itself, either as a secretion or as a modification of its substance, to form a cement-like material or matrix in which true chromatin is carried. The two primary constituents of a nucleus are chromatin and protoplasmic framework.

Nuclei, whatever their structure, are, as a general rule, bodies of spherical or ovoid form; but in some cases, especially amongst Infusoria, the nuclei exhibit very varied forms in different species. The nucleus may then be sausage-shaped, or in the form of a horseslioc, or resemble a string of beads (" moniliform "), or be branched 
in a complicated manner. In the remarkable Acinetan Dendrosoma radians a colony is formed by budding, which resembles superficially a hydroid colony, eaeh hydranth being represented by the head of an Acinetan individual with suctorial tentacles; the branched nucleus is continuous throughout the whole colony, passing uninterruptedly from one individual to another.

Typically the cell-body contains a single nucleus, but in many Protozoa two or more nuclei occur constantly. When there are

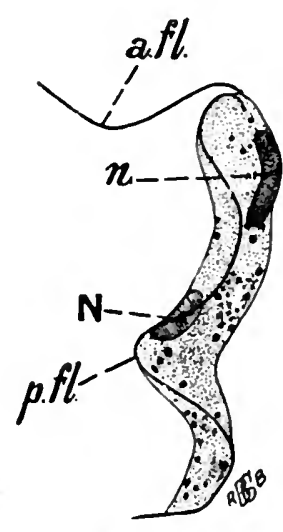

F1G. 36. - Trypanoplasina gurneyorum from the blood of the pike (E'sox lucius). a.fl., Anterior flagellum; $n$, kinetonucleus ; $N$, trophonucleus; $p$.f., posterior flagellum forming the edge of the undulating membrane, and continued beyond it as a very short free flagellum posteriorly. After Minchin (478), $\times 2,000$. more nuclei than one, they may be all alike and quite undifferentiated, or they may show differences in size, structure, and function. In many Sarcodina multiple nuolei without differentiation are found to occur constantly in certain species; for instance, two in Amœba binucleata and Arcella; several, perhaps a dozen or so, in Difflugia (Fig 16); from twenty to forty up to some five hundred in Actinosphorium (Fig 3); so also in Pelomyxa; and in the large plasniodia of Mycetozoa many thousands of nuclei are found.

Differentiation of nuclei, when it occurs, may be related to various causes. In trypanosomes and allied forms two nuclei occur constantly-a principal nucleus, or trophonucleus, so called because it appears to regulate the general metabolism and trophic activities of the cell-body; and a kinetonucleus, which is in special relation to the organs of movement, flagella, and undulating membrane. As a rule the kinetonucleus is smaller, in some cases very minute, and has a dense compact structure, while the trophonucleus has a vesicular structure; but in other cases (Trypanoplasma) the kinetonucleus is the larger of the two (Fig. 36).

A nuclear differentiation of totally opposite character is seen in the Infusoria, where two nuclei of different sizes, hence termed "macronucleus" and " micronucleus," are constantly present; the behaviour of these two nuclei in relation to sexual phenomena and reproduction (vide p. 153, infra) shows that the macronucleus is composed of vegetative chromatin, while the mieronucleus contains the reserve generative chromatin. In some cases-for example, in Myxosporidia (p. 403)-nuclei of different sizes occur in relation to sexual differences.

In some Protozoa - the so-called "Monera" of Haeckel-the nucleus has been stated to be wanting entirely ; but this statement 
is probably based on incomplete or erroneous observation, or on defective technique. In all Protozoa that have been examined in recent times, at least one nucleus has been found to occur without exception, though in some phases of the development the nucleus may temporarily disappear and resolve itself into chromidia.

There now remains for consideration the question of the centrosome, the centre of the kinetic activity of the nucleus. Of all the questions connected with the nuclear apparatus, those relating to the centrosome are the most difficult to handle in a general manner, largely on account of the minuteness of the bodies dealt with, and the consequent difficulty of ascertaining their structure and composition, even their presence, in many cases. Hence, in the literature of the centrosome, there is found considerable confusion in the terminology, different authors disagreeing entirely as to the precise structures to which the name centrosome should be applied, and opposed theories, which cannot be discussed adequately in a short space, have been put forward as to the nature and origin of the centrasome.

As the focus of the kinetic activities of the nucleus, the centrosome is most apparent and recognizable when the nucleus is in process of reproduction by division, and much less so when the nucleus is in the so-called "resting state." Hence the study of the nucleus during the process of division is alone decisive as to the presence of a centrosome in any given case; and since in many cases nuclear division appears to go on without centrosomes being present, it may be taken as equally probable that, in all such cases at least, no centrosome is present in the resting state of the nucleus. In many cases, however, the presence of a centrosome in, or in connection with, the resting nucleus can be ascertained clearly; it may then lie either outside or inside the nucleus.

When the centrosome lies outside the nucleus, as it usually does in the cells of Metazoa, it is found typically as a minute grain or pair of grains ("diplosome") close beside the nuclear membrane. Its presence may be indicated by the radiate structure of the surrounding protoplasm, giving the appearance of a system of rays centred on the centrosome; but such radiations are absent as a rule during the resting state of the nucleus, and the appearance of rays is often the first sign of impending activity and division of the nucleus. In many cases the centrosome is found lying in a mass of clear protoplasm termed archoplasm, a substance which differs, apparently, from the rest of the cytoplasm only in being free from granulations of all kinds. Archoplasm may, in short, be regarded simply as pure cytoplasm. and it appears either perfectly homogeneous, or traversed by striations which radiate from the centrosome, through the archoplasm, and even beyond its limits; 
the striations thenselves being the optical expression of a radiate arrangement of the protoplasmic alveoli (meshes of the "reticulum "), indicating lines of force or tension centred in the centrosome. In some cases it is probable that arehoplasm showing radiate striations may be present without any centrosome. In Aclinosphorium Hertwig showed that rays were formed in the archoplasm before a centrosome had been formed, and heralded its appearance.

When the centrosome lies within the nucleus, it is found most frequently, in Protozoa, within a plastiu-body or karyosome, a position which it may retain permanently during botl the resting and dividing conditions of the nueleus. The simple nuelei of the protokaryon-type probably contain in most cases a centrosomic grain lodged in the karyosome. In a few cases, however, an intranuelear centrosome occurs without a karyosome, or outsido the karyosome if one is present. On the other hand, there are many examples of the occurrence of extranuclear centrosomes in l'rotozoa ; but these are for the most part cases in which the centrosome is in relation, not only to the kinetic functions of the nucleus, but also to those of other cell-organs, as will be describer presently. Nuclei containing centrosomes have been termed "centronuclei" by Boveri.

The centrosome is seen, as a general rule, under the form of a minute grain, or centriole. This is the form in which it occurs invariably when it has an intranuclear position, lodged within the karyosome. But when it occurs outside the nucleus, it exhibits structural peculiarities which may vary at different periods, and it often presents cyclical changes corresponding to different phases of the activity of the nucleus. Thus, in Aclinosphcrium, Hertwig (64) describes the centrosome at its first appearance as a relatively large body of spongy structure, formed at one pole of the nucleus from extruded portions of the achromatinic reticulum (Fig. 37, $A-E$ ). At this stage, in which the centrosome is termed a centrosphere, it lies in a patch of archoplasm, and is the centre of a well-marked system of radiations. The centrosphere then gives rise, by condensation of its substance, to two centrioles, or to one which divides, and at the same time the archoplasmic radiations become fainter and disappear (Fig. 37, F, G). The centrioles then take part in the division of the nucleus, and when this process is complete they again become spongy centrospheres, which go through the same series of successive changes that have already been described. Analogous cyclical changes of the centrosome have also been described in other cases, and have led to a conflict of opinion as to whether the term "centrosome" should be applied to the whole centrosomic complex, as it may be termed, or to the centrioles, of which many 
may be present. It is simplest in theory, and probably correct in fact, to regard the centriole as the primary, in many cases the sole, constituent of the eentrosome-an element which may be capable, to a greater or less extent, of ehanges in size and structure, and which multiplies by division. To the primary centrosome or
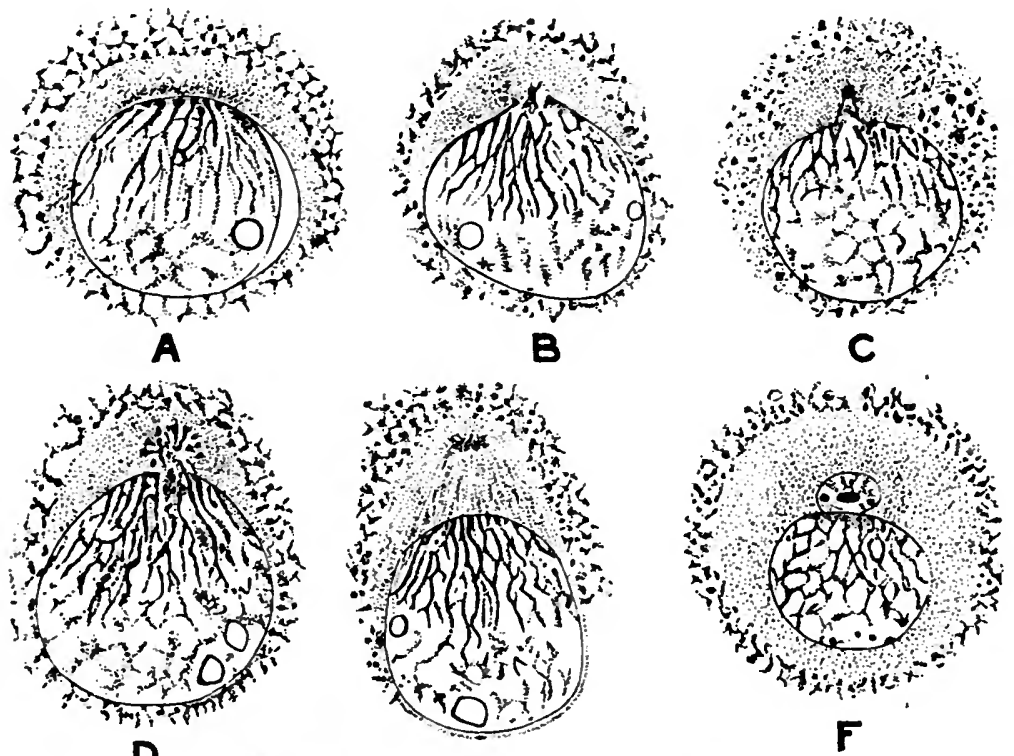

D

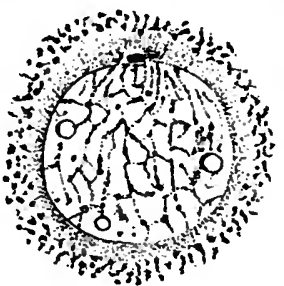

6

E

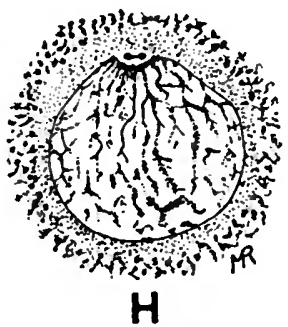

Fic. 37.-Actinospherium eichhorni : formation of the centrosome. A, Concentration of the nuclear reticulum towards one pole of the nucleus, near which the cytoplasm appears freo from granulations, forming tho archoplasm; $B, C, D$, passage of a portion of the nuclear reticulum to the exterior to form the "spongy centrosome" lying in the archoplasm; $E$, spongy centrosome with striations passing from it through the archoplasm to the nucleus; $F, G$, tho centrosome passes back again to the vicinity of the nucleus and undergoes a reduction of substance-tho archoplasm also diminishes temporarily in quantity ; $H$, division of the centrosome. After Hertwig (64).

eentriole there may be added adventitious elements of protoplasmic or nuclear origin, thus forming a eentrosomie complex which may attain a size relatively considerable in some cases.

So far the centrosome has been discussed only in its relation to 
the kinetic activities of the nucleus, a function which may be regarded as its primary and most characteristic rôle. It may act also, however, as the centre of other kinetic functions of the cellbody, especially in relation to motile organs such as flagella; it then appears as the so-called "basal granule," from which the flagella take origin. The basal granule appears as a thickening at the base of the flagellum. It rnay be continued farther into the cytoplasm, or connected with the nucleus, by means of one

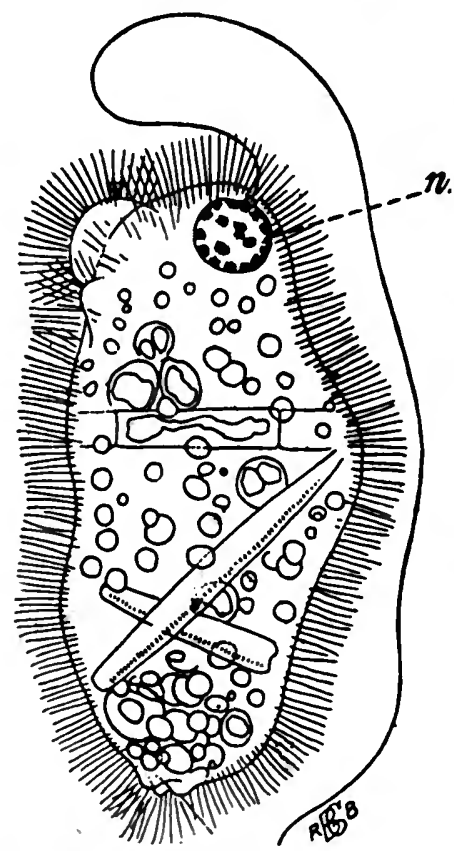

Fia. 38.-Mastigina setosa, after Goldschmidt (41). n., Nucleus from which the long flagellum arises; the body is full of distoms and other food. bodies. The surface of the body has a covering of short bristle-like processes. or more root-like processes known as the rhizoplast. A centrosome which is in relation to a motor cell-organ is termed generally a blepharoplast. The rhizoplast may have various origins; in some cases it represents the centrodesmose (p. 103) which connects the blepharoplast with the nuclear centrosome, or the remains of such a connection ; in other cases it represents the remains of the nuclear spindle of the previous nuclear division, as in the swarm-spores of Stemonitis flaccida (Jahn, 69) and

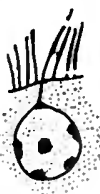

A

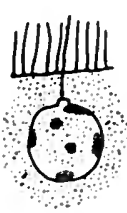

B

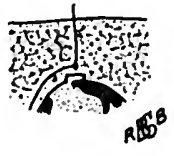

C
Fia. 39.-Connection of the flagellum and the nucleus in Mastigina setosa. $A$ and $B, A s$ seen in the living state; $C$, after fixation and staining. After Goldschmidt (41).

the collar-cells of Heterocœla (Robertson, 79); while in some instances it may be formed by outgrowth of root-like processes, of no special cytological significance, from the blepharoplast.

The relation of the nuclear to the kinetic apparatus is best studied in the Flagellata, where three principal conditions may be distinguished as follows :

1. The cell-body contains but a single centrosome, which functions also as a blepharoplast ; these two names, then, denote two different phases of activity of one and the same body, whish is a centro- 
some when it is active in relation to the division of the nucleus, and a blepharoplast when it is in connection with flagella or other motile organs during the resting state of the nucleus. In this, probably the most primitive state of things, there are, further, two different structural conditions found to occur in different cases. First, the centrosome-blepharoplast may be within, or closely attached to, the nucleus; secondly, it may be quite independent of the nucleus, and some distance from it in the cellbody, during the resting state of the nucleus. In the first case - of which an example is seen in Mastigina (Figs. 38, 39), paralleled by collar-cells in the Leucosoleniid type of calcareous sponges-the flagellum appears to arise directly from the nucleus; in the second case, exemplified by Masligella (Fig. 40), and by collar-cells of the Clathrinid type, the flagellum takes origin quite independently of the resting nucleus. In both cases alike, the flagellum generally disappears before division of the nucleus begins; the blepharoplast becomes the centrosome, divides, and initiates the division of the nucleus ; the new flagella of the daughtercells grow out from the two daughter - centrosomes during or after division of the

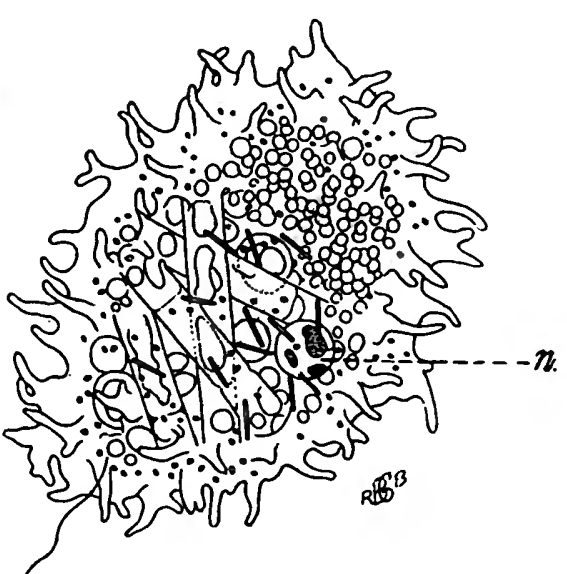
nucleus, and in either case, when the two daughter-cells are completely formed, their centrosomes, as blepharoplasts, remain as the basal'granules from which the flagella arise.

2. The cell-body contains more than one body of centrosomic nature-namely, a definitive centrosome, in relation to the single nucleus, and, in addition to this, one or more blepharoplasts in relation to motile organs. Then, when division of the cell takes place, one of two things may happen. 
In the first place, the flagellum or flagella may disappear, together with their blepharoplasts; the nuclear centrosome divides into two, which control the division of the nucleus in the usual way, and the centrosome of each daughter-nucleus divides again into two, one of which is the definitive centrosome, the other the blepharoplast, of the daughter-cell. The new flagella may either grow out from the daughter-centrosomes before they divide, and be carried off, as it were, by the product of division which becomes the

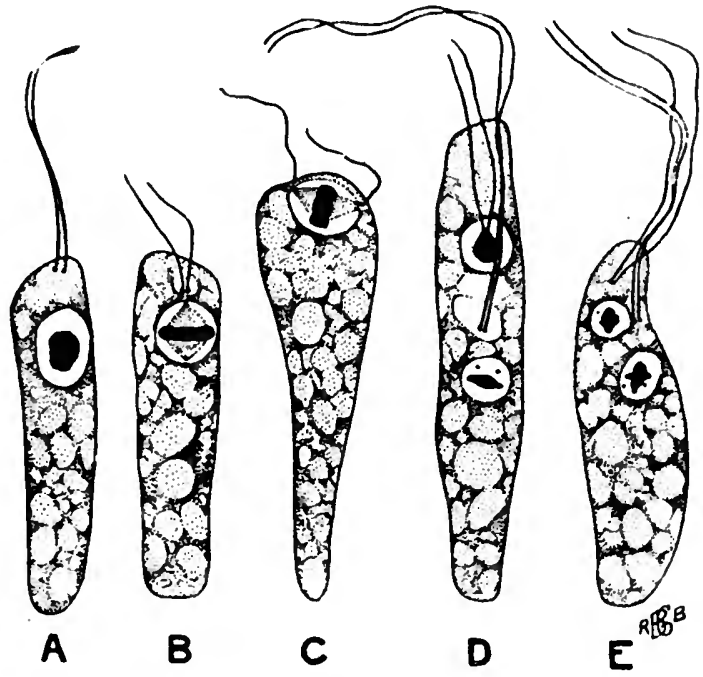

Fic. 41. - Stages in the division of Spongomonas splendida, to show different ways in which the daughter-flagella a rise. Compare the stages of $S$. vella (Fig. 42). $A$, Resting condition of rthe cell. $B$, Early stage of mitosis; the two flagella of the parent cell aro in process of absorption, together with their blepharo. plasts. $C$, Daughter-flagella arising at the poles of tho nuclear spindle; the flagclla of the parent have disappoared. $D$, Nucleus completely divided; one pair of daughter-flagella are seen arising from the karyosome of a daughternucleus, in which the blepharoplasts are still enclosed ; in the other daughternucleus the blepharoplasts have become distinct and the flagolla are given off from them. $E$, Similar stage ; the two pairs of blepharoplasts, from which the flagella arise, are quite independent of the two daughter-nuclei. After Hartmann and Chagas (62), magnification about 2,400 diameters.

blepharoplast (Fig. $41, C, D, E$; Fig. $42, C$ ), or they may not arise from the blepharoplasts until a later period, after they have separated off from the definitive centrosomes (Fig. 42, $D, E, F$ ). The examples figured show that these differences in the origin of the flagella may occur as developmental variations in one and the same species.

In the second place, the blepharoplasts and flagella may persist throughout the division of the cell; then either the old flagellum and blepharoplast are retained by one daughter-cell, while the other 
forms a new blepharoplast from its centrosome, and subsequently a new flagellum; or the blepharoplast of the parent cell divides independently to form the blepharoplasts of the daughter-cells (Fig. 43). In this last type, the blepharoplast, though obviously a body of centrosomic naturc, acquires a more or less complete independence of the definitive centrosome, and becomes a distinct cell-organ, permanent for at least a certain number of cell-generations; it may multiply and undergo various structural complications, to be described presently.
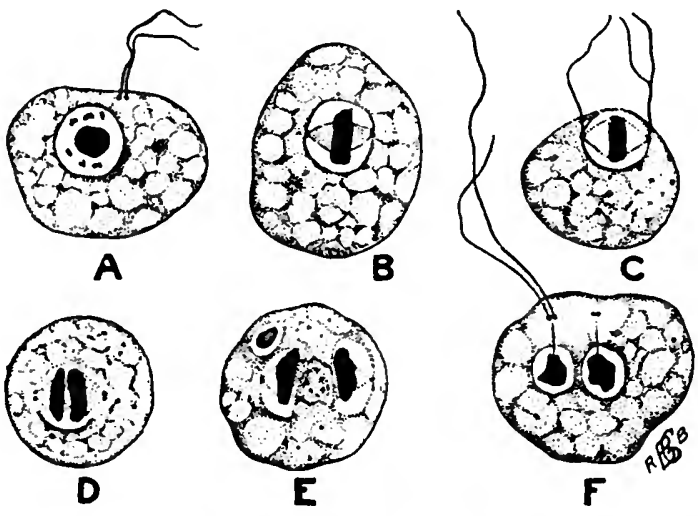

FIo. 42.-Stages in the division of Spongomonas uvella. A, Resting condition of the cell ; two flagella arise, each from one of a pair of blepharoplasts (diplosome); the nucleus contains a large karyosomo, in which the centriole is lodged, and a few irregular grains of peripheral chromatin in the nuclear cavity. $B$, Early stage of mitosis ; an achromatinic spindle is formed with the centrioles at tho poles, one contriole (on the right) having already divided into two; the chromatin, both periphcral and central, has united to form a dense equatorial plate in which separate chromosomes cannot be discernod; the flagella have disappeared, together with their blepharoplasts. $C$, Similar stage in which the daughter-flagella are growing out precociously from the eentrioles, ono on the left, two on the right. D, Later stago in which the erfiatorial plate has split into two daughter-plates, but no flagella havo as yet grown out from the centrioles, of which there are two at cach pole. $E$, jivision of the nueleus nearly complcte; no flagella. $F$, Nucleus completely clivided, daughter-nuclei in process of reconstruction; from each a pair of blephn roplasts has been budded off, still connected by a eentrodesmose with the centriole eontained in the karyosomo; a pair of daughter-flagella has arisen froul onc pair of blepharoplasls, but not as yet from the other. After Hartuanu and ('hagas (62), magnification about 2,400.

3. In certain flagellactes-for example, trypanosomes and allied forms (" Binuchata ") - - the cell-body contains two nuclei, as already noted: a trophonucleus and a kinetonucleus. To what cxtent these nuclei are provided with centrosomes is at present a little doubtful ; probably this point is one which varies in different cases (compare Wenyon, 84). There are, however; three chief possibilities: (a) There may be but a single centrosome, that of the linetonucleus, whieh acts both as blepharoplast and division-centre 


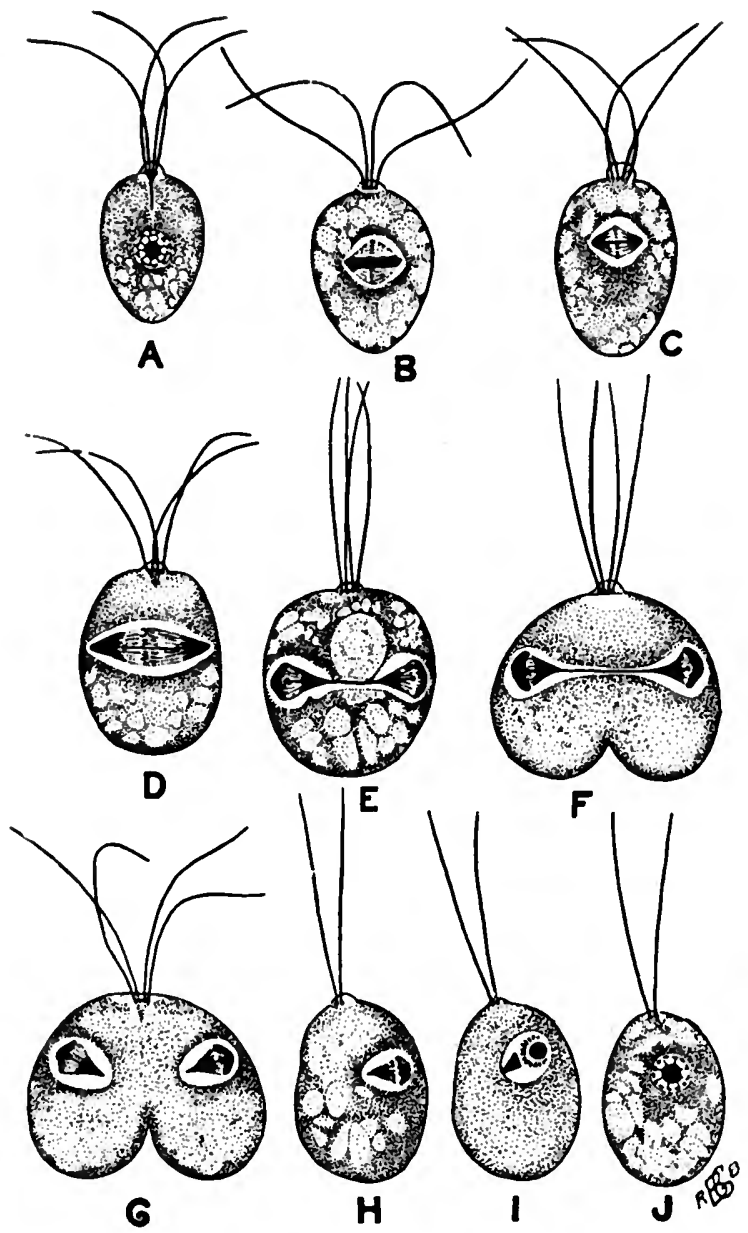

FIG. 43.-Stages in tho division of Polytomella agilis. $A$, Resting condition of the cell; the four flagella ariso from four blopharoplasts which are connected by a rhizoplast with the nucleus; in the nucleus is secn a largo karyosome, which contains the centriole and is surrounded by a peripheral zone of chromatin-grains in a nuclear reticulum. $B$, Early stago of mitosis ; the karyosome is dividing to form a bar of chromatin occupying the axis of the achromatinic spindlc, at the equator of which a plato of chromosomes is formed by the peripheral chromatin of tho last stage. $C$, Later stage; tho karyosome has divided complctely, forming two masses at the poles of the spindle connected by a centrodesmose. $D$, Tho spindle has become elongated, and the equatorial plate has split; the centrioles are seen connectod by tho centrodesmosc. $E$, Division advancing; the polar masses have become cap-shaped, and the daughter-plates of chromosomes have fused into conical masses; centrioles and centrodesmose still visible. $F$, Division of body beginning. $G$, Centrodesmose broken through, the two daughter-nucloi soparate. $H, I, J$, Division of ccll complete, one daughter-cell only ropresonted, to show the reconstitution of the daughter-nuclcus; the polar cap becomos the karyosome, enclosing the centriole; the conical mass formed in Stage $E$ by fusion of the chromosomes in the daughter-plates becomes resolved gradually into chromatin-grains again, and so forms tho peripheral zone of the daughternucleus; each daughter-coll has two of the four blepharoplasts and flagella of the parent, and the number is doubtless made up to four again by division after the daughter-cells aro set free. After Aragao (45). 
for the cell ; then, when cell-division takes place, the kinetonucleus first divides, and the two products of its division place themselves on each side of the trophonucleus and act as its centrosomes, as described by Franca and Athias $(56)^{*}$; $(b)$ the trophonucleus may have a centrosome of its own, lodged in the karyosome, in addition to the centrosome-blepharoplast in connection with the kinetonucleus ; this is probably the most usual condition with two subordinate variations, according as the centrosome-blepharoplast is lodged within the kinetonucleus, as in Leishmania tropica (Wenyon, 84), or is situated close beside it, as in most trypanosomes; in either case the kinetonucleus,and trophonucleus divide quite independently of one another, as commonly seen; $(c)$ it is possible, but perhaps not very probable, that in some cases there may be a blepharoplast for the flagellum distinct from the centrosomes of the two nuclei ; such a condition, perhaps, occurs in Trypanoplasma. In all cases alike, division is initiated by the centrosome from which the flagellum arises; next the kinetonucleus, and lastly the trophonucleus, divide.

The various forms of flagellar insertion described in the foregoing paragraphs admit of a simple and uniform phylogenetic explanation. Starting with a non-flagellated organism in which a simplo protokaryon contains a single centriole (Fig. 44, $O^{\mathrm{a}}$ ), we may suppose the flagellum at its first origin to grow out from the centriole in the nuclens $\left(O^{\mathrm{b}}\right)$. No such condition is actually known amongst flagellates, though it may be compared to the origin of the axopodia from a central grain in an Actinophrys-type of Heliozoon (see below); in the flagellates the controsomo-blepharoplast always, apparently, moves out of the nucleus, either remaining in close proximity to it $\left(1^{\mathrm{a}}\right)$ or becoming quite independent of it $\left(\mathrm{I}^{\mathrm{b}}\right)$, the two variations of the first type.

The second.type may be derived by division of the centrosome-blopharo. plast to form the definitive centriole and the blepharoplast; the latter may also remain in elose proximity to the nucleus $\left(2^{\mathrm{a}}\right)$ or become quite independent of it $\left(2^{\mathrm{b}}\right)$.

The third type may be supposed to ariso from the hypothotical primitivo condition $\left(O^{\text {n }}\right)$ by supposing that, not the blepharoplast-controsome alone, but the whole nucleus, divides to form two nuclei of unequal sizo and distinct function, the trophonucleus and kinetonucleus, oach with its own centriole $\left(3^{\mathrm{a}}, 3^{\mathrm{b}}\right)$. The centriolo of the kinetonucleus, which is at the same time the blepharoplast, may either remain within the kinotonuclous $\left(3^{b}\right)$ or come out of it $\left(3^{c}\right)$; its relations to the kinetonucleus are parallel to those of the centrosome-blepharoplast to the nucleus in types $1^{a}$ and $1^{b}$. Or, on the other hand, the centrosome-blepharoplast may divide into a definitive kinetonuclear centrosome and a true blepharoplast $\left(3^{\circ}\right)$. The condition with only a single centriole for both the nuclei may, if it exists, be derived from $3^{\mathrm{a}}$ or $3^{\mathrm{b}}$ by supposing that the trophonuclear centrosome becomes atrophiod.

When a blepharoplast exists independently of the nuclear apparatus, it may retain the form of a single grain or basal granule of the flagellum, when this organ is single, or it may multiply to

* The statements of Franca and Athias are not, however, eonfirmed by Lobedeff (468), and it may be doubted whether any species of trypanosome or other " binueleate" exists which has but a single division-eentre in the cell. 
form two or more grains when there are numerous flagella. Thus, in Lophomonas, which shows the extreme of complication, there are numerous basal granules corresponding to the tuft of flagella (Fig. 45). Each basal granule in this case is divided into a proximal

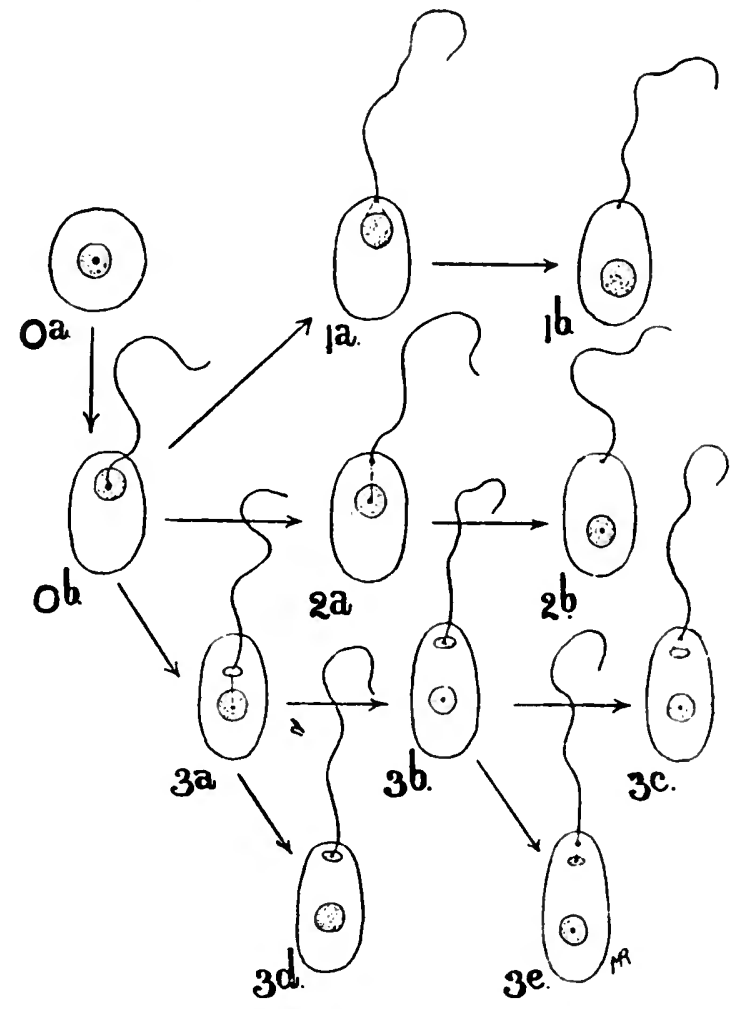

Fra. 44.-Diagranmatic representation of the possible phylogenetic origin of the different types of flagellar attachment in flagellates. For the salie of simplicity it is supposed that the animal has lout a single llagellun. ("), Nonflugellated cell with a centriole in the nuclous; $O^{1}$, in a cell like the last a flagellum arises from the centriole; $1^{\mathrm{a}}$, condition with a flagellum arising close beside the nucleus; $1^{b}$, condition with the blepharoplast quite separate from the nucleus; $2^{\mathrm{a}}$, division of the single centriole into a definitive centrosome and a blepharoplast, which becomes quite independent $\left(2^{i}\right)$ of the nucleus; $3^{4}$, division of both nucleus and centriole to form distinct kinetic and trophic nucloi, each with its own centriole ; $3^{\text {b }}$, the kinetonuclear centriole remains within tho nucleus; 3 , the kinetonuclear centriole becomes distinet from the nucleus; $3^{\mathrm{d}}$, condition with a single centriole in tlie cell $; 3^{\mathrm{e}}$, condition with a blepharoplast distinct from the centrioles of the two nuclei.

and a distal granule, and the pairs of granules are arranged in a ring, interrupted at one point ; the tuft of flagella takes origin from the distal granules of the ring. When the nucleus divides, the daughter-centrosomes give rise to new rings of blepharoplasts, 
from which daughter-tufts of flagella grow out; the old tufts, with their rings of blepharoplasts, persist for some time after the new ones have been formed (Fig. 45, $C$ ), but ultinately they degenerate and disappear. The ring of blepharoplasts in Lophomonas is

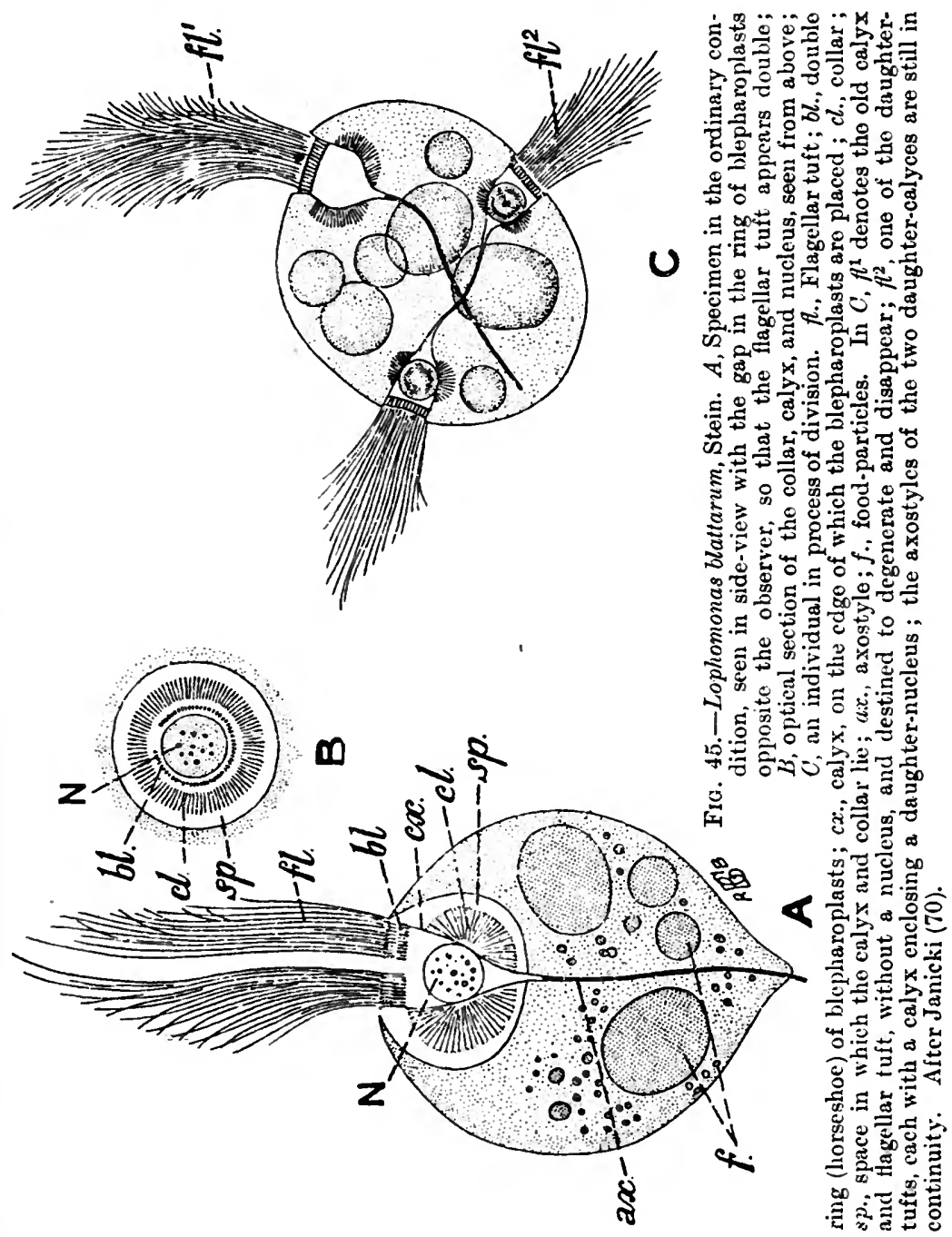

supported on the edge of a membranous structure, or "calyx," which in its turn is surrounded by a peculiar striated body, the "collar" of Grassi, or " parabasal apparatus" of Janicki (Fig. 45, cl.). Janicki (71) has found a corresponding parabasal apparatus in other flagellates, especially in Trichonymphidae; the significance 
of this peculiar structure remains for the present problematical. In the spores of Derbesia, Davis ("Annals of Botany," xxii., pp. 1-20, plates i. and ii.) has described a condition very similar to that of Lophomonas-namely, a double ring of blepharoplasts, which, however, fuse together to form a ring of homogeneous appearance. The blepharoplast-grains are given off from the nucleus.

Centrosomic bodies may be related, not only to flagella, but also to pseudopodia, especially in those cases in which the pseudopodia

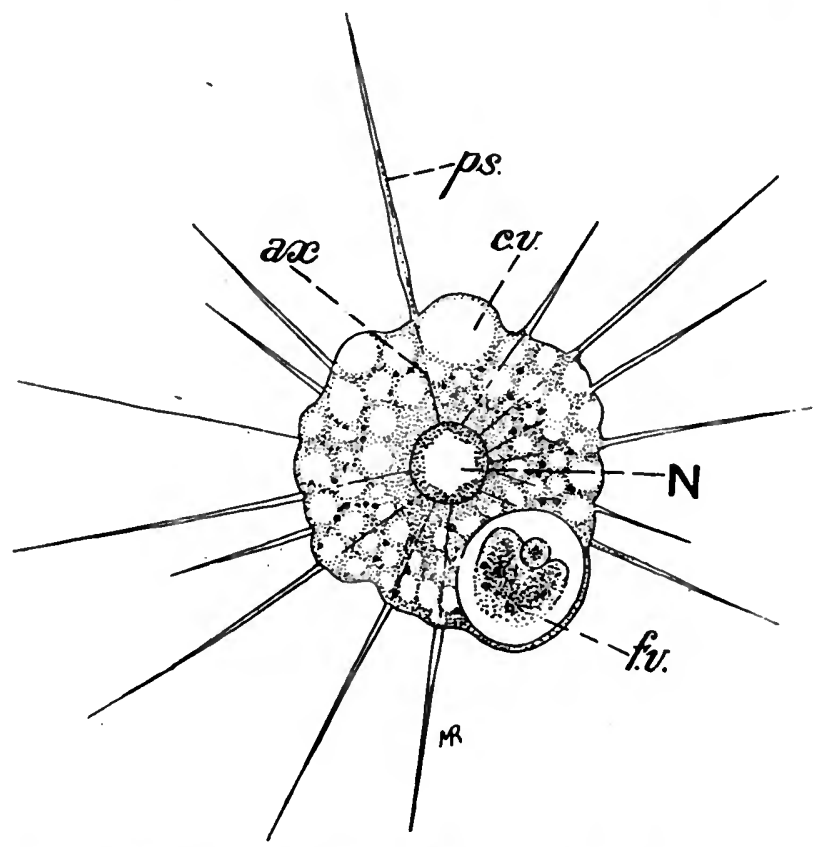

Frc. 46.-Actinophrys sol, showing the axial filaments of the pseudopodia centred on the nucleus. $N$, Nucleus ; ps., pseudopodia ; $a x$. , axial filament ; c.v., contractile vacuole; f.v., food-vacuole. After Grenacher.

have become specialized in form and movement, as in the Heliozoa. In this group the relationship of the nuclear apparatus to the pseudopodia exhibits two types of arrangement, which are analogous to the two arrangements described above in Mastigina and Mastigella respectively, and which may be explained by supposing that in the one case the kinetic centre lies within, in the other case without, the nucleus itself. Thus, in Actinophrys (Fig. 46) the numerous pseudopodia are all centred on the single nucleus, in which the centrosome is contained. A variation of this type is described by Schaudinn (43), in the peculiar multinucleate form 
Camptonema nutans, in which a pseudopodium arises directly from each nucleus (Fig. 47).* In Acanthocystis (Fig. 18) an example is seen of the second type, the evolution of which can be traced in the actual development; in the buds of Acanthocystis a centriole is contained in the karyosome of the nucleus, but during the growth

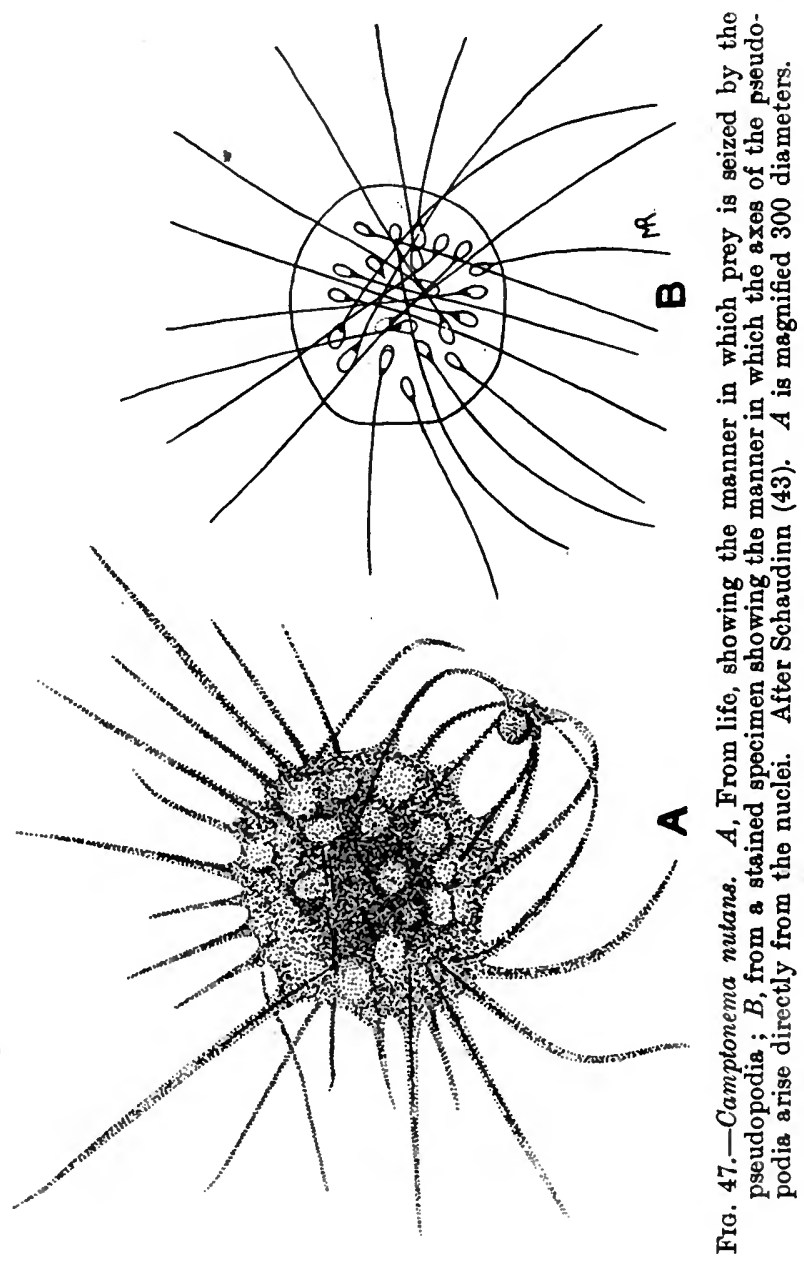

of the bud into the adult condition the centriole passes out of the nucleus, and bccomes the so-called "central grain" of the adult, a corpuscle which occupies the centre of the body, and upon which

* In Actinosphcerium, however, there is no relationship between the pseudopodia and the nuclei. From the researches of Hertwig (64), it is cvident that in this form the centrosomes are lost altogether during the vegetative life, and are formed only in eertain phases of the dovclopment (p. 115). 
the axial filaments of the pseudopodia are centred, while the nucleus is displaced to one side and beeomes excentric in position; when the cell enters upon division, the central grain beeomes the centrosome (Fig. 64).

From the condition soen in Acanthocystis, it is not difficult to explain tho stato of things which has been described by Zuelzer (86) in the remarkablo form Wagnerella (p. 246). Here also the buds formod possoss each a single nucleus containing a centriole; in this condition they may multiply by fission with mitosis, in which the centriole functions as a centrosome. When the buds develop into the adult form, a centriolo is extruded from the nucleus to form the central grain. The organism attaches itself, and the body becomes divided into three regions-liead, stalk, and basal plate (Fig. 48). The nucleus travels down into the basal plate, while tho central grain remains in the head and functions as tho kinetic centre of the pseudopodia, becoming very complicated in structure. It consists of a controsome surrounded by a sphere, which is perhaps of the nature of archoplasm, hut is stated to be rich in plastin; when the pseudopodia sre extended tho sphere shows wellmarkod radial striations. From the centrosome minute granules are buddod off, which pass along the striations of the sphere to its surface, and from these granulos arise the delicate axial filamonts of the pseudopodia; the basal granules are therofore comparable to the ring of blepharoplasts in Lophomonas. Whon the pseudopodia are rotracted, the basal granules lie within the sphore, inmodiately surrounding the centrosome, and the radial striations of the sphere vanish. The centrosome itself varies in structure at different times, going through cyclical changes, but usually showe a distinct contral granule or centriolo.

Whon Wagnerella divides by fission, the central grain and the nucleus divide independently, and the central grain does not act as a centrosome for the dividing nucleus, which contains its own centriole. In this form, therefore, the central grain, though centrosomic in origin and nature, losos its primitive relation to the division of the nucleus, and becomes specialized exclusively as a kinetic centre for the organs of locomotion, a course of evolu. tion perfectly parallel to that which hus boen traced abovo for the blepharoplasts in their relation to flagella.

While there can be but little doubt as to the centrosomic nature of the blepharoplasts or basal granules of the flagella, and of the central grains on which the pseudopodia of the Heliozoa are centred, the true nature of the basal grains of cilia, on the other hand, is less certain. The majority of those who have studied them in Ciliata are of opinion that they have nothing to do with centrosomes (compare Maier, 73, and Schuberg, 44, and see p. 443, infra); but there are certain observations which indieate that the basal granules of the cilia have a connection with (Collin, 50), or an origin from (Entz, 53), the nuelear apparatus, in which case they may be of the same nature as the multiple blepharoplasts of such a form as Lophomonas. Hertwig (66) considers that the basal grains of the cilia may be of centrosomic nature, and that, if they have no connection with the nucleus, they afford support for the view that centrosomes can arise from the cytoplasm as well as from the nuclear framework. In view of the great structural similarity between cilia and flagella in other respects, it seems 
hardly likely that the basal granules would be of a different nature in the two cases. The whole question of the nature of the basal granules has been discussed in a recent memoir by Erhard (54).

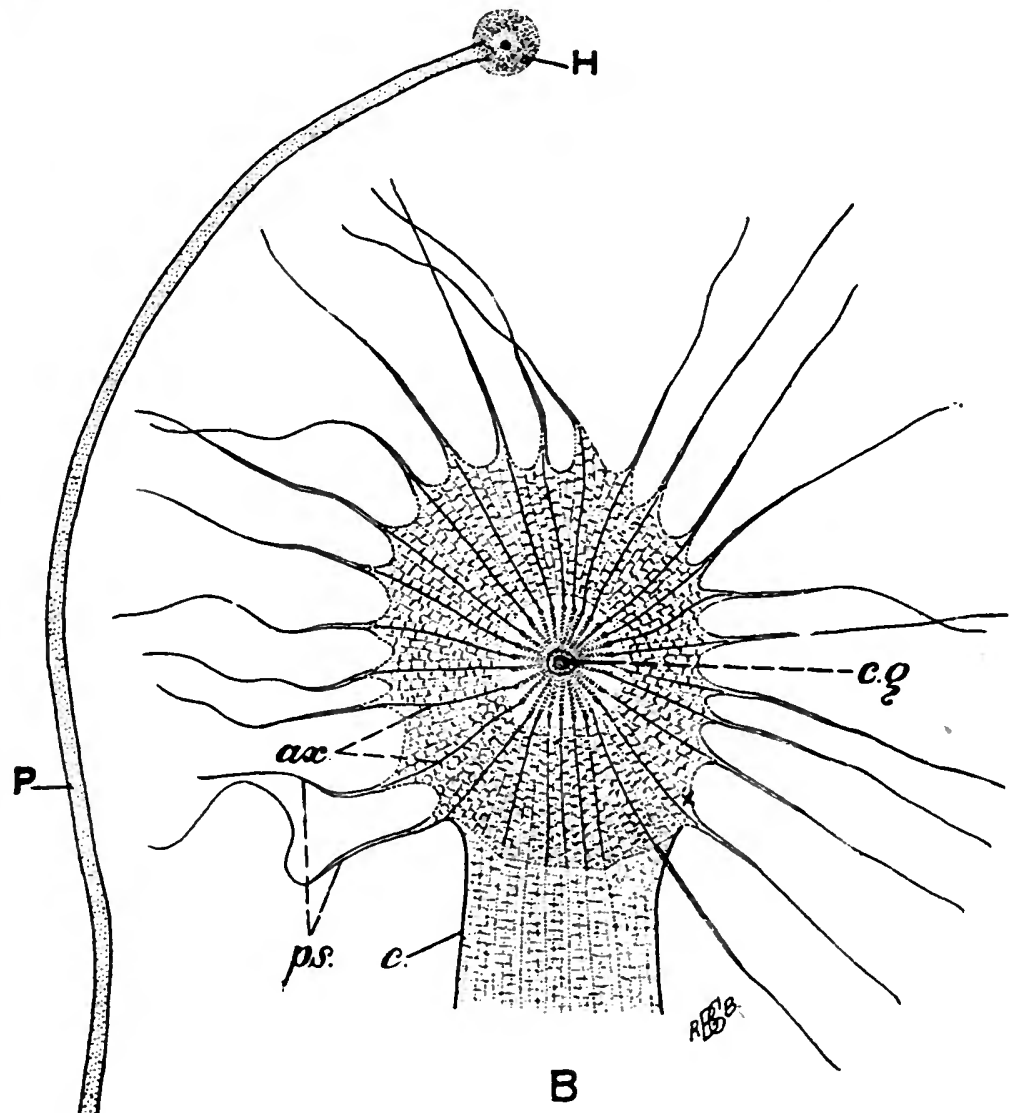

FIG. 48.-Wagnerella borealis, Mereschk. $A$, Whole specimen seen under a low magnification: $H$, head containing the central grain ; $P$, stalk ; $N$., nucleus contained in the basal plate of attachment. $B$, Enlarged view of the head, after fixation and staining with iron-hamatoxylin : c., cutielo of the stalk; p.s., pseudopodia; ax., axial filaments of the pseudoporlia, each urising from a basal granule; c.g., central grain. After Zuclzer (86).

Few problems in cytology have been more discussed than the question of the nature and origin of the centrosome, and three opposed views have been put forward which may be termed, respectively, the achromatinic theory, the nucleolo-centrosomic theory, and the nuclear theory. 
According to the achromatinic theory, the centrosome is "an individualized portion of the achromatinic nuclear substance" (Hertwig, 66), a kinetic centre on which the movements of the framework are focussed. The essential and primary constituent of the centrosome is the centriole, and so long as the centrosome remains intranuclear, as in perhaps the majority of Protozoa, it consists of the centriole alone. When, however, the centrosome becomes extranuclear, as in many Protozoa and almost universally
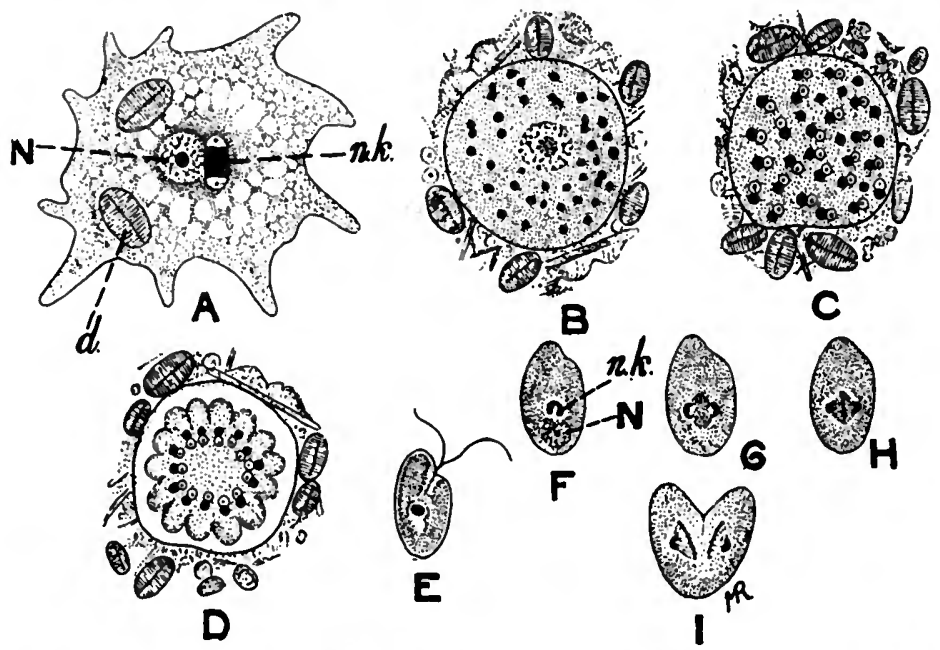

Fro. 49.-Paramoba eilhardi : stages of the life-cycle. A, Amœba in the vegetative stage: $N$., nuoleus ; n.k., "Nebenkern"; $d$., ingested diatom. $B, C, D$, Stages in the multiplication of the encysted amoba; in $B$ the Nebenkern has divided up, the nucleus is still undivided; in $C$ the nucleus has divided up into a number of daughter-nuclei, each of which has paired with a daughterNebenkern ; in $D$ the body has divided into a number of daughter-cells, each containing a nucleus and a Nebenkern. $E$, A free-swimming flagellula, dorived from one of the daughter-cells in $D$, and containing a nucleus and a Nebenkern. $F, G, H, I$, Four stages of the division of a flagellula ; in $F$ the Nebenkern is dividing; in $G$ the two halves of the Nebenkern have placed themselves on each side of the nucleus, which is preparing for division; $H$, stage of the nuclear spindle with the two halves of the Nebenkern at each pole; in $I$ the nnclear division is nearly complete, and the body is beginning to divide. After Schaudinn (81), all figures magnified about 500 diameters.

in the cells of the higher animals and plants, accessory cytoplasmic elements may be added to the centriole to form a centrosomic complex. A point still undecided, on the theory that centrosomes are of achromatinic origin, is whether or no these bodies can be formed, in some cases, in the cytoplasm also, as maintained by some authorities. On Hertwig's view, mentioned above, that the achromatinic substance of the nucleus is identical in nature with the ground-substance of the general protoplasm, it follows that material for the formation of the centrosome must be present in the cyto- 
plasm no less than in the nucleus. Bütschli (3) considers it possible that the centrosome might have been originally a cytoplasmic structure, which had nothing to do with the nucleus, but became mcluded in it when a nuclear membrane was formed.

Attention must be drawn here to the remarksble genus Paramoeba (Fig. 49) founded by Schaudinn for the species $P$. eilhardi (seo p. 228). In this form there is present beside the nucleus a body which was termed the "Nebenkern," consisting of a darkly-staining middle piece, at each end of which is a cap of clear substance. The Nebenkern has generally been considered to represent a centrosome, and Chatton (49) has put forward the suggestion that it may. correspond to a karyosome or a portion of a karyosome that has passed out of the nucleus with the centrosome. Recently, however, Janicki $(71 \cdot 5)$ has described two new species of Paramceba, and puts quite a different interpretation upon the Nebenkern. He regards the middle piece as chromatin, the clear caps as archoplasmic masses, each of which contains a centrosome; and he considers the entire structure "as a second nucleus, as it were, fixed in division, in which the state of division has become the permanent form." He proposes to replace Schaudinn's term "Nebenkern" by the term " nucleus secundus," and considers it especially comparable to the "sphere" of Noctiluca (Fig. 65). Division of the nucleus and Nebenkern takes place quite independently of one another.

On the nucleolo-centrosomic theory, the whole karyosome with the contained centriole, as found in many Protozoa, is compared with the complex extranuclear centrosome of the higher organisms. It is clear, however, that the karyosome consists chiefly of plastin which is impregnated to a greater or less extent with chromatin, and in which the centriole is imbedded. As Chatton (49) has pointed out, the three elements which compose the karyosome are independent of each other. When the centriole and chromatin have left the karyosome, the plastin-mass remaining behind is homologous in every way with the nucleolus of the metazoan cell, and the only element common to both the karyosome of Protozoa and the centrosome of Metazoa is the centriole.

The nuclear theory of the centrosome is associated especially with the names of Schaudinn and, in more recent times, of Hartmann and Prowazek (63). According to this view, the centrosome represents a second cell-nucleus, and every ccll is to be regarded as primarily binucleate. The starting-point of the evolutionary series would be such a form as $A$ moba binucleata, which possesses two similar and equivalent nuclei. In the nest stage of evolution one of the two nuclei became specialized more for kinetic, the other for trophic, functions ; examples of this stage would be furnished by Paramoeba (Fig. 49), with its nucleus and "Nebenkern," and by a trypanosome, with its trophonacleus and kinetonucleus, the Nebenkern of the first and the kinetonucleus of the second representing the kinetic nucleus. The central grain of the Heliozoa or the extranuclear centrosome of the Metazoa would represent the final stage of evolution, namely, a kinetic nucleus deprived of all 
chromatin-elements; while the cell-nucleus proper would represent the trophic nucleus deprived of all kinetic elements.

On the other land, the condition in amœbre and similar organisms, where the cell appears to contain but a single nucleus which includes the kinetic centres, is explained by supposing that here the kinetic nucleus is encapsuled in the trophic nueleus, and is represented by the karyosome with its centriole; henee the supporters of this theory term the type of nucleus characterized by a large karyosome an "amphinucleus" or " amphikaryon," and, in their descriptions of such nuclei, they speak of the onter nucleus (peripheral zone of chromatin) and the inner nucleus (karyosome).

The reasons against homologizing the karyosome and the extranuclear centrosome have been stated already. Against the theory of binuclearity it may be urged further-First, that to regard the protokaryon-type of nucleus scen in the most primitive forms of Sarcodina and Flagellata as a secondary condition is a complete inversion of what is, to all appearance, the natural scries of evolution of the nuclear apparatus; sccondly, that the binucleate condition of trypanosomes and allied forms is elearly, by comparison with other Flagellates, a specialized condition; the trophonucleus of trypanosomes also contains a karyosome and centriole, and would therefore be an "amphikaryon," on this theory; thirdly, that the binuclear theory still leaves the centriole as a kinetic centre of achromatinic origin, which is present in both trophonucleus and kinetonucleus of trypanosomes, in both nucleus and central grain of Heliozoa (Wagnerella), etc. All that the binuclear theory is capable of explaining is the secondary elements of the extranuclear centrosomic complex. That the centriole is a body of intranuclear origin and formation is shown clearly by its presence in nuclei of the primitive karyosomatic type which arise, not by division of pre-existing nuclei, but by aggregation and organization of clumps of chromidia. It should be added that, in its most recent exposition by Hartmann (61), the theory of binuclearity has undergone considerable modification and restriction.

Having considered now the structure and composition of the nucleus in its prineipal types and morphological variations, it remains to atteinpt to establish a more precise conception as to what exactly is meant by a nucleus. It is evident, in the first place, that the essential component of a nucleus, never absent, is chromatin ; but it is equally clear, in the second place, that a simple mass, or several such masses, of chromatin, do not by themselves constitute a nucleus in the true sense of the word. The word "chromatin" connotes an essentially physiological and biological conception, as it were, of a substance, far from uniform in its chemical 
nature, which has certain definite relations to the life-history and vital activities of the cell. The word " nucleus," on the other hand, as many authorities and more recently Dobell (52) have pointed out, is esscntially a morphological conception, as of a body, contained in the cell, which exhibits a structure and organization of a certain complexity, and in which the essential constituents, the chromatin-particles, are distributed, lodged, and maintained, in the midst of achromatinic elements which exhibit an organized arrangement, variable in different species, but more or less constant in the corresponding phases of the same species. If this standpoint be accepted, and the nucleus be regarded as an essentially morphological conception, it seems to me remarkable that Dobell, in his valuable memoir on the cytology of the bacteria, should apply the term " nucleus " to a single grain of chromatin, or to a collection of such grains, and should speak of a nucleus "in the form of chromidia scattered through the cell," or " in the form of a discrete system of granules (chromidia)," phrases which are self-contradictory on the principles that he himself has laid down.

Wc are confronted, nevertheless, with a considerable difficulty when we attempt to state exactly what amount of organization and structural complexity is-essential to the morphological conception of a nucleus. If, as is probable in phylogeny, and certainly occurs frequently in ontogeny (compare Fig. 32), the nucleus arises from a primitive chromidial condition of scattered, unorganized chromatin, at what point does the mass cease to be a chromidium and become a nucleus? This is a question very difficult to answer at present, a verbal and logical difficulty such as occurs in all cases where a distinction has to be drawn between two things which shade off, the one into the other, by infinite gradations, but which does not, nevertheless, render such distinctions invalid, any nore than the gradual transition from spring to summer does away with the distinction between the seasons. Hartmann and his school consider the possession of a centriole as the criterion of a nucleus (see Nägler, 76) ; but it cannot be considered as established, in the present state of knowledge, that all nuclei have centrioles or centrosomes. All that can be said is that, as soon as a mass or a number of particles of chromatin begin to concentrate and separate themselves from the surrounding protoplasm, with formation of distinct nuclear sap and appearance of achromatinic supporting elements, we have the beginning at least of that definite organization and structural complexity which is the criterion of a nucleus as distinguished from a chromidial mass.

In the first chapter of this book a distinction was drawn between organisms of the "cellular" gradc, with distinct nucleus and cytoplasm, and those of the "bacterial" grade, in which the 
chromatin does not form a distinct nucleus. In all Protozoa there is a true nucleus in at least the principal stages of the life-history, and it is obvious that the recognition of a cellular grade, characterized by the possession of a true nucleus, postulates that the first origin and evolution of the nucleus must be sought amongst those organisms which have been classed, speaking broadly, as the bacterial grade. We may expect, therefore, to find in organisms which stand on the plane of morphological differentiation which characterizes the bacteria the early stages of the evolution of the nucleus from the primitive chromidial condition, and even cases in which the condition of a true nucleus has been reached. The matter cannot be discussed further here, where it must suffice to establish the existence of true nuclei in Protozoa ; but Dobell (52) has described an interesting series of conditions whioh may be regarded as stages in the evolution of nuclei amongst bacterial organisms.

Since the possession of a true nucleus has been regarded here as the criterion of the cellular grade of organization, it is necessary to discuss briefly the meaning and application of the term "cell." By many, perhaps most modern writers, the cell has been regarded as the elementary vital unit, than which there exists nothing simpler apongst living beings. In this sense the word "cell" becomes synonymous with the term " micro-organism," "protist," or any other word used to denote living beings of the most primitive type : "tout ce qui vit n'est que cellules" (Delago and Hérouard, 6). The ,word "cell" was, however, applied originally to the elements that bnilt up the tissues of animals and plants. At first, as the word cell implies, it was used to denote only the enclosing membrane or framework; but when it became apparent that the membrane was of secondary importance, it was transferred to the contained stuff, and so came to signify a structural element in which the living substance, protoplasm, is differentiated into two distinot partsnucleus and cytoplasm. If the term "cell" is not to become so vague and indefinite in its significance as to be absolutely meaningless, it is best to restrict its application to living organisms which have reached this degree of differentiation. Dobell considers that all Protista are nucleated organisms; in the preceding paragraphs reasons have been advanced against accepting this proposition as a statement of fact, and from the point of view of phylogenetic speculation, I, at least, find it difficult to believe that the earliest form of life could have been an organism in which the living substance was differentiatcd $a b$ initio into distinct nucleus and cytoplasm.

In my opinion the cell, as defined above-that is to say, an organism in which the living substance, protoplasm, has become 
differentiated into two parts, a nucleus, in the morphological sense, distinct from the cytoplasm-does not represent the primary and universal form of the living organism or unit, but is to be considered as a stage in the evolution of living beings, a stage which many living beings have not reached. Thus a bacterial typé of organism, in which the chromatin is scattered through the protoplasmic body in the form of chromidial granules, and which there. fore does not possess a true nucleus, is not to be regarded as a cell, but as representing a condition antecedent to the evolution of the true cellular type of structure. In all Protozoa, on the other hand, the entire plan of the organization is founded on the type of the cell, which is to be regarded as the starting-point in the evolution of the entire animal and vegetable kingdoms (compare Minchin, 75). This point will be discussed further in a.subsequent chapter (p. 464).

Bibliography.-For references see p. 477. 


\section{CHAPTER VII}

\section{THE REPRODUCTION OF THE PROTOZOA}

THE methods by which reproduction is effected amongst the Protozoa vary greatly in matters of detail, as will be seen; but the obvious diversity in method throws into greater relief the underlying unity in principle. In Protozoa, as in Protista generally, reproduction takes place always by means of some form of fissionthat is to say, division or cleavage of the body into two or more parts, whieh are set free as the daughter-individuals. An essential part of the process is the partition amongst the daughter-individuals of at least some part of the chromatin-substance possessed by the parent. Hence fission of the cell-body as a whole is always preceded by division of the nucleus; and if chromidia are present, they also are divided amongst the products of the fission of the body. On the other hand, division of the nucleus is not necessarily followed at once by division of the body.

Considering the methods by which fission is effected from a general standpoint, we may distinguish three chief types of reproduction, each of which may show subordinate variations :

1. Division of the nucleus, or, if there are two differentiated nuclei, division of each of them, is followed by division of the body ; this is the commonest and most typical mode of reproduction, hnown as simple or binary fission.

2. Division of the nucleus or of each of two differentiated nuclei is not followed immediately by corresponding divisions of the hody, hut may be repeated several times, and so give rise to a multinucleate cundition of the body, which may be-

(a) Temporary, and soon followed by eleavage of the body into as many daughter-individuals as there are nuclei or pairs of differentiated nuclei; this method is known as multiple fission (Fig. 127) ; or it may be-

(b) Permanent, giving rise to a multinucleate body which is termed a plasinodium. Then division of the body may take place at any time by cleavage of the body into two or more inultinucleate parts ; this process is known as plasmotomy. Ultimately, however, in all cases a plasmodium breaks up by multiple fission into uni- 
nucleate individuals at the end of a longer or shorter vegetative existence during which it may have multiplied frequently by plasmotomy.

The process of fission must now be considered in more detail, beginning with-

1. Division of the Nucleus.-As in the case of the cell-body as a whole, the division of the nucleus is effected in various ways. Probably the most primitive type is that in which the nucleus becomes resolved into chromidia, from which, again, secondary daughter-nuclei are reconstituted. This type of division may be termed " chi omidial fragmentation." It is of comparatively rare occurrence, but examples of it are found among Sarcodina and Sporozoa. In Echinopyxis two daughter-nuclei are formed in this way (Hertwig, 66, p. 8). In other cases nuinerous daughter-nuclei may arise, as in the formation of the nuclei of the microgametes in Coccidium (Fig. 50), where the parent nucleus gives off into the cytoplasm a fine dust of chromidial particles which travcl to the surface of the cell and become concentrated at a number of spots to form the daughter-nuclei.

True nuclear division, in which the parent and danghter-nuclei retain throughout the process their individuality and distinctness from the cytoplasm, must be distinguished clearly from the abovementioned process of chromidial fragmentation. In the vast majority of cases the nucleus divides into two halves by simple or binary fission, which, as already stated, may be repeated several times before cell-division takes place ; but in a few cases the sucpleus divides simultaneously into a number of portions by $m$ ltiple fission.

In the cells of Metazoa true nuclear division alone occurs, and may follow one or the other of two sharply-marked types, termed comprehensively direct and indirect. In direct division the nucleus is constricted simply into two parts, without circumstance or ceremony. In indircet division, on the other hand, the nuclcus goes through a complicated series of changes, following each other in a definite order and sequence, the whole process being known as karyokinesis or mitosis. In spite, however, of the intricate nature of karyokinetic division, and the variations in matters of detail that it exhibits in different cases, the whole process is perfectly uniform in its general plan, and admits of being described without difficulty in generalized terms. Such a description is found in every textbook of biology at the present time, and need not be repeated here; it will be sufficient to analyze briefly the more important events that take place.

In the process of karyokinesis, the achromatinic elements of the nucleus furnish the active mechanisms, while the chromatin-sub- 
stance appears to be the passive subject of the changes that are effected. With the achromatinic nuclear elements, extranuclear cytoplasmic substanoes, such as archoplasm, may collaborate. After
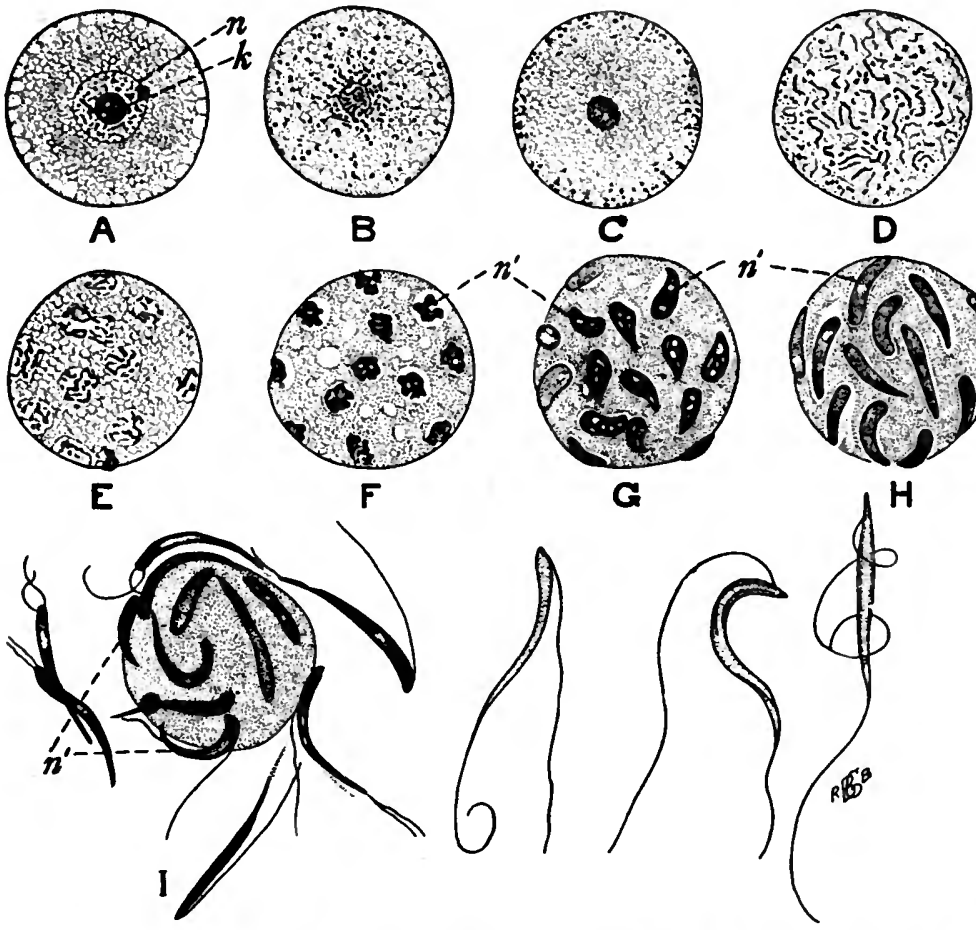

Fra. 50.-Formation of microgametes in Coccidium schubergi. A, Full-grown microgametocyte, with finely-granular cytoplasm and large nucleus containing a conspicuous karyosome ; freed from the host-cell. $B$, The nuclear membrane has disappeared, and the chromatin, in the form of minute chro. midial granules, is passing out into tho cell. $C$, The chromidia have collected at the periphery of the body ; the karyosome is left at the centro, and has become pale through loss of chromatin-substance. $D$, The chromidia, seen on the surface of the body, are collecting together into irregular streaks and clumps. $E$, The chromatin-streaks of tho preceding stage aro collecting together into patches. $F$, The patches of chromatin of the preceding stage have become dense and closely packed. $G, H$, The patches of chromatin take on a definite form as the futuro nuclei of the microgametes. I, Two flagella grow out from close to each microgamete-nucleus, and by their activity the microgametes, consisting almost entirely of chromatin, break loose from the body of the gametocyte and swim away. $J$, Three microgametes, more highly magnified; in each, two flagella ariso from the thicker ind ; one of the flagella (the shorter) becomes free at once, the other (the lnnger) runs along the body and becomes free at the hinder end. n., Nuclous, $\boldsymbol{k}$, karyosome, of the microgametocyte; $n^{\prime}, n^{\prime}$, nuclei of the microgametes. After Schaudinn (99); $A-E$ magnified $1,000, F-I$ magnified 1,500 , $J$ magnified 2,250.

disappearance, as a rule, of the nuclear membrane the achromatinic substance, or the combination of achromatinic and archoplasmic 
elements, assumes a characteristic bipolar form, like a spindle. At each pole of the spindle a centrosome or centriole is to be found, as a general rule. The two centrosomes have arisen by division of the originally single centrosome, and may remain for some time connected by a fibril or by a system of fibrils, forming what is often termed a "central spindle," but is better named a centrodesmose. The axis of the achromatinio spindle is formed by the centrodesmose, if it persists, and the remainder of the spindle is constituted by the socalled " mantle-fibres" running from pole to pole. The mantle-fibres are derived from the achromatinic reticulum of the nuclens and the archoplasm ; they are probably in most cases the optical expression of an arrangement of the protoplasmic alveoli in longitudinal rows, under the influence of tensions or forces centred at the poles of the spindle. Such an arrangement of the alveoli produces the optical appearance of fibrils connected by cross-junotions, the apparent fibril being formed by thickened walls of alveoli in line with one another, while the cross-junctions are the trensverse walls between consecutive alveoli. On this view the apparent fibrils of the achromatinic spindle are in reality merely the indication of lines of force in the protoplasmic framework ; but some authorities consider that in certain cases at least true fibrils are formed, which may be isolated from each other and without cross-connections (Hertwig, 64). The spindle-fibres, whether real or apparent, are centred at the poles of the spindle on the centrosomes, from which other striations may radiate out in all directions through the archoplasmic masses (" attraction-spheres"), and extend into the surrounding cytoplasm.

While the achromatinic spindle-figure is in process of formation, the chromatin of the nucleus has gone through a series of changes which may differ in different cases, but which result in the formation of a number of masses of chromatin termed chromosomes. The number, size, and shape, of the chromosomes vary greatly in different species, but in Metazos these characters are generally constant for the corresponding phases of the same species. Each chromosome, when formed, consists of a great number of minute grains of chromatin, chromidiosomes, cemented together in a matrix or ground-substance of plastin. The chromosomes arrange themselves at the equator of the achromatinic spindle in the form of a plate, hence termed the equatorial plate. The nucleolus disappears, being absorbed or cast out, and does not contribute to the karyokinetic figure, but a part at least of its substance probably furnishes the plastin ground-substance of the chromosomes.

At this phase, when the achromatinic spindle is fully formed, with the plate of chromosomes at its equator, the actual partition of the chromatin between the two future daughter-nuclei usually begins, 
though in some cases it is accomplished at an earlier stage; it takes place in one of two ways, known respectively as equating and reducing division. In equating division each chromosome divides into two daughter-chromosomes, a process which, in the finished and perfect karyokinesis of the higher organisms, is effected by a longitudinal splitting of the chromosome, and which may be interpreted as a simple division into two of each of the component chromidiosomes (compare Fig. 60). In reducing division, on the other hand, the individual chromosomes do not divide, but are sorted out, half of them going to one pole of the spindle, and eventually to one daughter-nucleus, the other half to the other; with the result, finally, that each daughter-nucleus has half the number of chromosomes possessed originally by the parent nucleus. Equating division is the usual type of karyokinesis seen in ordinary cellmultiplication; reducing division, on the other hand, is seen only in certain phases of the maturation of the germ-cells, as explained in the next chapter.

In either type of division, whether equating or reducing, the equatorial plate of chromosomes as a whole divides into two daughter-plates, which separate from one another and travel towards the poles of the achromatinic spindle. As the daughter-plates move away from each other, an achromatinic framework appears between them, in which a longitudinal striation or fibrillation is seen in line with, and continuing that of, the achromatinic spindle. Hence the achromatinic spindle as a whole consists now of the older terminal portions passing from the poles to the daughter-plates, and a new median portion passing between the two daughter-plates; the two terminal portions constitute together what may be termed conveniently the "attraction-spindle," the median portion the "separationspindle." As the daughter-plates travel further apart, the separation-spindle elongates more and more; the attraction-spindle, on the other hand, becomes shorter, usually to such a degree that the daughter-plates are brought close up to the poles of the attractionspindle, which consequently is obliterated and disappears. Whon full separation of the daughter-plates is attained, the separationspindle breaks down and disappears gradually, the middle part alone persisting in some cases; the chromatin of the daughter-plates becomes rearranged to form the daughter-nuclei, going through a series of changes similar to those by which the chromosomes arose from the parent-nucleus, but in inverse order. A nuclear inembrane is formed round each daughter-nucleus, and the process is complete.

In the Metazoa, direct and karyokinetic division stand out as the sole types of nuclear division, in sharp contrast and without intermediate or transitional forms of the proeess. In Protozoa, on the 
contrary, every possible form of nuclear division is found, from the most simple and direct to karyokinesis as perfect as that seen in the Metazoa. The nuclear division-processes of Protozoa are therefore exceedingly interesting as furnishing object-lessons in the gradual evolution of the mechanism of nuclear division; but the extreme diversity in these processes makes it very difficult to deal with them in the Protozoa in a general and comprehensive manner in a short space and without excessive detail. Speaking generally, the indirect nuclear division seen in Protozoa differs from that of the higher organisms in a number of points which indicate that it stands on a lower grade of evolution. As regards the achromatinic elements, the nuclear membrane is usually persistent throughout the process of division, a ciroumstance which enables a sharp distinction to be drawn between the portions of the division-mechanism derived from the nuclear framework and the cytoplasm respectively. In many cases it is then seen that the cytoplasm does not take any share in the process at all, but that the nucleus divides in a perfectly autonomous manner, spindle and centrioles remaining intranuclear throughout the whole process. As regards the chromatin, the chromosomes when formed are often irregular in form, size, and number; they often appear imperfectly separated from onc another ; they are not always arranged in a definite equatorial plate, but may be scattered irregularly along the spindle; and they do not always split in the exact manner characteristic of the nuclear divisions of the higher organisms, but divide irregularly and often transversely.

The principal types of nuclear division in Protozoa will now be described with the aid of a few selected examples. We may begin with those in which the division of the nucleus is autonomous, without co-operation of cytoplasmic elements.

Division has often been asserted to be direct in cases in which subsequent research has revealed a more elaborate type; nevertheless, many typical cases of amitosis occur among Protozoa. In some nuclei of the vesicular type, the chromatin appears to be concentrated entirely in the karyosome, which may contain a centriole also, and when the nucleus divides the karyosome becomes dumb-bell-shaped, and is finally constricted into two halves, the entire nucleus following suit; as an example of this, almost the simplest conceivable type of nuclear division, may be cited the nuclei of the Microsporidia and allied organisms (Fig. 173, p. 416).

A type similar in the main to that just described, but slightly more advanced in structural complication, is exemplified by the division of the nucleus in the schizogony of Coccidium (Fig. 51, $F-M$ ); here there is a peripheral zone of chromatin and a more distinct nuclear membranc. After division of the karyosome, the peripheral 
chromatin is halved irregularly; no definite chromosomes are formed, but the grains of peripheral chromatin form clumps and masses of various shapes and sizes. A definite achromatinic spindle

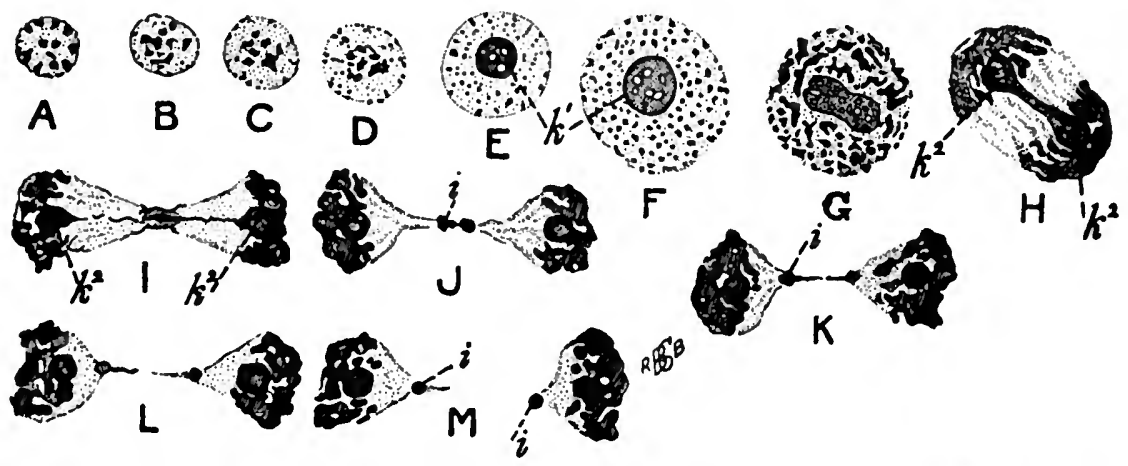

Fig. 51.-Formation of the karyosome and division of the nucleus in the schizont of Coccidium schubergi. $A$, Nucleus of the sporozoite, with scattered grains of chromatin but no karyosome. $B, C, D$, Nuclei of young schizonts in which larger grains of chromatin collect together at the centre to form the karyosome. $E$, Nucleus of older schizont with complete karyosome. $F$, Nucleus of full-grown schizont. $G-M$, Division of the nucleus of the schizont; the chromatin of the nucleus becomes aggregated into larger clumps and the karyosome becomes dumb-bell-shaped,, with masses of chromatin at each pole $(G$ and $H)$; the two daughter-karyosomes, at first connetted by a filament or centrodesmose, travel apart, taking the polar clumps of chromatin with them $(I)$; the centrodesmose breaks through and disappears, and the two daughter-nuclei travel apart, with formation of an intermediate body on the filament between them $(J-L)$; finally the connecting filament breaks down and the daughter-nuclei separate $(M)$. $k^{1}$, Karyosome; $k^{2}, k^{2}$, daughterkaryosomes ; $i$., intermediate body. After Schandinn (99), magnified 2,250.
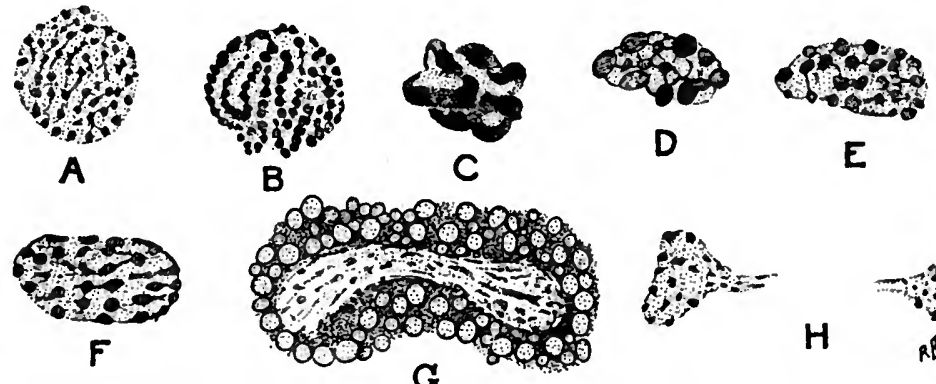

$\mathrm{H}$

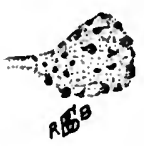

Fio. 52.-Direct division of the nuclei in the oöcyst of Coccidium schubergi. $A$, The resting nuclcus; $B, C, D$, clumping together of the chromatin-granules preparatory to division; $E, F, G$, the nucleus elongates and becomes dumbbell-shaped; $H$, the nucleus has just divided into two halves. After Sohaudinn (99), magnified 2,250.

also does not become differentiated. As the daughter-karyosomes, connected by a centrodesmose, travel apart, half the peripheral chromatin follows one karyosome, half the other. This method of 
division is a very common one in the nuclei of Protozoa, and may show a further advance towards a true mitosis in that the peripheral chromatin may shape itself into more or less definite chromosomes, as in Euglena.

Examples of granular nuclei which divide in the direct method are seen in the division of the nucleus of the oöcyst of Coccidium (Fig. 52) to form the nuclei of the sporoblasts (see p. 349, infra) and in the corresponding divisions of the nuclei of hæmogregarines (Fig. 53). In these two cases the presence of a centriole in the nucleus is doubtful, but is affirmed by Hartmann and Chagas (89) for hæmogregarines; a true nuclear membrane,

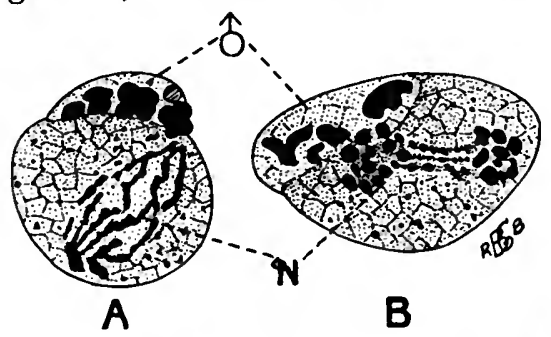

Fra. 53.-Direct division of the nucleus in the zygote of Homogregarina stepanoui. $\delta$, Degenerating male elements attached to the zygote; $N$., dividing nucleus of the zygote, two successive stages $(A$ and $B$ ). After Reichenow (78).

however, appears to be absent, and this form of division is not much advanced beyond the condition of chromidial fragmentation. In the macronucleus of Infusoria (Fig. 54), in which a distinct membrane is present, the division is also direct, and centrioles are stated to be absent as a general rule; in some cases, however, true centrioles appear to be present (Nägler). When centrioles are absent, the achromatinic framework of

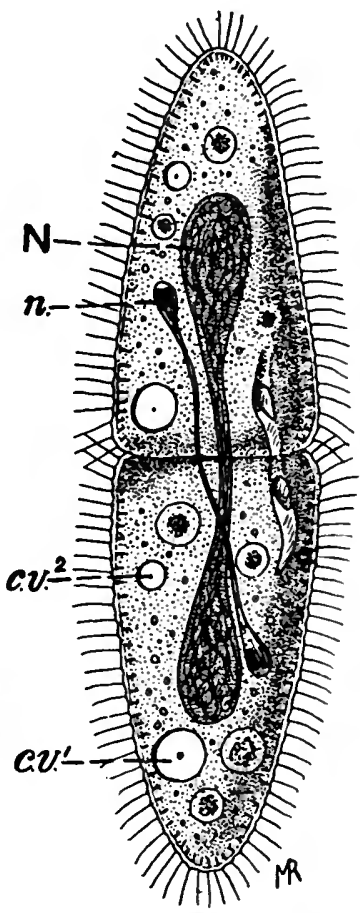

Fra. 54-Paramecium caudatum : division showing the macronucleus $(N)$ dividing without mitosis, the micronucleus $(n)$ dividing mitotically. c.v. 1 Old, and c.v. ${ }^{2}$, new, contractile vacuoles. After Bütschli and Schewiakoff, in Leuckart and Nitsche's Zoologische Wandtafeln, No. Ixv. the nucleus appears to be principally active in the division. In some cases the division of the macronucleus of Infusoria is not into two equal halves, but may take the form of budding off a smaller daughter-nucleus from the main mass. Remarkable instances if nuclear budding of this kind are seen in the Acinetaria, where it is related to the formation of buds by the parent individual. In some cases (Fig. 55), the nucleus may form a con- 
siderable number of buds simultaneously, each of which becomes the nucleus of a daughter-individual budded off from the parent.

The simplest types of mitosis show but little advance on the processes of direct division that have just been described. Taking first the vesicular type of nucleus with a large karyosome (" protokaryon "), the first stage in the process is the division of the karyosome, as in Coccidium; its centriole divides first, then the karyosome becomes constricted and divides, the two halves often plainly connected by the centrodesmose formed by the division of the centrioles. Next an achromatinic spindle is formed between the two daughter-karyosomes, and chromosomes make their appearance,

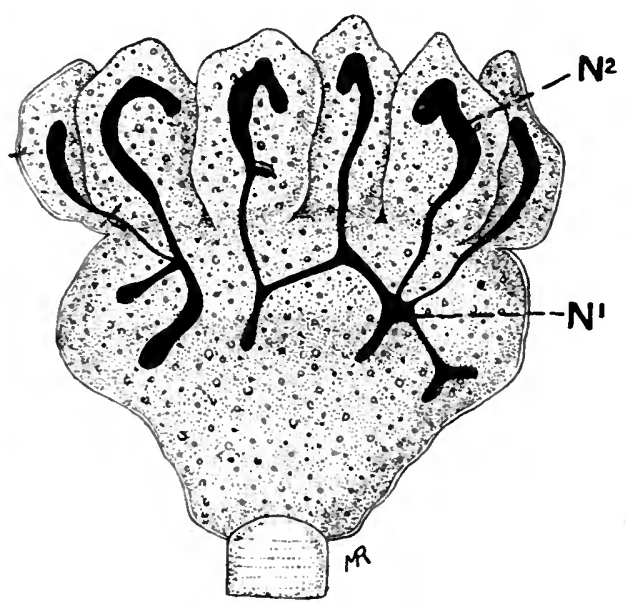

FI1. 55.-Budding in Podophrya gemmipara. The macronucleus of the parent has sent off a number of outgrowths, which extend into the buds and give rise to the nuclei of the daughter-individuals a bout to be budded off. N1. Parent-nucleus ; $\mathrm{N}^{2}$, nuclei of buds. After Hertwig. derived partly (perhaps in some cases entirely.) from the peripheral zone of chromatin, partly from the chromattn eontained in the karyosome. A good example of this mode of division has been described by Aragao (87) in an amœba named by him $A$. diplomitotica from the fact that two types of mitosis occur in this species. In the first type (Fig. 56, $A-G$ ), the little rod-like chromosomes are not arranged in a definite equatorial plate, but are scattered irregularly along the spindle; some travel towards one pole, some towards the other, and, after separation into two groups in this manner, the chromosomes of each group fuse together to form an apparently solid mass of chromatin, representing the daughterplates; these masses of chromatin follow each their respective karyosomes as they travel apart, and when the nucleus is finally constricted into two daughter-nuclei, the chromatin-masses break up again into their constituent chromosomes, which become distributed in the peripheral zone and karyosome of the daughternuclei, whore they can be distinguished plainly even during the resting state (Fig. $56, A$ ).

In the second type of mitosis seen in $A$. diplomitotica (Fig. 56, $H-K$ ), the chromosomes arrange themselves in a definite equatorial 
plate, which divides into two equally definite daughter-plates composed of distinct ehromosomes; whether this division is brought about by splitting of the individual chromosomes is not clear. When the nucleus is finally constricted into the two daughternuclei, the chromosomes are at first aggregated close beside their respective karyosomes, but soon distribute themselves in the manner already described.

The simple types of mitosis described in the two foregoing paragraphs are examples of the so-called "promitosis" (Nägler, 95)
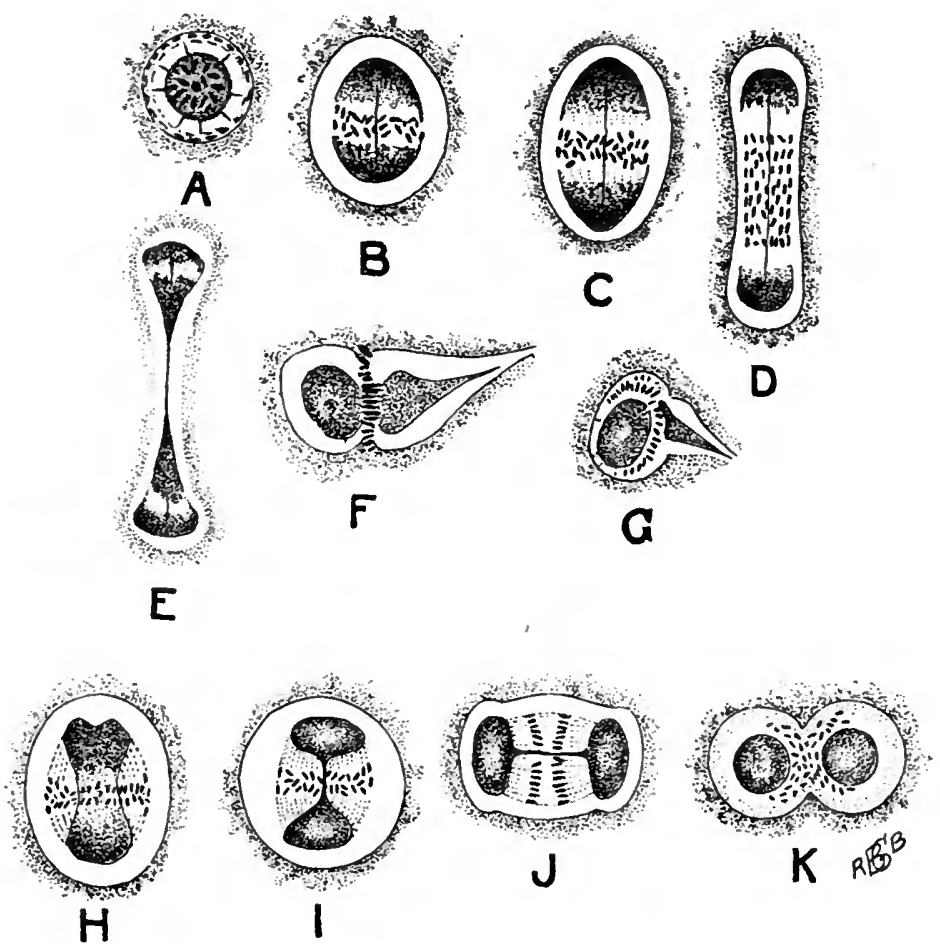

FIa. 56.-The two methuds of nuclear division in Amoba diplomitotica. A, Resting nucleus; $B-G$. first method; $H-K$, second method. In $F$ and $G$ only one of the tiwo halves of the nuclear figure is drawn. After Aragao (87).

seen commonly in nuclei of the protokaryon-type. The nuclear membrane in this type is n negligible quantity; it may be scarcely or not at all developed in the resting nucleus, and when a distinet membrane is present it may vanish entirely during the mitosis; as in the form just described. In any case, however, the entire mitosis goes on within the nuclear space. The chromosomes may show every possible condition in clifferent cases, from complete irregularity in form, number. arrangement, and mode of division, to the 
formation of a definite equatorial plate which splits into two daughter-plates. The most striking and salient feature of this type of mitosis is furnished by the relatively huge " polar masses," consisting of the daughter-karyosomes with their contained centrioles. In the division of the nucleus of Arcella (Fig. 57), however, the karyosome first breaks up into fine grains of chromatin, from which the polar masses and the equatorial plate are formed. The karyosome, as has been pointed out in the previous chapter, consists of three distinct elements-namely, plastin, chromatin, and centriole

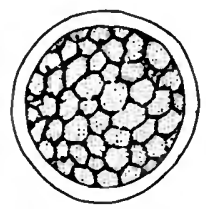

A

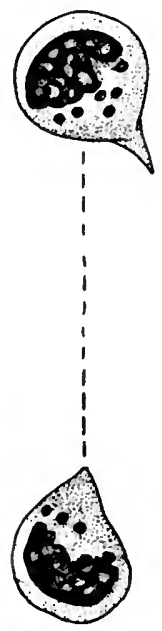

$\mathbf{F}$

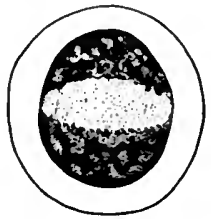

B

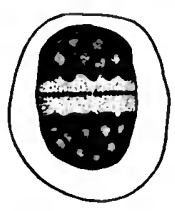

C

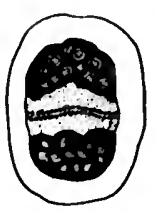

D

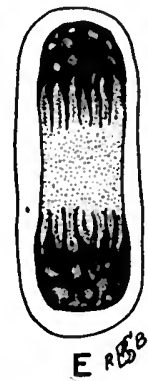

FIa. 57.-Nuclear division in Arcella vulgaris: karyokinesis of one of the two principal nuclel. $A$, Spireme-stage, resulting fron disruption of the karyosome; $B-D$, formation of an equatorial plate of minute chromosomes (?) which split; $F$, anaphase; $F$, the two daughter-nuelei shortly after division. After Swarczewsky (101), magnified 2,250 .

- each independent of, and separable from, the others. In proportion as the karyosome loses its plastin and chromatin elements, and becomes reduced to the centriole alone, so the primitive promitosis will approach more and more to the type of an ordinary mitosis. Such a reduction of the karyosome could take place during the mitosis if, as happens frequently, the whole of the chromatin contained in the karyosome passed out to join the peripheral chromatin in forming the chromosomes, the plastin-substance at the same time furnishing the required ground-substance of the chromosomes (Fig. 58). On the other hand, the karyosome may disappear from the resting nucleus also; Chatton (49) has brought together a number of instances of nuclei showing a gradual reduction of the karyosome in different species, and the evolution of a granular type of nucleus in which the chromatin is scattered through the achromatinic framework, leaving the centriole free or but slightly encumbered by other elements in the nuclear cavity. When a nucleus of this type divides by mitosis, a most typical and perfect karyokinetic 
figure may be produced, as in Euglypha (Figs. 59, 60), only differing from that of Metazoa in that the whole mitosis takes place within the nuclear membrane, and consequently without any co-operation of cytoplasmic elements. Chatton proposes for a mitosis of this type the term "mesomitosis," as distinguished from the more advanced type, or "metamitosis," in which a collaboration of cytoplasmic and nuclear elements is effected, and the entire karyokinetic
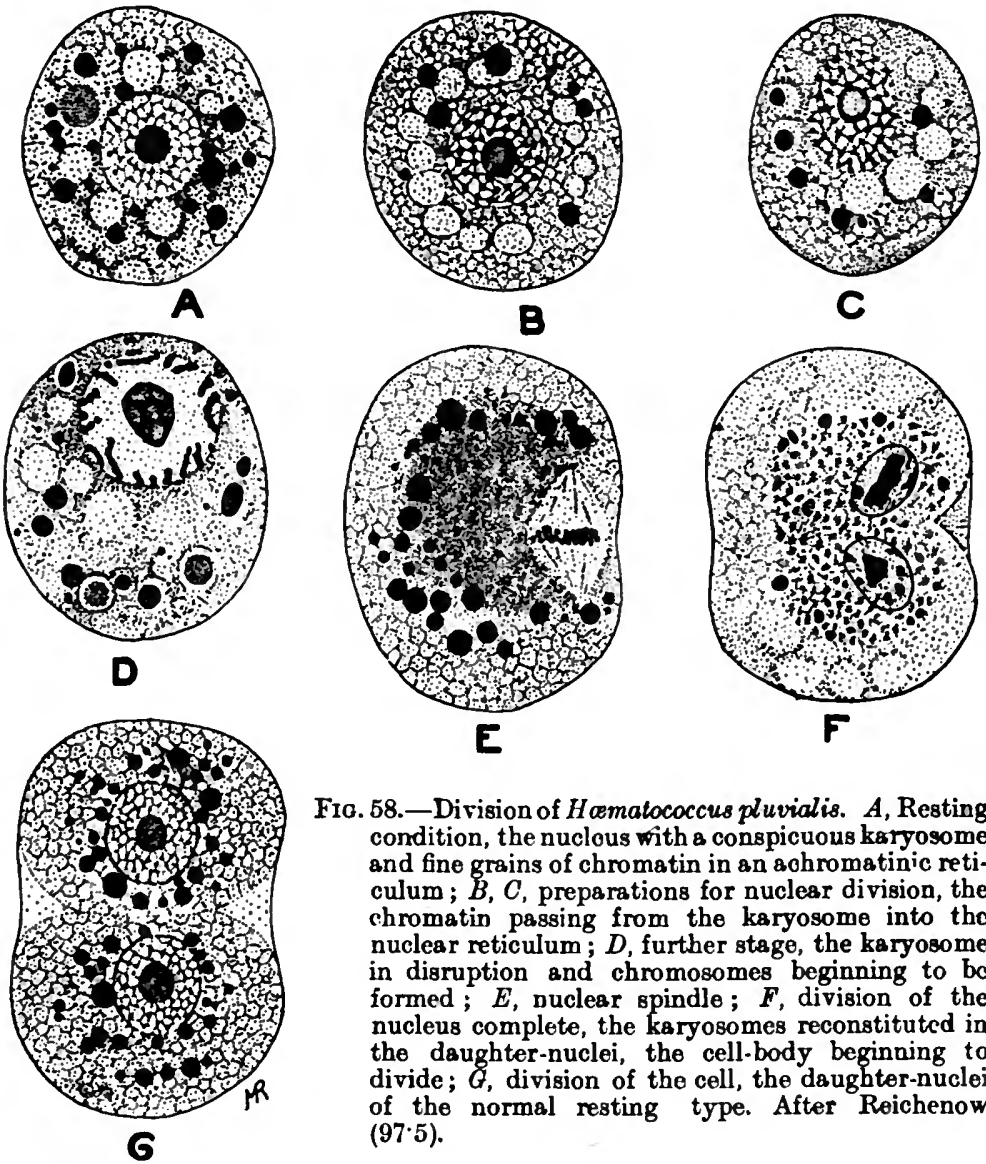

Fic. 58.-Dirision of Homatococcus pluvialis. A, Resting condition, the nuclous with a conspicuous karyosome and fine grains of chromatin in an achromatinic reticulum ; $B, C$, preparations for nuclear division, the chromatin passing from the karyosome into tho nuclear reticulum; $D$, further stage, the karyosome in disruption and chromosomes beginning to bo formed ; $E$, nuclear spindle; $F$, division of the nucleus complete, the karyosomes reconstituted in the daughter-nuclei, the cell-body beginning to divide; $G$, division of the cell, the daughter-nuclei of the normal resting type. After Roichenow $(97 \cdot 5)$.

figure lies free in the cytoplasm after disappearance of the nuclear membrane. Before passing, on, however, to this more advanced type, account must be taken of the more simple types of mitosis seen in granular nuclei.

Instructive examples of the division of nuclei, in which the chromatin is not concentrated into a karyosome, but distributed evenly throughout the achromatinic framework, are seen in the nuclei 


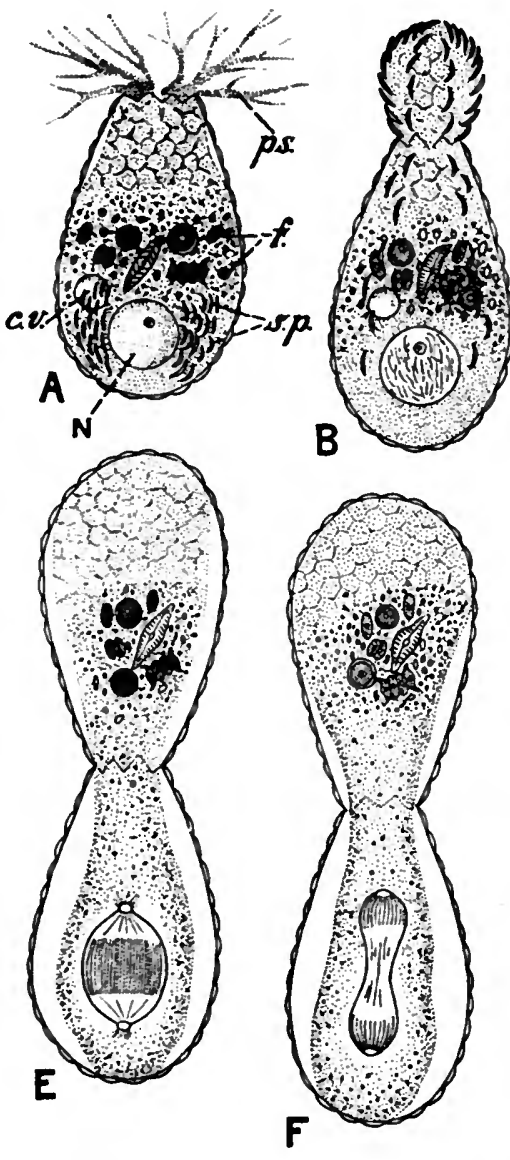

Fra. 59.-Division of Euglypha alveolata, as seen in the living animal.

$A$. Condition of tho animal when about to divide. The protoplasmic body shows three zones: (1) At the fundus of tho shell is clear protoplasm containing the nuclcus $(N$.$) and the reserve shell.$ plates (s.p.); (2) the middle region is ocoupied by granular protoplasm containing ingestod food-materials $(f)$.) and the contractilo vacuole (c.v.); (3) near the mouth of the shell is a zone of hyaline protoplasm from which the pseudopodia (ps.) are given off.

$B$, Early stage of division, about twenty minutes later than $A$. Tho protoplasm is streaming out of the shell-mouth to form the body of a daughterindividual, into which the reserve shell-plates are passing and arranging themsclres at its surface to form a daughter-shell. In the nucleus chromosomes are beginning to be formed.

$C$, About twenty-five minutes later than $B$. The body of the daughter and its shell are further advanced in formation; in the nucleus of the parent the equatorial plate is forming, and the two centrosomes are becoming visible on the two flattened sides of tho nucleus (the centrosomes are probably derived from the division of the karyosome, no longer visible in the nucleus at this stage, or from a centriole contained in the karyosome). [Continued at foot of $p .113$. ] 

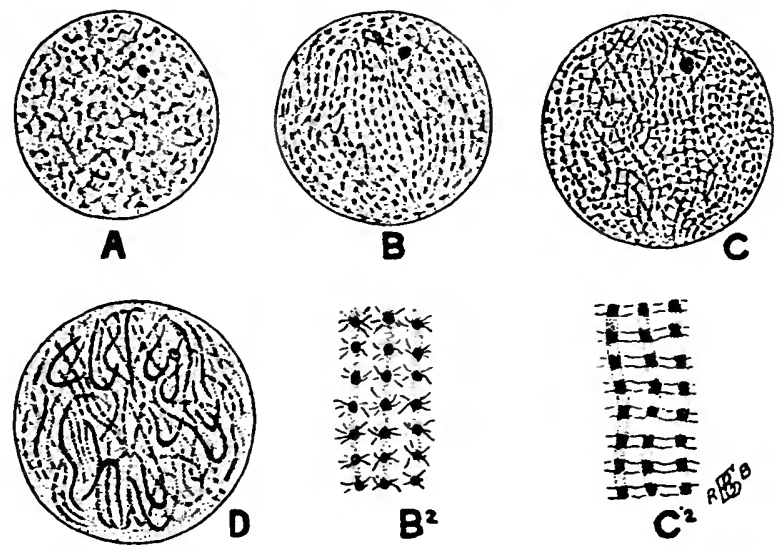

Fra. 60.-Details of the structural changes of the nueleus of Euglypha alveolata during karyokinesis, showing the formation of the chromosomes. $A$, Coarsely-meshed eondition of the nucleus; the chmmatin-granules aggregated at the nodes of tho retieulum. $B$, Later stage; tho nueleus beginning to show a fibrous structure as a result of tho irregular elumps of chromatin-granules of the previous stage becoming arranged in lincar series. $B^{2}$. Some of the fibrils of this stago more highly magnified. $C$, Later stage; the fibrils have become smoother and more parallel in arrangement. $\mathrm{C}^{2}$, Fibrils moro highly magnified; they consist, as in the last stage of darker and lighter parts (the former ehromatin, the latter probably plastin); between the individual fibrils are cross-connections, more regular in this stage than in the last (remains of the nuelear reticulum). $D$, Tho fibrils have become shorter and thieker, and are bending up to form the U-shaped chromosomes. After Sehewiakoff $(100)$; magnification of $A, B$, $C$, and $D$, about 1,200 diameters.

of ciliate Infusoria, such as Paramecium. The macronucleus divides without mitosis, as stated already, but the micronucleus exhibits a primitive type of mitosis (Fig. 61). When division begins, the

\section{Fra. 59-continued:}

$D$, About fiftoen minutes later than $C$. The daughter-shell is now completely formed, and the middle granular zone of the parcnt is passing over into it ; the nucleus of the parent has assumed its definitive orientation, with the centrosomes at the poles of an axis ooineident with the longitudinal axis of the animal, and the equatorial plato is definitely formed.

$E$, About thirty minutes later than $D$. The whole of the middle zone of the parent has passed over into the daughter-shell; the flattened form of tho nucleus is changing into an elongated spindle-form, and the equatorial plate is splitting to form the two daughter-plates.

$F$, About fire minutes later than $E$. The daughter-plates have travelled apart, and the division of the nucleus is beginning.

$G$, About five minutes later than $F$. The division of the nucleus is complete, and one daughter-nucleus has passed oven lit to the body of the daughterEnglypha.

$H$, About twenty-five minutes later than $G^{\prime}$ labout 125 minutes from the beginning). Some of the protoplasm of the middle zone flows back into the parent-shcll, and each individual has its own contraetile vacuole; the two daughter-nuclei are reconstituted, and the karyosome has reappeared in eaeh; pseudopodia are being protruded from the mouths of the shells; the division is complete, and the animals are beginning to separate.

After Schowiakoff (100); magnification about 470 diameters. 
amount of chromatin increases, and the nucleus becomes oval in form. The chromatin forms a number of chromosomes shaped like elongated rods or short threads, which arrange themselves at the equator. At the same time the achromatinic framework shows a longitudinal fibrillation or striation, the apparent fibrillæ being centred in thiokenings of the achromatinic framework which appear at the two poles of the nucleus within the persistent nuclear membrane, hence termed the "polar plates." Centrosomic grains are stated to be entirely absent, and their functions are performed by the polar plates. The nucleus continues to elongate, and the chromosomes divide transversely to their long axis to form the daughter-plates, which travel apart; as they do so the fibrillated
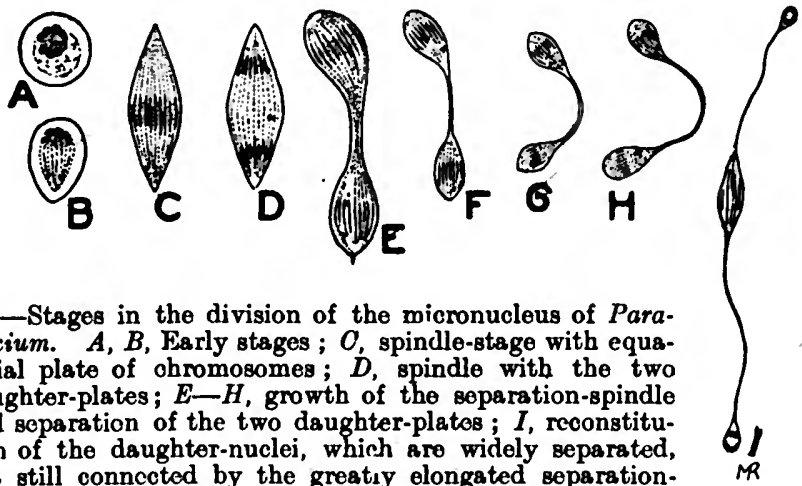

Fra. 61.-Stages in the division of the micronucleus of Paramecium. $A, B$, Early stages; $C$, spindle-stage with equatorial plate of ohromosomes; $D$, spindle with the two daughter-plates; $E-H$, growth of the separation-spindle and separation of the two daughter-platos; $I$, reconstitution of the daughter-nuclei, which are widely separated, but still connccted by the greatıy elongated separation. spindle, the contral part of which shows a dilatation prior to its final absorption. After Hertwig. Figs $A-B$ are drawn on a larger scalo than the other figures.

separation-spindle appears between them. The nucleus as a whole now becomes dumb-bell-shaped; the daughter-plates are lodged in the terminal swellings, while the rapidly-growing separation-spindle occupies the handle of the dumb-bell. The daughter-plates now break up and reconstitute the daughter-nuclei, but the connecting portion continues to elongate and to push the daughter-nuclei apart. It is clear that the separation is effected by intrinsic growth of the achromatinic framework constituting the separation-spindle, which is often curved úp into a horseshoe-figure, and shows behding or twisting of its fibrils, as the result of the inert resistance of the surrounding cytoplasm. Finally, however, a limit of growth is attained; the daughter-nuclei become constricted off completely from the connecting bond, which is absorbed and disappears. The nuclear membrane persists throughout the division.

In all the forms of nuclear division dealt with so far, nuclear elements alone have been active in the process. A most instructive series, showing how extranuclear elements come to collaborate in 
the mechanism of division, is furnished by some examples of the Heliozoa, and especially by the nuclear divisions of Actinosphorium, which have been the subject of extraordinarily thorough investigation by Hertwig (64). In this form there are three different modes of karyokinesis, which, however, for present purposes may be classified under two heads : karyokinesis without and with centrosomes. In the ordinary nuclear division during the vegetative life .of the organism, and also in the divisions by which the primary

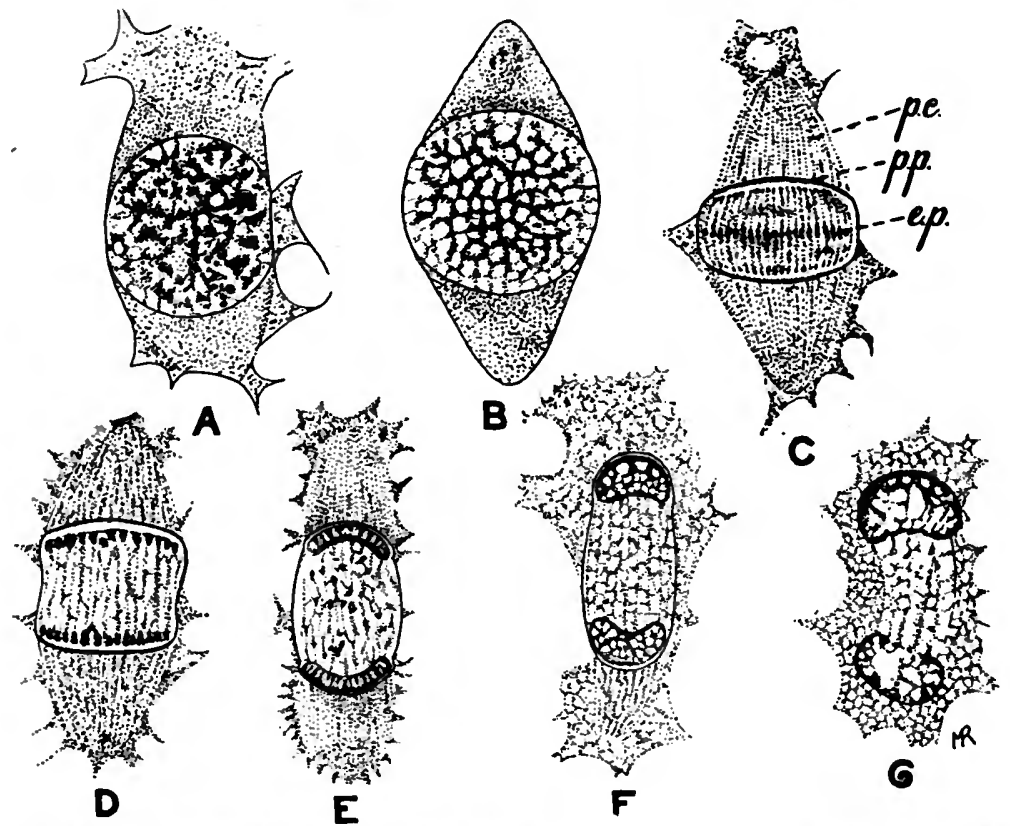

Fra. 62.-Actinospharium eichhorni: stages of tho ondinary, vegetativo nuclear division, without centrosomcs, of frecliving individuals (not encysted). $A, B$, Formation of tho chromosomes within tho nucleus, and of the proto. plasmic polar cones outside tho nucleus; $C$, spindle-stage with polar cones (p.c.), polar plates (p.p.), and cquatorial plato of chromosomes (e.r.); $D$, stage with daughtor-plates of chromosomes which havo travelled towards tho polar plates ; $E-G$, division of tho nucleus, rcconstitution of the daughter-auclei, and disappearanco of the polar cones. After Hertwig (64).

cysts divide into the secondary cysts (p. 138), centrosomes are absent, but they are present in the two divisions which produce the two reduction-nuclei thrown off from each secondary cyst.

In the ordinary karyokinesis of Actinosphuerium (Fig. 62) an equatorial plate is formed composed of a large number of small, rod-like chromosomes, imperfectly separated from one another, which divide transversely. The spindle arises from the achromatinic framework of the nuclcus, and terminates in two conspicuous polar 


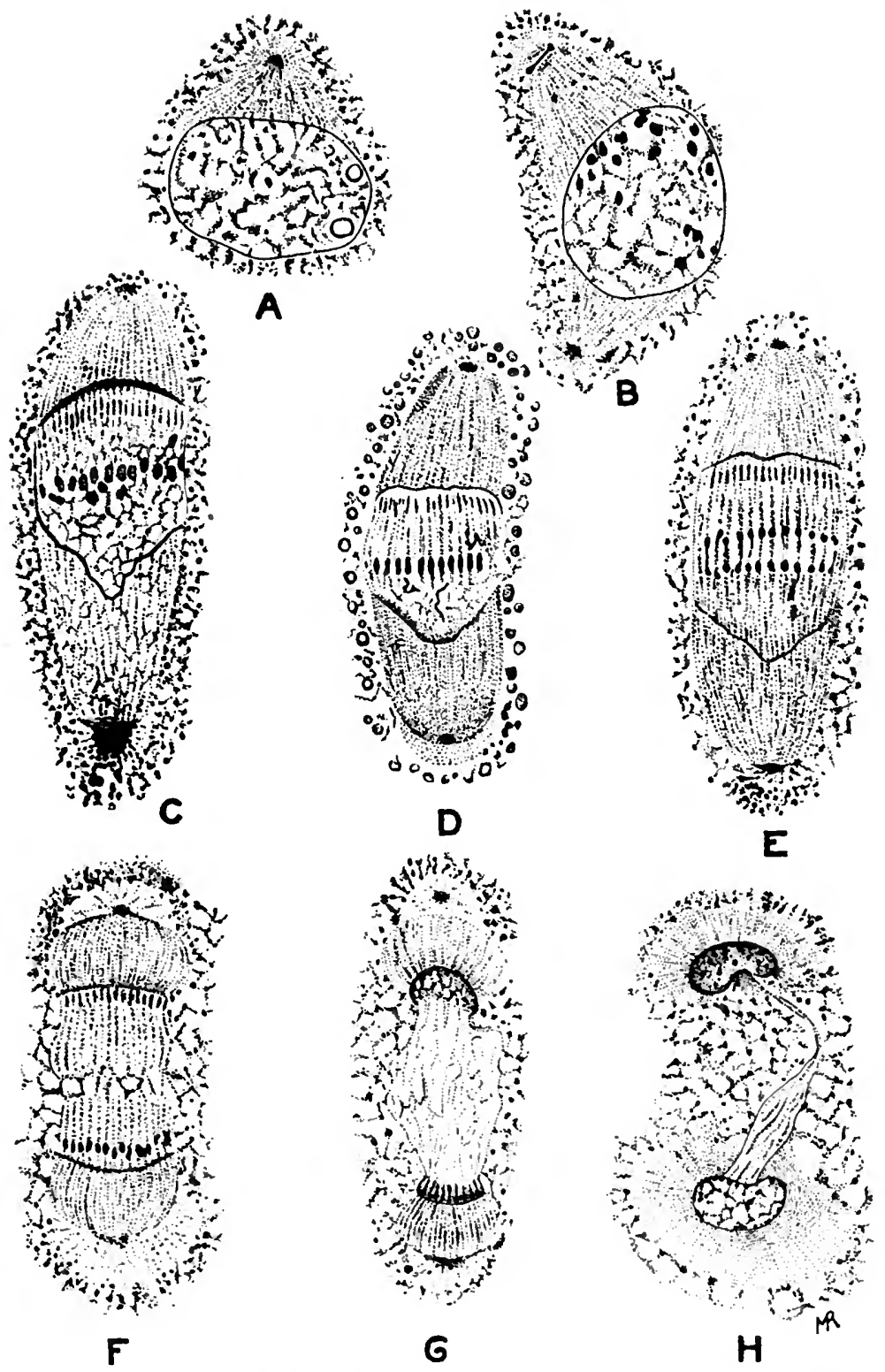

Frc. 63.-Actinospharıum eichhorni: first reduction-division, with centrosomes (the stages here shown follow those of the centrosome-formation in Fig. 37). $A$. Centrosome with radiations in a mass of archoplasm at one prole of tho nucleus; $B$, two centrosomes and archoplasnic cones, taking up positions on of posite sides of the nucleus, in which chromosomos a re begiming to appear; $C, D$, formation of the nuclear spindle and equatorial plate of chromosomes; $E$, division of the equatorial plate; $F$, division of the nucleus beginning; $G, H$, division of the nuclous and reconstitution of the dunghter-nuclei; one daughter-nucleus will degenerate and be rejected as a reduction-uncleus; the beginning of this is seen in $I$, whero tho upper darker daughter.nucleus is the one which degenerates. After Itertwig (64). 
plates lying within the persistent nembrane. External to the membrane are two large conical masses of archoplasm, termed the "polar cones." As in the micronucleus of Paramecium, the polar plates represent functionally the centrosomes, towards which the daughter-plates travel, and division of the nucleus is effected by growth of the separation-spindle. The archoplasmic polar cones appear to take little or no part in the mechanics of the division, since their apices maintain their distance from one another, and the growth of the separation-spindle pushes the daughter-nuclei into their substance.

The reduction-karyokinesis is heralded by the formation of a centrosome from the nucleus (Fig. 37 ; see p. 80, supra). The centrosomes are at first close to the nuclcus, external to its membrane, but when the karyokinetic spindle is formed the centrosomes travel to the apices of the cones. From the centrosomes radiations extend through the polar cones, continuing the direction of the longitudinal striations of the intranuclear spindle, though separated from them by the intervening nuclear membrane. During the division the apices of the cones move apart to a slight extent, but the separation of the daughter-nuclei is still mainly the work of the separation-spindle, which pushes them into the polar cones and brings them close to the two centrosomes again; hence the activity of the polar archeplasm can be but slight. 'The chromosomes in the reduction-divisions are more distinctly separated from each other as the result, apparently, of a reduction in the amount of the plastin forming the ground-substance. The nuclear membrane persists throughout the whole process.

In Actinophrys the karyokinesis appears to be of a type similar to that of Actinosphorium, with persistent membrane, but with more activity in the extranuclear archoplasmic elements. In Acanthocystis (Fig. 64), however, the nuclear membranc disappears completely from the karyokinetic figure, and it is no longer possible, in consequence, to distinguish the parts of the achromatinic spindle which are of intranuclear and extranuclear origin respectively. Nuclear and cytoplasmic elements are in complete co-operation, a condition of things which has apparently been brought about and rendered possible by the extrusion of the centrosome from the nucleus in the first instance.

From the foregoing cxamples, it is seen that amongst the Protozoa the material is to be found for illustrating the gradual evolution of the mechanism of karyokinetic division, from the starting-point of simple and direct division up to the most advanced type in which a perfect karyokinetic figure is formed by co-operation of nuclear and cytoplasmic substance. It is not necessary to suppose, however, that the course of evolution has always been in the direction 
of that type of mitosis found in the cells of Metazoa; it would be more reasonable to expect that in some cases at least other distinct types 'of division-mechanisms would have been evolved-side-
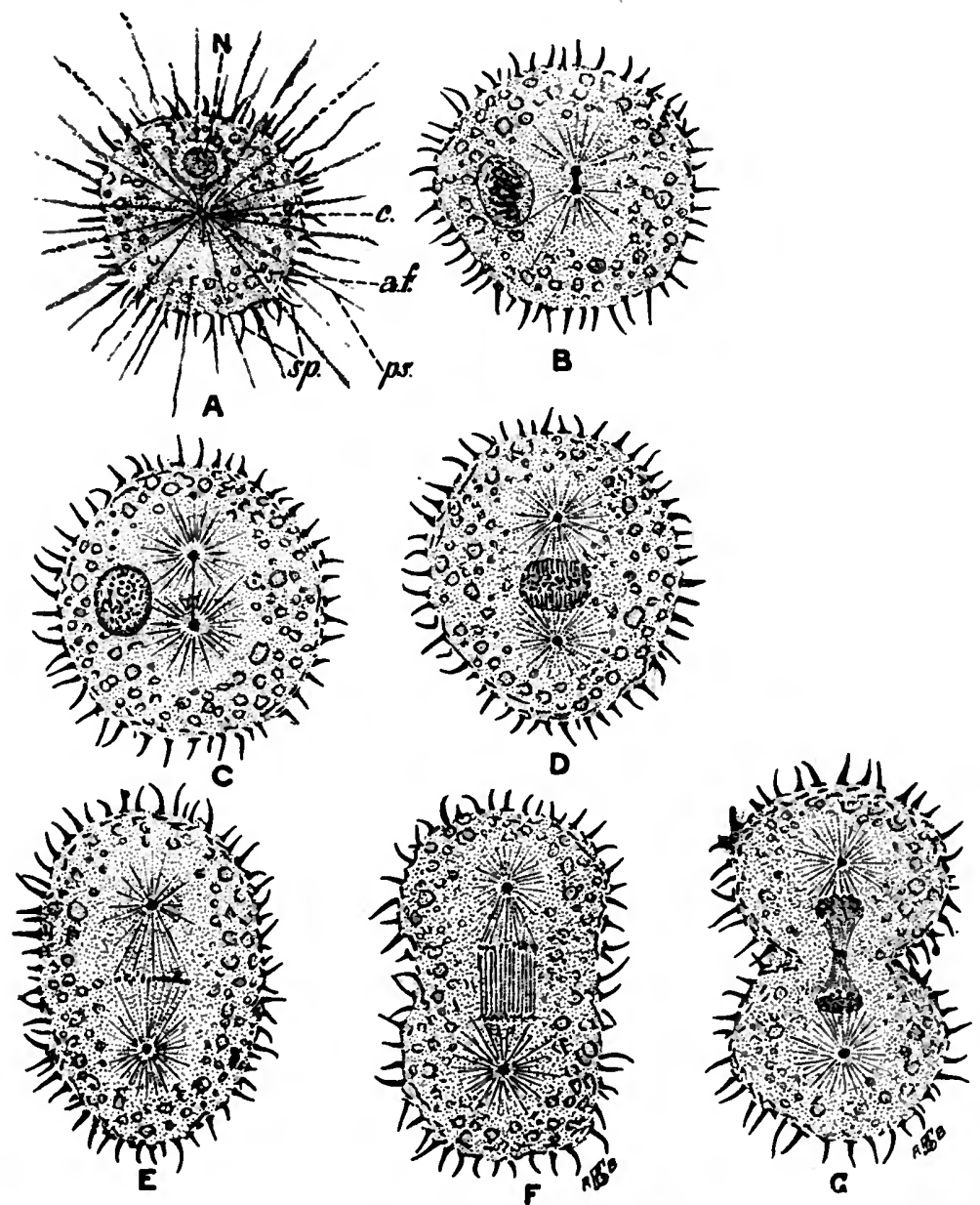

Fis. 64.-Division of Acanthocystis aculeata. A, Resting state of the animal. $N .$, Nucleus ; c., central grain ; a.f., axial filaments of the pseudopodia, ps. ; sp., spioules. $B$, Pseudopodia withdrawn; nucleus in the spirome-stage; central grain dividing. $C$, Division of the central grain further advanced; nucleus showing distinct chromosomes. $D$, Central grain completely divided into centrosomes, between which tho nucleus is placed; in the nucleus the membrane is becoming dissolved, the reticulum is becoming modified in arrangcment to form the achromatin:c spindlo (or a part of it), and the chromosomes are taking up their position in the equatorial plate. $E$, Complete nuclesr spindle, with centrosomes, achromatinic spindle, and equatorial plate. $F$, Later stage with daughter-plates and division of the cell-body beginning. $G$. Division of the nucleus and of the ccll-body ncorly complete. After Sohaudinn (82). 
branches, as it were, of the stem which culminates in the Metazoan type. An example of this is seen in the peculiar karyokinesis of Noctiluca (Fig. 65), in which the division is directed by a large "sphere," consisting of a mass of archoplasm containing the centrioles. The sphere divides and forms the axis of the karyokinetic figure, of which the nuclear portion is placed asymmetrically to one side.

In considering this remarkable process of evolution, consisting in the gradual elaboration of a highly complicated mechanism for division of the nucleus, the question naturally arises, What is the object of a process so elaborate? Or, if this method of posing the problem offends as being too teleological, we may alter the phraseology, and inquire, What is the result of the process? The answer is perfectly obvious. The result effented by equating karyokinesis
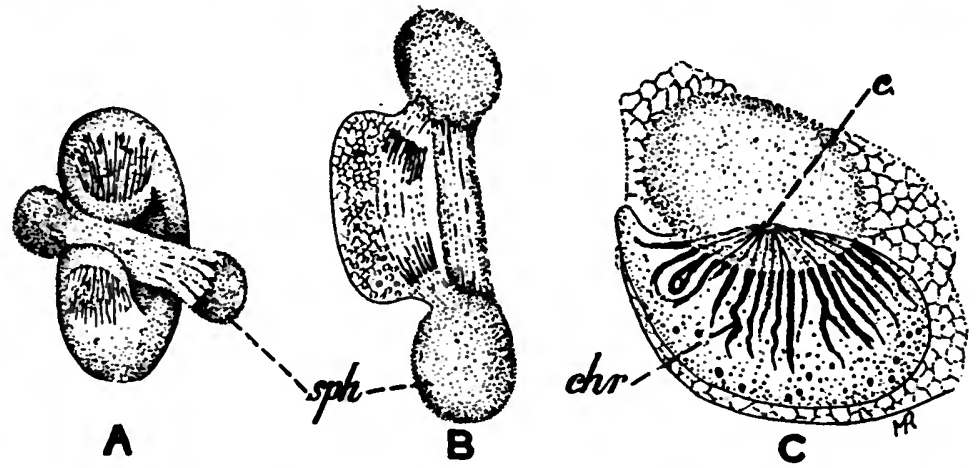

Fra. 65.-Stages in the nuclear division of Noctiluca miliaris. A, Eairy stage, the "sphere" (sph.) beginning to divide, the nuclcus wrapping round it; $B$, later stage, the sphcre ncarly divided, the two poles of the nucleas apindle in section attaohed to the two daughter-spheres; $C$, section aoross $B$; the sphere contains a centriole (c.), to which the chromosomes (chr.) are attaohod by achromatio fibrils. After Calkins (48).

in its most perfected forms is an exact halving, both quantitative and qualitative, of the chromatin-substance of the nucleus-quantitative, by ditision of each chromatin-granule or chromidiosome, and the partition of the division-products equally between the two daughter-cells ; qualitative also, if we suppose that different chromidiosomes may have different properties, and exert their own peculiar influence on the life and activities of the cell; then, since each daughter-cell contains finally the sister-chromidiosomes of those contained in the sister-cell, the qualities of its chromatin are the exact counterpart of those of the sister-cell and also of the original parent-cell. Hence karyokinesis may be regarded as insuring the transmission to the daughter-cells of the distinctive properties of the parent-cell, unimpaired and unaltered. The whole process indicates clearly the immense importance of the chromatin-substance 
in the life of the cell. It is probable, also, that the elaboration of the process of karyokinetic division in Protista was an indispensable antecedent to the evolution of multicellular organisms, since for the formation of a tissue it is necessary that all the eells which build it up should be perfectly similar in their constitution and properties, and this condition could only be brought about, probably, by karyokinetic division of the nuclei in the process of cellmultiplication.

In the foregoing paragraphs we have dealt only with simple (binary) nuclear division, but, as already stated, in some cases the nucleus divides by multiple fission into a number of daughternuclei simultaneously. A simple instance of direct multiple division of a nucleus, in which, apparently, no centrioles are present, has been described by Lebedew (93) in the nuclei of Trachelocerca (Fig. 66 ; see also p. 448). In this form partitions are formed within the nucleus between the grains and masses of chromatim, and finally the nucleus becomes segmented into a mulberry-like mass

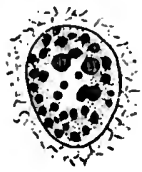

A

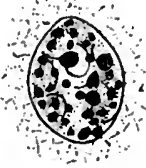

B

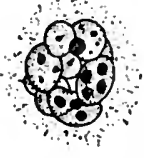

C

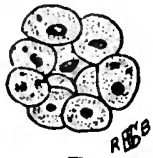

D

Fra. 66.-Four stages of direct multiple fission in the nuclei of Trachclocerca phoenicopterus. After Lebcdew (93). of daughter-nuclei, which separate from one another.

In most cases, probably, of multiple fission the nucleus contains a centriole, and the multiple fission is brought about in a manner analogous to the formation of a plasmodium by mutiplication of the nucleus in a cell which remains undivided-that is to say, the centriole multiplies by fission a number of times without the nucleus as a whole bccoming divided. Thus, in a nucleus of the simple protokaryon type, containing at first a single karyosonie and centriole, division of these structures may take place within the membrane without the nucleus as a whole dividing, so that the nucleus contains finally two or more karyosomes, each containing a centriole. The karyosomes are ultimately set free from the nucleus, either by being budded off singly from it, or by the nucleus as a whols breaking up ; then each karyosome becomes the foundation of a new nucleus. Division of this type, which may be termed a multiple promitosis, has been described by Zuelzer (86) in Wagnerella. In cases where the division of the nucleus is of the karyokinetic type, repeated divisions of the eentriole result in the formation of a complicated multipolar mitotic figure, leading to a multiple division of the nucleus, as seen in the divisions of the nuclei in the male sporont of Aggregata (Fig. 67), as described by Moroff (94). 
The presence of more than one centriole in a nucleus has led Hartmann (60) to formulate the theory that such nuclei are to be regarded as "polyenergid" nuclei.* Hartmann proposes to distinguish a nucleus with a single centriole as a "monokaryon" from a polyenergid nucleus or "polykaryon" containing many centrioles; he interprets many cases, in which a nucleus appears to become resolved into chromidia from which secondary nuclei are formed, as being really a setting free of monokarya from a complex polykaryon-an interpretation which certainly gets over the difficulty of the formation of centrioles in secondary nuclei (see further, p. 255 , infra).

In conclusion, mention must be made of the theory of cell-division and of the causes which bring it about, put forward by Hertwig (91, 92). This theory is based on the supposition, of which mention was made in the previous chapter (p. 70, supra) - that for the normal performance of vital functions a certain quantitative relation must be maintaincd between the nuclear substance and

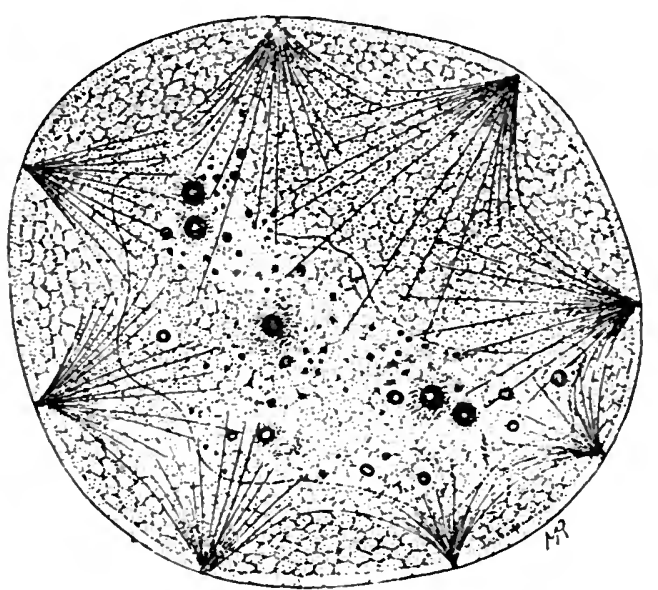

Fia. 67.-Multiplo nuclear division in the malc sporont of Aggregata jucquemeti. The nucleus, of which the outline has become irregular but is still visible, is surrounded by cight centrioles, from each of whieh striations pass towards and into the nucleus. After Noroff (94), magniliid 750 linear.

the cytoplasm. As a standard for the proportion of nuclear mass and cytoplasm ("Kcruplasma-Norm"), the individual immediately after fission may be taken. Exact measurements made on Infusoria show that, while the body grows continuously in size from one division to the next, the nucleus at first diminishes slightly

* The conception of "energids" is due to Saehs, who coined the term to denote " a single eell-nucleus with the protoplasm governed by it, su that a nucleus and the nrotoplasm surrounding it are to be conceived of as a whole, and this whole is an organic unity, both in the morphological and the physiological sense." He1 lwig (66) has criticized this coneeption, and liss shown its untenability in the casc of Protozoa, whieh behave as single individuals whether they possess one nucleus or many. Hartmann, considering the centriole as the criterion of individuality rather than the nucleus, has revived the encrgid theory in the manner described above. It Icads him to regard an ordinary Metazoan karyokinesis as the division of a polykaryon, in which each separate chromosone represents a distinct nuclear element or monokaryon-a conclusion which appears to lead rather to a reductio ad absurdum of the theory. 
in size, and then grows slowly until the acxt division-period is reached. As a result of the slow "functional growth" of the nucleus, a disproportion between the mass of the nuclear substance and that of the cytoplasm is brought about, producing a condition of tension between the nucleus and the cytoplasm ("KernplasmaSpannung "). When the tension reaches a maximum, the nucleus acquires the power of growing rapidly at the expense of the cytoplasm, and this "division-growth" leads to the fission of the cell, restoring the standard balance of nucleus and cytoplasm. Relative increase of the nuclear substance retards the cell-division, and brings about increase in the size of the cell ; relative decrease of the nuclear mass has the opposite effect.

2. Division of the Cell.-A distinction has been drawn above between binary fission, or division of the body into two, and multiple fission into many parts simultaneously. The daughter-individuals produced in either case may be similar to the parent-individual in all respects except size, or may differ from it in lacking more or fewer of its characteristic parts and organs, which are then formed after the daughter-individuals are set free. In extreme cases one or more of the daughter-individuals may possess, when first liberated, no structure more elaborated than the essential parts of a cell, cytoplasm and nucleus or chromidia ; in such cases the daughter is termed a "bud," and the process of fission by which it arises is termed " budding" or gemmation, distinguished further as "simple gemmation" when only one bud is formed at a time, and " multiple gemmation" when many arise simultaneously. In many cases of multiple gemmation the parent-organism does not survive the process, but breaks up almost completely into buds, leaving only a greater or less amount of residual protoplasm, which degeneates and dies off ; budding of this kind is termed sporulation.

In binary fission, when the organism is of simple structure, as in the case of amœbæ, the division is equally simple. After division of the nucleus, the two daughter-nuclei travel apart, and the body follows suit, by flowing, as it were, in two opposite directions, forming two smaller individuals each with a nucleus, and connected at first by a protoplasmic bridge, which soon snaps and is drawn in. The contractile vacuole, if present, is taken over by one of the two daughter-individuals, while the other forms a new vacuole ; in many cases the normal number of contractile vacuoles is doubled before division begins.

In forms of more complicated structure, the division also becomes a more complex process. Where the body-form is definite, the plane of cleavage bears usually a constant relation to it. Thus, in Ciliata the division of the body takes place typically transversely to its longitudinal axis, except in the order Peritricha. In Flagel- 


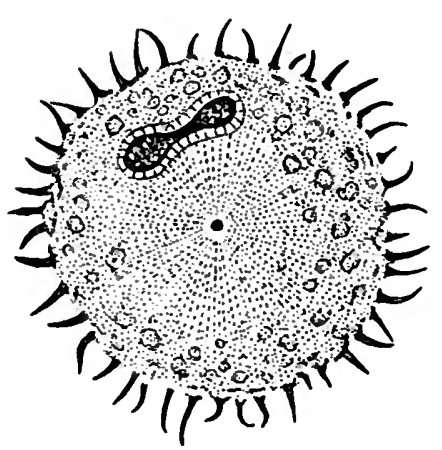

A
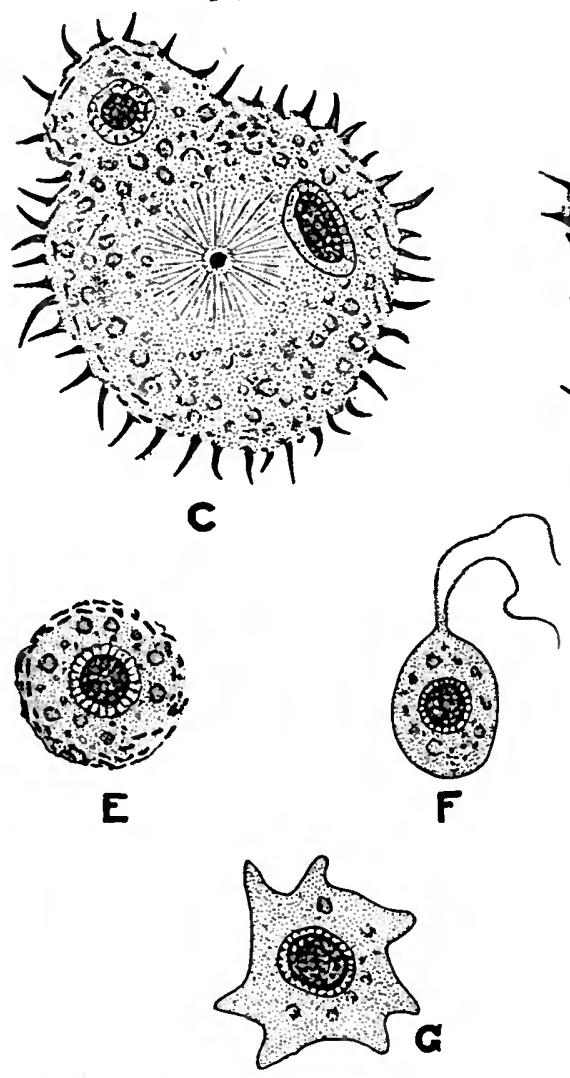

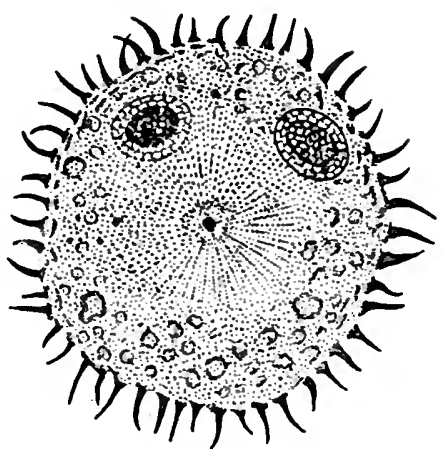

B

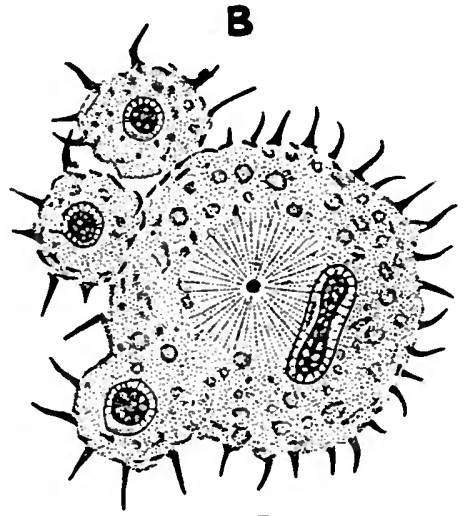

D

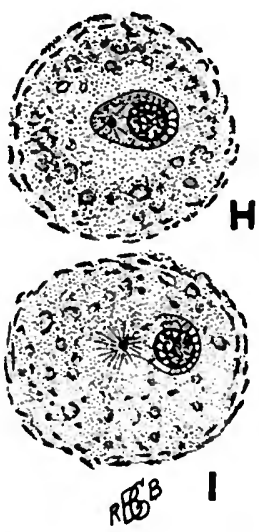

Fic. 68.-Budding of Acanthocystis aculeata (compare Fig. 64, A). A, B, Division of the nueleus, in which the central grain takes no part; $C$, extrusion of a bud; $D$, three buds in process of extrusion, the nucleus of the parent dividing again; $E$, free bud; $F$, flagellula, and $G$, amœbula, produced from buds; $I I$ and $I$, two stages in the extrusion of a centriole from the nucleus of a bud to form the central grain of the adult form. After Schaudinn (82). 
lata, on the other hand, the division of the body is usually longitudinal. In any case, the two products of fission may be equal or subequal in size, without perceptible difference of parent and young; or they may be markedly unequal, in which case parent and offspring can be distinguished clearly.

The various organs of the body may be doubled before division : either by splitting or new growth of one set; or, if there are many organs of a particular kind present, such as the cilia and trichoeysts of Cilihta, they may be simply shared between the two daughter-organisms; or, finally, any given organ present in the animal before division may be retained by one of the two daughterindividuals, while the other forms the organ in question anew after division. Thus, in Ciliata one daughter-individual retains the old peristome; the other forms a new one for itself. The greater the number of organs formed afresh in the daughter-individual, the more advanced is the transition from ordinary fission towards budding.

In typical gemmation small portions of the parent-organism grow out, into which pass either nuclei, the products of the division of the parent-nucleus (Fig. 68), or of budding from the nucleus of the parent (Fig. 55), or chromidia, alone or together with a nucleus. Such buds may arise on the surface of the parent-body, or they may be eut off in the interior of the cytoplasm of the parent, and may remain for some time within its body. Endogenous budding of this kind is seen in the Neosporidia (p. 325), in the Acinetaria, where it is combined with nuclear budding, and in Arcella (Fig. 80) and some amœbæ, where it is eombined with formation of secondary nuclei from chromidia.

Bibliography.-For references see p. 479. 
CHAPTER VIII

\title{
SYNGAMY AND SEX IN THE PROTOZOA
}

\author{
Kímę Bapeia.

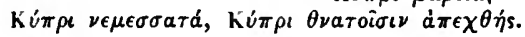

Ir is a matter of common knowledge that amongst all the higher animals and plants the phenomena of sexual generation and sexual differentiation are of universal occurrence. Reduced to its simplest terms, and stripped of all secondary complications, the sexual process in an ordinary animal or plant consists essentially of the following serfes of events : In the multicellular body certain cells are produced which may be termed comprehensively and universally the gametes. In the two sexes the gametes exhibit characteristic differences; those of the male sex, the spermatozoa, are typically minute, active, and produced in large numbers ; those of the female sex, the ova, are, on the contrary, relatively bulky, inert, and produced in far fewer numbers. The gametes are set free from the body, or, at least, from the organs in which they arise, and each male gamete, if it finds a partner and if circumstances permit, unites with a female gamete; their bodies fuse completely, cell with ccll and nucleus with nucleus, and the product is a "fertilized ovum," or zygote, a single cell which proceeds to multiply actively by cell-division, the final result being a new multicellular individual.

In the Protista belonging to what has been termed in the first chapter of this book the "cellular grade"-that is to say, in the Protozoa and the unicellular plants sexual phenomena are also of widespread, probably of universal, occurrence, and the process of sexual union differs only in unessential points from that seen in higher organisms.

In the first place, since the individual in Protozoa is a single cell, the gametes themselves are also complete individuals, modifications merely of the ordinary individuals of the species produced at certain periods or phases of the life-cycle.

Secondly, the differertiation of male and female gametes rarely attains to the high degree seen in the Metazoa, and may be nil; the two gametes may be perfectly similar in all perceptible features of structure or constitution, as, for example, Copromonas (Fig. 111). 
Sexual union of similar gametes is termed isogamy; of dissimilar, anisogamy. When the gametes are differentiated, then one gamote is generally smaller, more active, often with highly developed motor mechanisms, and without reserve food-material in the cytoplasm ; this is the microgamete, regarded as male. The other gamete, on the contrary, exhibits a tendency, more or less pronounced, to be large, inert, without motor mechanisms of any kind, and to store up reserve food-material in the cytoplasmthe macrogamete, regarded as female. The diffcrentiation of the gametes is seen to be a specialization of two kinds of cell-individuals, the onc rich in motile or kinetic protoplasm but poor in trophic substance, the other rich in trophic protoplasm but poor in kinetic substance. In some cases the sexual tifferentiation may affect also the mother-cells of the gametes, the gametocytes, or may be thrown back still farther in the series of generations preceding the gametes; in such cases a number of successive generations of gamonts exhibiting sexual differentiation terminate in a gametocyte generation from which the actual gametes arise.

Thirdly, in the process of sexual union, or syngamy, as it may be termed comprehensively, the bodies of the two gametes do not always fuse completely; in some cases the two gametes come together and merely interchange portions of their nuclear apparatus, remaining separate and retaining their distinct individuality. The nucleus which remains stationary in the one gamete then fuses with the migratory nucleus derived from the other gamete. Examples of this type of syngamy are seen in the Infusoria (Fig. 77). The type of syngamy in which the two gametes fuse completely is sometimes termed copulation (or total karyogamy); that in which they remain separate and exchange nuclear material, is known as conjugation (or partial karyogamy), and the two sexual individuals themselves as conjugants (they should not, perhaps, be termed "gametes," strictly speaking, for reasons explained below); but the term " conjugation" is often used quite loosely for either type and lacks precision.

These differences in the sexual process between Protozoa and the higher organisms enable us to give a wider significance, and at the same time a more precise definition, to the word "syngamy." However varied in detail, syngamy is cssentially nothing more than an intermingling of chromatin-substance derived from two distinct cell-individuals. Plus ça change, plus c'est la même chose. The chromatin that undergoes syngamic union may be in the form either of chromidia or of nuclei ; in the former case the process is termed chromidiogamy, in the second karyogamy. Chromidiogamy, though probably the most primitive type, is known to occur only in a few Sarcodina (Diffugia, p. 230; Arcella, p. 148). In the vast 
majority of Protozoa, as in all known cases amongst Metazoa and plants, syngamy takes the form of karyogamy. The nuclei of the gametes are termed pronuclei, and the nucleus that results from fusion of the pronuclei in the zygote is termed a synkaryon.

In many Protozoa (e.g. Coccidium, Fig. 69) the fusion of the two pronuclei is effected by means of a peculiar mechanism termed a "fertilizationspindle." When the two pronuclei are in contact, the female pronucleus first takes an elongated, fusiform shape, having its chromatin-grains spread over an achromatinic framework. The chromatin of the male pronucleus is then spread over the same structure. This mechanism has nothing to do with nuclear division, but merely effects a complete intermingling of the chromatin of the pronucloi, after which the synkaryon assumes its normal appearance and rounded form. In Infusoria the two pronucloi fuse in the condition of the karyokinotic spindle in many cases.
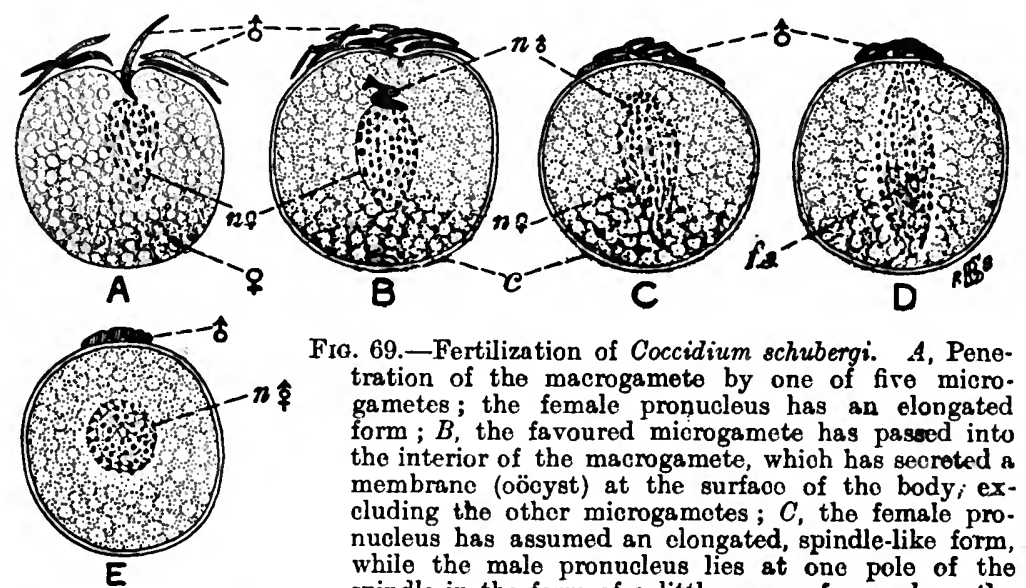

Fro. 69.-Fertilization of Coccidium schubergi. A, Penetration of the macrogamete by one of fire micro. gametes; the female pronucleus has an elongated form ; $B$, the favoured mierogamete has passed into the interior of the macrogamete, whioh has secreted a membrane (oöcyst) at the surfaco of tho body; excluding the other microgamotes; $C$, the female pronucleus has assumed an clongated, spindle-like form, while the male pronucleus lies at one pole of the spindle in the form of a little mass of granules; the excluded microgametes are degenerating; $D$, the granules of the male prontacleus liavo spread themselves over the spindle-figurs formed by the female pronucleus; $E$, the fortilization-spindle seen in $D$ has rounded itself off to form the synkaryon. and fertilization is complete. $\delta$, Microgametes; $q$, maerogamete; $n \delta$, male pronuclcus ; $n$ \% , female pronuclcus ; $f .8$. ., fertilization-spindle; $c$, oöcyșt ; $n \stackrel{\uparrow}{\uparrow}$. synkaryon. After Schaudinn (99), magnified 2,250.

True syngamy, as defined above, must be distinguished carefully from certain other phenomena which are likely to be confused with it; it must not be assumed that every fusion of cells, or evon of nuclei, is necessarily a case of syngamy. In some Protozoa tho mother-cells of tho gametos, the gamctocytes, enter into a more or less close association prior to the formation of gametes, which are produced in due course and then perform the act of syngamy in the normal manner. An example of such association is seen in gregarines (p. 330), where association between adult gametooytes is the rule. Sometimes the two gametocytes associate in the earliest stages of their growth, as in Diplocystis (Fig. $70, A$ ), and their bodies may then fuse completely into one; but their nuclei remain distinct, as in Cystobia (Fig. $70, B$ ), and give rise in due course to the pronuclei of distinct gametes. Forms in which precocious association of this kind occurs are described as being " neogamous" (Woodcock).

In many cases, union of distinct individuals can bo observed which have nothing to do with syngamy, since no fusion takes place of nuclei, but only 
of cytoplasm. Such unions are distinguished as plastogany (or plasmogamy) from true syngamy. Plastogamic union may bo temporary or permanent; in the latter case it loads to tho formation of plasmodia, as in tho Mycotozo: (p. 239). The significanco of plastogamy is obscure in many cases, but in some it may perhaps be comparable to the association of gametes already describod, and in this way may thruw light on some cases of so-callod "autogamy" (see p. 138, infra).

A further case of unions which aro not in any way sexual in nature is seen in the remarkable phenomena of agglomeration exhibited by some Protozoafor examplo, trypanosomes. In this case tho organisms adhere to each other by the posterior or aflagellar end of the body, apparently by means of a sticky secretion formed by the kinetonuclous, so that largc clumps are formed composed of numerous individuals. The phenomena of agglomeration are associated with conditions unfavourable to the parasite, and appear to be due to the formation of special substances, agglutinins, in the blood of the host. Similar phenomena are well known in bactcria as agglutination, since in this case the agglutinated individuals are unable to separato, whiln in

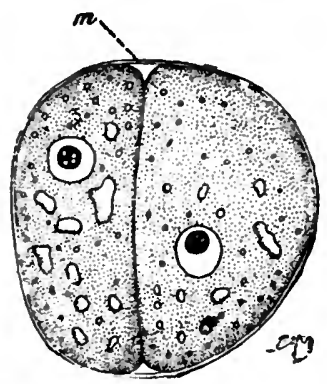

A

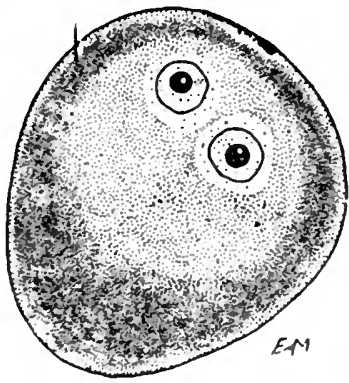

B

Fra. 70.-Precocious association and neogamy of gametocytes in gregarines. $A$, Diplocystis minor, parasite of the cricket:. $m$., common mombrane uniting the two associates; $g$., grains of albuminoid reserve-material. $B, C y s t o b i a$ holothuria, parasite of Holothuria tubulosa, showing the two nuclei in an undivided body. $A$ after Cuenot, magnified about 120 diameters; $B$ after Minchin.

the case of trypanosomes that are agglomorated it is possible for the individuals to become free again if the conditions are ameliorated. In other Protozoa, also, phenomena of the nature of agglomeration are scen in de. generating forms (see p. 209, infra).

Certain aspects of syngamy must now be discussed in more detail-namely, the relation of syngamy to the life-history as a whole ; its occurrence in the world of living beings; its significance for the life-cycle; and its effects on the species and the individual.

1. Syngamy in Relation to the Life-History of the Organism.In any living organism the principal manifestation of vital activity is the power of assimilation, resulting in growth. As a general rule, however, the growth of an organism is not indefinite, but has a specific limit; an individual of a given species does not exceed a certain size, which may be variable to a slight extent, but which is fairly constant for normal individuals of the species in question under similar environmental conditions. When the limit is 
reached the organism tends to reproduce itself. In Protista, as described in the last chapter, two principal types of reproduction occur-namely, simple or multiple fission. In either case the organism grows to its full specific size, and then divides into smaller individuals; the greater the number of daughter-individuals produced at each act of reproduction, the more minute those daughterindividuals. Following the act of reproduction comes a period of growth, during which the small forms grow up into full-sized individuals which reproduce themselves in their turn.

Thus the life-history of a Protist may be described as an alternation of periods of growth and periods of reproduction. If, however, the life-history consists of only these two events in alternating succession, it is an infinite series, not a cycle; continuous, not recurrent. Possibly such a condition, varied only by states of repose interrupting the vital activity of the organism is found in Bacteria and allied forms of life, where true syngamy apparently does not occur. But it is probable that in all Protozoa, as in all Metazoa and plants, the life-history is a recurrent cycle, of which an act of syngamy may be taken as the starting-point; this point will now be discussed.

2. The Occurrence of Syngamy in the Series of Living Beings.With regard to this question, there are two possibilities ; first, that syngamy and sexuality constitute a fundamental vital phenomenon, common to all living things; secondly, that it is an acquisition at some period or stage in the evolution of organisms, and not a primary characteristic of living beings. The sex-philosopher Weininger* has argued in favour of the first of these hypotheses, and goes so far as to regard all protoplasm as consisting primarily either of arrhenoplasm (male) or thelyplasm (female), standing in fundamental antithesis to one another, and combined in varying proportions in a given cell or sample of the living substance. A view essentially similar has been put forward by Schaudinn, and is discussed below.

It is beyond question that sexuality is a universal attribute of all living beings above the rank of the Protista, whether animals or plants. In Protista, however, syngamy has not been observed to occur with certainty in the Bacteria and organisms of a similar type of organization. It is true that certain rearrangements of the chromatin, observed in some larger organisms of the bacterial type at certain phases of their life-history, have been compared to sexual processes, but such an interpretation is, to say the least, highly doubtful. In Protozoa, syngamy. has been observed to occur in a vast number of forms, but by no means in all. In the 1906.

* Weininger, O., "Sex ańd Character," chapter ii. London: W. Heinemann, 
case of those species in which syngamy has not been observed, there are three abstract possibilities: first, that it does occur, but has not yet been seen; secondly, that it is secondarily in abeyance ; thirdly, that it is primarily absent-that is to say, that it has never occurred either in the form in question or in its ancestral lineage. On the whole, the first of these three possibilities is the most probable, though the second must, perhaps, also be taken into account, as will be shown later.

So far as a generalization is possible or permissible in the present state of knowledge, it appears that sex and syngamy are phenomena of universal occurrence in all truly cellular organisms, but we have no certain knowledge that they exist in any organisms of the bacterial type of organization. With the passage from the bacterial to the cellular type of structure, syngamy became, apparently, a physiological necessity for the organism, and was probably acquired once and for all.

3. The Significance of Syngamy in the Life-Cycle.-In order to appreciate the part that syngamy plays in the life-histories of organisms generally, it is necessary to compare briefly and in general outline the life-cycles of Metazoa and Protozoa in typical cases.

In the Metazoa the cycle starts from a single cell, the zygote or fertilized ovum, which multiplies by cell-division in the ordinary way. Thus is produced a multicellular individual, composed always of at least two classes of cells-tissue-cells (histocytes) and germ-cells. The histocytes are differentiated in various ways, related to various functions, to form tissues, and so build up the soma. The germ-cells are not differentiated for any functions but those of sex and reproduction, and occur primarily as a mass of undifferentiated cells constituting the germen; they are lodged in the soma and dependent upon it-parasitic upon it, so to speak -but in a sense distinct from it ; they draw their sustenance from the soma, influence greatly its development and activities, but contribute nothing to the work of the cell-commonwealth. Of these two portions of the Metazoan individual, the soma is necessarily mortal, doomed inevitably to ultimate senility and decay. The cells of the germen, on the other hand, are potentially immortal, since under favourable conditions they can separate from the soma and give rise in their turn to a new individual of the species with soma and germen complete again This type of generation is always found in every species, though non-sexual methods of generation may also occur in many cases.

In the life-cycle of the Metazoa, as sketched above in its most generalized form, two individualities must be clearly distinguished, the one represented by the soma together with the germen, con- 
stituting the complex body of a Metazoan individual; tho other represented by the single cells of which both soma and germen alike are built up. The phrase "reproduction," whether sexual or non-sexual, as applied to the Metazoa, refers only to the complex multicellular body as a whole, and not to its constituent cells, which reproduce themselves uninterruptedly by fission during the whole life-cycle.

In the comparison of a typical Protozoan life-cycle with that of the Metazoa, we may start in both cases alike from a single cellindividual which is the result of an act of syngamy. In Protozoa, also, the zygote multiplies, sooner or later, to produce numerous cell-individuals; but in this case the cells remain separate from one another and independent, so that no multicellular body is produced, except in the colony-building species, nor is there any distinction of somatic and germinal cells, save in rare cases, such as Volvox (p. 267). In Protozoa the phenomena of vital exhaustion, so-called "senility" (Maupas) or " depression" (Calkins, Hertwig), appear to be as inevitable as in the cells of the Metazoan body (see pp. 135 and 208, infra); but if the derangement of the bodily functions and the vital mechanism has not gone too far, the organism is able to recuperate itself ky self-regulative processes, of which the most important and most natural are those involved in the normal process of syngamy. Consequently no cell-individuals among Protozoa are doomed necessarily and inevitably to decadence and death, but all possess equally potential immortality-that is to say, the capacity for infinite reproduction by fission under favourable conditions. The Metazoan individual is represented in the Protozoa only by the entire life-cycle, from one act of syngamy to the next, and not by any living organic individual.

In the life-cycle of a Protozoon, as there is only one individuality, so thcre is only one method of reproduction-that, namely, of the cell, by fission ; and it must be made clear that the reproduction of the cell-individual is not in any special relation to syngamy in Protozoa, any more than in Metazoa.

It has been pointed out above that the life-history of a Protist organism consists of alternate periods of growth and reproduction. In those Protozoa in which syngamy has been observed, it is found to take place sometimes at the end of a period of growth and before a period of reproduction, sometimes at the end of a period of reproduction and before a period of growth, and sometimes there may be a difference between the two sexes of the same species in this respect. In the first case, syngamy takes place between full-grown individuals of the species, as in Actinophrys (Fig. 71)so-called macrogamy, which is almost always isogamous. In the second case, syngamy is between the smallest individuals produced 
by fission or gammation, as in Foraminifera (p. 235), Arcella (Fig. 80), etc.-so-called microgamy, which may be isogamous or slightly anisoge mous. In the third case, syngamy is between two individuals showing the utmost disparity in size, a tiny microgamete and a bulky macrogamete, as in Coccidium (Figs. 69, 152); the result being anisogamy of the most pronounced type.

From these facts, it is abundantly clear that syngamy in the Protista cannot be regarded as related specially to reproduction, but as a process affecting the life-cycle as a whole, related equally

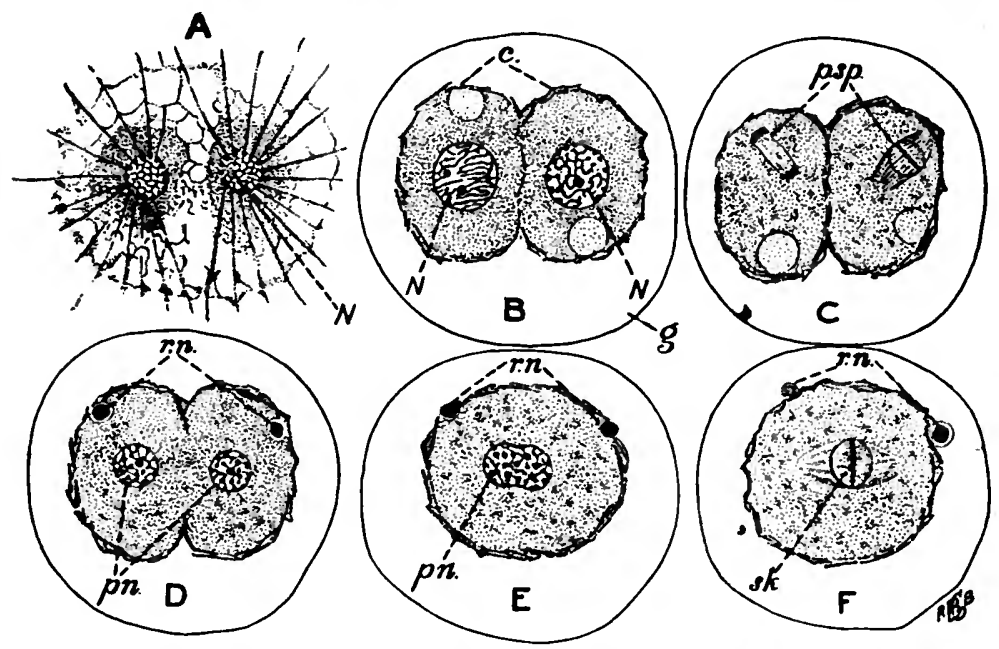

Fig. 71.-Copulation of Actinophrys sol. A, Two associated free-swimming individuals. $B$, The two individuals are beginning to encyst themselves; their nuclei $(N ., N$.) are preparing for karyokinesis; an outer gelatinous envelopo $(g)$ is secreted round the two gametes, and also round eaeh individual an inner eyst-envelope $(c$. ), incomplete at the surface of contact. $C$, The nueleus of esch gamete is dividing by karyokinesis (first polar spindle, $p . s p$.). $D$, Formation of the polar bodies or reduetion-nuclei $(r . n$.$) ; the reduced pronuclei (p n$. taku a central position in the body of the gamete; tho bodies of the gametes are beginning to fuse. $E$, The pronuclei are fuging; the reduction-nuclei have passed through the wall of the inner eyst. $F$, The synkaryon (sk.) is beginning to divide by karyokinesis; the degenerating reduction-nuclei have passed out of the inner eyst. $N ., N$., Nuclei of the gametes beforo reduction. After Schaudinn, magnified about 850 .

to all vital functions of the organism, and therefore only indirectly to reproduction-that is to say, only in so far as reproduction may result from renewed and invigorated vitality. This is equally true of the Metazoa, where, however, the life-cycle begins and ends with the production of a complex multicellular body, composed of soma and germen. Hence, in the Metazoa syngamy is brought into relation with the production of a higher individuality, the body, comparable to the whole Protozoan life-cycle, and it is in this sense that the phrase "sexual reproduction" must be under- 
stood; as already pointed out, syngamy has no special relation in Metazoa to cell-multiplication. In Protozoa sexual reproduction means simply reproduction following the sexual act; but sex and reproduction must be considered as two things entirely distinct.

The comparison instituted above between the life-cycles of the Protozoa and Metazoa, according to which an entire Protozoan individual is the morphological equivalent of a single constituent cell of a Metazoan body, is that which I personally have always held and taught. It is, I believe, the prevailing view among zoologists, and has beon enunciated clearly by Calkins (5). It has, however, been attacked vigorously by Dobell (110), who lays great stress on the physiological analogy between the single Protozoon, as a com. plete organism, and the entiro Metazoan body. On this ground he expresses the view that " a protist is no more homologous with one cell in a metazoon than it is homologous with one organ (e.g., the brain or liver) of the latter"; he considers it "incredible that anybody could advocate the view that the Metazoa have arisen from aggregatod Protozoa," and he puts forward the view that, if the Metazoa have arisen from protist forms, "it is far more natural to suppose that they did so by developing an internal cellular structure, and not by the aggregation of individuals to form a colony." Similar ideas have been put forward also by Awerinzew (890). From these and other considerations, Dobell draws the conclusion that the Protista are not to be regarded as unicellular, but as "non-cellular" organisms.

So far as the word "cell". is concerned, I have already expressed the opinion above that by the term should be understood a certain stage in the ovolution of the Protista, and that many protist organisms should not be termed " cells," but only those which have reached what may be considered as the truly cellular type of organization. I am not, therefore, concerned with Dobell's attack on his own conception of tho coll-theory so far as it concerns Protists generally, but only in so far as it applies to tho Protozoa.

It is not possible here to discuss in detail the ontogenetic development of the Metazoa. It must suffice to state that in all primitive types of embryonic development among Metazoa the cells which build up the body originate by repeated binary fission of a single cell, the fertilized ovum; and that the only cases in which the ovum breaks up into cells by the development of celllimits internally are those in which the development is modified by the presence of yolk, or where there is good reason to believe that yolk was ancestrally present in the egg. For confirmation of these statcments the reader must be referred to the ordinary textbooks of embryology. I must content myself with a single instance, that, namely, with which I am best acquainted by personal study.

In the development of a simple Ascon sponge, such ns Clathrina blanca or other species (see chapter "Sponges" in Lankester's " Treatise on Zoology," part ii., p. 68), the ontogany may be divided into four phases or periods, which indicate clearly, in my opinion, the general lines in the evolution of the Metazoa from Protozoan ancestors.

1. Starting with the fertilized ovum, strictly homologous with a Protozoan zygote, it divides by repeated binary fission into a number of cells (blastomeres), each similar to the ovum in every respect except size; the process is in every way comparable to the division of a Protozoan zygote into a number of individuals which remain connected to form a colony, as, for example, in many Phytomonadina.

2. Of the blastomeres thus formed, a certain number, variable in different species, but relatively few, retain their original characteristics, while the rest become differentiated into columnar flagellated cells forming the wall of a cavity (blastocoele). The undifferentiated blastomeres give rise to the archæocytes, from which ultimately the gorm-cells and gametes arise. The flagellated cells are the ancestors of the tissue-cells (histocytes) in the future sponge. At this stago, in which the embryo is hatched out as a froe-swimming 
larva, it is perfectly comparable to a colony of flagellates such as Volvox, in which the ordinary individuals have lost the power of becoming, or giving rise to, gametes, which can only arise from certain special individuals.

3. The free-swimming larva, composed mainly of flagellated cells, with the archæocytes either at the hinder pole or in the internal cavity, undergocs changes as it swims about, which consist in some of the flagellated cells losing their flagellum, becoming modified in structure, and migrating into the interior of the larva ; in this manner the two germ-layers are established, and the organism has then, so to speak, passed from the condition of a Protozoan colony to that of a true Metazoon.

4. When the germ-layers are established, the larva fixcs itself, and of the subsequent development it is sufficient to st3te that the cells of the two germ-layers become differentiated into the tissuos of the adult sponge, and that in the metamorphosis of the larva the cells undergo a complete rearrangement, which shows clearly that every cell has an individuality as distinct as that of any Protozoan individual, a conclusion fully borne out by the recent experiments of Wilson and Huxley (Phil. Trans., B., ccii., pp. 165. $189, \mathrm{pl}$. viii.) on the power of regeneration in sponges after complete separation of the cells from one another.

I am unable, therefore, to accept the standpoint of Dobell with regard to tho relations of Protozoa and Metazoa, but consider that the comparison of a Protozoan individual to a single cell in a Metazoan body is fully justified both morphologically and physiologically, and is a reasonable phylogenetic deduction from the ontogenetic data. The objection that there are no animals known which correspond to the four-cell, eight-cell, and blastula stages in embryological development misses the point and is not strictly true; the stage at which an embryo consists only of four or eight blastomeres is the homologue of a Protozoan colony, and in the Flagellata spocies are known in which the colony cansists only of four, eight, sixteen, or thirty-two cellindividuals (p. 275). To the query, "Has anyone ever found a metazoon which is composed of nothing but coherent gametes ?" it may bo replied that in many Volvocinex the colony also consists only in part of gamete. producing individuals. The theory that the Metazoa arise by cleavage of a multinucleato plasmodium, equivalent to a single Protozoan individual, has often been put forward, but has never found support from a general consideration of the facts of Metazoan embryology. In Protozoa the plasmodial phase is always tomporary, and onds sooner or later by breaking ap into separate uninucleato individuals.

4. The Effects of Syngamy-(1) upon the Individual, (2) upon the Specres. - 1. Of all Protozoa, the ciliate Infusoria are the group in which syngamy is most easily observed and studied-in the first place because in these organisms it is readily distinguished from simple fission, which is transverse, while in syngamy the two conjugants apply themselves laterally to one another; in the second place, owing to the fact that the species of Ciliata are practically monomorphic (p. 440), and can be identified without difficulty. Hence in this group elaborate and exhaustive experimental studies upon syngamy and its relation to the life-cycle have been carried out by many investigators, more especially by Maupas, Hertwig, Calkins, and Woodruff. The results of these investigators is briefly as follows: After syngamy the fertilized individuals appear vigorous, feed actively and multiply actively. After many generations of reproduction by fission, however, the race, if kept in an unchanged environment, becomes less vigorous 
and shows signs of enfeeblement and " senility" or " depression "a condition which, with continued isolation, reaches such a pitch that the organism is unable to assimilate, grow, or reproduce, but dies off inevitably unless conjugation with another individual takes place. At a result of syngamy, the vigour of the race is renewed, and the organisms once more grow and reproduce themselves actively until senility supervenes again. From these and many other facts it would appear as if syngamy produced a strengthening or re-organizing effect upon the organism, restoring vigour and activity to vital functions that have become, as it were, worn out and effete.

One very important discovery has resulted from the experiments of Calkins and Woodruff-namely, that the nocessity for syngamy can be greatly deferred by change of environment. A strain which has become senile and exhausted can be stimulated and revived by a change of food. Even this remedy appears to have its limits, however, a degree of exhaustion being reached sooner or later which nothing can restore to its pristine vigour. The animals may even reach a pitch of exhaustion so great that they are unable to conjugate, but die off in a helpless manner. Calkins explains such cases as due to the senility having affected not only the vegetative, but also the generative chromatin; producing generative senility, which is incurable, instead of mere vegetative senility, for which syngamy is a remedy. Nevertheless, the fact that the advent of senility and exhaustion can be deferred by the stimulation of changed conditions is a very important discovery. It must be remembered that the Ciliata are organisms of extremely complex organization, and it is not unre:sonable to suppose that in such forms the work thrown upon the vegetative chromatin is much heavier, and therefore the tendency to exhaustion much greater, than it would be in an organism of simpler constitution; in such a form the stimulus of change of environment might defer the advent of senility very greatly, perhaps even for an indefinite period (Woodruff, 141).* This suggestion applies particularly to parasitic forms, in which the organization is always greatly simplified, and in which change of environment from generation to generation is inseparable from their mode of life. It would not be surprising, therefore, if syngamy were found to be completely in abeyance in a parasitic form of simple structure.

It should be noted here that examples of syngamy being in abeyance are not wanting even in higher organisms. An instance

* In his most recent work on Paramecium, Woodruff (142) expresses the view that " most, if not all, normal individuals have, under suitable environmental conditions, unlimited power of reproduction without conjugation or artificial stimulation." Compare also Woodruff and Baitsell (143). 
is the banana-tree. In the wild-banana, seeds are produced from flowers of a normal type by fertilization, just as in any other flowering plant; in the cultivated banana, however, the flowers are sterile and incapable of fertilization, consequently the tree bears. fruit which are entirely seedless. Hence the cultivated bananatree is propagated entirely by a non-sexual method-namely, by the production of suckers growing up from the roots, and in no other way. Whether this complete abolition of sexuality will in time lead to exhaustion of the cultivated race of banana remains to be seen, but at present there seem to be no signs of loss of vigour under cultivation.

If syngamy can be entirely dispensed with in an organism relatively so high in the stale of life as a flowering plant, it seems probable in the highest degree that the same may be true in many cases for unicellular organisms of simple structure, and especially for those parasitic forms which live, like cultivated plants; in a medium rich in nutritive substances, and in an environment which is changed at least once in each developmental cycle. Instances of this are perhaps furnished by the various species of pathogenic trypanosomes, strains of which have been brought to Europe and propagated for many years from one infected animal to another by artificial inoculation, without the natural agency of an invertebrate host. If it be true, as is generally believed, that in trypanosomes syngamy takes place in the invertebrate host, then in the long-eontinued artifieial propagation of pathogenic trypanosomes sexuality has been in abeyance for a vast number of generations without any apparent loss of vital powers. The ease of the pathogenic trypanosomes cannot, however, be eited, in the present state of our knowledge, as an absolutely conclusive example of syngamy in abeyance, since it is by no means certain that this process does not take place in the vertebrate host, where its occurrence has frequently been affirmed (see p. 305, infra). But it is certain that in trypanosomes gonerally, whether pathogenic or non-pathogenic, syngamy is a rare phenomenon, since it has not yet been demonstrated satisfactorily in a single instance, either in the vertebrate or the invertebrate host, in all the many species that have been studied. It is possible that, in these and many other forms of life, sexual processes may intervene only at long intervals in the life-history, and by no means in every eomplete cycle of development or alternation of hosts. It then becomes necessary to distinguish a developmental eycle, consisting of a recurrent series of similar form-changes in regular succession, from a complete life-eycle marked by the occurrence of an act of syngamy. In such forms as the parasites of malaria, for example (p. 358), the life-cycle and the developmental eycle coincide-that is to say, 
syngamy occurs once for each complete cycle of development with alternation of hosts, though it must not be forgotten that the development in the vertebrate host comprises a vast and quite indefinite number of generations of the parasite. On the other hand, in such forms as trypanosomes, a complete lifecycle, from one act of syngamy to the next, may comprise, apparently, a great number of developmental cycles and alternations of hosts.

From the foregoing considerations it is evident that syngamy, though usually a necessity for the continued existence of unicellular no less than of multicellular organisms, can be dispensed with for a very large number of generations, perhaps even indefinitely, in some instances or under special circumstances. Two other phenomena of apparently widespread occurrence point to the same conclusion-namely, the phenomena of parthenogenesis and autogamy. Parthenogenesis is a mode of reproduction so common in Metazoa of various classes that it is unnecessary to cite instances of it here; it may be defined briefly as the power to develop without syngamy possessed by a sexually-differentiated gamete, which under nor-

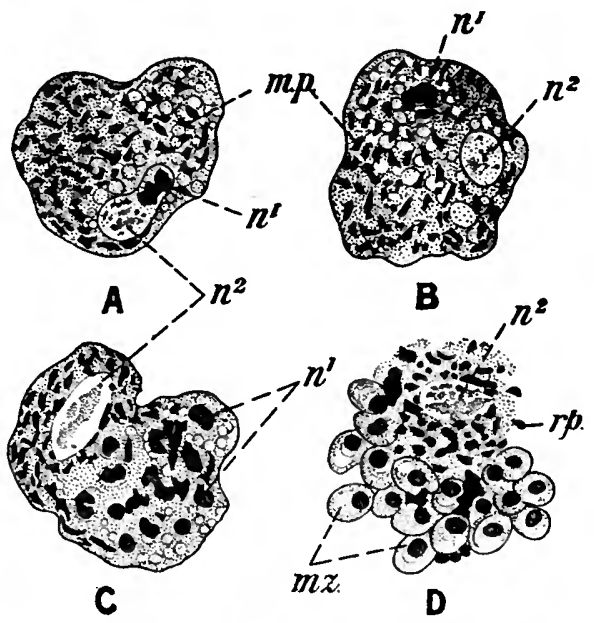

Fia. 72.-Parthenogenesis of Plasmodium vivax. $A$, A female gametocyte, of which the nueleus is dividing into a darker portion $\left(n^{1}\right)$ and $a$ lighter portion $\left(n^{2}\right) ; B$, the separation of the two parts is complete; $C$, the darker nucleus has divided into a number of portions; $D$, a number of merozoites are formed from the darker nuclei ; the lighter nucleus is abandoned in the residual protoplasm $(r . p$.$) containing$ the melanin-pigment. After Sehaudinn (130).

mal circumstances could do so only after syngamy with a gamete of the opposite sex. To this it must be added that the gamete which has this power is always the female; but this limitation receives an explanation from the extreme reduction of the body of the male gamete and its feeble trophic powers, rendering it quite unfitted for independent reproduction, rather than from any inherent difference between the two sexes in relation to reproductive activity. Parthenogenesis has been described by Schaudinn for the human malarial parasite (Fig. 72) and in Trypanosoma noctuce, and by Prowazek for Herpetomonas musca-domestica; none of these cases, however, are entirely free 
from doubt, and in any case parthenogenesis seems to be of much rarer occurrence among Protozoa than among Metazoa.*

Autogamy, on the other hand; is a phenomenon which has been frequently observed in Protozoa, chiefly, though not exclusively, among parasitic forms ; it may be defined as syngamy in which the two gametes, or at least the two pronuclei, that undergo fusion are sister-individuals derived by fission of the same parent cell or nucleus. Hartmann (116) has brought together the many cases of autogamy known to occur among Protozoa and other-Protist organisms, and has classified them under a complex terminology. It is sufficient here to mention two typical cases, those, namely,
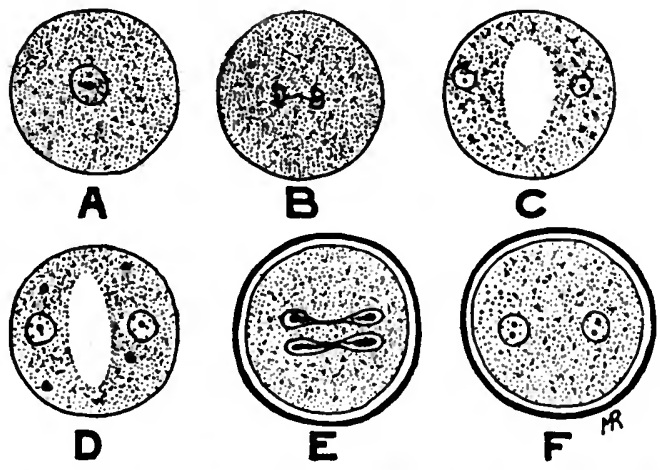

Fra. 73.-Autogamy in Entarnaba colv. A, The amœbs at the beginning of encystation with a single nucleus; $B$, the nuolens dividing; $C$, the two daughter-nuclei throwing off chromidia; a space has appeared between them; $D$, each nucleus has formed two reduction-nuclei, whieh are being absorbed; $E$, a resistant oyst-membrane has been secreted; the partial division in the protoplasm has disappeared, and the two reduced nuclei are each dividing into two ; $F$, each daughter-nucleus of the two divisions in the last stage has fused with one of the daughternuolei of the other division to form two synkarya. After Hartmann (116), drawn by him from the dosoription given by Schaudinn (131).

of Actinospharium and Entamoeba coli, made known by $R$. Hertwig (64) and Schaudinn (131) respectively.

\section{In Actinospharium} an ordinary individual (Fig. 3) becomes encysted as a multinucleate "mother-cyst," which becomes divided up into a number of uninucleate "primary cysts," after absorption of about 95 per cent. of the nuclei originally present. Each primary cyst then divides completely into two distinct cells"secondary cysts."

Each secondary cyst then goes through a process of nuclear reduction (see below), after which it is a gamete; the two gametes then fuse completely, cell and nucleus, to form the zygote.

* Prowazek (557) has desoribed in Herpetomonas musca-domestica a process interpreted by him as parthenogenesis ("etheogenesis ") of male individuals, but the correctness both of his observations and of his interpretations aro open to the gravest doubt. Aecording to Flu (536), the objects to which Prowazek gave this interpretation are in reality stages in the lifs-history of a quite distinot organism, named by Flu Octasnorea musca-domesticas, and now referred to the Microsporidia. It is grestly to be depreosted that interpretations of such uncortsin validity should be used, ss has boen done, to support general theories in the disoussion of the problem of syngamy. 
In Entamceba coli (Fig. 73) the process starts in like manner frosn a uninucleate individual, the nucleus of which divides into two, but the cell divides incompletely and only temporarily. Each nucleus then breaks up completely into chromidia and disappears from view. Some of the chromidia are absorbed, while from others a secondary nucleus is formed on each side of the cell, so that two nuclei reappear again in the cyst, but smaller than before and staining feebly. Each secondary nucleus now divides twice to form three nuclei on each side, two of which degenerate as reduction-nuclei, while the third in each caso persists as a gametenucleus. As soon as the process of reduction is complete, the incomplete separation of the two cells disappears, so that the two gamete-nuclei lie in a single cell, which at this stage forms a tough cyst. Now each gamete-nucleus divides into two pronuclei, those of the same pair being slightly different from those of the other, according to Schaudinn (133). Then a pronucleus of each pair fuses with a pronucleus of the other pair, so that two synkarya result. At a later stage each synkaryon divides twice, and eight amœbulm are formed by division of the cell within the cyst.

From these two examples, it is seen that autogamy is a process of extreme inbreeding as regards the gametes. In typical cases of syngamy the two gametes must be derived from two distinct strains, and those of the same strain will not conjugate; Schaudinn (131), for example, observed that the gametes of Polystomella crispa would only copulate when a couple came together in which each gamete was of distinct parentage. In a great number of Protozoa the differentiation of the gametes and their mode of formation makes it certain that the couple which join in syngamy are derived from different parents. On the other hand, in many cases of autogamy that have been described, it is equally certain that the conjugating gametes and pronuclei have a common parentage, and it is hardly possible to consider autogamy otherwise than as a degeneration of the sexual process, evolved in forms in which one feature of true syngamy-namely, the mixture of distinct strains-is, for some reason, no longer a necessity; we shall return to this point when discussing the nature and origin of the syngamic process. It is possible, moreover, to recognize progressive stages of the degeneration, as shown by the two examples selected. In the less advanced stage (Actinospharium) the parent cell divides into two complete cells, each of which, after a process of maturation, becomes a gamete. In the more advanced stage (Entamaba coli), the division of the parent-cell is checked, and only its nucleus divides, each daughter-nucleus becoming a pronucleus after reduction.

The occurrence of autogamy has been asserted in a number of cases which are, to say the least, oxtremely doubtful, as for example, the Myxosporidia 
(p. 407) and allied organisms, where it is far from certain that the two nuclei or colls, from which ultimately the pronuclei or gametes arise, have a common parentage. Autogamy has recently become very fashionable, and there is a tendency to regard as such, not only many cases which are probably truly heterogamous, but also nuclear fusions or appositions which are not in any way sexual (e.g., Schilling, 134).

The essential point to consider, in cases of autogany, whethor there is a union of chromatin derived from distinct strains-amphimixis-or from a common parentage-automixis. Thus, it has beon pointed out above that in gregarines two gametocytes may associate, and even fuso into cone body, but with the nuclei remaining distinct (Fig. 71, B). When gamete-formation takes place in a " neogamous" species of this type, the gametes of one sex derive their pronuclei from one gametocyte-nucleus, those of the opposite sex from the other, with subsequent syngamy, of a truly heterogamous type. If the fusion of the gametocytes were to go farther, a plastogamic, non-sexual union of the two nuclei might result, producing a single nucleus containing chromatin from two distinct sources; in that case, when gamete-formation took place, the syngamy would be, to all intents and purposes, a typical case of autogamy, and would certainly be so described if it were not known that the single gametocyte-nucleus had arisen by fusion of two distinct nuclei. If, however, in each couple of copulating gametes, one pronucleus contained chromatin derived from one of the two original gametocyte-nuclei, the other pronucleus, similarly, chromatin derived from the other nucleus, the result would be a true amphimixis, just as in ordinary heterogamy.

In Actinaspharium, plastogamic fusions of the ordinary vegetative, multinucleate individuals are stated to be of common occurrence; it is therefore possible that an individual which encysts may contain frequently nuclei from distinct sources. According to Brauer, fusion of nuclei takes place in tho mother-cyst to form the nuclei of the primary cyst. There is thorefore at least a possibility that the autogamy of Actinospharium may be, in some cases, combined with amphimixis.

In other cases, however, such as Entamceba coli and Amoba albida (Fig. 87), there seems little reason to doubt that the autogamy is a true automixis. Analogous cases of self-fertilization are well known in flowering plants, where they are sometimes the rule, sometimes an alternative to cross-fertilization. In freo-living Ciliata, also, syngamy has been observed between cousins, the descendants of an ex-conjugant after but four divisions (Jennings, 121), which is not far removed from automictic autogamy.

The conclusion put forward above, on experimental grounds, that syngamy has a strengthening or invigorating effect on the cell-organism, receives further support from the many instances in which it is observed to occur as a preliminary to the production of resistant stages destined to endûre unfavourable conditions of life. Thus, in free-living Protozoa syngamy occurs commonly in the autumn, previously to the assumption of a resting condition in which the organisms pass through the winter. In Difflugia, for instance, syngamy in the autumn is followed by encystment, and the cysts remain dormant until the spring. This is strictly comparable to the state of things known in many Metazoa, such as Rotifers, Daphnids, etc., where in the summer soft-shelled eggs are produced which develop parthenogenetically, but in the autumn hard-shelled winter-eggs are produced which require fertilization. In parasitic forms, such as Coccidia and Gregarines, syngamy is related to the formation of resistant cysts which pass out of the host 
and endure the vicissitudes of the outer world, until taken up by a new host in which the parasite is set free from its cyst and starts upon a fresh cycle of growth or multiplication without syngamy, under the most favourable conditions of nutrition.

2. As regards the effects of syngamy upon the species, it must be pointed out, in the first place, that a great difference exists between multicellular and unicellular organisms as regards the effects of external conditions of life upon the sexual process. In Metazoa the germ-cells, as already pointed out, are a race of cells apart, and are sheltered by their position in the body from the direct effects of external conditions-at least, to a very large extent. In Protozoa, on the other hand, there is no special race or strain of germ-cells, but any individual may become a gamete or the progenitor of gametes, and all alike are exposed to the direct action of the environment. If, now, Protist organisms placed under slightly different conditions of existence, tend to vary in their characters as a direct consequence of environmental influences, syngamy would check any such tendency, and would, on the contrary, tend to keep a given species constant and uniform in character, within narrow limits. Were there no intermingling of distinct strains, such as syngamy brings about, individuals of a species subject to different conditions of life would tend to give rise to divergent strains and races ; syngamy levels up such divergencies and keeps the tendency to variation within the specific limits (compare Eniriques, 112 and 113 ; Pearl, 124). If this supposition be correct, it would follow that no irue species could exist until syngamy had been evolred ; and if it be true that no syngamy occurs in organisms of the bacterial type of organizaition, then such organisms must be regarded as having diverged under direct environmental influences into distinct races and strains, but not as constituting true species. The "species" of bacteria would then be comparable to the races of the domestic dog, rather than to the natural species of the genus Canis. Not until syngamy was acquired could true species exist amongst the Protista, a condition which was probably first attained after the cellular grade of organization had been evolved.

The conclusions reached in the foregoing paragraphs may be summed up briefly as follows: Syngamy is a process of intermingling, in a single cell-individual, of chromatin derived from two distinct individuals, gametes, which may exhibit differentiation into "male" individuals, characterized by preponderance of kinetic activity, and "female," in which trophic activities are more pronounced. Syngamy is probably of universal occurrence in organisms of the cellular type of organization, and from them has been inherited by the higher plants and animals, but apparently 
it does not occur amongst organisms of the bacterial grade. Syngamy is related to the life-cycle as a whole, and not specially to cell-reproduction. In its effects on the cell-individual, syngamy appears to have an invigorating effect, renewing vital powers that have become effete and cxhausted; but in species that live in very favourable conditions of nutrition, etc., whether such conditions are due to artificial culture or to natural causes, such as parasitism. syngamy may be deferred for a very long time, and may even be completely in abeyance, or may degenerate into parthenogenesis or autogamy. In its relation to the race, syngamy tends to level down individual variations, and so produce true species amongst the Protista.

Before proceeding to discuss the nature and probable origin of the syngamic process, it is necessary to take into account a process which appears to be a universal concomitant of syngamy-namely, the process of nuclear reduction in the gametes. In all cases of syngamy that have been carefully studied, it has been found that the gametes differ from the ordinary cell-individuals of the species in having undergone a process of so-called "maturation" which consists essentially in nuclear reduction-that is to say, in a diminution of the normal quantity of the chromatin by so-called "reducing" divisions of the nucleus. Hence the pronuclei which undergo syngamic fusion differ in their constitution from the nuclei of cells not destined for this process, and do not multiply, as a rule, under normal conditions so long as they remain single. In some cases among plants, however, the cells that have undergone nuclear reduction may multiply by fission and produce a multicellular organism (gametophyte) from which gametes ultimately arise; in this way is brought about the well-known alternation of generations of the ferns and flowering plants. Since, moreover. in Metazoa, ova that have undergone nuclear reduction can be stimulated artificially to start their development without fertilization, it is clear that the nuclear reduction does not in itself inhibit further development or cell-multiplication.

True nuclear reduction in gametes must be distinguished clearly from the process of elimination of effete or vegetative chromatin which precedes the formation of the gametes or their nuclei, probably in every case. As has been stated above (p. 72), vegetative and generative chromatin may be combined in the same nucleus, or may occur, the one in the form of a nucleus, the other in the form of chromidia, or may constitute two distinct nuclei. When the two are combined in one nucleus, a necessary preliminary to gameteformation is the purification of the generative chromatin of all effete vegetative material. When the vegetative chromatin is already separate from the generative, the latter alone takes a 
share in syngamic processes, and the vegetative chromatin, whether as chromidia or a nucleus, disappears from the life-history.

Nuclear reduction, in the strict sense, concerns simply the nuclei composed of generative chromatin, and is a process which results in the reduction of the chromatin to half the specific quantity, a deficiency made up again to the full amount by the union of the two pronuclei to form the synkaryon. It is therefore a process which is seen in its most characteristic form in those cases where it is possible to gauge the amount of chromatin in the nucleus more or less accurately by the number of chromosomes formed during division.

In the Metazoa, where each species is characterized by possessing a number of chromosomes which is generally constant (the so-called "somatic number"), the process of reduction appears to be extremely uniform in its essential details throughout the whole series, from the Sponges and Cœlenterates up to man, and admits of a description in general terms. The gametocyte (oöcyte or spermatocyte), when at the full term of its growth, has a large nucleus which then goes through two maturative divisions in rapid succession. When the gametocyte-nucleus prepares for division, it appears with half the somatic number of chromosomes; but each chromosome is in reality bivalent, and produced by the fusion or close adherence of two separate somatic chromosomes. In the first reduction-mitosis, the two adherent chromosomes in each case separate from one another and travel to opposite poles of the spindle; hence this division is in reality a reducing, though it simulates in some of its features an equating, dirision. Immediately or very soon after the two chromosomes of each pair have separated, they split longitudinally in preparation for the next mitosis, which follows hard upon the first, and in which the two sister-chromosomes of each pair go to opposite poles of the spindle. Consequently the second reduction-division is in reality an equating mitosis, though on account of the precocious splitting of the chromosomes it may simulate a reducing division. Thus, to sum up the process briefly, the number of chromosomes in the germ-cells is reduced to half the somatic number by two successive mitoses, the first a reducing, the second an equating division. In the male sex, the spermatocyte divides into four gamete-cells of equal size, the spermatids, each of which becomes a spermatozoon. In the female sex the oöcyte-divisions are very unequal, producing the ovum, ripe for fertilization, and three minute sister-cells of the ovum which, as the so-called "polar bodies," are cast off and die away. By syngamy between a ripe ovum and a spermatozoon, each containing half the somatic number of chromosomes, the full somatic number is restored. 
In Protozoa the chromosomes are seldom so sharply defined as in Metazoa, and consequently it is difficult or impossible to determine their number. Many cases in wnich a fixed number of chromosomes is alleged to occur, as in Trypanosoma noctuce (Schaudinn, 132), cannot be accepted without question in the present state of our knowledge. On the other hand, in all groups of the Protozoa, where the sexual processes have been carefully studied, the union of the gamete-nuclei has been found to be preceded in a great many cases by two successive divisions of each nucleus, with one or the other of the following results: either the succcssive formation of two reduction-nuclei,* which are cast out of the cell or absorbed without dividing further, while the third persists as the pronucleus of the gamete; or the production of four nuclei, all of which, or only one of them, persist as pronuclei. These reducing divisions in Protozoa suggest forcibly a comparison with those of the Metazoa, and from this analogy it may be further inferred that in Protozoa also the chromatin of the conjugating pronuclei has undergone a reduction to half the specific quantity; but it is seldom possible to confirm this inference by accurate enumeration of the chromosomes. In the case which has been the most carefully studied of all others, that, namely, of Actinospharium, Hertwig (64) found the number of chromosomes in the first reductionspindle to be between 120 and 150 ; in the second reduction-spindle the number was about the same, but the chromosomes were about half the size of those in the first reduction-spindle. Moreover, in both the reducing divisions of Actinospharium the chromosomes in the equatorial plate divide to form the daughter-plates, as in ordinary karyokinesis, whereas in the reducing divisions of Metazoa the individual chromosomes are not divided, but merely sorted out. Hence it would appear that in Actinospharium, and probably many other Protozoa, the reduction of the chromatin in the pronuclei is effected by more direct, though perhaps less exact, methods than in the highly-perfected process seen in the Metazoa.

Nevertheless, a few cases are known among Protozoa in which the small number of chromosomes permits of their being accurately counted, and in which they are seen to be reduced to half the usual number in the maturation-divisions of the gametes. In Pelimyxa the first division reduces the chromosomes from eight to four ; the second division, however, is equating, and no further reduction takes place (p. 150). In some Infusoria it has been observed that

* These rednction-nuclei are sometimes termed "polar bodies," by analogy with the maturative process of the Metazosn ovum, but the term is to be avoided in this connection, as it places upon these divisions an interpretation which is at least highly doubtful ; the polar bodies of Metazos are sister-cells of the ovum; the reduction-bodies in Protozos are simply nnclei which are extruded or absorbed. It is certainly not justifiable in fact, and probably no more 80 in theory, to regard their formation as abortive cell-division. 
the first division of the micranucleus is an equating division, the second reducing; so in Opercularia (Enriques, 112), Chilodon (Enriques, 113), Carchesium (Popoff, 125), Didinium (Prandtl, 126), and Anoplophrya (Fig. 74); in the last named the second division of the micronucleus reduces the chromosomes from six to three, and anion of the pronuclei brings the number up to six again. In Carchesium the number of chromosomes is reduced from sixteen to eight. A similar reduction-process has been described by Mulsow (123) in gregarines (p. 335). Hence in these cases the pronuclei
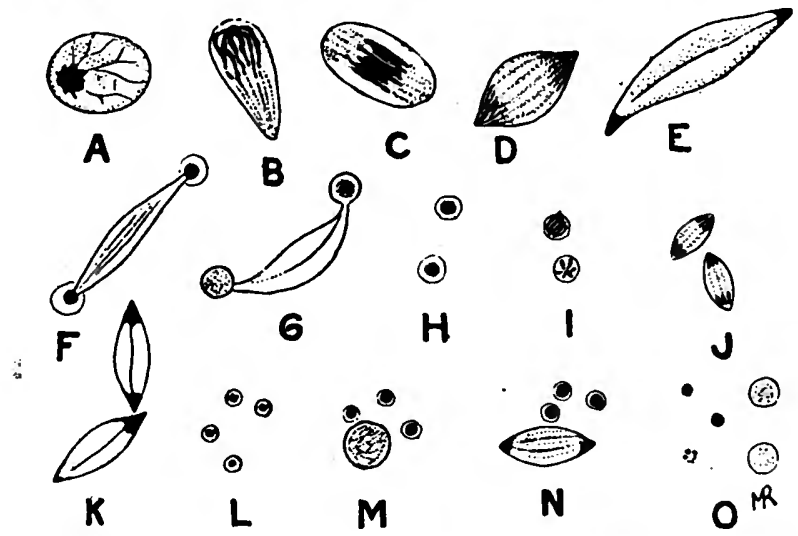

Fra. 74.-Behaviour of the micronucleus during sucoessive stages of the conjugation of Anoplophrya (Collinia) branchiarum. $A$, Micronuoleus of ono conjugant preparing for division ; $B$, later stage, with six chromosomes distinct; $O$, nuclear spindle, with an equatorial plate of six ohromosomes; $D$, diaster-stage, with six daughter-ohromosomes at cach pole of the spindle; $E$, lator stage, with the chromosomes at each pole fused into one mass; $F, G, H$, reconstruction of the daughter-nuclei ; the remains of the spindle between them disappears gradually; $I$, the two micronuclei preparing for division; appearance of six chromosomes in each (ono nucleus is seen in profile, the other from one pole); $J$, diaster-stages, showing three ohromosomes at each pole of the spindle (reduoing division); $K$, later stage, the chromosomes fused into masses of chromatin ; $L$, four granddaughter-microinuclei ; $M$, one of them grows in size, the other three begin to degenerate; $N$, division of the persistent micronucleus to form the two pronuolei; $O$, two pronuclei and three degenerating micronuclei. After Collin (50), magnification about 2.000 diameters.

have exactly half the amount of chromatin contained in the ordinary nuclei, just as in the Metazoa.

Doflein (111) and Hartmann (116) consider that a process of reduction is absolutely essential to the conception and definition of syngamy, and regard reduction as a criterion whereby true syngamic union of gametes and pronuclei can be distinguished from plastogamic and nuclear fusions which have nothing to do with the sexual process. "No fertilization without reduction" (Hartmann). But it must be acknowledged that in a great many cases of gamete-formation in Protozoa a reduction of the chromatin- 
substance of the conjugating pronuclei cannot be deduced from observation, and could only be inferred from analogy. In the gamete-formation of Coccidium schubergi, so carefully studied by Schaudinn (99), a large number of male pronuclei are formed simultaneously by local condensations of chromidia thrown off from the nucleus of the gametocyte, which is left behind in the residual protoplasm, with its conspicuous karyosome (Fig. 50); in the female gamete, also, the process of reduction appears to consist of a simple elimination of the karyosome (Fig. 75), a process which could be interpreted more naturally as elimination of effete vegetative chromatin than as a process of true nuclear reduction. In the case of Coccidium, as in others that might be cited, it must either be assumed that reduction-processes, in the strict sense of

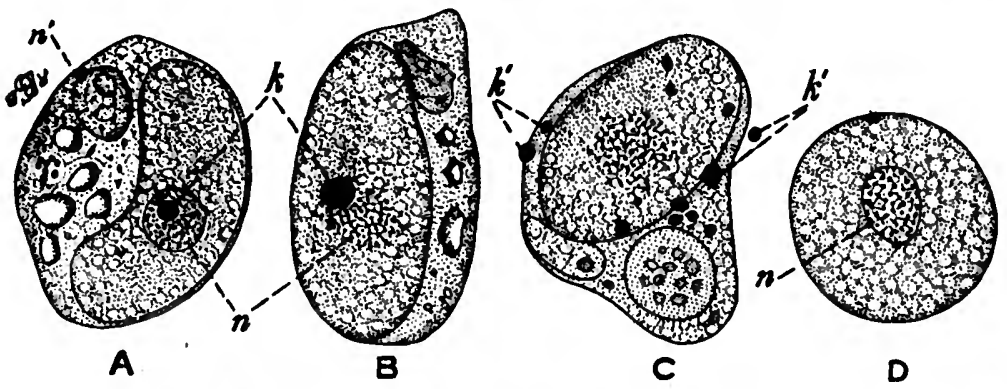

Fra. 75.-Four-stages in the maturation of the female gametooyte of Coccidium schubergi. $A$, Full-grown macrogametocyte contained in the host-cell; $B$, the macrogametoeyte is beginning to round itself off and to expel the karyosome from its nueleus; $C$, the karyosome expelled from the nucleus of tho macrogametocyte has reached the surface of the body and broken up into a number of fragments, which lie scattered in the body of the host-oell or are extruded from it; $D$, the macrogametocyto has now become a ripo macro. gamete, having rounded itself off, eliminated the ksryosome from its nuclens, and divestod itself entirely of the host-oell. n., Nucleus of the gametocyto; $k$., its karyosome ; $n .^{\prime}$, nuoleus of the host-eell ; $k .^{\prime}, k .^{\prime}$, fragments of ertrudud karyosome. After Schaudinn (99), magnified 1,000.

the phrase, occur but have been overlooked, or that the method of reduction is one that can only be brought into line with the typica] method by theoretical interpretation founded on analogy.

It must therefore remain an open question, in the present state of our knowledge, whether a process of nuclear reduction strictly comparable to the process seen in Metazoa is essential to the definition of true syngamy, or whether such a process has not been evolved and perfected gradually as a consequence of the sexual process. It is quite conceivable that syngamy may have been at its first origin merely a process of intermingling of chromatin of distinct cell-individuals; that in this crude and primitive form syngamy would tend to disturb the normal balance of nucleus and cytoplasm, since it would lead to quantitative excess of the 
nuclear substance; that, consequently, by a regulative process which may primitively have followed the syngamic union, the chromatin of the zygote was reduced to the normal quantity by elimunation of half of its mass; and that from this hypothetical primitive process of regulation of the nucleo-cytoplasmic balance a process of nuclear reduction preceding the syngamic act has been gradually evolved until it reaches its perfection in the form seen in the Metazoa. On this view, it is to be expected that in Protista a great diversity in the methods of nuclear reduction would occur, from those of the roughest type to others highly elaborated and perfected; and this expectation certainly receives justification from the data of observation. Hertwig (119), on the other hand, compares the reducing divisions in the maturation of the gametes to the so-called "hunger-divisions" in Infusoria, which exhibit a great disproportion in the relative mass of nucleus and cytoplasm as the result of starvation in artificial cultures; in such forms the body is smaller than in forms from a normal culture, but the nucleus is not merely relatively, but absolutely, larger than that of a normal form. The disturbance in the nucleo-cytoplasmic ratio (see p. 70, supra) can, however, be regulated by reducing divisions of the nucleus. On the ground of this comparison, Hertwig considers that the maturative processes of the gametes are to be regarded as the necessary consequences of antecedent events* in the life-history -as processes which in their turn bring about syngamy, and not such as have the object of preparing the nuclei for fertilization.

In order to give a more concrete idea of the processes of syngamy and reduction in Protozoa, a few typical examples will now be described, selected in order to illustrate the salient features of these processes. The most convenient method of classification of the examples chosen is to distinguish those cases in which chromidia are present in addition to nuclei from those in which nuclei alone are present.

1. Syngamy and Reduction with Nuclei and Chrominita.-In a great many Sarcodina, especially those belonging to the orders Amœbæa (p. 218) and Foraminifera (p. 231), chromidia may be present in the gamete-forming individuals as a permanent constituent of the body-structure. In such cases the chromidia represent, wholly or in part, the generative chromatin, and give rise, by formation of secondary nuclei, to the nuclei of the gametes. As an example Arcella may be taken, the life-cycle of which is described in a subsequent chapter. In this form two distinct forms of syngamy have been described.

* It is, of course, hardly necessary to point ont that starvation is by no means the only influence which can bring about a disturbance of the nucleo-cytoplasmic equilibrium ; over-nutrition, for example, may havo the same effect. 
(a) Karyogamy.-The body of an Arcella gives rise by multiple gemmation to a number of amœbulæ, each containing a secondary nucleus derived from the chromidia, while the primary nuclei of the parent-form degenerate (Fig. 80). The number and size of the amœbulæ vary, however, in different individuals. In one Arcella the number is less and the amobulæ are larger, eight or nine macramoboe being produced. In another the amœbulæ are more numerous and smaller, about forty micramoboe being formed. In either case the amœbulæ swarm out of the parent-shell and are the gametes. A micramœba copulates with a macramœba, the two fusing completely to form a zygote with a synkaryon. The amœboid zygote thus produced is the starting-point in the growth and development of an Arcella (Fig. 80, A).

In this example the karyogamy is a case of microgamy, which, like other such cases, precedes a period of growth and follows a period of active reproduction. It is possible that the syngamy of the gametes is preceded by reducing divisions of the nuclei of the amobulæ, but no such reduction has been observed in Arcella. In Foraminifera (p. 235), in which the syngamy is perfectly isogamous, each secondary nucleus formed from the generative chromidia divides twice to form the gamete-nuclei--divisions doubtless to be regarded as reducing divisions. In Centropyxis, according to Schaudinn (131), amœbulæ, all of the same size, are produced as in Arcella, by formation of secondary nuclei; but in some broods each amœbula divides into four micramœbæ (microgametes), whide in other broods the amœbulæ remain undivided as macramœbæ (macrogametes); copulation then takes place between two gametes of different size.

(b) Chromidiogamy (Fig. 80, $M-Q$ ).-Two ordinary adult Arcella come together and apply the mouths of their shells. The protoplasm of one individual flows over almost entirely into the other shell, taking with it both chromidia and primary nuclei, only so much protoplasm being left in the one shell as suffices to hold the two shells together. The primary nuclei now degenerate, and the chromidia derived from each conjugant break up into a fine dust of chromatin-particles and become intimately commingled. When this process is complete, the protoplasm with the chromidia becomes again distributed between the two shells, and the two conjugants separate. Then in each individual secondary nuclei are formed from the chromidia, and by a process of multiple gemmation a number of uninucleate amcbulæ are formed which swarm out of the shell, and, like the zygotes resulting from karyogamy, become the starting-point of a new Arcella.

Thus chromidiogamy is here a case of macrogamy which, like other similar cases, follows a period of growth and precedes a 
period of active reproduction. Chromidiogamy is a raro but very interesting form of syngamy which, from the standpoint of general notions with regard to the evolution of the nucleus, may be regarded as the most primitive type. It is known to occur also in Diffugia (Zuelzer, 85), where also copulation of swarm-spores takes place as an alternative method (p. 230).

A case must now be considered in which the chromidia represent vegetative, while the nuclei contain the generative, chromatin. An example of this state of things is furnished by Plasmodiophora brassica, a well-known parasite of cabbages, turnips, etc., in which
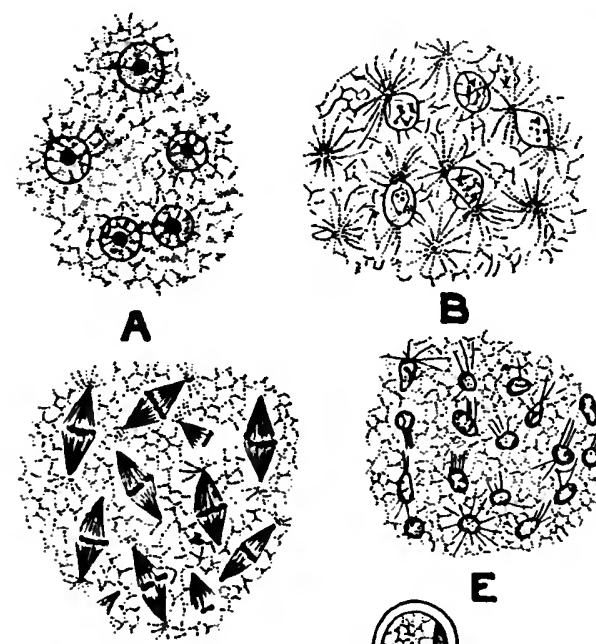

D
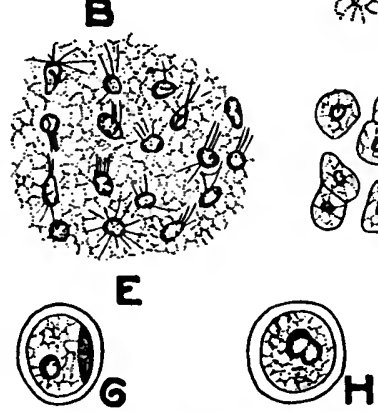
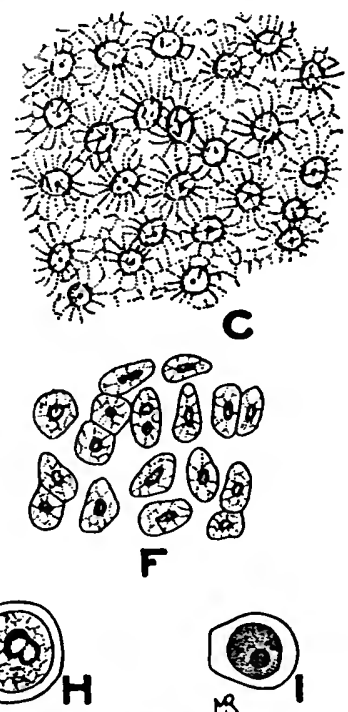

Fic. 76.-Gamete-formation and syngamy in Plasmodiophora brassicas. A, Normal vegetative nuclei of the myxamobø; $B, C$, extrusion of chromidia from the nuclei ; $D$, division of the nuclei by karyokinesis (first reducing division); $E$, wuclei after reduction; $F$, formation of gametes which are fusing in pairs ; $G$, spore (zygote) containing two nuclei, one of which is going through a further reduction-division; $H$, fusion of the two pronuclei within the spore; $I$, ripe spore with synkaryon and two centriolcs. After Prowazck (127), magnified abont 2,250 diameters.

it produces a disease known as "fingers and toes" (Kohlhernie). According to the investigations of Prowazek (127) and others, Plasmodiophora goes through a development which may bo briefly summarized as follows: At the end of the "vegevative" period of growth and multiplication, there are found within the cells of the infected plant a number of " myxamœbæ," amœboid individuals (plasmodia) each with many nuclei containing distinct karyosomes (Fig. 76, $A$ ). From the nuclei chromidia are given off into the cell, and during this process the karyosomes disappear and centrosomes make their appearance (Fig. 76, B, C). The chromidia are ab- 
sorbed and disappear, and the nuclei divide twice by karyokinesis (Fig. 76, D), so that their number is quadrupled. The myxamœba then undergocs multiple fission into as many cells as there are nuclei in the plasmodium (Fig. 76, F), and each of these cells is a gamete. The gametes now conjugate in pairs, and the zygotes become encysted to form the spores. Within the spores the nuclei of the gametes are stated to undergo a further process of reduction before they fuse to form the synkaryon (Fig. 76, $G$ ). The syngamy in Plasmodiophora is stated to be a case of autogamy, but this allegation assumes that the nuclei of the myxamœbæ are sisternuclei derived all from the division of one original nucleus; they may equally well be nuclei of different origins brought together by plastogamic fusions.

The two examples selected, Arcella and Plasmndiophora, show that the chromidia may represent generative chromatin in one casc, vegetative in another. Goldschmidt (57) has proposed to distinguish these two conditions by a special terminology, retaining the name " chromidia" (trophochromidia, Mesnil, 74) for those which are purely vegetative, and coining a new term, sporetia (idiochromidia, Mesnil) for those of generative nature. It is more convenient, however, to retain the term "chromidia" in its original significance, to denote simply excranuclear particles of chromatin, and to qualify the term by the adjectives "vegetative" and "generative" when required (see also Goldschmidt, 41, p. 130). The formation of vegetative chromidia, which are finally absorbed, is a common phenomenon in many Protozoa; it may take place as a purely regulative process, as in Actinosphorium during depression-periods (p. 208), when hypertrophy of the nuclear apparatus is corrected by the extrusion from the nuclei of chromidia, which ultimately degenerate and become converted into masses of pigment, and as such are eliminated from the protoplasm.

Tho account given by Bott (103) of gamete-formation in the common Pelomyxa (A maeboea nuda, p. 227) describes a condition in which chromidia, extruded from the nuclei, are partly vegetativo, partly generative; secondary nuclei are formed from them, which later cast out a portion of their chromatin, then give rise to the gamete-nucloi. After the secondary nuclei have been purified in this way of their vegetativo chromatin, the generative chromatin remaining in each of them forms a karyokinotic spindle with oight chromosomes, and a reducing division follows by which each daughter-nucleus obtains four chromosomcs. The " pronuclei of the first order," resulting from the first reducing division, divide again, forming a spindle with four chromosomes which split, so that the "pronuclei of the socond order" have also four chro. mosomes. From the nuclei that have undergono reduction in this manner the nuclei of the gametes arise in a somewhat remarkable fashion : tho pronuclei of the second order separate into two compact masses of chromatin; a vacuole is formed near them; and the chromatin of the two masses wanders, in the form of finely-divided granules, into the vacuolo to form the definitive pronucleus of the gamete, which forms a membrane when the process is completo. When formed the gametes wander out as Holiozoon-like indi- 
viduals, which copulate in pairs, and the uninucleate zygote grows up into the multinucleate Pelomyxa.

The conception of vegetative and generative chromidia has not been accepted universally or without criticism. Hartmann, as pointed out above, considers that many cases of generative chromidia are really the result of a disruption of a polyenergid nucleus; Awerinzew (47) is of opinion that, while all Protozoa possess vegetative chromidia at some stage at least in the lifecycle, generative chromidia are to be considered as a new acquisition, a hastening of the process of the formation of numerous gamete-nuclei; Doboll (51) puts forward a similar view. with regard to generativo chromidia. With regard to the latter criticism, it may be pointed out that nuolei may become resolved into chromidia in order to undergo simple binary fission. With regard to Hartmann's view, there is at present, at loast, little evidence that it is an adequate explanation of the many cases of formation of secondary generative nuclei from chromidia known amongst the Sarcodina. The question is discussed further below (p. 255).

2. Syngamy and Reduction with Nuclei only.-A very simple example is furnished by the common Actinophrys sol (Fig. 71), as described by Schaudinn (129). Conjugation takes place between two adult forms (macrogamy), which come together and become enclosed in a common cyst. The nucleus of each individual then divides by karyokinesis, and one nucleus of the pair thus produced is expelled from the body and undergoes degeneration as a reduction nucleus. The persistent nucleus of each individual then repeats the process and forms a second reduction-nucleus. The nucleus now remaining in each cell is the definitive pronucleus. The two gametes now copulate, their pronuclei fusing to form the synkaryon, after which the synkaryon divides by karyokinesis and the zygote divides into two individuals which later escape from the cyst and resume the free-living vegetative life. The course of syngamy in Actinophrys is exactly similar to that performed by the two " secondary cysts" derived from division of a "primary cyst" in Actinosphcerium (see p. 138, supra). In both cases alike the nucleus of the conjugants may be supposed to contain both vegetative and generative chromatin mixed together. It is possible that the vegetative chromatin is extruded from the nucleus in the form of chromidia prior to the reducing divisions, but no elimination of vegetative substance has been described.

The last example of syngamy in Protozoa that need be considered specially at this point is that of the Infusoris, which have been the subject of numerous investigations. These organisms present the highest degree of specialization of the body-structure and elaboration of the nuclear apparatus found in any Protozoa. Their syngamic processes vary in detail to some extent in different cases (see p. 448), but the whole process is essentially as follows (Fig. 77): Two individuals come together and adhere, placing themselves side by side. The two conjugants may be similar in visible constitution, or may differ to a greater or less extent, and 

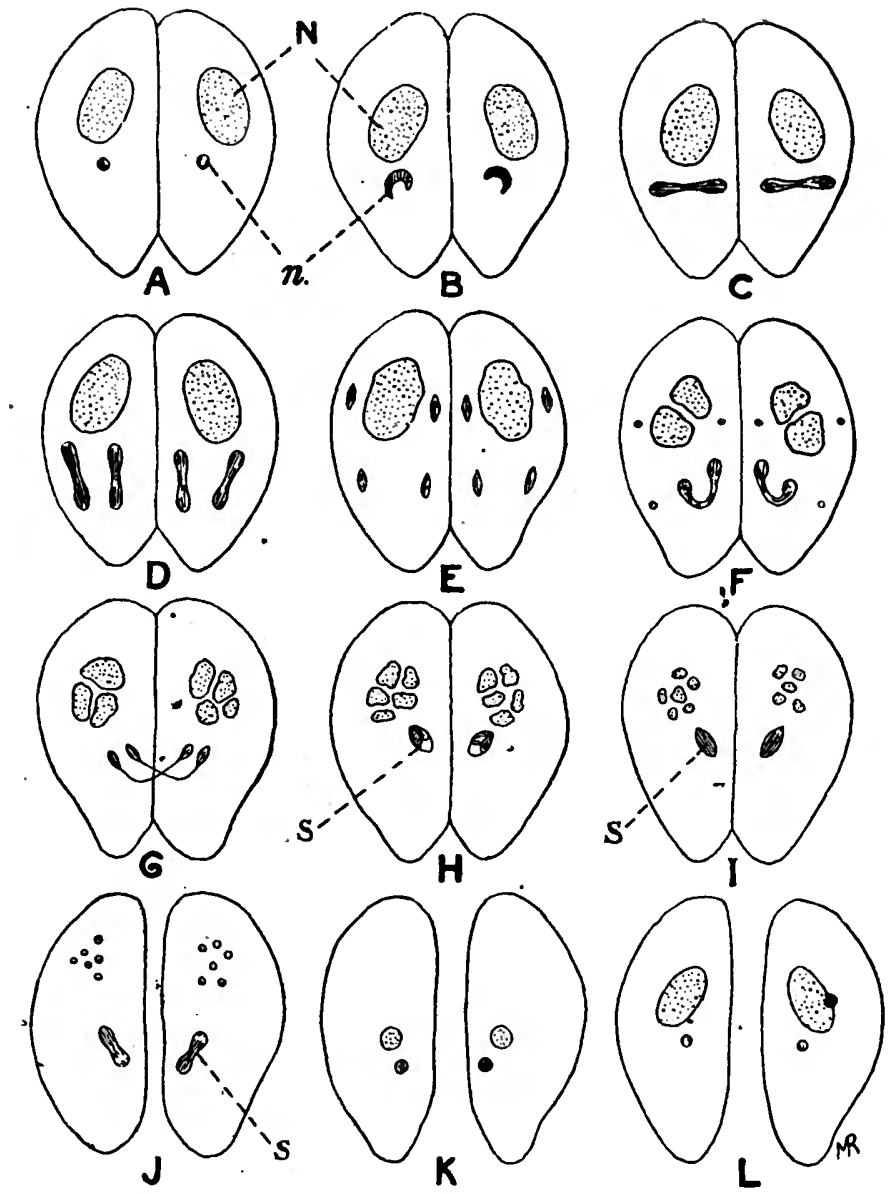

Fia. 77.-Diagram showing the suocessive stages of conjugation in Infusoria. $A$, The two conjugants attached, each with a macronucleus $(N)$ and a micronucleus $(n) ; B, C$, tho micronucleus of cach conjugant dividing; $D$, each conjugant has two micronuolei which aro beginning to divide again ; $E$, eaoh conjugant has four micronuclei; tho macronuclei are beginning to become irregular in form; in later stages they degenerate, break up, and are absorbed; $F$, three of the four micronuclei of each conjugant are degenerating and being absorbed ; the fourth is dividing ; $G$, one half of each dividing micronucleus of the preceding stage has travelled over into the other conjugant as the migratory pronucleus; $H, I$, fusion of the stationary pronucleus of each conjugant with the migratory pronucleus derived from tho other conjugant to form the synkaryon $(S) ;$.$J , the two conjugants now separate: in each$ ex-conjugant the synkaryon (S.) divides; the old macronuolei aro now almost complctely absorbed; $K, L$, the synkaryon has divided into two nuclei, one of whioh grows large and becomes the new macronueleus, the other remains small and becomes tho new micronuclcus, of each cx-conjugant. After Delage and Hérouard. 
are sometimes markedly different in size (Doflein, 111). The greatest amount of differentiation is seen in the order Peritricha (p. 448), where microconjugants and macroconjugants can be distinguished. Each conjugant has a micronucleus and a macronucleus. The macronucleus begins to degenerate, and finally disappears completely. The micronucleus, on the other hand, enlarges and divides by a simple form of karyokinesis (see p. 114, supra). The division of the micronucleus is repeated twice as a rule, but sometimes three times, and, as stated above, in one of these divisions the number of chromosomes is halved in a great many, possibly in all, cases. Of the four (or eight) micronuclei thus formed, all but one represent reduction-nuclei which are absorbed and disappear. The persistent micronucleus then divides by equating division into two pronuclei, which may be distinguished as migratory and stationary, respectively ; they sometimes exhibit distinct structural differentiation. At this juncture the cuticle of each conjugant is absorbed at the point of contact, and the migratory pronucleus of each conjugant passes over into the protoplasm of the other and fuses with its stationary pronucleus. The gap in the cuticle is now repaired and the two individuals separate, each "ex-conjugant" having a synkaryon constituted by a fusion of one-eighth (or onc-sixteenth) of its own original micronucleus with the same fraction of the micronucleus of the other partner. The synkaryon grows and divides into two nuclei, one of which grows and becomes the macronucleus, while the other remains small and becomes the micronucleus, of the ex-conjugant, which thereby becomes indistinguishable from an ordinary individual of the species, and proceeds to start on a course of vegetative growth and reproduction in the usual manner, until the next act of syngamy initiates a fresh cycle. It has been observed that the two ex-conjugants sometimes differ markedly in their capacities, one of them multiplying much faster than the other.

In the syngamy of Ciliata it is seen clearly that the macronucleus represents effete vegetative or "somatic" chromatin, which is eliminated bodily from the life-history of the organism, while the micronucleus represcnts reserve generative chromatin from which, after reduction, the entire nuclear apparatus is regenerated. The remarkable feature in the syngamy of Infusoria is the manner in which. the conjugants remain distinct, and merely exchange pronuclei (so-called "partial karyogamy"). Versluys (137), following Boveri, derives this from an ancestral condition of isogamic copulation-that is to say, a condition in which the two conjugants fused completely as gametes, both body and nucleus, after which the zygote divided into two individuals; on this view the final division of the micronucleus which gives rise to the two 
pronuclei is to be regarded as the equivalent of the division of the synkaryon which took place ancestrally after syngamy. While, however, there is a general agreement that partial karyogamy (conjugation) is to be derived from total karyogamy (copulation), it is very doubtful if the two conjugants in Infusoria represent simple gametes; it is more probable that the type of syngamy characteristic of Infusoria is derived from an ancestral condition in which each conjugant produced a number of minute gametes (swarm-spores) which copulated (compare especially Popoff, 125, and Hartmann, 116, and see p. 453, infra). On this view the divisions of the micronucleus represent a primitively much larger number of divisions which produced the numerous gametes, and the conjugants themselves are not to be regarded as true gametes, but rather as gametocytes or gamonts.

Having now illustrated by typical examples the various forms which the syngamic process takes in Protozoa, we may conclude this chapter by a consideration, necessarily brief, of the problem of the significance and origin of syngamy and sex. This is a problem which has a vast literature, and it is only possible here to indicate in outline some of the theories that have been put forward, none of which can claim to be a complete solution of one of the profoundest mysteries of the living substance and its activities.

Considering first the fertilization of the Metazoa, it is evident that the union of the spermatozoon with the ovum has two principal results. In the first place the spermatozoon brings with it a pronucleus, the equivalent of that contained in the ovum, but derived from a distinct individual, and therefore possessing different hereditary tendencies acquired from its own particular ancestral history. The union of the male and female pronuclei brings about, therefore, a process for which Weismann has coined the term amphimixis - that is to say, a mingling of different hereditary tendencies in one and the same individual. In the second place the spermatozoon produces a result which may be termed briefly " developmental stimulus" (Entwicklungserregung)—that is to say, it produces a disturbance in the equilibrium of the protoplasmic body of the ovum which causes it to start on a course of cell-division oft-repeated, a process of cleavage which converts the unicellular ovum into the mass of cells which supplies the material for the building up of the multicellular body. It is very probable that the developmental stimulus is supplied by the greatly-developed centrosome of the spermatozoon, that of the ovum having completely atrophied, apparently, after the completion of its maturative processes.

The introduction of a male pronucleus-that is to say, the process of ampllimixis - can be effected only by the spermatozoon. But the researches of Loeb and others have demonstrated fully that the 
spermatozoon is not indispensable for supplying a developmental stimulus ; an unfertilized ovum can be induced by artificial stimuli of various kinds to start upon a course of development similar to that initiated, under natural circumstances, by fertilization with a spermatozoon. Hence, of the two results produced in the fertilization of Metazoa, amphimixis alone would appear to be that which is essential and peculiar in the process, and which only fertilization can bring about.

From the above considerations, amphimixis is regarded by many thinkers as the essence of syngamy, a necessity for the evolution of living beings in that it supplies, by the intermingling of different hereditary tendencies, the conditions required for the production of " innate" variations in organisms in which the germinal substance is shielded from the direct influence of external conditions by its position within a multicellular body. Apart from the question, however, whether any such innate variations exist in the Protozoa, where all cells alike are exposed equally to the direct action of the environment, the criticism has often been made that amphimixis gives only a teleological explanation of the sexual process, and as such cannot be invoked as a causal explanation of its origin. The intermingling of distinct hereditary tendencies, however useful to the organism or important in the evolution of living beings generally, cannot be regarded as the incentive to syngamy at its first appearance in the Protista. In other words, amphimixis must be regarded as a secondary consequence not as a primary cause, of syngamy.

It is necessary, therefore, to seek some explanation for the first origin of syngamy other than the benefits which it may confer through amphimixis, and it is undoubtedly among Protist organisms that the conditions under which syngamy first arose must be sought. It has been pointed out above that syngamy appears to have a strengthening or recuperating effect upon the cell-organism, and upon such grounds has bcen founded the theory of "rejuvenescence" (Verjüngung). According to this theory, connected chiefly with the name of Maupas, the cell-protoplasm, after many generations of reproduction by fission, tends to become effete and senile to an ever-increasing degree, a condition which, if not remedied, ends in the death of the organism; the natural $r \in$ medy is furnished, however, by the process of syngamy, which has the effect of renewing the "youth" of the cell and starting it upon a fresh series of generations, until senility, once more supervening, necessitates syngamy again.

The rejuvenescence-theory has been criticized by many critics who have themselves done little more, in some cases, than give a more precise meaning to the terms "youth" and "old age," terms that certainly stand in need of further explanation, since 
it can hardly be supposed that the time-factor alone can account for the exhaustion or depression of the vital faculties. It is generally admitted that unicellular organisms, such as the Protozoa, tend, after a greater or less number of generations, to exhibit a certain degree of exhaustion in their vital properties, or, it may be, of derangement in their organization and vital mechanisms. Hertwig (164) is of opinion that " the conditions of death exist in the living substance from the beginning, and are a necessary consequence of its vital function" - a generalization which may be accepted for those Protista in which the body exhibits the degree of specialization and structural complication proper to a true cell (as the term is understood in this book-see p. 98) ; but it is very doubtful if it is true also for the simplest forms of life, such as the bacteria and allied organisms. If it be further admitted that syngamy is the natural remedy in unicellular organisms for a natural disease, the prohlem before us is to discover, if possible, the precise nature of the derangements, and of the method by which the remedy restores them to the normal functional condition.

At the outset, attention must be drawn to a very constant and general preliminary to syngamy in Protozoa-namely, the elimination of a large amount of chromatin which appears to have been regulating the vital activities during previous generations (vegetative chromatin), and its replacement by chromatin which has been inactive and lying in reserve (generative chromatin). This process is seen in its most striking form in the Ciliata, where the macronucleus is entirely eliminated during the act of syngamy, and is replaced in subsequent generations by a new macronucleus derived from the micronucleus formed by fusion of portions of the micronuclei of the partners in syngamy. Hence it might seem as if the chief result of syngamy was to replace effete vegetative chromatin by fresh generative substance which through inactivity has retained its powers unimpaired. But in the first place it must be pointed out that, to effect a replacement of this kind, the union of two individuals is not necessary; it would be sufficient for a single individual to form a new nucleus from its store of generative chromatin, and to get rid of its old, effete vegetative chromatin. If we regard the chromidia of Arcella as composed of generative chromatin, the buds produced by formation of sccondary nuclei from the chromidia would represent nuclear regeneration of this kind. Secondly, it is open to doubt how far the theory of vegetative and generative chromatin can be applied throughout the whole series. In such forms as Arcella the chromidial mass, although it furnishes the gamete-nuclei, is a ccll-element in a functional condition, and in the more primitive forms the distinction between vegetative and generative chromatin cannot be pressed so far as 
in highly-organized forms, such as the Ciliata. Hertwig (68) considers that the separation of two kinds of chromatin is an adaptation to particular conditions of life, evolved progressively, and attaining its greatest perfection in the Ciliata; whereby chromatin which has become functionally effete is separated from that which has retained its constitution.

According to the view put forward by Hertwig (118), syngamy remedies the effete condition of the cell chicfly by regulating the necessary quantitative balance between the nucleus and the cytoplasm. Such regulation may be effected also by internal rearrangements of the nucleas substance or by plastogomy, but is brought about most efficiently by syngamy, since the definite and necessary mutual relations between nucleus and cytoplasm are better maintained by " arrangements which prevent disturbance, than by arrangements which compensate for disturbances that have already set in." The obvious criticism of this theory is that it is difficult to understand why an internal regulative process of the cell should require the co-operation of two individuals, and the reason contained in the sentence just quoted from Hertwig scarcely seems an adequate explanation.

The fact that two cells participate in syngamy indicates in itself that the necessity for syngamy depends on a loss of balance between two constituents or substances in the cell, and that the union of the two gametes restores equilibrium. As Hertwig (119) has pointed out, the quantitative relation of nucleus to cytoplasm is more altered in the gametes of Metazoa than in any other cells, and to opposite extremes in the two sexes ; in the orum the quantity of cytoplasm is enormous in proportion to the nucleus, while in the spermatozoon the exact reverse is the case. The same argument applies to a greater or less degree in the case of anisogamous gametes of Protozoa. It would not, however, apply to the many cases of isogamy in Protozoa where the quantitative relations of nucleus and cytoplasm are the same in each gamete; in such cases union of the gametes would leave the nucleo-cytoplasmic relation exactly what it was before.

A theory of a different kind has been put forward by Schaudinn (133) and his followers Prowazek (128) and Hartmann (116), which is based on the notion that sex and sexual differentiation are primary characteristics of living matter. A normally functioning cell is regarded as hermaphrodite, having male and female elements equally balanced. The differentiation which leads to the formation of gametes arises, as Bütschli originally suggested, from inequalities in the results of cell-division, which may be supposed to lead always to more or less imperfect partition of the qualities of the parent-cell between the daughter-cells. As a result 
of the defects in the process of cell division, some cells acquire more " male" properties, other more "female"; the cells preponderatingly male show greater kinetic and motile energy, those that have more female qualities show greater trophic activity. With continued cell-division these opposite tendencies tend to accumulate in certain cells which in consequence become altogether one-sided in thcir vital activities. Thus a want of balance in the vital functions is brought about, which may reach such a pitch that the organism is unable to continue to assimilate and reproduce, and must die unless the balance is resorted by syngamy with an individual that has become specialized in the opposite direction. By the union of two gametes differentiated in this manner, equilibrium is restored and the vital functions are reinvigorated. No gametes, however, whatever their degree of specialization, are to be considered as perfectly unisexual, but only relatively so; a male gamete will always contain a certain amount of female substance, and a female gamete a certain amount of male substance, thus accounting for the possibility of parthenogenesis. Schaudinn's theory of sex is thus very similar to that developed by Weininger on purely psychological grounds.

Schaudinn, whose work on Protozoa must secure full consideration for any statement of his observations, however inherently improbable the facts or the interpretations based upon them may seem, founded his theory chiefly on data alleged to have been observed by him in the development of Trypanosoma noctuce (Schaudinn, 132). According to him, an "indifferent" oökinete might give rise either to male or female forms. In the formation of males, certain nuclear elements were separated out to become those of the daughter-cells, while certain other nuclear elements remained behind and degenerated together with a quantity of residual protoplasm. In the formation of females, the same two sets of nuclear structures were separated out, but those proper to the male sex degenerated, while those of the female sex, which were just those which degenerated in the formation of males, in this case persist and become the nucleus of the female gamete. Thus the indifferent oökinete was supposed to be really hermaphrodite, containing male and female elements mixed together, and giving rise to individuals of one or the other sex by persistence of one set of characters and atrophy of the other. It imust be noted here that these observations of Schaudinn's are entirely unconfirmed, nothing similar having as yet been found by other investigators, either in trypanosomes or in any other Protozoa ; and further that, even if Schaudinn's observations be accepted as exact in every detail, they will not bear the interpretations which he places upon themnamely, that the small and large forms produced as he describes are males and females, since, as he himself admits, they do not, 
when developed, perform any act of syngamy. The alleged sexuality of the forms described by Schaudinn lacks the only decisive criterion of sexual differentiation-namely, sexual behaviour ; and the differentiation exhibited by the two forms of trypanosomes described by Schaudinn admits of an entirely different and far lesss forced interpretation (see p. 176, infra).

There are two further criticisms that may be made of Schaudinn's theory. The first concerns the alleged universality of sexual differences in living matter It must be pointed out that, as stated above, at the present time we have no evidence whatever of the occurrence of true syngamy in any organisms of the bacterial grade. The processes that have been interpreted by Schaudinn as autogamy in certain bacteria may be much more easily regarded as processes of internal regulation of the chromatin-substance. Nowhere yet has the union of two distinct gametes been observed in any bacterial organisms. The theory that sex is a universal characteristic, and syngamy an elementary function, of living things, does not rest at the present time on any basis of established fact.

The second criticism is that the terms "male" and "female" require definition and explanation, without which they remain meaningless, connoting merely unknown, mystic properties, nc: further analyzable, of the living substance. The characteristic feature exhibited by male cells is the preponderance of kinetic activity, and by female cells, of trophic functions, as Schaudinn and many others have pointed out. Before Schaudinn, the same idea was expressed in different language by Geddes and Thomson (114), who regarded the male sex as characterized by katabolic, the female sex by anabolic activities. It we suppose that these two manifestations of physiological activity have each a distinct material basis in the living cell, then it can easily be imagined that the imperfections of cell-division may lead to the production of cells in which one or the other substance predominates. This is the view that Doflein (7) has developed in his very interesting critical summary of the views that have been put forward upon the sexual problem. He supposes, further, that these two different physiological qualities depend upon substances which have intense mutual interactions and attract each other strongly, and that a certain equilibrium between them is necessary for the normal life of the cell. When, therefore, one or the other substance preponderates greatly in a cell, a functional derangement results ; but since cells differentiated in opposite directions attract each other strongly, they tend to unite, and by their union to restore equilibrium.

The question of the sexual differentiation of the gametes is one that will be discussed at greater length in the next chapter. It is only necessary to point out here that a clear distinction must be 
drawn between intrinsic differences, not necessarily visible, and structural or other differences which are more or less obvious. The fact that gametes and pronuclei tend to unite proves that in all cases there must be intrinsic differences between them which stimulate them to do so; in this sense, at least, we may endorse fully the dictum of Hertwig, that "fertilization depends on a fusion of sexually-differentiated cell-nuclei." On the other hand, gametes of opposite sexes exhibit every possible condition from complete similarity in structure and appearance to the greatest possible contrast in every feature of their organization. There can be no doubt that visible differentiation of the gametes is largely, if not entirely, an adaptation to the functions that they have to perform; and this conclusion is by no means weakened by the fact that there are many cases of isogamy which are undoubtedly secondary, in which a more primitive and phylogenetically older structural differentiation has gradually become annulled, under circumstances in which adaptive differences in the gametes are no longer necessary-as, for example, in gregarines (p. 173).

In Metazoa it is generally recognized that the two pronuclei that undergo fusion are perfectly equivalent,* and that the differences seen between them in the gametes are temporary and, in the case of the spermatozoon, an adaptation to circumstances; here the real differentiation of the gametes affects only cytoplasmic characters. In Protozoa, on the other hand, the conjugating pronuclei often exhibit differences of structure when the cells themselves appear perfectly similar. In the Infusoria, for instance, differences have been noted between the migratory and stationary pronuclei; how far these differences may be correlated directly with the differences in their activities must remain an open question.

In the foregoing paragraphs we have set forth and discussed some of the attempts that have been made to solve the problem of sex. It cannot be said that a perfectly satisfactory solution has been attained, but at least certain conditions of the problem may be laid down. In the first place, no theory of sex is satisfactory which does not explain why the union of two cells should be necessary in syngamy. In the second place a teleological interpretation, such. as amphimixis, can only state a secondary consequence, not a primary cause, of sexual union; but such a consequence may suffice to explain the retention and persistence of sexual phenomena after the conditions have ceased to exist under which they came into existence.

In the simplest Protista of the bacterial grade, it may be supposed, either that the living matter is not differentiated into localized substances having distinct physiological qualities, or that in such

- Apart, that is to say, from the much-disoussed question of the supernumerary chromosome. 
minute bodies reproduction by fission does not produce differentiation in the fission-products. With increased size such differences may arise, at first to a minor extent, and capable of being adjusted by internal rearrangements of the living substance such as have been described in the larger Bacteria. Not until the process of natural evolution had gone so far as to produce the full complication of structure seen in a true cell would localized differences in the living substance be brought about to a sufficient extent to lead to differences between the daughter-cells produced by fission, as a consequence of the imperfections of the process of cell-division. The differences produced in this way might be changes in the nucleo-cytoplasmic balance, as Hertwig supposes, or in the relative proportions of substances exerting different physiological activities, as suggested by Bütschli, Geddes and Thomson, Schaudinn and Doflein, or possibly of all these and other changes yet unknown. In any case it is reasonable to suppose that the imperfect character of the primitive types of cell-division, described in the last chapter, might produce accumulated material or structural inequalities in the daughter-cells, such as could only be rectified by the union of two cells differentiated in opposite directions, thus making syngamy a necessity for the continued existence of the species. This theory explains the necessity for syngamy recurring with greater frequency in forms having a high degree of structural differentiation than in forms of a primitive and simple type of organization.

With increasing perfection in the process of the division of the cell, and especially of the nucleus, the primary cause of, or necessity for, syngamy might be expected to disappear ; but at this stage in evolution other benefits to the species consequent on the process of amphimixis might be a sufficient cause for the retention of a process already well established. This conclusion appears to receive some support from the fact that intensive culture, whether artificial, or natural as in parasitism, seems to diminish the necessity for syngamy. It can hardly be supposed that intensive culture can diminish consequences arising from defective cell-division; but it might conceivably produce a strengthening effect equal to, and capable of supplanting, the benefits derived from amphimixis. Enriques (113) has stated that in Infusoria ex-conjugants may proceed to conjugation again, so that between one act of syngamy and the next there may not be a single cell-division intervening. In this case neither cell-division nor any consequences of celldivision can be the factor bringing about sexual union, but some other explanation must be sought. Enriques considers that the function of syngamy in Infusoria is to maintain the fixity of the species. 


\section{CHAPTER IX}

\section{POLYMORPHISM AND LIFE-CYCLES OF THE PROTOZOA}

\section{A. Polymorphism.}

ONE of the most striking peculiarities of living beings is the infinite variety of form, structure, and appearance, which they present. There is, perhaps, no living individual of any kind which is exactly similar, in all respects, to any other. Nevertheless, the most uncultured intellect cannot fail to recognize that, in the case of all ordinary, familiar plants and animals there is a pronounced tendency to segregation into distinct kinds or species-that is to say, natural groups of individuals which, though they may vary greatly amongst themselves, yet resemble one another far more than they do the individuals of another species. It is not necessary to point out that species are not to be regarded as permanent or immutable entities. It is certain that a species may in course of time become modified so as to acquire characters different from those it originally possessed, thus giving rise to a new species, or that a single parent-species may become split up into a number of groups which, by a similar process of modification, became so many daughter-species differing from one another and from the parentspecies to a greater or less degree. The problem of the origin of species is one that it is not necessary to discuss here ; it is sufficient to point out that the mutability of species often makes it very difficult to define or delimit a given species exactly, of which a striking example is seen in the pathogenic trypanosomes of the brucii-group, probably to be regarded, as pointed out abovo (p. 27), as instances of species in an incipient or nascent condition. Some species are sharply marked off from others, some are much less so, and some are of questionable rank, regarded by one naturalist as distinct, by another as mere races or varieties a state of things perfectly intelligible if existing species are regarded as having arisen by descent, with modification, from pre-existing species.

In the Protozoa the existence of distinct species is just as marked as in the higher plants and animals, and is universally recognized. As has been pointed out in the provious chapter, it is probably syngamy which is responsible for the segregation of individuals 
into specics, by blending the divergent characters that may be supposed to arise from the influence of different conditions or circumstances of life. Thus, syngamy in unicellular organisms appears to have an effect which is the opposite, to a large extent, to that which it produces in multicellular organisms, in which there are special germ-cells, sheltered to a greater or less degree from the direct influence of the environment, and in which amphimixis appears rather to be a means by which variations arise.

The conception of a species is by no means incompatible with the occurrence of a number of distinct forms in its life-history. Taking well-known instances from the Metazoa, there may be, in the first place, ontogenetic or developmental differences; not only may the individuals of the same species differ in size at different periods in the development, but they may differ so greatly in appearance and structure that only a knowledge of the life-history enables us to assert that they belong to the same species-as, for example, a caterpillar and a butterfly, or a hydroid and a medusa. Secondly, the adult individuals may differ to an enormous extent in the two sexes. Thirdly, there may be in many cases differences between individuals of a species related to differences in the functions which they perform, not merely at successive phases in the life-history, as in some cases of ontogenetic differentiation already mentioned, but even at corresponding phases of the life-history-a phenomenon best seen in social or colony-forming organisms, as in the case of ants and termites, or in the colonies of Hydrozoa.

In Protozoa, similarly, a given species may show distinct phases or forms at different or corresponding periods of its life-history to a greater or less extent. In some species the form-changes are very slight, and the individuals occur always under a similar form and aspect, at least during the active state, and are therefore recognizable without difficulty as regards their specific identity; such forms may be termed monomorphic, and as examples the species of ciliate Infusoria can be cited. Other Protozoa, on the other hand, are extremely polymorphic - that is to say, they occur under a variety of widely-differing forms at different stages in the life-cycle or in response to variations in the conditions of life. Hence it is often difficult or impossible to refer a given form to its proper species without tracing out its life-history and following its development step by step. The unravelling of the complicated life-cycles of Protozoa is attended by far greater difficulties than in Metazoa, since one important criterion fails us altogether in the Protozoa, that, namely, of sexual maturity. A naturalist has no hesitation in pronouncing a trochophore to be a larval form, and a rotifer to be an adult organism, from the fact that the former is sexually immature, while the latter produces ripe generative cells. In the 
Protozoa, however, there is no visible criterion of any similar state of maturity or the opposite which might be a guide in estimating the significance of a particular form. It is certain that with increasing knowledge many species of Protozoa now regarded as distinct will prove to be developmental stages of others, as has happened so frequently in the case of Metazoa.

The polymorphism of the Protozoa may be related directly or indirectly to a variety of causes, which may be grouped generally under three headings-life-conditions, growth and development of the individual, and sex,

1. Polymorphism in Relation to the Conditions of Life.-Under this heading are included all those cases where the individual is forced to adapt itself to inevitable changes in the environment, or else succumb to their effects; hence this type of polymorphism may be termed briefly adaptive. The animal may adapt itself to such changes in one or the other of two ways : passively, by passing into a resting state, in which vital activities are temporarily suspended ; or actively, by changes of form, structure, and function, adapted to the changed conditions.

Methods of passive adaptation to unfavourable conditions occur probably in all Protozoa-perhaps it might be said in all Protista, so that no species can be said to be absolutely monomorphic. The commonest form of such adaptation is the process of encystment, whereby the organism protects itself by secreting a firm, resistant envelope, or cyst, round its body.

The first preliminary to encystment in Protozoa is usually a rounding off of the body-form. In the case of naked amœboid forms such a change of form follows naturally, as pointed out above, from cessation of the locomotor activity. It is, however, also observed that a similar change takes place in corticate forms, a phenomenon which indicates that the cuticle or cortex must be absorbed or softened, and that any internal form-giving elements must be dissolved, so that the protoplasm is free to conform to the natural physical tendencies of a fluid body. In the great majority of cases, an individual in process of encystment becomes perfectly spherical, whatever may have been the form of its body in the active state, but in some cases the spherical form is not fully attained, and the body becomes ovoid or pear-shaped. During the process of rounding off, any food-particles or foreign bodies contained in the cytoplasm are rejected or absorbed, as a rule; the contractile vacuoles, if there be any, cease to be formed and vanish; and all locomotor organs, such as cilia, flagella, and of course pseudopodia, are absorbed or cast off. At the same time the protoplasm of the organism becomes less fluid and more opaque, and usually diminishes appreciably in bulk, probably through loss of water; it 
thereby becomes denser in consistence, but of less specific gravity Lastly, the cyst-membrane itself appears round the body, if it has not already done so ; it generally stands off distinctly from the surface of the body, and may vary in nature in different cases, from a soft, slimy or gelatinous coat to a firm membrane of variable thickness, often exceedingly tough and impervious.

In the encysted state, Protozoa are able to withstand the many vicissitudes to which they are naturally subject. They can then be dried up, frozen, or sun-baked ; and since the protoplasm becomes much lighter, they can be trensported great distances by winds, a fact which accounts for the appearance of Protozoa in infusions exposed to the air in any situation-a peculiarity from which the name Infusoria is derived. In general the function of encystment is to protect the organism against unfavourable conditions or violent changes in the environment-for instance, in freshwater forms, against drought and climate, the cold of winter or the heat of a tropical summer. In parasitic forms it is an adaptation commonly coinected with a change from one host to another.

In parasites two types of cysts can be distinguished. In the first place, full-grown forms may produce relatively large, resistant cysts (Dauerzysten) of the ordinary type, almost invariably spherical or ovoid in form. In the second place, the smallest forms in the developmental cycle, the products of multiple fission or "sporulation," may secrete round themselves tough, resistant envelopes, within which they may multiply further; in this case the envelope is termed a sporocyst, and the entire body a spore.*

* The word "spore" has come to be used in two distinct senses, as applied to Protozoa, thereby producing a regrettable confusion and ambiguity. The word itself is derived from the Greek $\sigma \pi \delta p o s$, a seed, and was applied by botanists to those cases. where plants produce seed-like bodies which are not true seeds; for instance, the seed of an ordinsry flowering plant is a complete embryo, with root and shoot distinct, encapsuled in protective envelopes, but the "soed" of a fern is merely a single cell enclosed in a protective membrane. Consequently the term "spore" was used to distinguish the " seeds" of ferns, fungi, eto., from the true seeds of flowering plants.

It was observed at a very early period that many parasitio Protozoa produced minnte seed-like bodies, which conveyed the infection; for those of Myrosporidia Johannes Müller coined the term " psorosperms," but in general the term " spore" was used for these bodies, and the group in which the production of such spores is a very characteristic feature was named the Sporozoa.

With the progress of further investigation, it was found that in a great many cases the essential part of the spore-namely, the encapsuled protoplasmic bodyarose by a process of multiple fission, hence termed "sporulation," from a larger parent-body ; consequently the term "spore" has been used by many in a secondary sense to denote a minute germ formed by multiple fission, as in the merozoites of the malarial parasites. It is preferable to retain the word "spore" in its original significance as a seed-like body contained in a resistant envelope or sporocyst, and to use the word "germ " (equivalent to the German word Keim) for the protoplasmic body formed by sporulation, whether enolosed in a sporocyst or not. Unfortunately the word " germ" has become very much misused in popular languagc, and a less ambiguous term would perhaps be the word gymnospore for naked germs not enclosed in a protective cnvelope.

There is no essential difference between a cyst and a spore, except their relation 
Sporccysts are often simply rounded or oval bodies, like cysts, but in some cases they exhibit special forms, and may be prolonged into spikes, tails, or processes of various kinds.

In many cases the purely protective uses of the cyst may be combined with the performance of some special function within it. The contained organism may remain merely in a resting state within the cyst (hypnocyst); or it may utilize its leisure for the digestion of large quantities of ingested food-material, or for carrying on processes of reproduction or syngamy. As a process of similar nature to encystment, the formation of "sclerotia" in the Mycetozoa must be noted (see p. 240, in/ra).

Active adaptation to changed conditions is seen in those forms in which the mode of life is bound up with changes of environment during different periods of the life-history-that is to say, more especially in parasitic forms, in which a change of hosts is necessary for the continuance of the species. In such forms there are in general two functions for which provision must be made : the first is that of multiplying in the host itself and keeping up a stock of the parasites in it ; the second is that of infecting a new host sooner or later (see p. 20, supra). In the most primitive types of parasitic Protozoa there is no differentiation of form or structure corresponding to these two distinct functions; but as a general rule a given parasite in a given host exhibits usually two forms or series of forms, which may be termed " multiplicative" and "propagative" respectively (Doflein). Multiplicative forms may be wanting in some cases, as in the Eugregarines, but propagative forms are always found, being an absolute necessity for the continuance of the species.

As examples of multiplicative and propagative forms, we may consider first species which are parasitic only on a single host in the course of the entire life-cycle. A typical example is seen in Coccidium (p. 342, Fig. 152), in which adult forms, "schizonts," multiply rapidly in the host by a process of multiple fission, "schizogony," a process which takes place unaccompanied by any sexual phenomena, and in which no resistant cysts are formed, since they are quite unnecessary. Sooner or later, however, generations of individuals, "sporonts," appear which do not multiply like the schizonts, but which, as gametocytes, give rise to the gametes. After a process of syngamy the zygote forms a resistant cyst within

to a developmental oycle; the "spores" of Bacteria are for the most part simply cysts, but are called spores on account of their small size.

In this book the word "spore," when not qualified by any prefix, will be used to denote a resistant seed-like body protected by a tough envelope, or sporocyst, and the production or development of such bodics will be termed " spore-formation." On the other hand, the produotion of numerous small cells or germs by multiple fission will be termed " sporulation." 
which it multiplies to form a number of germs, which may or may not be enclosed in sporocysts, in different species. Cysts and spores pass out of the host, and do not develop further unless they are devoured by a second host of a species in which they are able to establish themselves ; if this event takes place, the spores germinate in the new host and produce a fresh cycle of infection, each germ when set free growing up into a schizont. In this case it is seen that the schiz snts represent the multiplicative, the sporonts the propagative, phase, and that in the latter resistant cysts are produced as a protection against the vicissitudes of the outer world, to which the parasite must expose itself during this phase of its life-history.

An example of a parasite which infects two distinct species of hosts in the course of its life-history is furnished by the malarial parasites (p. 360, Fig. 156). In this case there are first of all schizonts which, like those of Coccidium, reproduce themselves by multiple fission, this part of the life-cycle being passed in the blood of a vertebrate host. Later, sporonts are generated which under normal circumstances are incapable of multiplication in the vertebrate host, or, indeed, of any further development, unless taken up by another host, in this case a mosquito, which takes them from the vertebrate host by sucking its blood. In the stomach of the new host the sporonts behave in a similar manner to those of Coccidium - that is to say, they give rise as gametocytes to gametes, which by syngamy produce zygotes. The zygotes grow and reproduce themselves by multiple fission, forming an enormous number of minute germs or sporozoites, which do not develop further unless they pass from the mosquito back into the blood of a suitable vertebrate host, in which they start a fresh developmental cycle.

The life-cycle of the malarial parasites shows that a given phase of a parasite is only to be regarded as multiplicative or propagative in relation to a particular host. In the vertebrate blood the schizont is the multiplicative, the sporont the propagative, phase. As soon, however, as the sporont passes into the mosquito, it becomes there the multiplicative phase which gives rise ultimately to the sporozoites, representing the propagative phase in the mosquito. The sporozoites in their turn, when they reach the blood of the vertebrate, develop there into schizonts. Thus one and the same stage in the life-cycle represents one phase in one host and another in another, according to circumstances. It should be noted further that in the life-cycle of the malarial parasites resistant cysts are unnecessary, since the parasite never comes out into the open, but passes the whole of its existence in one or the other of its two hosts ; consequently such cysts are not formed at any stage of the lifecycle in these forms. 
Another example of a parasite with alternation of hosts, in whioh the course of events is different from that of the malarial parasites, is furnished by the species of the genus Aggregata (p. 353). Here the schizonts are parasitic in crabs, and reproduce themselves by multiple fission without encystment to form naked germs, merozoites, which grow up into schizonts, and multiply again in the same way. If, however, the orab is devoured by a Cephalopod, the merozoites adapt themselves to their new surroundings and become sporonts, which produce gametes. The zygotes form resistant cysts in which they multiply to form spores enclosed in tough sporocysts. The resistant phases pass out of the Cephalopod in its fæces, and to develop further they must be devoured by a crab, in which they become schizonts again. In this case there is no special differentiation of propagative phases in the crab, but the same stage can serve both functions; on the other hand, in the Cephalopod there is no multiplicative phase, but only a propagative phase with resistant cysts.

2. Polymorphism in Relation to Growth and Development of the Individual.-In Protozoa which multiply only by equal binary fission, as, for example, many Infusoria, there is practically no difference between young and old forms beyond a slight variation in size. An individual feeds, and in consequenco grows slightly beyond the size characteristic of the species to which it belongs. It then divides by equal binary fission into two individuals each slightly below the specific size, and they in their turn feed and grow and reproduce themselves by fission in due course.

In other cases, however, young and adult forms of a species can be clearly distinguished, and may differ in structure as well as in size. Beginning with reproduction by binary fission, the simplest case is where the adult individual divides into two unequal portions, so that parent and daughter can be distinguished, the former not appreciably smaller than ordinary full-grown indıviduals, the latter, however, very much smaller; it may be relatively minute. Examples of this type of reproduotion are furnished by trypanosomes, a group in which all gradations may be found between equal and very unequal fission (Fig. 127). Still greater differences between parent and young individuals are seen in cases of gemmation-that is to say, where the offspring is set free in an undifferentiated condition, and acquires after separation from the parent the characters of the adult, as in Acinetaria.

The greatest differences between young and old forms are seen, as might have been expected, in cases of reproduction by multiple fission or gemmation. In such cases the young forms produced often differ from the adult in structuro and appearance, as well as in size. An example of multiple fission is furnished by the common 
Trypanosoma lewisi of rats, in which two types of such fission are seen : either the multiplication of a small individual by repeated binary fission to form a "rosette" composed of several daughterindividuals (Fig. 127, $J, K$ ), or the separation of several small daughter-individuals from a large one (Fig. 127, $F, G, H$ ). In both cases the multiple fission is simply rapid and repeated binary fission. The young individuals resulting from the fission are sometimes crithidial in type (p. 294), and grow into the adult trypanosomeform.

In multiple gemmation (sporulation) the parent body breaks up into a number, sometimes very large, of small or even very minute individuals, buds, or germs, usually given off from a more or less considerable mass of residual protoplasm, which degenerates and dies off. The buds when set free may become active at once, or they may pass first into a resting state to which an active state succeeds at a later period. In the latter case they may form sporocysts, and become the spores already described. Within the sporocyst the minute germ may multiply furthereby fission. In the subclass Telosporidia of the Sporozoa, the contents of the spore may divide up in this way to form a variable number of slender sickle-shaped germs, for which Aimé Schneider coined the term sporozoites, a term which has since been frequently applied in senses quite different to its original meaning.

An active germ produced by sporulation is termed a swarm-spore or zoospore, whether or not the active phase is preceded by a resting spore-stage. The swarm-spores of Protozoa may be of various types in different cases. The swarm-spore may be amœboid and creep about by the aid of pseudopodia; it is then termed an amoebula (or pseudopodiospore). It may be provided with one or more flagella as organs of locomotion, and is then termed a flagellula (or flagellispore). It may have a coat of cilia, as in the young stages of Acinetaria, and may then be termed a ciliospore. Lastly, the swarm-spore may be without organs of locomotion, whether permanent or temporary, and may progress by twisting and wriggling movements of the body as a whole, or by gliding forwards on its long axis in a manner similar to the gliding movements of gregarines; swarm-spores of this type are specially characteristic of the Telosporidia amongst the Sporozoa, arising either by sporulation of a schizont (merozoites) or in the process of spore-formation after syngamy (sporozoites), and may be termed gregariniform swarmspores or gregarinulce comprehensively.

In some cases the swarm-spore may pass through more than one active phase, and exhibit different modes of locomotion in each. This is well seen in the Mycetozoa (p. 239), wherc the germination of the spore produces an amobula, which may acquire a flagellum 
and become a flagellula; after a time the flagellula settles down and becomes an amoebula again after loss of the flagellum.

A very interesting point, in connection with the question of young and adult forms of Protozoa, is the occurrence of stages in the development which may be interpreted as recapitulative in the phylogenetic sense-that is to say, as representing past stages in the evolution of the species, in a manner comparable to the recapitu lative larval or embryonio stages in the development of Metazoa. It is probable that suoh recapitulative stages are commoner in the development of Protozoa than has been generally supposed (compare Awerinzew, 47). The best-known instance is furnished by the ciliated larvæ of Aoinetaria (p. 459), indicating that this order is descended from a ciliate ancestor of the order Peritricha, a relationship fully confirmed by the similarity of their reproductive processes to those of other Infusoria. The crithidial phase that occurs so constantly in the development of trypanosomes (p. 299) is probably to be regarded as a recapitulative form representing a type of structure antecedent in evolution to that of the typical trypanosomeform. The frequent occurrence of flagellated swarm-spores in the development of Sarcodina (Foraminifera, p. 235 ; Radiolaria, p. 254) probably has a phylogenetio significance, as pointed out by Bütschli. Finally attention may be drawn to the remarkable series of forms in the ontogeny of Arcella desoribed in the next chapter ; first the amobula, then the Nuclearia-stage, followed by the Pseudochlamysstage, which grows finally into the adult Arcella-form. In the many cases where young forms are markedly different from the adult, it may be a difficult matter, as it often is in the oase of Metazoa, to decide whether a given larval form is to be interpreted as recapitulative or merely adaptive; but even in cases where the characters of a larval form have an obvious adaptive importance, as in the ciliated larvæ of Acinetaria, atavism may be nevertheless a factor determining the particular form taken by the adaptive characters in question-that is to say, by the organs of locomotion in the example chosen.

3. Polymorphism in Relation to Sex.-The phenomena of sexual differentiation consist primarily of differences in size, structure, and other oharacteristics between the gametes, the cells which are concerned in the act of syngamy. Secondarily such differences may extend to other oell-individuals, both in the life-cycle of a Protozoon or in the body of a Metazoon. In the previous chapter it has been pointed out that, while in Metazoa the gametes at least are sharply differentiated in all cases, in the Protozoa every condition is found from perfect isogamy to a differentiation nearly as pronounced as that in the Metazoa. The question has been discussed in the last chapter whether or no sexual differentiation is to be regarded as 
an inherent property of all living beings, as maintained by many high authorities.

Whatever view be held with regard to the existence or nonexistence of inherent, intrinsic sexual differences in living organisms, it seems clear that the apparent sexual differentiation of the gametes is largely, perhaps purely, adaptive, and furnishes good examples of the principle of morphological differentiation of structure in relation to physiological division of labour. One gamete, termed "female," tends to be bulky and inert, storing up reserve-material in greater or less quantity, a provision (sit venia verbo /) for future requirements; it is economical of substance, and but few are produced. The other gamete, termed " male," develops in the opposite direction in every respect; it tends to be small and active, not weighted with superfluous material of any kind, but with motor mechanisms strongly developed; it is prodigal of substance, and many are produced, but few are favoured by destiny. In extreme cases the female gamete is a relatively huge, inert cell, incapable of movement, crammed with foodstuffs; the male is excessively minute, and is practically nothing but a nucleus which has its constituent parts packed into the smallest possible space, and with motor mechanisms attached to it.

In reviewing the progressive differentiation of the gametes in Protozoa, it is convenient to treat separately those forms in which there is little or no ontogenetic differentiation from those in which there is a more or less pronounced difference between the young and adult forms. An example of the first type is seen in Copromonas (Fig. 111), in which the gametes are ordinary individuals of the species, only differing in that their nuclei have undergone a process of reduction. Good examples of monomorphic forms are furnished also by the Infusoria, a group in which a species may be free-swim. ming, or may be more or less permanently attached and sessile in habit.

In the free-swimining ciliate Infusoria, sexual differences in the conjugants are frequently not discernible ; if they exist, they can only be inferred from the fact that syngamy takes place, or from subsequent behaviour of the individuals after conjugation, as, for instance, the fact observed by Calkins, that in Paramecium one exconjugant multiplies much more rapidly than the other. In other cases differences of size more or less pronounced are exhibited by the conjugants (Doflein, 111). As pointed out above, differences of structure have also been noted in some cases between the stationary and migratory pronuclei produced by a conjugant. Collin (50), however, was unable to find the slightest morphological differentiation of the conjugating pronuclei of Anoplophrya.

In the sedentary Infusoria, sexual differentiation may be as little 
apparent as in the free-swimming species, as, for instance, in Acinetaria, where conjugation can take place between two adjacent individuals each on its own stalk. But in the Vorticellids special free-swimming individuals, microconjugants, are devoloped which are budded off from a sedentary individual, and then acquire cilia, swim off, and conjugate with another sedentary individual

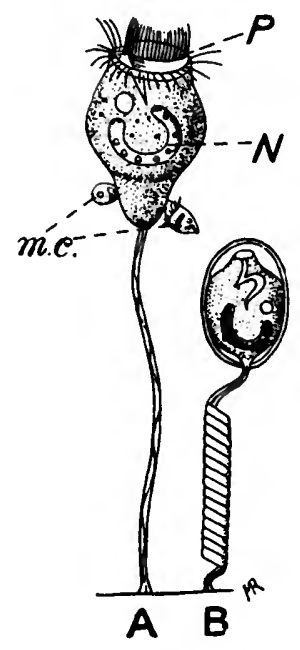

Fig. 78.-Vorticella microstoma, Ehrb. On the left an ordinary, sedentary individual (macroconjugant) with two microconjugants (m.c.) attached to it, one of which (to the left) is in the act of conjugation. On the right is an individual with the stalk contracted and the body enclosed in a cyst. $N$, Macronucleus; $P$, peristome and adoral ciliary spiral. After Hickson.

(Fig. 78). It seems obvious that this state of affairs is an adaptation to the exigencies of a sedentary life to insure crossfertilization analogous to the formation of complemental males in the Cirripedes. The free-swimming microconjugants of Vorticellids are commonly termed " males," but it is open to question whether, strictly speaking, they deserve that title.

It is in species with marked differences between young and adult forms that the greatest differentiation of the gametes occurs, though by no means universally even in such forms. In polymorphio species of this type, three different conditions can be distinguished, to which reference has been made in the previous chapter.

1. Macrogamy - that is to say, syngamy between full-grown individuals of the species. In this type the gametes appear to be always perfectly similar, so far as is known; examples are seen in Actincphrys (Fig. 71), the ohromidiogamy of Arcella (Fig. 80), and possibly Noctiluca (p. 279).

2. Microgamy - syngamy between the youngest individuals, products of the rapid multiplication of an adult. Conjugation of swarm-spores is by far the commonest type of syngamy in Protozoa, and may be regarded as the normal type. In this case there is usually complete iscgamy, as in Foraminifera (p. 235), sometimes slight anisogamy, as in Radiolaria (p. 254, Fig. 108).

3. Mixed microgamy and macrogamy - that is to say, syngamy between a full-sized adult individual on the one hand and a minute individual, a swarm-spore, on the other hand. This type may be regarded as derived from microgamy by progressive, and finally complete, inhibition of the divisions that produce the swarm-spores in one sex-possibly also with an enhanced tendency to such divisions in the other sex. Thus in Arcella, as described in the previous 
chapter, the macramœbæ produced are fewer than the micramœbæ, showing that the tendency to division is more restricted in the former case than in the latter. Again, in the development of Centropyxis, as described by Schaudinn (131), formation of gametes is initiated by a process of multiple fission sombined with formation of secondary nuclei from chromidia, as in Arcella, and in this way a number of amœbulæ are produced. The amœbulæ from one Centropyxis remain undivided, as macramœbæ, while those produced from another adult divide each into four micramœbæ; syngamy takes place later between a micramœba and a macramœba, after each has secreted for itself a shell.

When the inhibition of the gamete-forming divisions is quite complete in one sex, the result is the most pronounced type of anisogamy occurring in Protozoa ; and, conversely, it may be said that all cases of extreme anisogamy in Protozoa are of this type. In Metazoa the disproportion in the size of the gametes is mainly due to the relatively enormous growth of the gametocyte, partly also to the inequality of the four cells produced by the reducing divisions, in the female sex. In Protozoa with extreme differentiation of gametes, on the other hand, such as the Coccidia and Hæmosporidia, the gametocytes do not differ greatly, sometimes not at all, in size, though the female gametocyte may contain more reserve food-material, and consequently less protoplasm. The disproportion of the gametes is due almost entirely to the fact that in the female sex the gametocyte does not divide, but becomes a single macrogamete, while the male gametocyte sporulates to produce a larger or smaller number of microgametes.

Very instructive in this respect is the comparison of the formation of the gametes in the gregarines (p. 331) and the coccidia (p. 346) respectively, two groups of Protozoa which are certainly closely allied to one another. In such a form as Coccidium (Fig. 152), the gametocytes remain separate one from the other, and the male gametocyte forms numerous minute microgametes which swarm away ; the female gametocyte, on the other hand, becomes a macrogamete after going through a process of reduction, and is fertilized by a single microgamete. In gregarines, however, the gametocytes associate in couples, either before or after attaining their full size, and become surrounded by a common cyst, within which each gametocyte sporulates to produce a large number of small gametes. The gametes of gregarines can be arranged in a series, showing marked anisogamy at one end, complete isogamy at the other. Thus in Pterocephalus (Fig. 79, $A, B$ ) the gametes are very unequal in.size, and the microgametes are motile, the macrogametes not so. In Stylorhynchus the gametes of opposite sexes are equal in size, but in one sex the gametes are motile, in the other not (Fig. 79, 
$C, D$ ). In Monocystis (Fig. 79, G-L) tho gametes differ slightly in size in the two sexes, but have no organs of locomotion in either case. In Urospora (Fig. 79, $E, F$ ) the gametes are not appreciably different in size, but in those of one sex the nuclei are slightly smaller than in those of the other. Finally, in Gregarina, Diplodina, and many other genera, no difference whatever is perceptible between the two gametes that perform syngamy. In those gregarines which have dimorphic gametes, syngamy is always between two dissimilar individuals of distinct parentage, and it may be inferred, therefore, that in all cases alike the gametes that unite are derived from distinct gametocytes.

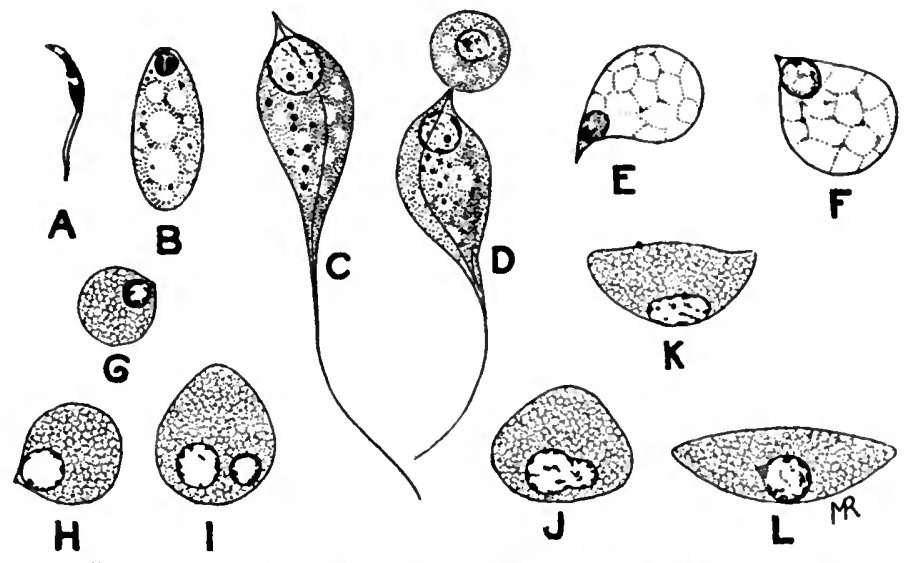

Fic. 79.-Gametes of different species of gregarines. $A$, Malo, $B$, female, gamete of Pterocephalus (Nina) gracilis. $C$ and $D$, Stylorhynchus longicollis : $C$, malo gamete; $D$, male gamete attaching itself to a female. $E$, Malo, $F$, femule, gamete of Urospora lagidis, showing differences in the size of the nuclei. $G-L$, Monocystis sp.: $G$, male gameto; $H$, female; $I$, union of the two gametes, the nuolei still separate; $J$, the two nuclei fusing; $K$, the zygote becoming elongated; $L$, the zygote has taken the form of the spore, and in the synkaryon a centrosome has appeared, preparatory to division. $A$ and $B$ after Leger and Duboseq; $C$ and $D$ after Leger; $E-L$ after Brasil.

From a comparison of the life-cycles of the Coccidia and the Gregarines respectively (see p. 354, infra), it is highly probable that in the common ancestor of the two groups the gametocytes were separate, as in Coccidium, and each produced numerous gametes, as in Gregarines. Sinco the gametes had to find each other, by a process of adaptation, those of one sex became smaller and more motile (microgametes), while those of the other sex were more bulky and inert (macrogametes).

In the course of their evolution fron this primitive ancestral type, the Coccidia, with some exceptions presently to be noted, retained the habit of the gametocytes, remaining separate, and the specialization of the gametes became greatly increased, as an adap- 
tation to this condition, the female gametocyte ceasing to divide and becoming a single macrogamete, while the male gametocyte produced a swarm of minute, motile microgametes. Only in a few Coccidia, exemplified by the genus Adelea (Fig. 154), did the gametocytes acquire the habit of association before forming gametes, a habit which led in this case to a reduction of the number of microgametes produced to four, of which one fertilizes the macrogamete, while the other three perish. It is clear that the formation of microgametes in close proximity to the macrogamete increases vastly the chance of the gametes finding each other, and renders unnecessary the production of a swarm of microgametes.

In the gregarines, on the other hand, the gametocytes acquired the habit of associating and forming their gametes in a common cyst. Under these oircumstances it becomes a certainty that a gamete of either sex will find a partner if the gametes of each sex are in equal numbers. Consequently there is seen in gregarines a progressive tendency, illustrated by the examples cited above, to disappearance of those characters of the gametes which are an adaptation to the necessity of the sexes coming together, culminating in production of gametes of opposite sexes which are perfectly similar. On this view the isogamy seen in many gregarines is a secondary condition brought about by the gradual obliteration of adaptive differences between the gametes of opposite sexes, under circumstances which render such differences unnecessary.

The comparison of the gamete-formation in different species of gregarines furnishes an instance of a progressive levelling-down of struotaral differentiation of gametes, under conditions in which no such differentiation is required, until an anisogamy undoubtedly primitive has been reduced secondarily to a perfoct isogamy. This has led to the view expressed in many quarters, that anisogamy is in all cases a primitive, isogamy a secondary, condition. The case of the gregarines is by no means adequate, however, to support so swooping a generalization; the only conclusion that can be drawn from it is that adaptive differences tend to disappear when the conditions to which they are an adaptation no longer exist; and the very fact that the obvious structural differentiation between the gametes vanishes in such a case is of itself a proof that such differentiation is not the expression of intrinsie constitutional differences between the gametes, for such differences could not be annihilated merely by changed conditions of environment.

There can be no doubt that anisogamy in tho form of visible structural differences between the gametes of opposite sexes must have been acquired very early by gametes as an adaptation to their functions. On the other hand, it is highly improbable, to say the least, that tho earliest gametes, whon the sexual process was first invented, so to speak, were structurally differentiated. It must, of course, be postulated that the gametes possess such intrinsic constitutional differences as would account for their behaviourthat is to say, their mutual attraction and union; and in this sense anisogamy may be considered as a universal and primitive phenomenon. Bul the number of cases in which gametes are perfectly isogamous, as regards visible structural or othor differences, is a sufficiont proof that purely constitutional anisogamy does not necessarily oxpress itself in perceptible differentiation of the gametes. 
So far only primary sexual differences-that is to say, those between the actual gametes-have been discussed; but, as has been stated above, the sexual differentiation may be thrown back, as it were, into generations preceding the gametes. Thus, it is by no means uncommon, especially in Coccidia and Hæmosporidia, for the gametocytes to be clearly distinguishable according to sex, the female gametooyte having the cytoplasm loaded with reserve foodmaterial, and usually with a smaller nucleus, while the male gametocyte has the cytoplasm clear and free from inclusions, and the nucleus is relatively large. In Adelea the male gametocyte is very much smaller than the female (Fig. 154). In Cyclospora caryolytica, parasitic in the mole, the sexual differentiation is carried back through generations antecedent to the gametocytes, and, according to Schaudinn (147), male and female merozoites can be distinguished.

The various types of polymorphism that have been discussed in this chapter may be classified as follows :

1. Adaptive polymorphism.

(1) Passive.

(2) Active.

2. Ontogenetic polymorphism.

(1) In size alono.

(2) In structure also.

(a) Recapitulative.

(b) Adaptive.

3. Sexual polymorphism.

(1) Primary (of gametes).

(2) Secondary.

(a) Of gametocytes alone.

(b) Of other generations also.

In the task of unravelling the complicated life-cycles of Protozoa, it is of the,greatest importance to distinguish clearly the significance of the various forms that are seen, and there can be no doubt that failure to do so has often been a source of error. With some writers it is an obsession to ascribe all differences to sex, and to interpret, for instance, in the development of trypanosomes, all bulky forms as females, and all slender, active forms as males, quite regardless of the behaviour of the forms thus designated. It is far more probable that in the majority, at least, of such cases the bulky forms are related to the multiplicative, the slender, active forms to the propagative function, respectively, and that the differences between then have no relation whatever to sexual functions, either in the forms themselves or in their descendants. 


\section{B. LIFE-Cyches.}

In the foregoing section the various forms have been described under which one and the same species of Protozoon may occur in the course of its life-history, and in response to the conditions of its particular mode of life. In some species it has been seen that the changes of form and structure are so slight that the species are practically monomorphic, in the sense that they can be identified without difficulty in any active phase of life ; no species is absolutely monomorphic, since, in addition to resting states, differences in size due to growth, at least, will always bo found. Other species, on the other hand, are polymorphic to such an extent that their specific identity in different phases can only be determined by tracing their development in a continuous sequence; and in extreme cases of polymorphism the life-history becomes a varied pageant of dissimilar forms succeeding each other in more or less regular order, determined largely, if not entirely, by the conditions of the environment. In a former chapter the distinction has been drawn between a developmental cycle, consisting of a recurrent series of different forms, and the complete life-cycle, consisting of the whole series of forms or phases which appear between one act of syngamy and the next. The complete life-cycle may comprise many developmental cycles.

As a concrete example of a life-cycle comprising a great number of different forms, and in which also the development may follow more than one course, the life-cycle of Arcella vulgaris may be selected (Fig. 80). The life-history of this form has now been made known in detail by the combined labours of many investigators, amongst whom Hertwig (65), Elpatiewsky (144), Swarczewsky (101), and Khainsky (145), must be specially mentioned.

The form which may be taken as the starting-point of the lifecycle is a minute, amœba-like form, with a single nucleus (Fig. 80, $A$ ). The amœbula, when set free, feeds, grows, and becomes after a time spherical in form with radiate pseudopodia (Fig. 80, $B$ ); in this stage it resembles a species of the genus $N$ uclearia. After a time the Nuclearia-form secretes a shell, and now resembles an example of the genus Pseudochlamys (Fig. 80,C). With further growth, chromidia are given off from the nuoleus into the cytoplasm, the nucleus divides into two, and the animal thus assumes gradually the characters of the adult Arcella (Fig. 32 ; Fig. 80, D). It has a chitinous shell, circular in outline, flattened in profile-view, and slightly concave on the under-side, in the centre of which is a large circular aperture through which the pseudopodia stream out. The body-protoplasm contains two nuclei situated approximately at 


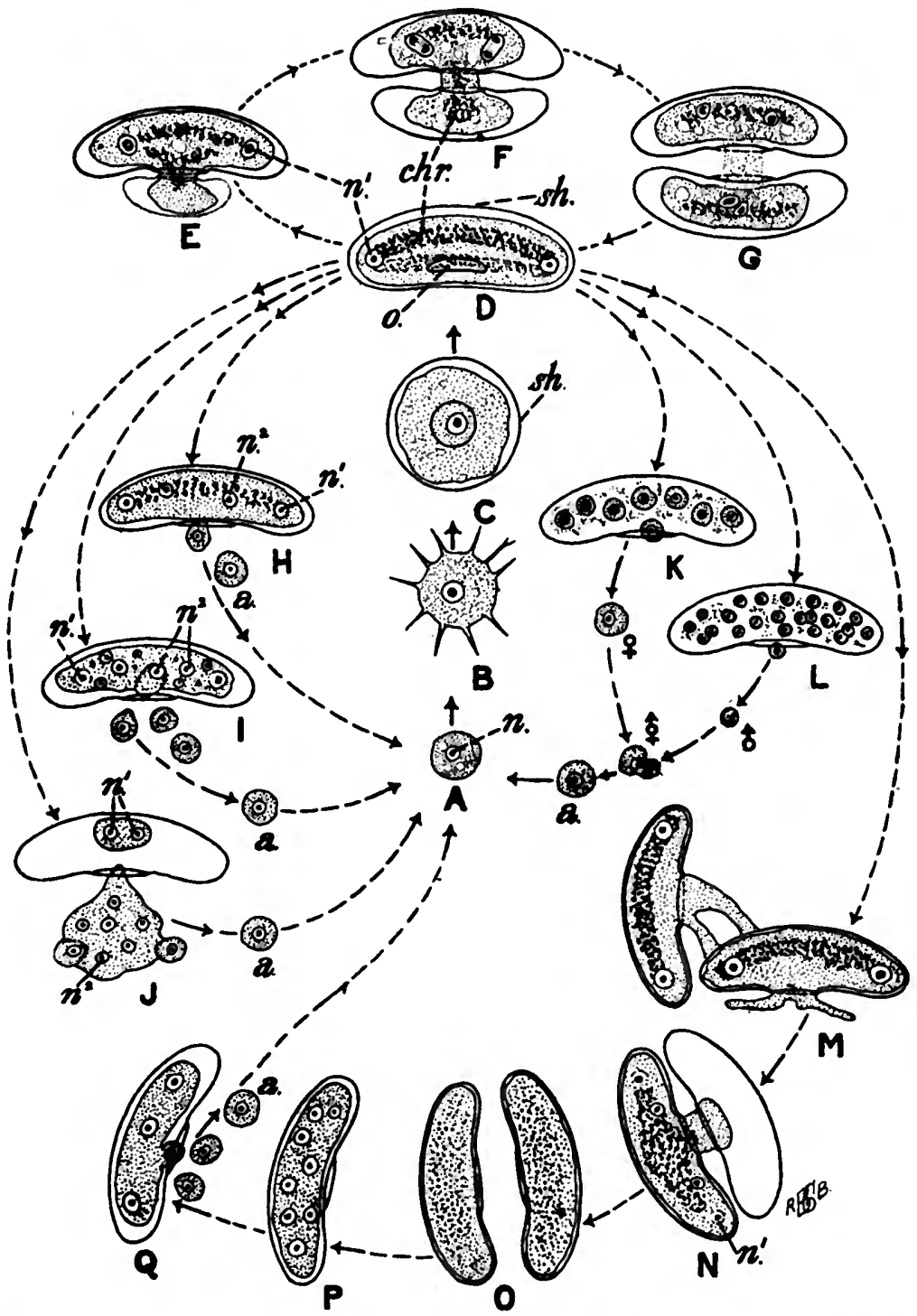

Fro. 80.-Combined diagram to show the different methods of reproduction and syngamy in the life-cyole of Arcella.

$A-D$, The four stages in the ontogeny: $A$, the amcobula; $B$, the Nuclearia-form; $C$, the Pseudochlamys-form; $D$, the adult Arcella.

$D-G$, Stages in the vegetstive reproduction by fission: $E$, the protoplasm beginning to stresm out of the shell of the parent-individual; $F$, division of the nuclei of the parent, and formation of the shell of the daughter; $G$, migration of the daughter-nuclei into the daughter-individual and completion of the division.

[Continued at foot of p. 179. 
the opposite ends of a diameter of the circular body, and an irregular ring of chromidia forming a dense chromidial net. Under certain conditions Arcella becomes encysted, forming a spherical oyst with a tough impervious membrane within the shell, closing the mouth of it.

The adult Arcella reproduces itself by a variety of methods, which, however, may be reduced to two principal types : binary fission, producing daughter-individuals (Arcella) of approximately equal size; and gemmation, producing small amobulæ such as have been described above as the starting-point of the ontogeny. The production of the amœbulæ may or may not be in relation to syngamy, which, when it occurs, may be of one or the other of two distinct types-karyogamy between amœbulæ, or chromidiogamy between adult Arcelloe.

Binary fission (Fig. 80,D-G) is the ordinary type of reproduction during the "vegetative" life in the summer months, when the animal is actively feeding, growing, and reproducing itself. In the process of binary fission, the two nuclei divide by a form of karyokinesis (Fig. 57, p. 110). A quantity of the body-protoplasm streams out through the mouth of the shell, together with some of the chromidia, and one of the two daughter-nuclei of each pair also passes out of the shell. The daughter-Arcella thus formed secretes for itself a new shell, and separates from the parent-individual, which retains the old shell. Thus in binary fission both nuclei and chromidia take part, the former dividing by mitosis, while the latter are subjected to a roughly equal partition.

The ordinary binucleate form of Arcella may become multi-

Fio. 80-continued:

All the figures below the level of $D$ represent reproduction by gemmation : those to the left are reproductive processes not combined with syngamy; those on the right show the methods of syngamy.

$H$, Formation of secondary nuclei and buds which are liberated singly from the parent as amcobula (a.).

$I$, Rapid bud-formation, leading to almost the whole protoplasm of the parent being used up to form them.

$J$, Bud-formation external to the shell ; the protoplasm has streamed out, leaving only a small residual portion, containing the primsry nuclei, in the shell ; the extruded protoplasm producing buds with formation of secondary nuclei.

$K, L$, Formation of gametes and karyogamy : $K$, formation of macramobæ $(q) ; L$, formation of micramœbæ ( $\delta)$; the gametes ( $q$ and $\delta$ ) pass out of the shell and copulate ( $\left.\begin{array}{l}\hat{\phi} \\ +\end{array}\right)$ to produce the zygote or amcebula $(a$.$) .$

$M-Q$. Chromidiogamy : $M$, two Arcella coming together ; $N$, the proto. plasm, with the chromidia and degenerating primary nuclei, of the one passes over into the shell of the other; $O$, after intermingling of the chromidia, the protoplasm becomes equally distributed between the two shells; $P$, the chromidia give rise to secondary nuclei; $Q$, buds (amobulm, $a$.) are formed and liberated.

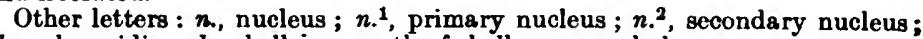
chr., chromidia ; sh., shell ; o, mouth of shcll ; $a$., amœbulæ.

Modified from a diagram by Swarozewsky. 
nucleate by formation of secondary nuclei from the chromidia, as described above (Fig. 32, p. 67). The secondary nuclei are entirely distinct in their origin from the primary nuclei, which degenerate when the secondary nuclei are formed. A multinucleate Arcella may reproduce itself by binary fission after division of each secondary nucleus by karyokinesis ; of each pair of secondary daughternuclei, one goes to one daughter-Arcella, the other to the other, so that each daughter-Arcella has the same number of nuclei exactly (Hortwig, 65).

Gemmation takes place in multinucleatc forms containing a number of secondary nuclei. A portion of the body-protoplasm bccomes centred round each secondary nucleus, and thus a small cell is formed, which becomes amœboid, quits the parent-body, and either grows directly into an adult Arcella by the successive stages described above, or before doing so performs an act of syngamy.

Gemmation, as above described, takes place in three different ways, as follows :

1. The buds are formed one at a time, and the parent-individual persists and continues to reproduce itself (simple gemmation, Fig. 80, $H$ ).

2. The whole budy of the Arcella breaks up into numerous buds which swarm out of the shell, leaving behind in it the two primary nuclei, with a small quantity of residual protoplasm. The parentindividual then dies off, apparently, but it is possible that it may in some cases regenerate the body again. This process of multiple gemmation differs only. from the simple gemmation described in the previous paragraph in being, as it were, greatly intensified, taking place with such rapidity as to use up almost the entire protoplasm at once (Fig. 80. I).

3. The protoplasm of the Arcella, with the chromidia, streams out of the shell, leaving in it only the degenerating priniary nuclei. Outside the shell the amœboid body forms secondary nuclei, and breaks up by multiple fission into a number of amœbulæ. This process differs from that described in the foregoing paragraph only in taking place outside the shell (Fig. 80, $J$ ).

As already stated, the amœbulæ formed by multiple gemmation may either be agametes, which develop directly into the adult form, or gametes, which first go through a process of syngamy which has been described in the previous chapter (Fig. 80, $K, L$ ). Both agametes and gametes arise in the same manner; the gametes, however, show sexual differentiation as regards size. The zygote is an amœbula which develops into the adult form in the same way as an agamete. In addition to syngamy (karyogamy) between amœbulæ, chromidiogamy between adult Arcelloe also occurs, as already described; the result in this case also is the formation of a 
number of amœbulæ which develop into the adult in the usual way (Fig. 80, $M-Q$ ).

Arcella thus furnishes a surprising example of diversity both in the courses taken by the development and in the methods of syngamy. We may now consider some further complications of the life-cycle, which in other Protozoa takes usually a more definite and stereotyped course, less liaile to the variations in one and the same species seen in Arcella.

One of the commonest complications introduced into the lifecycles of Protozoa is the differentiation of sexual and non-sexual cycles. In the account given above of the life-cycle of Arcella, it has been seen that an adult may produce amœbulæ which as agametes can grow up directly into the adult form without syngamy, or which as gametes copulate before developing further. The adult Arcella, however, do not, so far as is known, exhibit any differentiation in relation to these developmental differences, the form that produces gametes being perfectly similar to that which produces agametes. But in other cases there may be two distinct forms of the adult individuals: the one, known as the sporont or gamont, which gives rise to gametes; the other, termed the schizont or agamont, which produces agametes.* In this way an alternation of generations is brought about in which the life-cycle as a whole becomes a combination of two distinct types of developmental cycle -one known as schizogony, in which no sexual processes occur; the other as sporogony, in which at one stage gamete-formation is followed by syngamy.

An example of alternation of generations in a free-living form is seen in the life-cycle of Trichospharium (Fig. 81), as described by Schaudinn (146). The adult phase is a relatively large amoeboid form, approximately spherical in contour, and having the body surrounded by a gelatinous envelope in which at intervals there are apertures through which the lobose pseudopodia are extruded; the

* The word " sporont" was a modification suggested by Bütschli for the term "sporadin," originally coined by Aime Schneider to denoto the adult sporcforming phase in the cephalino Gregarines (p. 339), and to distinguish it from the earlier phaso which still bears the epimerite, known as a cephalont (" cephalin," Schneider). Since the production of resistant spores in Gregarines and allicd orders, such as the Coccidia, is accompanied by sexual phenomena, the word "sporont" has undergone both an extension and a change in its original meaning, and has como to be used to denoto a gamete-producing form. In his memoir on Trichospharium, Schaudinn used the word "sporont" in this sense, and coincd the term schizont to denote the agamete-producing form, and further coined the words "schizogony" and "sporogony" to denoto the non-sexual and sexual oycles respeatively. Since the word "sporont "in the secondary meaning thereby given to it has reference solely to the occurrence of syngamy and not to the formation of resistant spores, and since these two processes are not always, though frequently, combined in the same series of generations, it would perhaps be better to replace the terms " schizont " and "sporont" by " agamont " and "gamont" respectively, were it not that this leads to the substitution of the extremely cacophonous words "agamogony" and "gamogony" for "schizogony" and "sporogony." 


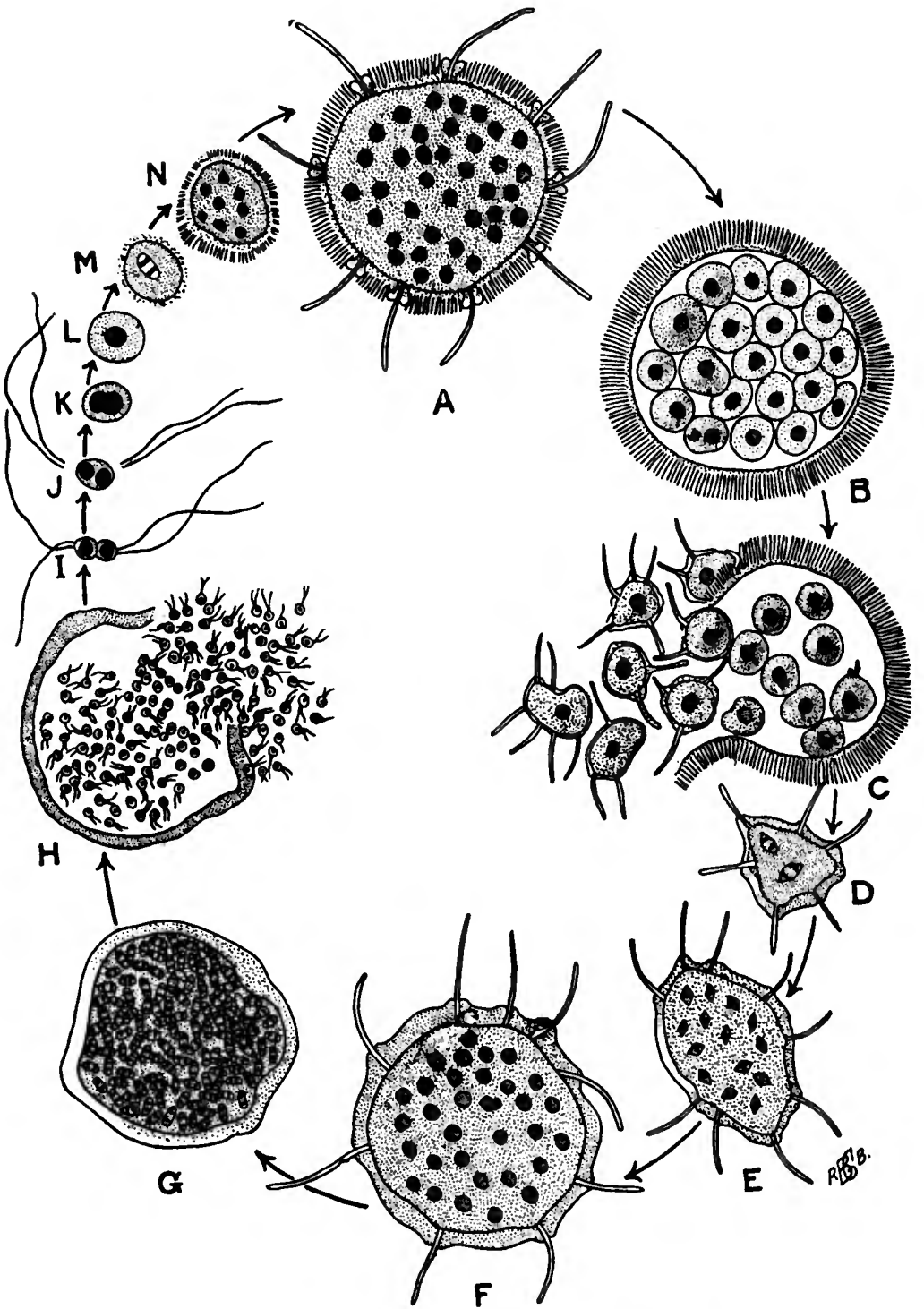

F19. 81.-General life-cycle of Trichosphcerium sieboldi, as an example of dimorphism in the adult condition combined with alternation of generations. $A$, Sobizont or non-sexual form, distinguished by tho possession of rod-like bodies in the envelope (compare $F$ ); this form may multiply by simple or multiple fission (plasmotomy) in a "vegetativo" manner, or by the process of sporulation (schizogony) seen in $B$ and $C$, in order to give rise to the gameteproduoing form ; $B$, division of the body of the schizont into as many cells ("sporogonia") as there are nuclei ; $C$, rupture of tho envelope and escape of the sporogonia as active amøbulæ, each of whioh forms an envelope for 
protoplasmic body is a multinucleate plasmodium. There are two forms of the adult-the schizonts (agamonts), which aro distinguished by the presence of rod-like spicules in the envelope (Fig. 81, $A$ ); and the sporonts (gamonts), which have no spicules (Fig. 81, F). The schizonts reproduce themselves either in the free state or after encystment. In the free state the reproduction is by simple or multiple plasmotomy - that is to say, by division of the plasmodium into two or more portious. In the encysted condition the schizonts divide by multiple fission into as many daughtercells as there are nuclei in the plasmodium (Fig. 81, $B$ ), and each daughter-cell is set free as an amœbula (agamete), which may either grow up into a sporont, or into a sohizont which repeats the process of multiplication by schizogony.

The sporont may reproduce itself in the free state in the same manner as the schizont, by plasmotomy, or it may become encysted, and then it multiplies in a manner totally different from that seen in the corresponding phase of the schizont. The nuclei of the encysted sporont multiply rapidly by karyokinesis (Fig. 81, $G$ ) until there are a very large number of minute nuclei ; very probably the final divisions in this process of multiplication are reducing divisions. The protoplasmic body then becomes divided up into as many minute cells as there are nuclei, and each of the daughter-cells acquires two flagella, and is set free as a flagellula or gamete (Fig. 81, $H$ ). The gametes, which are not differentiated in any way, copulate with those derived from another sporont, and lose their flagella (Fig. 81, $I-L$ ); the zygote is a small amœbula which grows up into a schizont (Fig. 81, $L, M, N, A$ ).

An alternation of generations similar to that of Trichosphoerium occurs also in the Foraminifera (p. 234). Here the schizont contains numerous nuclei, which multiply by fission as the animal grows, and also chromidia ; it reproduces itself by a process of multiple fission, breaking up into a number of amœbulæ (agametes), each with a nucleus and chromidia. The amœbulæ creep out of the old shell, which is abandoned, and each amœbula secretes a shell for itself,

Fig. 81 continuel:

itself and grows, with multiplication of the nuclei $(D$ and $E)$ into the gameteproducing form or sporont $(F)$, similar in general structure to the schizont $(A)$, but without rods in the envelope ; the sporont may also multiply in a vegetative manner by simple or multiple-fission, or it may form gametes in the manner seen in $G$ and $H ; G$, active multiplication of the nuolei of the sporont to form a great number of very small nuclei, after which the body divides up into as many minute cells as there are nuelei ; these cells are the gametes, and each gamete acquires two flagella; $H$, rupture of the envelope to set free the gametes, which swarm out and conjugate ; $I$, conjugation of two gametes, more highly magnified; $J$, after fusion of the bodies of the gametes the flagella are thrown off ; $K$, fusion of the two pronucloi ; $L$, eomplete zygote. which forms an envelope and grows, with multiplication of the nuolei $(\mathbb{N}, N)$ into the sohizont $(A)$, which was taken as a starting-point of the life-oyole. After Schandinn (146). 
and grows up either into a sporont or into a schizont again. The sporont pessesses only a single large nucleus, the primary nucleus originally present in the amcebula, and a great number of chromidia. When the sporont enters upon the reproductive phase, the primary nucleus degenerates, and an immense number of secondary nuclei are formed from the chromidia. Then the protoplasmic body divides up to form as many cells as there are secondary nuclei. The cells thus produced are the gametocytes, each of which divides by mitosis to form four small cells, the gametes, which acquire flagella, swim off, and copulate with gametes produced from another sporont; there appear, however, to be no differences exhibited by the gametes of opposite sexes. The zygote forms a shell and grows into a sporont. Since the zygote is very much smaller than the amcbula produced by schizogony, the shell formed by it is also smaller. This shell is later the initial chamber of the polythalamous adult, and thus leads to a dimorphism in the adult shells, so-called " microsphæric" and "megalosphæric" forms (p. 235)-a dimorphism related, in this case, not to the manner in which the adult individuals reproduce themselves, but to the manner in which they have been reproduced.

In free-living forms the alternation of generations is related to external conditions of the environment, as, for example, seasonal changes; the sexual generation may appear in the autumn, while the non-sexual generations are found in the spring and summer. In parasitic forms, on the other hand, alternation of generations is of common occurrence in relation to a change of hosts. Thus, in the life-cycle of the Coccidia (Fig. 152), described above, the multiplicative phases reproduce non-sexually by schizogony; as the socalled "endogenous cycle"; the propagative phases are preceded by gamete-formation, leading to spore-formation, the so-called "exogenous cycle." In Hæmosporidia, such as the malarial parasites, for example (Fig. 156), the alternation of gelierations is related to an alternation of hosts; the non-sexual, schizogonous generations take their course in the blood of the vertcbrate host, in which the gamonts are produced, but do not develop further unless taken up by the invertebrate host, in which alone gametes are formed and sporogony takes place.

The phrase " alternation of generations" must not be construed into meaning that the sexual and non-sexual generations succeed each other in a regular alternation. On the contrary, such regular alternation, if it occurs at all, is rare, and as a rule a single sexual generation is followed by several, or it may be by an immense number, of non-sexual generations before the sexual cycle recurs. The malarial parasite can multiply non-sexually in the blood for many years without dying out ; and if propagated artificially from one 
vertebrate host to another, it is probable that itoould dispense altogether with the sexual cycle, which occurs only in the invertcbrate host, so far as is known. In the suborder Eugregarinæ of the Gregarinoidea an opposite condition occurs, since these forms possess only the sexual cycle, sporogony, and there is no non-sexual schizogony. Whether this condition is to be regarded as a primitive state of things, or whether the Eugregarines are to be regarded as having dispensed with the non-sexual process of schizogony seen in the allied suborder Schizogregarinæ, must remain an open question.

A further caution is also necessary with regard to the alternation of generations in Protozoa. From the known facts of the malarial life-cycle, in which an alternation of sexual and non-sexual cycles is correlated with an alternation of hosts, it has often been assumed, implicitly or explicitly, that a similar alternation of sexual and nonsexual cycles must occur in other cases where there is an alternation of hosts, as in the case of trypanosomes, and in particular that the sexual cycle must occur in the invertebrate host. This assumption is by no means justified, however, and has been the cause of much unsound or unwarranted interpretation of the facts, especially as regards the significance of the various forms of trypanosomes, which are continually ascribed to sexual differentiation on no other ground than the bare fact of form-differentiation, as pointed out in the previous chapters. Up to the present there is not a single case in which sexual phenomena in trypanosomes have been described in a perfectly satisfactory manner, free from all doubt; and, on the other hand, it has been asserted that the syngamy occurs in the vertebrate host in these parasites (Ottolenghi, 492).

Bibliography.-For references see p. 480. 


\section{CHAPTER $\mathrm{X}$}

\section{THE GENERAL PHYSIOLOGY OF THE PROTOZOA}

The Protozoa, as has been seen in the previous chapters, exhibit a wide range of structural differentiation, from forms which exemplify a cell reduced to its simplest essential parts, nucleus and cytoplasm, to others in which the cytoplasmic elements give rise in different parts of the body to a great variety of structures and organs, each subservient to some special function. In the Protozoa of simplest structure, therefore, the study of the physiological activities of the organism coincides, more or less, with that of the elementary properties of the living substance, protoplasm, its peculiar powers of metabolism and transmutation of energy ; while in Protozoa of complicated organization the mechanism and mode of action of the various cell-organs must be considered in relation to their structure, so far as it can be made out.

It is not possible to discuss adequately, in the limited space of a chapter, the intricate problems, for the most part still very obscure, of the vital mechanisms of elementary organisms. The matter can only be dealt with here on broad general lines, and those desirous of studying the subject further must consult the references given to special works or memoirs.* On the other hand, the special functions and mechanisms of the various cell-organs ("organellæ") have been considered in describing the structure of the organs themselves. In this chapter, therefore, it is intended rather to fill the gaps left in previous chapters; and the physiological problems presented by the Protozoa will be sketched in brief outline under the following headings : (1) Nutrition and Assimilation ; (2) Respiration ; (3) Secretion and Excretion; (4) Transmutation of Energy ; (5) Reactions to Stimuli and to Changes of Medium or Environment ; (6) Degeneration and Regeneration.

* For works dealing with the physiology of Protozos in a general way the student should consult especially Verworn, "Allgemeine Physiologie," Jena, 1907 (a translation of the second German edition, under the title "General Physiology," was published by Macmillan, 1899) ; Prowazek, "Einführung in die Physiologie der Finzelligen," Leipzig (Teubner), 1910 ; the chapter on the general physiology of the Protozoa in Doflein's "Lehrbuch der Protozoenkunde"; and the excellent: summary of methods and results of physiological investigations upon Protozoa given by Pütter in Tigerstedt's " Handbuch der Physiologischen Methodik." 
1. Nutrition and Assimilation.-Living organisms, considered generally, exhibit a great variety of methods of nutrition, which may be classified into two main groups ; bearing in mind, however, that in all classifications of living beings, or of their vital properties, any groups or classes that can be distinguished are always connected by gradual and imperceptible transitions, and that consequently forms will present themselves which, owing either to their transitional nature or to the imperfect state of our knowledge concerning them, can only be assigned to one or the other group in a manner as arbitrary as the statement that the 2lst of June is the first day of summer-a difficulty which in no way invalidates the distinction between spring and summer.

In the first place, inany organisms can build up the complex protein-substances, of which the living protoplasm is composed, from simpler chemical materials. Of this type there are found among Protozoa, as already stated, two types of nutrition : first, the holophytic, or plant-like, in which the organism is able, by means of special cell-organs, to utilize the energy of the sunlight in order to synthesize its body-substance from the simplest chemical materials, such as water, carbon dioxide, and mineral salts, through a series of substances in an ascending scale of che uical complexity; secondly, the saprophytic type, in which the body contains no visible organs subserving the function of nutrition, but the organism is able to build up its protoplasm from food-materials consisting of organic substances in solution which are far less complex chemically than the body-proteins.

In the second place, many organisms cannot build up their bodysubstance from materials of simpler chemical constitution, but are entirely dependent on a supply of protein-substance ready-made, which they obtain either by ingesting and digesting other living organisms in the holozoic method, or by living as parasites at the expense of other creatures. These two methods graduate into one another, since many parasites simply devour portions of the bodies of their hosts in a holozoic manner, but the majority of parasites absorb fluid nutriment from their hosts in an osmotic manner; hence it is convonient to distinguish holozoic and osmotic parasites.

Considering these various methods of nutrition, it is seen that, from the point of view of the nature of the food, those which ingest solid food-particles (holozoic forms). can be distinguished from those which absorb their food in a diffused or dissolved condition (holophytic and saprophytic forms and osmotic parasites). From the point of view of the structure of the organism, those which possess special organs of nutrition (holozoic and holophytic forms) can be distinguished from those which possess none (saprophytic forms and osmotic parasites). 
(a) Holophytic Nutrition.-The characteristic of this type of nutrition is that the organism contains special pigments by means of which it is able to decompose $\mathrm{CO}_{2}$ in the sunlight, setting free the oxygen and retaining the carbon, which is built up in union with other clements derived from water and mineral inorganic salts. The pigments, termed comprehensively chromophyll, are contained in bodies termed " chromatophores," which occur in diverse forms and varying numbers in different species, and which multiply by division when the cell divides. The chromophyll-pigments are of various tints - yellow, brown, green, blue-green, etc. - but the commonest tint is the green chlorophyll, similar to that characteristic of plant-cells. A blood-red pigment, termed hoematochrome, occurs in some flagellates-e.g., Hamatococcus; it appears to be a modification of chlorophyll produced under certain conditions (seo Reichenow, 97:5).

For the details of the complicated process of the synthesis of chemical substances in the holophytic mode of nutrition, the student is referred to botanical textbooks dealing with plant-physiology. There appears to be no essential difference between the assimilative processes of holophytic Protozoa and of ordinary plant-cells. A characteristic product of holophytic nutrition is seen in the formation of anyloid substances, the most important of which are starch (amylum), and an allied substance known as "paramylum," which differs from starch in some of its reactions, notably in that it is not coloured blue with iodine. Paramylum is of more frequent occurrence in Protozoa than true starch. The amyloid substances occur in characteristic masses in the cytoplasm (see especially Bütschli, 153).

The chromatophores of Protozoa contain usually small refringent bodies termed pyrenoids, which also multiply by division. The pyrenoids are often surrounded by a coat or envelope of paramylum, and appear to be the centres of the production of amyloid substance.

Many flagollates with green chromatophores combine holophytic with saprophytic nutrition. Examples of such "mixotrophic" forms are seon in the genus Euglena (Zumstein, 223), the species of which flourish best in a medium containing organic substances, and cannot maintain thomsolves in pure water. Euglena viridis was shown by Khawkine to be able to live for a considerable period in the dark in media containing organic substances, but did not lose its green colour and did not multiply. $E$. gracilis, on the other hand, in Zumstein's experiments, lost its green colour and passed into an Astasia-like phase in the dark, or even in tho light when placed in solutions vory rich in organic substances, nourishing itself as a saprophyte. When tho Astasia-form was exposed to the light, in solutions containing a small amount of organic matter, it became green again and passed back into the Euglenaphase. The degree to which the species of Euglena can adapt themselvos to a purely saprophytic life would appear to vary in different cases. In tho colourless forms the chromatophores lose their chlorophyll, and remain as colourless leucoplasts.

The combination of holozoic and holophytio nutrition has been noted above (p. 15). 
(b) Holozoic Nutrition.-In this type of assimilation three series of events must be distinguished, each of which may be effected by means of special organs : the capture and ingestion of the prey ; its digestion; and lastly the rejection from the body of the nonnutritive residue (defæcation).

The methods of food-capture and ingestion have been dealt with above in a general way. As regards food-capture, methods of prehension by means of pseudopodia, or by special adhesive organs, such as the suctorial and raptorial tentacles of Acinetaria (p. 457) the tongue of Didinium (p. 442), etc., must be distinguished from methods whereby the food is wafted towards the body in currents produced by special vibratile organs such as flagella and cilia. As regards ingestion of food, a distinction is imposed by the nature of the outer surface of the body-protoplasm, whether naked or invested by a firm cortex or cuticle.

In naked forms the food is ingested at any point, by methods which vary in different forms. In Amceba proteus the hinder end of the body is most active in ingestion ; in Actinosphorium all points on the surface are equally active. Rhumbler (204) distinguishes four methods of food-ingestion in amœbæ : (1) By " import," when the food is drawn into the protoplasmic body as soon as it comes into contact with it, and with scamely any movements on the part of the amœba (Fig. 23) ; (2) by flowing round, "circumfluence," in which the protoplasm, as soon as it comes into contact with the food-particle, flows round it on all sides and engulfs it; (3) by " circumvallation," when the amøba, while still at some distance from the object, sends out pseudopodia which. flow towards each side of the prey, and ultimately meet round it and surround it completely, without ever having been in actual contact with it ; (4) by "invagination," in which the amcba touches and adheres to the object, and the portion of the ectoplasm in contact with it is invaginated into the endoplasm like a tube, the walls of which become liquefied and fused together, so that the food-particle is, as it were, sucked into the endoplasm (Fig. 82). Of these various methods, the process of circumvallation is most suggestive of a conscious and purposeful act on the part of the amceb; but a remarkable parallel to it is seen in the penetration of Lankesterella into a red blood-corpuscle, as described by Neresheimer (see p. 378, infra). In this case, as soon as the parasite comes within a certain distance of the corpuscle, the latter opens its arms, as it were, to the parasite, and engulfs it in a manner very similar to the ingestion of food by circumvallation on the part of an amcba. In both cases the object that is ingested must give off some substance which exerts at a certain distanoe an effect on the protoplasm of the cell which ingests it. 
According to Rhumbler (204), with a more fluid condition of the ectoplasm, the food is ingested by import or circumfluence; when the ectoplasm is stiffened to a membrane-like oonsistence, the ingestion is effected by circumvallation or invagination. Rhumbler maintains that all known methods of food-ingestion by amobæ, as well as their movements, can be explained mechanically by differences of surface-tension in colloidal limiting membranes, and can be imitated artificially in substances that are not living.
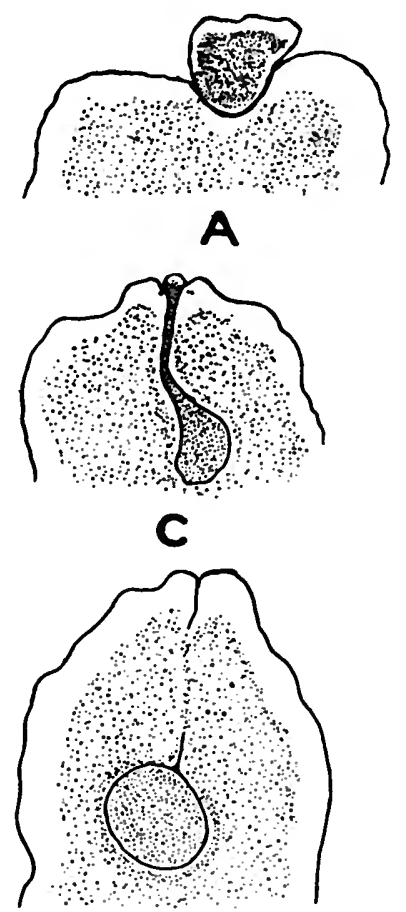

$E$
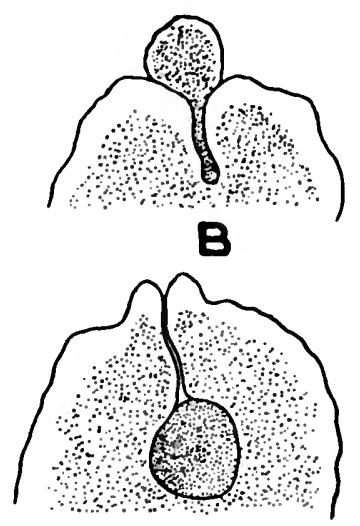

D

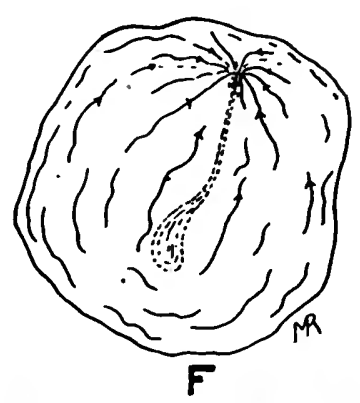

Fig. 82.--Ingestion of a food-particle by "invagination" in Amoeba terricola. $A-E$, Five stages of the process, semi-diagrammatio ; $F$, diagrammatic figure to show the direction of the currents on the surface of the body of the a moeba during the process of ingestion. After Grosse-Allermann (245).

In corticate forms the ingestion of food is limited to one or more special openings or organs, in which a direct communication is cstablished between the fluid endoplasm and the surrounding medium, as in the cytostomes of Flagellata and Ciliata and the suctorial tentacles of Acinetaria.

The digestion of the food is effected within the protoplasmic body, and as a rule the prey is taken bodily into the cytoplasm; but the Acinetaria have the power, not fully explained, of sucking out 
the body-substance of their prey, probably by the aid of secreted ferments. Together with the food a certain amount of water is ingested, forming a drop or food-vacuole in which the actual digestion takes place. The quantity of water ingested with the food varies considerably, and, speaking generally, is inversely proportional to the size of the object that is devoured ; that is to say, small food-particles, such as bacteria, lie as a rule in a very distinct vacuole, but large bodies, such as diatoms, usually appear as if imbedded in the cytoplasm, with no liquid vacuole visible around them. Amœbæ not infrequently devour organisms larger than themselves, so that the cytoplasm of the amœba appears like a thin skin or envelope over the surface of the prey. According to Greenwood (161), Amoeba proteus takes in but little fluid when it ingests quiescent solid matter, such as starch-grains or yeast-cells, but when actively-moving prey is dealt with an area of water not inconsiderable surrounds it; on the other hand, non-nutritious particles are not surrounded by fluid when they lie in the endoplasm.

In forms in which food is ingested through a cytostome, as in Ciliata, the food-particles, usually of small size, are wafted down the cesophagus and collect at its proximal blind end, where a depression arises in the endoplasm, which gradually deepens, and finally closes over and separates from the cesophagus as a closed vacuole containing the food. According to Nirenstein (181), the foodvacuole is detached from the œesophagus by suction of the endoplasm, like a process of swallowing ("Schlingvorgang"). The vacuole is at first immured in a thin layer of less fluid protoplasm, doubtless as the effect of contact with water (see p. 44); consequently the vacuole is not at first circular, but often spindle-shaped in its contours; it soon, however, assumes a spherical form, indicating that its protoplasınic envelope has become liquefied.

In cases where actively-motile organisms are devoured-as, for example, flagellates by amœbæ-the prey can often be seen to perform violent movements within the vacuole; but soon the movements become feebler and cease entirely. Bacteria ingested by Paramecium become immobile about thirty seconds after the vacuole has become detached from the œsophagus. In many cases, however, the proy is killed when seized by the pseudopodia, and before being ingested, as in Heliozoa and Foraminifera. After the prey is killed it is slowly digested within the food-vacuole.

During the process of digestion the food-vacuole may perform definite migrations within the body of the animal. In a mobæ the vacuoles are carried about by the currents of the protoplasm, without, however, pursuing any definite course, and they tend to become aggregated in the hinder ond of the body, when the animal is moving in a definite direction. In the Infusoria, on the other 
hand, the endoplasm shows a constant rotating movement, known as "cyclosis." In Paramecium the vacuoles are carried round by the current of the cyclosis, and each vacuole may either do a short course or a long course; the short course is simply round the nucleus, keeping close to it, while the long course travels the whole length of the body, up one side and down the other. As a rule a vacuole goes a short course two or three times, and then does a long course (Nirenstein, 181). The path of the vacuole varies, according to the nature of the contents; but the tendency is to keep them in the region posterior to the nucleus, where the contents are either cast out through the anal pore, or the vacuole circulates again in the cyclosis. In Carchesium the food-vacuoles, when formed at the base of the osophagus, pass down to one end of the horseshoe-shaped nucleus, and then glide close along its concave margin, passing round and up to the opposite end of the horseshoe into the region near the upper end of the vestibule, from whence the vacuole is finally emptied through an anal pore into the vestibule itself (Greenwood, 162).

The process of digestion within the food-vacuole has been studied by a number of investigators, amongst whom Le Dantec, Greenwood (162), Metschnikoff (180), Metalnikoff (179), Nirenstein (181), and Khainsky (170.5), must be specially mentioned. Their results are not always in agreement, indicating that the process of digestion is not always the same in different cases, even in the food-vacuoles of one and the same species. According to Nirenstein (181), the food-vacuoles of Infusoria exhibit changes which can be divided nto two periods : in the first the vacuole shows an acid reaction, and the ingested organisms are killed; in the second the vacuole has an alkaline reaction, and the albumens are digested. According to Khainsky (170.5), however, the reaction of the food-vacuoles of Paramecium is acid during the entire period of the proteolytic process, and only becomes neutral and finally alkaline when the solution of the food-substance is at an end.

In the first or acid period, according to Nirenstein (181), the ingested food-particles-e.g., bacteria-after being rendered immobile, are clumped together, enveloped in a turbid substance which makes their outlines indistinct. The reaction of the vacuole is strongly acid, due to the presence of mineral acid in the vacuole. During this period, which lasts from four to six minutes, the vacuole diminishes in size, till it is not more than one-third of its original size. When the vacuole was first formed, its wall was surrounded by a number of granules which stain very distinctly with neutral-red; these granules pass suddenly into the interior of the vacuole after it has become diminished considerably in size. Nirenstein regards the red-staining granules as bearers of a tryptic ferment. 
In the second or alkaline period the vacuole enlarges rapidly, to more than its original volume. The red colour produced by staining with neutral-red disappears. The clumped food-mass breaks up into smaller particles again. From the red-staining granules of the first period deeply-staining spheres arise, homogeneous, refractile, and apparently fluid (Nirenstein, 181). According to Khainsky (170.5), the grains or droplets which are formed gather at the surface and pass out into the endoplasm; they represent the first products of the assimilatory process in the vacuole, and their further chemical transformation takes place in the endoplasm itself (compare the refringent bodies formed in the process of digestion in acinetans, p.458). According to Nirenstein, however, the spheres become smaller and smaller, being reduced to tiny grains which vanish completely, dissolved in the vacuole-contents. The vacuole now diminishes in size a second time, and passes to the anal region, where it fuses with other similar vacuoles, and is finally rejected from the anal pore.

In other cases, however, no acid reaction has been demonstrated in the vacuoles at any time, as, for example, in Actinosphorium $\rightarrow$ peculiarity which is perhaps to be correlated with the fact that in this form the prey is killed when seized by the pseudopodia. It may be supposed that the processes which, in Infusoria, etc., go on during the first or acid period of the food-vacuole, take place in Actinosphorium and some other forms before the vacuole is formed, in which case the vacuole itself shows only the second or alkaline phase of the digestion.

According to Greenwood and Saunders (163), any ingested particles excito tho secretion of acid, but the true digestive vacuole is only formed under the stimulus supplied by nutritive matter. Metalnikoff (179), however, found that in the same individual some of the food-vacuoles are first acid and then alkaline, while others are alkaline throughout in their reactions, and others again, but rarely, show an acid reaction throughout; he concludes that the living cell has the capacity of adapting itself to the food supplied, and of altering the properties of its digestive juices in accordance with its requirements. The process is perhaps comparable to the manner in which the bloodcells produce different anti-bodies when brought into contact with different pathogenic organisms or toxins.

The variety of ferments that have been isolated from different Protozoa also indicates that the digestion takes a different course in different cases. In the plasmodia of Mycetozoa, a peptic fermont, which when acidulated dissolves fibrin, has been isolated; but since the protoplasm of the plasmodium has a distinctly alkaline reaction, it was thought by some that the ferment must be without function. Metschnikoff (180) showed, however, that the food-vacuoles formed in the plasmodium had a strongly acid reaction, in contrast to the protoplasm, and thus demonstrated the function of the peptic ferment in the digestion. In other cases tryptic ferments have been isolated (" amobodiastase," etc.). 
Some doubt has existed as to the power possessed by Protozoa of digesting fats, and, according to Staniewicz (208), no digestion of fat takes place in Infusoria. According to the recent investigations of Nirenstein (182), however, Paramecia under natural conditions contain fat in more or less considerable quantities. By choice of suitable food, the quantity of fat in the endoplasm oan be increased greatly. The fat-granules serve as reserve-nutriment, and disappear under starvation. Paramecia which have lost their fat in this way, if then $d$ with milk, oil-emulsion or yolk of egg rubbed up in water, show in a few hours the endoplasm full of fatgranules; if fed with starch or particles of egg-albumen, the same result is obtained, but not to anything like the same extent. Experiments on fatty substances ingested by the animals showed that the fat remains unaltered during the first (acid) period of the digestion in the food-vacuole, and is digested during the seoond (alkaline) period. Feeding with fatty acid and glycerine also leads to storage of fat in the endoplasm. If fed with oil-globules stained with Soudan III., unstained oil-globules appear in the endoplasm. Nirenstein concludes from his observations that the fat is broken up into its soluble components in the vacuole, and synthesized again to neutral fat in the endoplasm.

The indigestible residues of the food are ejected from the body either at any point on the surfice, in amœboid forms, or through a definite aperture, in corticate forms. A great accumulation of fæcal matter may take place in some cases, as in the "stercome" of Foraminifera (p. 233), of which the animal purges itself periodically.

(c) Saprophytic and Parasitic Nutrition. - In this type the organism absorbs its nourishment by diffusion through the surface of the body without the aid of any visible organs or struotural differentiations of any kind. Practically nothing is known of the mechanism by which this is effected or of the chemical processes involved, but it is probable that enzymes secreted by the organism reduce the nutritive partioles to a soluble form prior to absorption. There is reason to believe that the nucleus is specially concerned in the produotion of enzymes, and in many species, parasitio or otherwise, the behaviour of the nucleus indicates a relationship between it and the process of absorption of food-substance. In Carchesium, as already stated, the path along whioh the foodvacuoles travel runs elose along the inner edge of the horseshoe-shaped macronucleus (Greenwood, 162); in Euplotes, similarly, the large macronucleus enoloses an area containing all the food-vacuoles (Fig. 182). According to Wallengren (214), the reactions of the food-vacuoles of Paramecium change as they pass the nucleus, and the function of the cyclosis in the endoplasm is to bring the food- 
vacuoles near, and under the influence of, the nucleus. In the coccidian parasite Caryotropha (p. 352), the nucleus of the parasite is connected by a kind of protoplasmic canal with the nucleus of the host-cell (Siedlecki, 653). In the astomatous Ciliata (p. 451) a diffuse nucleus is very commonly found, probably in relation to absorption of nutriment by the osmotic method.

The process of nutrition in Protozoa may lead in some cases, not to growth of the protoplasmic body directly, but to the production and storage of reserve food-substances, which are precipitated in the cytoplasm, and are utilized at a later period for rapid growth during reproductive phases. The reserve-materials deposited in this way vary considerably in nature in different cases. Examples are the paramylum-grains of many flagellates; the paraglycogengrains of gregarines and ciliates, similar in nature to glycogen, but with certain distinctive reactions; the plastinoid granules of coccidia (p. 346) ; and other similar substances. In Radiolaria oilglobules and albumen-spheres occur. An important substance, acting apparently as reserve-material for the growth of the nucleus, is volutin (p. 68).

The effects of starvation on Protozoa have been studied by a number of investigators, most recently by Lipska (173), who gives a complete bibliography and résumé of previous work on the subject. Lipska found that Paramecium died after five to seven days, a much shorter period than allowed by Wallengren (214) and others, indicating that Lipska's methods were more drastic and sources of food were more thoroughly excluded in her experiments. In the first period of starvation the reserves in the endoplasn are used up, first the food-vacuoles and their contents, then the smaller endoplasmic granules. After the fourth day the animal becomes deformed. Its dimensions diminish progressively, and death supervenes when it has lost half its initial volume. The ectoplasm with its cilia and trichocysts undergo no change, but the endoplasm loses its food-vacuoles and a part of its crystals, and becomes very transparent. The macronucleus becomes enlarged and breaks up into two halves. The micronucleus undergoes no change of any kind. Death is preceded by a progressive enfeeblement of all functions, such as movements of the cilia and pulsation of the contractile vacuoles. According to Wallengren, the reactions of the Paramecium (gcotaxis, thermotaxis, galvanotaxis) remain normal to the last. Wallengren described an exccssive vacuolation of the endoplasm as the result of starvation; but according to Lipska this phenomenon is not due to starvation, but to the chemical action of ammoniacal products generated by bacteria present in the infusions, and does not occur if they are excluded. Other observers noted the occurrence of numerous conjugations during the first few days of starvation, but Lipska was unable to eonfirm this; in her experiments, however, the number of Paramecia placed in each tube was small, not more than ten. Paramecia containing symbiotic algæ were more resistant to starvation than thoso without them.

2. Respiration.-By respiration in its widest sense must be understood all processes in the organism whereby the potential energy stored up in chemical compounds of high complexity is set free to furnish the energy required by the organism for its vital activities. This object may be effected in two ways-by processes of oxidation, 
or by the splitting up of complex chemical substances; the result in either case is the production of energy in various forms and of simple chemical substances, such as water and carbon dioxide (compare Barratt, 148). For the processes of oxidation the organism may either absorb free molecular oxygen from its environment, or may produce it by internal molecular changes of substances contrined in its own body, as in anaerobic organisms living in a medium in which free oxygen is lacking.

Many free-living Protozoa require oxygen, and are visibly and rapidly affecter by the lack of it, especially in their powers of movement. No special organs of respiration are found in any Protozoa, being unnecessary in animals of such small bulk, and in which, consequently, the surface of the body is considerable in proportion to the mass. The contractile vacuoles, when present, are doubtless a means of eliminating carbon dioxide, together with other waste products, from the body. It must be supposed, therefore, that as a general rule oxygen is taken up from the surrounding water by the protoplasm, of which the limiting membranes are freely permeable; and that the carbon dioxide is given off in a similar manner. The experiments of Verworn (211) on Spirostomum show that the respiratory processes take place in the cytoplasm, independently of the nucleus, which takes no share in respiration.

On the other hand, many sapropelic (p. 14) and parasitic forms inhabit media lacking in free oxygen, and are anaerobic; in such forms the respiratory processes of the protoplasm can only take place by intramolecular changes, in which the stored-up reserve-materials are probably split up to supply the required oxygen.

The experiments of Pütter (201) on a number of species of Ciliata, both free-living and parasitic, showed that, when these animals were placed in an anaerobic environment, different individuals of the same species reacted very differently to the conditions, some dying very rapidly, others being quite unsffected for a long time. It was shown further that this difference was related to the amount of reserve-materials present in the body (proteins and glycogen), which can be observed to vary greatly in different individuals from the same culture. If Paramecia were first starved for some days and then placed in anaerobic conditions, they succumbed much more rapidly than normal individuals. Moreover, under anaerobic conditions tho reservematerials were used up much more rapidly than under normal conditions, and without resulting in increased production of energy. Opalina, when placed in a culture-medium to which albumen was added by boiling up dried white of egg in salt-solution, was able to make use of the energy of the albumen without the help of free oxygen, and so to live for a much longer time. The ciliates were found to succumb much more rapidly to the effects of anaerobic conditions in smaller than in larger quantities of water, as the result of autointoxication in consequence of the defective excretion of the products of anaerobic metabolism. Spirostomum was found to be more affected by anaerobic conditions in small quantities of water than Paramecium. The differences between the two forms is to bo ascribed to the system of the contractile vacuoles, which is far more efficient in Paramecium than in Spirostomum; the contractile vacuoles tend to removo from the body the 
produets of metabolism, a primary necessity of anaerobic life. The question of size is also a factor, since deleterious substances may diffuse from the surface of the body, and in a small body the surface is greater in proportion than in a larger one. Consequently the conditions are more favourable for a smaller species, such as Paramecium, than for a large form, such as Spirostomum.

Excess of oxygen was found by Pütter (198) to have an injurious effect on Spirostomum, affecting, however, only the cytoplasm, and not the nucleus, in the first instance.

On the current view that the symbiotic vegetable organisms present in many Protozoa aid in the respiratory processes by absorbing the carbon dioxide, breaking it up, and setting free the oxygen, the experiments of Lipska (173) on a culture of Paramecia which contained green algæ (Protococcaceæ) in their endoplasm are of considerable interest. In two glase vessels of equal size there were placed, in the one Paramecia with, in the other without, the algæ in their body. Hydrogen was circulated through the vessels to drive out the air, after which they were hermetically sealed and exposed to the same conditions of light and temperature. After fifty hours the vessels were opened. The Paramecia without algæ were dead, but those containing algæ were still alive, though feeble in their movements, and they revived completely in about twenty-four hours after air had access to them. In another experiment two batches of Paramecia were kept in the dark; after eight days those without algæ were dead, while those containing algæ were perfectly normal. Old cultures of Paramecia containing algæ showed no conjugation; Lipska explains this as due to the influence of the algæ, since, by setting free oxygen, they prevent the development of anaerobic bacteria which produce substances toxic to the Infusoria.

According to Popoff (185), the depression-periods of Protozoa (p. 208) are partly due to derangements of the respiratory processes and to accumulation of products of metabolism in the cell.

3. Excretion and Secretion.-The waste substances excreted from the protoplasm may be either soluble or insoluble in nature. If soluble, they may either pass out of the protoplasmic body by diffusion from the surface, or may be removed by the agency of the contractile vacuoles.

Contractile vacuoles are of common occurrence in free-living fresh-water Protozoa, but are usually wanting in marine forms, or, if they occur in them, they pulsate very slowly. They are generally absent also in entozoic and parasitic Protozoa, but are found, however, in some internal parasites-for example, in all Anoplophryinoe (p. 452 ; Cépḋde, 831).

Some authors (e.g., Degen, 154) have described an investing membrane to the contractile vacuole, but it is practically certain that no such membrane exists, and that the vacuole is simply a drop of watery fluid lodged in, and bounded by, the more viscid protoplasm, without any special structural differentiation (compare Khainsky, 170.5). The contractile vacuoles were believed at one time to empty themselves internally, and to function simply as circulatory organs; but in all cases in which they have been studied carefully, it has been proved that they empty themselves to the exterior (compare Jennings, 167, Khainsky, 170.5).

The effect of changes of temperature is noted below (p. 206). Increased pressure makes the pulse slower (Khainsky, 170.5). Degen (154), experimenting with Claucoma colpidium, found that oxygen produced at first an increase in the frequency of the pulse, which soon became normal again. Hydrogen and carbon dioxide diminished the frequency and caused a dilata. tion of the vacuole; both these gases were lethal in their effect, especially carbon dioxide. Isotonic solutions of neutral salts had a retarding effect. 
Substances that precipitate albumens have a retarding effect combined with dilatation of the vacuole. Degen, following Hartog, regards the vacuole as primarily a mode of compensation for the tendency of the protoplasm to take up water by imbibition, a tendency checked or inhibited by changes in the tonicity of the medium. Thus Zuclzer (222) found that Amoba verrucosa, if transferred gradually from fresh water to sea-water, lost its contractile vacuoles; at the same time its protoplasm shrank and altered in character, and the nucleus acquired a different structure and appearance. When rostored to fresh water, the contractile vacuoles reappeared, and the nucleus and cytoplasm became of normal character. These experiments indicate that the formation of the contractile vacuoles depends on differences in the tonicity of the protoplasm and the surrounding medium; they also raise the suspicion that many species of marine Protozoa may be only different forms, due to change of medium, of fresh-water species, or vice versa.

For the excretory vacuole-system of Opalina, see p. 447.

Insoluble excretion-masses are often formed in great quantity in the bodies of Protozoa. Such substances take the form of crystals or grains of various kinds, and often of pigment. An example of such a substanee is the melanin-pigment of the hæmamœbæ (p. 359), which appears to be a derivative of the hæmoglobin of the infected blood-corpuscle. Pigment may arise also by degeneration of superfluous chromatin extruded from the nucleus, as in Actinosphcerium (p. 209), or by degeneration of nuclei, as in abnormal oöcysts of Cyclospora caryolytica (p. 361).

The cytoplasm of Paramecium contains crystals which have been studied by Schewiakoff (206), who finds that they consist of calcium phosphate, either $\mathrm{Ca}_{3}\left(\mathrm{PO}_{4}\right)_{2}$ or $\mathrm{Ca}_{2} \mathrm{H}_{2}\left(\mathrm{PO}_{4}\right)_{2}$. When the Paramecia were starved, the crystals disappeared completely in one or two days; if then the Paramecia were supplied with food again, the crystals reappeared. Schewiakoff was never able to observe that the crystals were ejected from the anus, but they were seen to collect round the contractile vacuole. He is of opinion that the insoluble phosphate is dissolved in the enchylema, or is converted into the soluble form $\mathrm{CaH}_{4}\left(\mathrm{PO}_{4}\right)_{2}$, and then eliminated by the contractile vacuole.

Insoluble exeretion-masses may be simply extruded from the body, a process which commonly takes place at certain orises, as, for example, prior to encystment. Or, on the other hand, they may remain in the protoplasm, and are finally abandoned in the residual masses left over during reproductive phases, as seen commonly in the sporulation of various types - for example, the hæmamœbæ already cited and other Sporozoa. In such cases the young individuals are formed of protoplasm free from the coarse excretion-granules, and the body of the parent, so much as is left of it, dies off and disintegrates. In some cases, however, the young individuals formed contain enclosures derived from the parent-body, as, for example, the crystal-bearing swarm-spores of Radiolaria (p. 254); but in such cases the enclosure is probably of the nature of reserve-material.

Secretion, more or less rapid, of various substances can be observed without difficulty in various Protozoa. Examples are the 
spicules and various skeletal structures; the shells, houses, etc. adhesive substances or stalks in sedentary forms, as, for example, the non-contractile stalks of many Vorticellids (p. 441); and the cysts or envelopes secreted round the body, such as the sporocysts, etc. The pseudopodia of many Amœbæa, such as Diffugia, are covered by a sticky slime which enables the animal to adhere to surfaces over which it creeps, and which can be drawn out by contact with a glass rod into threads, like the mucus of a snail (Rhumbler, 34). In Foraminifera and Heliozoa the pseudopodia appear to secrete a substance which holds the prey fast, and at the same time kills it, as already mentioned. Some Protozoa-for example, gregarines-leave a trail of mucilaginous substance behind them as they move forwards, and by some authors this serretion has been regarded as the mechanism by which locomotion is effected (p. 327). Internal secretions in connection with the digestive function have been mentioned in a previous section. Arcella has the power of secreting gas-bubbles in its protoplasm for hydrostatic purposes (compare also the Radiolaria, p. 252).

4. Transtormation of Energy-(a) Movement.-The different motile organs of Protozoa have been described above. Considered from a morphological standpoint, the protoplasmic body may exhibit, in the first place, no specially differentiated organs of movement, which then takes the form of currents and displacements in the fluid protoplasm itself, manifested externally in the form of pseudopodial processes or flowing movements of the entire body, internally as streaming movements in the protoplasm. Secondly, there may be special organs of movement, either external, in the form of vibratile organs, such as cilia, flagella, or undulating membranes; or internal, in the form of contractile fibrils or myonemes.

Different as pseudopodia may appear at first sight from vibratile organs, such as cilia or flagella, there is nevertheless a very gradual transition from the one type to the other (see p. 53, supra). Of pseudopodia there are two chief types of structure-the lobopodia, in which a fluid core of endoplasm is enveloped by a superficial layer of stiffer ectoplasm; and the axopodia, in which, on the contrary, a secreted axis of rigid or elastic nature is covered by a more fluid layer of protoplasm. The axopodia are connected by transitions both of structure and movement with organs of the vibratile type. In both flagella and cilia the structure consists of a firmer elastic axis covered over by a more fluid superficial layer (pp. 52, 54); many axopodia exhibit swinging, nutating, or bending movements differing only in degree from those of flagella (p. 5l). There are grounds for believing the one type of organ to have been derived phylogenetically from the other.

The streaming movements of protoplasm have been tho subject 
of much investigation and discussion. The older view, which ascribed them to contractility and assumed a complicated structure in the protoplasm, has now been superseded generally by the theory connected more especially with the names of Quincke, Berthold, Bütschli (37), and Rhumbler (34, 35, 40, etc.), according to whioh differences of surface-tension are regarded as the efficient cause of the streaming movements of the pseudopodia and the protoplasm. The living substance is in a state of continual chemical change in every part; such changes are sufficient to account, in one way or another, for the origin of local differences in the physical nature (adhesion) of the surface of the body in contact with the surrounding medium, or of internal protoplasmic surfaces in contact with vacuoles or cavities filled with fluid ; and the resulting differences in surface-tension cause flowing movements both in the protoplasm and in the fluid with which it is in contact. The relation of such currents to the movements of pseudopodia has been discussed above (p. 47). Similar movements have been imitated artificially by Bütschli and Rhumbler in a manner which can leave no doubt tbat the physical analogy is a reasonable interpretation of the mechanism . of amœiboid movement.

The close structural similarity between flagella and cilia on the one hand, and the axopodia on the other, makes it highly probable, to say the least, that the same explanation of the movement applies to both. The axis of the vibratile organ is commonly regarded as a firm, elastic, form-determining structure; the more fluid sheath as the seat of the motile activity. Chemical differences set up in the limiting membrane, causing differences in the surface-tension of the sheath along certain lines, have been supposed to be responsible for a deformation of the sheath, bending the axis and the whole organ with it ; with equalization and disappearance of such differences, the elastic axis straightens itself again. How such chemical differences are set up remains to be explained; possibly they originate in chemical changes taking place explosively in the basal apparatus of the vibratile organs ; in any case it is clear that, as compared with pseudopodia, they act with extreme rapidity, and, further, that they are localized on the surface of the flagellum or cilium. From the movements of these organs, the contraction appears to run a spiral course as a general rule-at least in cilia (p. 54); flagella, however, appear to be capable of various kinds of movements (p. 52).

According to Prowazek (192), the flagellum of a trypanosome only retains its motility so long as it remains in connection with the kinetonucleus. Werbitzki (526), however, has succeeded in producing strains of trypanosomes without kinetonuclei, and with apparently no resulting loss of motility. It has been observed frequently that detached cilia or flagella continue to contract, for a time at least; and Schuberg (44) denies that the basal granules of the cilia function as centres of kinetin activity. 
With regard to the contractility of the myonemes, no detailed explanation can be offered at present. Bütschli (37) has shown the possibility of explaining the contractile mechanism of such structures by differences in surface-tension arising between the walls and the contents of protoplasmic alveoli which are disposed with a definite arrangement.

(b) Other Forms of Energy.-Light-production or phosphorescence is a common phenomenon in marine Protozoa, a property expressed in such names as Noctiluca (p. 279) and Pyrodinium (p. 278). The magnificent phosphorescent effects often seen at night, especially in warmer seas, is to be referred chiefly to swarms of Protozoa. The source of the luminosity appears to reside in small globules of fat or oil, and is probably the result of oxidation. It is easy to observe that the production of light is stimulated by agitating or stirring the water. For a general diseussion of luminosity in living organisms, see Pütter (200).

From the analogy of the known facts in the physiology of animal and plants, it may be inferred that in Protozoa also the vital activities are accompanied by the production of heat and by electrical changes; but no exact determinations of such changes have been made.

5. Reactions to Stimuli and Environment.-It can easily be observed that Protozoa react in a definite manner to stimuli, and behave in a particular way under certain conditions. In most cases, however, these responses to external conditions must be regurded as fundamental properties of the living protoplasm, and not as functions of specially differentiated organs of the body. This is well seen, for example, in amœbæ, some species of which are very sensitive to light, and cease feeding when exposed to the bright illumination of the stage of the microscope (Rhumbler, 34). In Arcella the nuclear division is stated to take place only at night, between 1 and 5 a.m. (Khainsky, 145). In such cases, however, there is nothing which can be identified as a special light-perceiving organ.

In other cases Protozoa may possess organs which must be regarded as sensory in nature. Pseudopodia appear to possess in many cases a tactile or sensory function to a marked degree, and gometimes to be specialized for such functions, as, for example, the anterior pseudopodia of some Myxosporidia, such as-Leptotheca agilis (Fig. 165). The same is true to a much greater degree of flagella and cilia ; anteriorly-directed flagella are perhaps always sensory in function, especially when they are not the sole means of locomotion, as in such forms as Rhizomastigina (p. 268) or Bodonidae (p. 270); and in many Ciliata stiff tactile bristles occur (p. 446). In many flagellates organs are found which appear to be specially sensitive to light, in the form of pigment-spots or stigmata, which are described further below. 
The occurrence of a conducting nervous apparatus is more doubtful; it has been affirmed for Stentor by Neresheimer (p. 446), but is not confirmed by other observers. It can at least bo asserted that in the more highly organized Ciliata a stimulus may lead to sudden movements in which different sets of contractile structures take a concerted part.

The reactions of Protozoa to stimuli have been the subject of a great deal of experimental research by many investigators, amongst whom Verworn, Loeb, Jennings (165), and Pütter (199), deserve special mention. The results of these investigations can only be summarized briefly here. The various reactions are classified in the first instance, according to the nature of the stimulus, by the use of a terminology in whioh each principal category is denoted by a word terminating in taxis, or in adjectival form-tactic. Thus we can distinguish-(a) Chemotaxis, or reactions to chemical stimuli ; (b) Phototaxis, or reactions to light; (c) Thermotaxis, or reactions to heat or cold ; $(d)$ Barotaxis, or reactions to mechanical stimuli ; and (e) Galvanotaxis, or reactions to electrical stimuli. A given Protozoon may be quite unaffected by a particular stimulus; or, on the other hand, it may be affected by it in such a way that it tends to move towards the source of the stimulus (positive taxis) or away from it (negative taxis). The result depends, in many cases, on the intensity of the stimulus applied; thus, a Euglens will move towards a moderate light (positive phototaxis), but away from a too intense illumination (negative phototaxis). In each case an optimum condition exists, in which the positive taxis reaches its maximum.

In such experiments the Ciliata are the objects of ehoice, on account of the definite polarity of their movements as compared with forms less highly organized, such as amœba. In the Ciliata a negative taxis results in an "avoiding reaction" (Schreckbewegung), in which the animal shrinks back with reversal of the ciliary movements, "turning towards a structurally-defined side, followed by a movement forward" (Jennings). Repeated experiments have shown that the forms taken by the avoiding movements do not depend on the nature of the stimulus, but on the organization of the animal itself, and are always the same for a given species. An Oxytricha, for example, turns always to the right, whatever the direction from which the stimulus comes. The movement is determined automatically by the structure of the body. "The same symptom can be called forth by the most diverse stimuli " (Pütter, 199).

The various taxes may now be considered briefly:

(a) Chemotaxis and Effects of Environment.-This category includes reactions to liquids or gases diffused in the water; reactions 
to gases may be considered as equivalent to a sense of smell in higher organisms (osmotaxis).

It has been shown by many experiments that a given specles is attracted towards certain chemical substances, repelled by others. Thus, Paramecium is attracted towards weak acids, but repelled by them in greatcr concentration. If a drop of acid of suitable strength is placed in the midst of a number of Paramecia distributed evenly in the water under a cover-slip on a slide, they tend to gather round the drop. As the drop diffuses in the surrounding water, the Paramecia arrange themselves in a ring in the region of optimum concentration. If, however, the drop of fluid employed is of a strength which represents the optimum of chemotaxis for the species,

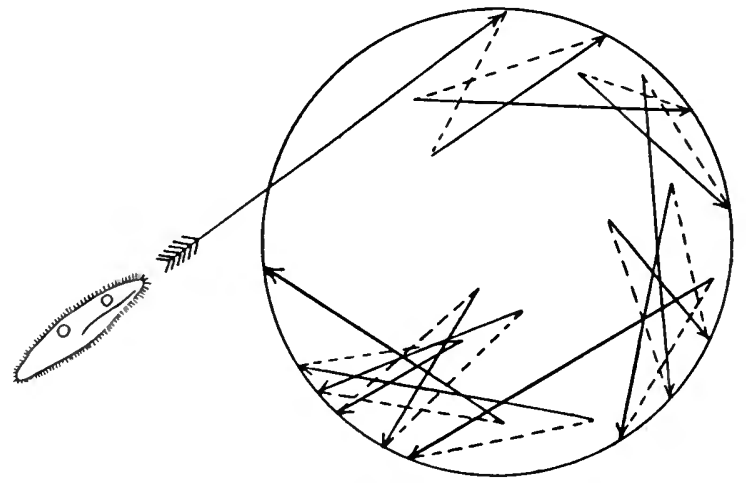

FIG. 83.-Diagram showing the course taken by a Paramecium which has entered a drop of fluid to which it is positively chemotactic. The forward movements of the Paramecium are indicated by arrows; its backward movements by dotted lines; the outline of the drop of fluid by a circle. Each time the Paramecium, in its forward movement, reaches the confines of the drop, it conies into contact with fluid which is less positively chemotactic than the drop into which it has entered; it then shrinks backward (avoiding reaction), after which it moves forward again with the same result every time it reaches the edge of the drop. After Lang (10).

the Paramecia gather within it, and in such a casc the position taken up by each Paramecium depends on the avoiding reaction made by it when it comes in contact with a less attractive medium. Thus, if a Paramecium, swimming in a straight line, enters a drop of fluid which is positively chemotactic to it, when it has crossed the drop to its opposite boundary it comes to the region where it meets with fluid which is less chemotactic to it; it then shrinks back with an avoiding movement; after a time it again moves forward, and comes again into the negatively chemotactic region, with the same result as before. Thus its movements are as if eaught in a trap (Fig. 83), in which it is held by the automatic movements called forth by the difference between the more and the less chemotactic fluids, unt:I 
the differences slowly disappear by the diffusion of the one liquid into the other.

Chemotaxis is a phenomenon which is obviously of the greatest importance in the natural life of the organism. It comes into play in the search for food and in sexual attraction, for example. It has long been known that certain Protozoa are attracted towards foodsubstances, especially those species which feed more or less exclusively upon certain particular foods. Plasmodia of Mycetozoa, for example, "scent" their food from a considerable distance, and move towards it.

Rhumbler $(34,204)$ has studied the ingestion of food by amœbæ, and has made a number of experiments on the manner in which drops of fluid take up or cast out solid particles. Thus, 8 drop of chloroform suspended in water draws into its interior a glass splinter coated with shellac when brought into contact with it ; after a time the coating of shellac is dissolved in the chloroform, and the glass splinter is then ejected from the drop. This experiment furnishes data for a mechanical explanation of the ingestion of food and ejection of fæcal mauter; and it might be expected that amœbæ in Nature would ingest mechanically, and as it were helplessly, many substances of a useless kind with which they are brought into contact. This may occur experimentally when amœbæ are brought into contact with substances of no nutritivo value; Rhumbler observed an amœba which ingested carmineparticles until it died. In Nature, however, there can be no doubt that amœbæ exercise a certain choice or selection in the food they ingest, doubtless as the effect of rhemotactic reactions (compare Jennings, 168). In the Ciliata, however, there appears to be no selection of the food-particles wafted down the cesophagus except as regards their size (compare Greenwood, 162). Purely mechanical reactions, on the other hand, may possibly explain the apparent selection which many Protozoa exhibit in building up houses of certain special materials (p. 34).

Chemotactic reactions to particular substances must play a large part in determining the migrations of certain parasitic Protozoa towards particular organs of the body in which they are parasitic, in so far as such migrations are not purely passive on the part of the parasite, or determined to some extent by rheotaxis (see below).

The attraction of gametes to one another can hardly be effected by anything but chemotaxis. It is well known that the antherozoids of the fernprothallus are positively chemotactic to malio acid, which is secreted by the oögonium. In Coccidium schubern', Schaudinn (99) observed that the macrogamete, as. soon as it had expelled its karyosome, but not before, became attractive to the microgamete.

The effects of drugs and reagents on the activities of the Protozoa is a field of investigation which cannot be dealt with in detail here. Some reagents have a quickening effect on the movements, others the contrary. Narcotics, on the other hand, such as alcohol, ether, etc., may at first have a stimulating, later a deleterious, action on the vital activity. Minute doses of alcohol, according to Woodruff (216), diminish the rate of division at one period, augment it at another, of the life-cycle, but in the latter case the rate is not continuous, but decreases again; increase in the amount of alcohol will, however, again cause a more rapid cell-division for a limited period Thyroid extract is stated to have an attractive effect on Paramecrum, and also increases its capacity for reproduction (Nowikoff, 183). For the effects of other drugs and poisons, see Giemsa and Prowazek (159), and Prowazek (191, 192, and 195). In the same culture different individuals often exhibit different powers of resistance to the effects of reagents. 
(b) Phototaxis and Effects of Light and Other Rays.-Many Protozoa appear quite indifferent to light-at least of ordinary intensity ; others show a very decided reaction, as already mentioned, either negative or positive. Thus many amœbæ, Pelomyxa, etc., are negatively phototactic, and pass at once into a condition of rest and inactivity when exposed to light. According to Mast (176), a sudden increase in the intensity of the illumination inhibits movement in Amoba proteus; but if the illumination remains constant, movement begins again in a few moments. If the illumination is very gradually increased, it produces no response. In strong light $A m c b a$ proteus orientates itself, producing pseudopodia only on the less illuminated side.

Many flagellates, on the other hand, especially the holophytic forms such as Dinoflagellates, Phytomastigina, Euglenoids, etc., show the opposite reaction, moving towards the light or becoming active when exposed to it, and passing into a resting state in the dark. The positive phototaxis of the holophytic Protozoa has an obvious bionomical significance, since the holophytic nutrition can only proceed in the presence of light.

In the majority of holophytic flagellates the phototactic reaction is associated with the possession of a special organ, the etigma or "eye-spot." The stigma of Euglena consists of a protoplasmic ground-substance forming a fine network, in which is embedded pigment in the form of drop-like bodies. The pigment granules are brightly refractile, with a distinct outline, and form a single layer. In some cases the granules are spherical and all of the same
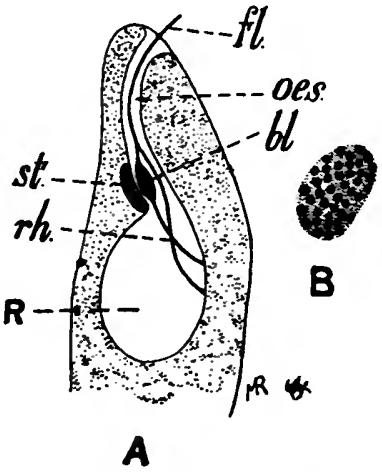

Fia. 84.- $A$, Anterior end of Euglena viridis. fl., Flagellum ; œes., œsophagus ; bl., thickening (blepharoplast?) on one of the two roots of the flagellum ; st., stigma ; $r h$, the two roots of the flagellum passing through the reservoir $(R)$ of the contractile vacuoles, two to be attached to its opposite side. $B$, Stigma in surface view, highly maguified, showing the pigmentgrains imbedded in a protoplasmic basis. After Wager (213).

size; in others they are more irregular in form and of different sizes. The pigment appears to be a derivative of chlorophyll. The stigma is in close contact with a well-marked thickening on one of the two branches into which the flagellum bifurcates at its base. Wager (213) suggests that this thickening (blepharoplast ?) is a specialized sensitive organ which is stimulated by the light-absorbing pigment-spot, the stigma, and that in this way the reaction of Euglena to light is determined. Euglena swims towards a moderate light, but away from strong sunlight. If kept in bright sunlight it comes to rest, rounds itself off, and ultimately becomes encysted.

The blue and violet parts of the spectrum exert the strongest stimulus on flagellates. In the case of Amoba proteus, Mast (176) found the blue rays nearly as efficient as white light in causing reactions, but violet, green, yellow, and red, to be but slightly active. Paramecium and some other Protozoa are stated to react only to the ultra-violet rays.

The effect of radium-rays upon various Protozoa has been investigated by Zuclzer (221). Some species are more affected by them than others; 
Amaba limax, for example, was very resistant to the rays, while other Protozoa wore very soon injured by them. In all cases long cxposure to the rays was fatal. The first effect of the rays was generally to quicken the movements; the next was an injurious action. The rays appear to act more particularly upon the nucleus in the first instance, with subsequent gradual deleterious effects upon the cytoplasm.

In experiments on the effect of Röntgen-rays on Paramecium aull Volvox (Joseph and Prowazek, 169), these forms wore found to exhibit a ncgative taxis, collecting in ten to fifteen minutes in a part not exposed to the rays. Exposure of Paramecium to the rays caused the pulse of the contractile vacuoles to become slower to a marked degree as a rule, but individual variations were observed in this reaction, the effcct being inconsiderable in some cases; and the animals gradually regain the normal pulse. Intra vitam staining of the nucleus of Paramecium exposed to the rays gave a result similar to that obtained by staining Paramecia fatigued by being shaken evenly and continuously for two hours. Long-continued action of the rays killed the organisms.

(c) Thermotaxis c $n d$ Effects of Temperalure.-For a given species of the Protozoa there is an optimum temperature at which its vital activity is at its highest pitch, and above which the activity is diminished until i1. reaches a point at which the vitality is impaired and the animal is finally killed. A temperature, however, at which the animal succumbs sooner or later may at first have a quickening effect upon the ital functions. Thus, many experiments have shown that a rise of temperature increases greatly the rapidity and frequency of the pulsstions of the contractile vacuoles; and in the case of Glaucoma colpidium Degen (154) found that, although the animal was killed by a temperature above $30^{\circ} \mathrm{C}$., the maximum frequency of the pulsations was produced temporarily by a temperature of $34^{\circ} \mathrm{C}$., above which the frequency was rapidly diminished (compare also Khainsky, 170.5).

The optimum temperature may, however, be different at different stages in the life-cycle, as in parasitic Protozoa which infest a warmblooded and a cold-blooded host alternately; in such cases a change of temperature may perhaps be a factor in bringing about developmental changes. In free-living Protozoa the phases of the life-cycle are often related to seasonal changes, and are probably induced largely by conditions of temperature.

Experimentally it has been shown that Protozoa tend to move towards regions of more favourable temperature, and away from those less favourable. Khainsky $(170.5)$ found that rise of temperature produced a quickening of the digestive processes in Paramecium, very marked at $24^{\circ} \mathrm{C}$. or above. At $30^{\circ} \mathrm{C}$. and above Paramecium takes up scarcely any more food; the contents of the food-vacuoles, which continue to be formed, then consist almost entirely of water.

The effects of temperature on the development in cultures are very marked. Popoff studied the growth of Frontonia leucas in cultures kept at $14^{\circ} \mathrm{C}$. and $25^{\circ} \mathrm{C}$. respectively; at the lower temperature the animals divide once in about eighty or ninety hours, in the warmer culture once in about seventeen hours; in the cold both the nuclcus and the body grow to a size absolutely larger than in the warmth, but in the former case the nucleus is about 3 , in the latter about $\frac{1}{64}$, the bulk of the whole body (Hertwig, 92). In the oase 
of Actinosphcerium, the experiments of Smith (207), Mackinnon (174), and Boissevain (15l), show that increased temperature hastens on encystment, and causes fewer and larger cysts to be formed in which the nuclei are larger but poorer in chromatin; while at lower temperatures the encystment is retarded, and finally inhibited altogether, and the cysts produced are smaller and more numerous, with nuclei smaller than the normal but rich in chromatin.

(d) Barotaxis and Effects of Mechanical Stimuli.-This category includes Geotaxis, or reactions to gravity ; Thigmotaxis, or reactions to the mechanical contacts of hard surfaces; and Rheotaxis, or reactions to the pressure of currents in the surrounding medium. The influence of gravity is seen in the manner in which many Protozoa, when placed in a vessel, seek of their own accord the bottom in some cases, the surface-film in others. The plasmodia of Mycetozoa exhibit often a well-marked rheotaxis, and move in the opposite direction to currents of water. It has been suggested that a similar rheotaxis may explain the passage of blood-parasites from the invertebrate to the vertebrate host during the act of bloodsucking; but it is probable that such migrations are purely passive, so far as the parasites are concerned.

Contact-stimuli acting from one side often have a marked effect on the movements of Protozoa. An amœba tends to adhere to, and spread itself over, a firm surface with which it comes in contact. The movements of Ciliata often cease when they come in contact with a firm substance, and the animal remains still; Pütter (197) has shown that the contact-stimulus may be sufficient to prevent a Paramecium from reacting to thermal or electric stimuli, which would otherwise produce a marked effect upon its movements.

Unider effects of mechanical stimuli must be included those brought about by changes in the tonicity of the surrounding medium. Such effects have already been discussed above as regards their action on the contractile vacuoles. For the remarkable experiments of Verworn on the change in body-form and in the nature of the pseudopodia exhibited by amœbæ under the action of different media, see p. 217, infra. Free-living Protozoa are probably seldom if ever subject to such changes, though they might well occur in the environment of marine forms living near the upper limit of the tide-marks, in rock-pools, or other places where the tonicity of the medium might be lowered temporarily by influx of fresh water, as the result of rain or other natural causes. On the other hand, parasitic forms, and especially those which pass from one host to the other, may be subject to rapid changes of tonicity in their environment. In this connection special interest attaches to the experiments of Robertson (503) on fish-trypanosomes; it was found that in undiluted blood or in blood diluted with isotonic solutions the trypanosomes underwent no change in vitro, but that when the blood was diluted with water the trypanosomes multiplied by division, and went through changes similar to the first stages of the natural development in the leech. It was concluded, therefore, that the principal stimulus which initiates the developmental changes in the organism was a lowering of the osmotic tension, with consequent absorption of water by the protoplasm. Neumann (677) also found that the "exflagellation" of the Proteosoma-parasite of birds was greatly furthered by addition to the blood of not more than one-fifth of its volume of water. 
(e) Galvanotaxis and Effects of Electrical Stimuli.-Protozoa placed in an electric fleld-that is to say, in a drop of water between the two poles of a battery under a cover-glass on a slide-are affected to a marked degree, but with opposite results in different species. Opalina places itself parallel to the direction of the current, with its anterior end towards the anode. With a current of moderate intensity it swims towards the anode; but with a stronger current the speed at which the animal moves is diminished, and with still more increased strength of current it is carried passively towards the kathode, with its hinder end forward, as the result of kataphoric action (Wallengren, 215). Chilomonas behaves in a similar manner. Paramecium and Colpidium, on the other hand, move towards the kathode. Spirostomum with a moderate current also moves towards the kathode, but with stronger currents it first contracts its myonemes spasmodically, and then takes up a position transverse to the direction of the current, and remains still.

According to Wallengren (215), the apparently different galvanotactic phenomena exhibited by different ciliates admits of a uniform explanation, by a combination of two effects. In the first place, in the half of the body turned towards the kathode the expansionphase of the ciliary movement is stimulated; in the anodic half of the body, the contraction-phase is stimulated. In the second place, the turning movements of the ciliates are determined mechanically (compare the "avoiding reactions" mentioned above), and may be effected either by the expansion or by the contraction of certain cilia. Consequently, if the turning movements are effecte' 1 by beats of expansion, the animal places itself automatically in a position in which it moves towards the anode; if beats of contraction are effective in the turning movement, it moves towards the kathode. According to Statkewitsch (209), the galvanotactic reaction is one which overcomes chemotactic stimuli, and leads the animals irresistibly into toxic media in which they are killed.

6. Degeneration and Regeneration.-The fact that under certain conditions Protozoa undergo a process of physiological degeneration, which may end in death, has been observed frequently by all those who have kept cultures of Protozoa under observation for a long time. It has been pointed out in a previous chapter (p. 135) that the life-cycles of Protozoa exhibit depression-periods (Calkins) which are characterized chiefly by cessation of feeding, metabolism, growth, and reproduction, together with increase in the size of the nucleus, and tendency to deposition of grains of fat or other substances in the protoplasm, giving the body a characteristic darkgrey appearance. Such periods recur regularly and apparently normally in the life-cycles both of Protozoan and Metazoan cells 
(Popoff, 184); they may also be induced artificially in various ways by unfavourable conditions, such as overfeeding or starvation, changes of temperature, or treatment with reagents (compare Smith, 207 ; Popoff, 186 ; Boissevain, 151).

A state of depression may be regulated naturally by conjugation, or by restoration of the nucleo-cytoplasmic balance through a process of self-regulation on the part of the organism. The regulative processes consist of absorption of a large part of the superfluous chromatin, so as to restore the normal quantitative relation of the nucleus and cytoplasm. On the other hand, the depression may lead to complete degeneration of the organism without possibility of recovery, and death ensues by a process of disruption of the protoplasm into granules-so-called "granular disruption" (körniger Zerfall). Some examples are given below :

Actinospharium can be brought into a condition of depression either by starvation or overfeeding (Hertwig, 164). In the depressed state a great quantity of chromatin is extruded from the nuclei in the form of chromidia which degenerate into pigment, so that the animal during a depression-period has a characteristic brownish tint, more or less pronounced in proportion to the degree of depression. In extreme cases the protoplasm is bereft of its nuclei, and becomes incapable of continuing to live. The nuclei may become entirely resolved into chromidia ; or some of the nuclei grow to a relatively gigantic size and sre cast out, while other nuclei break up; or the entire medullary layer surrounding the enlarged nuclei may be thrown off. The pseudopodia may disappear altogether or become deformed in various ways, the difference between cortical and medullary substance may be annulled or abnormally increased, and the metabolism may be modified, all these changes being in relation to nuclear alterations.

In Opalina, according to Dobell (155), physiological degeneration can be induced by starvation of its host, the frog. The degenerating Opalince lose their cilia and become irregular in form; peculiar refringent eosinophile globules appear in the cytoplasm; the nuclei undergo increase in size and modification in structure, give off chromatin, and undergo irregular fusions; and the body divides irregularly, sometimes producing buds which contain no nucleus. Ultimately the Opaline disintegrate.

Prandtl (187) has described the degeneration of Amoeba proteus. "The nucleus increases in size and becomes hyperchromatinic. Chromidia are extruded into the cytoplasm, and may there degenerate, with formation of numerous small crystals. The chromatin in the nucleus also degenerates to form a mass of brown pigment, which is extruded en bloc into the oytoplasm, or forms a ring of fine granules round the nucleus. The pigment may also spread through the whole cytoplasm, giving it a brownish tinge. Finally the nucleus breaks up and disappears altogether. Degenerating amœb» are subject to the attacks of parasites. A noteworthy feature is the tendency of the degenerating amcobæ to associate in clumpe, and plastogamic fusion of two amoba was observed by Prandtl. The tendency to fusion may be compared with the agglomeration of trypanosomes, etc. (p. 128), which is common also in degenerating forms or under unfavourable conditions.* It is not improbable that many of the plastogamio unions of Sarcodina often

* The "conjugations" observed by Pütter (201, p. 582) in Opalince kept without oxgyen must have been also phenomens of the nsture of agglomeration, since in Opalina syngamy takes places between opecial gametes, and not in the form of conjugation of adult forms 88 in other Ciliats (p. 453). 
described may be phenpmena of agglomeration associated with a similar condition.

In Radiolaria, Borgert (152) describes fatty degeneration affecting the nucleus as well as the protoplasm, both endoplasm and ectoplasm. The nucleus becomes converted entirely into a vesicle filled with a mass of fatglobules, or into a number of such vesicles.

In Tocophrya quadripartita subjected to starvation, after the refringent bodies (p. 458) have been absorbed, the nucleus becomes modified in structure, the tentacles are retracted, active budding takes place, and with the last bud formed the nucleus disappears and the remaining protoplasm dies away.

From a consideration of the various examples of degeneration from different causes, it appears that the first part to be affected is always the nucleus, and that the other derangements of the structure and functions of the body are secondary consequences of an abnormal condition of the nucleus.

The regeneration of lost parts of the cell-body of Protozoa has been the subject of experiment by a great number of investigators. The methods employed have consisted mainly in mutilating the body or cutting it up in o a number of pieces, in order to find out to what extent the fragments possess the power of regenerating the lost parts. The experiments have led to one very definite result, which can be expressed briefly: no separate part of the body is capable of continuing its vital activities indefinitely, or of regenerating any of the deficiencies in the structure of the body, if it does not contain the nucleus or a portion of the nucleus. Non-nucleated fragments may continue to live for a certain time; in the case of amœba such fragments may emit pseudopodia, the contractile vacuole continues to pulsate, and acts of ingestion or digestion of food that have begun may continue; but the power of initiating the capture and digestion of food ceases, consequently, all growth is at an end, and sooner or later all non-nucleate fragments or enucleated bodies die off. A Polystomella which possesses a nucleus can repair breakages to the shell; an individual deprived of its nucleus cannot do so (Verworn). On the other hand, an isolated nucleus, deprived of all protoplasm, dies off ; but a small quantity of protoplasm containing the nucleus or a part of it is able in some cases to regenerate the whole body, and to produce a complete individual of small size.

In experiments on regeneration the Ciliata are the objects of choice; their complicated structure permits the regeneration that has taken place to be estimated accurately ; their size renders the mutilation more easy to perform; and the large sizo and frequently extended form of the nucleus makes it possible to divide up this body also. In recent experiments Lewin (171) has succeeded in dividing Paramecium into a number of fragments (" merozoa"), containing each a portion of the macronucleus. Only one of the merozoa obtained in this manner contains the micronucleus, which is too minute to be divided by a mechanical operation. Except when the Paramecium was in process of division, only one merozoon recovered the normal bodyform and proceeded to divide; and the interesting result was obtained that 
the merozoon which survived was not necessarily the one which contained the micronucleus. Regenerated individuals multiplied for a number of genera. tions, producing a culture of "amicronucleate" Paramecia. If, on the other hand, a Paramecium in process of division was halved, each half regenerated the entire body and was capable of division. These experiments indicate that Paramecium contains a division-centre independent of the nuclei, and that its presence is necessary for regeneration of the body.

Prowazek (189) observed occasionally a certain power of regeneration in non-nucleated fragments of Stentor, but considered it possible that extranuclear chromatin might have been present. The same author (190) observed abnormal regeneration, leading to monstrosities with three hinder ends, in a culture of Stylonychia mytilus during a depression-period which led finally to the extinction of the culture. The recent experiments of Lewin (172) on Stylonychia mytilus show that, in the regeneration which follows artificial mutilation, multiplication of micronuclei may occur, with the result that the regenerated individual may have more micronuclei,than the number typical of the species or race.

Bibliography.-For references see p. 481. 


\section{CHAPTER XI}

\section{SYSTEMATIC REVIEW OF THE PROTOZOA : THE SARCODINA}

As stated in Chapter I., the Protozoa are commonly divided into four principal classes. Of these, two-namely, the Sarcodina and Mastigophora-may be regarded as the more primitive groups, comprising the main stock of less specialized and typical forms from which the other two classes have been evolved. The Sporozoa are an assemblage of exclusively endoparasitic forms exhibiting clearly the modifications and adaptations induced by, or necessary for, their particular mode of lffe ; and it is practically certain that the Sporozoa are not a homogeneous class showing mutual affinities based upon a common ancestry, but that one section of the group is a specialized offshoot of the Mastigophora, the other of the Sarcodina, and that the two sections are united only by characters of convergence due to the influence of a similar mode of life. The Infusoria, on the other hand, are a specialized group in which great complexity of organization has been attained; they are the highest class of the Protozoa, and furnish examples of the most extreme degree of structural differentiation of which a unicellular organism is capable.

While there is but little difficulty, as a rule, in defining the classes Sporozoa and Infusoria, or in assigning members of these groups to their proper systematic position, the case is different, very often, when we have to deal with the other two classes. The verbal distinction between them is based chiefly on the use of the word " adult": Sarcodina are Protozoa which have no permanent organs of locomotion in the adult condition, but move by means of pseudopodia extruded from the naked protoplasmic body ; Mastigophora, on the other hand, bear organs of locomotion in the form of flagella in the adult condition, whether the protoplasmic body is naked and amoboid or corticate and of definite form. In both classes the youngest stages may be flagellate; if, in an amoboid form, the flagella are retained in the adult, the organism is classed in the Mastigophora ; if lost, in the Sarcodina.

The word "adult" when applied to the Metazoa has a meaning which can be defined clearly, as a rule, by the criterion of sexual 
maturity. In the Protozoa no such criterion is available, and the distinction between young and adult is based on differences in size and growth, or on phases of the life-cycle selected in an arbitrary manner. In many cases the distinction presents no difficulty; it is perfectly easy to distinguish young from adult stages in such forms as the Foraminifera and Radiolaria among Sarcodina, or the genus.Noctiluca among Mastigophora. But in other cases it is purely a matter of opinion which phase in the life-cycle is to be regarded as adult. Such a form as Pseudospora has a flagellated and an amcoboid phase (Robertson), and can be placed in either the Sarcodina or the Mastigophora with perfect propriety: The amceba-like genus Mastigamoeba is placed in the Mastigophora because the flagellum is retained; but if any species of this genus were to lose its flagellum when adult, rigid adherence to verbal definitions would necessitate its being classed in the Sarcodina.

The difficulty of separating and defining the stems of the Sarcodina and Mastigophora at their root is only to be expected on the theory of evolution. The two classes are undoubtedly descended from a common ancestral type, which has become modified in two divergent directions, giving rise to two vast groups of organisms which may differ from one another very slightly or very greatly in selected examples. The systematist may meet with many obstacles when it is required to lay down verbal distinctions between the two classes, but it is easy to recognize, in a general way, two principal morphological types, round which each class is centred, and which may be realized to a greater or less extent in given cases.

1. Sarcodine Type.-Protozua which grow to a relatively large size; in the so-called "adult phase" permanent organs of locomotion are wanting, and the naked protoplasmic body moves or captures food by means of pseudopodia; the young stages may be flagellate or amoeboid.

2. Mastigophoran Type.-Protozoa usually of minute size, seldom with a large adult phase (as, for example, Nocti uca); flagella retained throughout active life, only lost in resting phases; body, amoboid or corticate.

\section{THE SARCODINA.}

The name Rhizopoda is sometimes used for this class but this name is only applicable, strictly speaking, to the first four orders recognized below, in which the pseudopodia are more or less rootlike, and not to the orders Heliozoa and Radiolaria, characterized by stiff radiating pseudopodia.

General Characteristics.-As stated above, the Sarcodina are Protozoa for the most part of relatively large size. Many Sarcudina 
are visible to the naked eye, and some of the Radiolaria, Foraminifera, and Mycetozoa, attain to a size that must be considered gigantic for Protozoa. The more primitive forms, on the other hand, are often very minute.

The body-form is of two principal types, related to-distinct habits of life-namely, the amœboid type, characteristic of forms that creep on a firm substratum; and the radiate type, seen in floating forms. Amœboid forms are found aquatic, semiterrestrial, and parasitic ; radiate forms are for the most part pelagic, living floating or suspended in large masses of water, marine or fresh-water.

The protoplasmic body is in many cases distinctly differentiated into clear motile ectoplasm and granular trophic endoplasm. The surface of the protoplasm is naked, or may be covered in rare instances (Amoba verrucosa, $A$. terricola, etc.) by a very thin pellicle which modifies, but does not restrain, the amœboid movements. A resistant cuticle or cell-membrane investing the body is not formed, but an external shell or internal supporting skeleton is frequently present.

The locomotor organs in the adult are always pseudopodia, which may be of various types-lobose, filose, or reticulose (Chapter V., p. 46) ; they may lie in one plane, as in creeping forms, or may be given off on all sides, as in pelagic forms. The youngest forms (swarm-spores) may be flagellate or amœboid. In some cases the pseudopodia of the young forms may differ markedly in character from those of the adult; for example, the adult Amoba proteus has fluid protoplasm with thick lobose pseudopodia, but the young amœbula produced from the cyst of this species has viscid protoplasin with sharp, spiky pseudopodia (Scheel).

The free-living Sarcodina are almost without exception holozoic, capturing other organisms by means of their pseudopodia, and devouring them; but the remarkable genus Chlamydomyxa (p. 243) has chromatophores, and can live in either a holozoic or holophytic manner, like some flagellates; and the genus Paulinella, allied to Euglypha, also possesses chromatophores; and is capable of holophytic nutrition (Lauterborn).

The nuclear apparatus consists of one or more nuclei, in addition to which chromidia may be present. A single nucleus is characteristic of the majority of species, even of many which grow to very large size, such as many Radiolaria, in which the nucleus also zttains to proportions relatively gigantic. In other cases increase in the size of the body is accompanied by multiplication of the nuclei ; there may be two nuclei constantly, as in Amoba binucleata (Schaudinn), or several, as in Diffugia urceolata, or many hundreds, as in Actinospharium and Pelomyxa, or even thousands, as in the Mycetozoa. In such forms the adult is a plasmodium, but the 
numerous nuclei show no differentiation amongst themselves, and appear to be perfectly equivalent both in structure and function. Chromidia may be present as a permanent cell-constituent in many Amœbæa, such as Arcella, Diflugia, and the Foraminifera; in other cases they are formed temporarily, as extrusions from the nucleus, during certain phases of the life-cycle, either as a preliminary to reproduction or as a regulative process under certain physiological conditions.

The reproduction of the Sarcodina is effected either by binary or multiple fission. Binary fission may be absent in some of the larger, more specialized forms, as in many Foraminifera and Radiolaria, but in most cases it is the ordinary "vegetative" method of reproduction during the active trophic life of the organism. In plasmodial forms it takes the form of plasmotomy (p. 100). Multiple fission or gemmation (sporulation) is in some cases the sole method of reproduction; in other case it is combined with binary fission, and occurs only at certain crises in the life-cycle, in relation to seasonal changes, or as a preliminary to syngamy. In this type of reproduction the organism, breaking up rapidly into a largeoften an immense-number of minute individuals, is necessarily put hors de combat as soon as the reproduction begins; hence it is not uncommon for the sporulation to take place within a cyst, when a shell or protective envelope is not present, as in Amoeba proteus (Scheel). The minute germs produced by sporulation may be set free at once as swarm-spores; or they may form a protective envelope or sporocyst, and be liberated as resistant spores which are disseminated passively, and germinate when conditions are favourable, as in parasitic forms and in the semi-terrestrial Mycetozoa.

The swarm-spores, whether produced directly by sporulation of an adult or indirectly by germination of a spore, may. be either flagellulæ or amobulæ. In many forms two types of sporulation occur-schizogony producing agametes, and sporogony producing gametes. The agametes may be structurally or morphologically distinguishable from the gametes. Thus in Foraminifera the agametes are amœbulæ, the gametes.are flagellulæ. In Radiolaria both alike are flagellulæ, but the agametes produced in schizogony - the "isospores"-are distinguishable from the gametes produced in sporogony- " anisospores."

In this class syngamy takes place rarely between adult individuals ; but examples of this are seen in Actinophrys, where it takes the form of karyogamy within a cyst (Fig. 71), and in Arcella (p.' 148) and Diffugia, where it takes the form of chromidiogamy between free individuals, followed in Diffugia by encystment. In the great majority of Sarcodina the syngamy is microgamous, and takes place 
between swarm-spores, either amœbulæ or flagellulæ. The microgamy is isogamous or slightly anisogamous ; macrogamy, as in other cases, is perfectly isogamous. Microgamy occurs, as has been seen (p. 148), in Arcella in addition to chromidiogamy ; and, according to a recent note of Zuelzer $(86, p .191$, foothate), syngamy between free swarm-spores occurs in Difflugia also.

As regards the life-cycle of the Sarcodina, there remains still so much to be discovered that to generalize is both difficult and dangerous. Even in the commonest forms, such as Amoba proteus, the complete life-cycle has not been yet worked out. In some
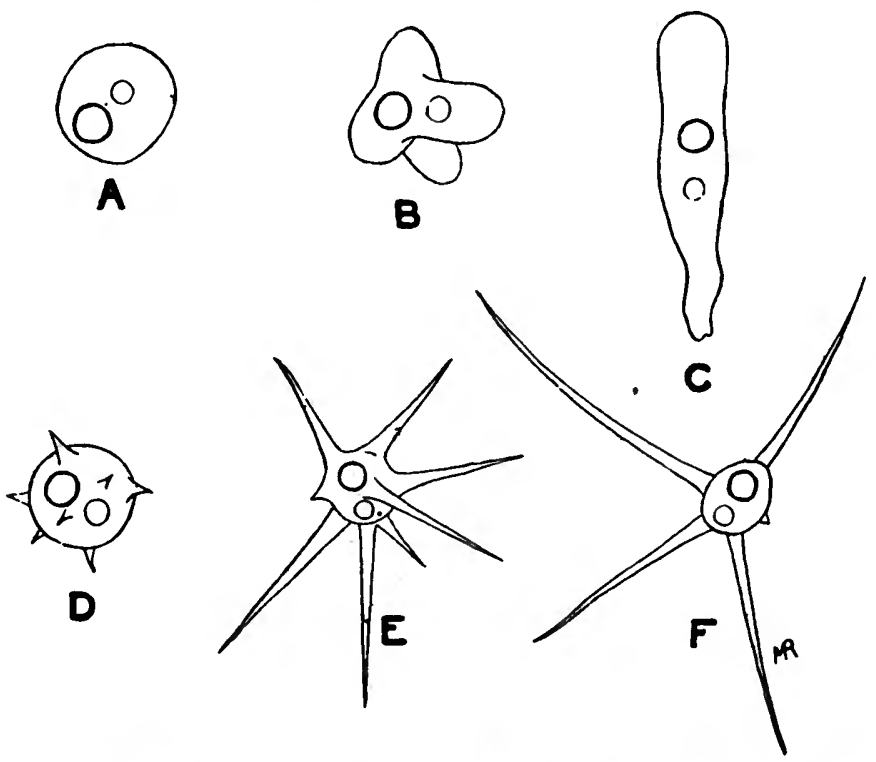

Fra. 85.-Changes in the form of an amœba under the influence of differences in the (surrounding medium. $A-C$, In its natural medinm (water): $A$, contracted ; $B$, beginning to throw out pseudopodis ; $C$, limax-form. $D-F$, Forms assumed after addition of potash-solution : $D$, contracted, beginning to throw out pseudopodia ; $E, F$, radiosa-forms. After Verworn.

cases the life-cycle appears to be of comparatively simple type, and the species is monomorphic or nearly so, as in Actinosphaerium; in other cases there is a well-marked alternation of generations, with dimorphism in the adult condition, as in Trichosphaerium (p. 182), the Foraminifera, etc.

Classification.-The Sarcodina are subdivided into a number of orders, the distinctions between which are based principally on the characters of the pseudopodia and of the skeleton, when present; in more highly differentiated forms, such as Radiolaria, the internal structure of the body is also taken into account. In the primitive 
forms of simple structure, however, in which no skeleton is present, the subdivisions are defined entirely by the characters of the protoplasmic body and the pseudopodia, which furnish distinctions of very doubtful validity. Not only may the characters of the pseudopodia vary in different phases of the life cycle, as already stated in the case of Amoba proteus, but even in the same phase under the influence of different media. Thus, no two forms of amœba could appear more distinct at first sight than the limax and radiosa forms, originany regarded as distinct species. In the limax-form the whole body flows forward as a single pseudopodium, gliding along like a slug; in the radiosa-form the spherical body becomes star-like, sending out sharp-pointed pseudopodia on all sides. Nevertheless. Verworn showed that the one form could be changed into the other by differences in the medium (Fig. 85). Doflein (238) obtained similar form-changes in Amoba vespertilio, and showed that the body-form and character of the pseudopodia were quite inadequate features for distinguishing the species of amcba, depending as they do upon the conditions of the environment and the nature of the medium. Compare also Gruber (246) on form-varieties of Amoba proteus.

In view of the protean nature of these organisms, it is not surprising that much diversity of opinion prevails as to the arrangement of the groups and the exact position of some of their members. It is usual to put a number of primitive organisms together in a group termed Proteomyxa, the members of which probably have more affinities with various members of other groups than with one another. On the other hand, the more highly organized Sarcodina are classified without difficulty into well-characterized orders; such are the Foraminifera, Mycetozoa, Radiolaria, and Heliozoa, though even in these groups there are forms near the border-line and of doubt ul position.

'The classification adopted here is mainly that of Bütschli (2), with the addition of some forms not included in his great work as follows:

A. Subchass Rhizopoda. - Typically creeping forms with branched, root-like pseudopodia.

I. Order Amobaea.-Amœboid forms of simple structure ; skeleton lacking or in the form of a simple shell.

1. Suborder Reticulosa (Proteomyxa).-With filose or reticulose pseudopodia, without shell.

2. Suborder Lobosa.-With lobose pseudopodia.

(a) Section Nuda, without shell or skeleton.

(b) Section Testacea, with shells.

II. Order Foraminifera.-With reticulose pseudopodia and shells. 
III. Order Xenophyophora.-With skeleton of foreign bodies and a peculiar internal structure.

IV. Order Mycetozoa.-Semi-terrestrial forms with reproduction by resistant spores and formation of plasmodia.

B, Subclass Actinopoda (Calkins).-Typically floating forms with radiating, unbranched pseudopodia.

V. Order Heliozoa.-Principally fresh-water, without a "central capsule."

VI. Order Radiolaria.-Exclusively marine, with a central capsule.

\section{Amcbera.}

1. Reticulosa.-In this suborder are comprised a number of forms of doubtful affinities, sometimes ranked as a distinct order, Proteomyxa. The only positive character which they have in common is the possession of filose or retioulose pseudopodia, with which is combined the absence of a shell and skeleton. Hence it is not surprising that the position of many forms referred to this suborder is extremely dubious, and some of them are referred to distinct orders by many authorities.

In general two types of organisms are referred to this suborder:

(a) Large marine plasmodial forms; an example is Pontomyxa flava, described by Topsent from the Mediterranean and British Channel. Pontomyxa is a multinncleate plasmodium of yellow colour. It sends out branching root-like pseudopodia, which may spread out and form a network extending over two or three inches in length. Nothing is known of its development or life-cycle.

(b) Small forms with a single nucleus, marine or fresh-water. which reproduce by process of multiple fission forming swarmspores. These forms have been subdivided into two families, according to the type of swarm-spore found-Zoosporida, producing flagellulæ; and Azoosporidø, producing amœbulæ. An example of the Zoosporidoe is furnished by the genus Pseudospora. which preys upon algæ, diatoms, Volvocineæ, etc. The adult phase is amœboid, flagellate, or even Heliozoon-like. It feeds on the cellsubstance and chlorophyll of the prey, and multiplies by binary fission. It can also break up by multiple fision into flagellate swarm-spores, with or without previous encystment. Robertson has observed syngamy between flagellulæ thus formed, which are therefore gametes; in other cases the flagellulæ are perhaps agametes. As already pointed out above, the position of this form amongst the Sarcodina is doubtful; by many authorities it is classified in the Mastigophora.

An example of the Azoosporida is furnished by Vampyrella, a 
small amœboid form which, like Pseudospora, preys upon algæ (Fig. 86), devouring the contents of the cell, and multiplying in the free state by binary fission. It also encysts and breaks up within the cyst by multiple fission to form a number of amœbulæ, which creep out and grow up into the adult form.

A large number of other genera are referred to the Reticulosa, for the most part so little investigated as regards their development and life-history that it is impossible to deal with them comprehensively in a brief space. For an account of them see Delage and Hérouard (6, p. 66), Hickson (248), and Rhumbler (288).

2. Lobosa.-This suborder comprises a great number of organisms, which it is convenient to subdivide into-(a) Nuda (Gymnamœbæ),

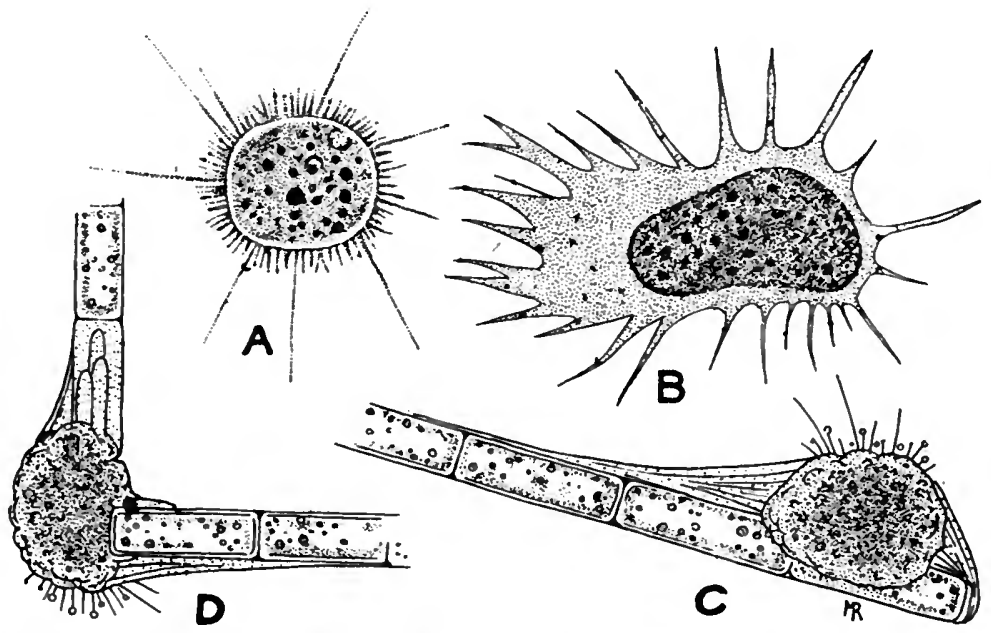

Fig. 86.-Vampyrella lateritia: various forms. $A$, Frec Heliozoon-like phase ; $B$, creeping amœboid phase; $C$, amœboid form attached to a Conferva-filament ; $D$, a similar form ; it has broken the algal filament at a joint, and has emptied one cell of its contents. $A$ and $B$ after Hoogenraad; $C$ and $D$ after Cash and Hopkinson.

with no shell; and (b) Testacea (Thecamœbæ, Thalamophora), with a shell or house.

General Characters.-Familiar examples of the Lobosa Nuda are furnished by the species of the genus $A$ moeba and allied forms. A very large number of free-living amœbæ have been described and named, but it is very doubtful how far they are true species; some of them, with pronounced and constant characteristics, such as Amoba proteus (Fig. 2) and $A$. verrucosa (Fig. 23), are probably "good" species; others, such as $A$. limax and $A$. radiosa, are probably forms that may occur as phases in the development of other species of amœbæ or of other organisms, such as Mycetozoa. 
At the present time the life-history has been worked out satisfactorily in but few free-living amœbæ, but in such protean organisms it is quite unsafe to attempt to characterize or dcfine a species without a knowledge of the whole life-cycle. As regards the familiar Amoba proteus, for example, practically all that is known of its life-cycle is that it encysts and multiplies within the cyst to form a great number of small amœbulæ, very different in appearance from the parent-organism; the amœbulæ creep out of the cyst, and probably grow up into the adult form (Scheel). Calkins adduces arguments in favour of the occurrence of a sexual cycle, which remains at present, however, purely conjectural.

The majority of free-living amœbæ are aquatic in habitat. A certain number, however, are semi-terrestrial, inhabiting damp earth, moss, etc. Such is Amoba terricola (vide Grosse-Allermann). The " earth-amœbæ," like other terricolous Protozoa, probably play a great part in keeping down the numbers of the bacteria and other organisms in the soil and thereby lessening its fertility from an agricultural standpoint (compare Russell and Hutchinson, 24 ; Goodey, 16).

A great many species of amcbæ are found living within the bo ties of animals of all kinds, for the most part in the digestive tract. The entozoic amœbæ are commonly placed in a distinct genus, Entamceba, distinguished from the free-living forms by little, however, except their habitat and the general (but not invariable) absence of a contractile vacuole. A common example is Entamoeba blatta, from the intestine of the common cockroach; others are $E$. ranarum of the frog (Dobell, 236, 237); E. muris of the mouse (Wenyon); the species parasitic in the human intestine, presently to be mentioned; $E$. buccalis (Prowazek), from the human mouth ; and many others. Chatton has described a species, Amoba mucicola, ectoparasitic on the gills of Labrida, and extremely pathogenic to its host.

Life-History.-So far as it is possible to generalize from the scanty data available at present, the development of many free-living species of amobæ appears to be of a type very similar to that of Arcella, described in a previous chapter (p. 179). In the free state the organisms reproduce themselves in two ways : first, "vegetatively," by simple binary fission, preoeded by a division of the nucleus, which varies in different cases from a promitosis (p. 109) of the simplest type to very perfect mitosis ; secondly, by formation of chromidia and subsequently of secondary nuclei, round which the cytoplasm becomes concentrated to form a number of internal buds, destined to be set free as amœbulæ, agametes, which grow up into the adult form. In addition to these two methods of reproduction in the free state, the animal may become encysted, 
and produce within the cyst a number of gametes in the same manner as the agametes already described, but with the following differences of detail : the principal nucleus degenerates as soon as the chromidia are formed ; the number of secondary nuclei produced is much larger, and the gametes are much smaller than the agametes ; and the cytoplasm of the parent is entirely used up in their formation. The gametes are ultimately set free from the cyst as amobulæ, and pair ; the zygote grows into the adult form of the amœba. Such a cycle has recently been described by Popoff (264) for a species named by him Amcba minuta; the gametes in this species are isogametes, without any sexual differentiation as in Arcella. This type of life-cycle is probably very common in many amœbæ,

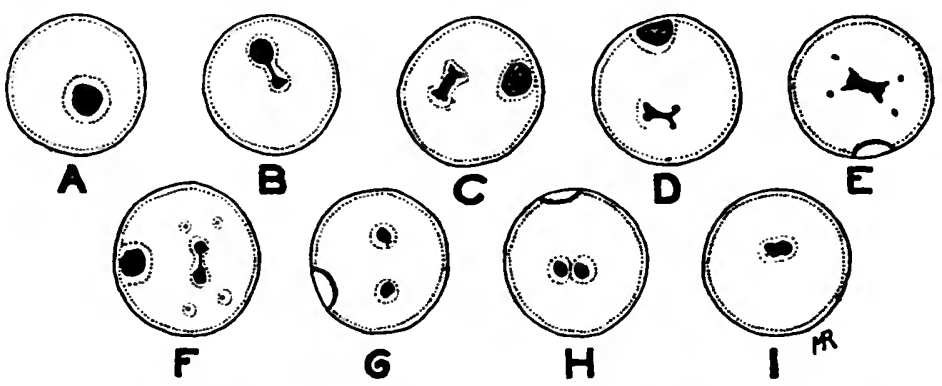

Fia. 87.-Amaba abbida : antogamy in the encysted condition ; drawn in ontline, with nuclear detsils only. $A$, Enoysted amceba; $B$, the nucleus of the amoba divides unequally into a larger vegetative and a smaller generative nucleus; the vegetative nuoleus, as seen in the subsequent figures, travels to the surface of the oyst, degenerates, and disappears; the generative nucleus gives rise to the gamete-nuclei; $C$, inoomplete division of the generative nucleus; $D$, one half of the generative nucleus is budding off two reduction-nuclei (on the right); $E$, four reduction-nuclei have been budded off, two from each pole of the incompletely divided generative nuclens; $F$, the reduced generative nuoleus completes its division; the four reduction-nuolei aro degenerating ; $G$, the two pronuclei far apart; $H$, the two pronuclei coming together ; $I$, the pronuolei fusing. After Nägler (95).

with specific differences of detail in different cases, of which the most important are, that in some cases, probably, the nucleus divides to form the gamete-nuclei, instead of becoming resolved into chromidia, and that autogamy within the cyst may occur, instead of free gametes being formed, as A. albidx (Fig. 87). According to Nägler (95), autogamy of this type is characteristic of all amœbæ of the limax-group ; in such cases only two gametenuclei are formed in the cyst, which after going through reducing divisions fuse to form a synkaryon. The zygote then leaves the cyst and begins a fresh vegetative cycle.

A different type of life-cycle is exemplified by that which Schepotieff has described in the case of a marine amoba identified by him as $A$. flava. In this case also the ordinary vegetative form is a 
uninucleate amœba, which reproduces itself by binary fission of the ordinary type; but large multinucleate forms occur which become encysted. Within the cyst the nuclei break up into chromidia, from which a great number of secondary nuclei are formed. The protoplasm becomes concentrated round the secondary nuclei to form a number of small cells, which acquire flagella and are set free from the cyst as flagellulæ, believed to be gametes and to copulate ; the zygote is at first encysted, but becomes free from the cyst, and develops into the uninucleate amœba. The life-cycle of $A$.proteus is possibly of this type, since in this species also multinucleate amœbø are commonly observed (see especially Stole; compare also Paramoba (Fig. 49).

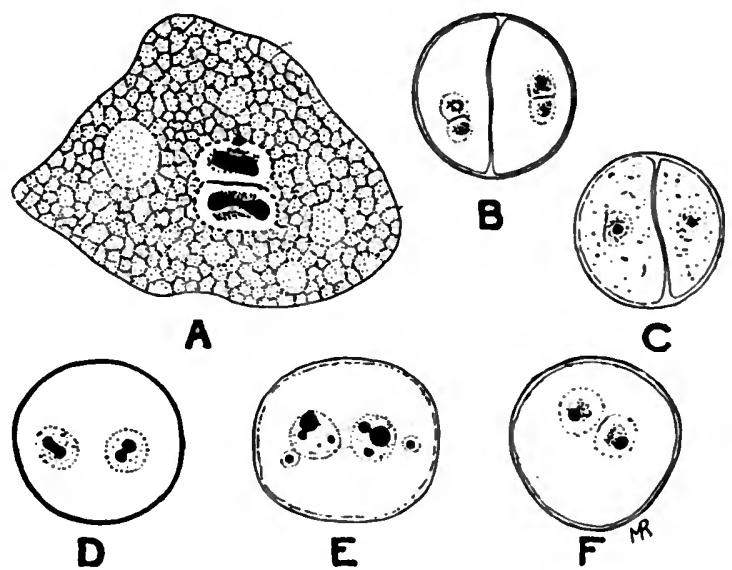

F1a. 88.-Ameba diploidea. $A$, The amoba in the vegetative condition, with its two nuclei ; $B-F$, the sexual processes within the cyst, drawn in ontline on a reduced scale; $B$, two a mobr, each with its two nuclei, encysted together, the nuolei beginning to give off chromidia ; $C$, the two nuclei of each amœba fused, numerous vegetative chromidia in the cytoplasm; $D$, the bodies of the amœbre fused, each synkaryon beginning its reduction-process; $E$, the synkarya giving off reduction-nuclei which are degenerating; $F$, the reductionprocess complete; the cyst contains a single amœba with two nuclei (synkarya), ready to emergo and begin its vegctative free life. After Nägler (95).

Metcalf (257) describes "gemmulcs" budded from small free amœbæ of the proteus-type, each gemmule becoming detached and developing into a flagellated gamete of a cercomonad type. The flagellula were observed frequently to lose their flagella and become amoboid. Copulation of two flagellulæ took place to form an amœboid zygote. Metcalf's observations upon the syngamy in this case recall strongly the observations of Jahn (294) on the sexual processes of Mycetozoa (p. 242). It is possible that the syngamy observed by him did not form a part of the lifc-cycle of the amoba, but of some other organism.

The sexual process described by Nägler (95) in Amoba diploidea is of a remarkablo kind (Fig. 88). In the ordinary vegetative condition the amœba possesses normally two nuclei, which divide simultaneously each time the 
animal reproduces itself by fission. The sexual process begins by two such amœbæ coming together and surrounding themselves with a cyst in common. Within the cyst their nuclei first give off vegetative chromidia, which are absorbed, after which the two nuclei in each separate amcba fuse together to form a single nucleus, a synkaryon. The protoplasmic bodies of the two amœbæ now fuse completely into one, after which each synkaryon goes through two reducing divisions, producing each two reduction-nuclei, of which the first may divide again, so that there may be in the cyst six reduction. nuclei altogether, which are gradually absorbed. The two persistent synkarya, after undergoing this process of reduction, approach each other, but remain separate, and the amoba is hatched out of the cyst to begin its regetative life with two nuclei representing gamete-nuclei that have undergone reduction - that is to say, pronuclei-which remain separate and multiply by fission throughout the vegetative life, and do not undergo syngamic fusion until the end of it.

In Amaba binucleata, described by Schaudinn, the vegetative phase also contains two simjlar nuclei which multiply simultaneously by division each time the animal divides; but in this case the complete life-cycle is not known.

Gwing to the practical importance of the entozoic amœbæ, and the attention that has been directed to them in consequence, their life-cycles have been more studied and are better known than those of the free-living species. According to Mercier, Entamaba blettce multiplies by binary fission in the gut of its host, and later becomes encysted, passing out of the body of its host in this condition. Within the protective cyst it breaks up by multiple fission, following repeated division of the nucleus, into a number of amobulæ, which are set free from the cyst when it is devoured accidentally by a new host. The amœbulæ are gametes which copulate after being set free, and the zygote grows into the ordinary vegetative form of the amœba. $E$. blattce thus furnishes a very characteristic and primitive type of the life-cycle of an entozoic amœba, and one which differs only in points of specific difference from that of Amoba minuta, described above.

- The question of the human entozoic amœbæ is at present in a somewhat confused state. The occurrence of amobæ in the hinder region of the human digestive tract, especially the colon, has long been known, and the name Amoba coli was given by Lösch to such organisms (synonym, Entamcba hominis, Casagrandi and Barbagallo). It is, however, certain that more than one species of amœba occurs in the human bowel, and Lösch's name must therefore be restricted to one of these.

An epoch in the study of human entozoic amœbæ was marked by the researches of Schaudinn (131), who distinguished two species. The first, to which he restricted the name Entamaba coli, occurs commonly in Europo and elsewhore as a harmless inhabitant of the intestine-that is to say, like $E$. blattoe and many others, it is not, under normal circumstances at least, a parasite in any sense of the word, but a simple scavenger, feeding on bacterial and other organisms, detritus, etc., in the colon and rectum. The second species, to which Schaudinn gave the name $E$. histolytica, ${ }^{*}$ is, on the contrary,

* Lüho has proposed to place $E$. histolytica in a separate genus, Poneramaba n. g. (Schr. Physik. Ges. Königsberg, vol. xlix., p. 421). 
a parasite of a dangerous kind, which occurs in tropical and subtropical regions, and is the pathogenic agent of amcebic dysentery and liver-absoess; it attacks and devours the tissues of the host, destroying the wall of the intestine, whence it penetrates into the blood-vessels and is carried to the liver, where it establishes itself and gives rise to liver-abscesses. These two species of amcebæe are distinguishable by structural characters. $E$. coli has a relatively fluid body, with ectoplasm feebly developed and with a fairly large spherical nucleus (or nuclei) lodged in the endoplasm. $K$. histolytica,

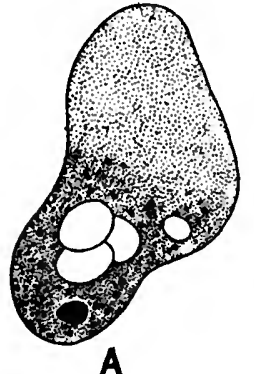

A
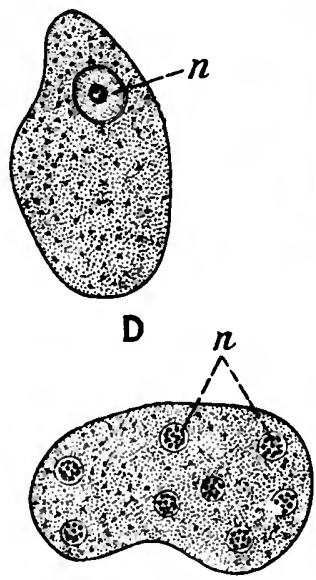

G

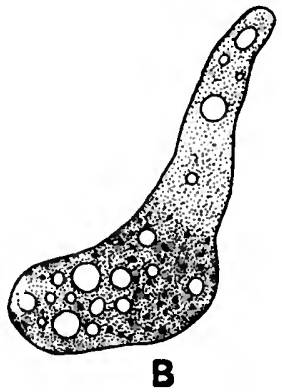

B
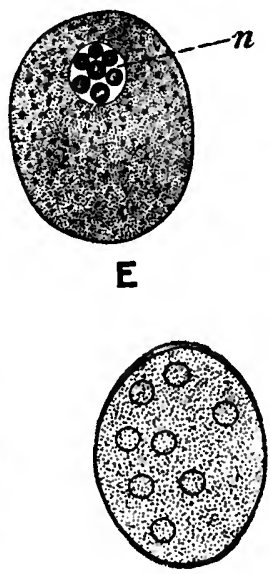

H
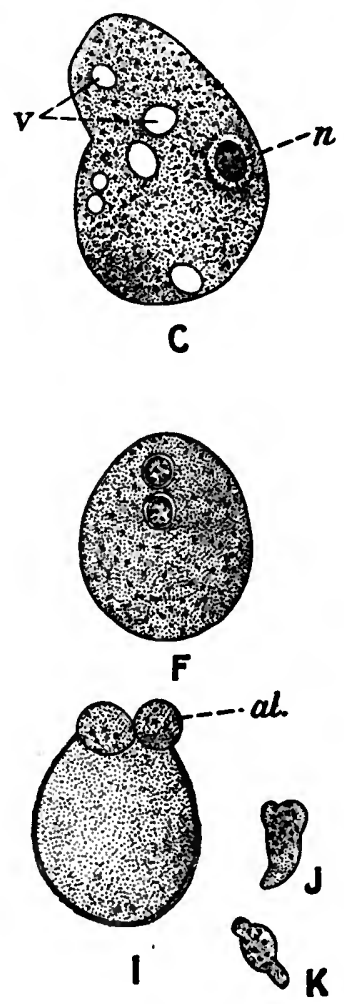

Fio. 89.-Entam ceba coli. $A$ and $B$, Living amobro showing ohanges of form and vacuolation in the endoplasm; $C, D, E$, amœbæ showing different oonditions of the nucleus $(n) ;$.$F , a specimen with two nuclei preparing for fission;$ $G$, a speoimen with eight nuolei preparing for multiple fission; $H$, an enoysted amobs containing eight nuclei; $I$, a oyst from which young amobr (al) are escaping; $J, K$, young amcom free. After Casagrandi and Barbagallo.

on the contrary, has a relatively viscid body with greatly-developed ecto. plasm, as is seen clearly in the formation of pseudopodia, which may consist entirely of ectoplasm ; it is smaller than $E$. coli, and its nucleus has a com. pressed form, stains feebly, and is lodged in, or immediately below, the superficial ectoplasmic layer. The life-cycles of these two species are also very different, as described by Schaudinn.

$E$. coli, in the amcoboid multiplicative phase, reproduces itself by binary 
fission of the ordinary type, and also by a process of multiple fission is which the nucleus divides until there are eight nuclei in the body; the characteristic 8-nucleate plasmodium then divides up into eight small amœbæ, each of which grows into an ordinary adult form. Hence it in characteristic of $E$. coli to occur in various sizes, from very small to full-grown amœbæ.

The propagative phase of $E$. coli is initiated by the formation of a gelatinous envelope round a full-sized amceba possessing a single nucleus. The nucleus then divides into two, and the process of maturation and autogamy takes place that has been described on p. 139, supra (Fig. 73). When it is complete, a tough resistant cyst is formed within the soft gelatinous envelope, and each of the two synkarya divides twice to produce four nuclei. Thus is formed the 8-nucleate resistant cyst which is characteristic, perhaps diagnostic, of this species. Within the cyst no further changes take place until it is swallowed by a new host; then it is believed that the contents of the cyst divide up into eight uninucleate amcobulæ, which are set free in the colon and are the starting-point of a now infeotion. Schaudinn was able to infect himself by swallowing the 8-nucleate cysts of the amœba.

Prowazek (A.P.K., xxii., p. 345) has described a variety of $E$. coli under the name $E$. williamsi.

$E$. histolytica reproduces itself in the am oboid phase by binary fission and by a process of gemmation in which the nucleus multiplies by division, and then small amcobulæ, each with a single nucleus, are budded off from the surface of the body. In the process of gemmation, however, the number of nuclei in the body is irregular, and not definitely eight, as in $E$. coli. In its propagative phase $E$. histolytica does not form a oyst round the whole body, but its nnoleus beoomes resolved into chromidia, which collect in patohes near the surface of the body. Little buds are then formed as outgrowths of the body, each bud oontaining a clump of chromidis. Round each bud a sporocyst is formed of so tough and impervious a character that no further cytological study of the

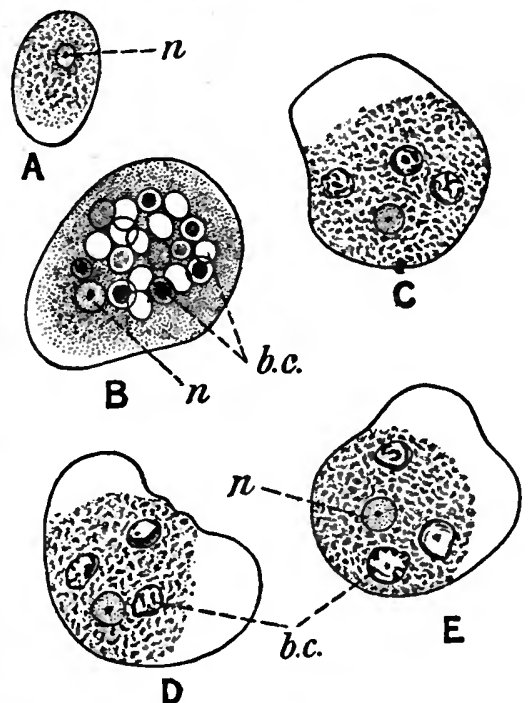

Fia. 90.-Entamobahistolytica. A, Young specimen; $B$, an older spenimen crammed with ingested blood-corpuscles; $C, D, E$, three figures of a living amoobs which oontains a nucleus and three bloodcorpuscles, to show tho ohanges of form and the ectoplasmio pseudopodia : $n$., nucleus; b.c., blood-corpuscles. After Jürgens.

bud is possible. The resistant spores formed in this way separate from the body, of which the greater part remains as residual protoplasm and dies off. The minute spores are the means of infecting a new kosi, as shown by Schaudinn in experiments on cats, which are particularly susceptible to the attacks of this amœba.

Sohandinn's investigations, of which a brief summary has been given in the forogoing paragraphs, first introduced clear ideas into the problem of the human entozoio amœbæ. Many of the works of subsequent investigators have tended, however, rather to confuse and perplex the question, for various reasons. In the first place, in oultures made from human fæces, free-living. non-parasitic species of amcbo make their appearance, which have passed through the digestive tract in an encysted condition, and emerge from their 
cysts in the cultures; $*$ such amcebæ, for the most part of the limax-type, have been confused with the true entozoic amceba, and have given rise to erroneous ideas. Secondly, it is certain now that the two species of amcbo recognized by Schaudinn does not exhaust the list of human entozoic amobæ. Thirdly, it is possible that Schaudinn did not see the entire life-cycle of the forms studied by him, or that in some cases he confused stages of different specics in the same life-cycle (compare Hartmann, 247).

It is still doubtful how many species of entozoic amœbæ occur in man. Hartmann recognizes two dysenteric amœbæ, in addition to the harmless $E$. coli : $E$. histolytica, Schaudinn, and $E$. tetragena, Viereck (synonym, $E$. africana, Hartmann). E. tetragena has been described from various parts of the tropics; it differs from $E$. histolytica in its characters, and more nearly resembles $E$. coli, but is distinguished by the formation of resistant cysts containing four nuclei. In addition to these species, many others have been described by various investigators-for example, $E$. minuta, Elmassian, which, according to Hartmann, is merely a variety of $E$. coli. A summary of the various amœbæ described from the human intestine is given by Doflein (7) and Fantham (241). In Cochin China, Noc obtained from liverabscesses and dysenteric stools a small amcoba (not named) which in the multiplicative phase reproduces in two ways : by binary fission of the ordinary type; and by budding off small amœbulæ containing secondary nuolei formed from chromidia. In the propagative phase Noc's amœba encysts and breaks up into amcebulæ. Greig and Wells, in Bombay, obtained results very similar to those of Noc. In cultures from liver-abscesses from Bombay, Liston found two distinct forms of amœbæ-a larger form contsining a single nucleus and numerous chromidia, and a smaller form containing a nucleus only. The larger amœba multiplies either by binary fission, with karyokinesis of the nucleus and partition of the chromidia ; or by the formation of endogenous buds containing chromidia from which a secondary nucleus is formed, the bud being finally set free as a small amœba with a nucleus and a number of chromidia. The small amœba multiples only by binary fission, preceded by amitotic division of the nucleus. Both large and small amoebre form resting cysts, in which, however, they remain unchanged, and from which they emerge when circumstances are favourable. It is evident that much of the iife-cyole of these liver-abscess amœbæ remains to be worked out.

From the foregoing it is clear that, with regard to the human pathogenic amcbæ, many important problems remain to be investigated, especially as regards their specific distinctions, distribution, and life-history. Much recent work has been carried on by culture-methods, with valuable results, which, however, should be interpreted with caution, since it remains to be ascertained whether the forms and phases assumed by ihese organisms in cultures are identical in character with those which they exhibit under natural conditions; and until this point has been cleared up it is not safe to describe the characters of a species of an amoba, any more than of a trypanosome, from cultural forms alone.

With regard to the life-cycle of the pathogenic amcbæ, it is most important to discover what are the phases of development or conditions of life under which they occur outside the human body; whether they exist only in an encysted, resting condition, or in an active state also; and, in the latter case, whether as free-living organisms or within some other host. On general grounds it is unlikely that an organism adapted to an entozoic life should be capable also of living free in Nature, and it is more probable that the pathogenic amœbæoutside tho human body occur only in the condition of resting cysts or spores, which produce infection through being accidentally swallowed with food or water (compare Walker, $276^{\circ}$ ). In that case، unfiltered water, uncooked vegetables

* Whether this also applies to oultures made from the pus of liver-abscesses, as asserted by Whitmore (279) and Hartmann (247), may well be doubted; it is not easy to understand how an encysted amoba could be transported passively from the intestine into a liver-absoess. 
and herbs, or fruit that grows near the ground, are likely sources of infection by becoming contaminated with the resting stage of the amœbæ scattered on the ground or in manure. In this connection the further question arises whether the human entozoic amœbæ are specific parasites of man or not, and consequently whether their infective stages would be derived only from human fæces, or trom the excrets of other animals also. From general considerations of parasitism in Protozoa, it seems probable that the harmless $E$. coli is a specific parasite of man, but that the pathogenic forms are parasites of other animals also, and perhaps only occasionally find their way into the human body; in which case garden-manure might be a fruitful source of contamination, through the medium of vegetables habitually eaten uncooked, such as lottuce, celery, etc. None of these questions can be answered decisively at present, however, and there is a wide field of investigation open.

Greig and Wells found that in Bombay amœbic infection shows a marked seasonal variatiou, closely associated with variations in humidity, but not corresponding with those of temperature, and reaching its maximum in August.

In addition to the various species of $A m o b a$ and of allied genera and subgenera, a number of other genera are included in the section under consideration, for an account of which the reader must be referred to the larger treatises; but two deserve special mention-namely, the genera Pelomyxa and Paramaba.

The species of Pelomyxa (Fig. 91) aro freshwater amœbæ of large size and "sapropelic" habit of life (p. 14). The body, which may be several millimetres in diamotor, is a plasmodium in the adult condition, containing some hundreds of nuclei ; it is generally very opaque, owing to tho animal having the habit of loading its cytoplasm with sand and débris of all kinds, in addition to food in the form chiefly of diatoms. The pscudopodia are of the lobose type, blunt and rounded, but tho animal may also form slender retioulose pseudopodia under certain conditions (Veley). The cytoplasm is very vacuolated, and contains a number of peculiar refringent bodies ("Glanzkörper") of spherical form, with an cnvclope in which bacterial organisms (Cladothrix pelomyxa, Veley) occur constantly. The bacteria multiply by fission in a linear series in tho form of jointed rods, which may branch; as a rule they have five or six jonits, or less, but at least two. The refringent bodies aro of albuminous nature (Veley). According to Gold. schmidt (57), the refringent bodies ariso from the nuclei when they give off chromidia; in this

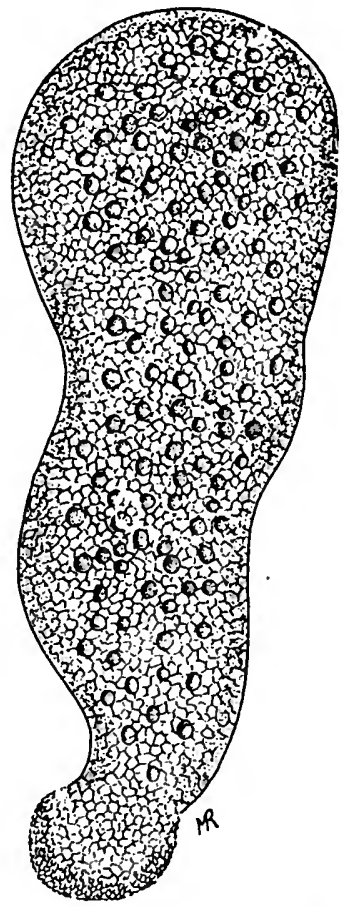

Fia. 91.-Pelomyxa palustris: a specimen in which the body is transparent owing to the absence of food-particles and foreign bodies, showing the vacuolated oytoplasm and the numerous nuclci and refringent bodies (tha refringent bodies aro for the most part larger than the nuclei) in the living condition. After Greeff, magnified 60 . process the chromatin is given off into tho cytoplasm, and the plastin-basis of the karyosomo is loft as a spherical mass which becomes tho refringent body. At first the plastin-sphero is surrounded by the remains of the nuclear membranc, which disappears, and the refringent body grows in size. Re. fringent bodics, with their bactcria, are seen frequently to be cjected by the animal during life. Bott (103), on the other hand, states that the refringent bodies are reserve food-stuff, their contents of tho nature of glycogen, and 
that they arise in the cytoplasm independently of the nuclei; but their rejection by the animal is more in favour of the view that they are wasteproducts of the metabolism (Veley). It is not clear what is the rôlc of the bacteria, whether they are parasites or symbionts.

Pelomyxa reproduces itself by simple fission or by formation of gametes. The sexual process, according to Bott, begins with extrusion of chromatin from the nuclei into the cytoplasm to form chıomidia, which may take place so actively that sometimes the nuclei break up altogother. A similar extrusion of chromidia may take place as a purely regulative process under ccrtain conditions, such as starvation; -but the vegetative chromidia formed in this way, and absorbed ultimately in the cytoplasm, must be distinguished from

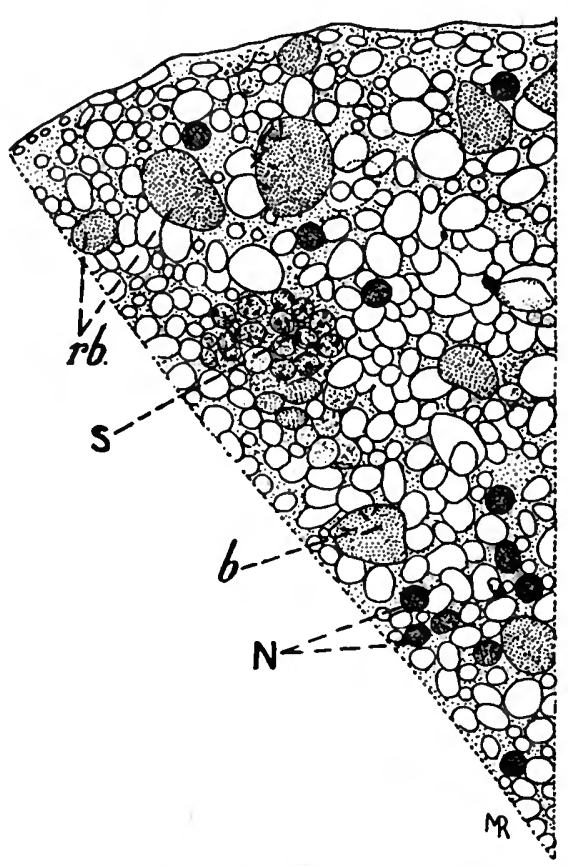

Fio. 92.- Portion of a section through the body of Pelomyxa. N., Nucleus ; r.b., refringent bodies; $b$., bacteria on the refringent bodies; s., sand and débris in the protoplasm. After Gould. this phase the amoba multiplies by binary fission accompanied by division both of nucleus and Nebenkern. It also becomes encysted and goes through a process of multiple fission, which shows three stages; in the first the Nebenkern multiplies by repeated division, the nucleus remaining unchanged; in the second the nucleus divides repeatedly to form as many small nuclei as there are Nebenkerne present, and each nucleus attaches itself to a Nebenkern ; in the third the protoplasmic body undergoes radial super. ficial cleavage into a number of cells, each containing a nucleus and a Nebenkern. Each of the cclls thus formed becomes a swarm-spore with two flagella.

* The amceba from the human intestine described by Craig under the name Paramceba hominis certainly does not belong to this genus. See Doflein (7), pp. $602,603$. 
The swarm-spores are liberated from the cyst and live freely, feeding and multiplying by binary fission, in whith the nucleus divides by mitosis and the Nebenkern acts like a centrosome. After a time, however, the swarm-spores lose their flagella, and become amœbulæ which develop into the adult phase. Syngamy was not observed, but probably takes place between the flagellulæ. Two new parasitic species of Paramceba havo been described recently by Janicki (71·5) ; see p. 95.

To the order Amœbæa should be referred, probably, the parasite of the Malpighian tubules of the rat-flea (Ceratophyllus fasciatus), described by Minchin under the name Malpighiella refringens, and the parasite of Ptychodera minuta, described by Su: under the name Protoentospora ptychodera.

The section Lobosa Testacea or Thecamœbæ contains a number of free-living forms familiar to every microscopist, such as the genera Difflugia (Fig. 16), Centropyxis, Arcella (Fig. 32), etc. The majority of these forms inhabit fresh water, but Trichosphorium (Fig. 81) is marine. Their common distinctive feature, in addition to the possession of lobose pseudopodia, is the formation of a shell or house into which they can be withdrawn entirely. The shell may be secreted by the animal, and then is chitinous (Arcella) or gelatinous (Trichospharium), or may be made up of various foreign bodies cemented together (Diffugia). Typically the house has the form of a chamber with a single large opening, through which the pseudopodia are extruded at one pole.

When the animal multiplies by fission, the protoplasm streams out through the aperture, and forms a daughter-shell external to the old one, after which division of the nucleus takes place and the two sister-individuals separate. In Trichospharium, however, the house has the form of a gelatinous investment to the body, with several apertures through which pseudopodia protrude, and when the animal divides the investing envelop divides with it.

The protoplesmic body contains typically one nucleus-sometimes more than one-surrounded by a ring of chromidia. In Arcella vulgaris there are constantly two primary nuclei; in Difflugia urceolata, from ten to thirty. Trichosphoerium possesses many nuclei, but no chromidia.

The life-cycle, so far as is known, is of various types; those of Arcella and Trichosphorium are described above (p. 177, Fig. 80, and p. 181, Fig. 81). The latter, with an alternation of generations combined with dimorphism in the adult condition, approaches that of the Foraminifera in character.

In the testaceous amcebæ the method of division varies in accordance with the nature of the shell. In those in which the shell is soft and yielding, as, for example, Cochliopodium and Cryptodiffugia, the division is longitudinal -i.e., in a plane which includes the axis passing through the mouth and apex of the shell (Doflein, 239); in Cryptodiffugia rapid division of this kind may lead to colony-formation. In forms with a rigid shell, on the other hand, such as Diffugia, Arcella, Centropyxis, otc., the shell sets a limit to the growth of the animal, which, when it has filled the shell, ceases to grow for a while 
and stores up reserve-material. Prior to division a sudden and rapid growth takes place at the expense of the reserve-material and by absorption of water; as a result the protoplasm grows out of the shell-mouth, a daughter-shell is formed, and the animal divides transversely (Fig. 50).

In Difflugia urceolata, Zuelzer (85) has described a process of chmmidiogamy. Two animals come together with the mouths of the shells in contact, and tho entire contents of one shell flow over into the other, the empty shell being asst off. The chromidia of the two animals fuse into a single mass; the nuclei, however, remain separate. Copulation of this kind is a preliminary to encystment, which takes place in Nature at the end of October or the beginning of November. Prior to encystment the pseudopodia are retracted, all foreign bodies, food-remains, excreta, etc., are cast out, and the protoplasmic body rounds itself off in the shell, and diminishes to about a quarter of its former volume, becoming denser and more refractile. Tho cystmemlerane is then secreted at the surface of the body. The old nuclei are gradually absorbed, and new nuclei are formed from the chromidial mass. The reconstitution of the nuclear apparatus takes place from January to April ; in the spring the cyst is dissolved, and the rejuvenated Diffugia begins to feed and to enter upon a summer course of vegetative growth and repro. duction. In a recent note $(86$, p. 191 , footnote) Zuelzer states that conjugation between free gametes also occurs in this species:

In Centropyxis aculeata, according to Schaudinn (131), the ordinary vegetative reproduction is by fission, the new shell that is formed being larger than the old one, until the maximum size is reached. Sexual processes are initiated by degeneration of the primary nucleus, which is single is this species. Then the protoplasm with the chromidia creeps out of the shell, and divides into a number of amœbulæ, each containing chromidis whioh condense into a single nucleus. Some amœbulæ form a shell at once; others before doing so divide into four smaller amœbulæ, and then form a shell. The larger are macrogametes, the smaller the microgametes; they copulate and abandon their shells. The zygote forms a new shell, chromidia appear, and a fresh vcgetative cycle is started.

In a species of the genus Cryptodiffugia ("Allogromia") a remarkable type of life-cycle has been described by Prandtl (265); see also Doflein (7.), p. 310, Fig. 283. In this form also the organism, at the time of gameteformation, quits its shell and penetrates into some other Protozoan organism, such as Amaba proteus, in the body of which it becomes parasitic and goes through the process of gamete-formation. The nucleus bresks up into chromidia, from which secondary nuclei are formed, producing a multinucleate plasmodium which multiplies by plasmotomy until the host is full of them. Ultimately the plasmodia break up into uninucleate cells, the gametes, which are set free and copulate. The zygote becomes a flagellated Bodo-like organism, with two flagella, one directed forward, the other backward as a trailing flagellum (p. 270, infra); it feeds and multiplies in this form for - coveral generations in the free state, bnt ultimately it loses its flagella, becomes amœboid, forms a shell, and develops into an adult Cryptodiffugia. Noteworthy in this development are the alternation of generations between the flagellated and the amcoboid phase, as in Pseudospora (p. 218), and the parasitism in the gamete-forming phases; if, however, the Cryptodiffugia does not succed in finding a suitable host, the gamete-formation may take place in the free state.

From the life-cycles and sexual processes of Arcella, Diffugia, Centropyxis, etc., it is seen that the primary nuclei of all these forms are vegetative in nature, while the chromidia give rise to the gamete-nuclei, and consist of, or at least. contain, the generative chromatin. The marine Trichospharium, however, stands apart from the fresh-water genera in regard to its structure, sexual processes, and life-cycle, in all of which it shows more similarity to the Foraminifera. 


\section{Foraminifera.}

General Characters-Shell-Structure.-The characteristic features of this group are the possession of reticulose pseudopodia and of a shell or test. The Foraminifera are typically creeping forms, moving slowly, and using their net-like pseudopodia chiefly for foodcapture. Certain genera, however, such as Globigerina, have taken secondarily to a pelagic existence, and float on the surface of the ocean, spreading their nets in all directions around them. On the other hand, some forms have adopted a sedentary life, attaching themselves firmly to some object. An example is seen in the genus Haliphysema (Fig. 17), once believed to be a sponge, and in the remarkable genus Polytrema and allied forms, recently monographed by Hickson (282)-organisms which in many cases have a striking and deceptive resemblance to corals.

The test may be secreted by the animal itself, and then is usually either chitinous or calcareous, rarely siliceous or gelatinous (Myxotheca) ; or it may be made up of foreign bodies cemented together, as in Haliphysema (Fig. 17), and is termed generally " arenaceous," but the materials used may be of various kinds, and the organism sometimes exhibits a remarkable power of selection (see p. 34, supra).

The typical form of the shell, as in the Amœbra Testacea, is a chamber with a wide aperture-sometimes more than onethrough which the pseudopodia are extruded, as in Gromia (Fig. 21). In addition to the principal aperture, the wall of the shell may be perforated by numerous fine pores, through which also the protoplasm can stream out to the exterior. Hence the shells of Foraminifera are distinguished primarily as perforate and imperforate, the former with, the latter without, fine pores in addition to the principal opening.

Whether perforate or imperforate, the shell remains a single chamber in the simple forms, as in the Amœbæa Testacea. In some cases, when the animal reproduces itself by binary fission, the protoplasm streams out through the principal aperture to give rise to the body of the daughter-individual, which forms a shell for itself, and, when the division is complete, separates completely from the mother, which retains the old shell. Division of this type is seen in Euglypha (Fig. 59). But in many species, when the animal outgrows its original single-chambered shell, the protoplasm flows out and forms another chamber, which, however, is not separated off as a distinct individual, but remains continuous with the old shell, so that the animal, instead of reproducing itself by fission, remains a single individual with a two-chambered shell. By further growth, third, fourth, ...nth chambers are formed successively, each newlyformed chamber being, as a rule, slightly larger than that formed 
just before. Hence a distinction must be drawn between monothalamous or single-chambered shells and polythalamous shells, made up of many chambers formed successively. In the latter type the new chambers may be joined in various ways to the old,

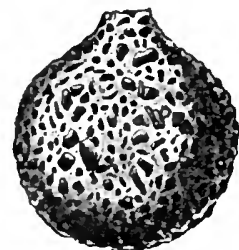

1.Saccammina

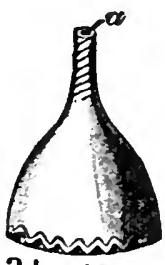

2.Lagena

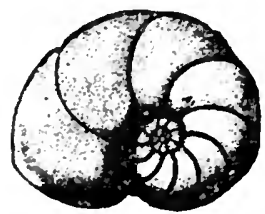

7. Discorbina

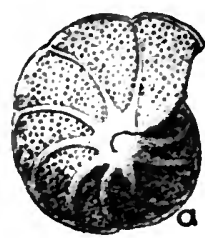

9.Planorbulina

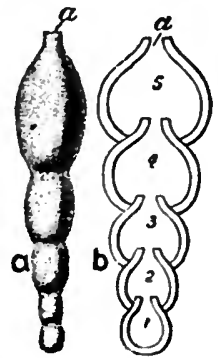

3. Nodosaria

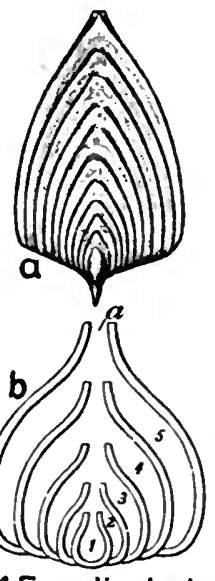

4.Frondicularia
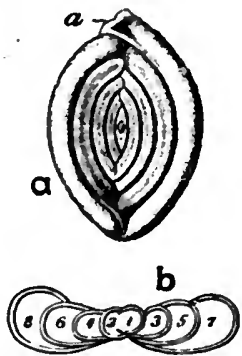

5. Spiroloculino

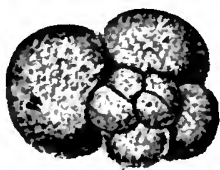

6. Clobigerina
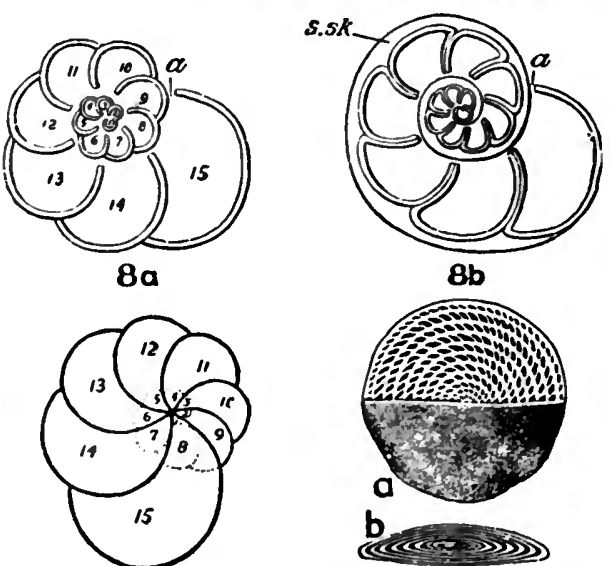

11 Nummulites

Fia. 93.-Shells of varions genera of Foraminifera. In 3, 4, and 5, $a$ shows the surface-view, and $b$ a section; $8 a$ is a diagram of a coiled shell without supplemental skeleton; $8 b$, of a similar form with sujplemental skeleton (s.sk.); 10 , of a form with overlapping whorls; in $11 a$ half the shell is shown in horizontal section; $b$ is a vertical section. In all the figures $a$ marks the aperture of the shell ; 1 to 15 , the successive chambers, 1 being always the oldest or initial chamber. From Parker and Haswell.

producing usually either a linear or a spiral series, and the utmost variety of shape and pattern results in different species (Fig. 93). Some polythalamous species exhibit a peculiar dimorphism (Fig. 94) 
in some individuals, hence termed microspheric, the initial chamber of the shell is smaller than in others, which are known as megalospheric. This point will be discussed further under the reproduction.

It may be noted that if, in this order, a species were to form no shell, whether from having secondarily lost the habit or as a primitive form which had never acquired it, then such a species would be classed in the order Amobæa Reticulosa. It is very probable that many of the large marine "Proteomyxa" are allied to the true

$a$
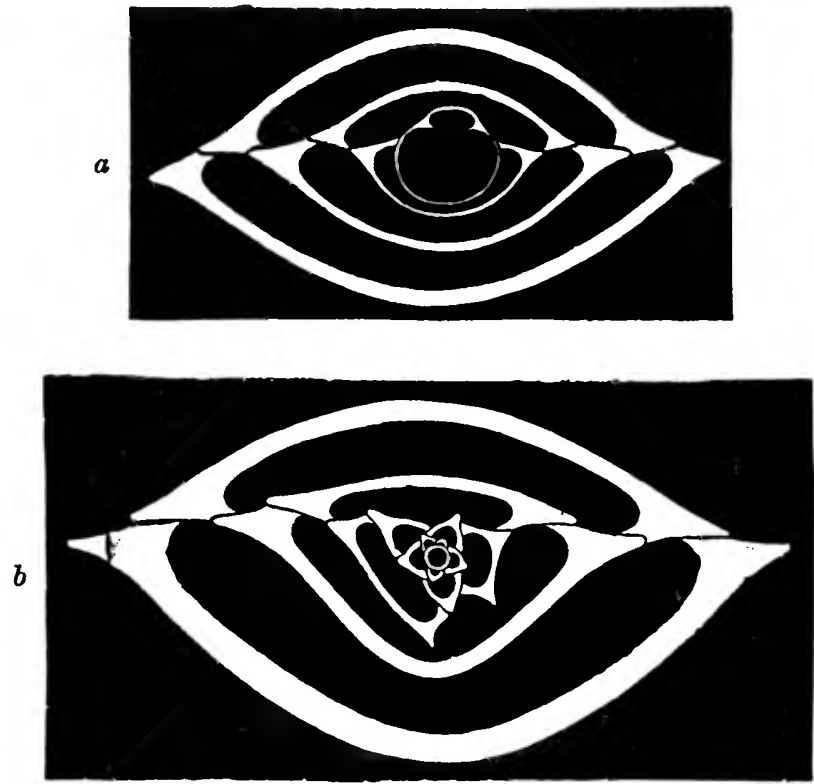

Fia. 94.-Biloculina depressa: transverse sections of $(a)$ the megalospheric form, magnified 50 diameters, and $(b)$ the microspheric form, magnified 90 diameters. After Schlumberger, from Lister.

Foraminifera, as forms either primitively or secondarily without a test; and Rhumbler unites the Foraminifera proper with the naked forms in the section Reticulosa.

The body-protoplasn exhibits no marked distinction of ectoplasm and endoplasm. Contractile vacuoles are present in some of the fresh-water genera, but are not found in marine forms. The protoplasm contains metaplastic bodies of various kinds, and may become loaded with fæoal matter in the form of masses of brown granules, termed by Schaudinn the " stercome " (compare also Awerinzew, 281). Periodically a process of defæcation takes place, whereby the protoplasm is cloured of these accumulations, often as a prelude to the formation of a new chamber (Winter, 28). The 
nuclear apparatus varies in different forms, even in the same species, as will be seen in the description of the reproductive processes.

The marine Foraminifera, so far as they have been investigated, show a well-marked alternation of generations in their life-history,
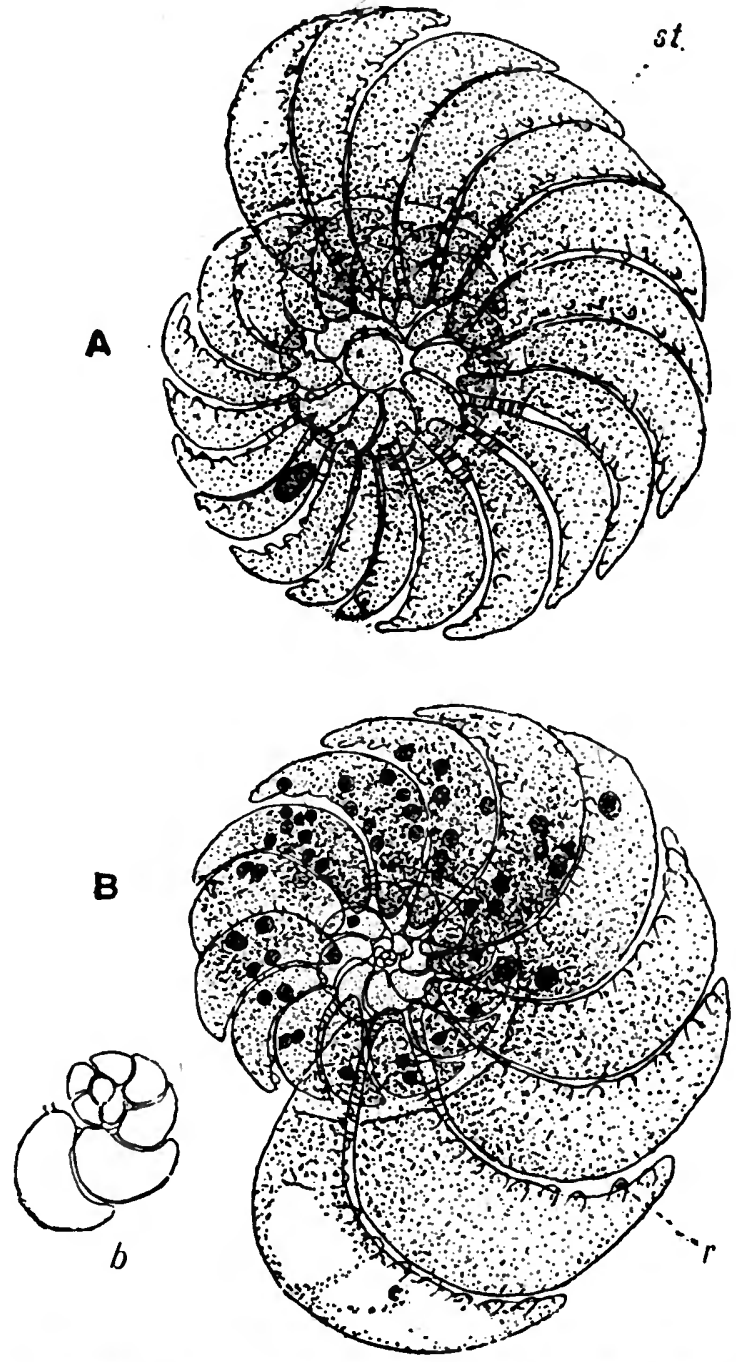

Fic. 95.-Polystomella crispa: desalcified speoimens to show the struoturo of the two forms. $A$, The megalospherio type; $B$, the microspherio type: $b$, the central chambers of the latter more highly magnified; r., retral processes ; st, communications between the chambers. From Listor.

combined with dimorphism in the adult condition. An example is Polystomella, which has been investigated by Lister (285) and 
Schaudinn (131); their results have been confirmed in the case of Peneroplis by Winter, who gives a useful combined diagram of the life-history (28, p. 16, text-fig. A). The microspheric form (Fig. 95, B.) has many nuclei, which multiply by fission as the animal grows, and which also give off chromidia into the bodyprotoplasm. When reproduction begins, the nuclei become resolved entirely into chromidia, and the protoplasm streams out of the shell, which is abandoned altogether. Secondary nuclei are formed from the chromidia, and the protoplasmic mass divides up into a swarm of about 200 amœbulæ (Fig. 96). Each amobula contains a nucleus and chromidia, and secretes a single-chambered shell, which is the initial chamber of a megalospheric individual. The amœbulæ separate, ar $\mathrm{d}$ each one feeds, grows, forms new chambers successively, and becomes a megalospheric adult. Thus the microspheric form is seen to be an agamont or schizont, which gives rise by a process of schizogony or multiple fission to agametes (amœbulæ). The megalospheric form, when full grown, has a single large nucleus and numerous chromidia (Fig. 95, $A$ ). The nucleus is that of the amœbula which was the initial stage in the development of this form ; as it grows the nucleus passes from chamber to chamber, and at the same time gives off chromidia into the cytoplasm. Finally the primary nucleus is resolved entirely into chromidia, from which a great number of secondary nuclei are formed. Round each such nucleus the protoplasm becomes concentrated to form a small cell, which may be termed a gametocyte. By two divisions of the nucleus and cell-body of the gametocyte four gametes are formed, each of which acquires two flagella, and is set free as a biflagellate swarm-spore. In Peneroplis, however, the gametes have a single flagellum, and in Allogromia ovoidea the gametes are amœbulæ (Swarczewsky). Gametes produced by different individuals copulate, losing their flagella in the process, and the zygote secretes a minute single-chambered shell, and thus becomes the starting-point of the growth of a microspheric individual.

From the foregoing it is seen that the megalospheric form is the gamont, which by multiple fission produces the gametocytes, and ultimately the gametes. Thus, if $m$. represents the microspheric form and $M$. the megalospheric, $a m$. the amœbulæ (agametes), and $f$. the flagellulæ (gametes), the life-cycle may be represented thus:

$$
\text { m. }-a m .-M .-(f .+f l .)-m .-a m . \text {. . }
$$

In some cases, however, the life-cycle does not present a regular alternation of sexual and non-sexual generations, but a number of non-sexual generations may tako place before a sexual generation intervenes; that is to say, tho megalospheric forms may produce agametes and other megalospheric forms again for several generations, before gametes are produced and the sexusl processes occur. Then the life-cycle may be representod thus :

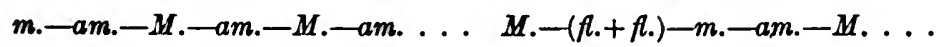



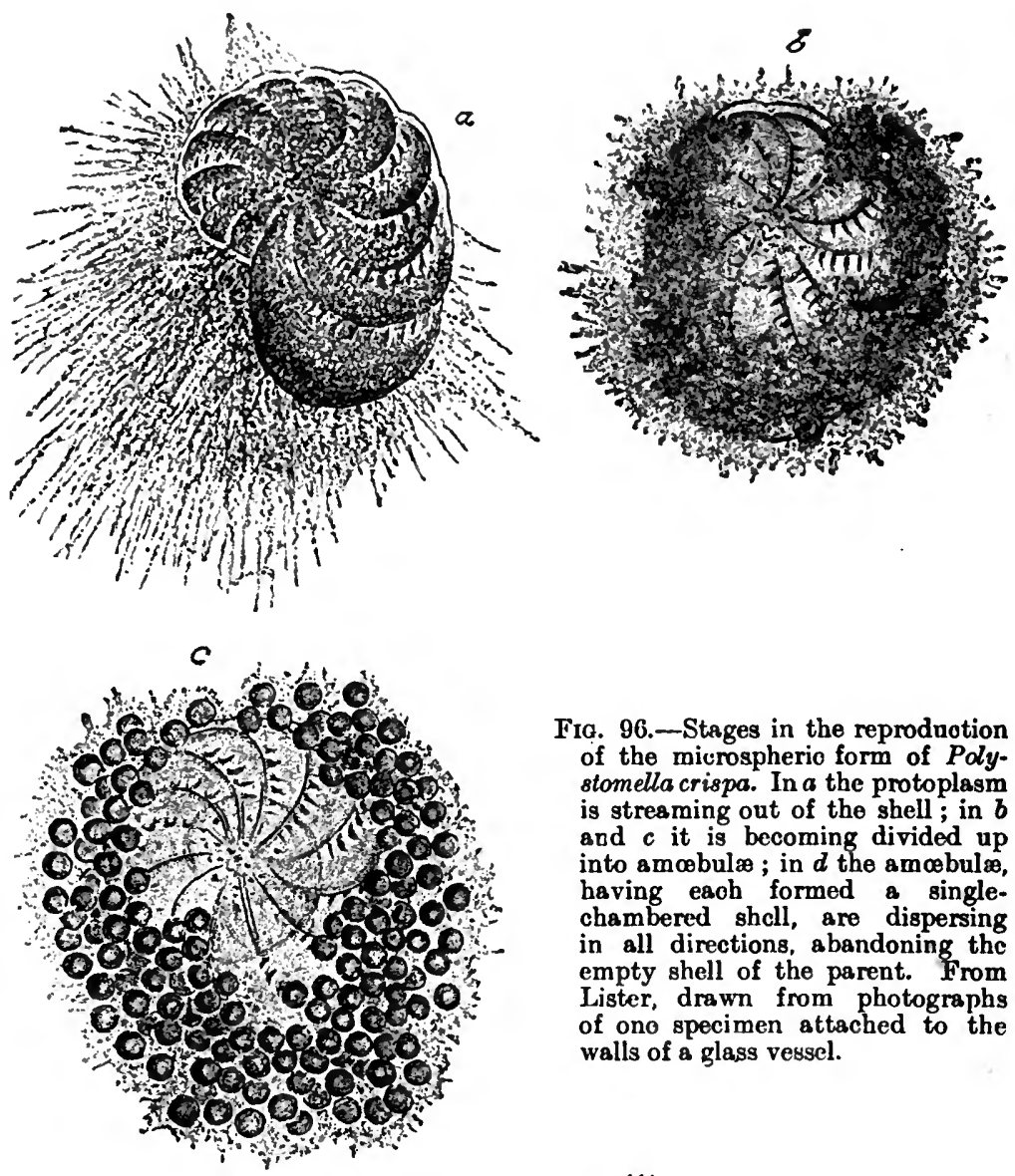

Fig. 96.-Stages in the reprodnotion of the microspheric form of $P d y$. stomella crispa. In $a$ the protoplasm is stresming out of the shell ; in $b$ and $c$ it is becoming divided up into amcobulæ; in $d$ the amœbulæ, having each formed a singlechambered shcll, are dispersing in all directions, abandoning the empty shell of the parent. From Lister, drawn from photographs of ono specimen attached to the walls of a glass vessel.

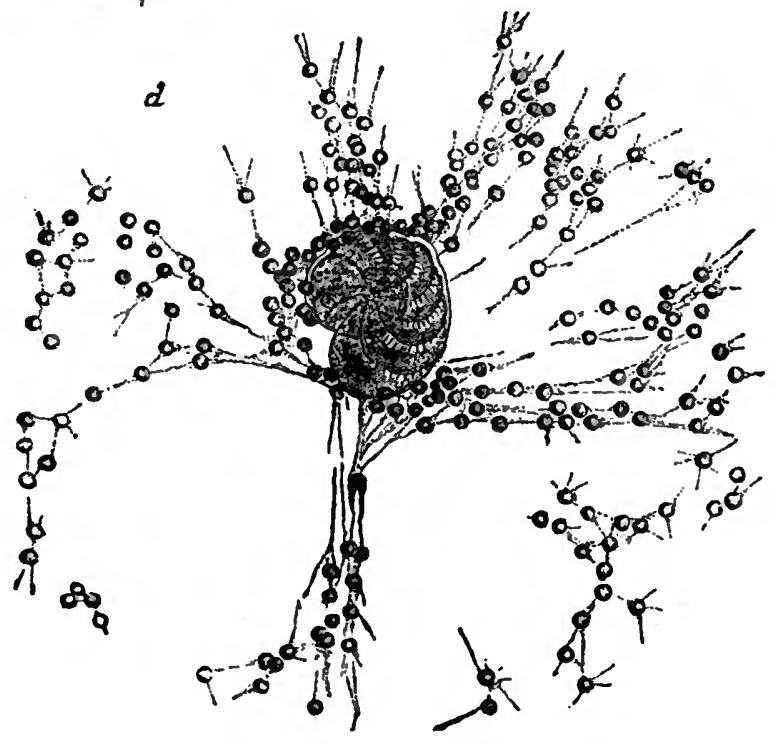


Hence the dimorphism of the adults is due to their parentage, and is not necessarily related to the manner in which they reproduce. A microspheric form is produced sexually, and is always an agamont; a megalospheric form is produced non-sexually, and may be either a gamont or an agamont.

Very little is known of the life-cycle of the non-marine genera. The only form of which the cycle is known with any approach to completeness is Chlamydophrys stercorea, the only entozoic member of the order, which is found in the fæces of various vertebrates; a second species, $C$. schaudinni, is distinguished by Schüssler (A.P.K., xxii., p. 366). The adult form has a chitinous single-chambered shell, and its protoplasm contains a single nucleus and a ring of chromidia. It reproduces itself vegetatively by binary fission, and also by multiple fission producing gametes. In the gamete-formation, according to Schaudinn (131), the nucleus is ejccted from the shell together with all foreign bodies, food-particles, etc. In the shell is left a small quantity of protoplasm containing the chromidia, from which about eight secondary nuclei are formed, and then the protoplasm concentrates round each nucleus and divides up into as many cells, the gametes, each of which becomes a biflagellate swarm-spore, and is sot free. The gametes copulate and the zygote encysts. In order to develop further, the cyst must be swallowed by a suitable host and pass through its digestive tract. If this happens, the cyst germinates in the hind-gut, setting free an amcbula which forms a shell and becomes a young Chlamydophrys, living as a harmless inhabitant of the hindgut, and feeding on various organisms or waste products occurring there; bnt according to Schaudinn it may, under circumstances not yet defined or explained, pass from the digestive tract into the peritoneal cavity, and multiply there as an amoboid form without a shell, thus giving rise to the organism described by Leyden and Schaudinn, from ascites-fluid, under the name Leydenia gemmipara.

The Foraminifera as a group comprise a vast number of genera and species, both recent and fossil, for an account of which the reader must be referred to the larger works. They are classified by Lister (286) into ten orders (suborders ?), containing in all thirtytwo families; Rhumbler (288) recognizes ten families in all. The vast majority are marine, but some of the simpler forms, such as Euglypha, are found in fresh water, and car scarcely be separated from the Lobosa except by the characters of their pseudopodia, a feature upon which great weight cannot be laid as an indication of affinity. Until the life-histories of these simpler forms have been studied, their true systematic position must be considered as somewhat uncertain. But the affinities of such genera as Euglypha and Chlamydophrys would seem to be with the Lobosa Testacea rather than with the Foraminifera.

\section{XENOPHYOPHORA.}

This group was founded by F. E. Schulze (290) for a number of curious organisms of deep-sea habitat, the zoological position of which was a matter of dispute. By Haeckel they were believed to be sponges allied to Keratosa; such as Spongellidae, horny sponges which load the spongin-fibres of the skeleton with foreign bodies of various kinds. Schulzc established definitely the ir relationship to 
the Rhizopoda by showing that the soft body was a plasmodium containing numerous nuclei and chromidia, and forming a pseudopodial network, but with no cell-differentiation or tissueformation.

The body consists principally of a network of hollow tubes in which the plasmodium is contained. The wall of the tubes consists of a hyaline organic substance resembling spongin. In the interspaces between the tubes great numbers of foreign bodies (" xenophya," Haeckel) are deposited, such as sand-grains, spongespicules, Radiolarian skeletons, and so forth. In one family (Stannomida) the xenophya are held together by a system of threads, "linellæ," in the form of smooth, refringent filaments, approximately cylindrical, which pass from one foreign body to another, and are attached to them by trumpet-like expansions of their ends. The substance of the linellæ is doubly refractile and allied to spongin in its chem:cal nature. Schulze compares them to the capillitium of the Mycetozoa (see p. 241, infra).

The protoplasmic body within the tubes contains, in addition to nuclei and chromidia, enclosures of various kinds. Many tubes, distinguished by the darker colour of their walls, contain quantities of brown masses, apparently of fæcal nature, and comparable to the stercome of the Foraminifera (p. 233). In other tubes, lighter in colour, there are found small, oval, strongly-refractile granules, or "granellæ," which consist chiefly of barium sulphate. Schulze terms the system of stercome-containing tubes the "stercomarium," and those that contain granellæ the "granellacium." The tubes of each system are distinguishable by their mode of branching, as well as by their colour and contents. In the tubes of the granellarium the protoplasmic bodies are often found to contain isolated cells or groups of cells, each with a single nucleus, which are probably stages in the formation of swarm-spores. Hence the stercomarium probably represents the purely vegetative part of the body, in which the waste products of metabolism are deposited, while the granellarium is a differentiated region of the plasmodium in which the reproductive elements are produced.

Nothing is known of the actual life-cycle of these organisms, but from the appearances already described, seen in preserved specimens, Schulze conjectures that they reproduce by formation of swarm-spores, much as is known to take place in the Foraminifera.

The affinities of the Xenophyophora are seen to be with the Foraminifera. In their habit of forming a skeleton of foreign bodies they resemble the arenaceous Foraminifera, in which, however, the foreign bodies build up the house which directly encloses the soft body, while in the Xenophyophora the soft body is en- 
closed actually within the system of tubes. Nothing similar to the linello is known in any Foraminifera.

For the classification of the Xenophyophora and their genera see Schulze (290).

\section{MYcetozos.}

The Mycetozoa are a group of semi-terrestrial Rhizopods occurring in various sutuations, especially on dead wood or decaying vegetable matter of various kinds. Their most characteristic features are the formation of plasmodia, which represent the adult, vegetative phase of the life-history, and their method of reproduction, consisting in the formation of resistant spores very similar to those of fungi. The Mycetozoa were originally classified amongst the Fungi as a group under the name Myxomycetes, but the investigations of de Bary first made clear their Rhizopod affinities.

The life-history of a typical member of this group exhibits a succession of phases, the description of which may conveniently begin with the spore. Each spore is a spherical cell with a single nucleus, enclosed in a tough protective envelope which enables it to resist desiccation. It may be dormant for a considerable period, and germinates when placed in water. The envelope bursts, and the contained cell creeps out as an amcbula with a single nucleus (Fig. 97), the so-called "myxamœba." After a time the amœbula develops a flagellum, and becomes a flagellula or zoospore ("myxoflagellate"), which feeds and multiplies by fission. The flagellula (Fig. 98) retains its amoboid

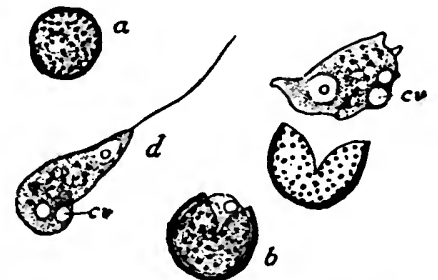

Fro. 97.-The hatching of a spore of Fuligo seplica. a, Spore; $b$, $c$, contents emerging and undergoing amcoboid movements prior to the assumption of the flagellula-stage; $d$, flagellula. c.v., Contractile vacuole. After Lister, magnified 1,100 .

form, and sometimes also the amoboid method of locomotion, the flagellum appearing to act as a tactile organ. It captures bacteria and other organisms by means of its pstudopodia, nourishing itself in a holozoic, perhaps also in a saprophytic, manner. It also may become temporarily encysted.

The flagellate phase is succeeded by a second amcboid stage, the flagellum being lost. The amœbulæ of this stage tend to congregate together in certain spots, and the groups thus formed fuse together (their nuclei, however, remaining separate) to form the plasmodium, the dominant vegetative stage, which feeds and grows, its nuclei multiplying as it does so, until from the small mass of protoplasm formed originally by the amobulæ, with relatively few nuclei, it becomes a slieet or network of protoplasm, which may 
be several inches across and contain many thousands of nuclei. The plasmodium moves about in various directions, showing

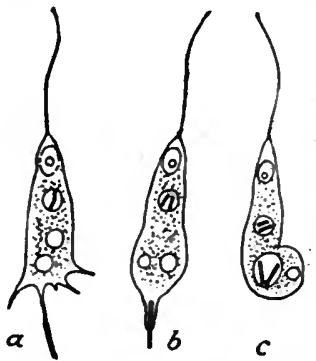

Fra. 98. - Flagellula of Stemonitis fusca, showing successive stages in the cepture of a becillus. In $a$ it is captured by one of the pseudopodia at the hinder end ; in $c$ it is enclosed in a diges. tive vacuole. Another bacillus is contained in an anterior vacuole. From Lister, magnified 800.

exquisite streaming movements of the protoplasmic body (Fig. 99). The nature of the food varies in different species ; the majority feed on dead vegetable matter, but some attack and devour living fungi. The mode of nutrition is generally holozoic, but in some cases perhaps saprophytic. Contractile vacuoles are present in large numbers in the protoplasm, in addition to the innumerable nuclei, which are all similar and not differentiated in any way. The plasmodia are often brightly coloured.

From their mode of life, the plasmodia are naturally liable to desiccation, and when this occurs the plasmodium passes into the sclerotial condition, in which the protoplasm breaks up into numerous cysts, each containing ten to twenty nuclei. When moistened, the cysts germinate, the contained masses of protoplasm fuse together, and so reconstitute the active plasmodium again.

The plasmodium represents the trophic, vegetative phase, which is succeeded by the reproductive phase, apparently in response to external conditions, such as drought, but more especially scarcity of food. The reproduction begins by the plasmodium becoming concentrated at one or more spots, where the protoplasm aggregates and grows up into a lobe or eminence, the beginning of the sporangium (Fig. 100), the capsule in which the spores are found. The sporangium is modelled, as it were, on the soft protoplasmic body, and

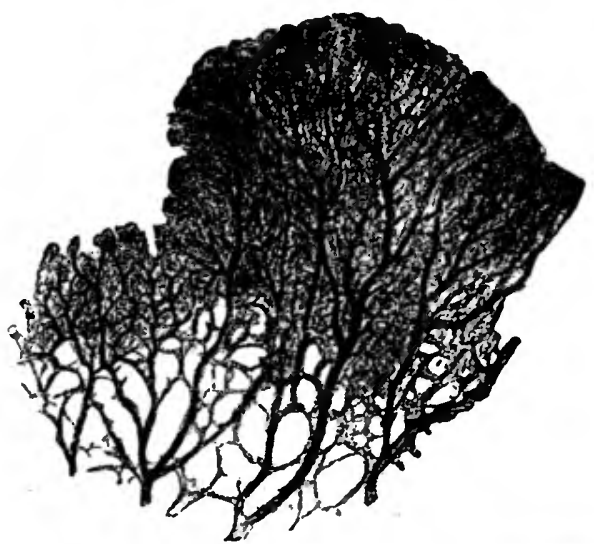

Fra. 99.-Part of a plasmodium of Badhamia utricularis expanded over a slide. From Lister, magnified 8 diameters. takes the form of a rounded capsule, attached to the substratum by a disc-like attachment known as the hypothallus. Between the sporangium 
proper and the hypothallus the body may be drawn out into a stalk.

The first events in the reproductive process are the formation of the protective and supporting elements of the sporangium. Over the surface of the lobe a membrane or envelope is secreted, the "peridium," and in the interior of the protoplasmic mass a network, or rather feltwork, of filaments, the "capillitium," is produced, of similar nature to the peridium, and in continuity with it; peridium and capillitium contain cellulose or allied substances and the former may contain carbonate of lime in some species.

During the formation of the protective peridium and the supporting capillitium the protoplasmic mass remains in the plasmodial condition, but when the accessory structures are completely formed the actual sporeformation begins. According to recent investigations, spore-formation is initiated by the degeneration of a certain number of the nuclei; the nuclei that

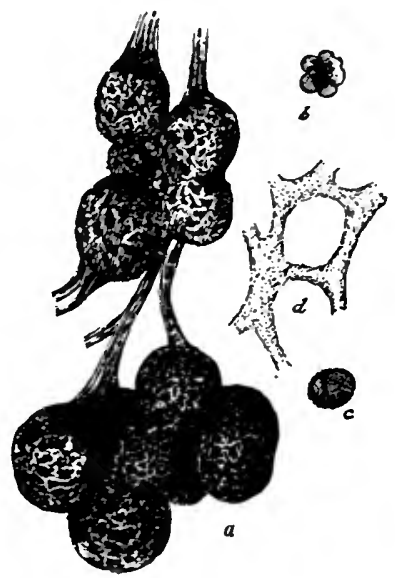

Fig. 100.-Badhamia utricularis. $a$, Group of sporangia, magnified $12 ; b$, a cluster of spores ; $c$, a single spore; $d$, part of tho capillitium containing limegranules: $b$ and $d$ magnified 170. From Lister. persist then divide by karyokinesis simultaneously throughout the whole plasmodium. The protoplasm then becomes divided up,

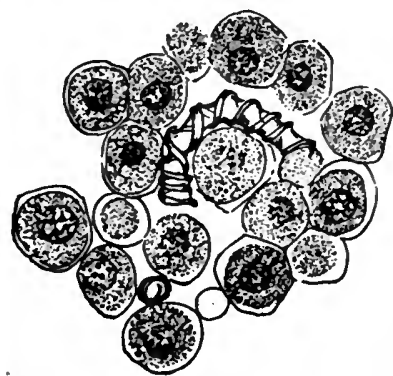

FiG. 101.-Trichia varia : part of a section through a sporangium after the spores are formed; threads of the capillitium are seen in longitudinal and transverse section. From Lister, magnified 650 diameters. directly or indirectly, into as many masses as there are nuclei. The cells thus produced, lying in the interstices of the capillitium, become surrounded each with a tough membrane, and are the spores (Fig. 101). They are liberated hy bursting of the peridium, and the hygroscopic properties of the capillitium are the cause of movements in it which assist in scattering the spores. With the formation of the spores the life-cycle has been brought round to the starting-point that was selected. The spores are scattered in all directions by the wind, and germinate in favourable localities.

The account given above may be taken as describing the typical series of ovents in the life-history, which is liable to considerable variations in particular 
types. In the subdivision termed the Sorophora or Acrasim there is no flagellula-stage in the life-history, and the amœbulæ which are produced from the spores aggregate together, but form only a pseudo-plasmodium, in which the constituent amcebulæ remain distinct, without fusion of their protoplasmic bodies, each amcbula multiplying independently. The details of the reproductive process also vary greatly. In the division known as the Exosporeæ; represented by the genus Ceratiomyxa, no sporangium is formed, but the plasmodium grows up into antler-like processes, sporophores, over the surface of which the plasmodium divides up into a mosaic of cells, each containing a single nucleus of the plasmodium. Each cell becomes a spore, which is produced on the free surface of the sporophore, and drops off when ripe. In the Soronhora the amœbæ associated in the pseudo. plasmodium are not all destined to become reproductive individuals; some of them join together toj secrete a stalk, and develop no further; others form clusters (" sori ") of naked spores on the stalk.

The cytological details of the life-history of the Mycetozoa have been the subject of a series of studies by Jahn, who, however, in his latest investigations, has come to conclusions different from those at which he arrived in his earlier works. According to the earlier accounts given by Jahn and Kränzlin, the spore-formation was preceded by a fusion of nuclei in pairs throughout the sporangium, a process which was regarded as the true sexual karyogamy, and was followed by reducing divisions. According to Jahn's latest investiga. tions (294), however, the nuclear fusions observed in the sporangium take place only between degenerating nuclei, and are to be interpreted as purely vegetative phenomena which have nothing to do with the true sexual process, which is stated to be as follows: The nuclear division which immediately precedes spore-formation is a reducing division, whereby the number of chromosomes is reduced from sixteen to eight. Consequently the nuclei of the spores, and also the swarm-spores produced from them, both flagellulie and amcbulæ, have half the full number of chromosomes. In Physarum didermoides the amcobulæ multiply by fission, with mitoses showing eight chromosomes. After a certain number of such divisions, the amcbulæ copulate in pairs as gametes. The zygotes thus formed are the foundation of the plasmodła ; when one zygote meets another it fuses with it, the nuclei remaining separate, and by repeated fusions of this kind the plasmodia are formed. When, on the other hand, a young plasmodium or a zygote meets an amœbula (gamete), it devours and digests it. The nuclei of the plasmodia multiply by mitoses which show sixteen chromosomes.

In Ceratiomyxa the reduction-division preceding spore-formation is followed by degeneration of one of the two daughter-nuclei; the other becomes the nuclelis of the spore. Within the spore the nucleus divides twice, forming four nuclei, and as soon as the spore germinates the contents divide into four amœbulæ, which adhere in the form of a tetrahedron. Each amcobula has eight chromosomes in its nucleus, and divides into two amcbulæ, also with eight chromosomes. Each of the amœbulæ develops a flagellum and swims off. Possibly in this genus the syngamy takes place between flagellulæ.

From the investigations of $\mathrm{Jahn}$, it is clear that the swarm-spores of Mycotozoa, like those of other Sarcodina, are the gametes ; their nuclei have undergone a process of reduction, and represent pronuclei, which after a certain number of divisions give rise by syngamy to synkarya, from which the nuclei of the vegetative phase, the plasmodium, takes origin.

The Mycetozoa are classified by Lister (297) as follows :

SUBORder I. : EUPLASMOdIda (Myxogastres, Myxomycetes sens. strict.).Mycetozoa with a flagellula-stago and a true plasmodium formed by plasto. gamic fusion of amobulæ. This suborder comprises forms with the full lifecycle described above.

Sectian 1. Endosporea. - Spore-formation within a sporangium. Examples: Badhamia, Fuligo (AEthalium), etc.

Section 2. Ectosporea. Spores formed on the exposed surface of sporophores. Example: Ceratiomyxa. 
SUborder II.: Sorophora (Acrasiæ, Pscudoplasmodida). - With no flagellate stage in the life-history; the amœbulæ do not fuse completely to form a true plasmodium; the spores are formed in clusters (" sori "). Here belong various genera, for the most part found in dung, such as Dictyostelium and Copromyxa. Acrasis occurs in beer-yeast.

In addition to the typical Mycetozoa belonging to these two suborders, there are a number of forms on the border-line, referred by some authorities to the Mycetozoa, by others to other orders, such as the Proteomyxa. It is only possible to refer very briefly to these genera here.

In the first place, there are a number of parasitic forms, placed together by Doflein in the suborder Phytomyxina, Schröter. In this suborder no sporangium is formed, the process of spore-formation being simplified, probably, in correlation with the parasitic mode of life. The typical members of this group are parasites of plants, but some recently-described parasites of insects have been assigned to Phytomyxince. The best known example of the group is the common Plasmodiophora brassica, which attacks the roots of cabbages and other Cruciferæ, producing a diseaso known as "Fingers and Toes" ("Kohlhernie"), characterized by knotty swellings on the roots. Other genera parasitic on plants are Tetramyxa and Sorosphora.

In Plasmodiophora the spores germinate to produce flagellulæ, which are liberated in water or damp earth, and which in some way penetrate into the cells of the plant, and there appear as the myxamœbæ after loss of the flagellum. The youngest myxamœbæ seen have two nuclei. They grow in the cell-contents with multiplication of their nuclei, and fuse with one another to form plasmodial masses which fill the cell after absorption of its contents,

In a diseased plant a number of cells are attacked by the parasite, and it is not certain whether the myxamœbæ can pass from one cell to another, and so spread the infection, or whether all the infected cells are derived from the multiplication of the first cell infected. The second view, maintained by Nawaschin, is supported by Prowazek, and also by Blomfield and Schwartz, with regard to the allied genus Sorosphara.

When the host-cell is exhausted, the reproductive phase begins, according to Prowazek (127), by the nuclei of the plasmodium throwing out numerous chromidia, and becoming in consequence very indistinct. In Sorosphara at this stage (Blomfield and Schwartz) the nuclei disappear altogether, being entirely resolved into chromidia from which socondary nuclei are formed. Spore-formation, preceded by sexual processes, takes place in the manner described above (p. 149, Fig. 76). In Sorosphora, Blomfield and Schwartz found that, after reconstitution of the generative nuclei, the plasmodium divides up into uninucleate cells, each of which divides twice by karyokinesis ; after these divisions the cells become arranged as a hollow sphere, the "sorosphere," and each cell bccomes a spore. No cell-fusions or syngamic processes were observed.

As stated above, certain parasites of inscets are referred to this order by Léger. Such are the genera Sporomyxa, Léger (295), Mycetosporidium, Léger and Hesse, and Peltomyces, Léger (C.R.A.S., cxlix., p. 239). Zoomyxa legeri, Elmassian (637), parasitc of the tench, is perhaps also to be referred to the Mycetozoa. The position of these forms must, however, be considered somewhat doubtful at present. Chatton has thrown out the suggestion that the affinities of Peltomyces are rather with the Cnidosporidia (p. 409), through the genus Paramyxa recently found by him (761).

Lastly, mention must bo made of the remarkable genera Chlamydomyxa, Archer, and Labyrinthula, Cienkowski, the affinities of which are still obscure. By Lankester (11) they wero ranked as an independent order of the Sarcodina under the name Labyrinthulidea; by Delage and Hérouard (6) and others they are placed as a suborder, Filoplasmodida, of the Mycetozoa.

Chlamydomyxa is a fresh-water genus occurring either free or encysted. Its most remarkable feature is the possession of chromatophores which enable it to live in a holophytic manner, and consequently to assimilate and grow when encysted. On the other hand, when free it forms a network of long, 
filamentous pseudopodia, by means of which it is able to digest food in the ordinary holozoic manner. The body is a plasmodium containing, in addition to numerous nuclei, chromatophores, and peculiar "oat-shaped bodies," "spindles," or " physodes," stated to consist of phloroglucin. The cystonvelopo consists of cellulose, and has a stratified structure. In addition to reproduction by fission (plasmotomy), Chlamydomyxa appears to form flagellate swarm-spores, possibly gametes.

Labyrinthula occurs in marine and fresh water. In the active state it has the form of a network of filaments, 1 millimetre or so in extent, over which travel a great number of " units," each a nucleate cell or amcbula, sometimes brightly coloured. When dried, each unit encysts and hatches out again separately. The units multiply by fission. They were formally compared erroneously with the "spindles" of Chlamydomyxa. Lister (298) regards Labyrinthula as a colonial organism of which the units remain in connection by their pseudopodia. He considers these two genera as related in one direction to certain members of the Foraminifera (Gromiida), in other drections to the Heliozoa and the Proteomyxa.

\section{HeLtozoA.}

The Heliozoa are characterized, as a group, by their spherical form and stiff, ridiating pseudopodia, whence their popular name of "sun-animalcules." As in the case of the Radiolaria, these peculiarities of form are generally correlated with a floating habit of life, though in a few cases the animal is sedentary and attached to a firm support. In contrast with the Radiolaria, a "central capsule" (p. 250) is absent from the body-structure. A skeleton may be present or absent. The majority of species inhabit fresh water, but a few are marine.

General Characters.-As in other orders of Sarcodina, a concise statement of the characteristic features of the group is rendered difficult by the occurrence of border-line forms, of which the exact position is doubtful. It is best, therefore, to consider first typical forms of which the position is incontrovertible, and then those which link the Heliozoa to other groups of Protozoa.

The body-protoplasm exhibits commonly a vacuolated, frothy structure, with distinct cortical and medullary regions. The cortical zone, distinguished by vacuoles of larger size, disposed in a radiating manner, is regarded as ectoplasm; the medulloury region, with smaller vacuoles irregular in arrangement, as endoplasm; but it is open to doubt if these two regions correspond truly to the ectoplasm and endoplasm of an amoba. The cortex contains the contractile vacuoles, and gives off the pseudopodia, which are typically stiff, straight, and filamentous, ending in a sharp point and supported by an axial organic rod (p. 48); but in some genera the supporting axis is wanting. In the medulla are lodged the nuclear apparatus, the food-vacuoles, and frequently also symbiotic organisms, which are probably in most cases vegetative, nonflagellate phases of holophytic flagellates (Chlamydomonads).

As regards the nuclear apparatus, there are two types of arrange- 
ment (compare p. 90). In the first or Actinophrys-type (Fig. 46) the nircleus is central, and the pseudopodia arc centred on it. Actinosphorium (Fig. 3) can be derived from this type by multiplication of the nucleus, originally single, until there may be some hundreds present in large specimens. The marine form Camptonema nutans, Schaudinn, is perhaps also to be referred to this type of structure ; it has as many pseudopodia as there are nuclei present, each pseudopodium arising directly from a nucleus (p. 91, Fig. 47).

In the second or Acanthocystis-type (Figs. 18, 64) the centre of the spherical body is occupied by a "central grain" (p. 91), on which the axial rays of the pseudopodia are centred. The nucleus, on the other hand, occupies an excentric position in the body. In this type there is a tendency to a sessile habit of life, the animal being attached by the surface of the body, which may grow out into a stalk, as in Clathrulina (Fig. 19). In the interesting marine genus Wagnerella (Fig. 48), the surface of attachment has become drawn out in such a way that the body is divided into three partsbasal plate, stalk, and head. The nucleus is situated in the basal plate. The head contains the central grain, from which the pseudopodia radiate. Thus, in this genus the excentric posicion of the nucleus is carried to an extreme; it may be regarded as having grown out from the body in a lobe or prolongation which forms the basal plate and stalk, while the original body remains as the head with the central grain and pseudopodia.

The skeleton, when present, may take various forms. It may be a simple gelatinous investment, or may contain mineral (siliceous) substance either in the form of loose, radiating spicules, as in Acanthocystis, or of a continuous lattice-like investment, as in Clathrulina. In Wagnerella the basal plate and stalk are protected by a tough yellowish organic membrane, replaced in the head by a coloukless gelatinous layer, and both head and stalk are further protected by siliceous spicules, which are formed in the protoplasm and transported by protoplasmic currents (Zuelzer, 86).

Life-History. - Reproduction in the free vegetative phase is effected by binary fission or gemmation. Imperfect binary fission may lead to colony-formation, as in Rhaphidiophrys. The sexual phases are only known accurately in a few cases. In Actinophrys, Schaudinn described copulation within a cyst (p. 132, Fig. 71), with subsequent division of the zygote and liberation of two individuals from the cyst. In Actinosphorium (Hertwig), encystment of a large multinucleate individual is followed by degeneration of about 95 per cent. of the nuclei; the remainder appear to fuse in pairs, and the body then divides into as many cells as there are nuclei. Round each cell a separate "prinfary" cyst is secreted within the gelatinous " mother-cyst" originally formed round the whole mass. 
Each primary cyst then divides into two secondary cysts, which after nuclear .reduction become the gametes and copulate. The zygote develops into a young Actinospharium with several nuclei, which emerges from the cyst and begins a vegetative life, but appears to divide frequently at the start into uninucIeate, Actinophrys-like forms.

In other genera, on the other hand, and especially in those of the Acanthocystis - type (Acanthocystis, Clathrulina, and Wagnerella), flagellate swarm-spores are formed, which probably represent gametes, as in many other Sarcodina.

The life-history of Wagnerella has recently been studied in detail by Zuclzer (86); her investigations reveal a diversity in its modes of reproduction almost as great as that seon in Arcella, and indicate that there is much yet to be discovered with regard to the life-oycles of other forms.

Wagnerella exhibits, according to Zuelzer, dimorphism correlated with alternation of generations. In June and July stout forms are observed, which are believed to arise from the conjugation of gametes; they reproduce by binary fission, and by a process of schizogony giving rise to amcebulæ (agametes). The more usual form, on the other hand, is smaller and more slender, and multiplies by binary fission, gemmation, and formation of flagellate swarm-sporcs. Hence this peculiar form reproduces in a variety of ways. In the process of binary fission the nucleus migrates from the. base up the stalk into the head, and places itself beside the central grain, which divides, its two halves passing to opposite sides of the nucleus; then the nucleus follows suit and divides also. Divisions of the central grains, and subsequently of the corresponding nuclei, may be repeated until eight to ten nuclei and as many central grains are present. Each nuclear division is followed by division of the head, at first incomplete, so that a condition results resembling the colonial form Rhaphidiophrys, a number of daughterindividuals united together, and each sending out pseudopodia (Fig. 102, D). After a time the colony breaks up, the daughter-individuals separate, and each one fixes itself and grows into the adult Wagnerella-form.

Bud-formation in Wagnerella (Fig. 102, $A-C$ ) is initiated by division of the karyosome within the nucleus, which retains its position in the base. The process is repeated until the nucleus contains a number of karyosomes, each with a centriole. The nucleus then buds off one or more small daughternuclei, each containing a single karyosome. Sometimes the nucleus breaks up entirely into as many daughter-nuclei as there are karyosomes, in which case the parent-individual dies off, in a manner similar to Arcella (p. 180), after liberation of the buds. Each daughter-nucleus migrates up the stalk into the head, where it becomes surrounded by a layer of protoplasm to form the bud, which is set free at first as an amoboid body. Before or after being set free, the bud may multiply by binary fission with mitosis, in which the centriole in the karyosome acts as a controsome. Finally each amœboid body develops into a Wagnerella, and in the process the centriole passes out of the nucleus and becomes the central grain, while the nucleus becomes displaeed from the centre. In the process of gemmation the central grain of the parent-individual takes no share whatever.

In the formation of the swarm-spores, minute secondary nuclei arise from chromidia near the principal nucleus in the base. Each secondary nucleus forms a centriole and divides by mitosis; the division is repeated until the wholo body, stalk and bead as well as base, is filled with small nuclci, while the primary nucleus degenerates. The body then divides up into as many cells as there are secondary nuclei, each cell becoming a biflagellate swarmspore which is set free, while the parent-individual degenerates. The destiny of the swarm-spores is uncertain, but they are believed to be gametes. 
In the " schizogony " of the stout forms the nucleus breaks up into a number of daughter-nuclei, as in gemmation ; each daughter-nucleus grows, its karyosome multiplies by fission, and it breaks up in its turn into granddaughternuclei. Continued multiplication of the nuclei in this manner proceeds until the body is filled with vesicular nuclei ; it then breaks up into as many amœbulæ, which are set free, leaving a residual body with the central grain, which degenerates.

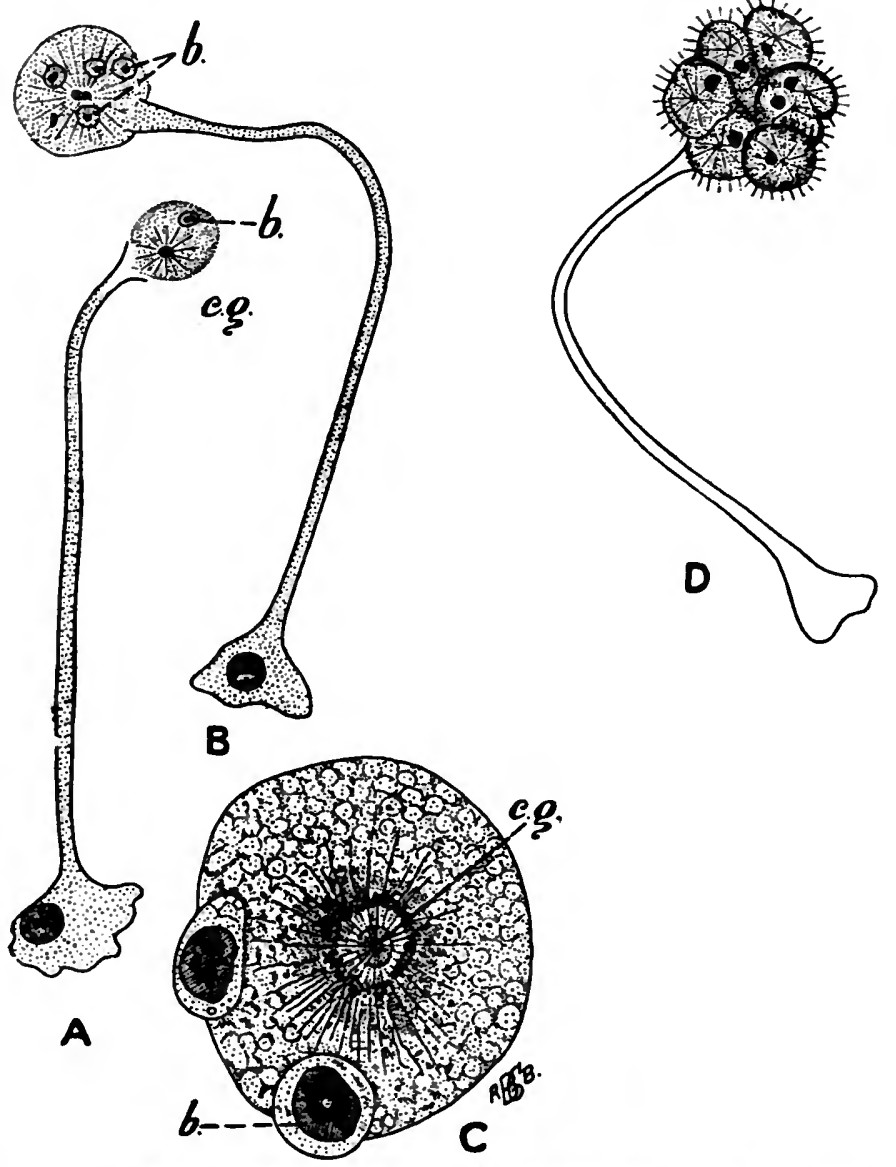

Fta. 102.-Wagnerella borealis, showing budding and fission. $A$, Speoimen with a single bud $(b)$ : c.g., central grain ; $\mathrm{B}$, specimen with four buds $(b)$; $C$, enlarged view of the head of a speoimen containing two buds $(b)$ in process of extrusion; $D$, speoimen in which the head has multiplied by fission to produce a Rhaphidiophrys-like colony; six individusls are seen, five of them eaoh with nucleus and central grain, the sixth in process of fission, with two nuclei and two oentral grains. After Zuelzer (86).

The Heliozoa are classified into four suborders :

SUborder I. : Aphrothoraca.-Body naked in the active state; envelopes, sometimes with siliceous spicules, only formed during 
encystment. Examples: Actinophrys (Fig. 46), Actinospharium (Fig. 3), Camptonema (Fig. 47), etc.

SUboRder II. : Chlamy dopHora.-Body protected by a soft gelatinous envelope, but without solid skeletal elements. Example : Astrodisculus.

Suborder III. : Chalarothoraca.-Body invested by a soft. envelope containing isolated spicules, usually siliceous, sometimes chitinous. Examples: Acanthocystis (Figs. 18, 61, 68), Wagnerella (Figs. 48, 102), He!erophrys (Fig. 103).

SUborder IV. : Desmothoraca.-Body invested by a continuous, lattice-like skeleton. Example : Clathrulina (Fig. 19).

Fro. 103.-Heterophrys fockei, Archer. c., c., Contractile vacuoles ; 8., radial chitinous spines surrounding the envelope. A nucleus is present in the body, but is not shown; the bodies in the protoplasm represent zooxanthella. From Weldon and Hickson, after Hertwig and Lesser.

A certain number of genora must be mentioned which are of doubtful position, referred by some authorities to the Heliozoa, by others to other orders. Some of these genera perhaps do not represent independent, " adult" forms, tut may be only developmental phases of other genera. Nuclearia, classed by some in the Aphrothoraca, by others in the Proteomyxa, has an amcoboid body and pseudopodia without axes. As described above (p. 177 and Fig. 80), a Nuclearia-stage occurs in the development of Arcella.

Especially remarkable are certain genera which indicate a close relationship between Heliozoa and Flagellata. An account of several such forms is given by Ponsrd (302), in addition to which the following may be noted: Ciliophrys, Cienkowski, has two phases; in the one it appears as a typical Heliozoon with stiff radiating pseudopodia; in the other it is a typical flagellate. In the process of transformation the Heliozoon-form retracts its pseudopodia, its body becomes amœeboid, and a flagellum grows out; finally 
the animal becomes a pear-shaped flagellate swimming by męans of its flagellum (Schowiakoff, 863 ; Caullery, 300). Ciliophrys thus recalls Psendospora in its two pnases (p. 218), and there can be little doubt that the two forms are closely allied.

Dimorpha nutans, Gruber (Fig. 104), has radiating pscudopodia strengthened by axial rods, and in addition a pair of flagella arising close together at one pole of the body. Both flagella and pseudopodia arise from a centrosome situated near the flagellated pole; the single nucleus is also excentric and placed close beside the centrosome. The animal uses one of its flagella for attachment, while the other remains free (Schouteden).

These facts appear to indicate an origin for the Heliozoa from Flagellates such as those of the genus Multicilia (p. 270, Fig. 113), in which the body bears radiating flagella planted evenly over the surface; transformation of the flagella into stiff pseudopodia would produce the Heliozoon-type of organism. On such a view two peculiarities of the Heliozoan pseudopodia receive explanation: the power of nutation and bending which they fro-

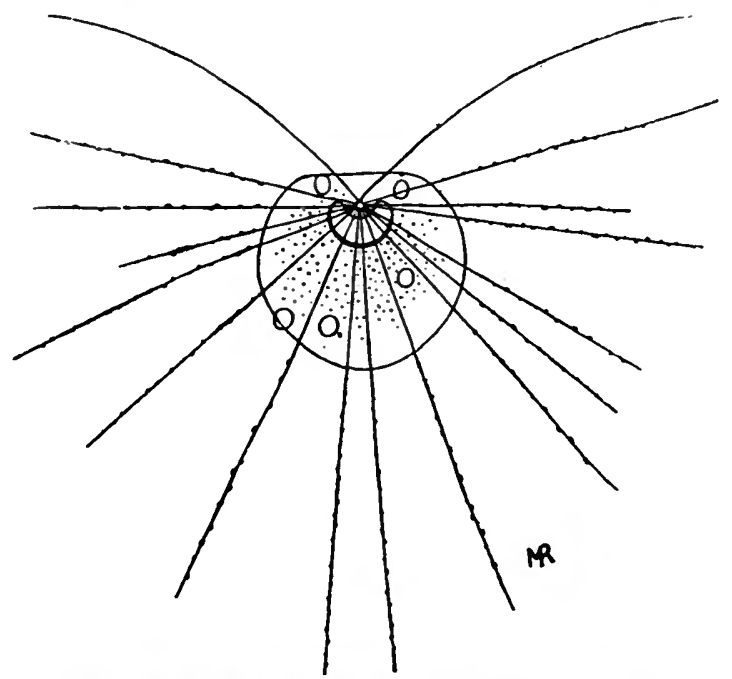

Fio. 104.-Dimorpha nutans. After Schouteden.

quently possess; and their insertion on a "central grain," which would then represent the blepharoplast, pure and simple, of a flagellate. On this view the pseudopodia of the Heliozoa would appear to be structures quite different in nature from the similarly-named organs of Lobosa.

On the other hand the Heliozoa also show affinities towards forms classed among the Reticulosa or "Proteomyxa," as already noted in the case of Ciliophrys and Pseudospora. Przesmycki has described a species, Endophrys rotatorium, parasitic in Rotifers, which he considers as a connecting-link between Nuclearia and Vampyrella. The exact systematic position of such geners must be considered at present an open question.

\section{RADIOLARIA.}

General Characters.-The Radiolaria are characterized, speaking generally, by the same type of form and symmetry that is so marked a feature of the Heliozoa, though in many cases the internal 
structure of the body, and especiallv the skeleton, may depart more or less widely from the radiate symmetry which is to be regarded, probably, as primitive for the group. Hence three principal types of symmetry can be distinguished in these organisms : (1) Homaxon (Figs. 13, 105, 107), in which all axes passing through the centre are morphologically equivalent, the symmetry of the sphere; (2) monaxon (Fig. 109), in which the body has a principal or vertical axis round which it is radially symmetrical, the type of symmetry of the cone; (3) bilaterally symrretrical (Fig. 106), in which the body

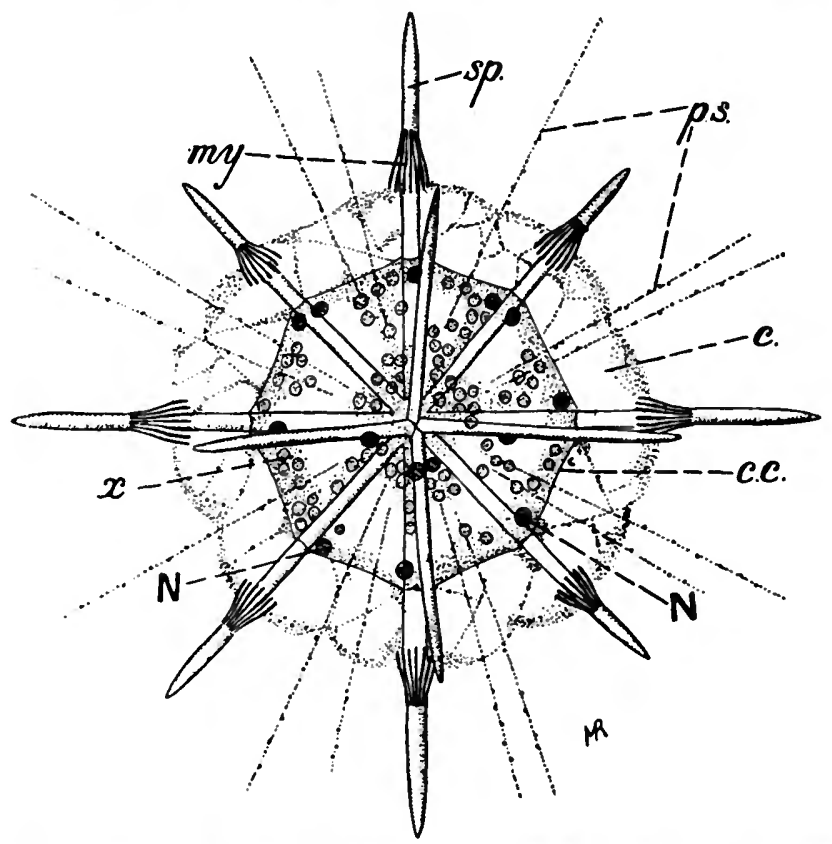

Fio. 105.-Acanthometra elastica, Haeckel. sp., Radiating spines of the skeleton (twenty in number, but only twelve are seen in the figure); ps., pseudopodis ; c., calymma ; c.c., central capsule ; $N$, $N$., nuolei ; $x$, yellow cells ; $m y$., myophrisks. After Biitsohli, Leucksrt and Nitsche's "Zoologisohe Wsndtafeln."

can be divided along a principal plane into equivalent right and left halves. With further modification the body may become asymmetrical. Sedentary forms are not known in this group, the species of which are exclusively marine, and occur on the open surfaces of seas and oceans, reaching in many instances a re atively large size and a very high degree of structural differentiation.

In the internal structure, the most salient feature is the division of the body by means of a membranous structure, termed the central capsule (Fig. 13, $C K$ ), into a central modullary region and a peripheral cortical zone-hence distinguished as the intracapsular 
and extracapsular regions of the body. The intracapsular medulla contains the nucleus or nuclei, and is the seat of reproductive processes. The extracapsular cortex is the seat of assimilation, excretion, food-capture, and of such locomotor processes as these organisms are able to perform, consisting chiefly of rising or sinking in the water by means of changes in a hydrostatic apparatus presently to be described.

The Radiolaria are an exceedingly abundant group represented by a great number of species both at the present time and in past ages; over vast tracts of the ocean-floor their skeletons are the principal, almost the cole constituents of the ooze; and the same must have been true in past times, since in many geological deposits the rocks are composed of the same materials. Every microscopist is familiar with their skeletons, which on account of their beauty and variety of form are favourite objects for microscopic study and demonstration. Corresponding with the variety of forms and species, the internal structure shows a range of variation and differentiation which it is impossible to deal with adequately in a short space; it must suffice, therefore, to describe here the main structural peculiarities of this group in a general manner, and to indicate briefly the principal variations of structure which are of importance for the classification of the group For further information the reader must be referred to the larger treatises and special monographs.

Siructure.-The central capsule, absent in rare cases, may be a thin, delicatc structure, visible only after treatment with reagents, or may be fairly thick. In homaxon forms it is generally spherical, but may assume various shapes correlated with the general bodyform, and even may be lobed or branched. It is perforated by openings which place the intracapsular protoplasm in communication with the extracapsular; the openings may take the form of fine pores scattered evenly over the whole surface (Peripylaria); of similar pores aggregated into localized patches, pore-areas or poreplates (Acantharia); of a single pore-plate at one pole of an asymmetrical capsule (Monopylaria, Fig. 106) ; or of one principal and two lateral apertures (Tripylaria).

The intracapsular protoplasm contains the nuclear apparatus, either oné nucleus of very large size or a number of smaller nuclei (Fig. 105). In addition, various bodies of metaplastic nature, serving as reserve-material for the reproductive processes, are found in this region, in the form of fat-globules, oil-drops, concretions, crystals, etc.

The extracapsular region consists of three zones, from within outwards: (1) an assimilative layer or matrix immediately surrounding the capsule; (2) a vacuolated layer, known as the "calymma," hydrostatic in function; (3) a protoplasmic layer from which the pseudopodia arise.

1. The assimilative layer contains pigment, representing excretory substances and ingested food-material in the shape of small 
organisms captured by the pseudopodia and passed into the body, to be digested in this region. In the Tripylaria an aggregation of food-material and excretory substances produces a characteristic greenish or brownish mass concentrated round the main aperture of the central capsule, and known as the phoodium, whence this suborder is sometimes known as the Phæodaria.

2. The calymma is composed for the most part of a great number of vacuoles containing fluid, the function of which is hydrostatic ; the contents of the vacuoles are stated to be water saturated with carbon dioxide, causing the animal to float at the surface, and

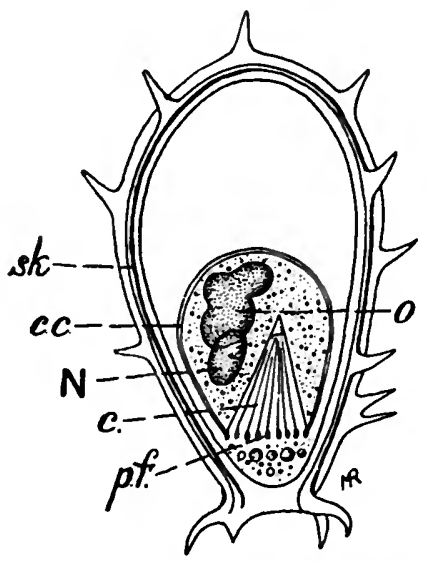

Fug. 106. - Lithocircus productus, Hertwig, showing a bilaterally symmetrical skeleton consisting of a simple siliceons ring prolonged into spicular processes. sk., Skeleton ; c.c., central capsule ; pf., pore-area, surmounted by a conical structure $(c$.$) , the$ so-called "pseudopodial cone"; $N$., nucleus ; $o$. , oil-globule. After Bütschli, Leuckart and Nitsche's "Zoolngisohe Wandtafeln." enabling it to regulate its position in relation to conditions of environment. In rough weather the vacuoles burst or are expelled from the body, and the animal sinks into deeper and quieter layers of water; there fresh vacuoles are formed, enabling it to return again to the surface if the conditions are favourable. Contractile vacuoles of the ordinary type are not present.

In addition to the vacuoles, the calymma contains numerous "yellow cells," generally regarded as symbiotic organisms of vegetable nature, and named "zooxanthellæ" or "zoochlorellæ," according to their colour. Absent in the Tripylaria, these yellow cells are found, as a rule, in the calymma, but in Acantharia they occur in the intracapsular protoplasm (Fig. 105, x). The nature of the yellow cells of Acantharia has been much disputed, and many observers have régarded them as an integral part of the organism itself; this view has recently been revived by Moroff and Stiasny, who bring forward evidence to prove that the yellow cells of Acantharia are a developmental phase of the organism. Still more recently this view has been extended by Stiasny to the colony-forming Sphærozoa in the first place, and then to Radiolaria generally. The difficulty in the way of such an interpretation which arises from the co-existence, in Thalassicolla and other genera, of yellow cells in the calymma, with an undivided nucleus in the hostorganism, is met by supposing-that in such cases developmental 
stages of other Radiolarians have penetrated into the calymma, and live there symbiotically-a supposition which is certainly in need of further proof bafore it can be accepted.

3. The most external layer of the body is a protoplasmic envelope from which the pseudopodia radiate. In Radiolaria, speaking generally, the pseudopodia are straight, slender, and filamentous, composed of motile protoplasm entirely ("myxopodia"); but in Acantharia some of the pseudopodia are, like those of Heliozoa, axopodia supported by stiff axial rods of organic substance, which originate deep within the central capsule and pass through the calymma along the axis of the pseudopodium, but without reaching as far as its distal extremity. In some Acantharia (Acanthometrida) are found also peculiar modifications of the bases of certain of the pseudopodia in the form of groups of rod-like bodies, " myonemes" or " myophrisks" (Fig. 105, my.), clustered round each of the spicules of the skeleton. As their name implies, the myonemes are contractile elements which, by their contraction or expansion, alter the hydrostatic balance of the organism, and enable it to rise or sink in the water. According to Moroff and Stiasny, the myonemes are formed in the interior of the central capsule, and are derived from nuclei.

In a certain number of Radiolaria a skeleton is absent altogether. The Acantharia have a skeleton composed of a substance which was formerly supposed to be of organic nature, and was termed acanthin by Haeckel, but which consists of strontium sulphate according to Bütschli (310). In other Radiolaria the skeleton, when present, is siliceous. In Acantharia the skeleton invades the intracapsular region, and consists typically of a system of twenty spines or spicules radiating from the centre of the body (Fig. 105). It is a simple and enticing view to regard such a skeleton as originating phylogenetically from a modification of the axis of pseudopodia. Union of outgrowths from radially-directed spicules gives rise to a lattice-work forming a spherical perforated shell, and as the animal grows in size several such concentric spheres may be formed, one within the other, supported by radial bars which represent the original radiating spicules (Fig. 107). In Radiolaria other than Acantharia the skeleton is usually entirely extracapsular, and exhibits a variety of form and structure which cannot be discussed further here. In some of the Tripylara foreign bodies are utilized for building up the skeleton, cither to form the basis of spines secreted by the animal or to construct a coat of armour on the exterior of the body (Borgert).

Life-History. - Reproduction of the Radiolaria is effected in some instances by binary fission-namely, in those forms in which a skeleton is lacking or consists of loose spicules. The nucleus 
divides by a mitosis remarkable for the vast number of chrumosomes, of which there may be over a thousand, and the apparent absence of a centrosome. The more usual method of reproduction, however, is formation of flagellated swarm-spores by a process of rap:d multiple fission within the central capsule. Two kinds of swarm-spores are produced, which are known respectively as " isospores" and " anisospores." The isospores (Fig. 108, $A$ ), which are probably agametes, are all similar in size and appearance, and frequently contain a crystal in their protoplasm, and are hence sometimes termed "crystal-spores." The anisospores (Fig. 108,

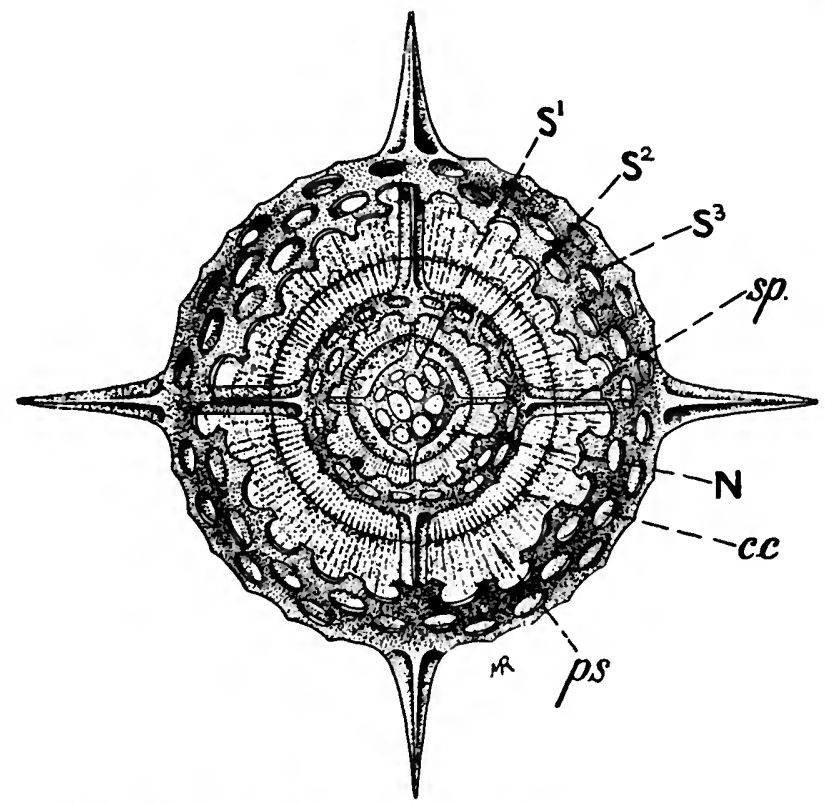

Frg. 107.-Actinomma asteracanthion: semi-diagrammatic to show the mode of growth of the skeleton. $S .{ }^{1}, S .^{2}, S .^{3}$, Three concentrie lattice-work shells, comnected by $s p$., radial bars which a re prolongel beyond the outermost shcll as spikes ; $N$., nucleus ; c.c., central ea psule ; ps., pscudopodis. After Bütschli, Leuckart and Nitsche's "Zoologische Wandtafeln."

$B, C)$, probably gametes, are of two kinds, smaller microspores and larger macrospores; they differ in structure from the isospores, and lack the characteristic crystal. The swarm-spores vary in structure in different species, but usually have two flagella. Isospores and anisospores are formed in different individuals, but it is still a moot point whether an alternation of generations occurs. Microspores and macrospores may be formed in the same individual in some species; in others they are produced by different individuals. Previous to formation of the swarm-spores the extracapsular region of the body disintegrates, and the central capsule with its contents 
sinks to a considerable depth. The swarm-spores are liberated by the breaking-up of the central capsule. The subsequent development of the swarm-spores when set free has not been made out.

While the main features of the reproductive process are as stated above, the cytological details of the formation of the swarm-spores is still a matter of dispute. The subject is dealt with in the recent memoirs of Moroff on the one part, and Hartmann and Hammer, Hartmann (60), and Huth, on the other. The formation of the anisospores is generally regarded as a breaking. up of the primary nucleus into chromidia, from a part of which the secondary nuclei arise, which become those of the swarm-spores (compare Foraminifcra). But according to Hartmann and his adherents, the huge primary nuclei scen in many Radiolaria are polyenergid nuclei or polykarya (p. 121) containing a vast number of nuclear energids or monokarya, consisting each of chromatin, in the form of a twisted thread or so-called "chromosome," and a centriole. In the gamete-formation a great number of such monokarya are set free from the primary nucleus to become the gamete-nuclei; hence the so-called "generative chromidia" set free from the nucleus are interpreted as secondary nuclei or monokarya already formed within the primary nucleus. A similar interpretation is given to the mitosis seen in the process of binary fission; the huge mitotic figure, composed of more than a thousand chromosomes, is interpreted as being in reality made up of as many mitotic figures as there are chromosomes, since each so-called "chromosome" is regardedeas a single
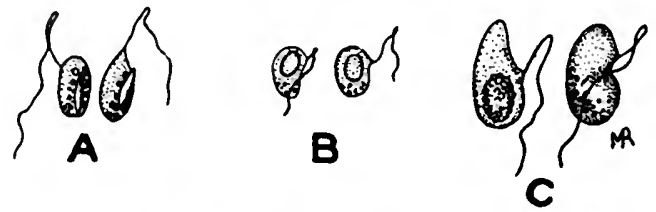

Fia. 108.-Swarm-spores of Collozoum inerme. A, Crystal-besring swarm-spores, agametes ; $B, C$, swarm-spores without crystals, gametes ; $B$, miorospores (microgametes); $C$, macrospores (macrogametes). After Hertwig.

nuclear energid or monokaryon with its own centriole, the whole number of energids dividing independently but synchronously to form the supposed mitotic figure.

According to Moroff and Stiasny, in Acanthometra pellucida a process of multiplication is proceeding continually within the central capsule, until it is entirely filled up with cells, from which the swarm-spores arise. In this multiplication, termed by the authors "schizogony," trophic nuclei (" macronuclei") and generative nuclei ("micronuclei") are formed. The trophic nuclei are the "yellow cells," which ultimately degenerate. Hence the Acantharia are considered not to be single individuals, but colonies of animals which have the extracapsular protoplasm, pseudopodia and skeleton in common.

Finally, attention must be drawn to the peculiar organisms found in certain Radiolaria, and regarded by some authorities as parasitio Flagellata (Silicoflagellata, Borgert), by others as developmental stages, of the Radiolaria themselves. See Delage and Hérouard (6, p. 371).

The Radiolaria ane classificd as follows:

Suborder I. : Peripylaria seu Spumellaria.-Central capsule spherical, perforated by evenly-distributed pores. Extracapsular region well developed. Skeleton wanting or consisting of scattered spicules or of lattice-work shells, developed in the extracapsular region, siliceous.

Legion 1 : Collodaria. -Skeleton wanting or simple in structure; monozoic forms. Five families. Examples: Thalassicolla (Fig. 13), Thalassophysa.

Legion 2: Spherellaria.-Skeleton complex, usually with lattice-work shell; monozoic, generally small. Four families. 
Legion 3: Sphcerozoa seu Polycyttaria.-Colonial forms consisting of numerous individuals embedded in a common jelly; their central capsules are distinct, but their extracapsular regions anastomose. The colonies reach a length of several contimetres. Two families. Example : Collozoum.

SUBORDER II. : AcaNTHARIA.- Skeleton composed of strontium sulphate, typically in the form of spicules radiating from the centre of the body, within the central capsule ; in addition lattice-work shells may be developed. Central capsule with pores evenly developed, or grouped in areas.

A number of families are recognized, grouped in different ways by different authorities. Example: Acanthometra (Fig. 105).

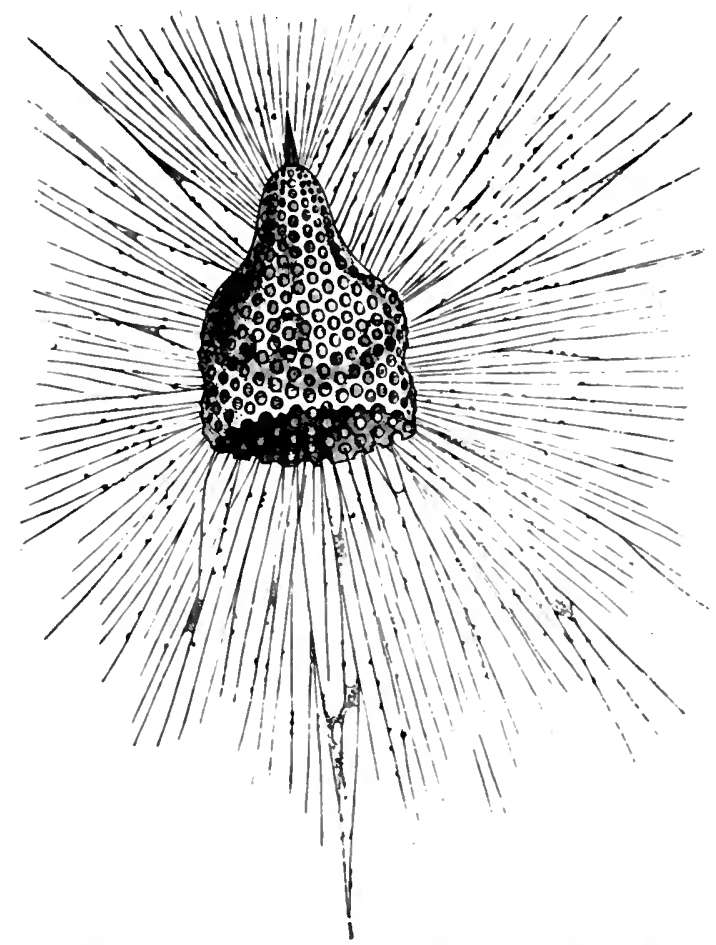

F1a. 109.-Eucyrtidium cranioides, Haeckel: entire animal as seen in the living condition. The central capsule is hidden by the beehive-shaped siliceous shell within which it is lodged. From Gamble, magnified 150.

Suborder III. : Monopylaria seu Nassella Ria.--Central capsule monaxon in form, with the pores aggregated at one pole into a pore-plate, and the walls of the pores thickened to form a conical structure directed inwards into the central capsule. Several families. Examples : Lithocircus (Fig. 106), Eucyrtidium (Fig. 109).

Suborder IV.: Tripylaria seu I'Heodaria.-Central capsule with a principal aperturo (astropyle) and two accessory apertures (parapyle). A mass of pigment (phæodium, p 252) surrounds the principal aperture. Divided by Häcker into six legions and numerous families. Example: Aulacantha. 


\section{CHAPTER XII}

\section{SYSTEMATIC REVIEW OF THE PROTOZOA : THE MASTIGOPHORA}

The distinctive feature of the class Mastigophora is the possession of one or more flagella as organs of locomotion and food-capture, not merely during early stages of development, but in the active phases of the adult organism also. In other classes, as has been pointed out in a previous chapter, flagella may be present in the young stages, but are absent in the adult phases. In the Mastigophora a flagellum is a permanent feature of the organization, though even in this class it may be temporarily lost, either in active phases, when the animal may become amcoba-like, or in resting phases, especially in parasitic forms of intracellular habitat.

The Mastigophora are divided into three subclasses, of which the first, the Flagellata, contains the more typical forms, and constitutes the nucleus, so to speak, of the class ; while the two remaining subclasses, the Dinoflagellata and Cystoflagellata, may be regarded as specialized offshoots of the primitive flagellate stem. It is convenient, therefore, to deal with the Flagellata in a general manner first, and then to describe the special features of the other two subclasses.

\section{Subclass I. : Flagellata (Euflagellata).}

General Characters.-The members of this group are for the most part of minute size, and seldom attain to considerable dimensions ; forms of relatively large size, such as the species of Euglena and allied genera, are small as compared with the larger species of the Sarcodina and other classes. As a rule the Flagellata are freeswimming organisms; a certain number, however, are sedentary in habit, attaching themselves to a firm basis, and using their flagella for food-capture alone. There is a great tendency to colonyformation in this group. In the process of multiplication by fission of the ordinary type, separation between the daughter-individuals may be incomplete, so that they remain connected together, either by means of a common envelope, house, or gelatinous matrix, or by organic, protoplasmic union, or in both ways. Repeated fission 
of this kind leads to the formation of a colony which may attain to dimensions relatively large, though composed of individuals of minute size. The colony may be free-swimming or fixed, and in the latter case is frequently arborescent in form. In many cases the colonies of Flagellata show a differentiation of the constituent individuals into vegetative and generative individuals-the former not capable of reproduction, but purely trophic in function; the latter destined to be set free, and to produce new colonies, with or without going through a process of syngamy.

Bionomics.-In their modes of life the Flagellata exhibit all the four types described in Chapter II. (p. 13), different forms being holozoic, holophytic, saprophytic, or parasitic ; and one and the same form may live in different ways during different periods of its life-history, according to circumstances.

The parasitic flagellates have attracted a great deal of attention of recent years, on account of their importance in causing disease in man and animals. Ectozoic parasites may occur in aquatic forms, as for example Costia, parasitic on the skin of fishes. The entozoic forms are parasitic for the most part in the digestive tract, or in the blood and lymph of their hosts. Parasitio flagellates are found in the intestines of practically all olasses of the Motazoa, and especially in arthropods and vertebrates; those parasitio in blood and lymph are found especially in vertebrates, and constitute an important group commonly termed as a whole the Hæmoflagellates, to which a special chapter will be devoted. From forms which were probably parasitic originally in the blood have arisen secondarily forms parasitic in cells which in their intracellular phase lose their flagellum entirely (Leishmania).

Many of the intestinal flagellates, especially in vertebrates, are probably not true parasites at all, but for the most part scavengers. In any case their pathogenic rôle appears to be very limited ; but in some cases a pathological condition of the host may be combined in a suspicious manner with great numbers of the parasites (compare Bohnc and Prowazek, Noc). It is worthy of note that in some cases an intestinal parasite may pass from the intestine into the blood or lymph under pathological conditions of the host. This condition seems to have been noticed first by Panilewsky, who described cases of frogs and tortoises which had been kept long in captivity and were in bed condition, thin, and with codematous swellings in the muscles and transudation of lymph into the peritoneal cavity; in such animals there were found in the blood and lymph, espocially in the codemata and transudations, abundant flagellates of the genus Hexcamitus (=Octominus, Fig. 116), of a species which in normal, healthy animals is found only in the intestine. A number of similar cases have been recorded by Plimmer (383, and Presidential Address to the Royal Microscopical Society, 1912), who found both Octomitus and Trichomonas in the blood of various batrachia and reptiles. The conditions under which these intestinal parasites pass into the blood appears to be striotly comparable to those under which the Leydenia-form of Chlamydophrys passes into the ascitio fluid (p. 237). Whether in such cases the migration of the parasite is the cause of the diseased state of the host, or whether, as seems more likely, the abnormal condition of the host gives the parasite an opportunity of spreading into fresh pastures, must remain for the present an open question; but, according to Plimmer, the presence of intestinal flagellates in the blood-circulation is associated with definite and recognizable lesions of the intestinal wall. In any case, the fact that intestiual flagellates can pass into the blood is a point which is prabably of phylogenetio as well as of practical importance (p. 322). 
Structure.-The body-form is of three principal types: (1) An envelope or tough cortex may be entirely absent, and the body is then amœboid, as in the Rhizomastigina (Figs. 38, 40); (2) a thin cuticle may be present, insufficiently rigid to inhibit changes of body-form due to contractility of the living substance (Fig. 15); (3) a thicker cuticle necessitates a constant body-form, which is either rigid and unalterable or sinuous and permitting movements of flexion and torsion. In the second type are comprised forms termed commonly " metabolic," on account of the changes of form they exhibit; contractions of the superficial layer of the body pass, as it were, in waves from the anterior to the posterior end of the body, in a manner similar to the peristaltic contractions of the intestine, producing rhythmic form-changes in the body.

In species in which the cuticle is thin or absent, a constant bodyform may nevertheless be maintained by internal form-giving organs, such as the axostyle of Trichomonas (Fig. 5), Lophomonas (Fig. 45), etc. True internal skeletons, however, do not occur. An external shell or house may be present, enclosing the whole body.

The protoplasmic body shows, in the amœboid forms such as the Rhizomastigina (p. 268), distinct ectoplasm and endoplasm. But as a general rule the thin ectoplasm is converted into a firm cuticle, or periplast, enclosing the body and containing contractile elements-myonemes. Hence the ectoplasm appears at first sight to be absent, and the protoplasmic body to consist of endoplasm alone. In larger forms the myonemes can be made visible by suitable treatment (Fig. 28), but as a general rule in such minute organisms the existence of myonemes or other contractile mechanisms can only be inferred from the movements of contractility or flexibility which the body exhibits.

The flagella may perform various functions in different cases; they may serve as organs of locomotion and of food-capture, as organs of temporary attachment, and as tactile organs. As stated above (p. 52), they may be distinguished by their relation to the progression of the organism, as tractella, anterior, and pulsella, posterior in movement. The flagella vary in number and in arrangement in different species, and for the different types of the flagellar apparatus a number of technical terms are in use : monomastigote, with a single flagellum (Fig. 38); isomastigote, with two or four flagella of equal length (Fig. 43); paramastigote, with one long principaI flagellum and a short accessory flagellum (Fig. 15); heteromastigote, with one or more anterior flagella directed forwards, and a "trailing flagellum" directed backwards (Figs. 5, 25); polymastigote, with a tuft of flagella (Fig. 45); and holomastigote, with numerous flagella scattered evenly over the body (Fig. 113). Of these various types of arrangement, the heteromastigote con- 
dition, with a backwardly-directed trailing flagellum ("Schleppgeissel "), deserves special attention, since by attachment of the trailing flagellum to the body an undulating membrane (p. 56) may arise; and that it has actually so arisen in some cases is indicated by the existence of pairs of similar forms, in which a
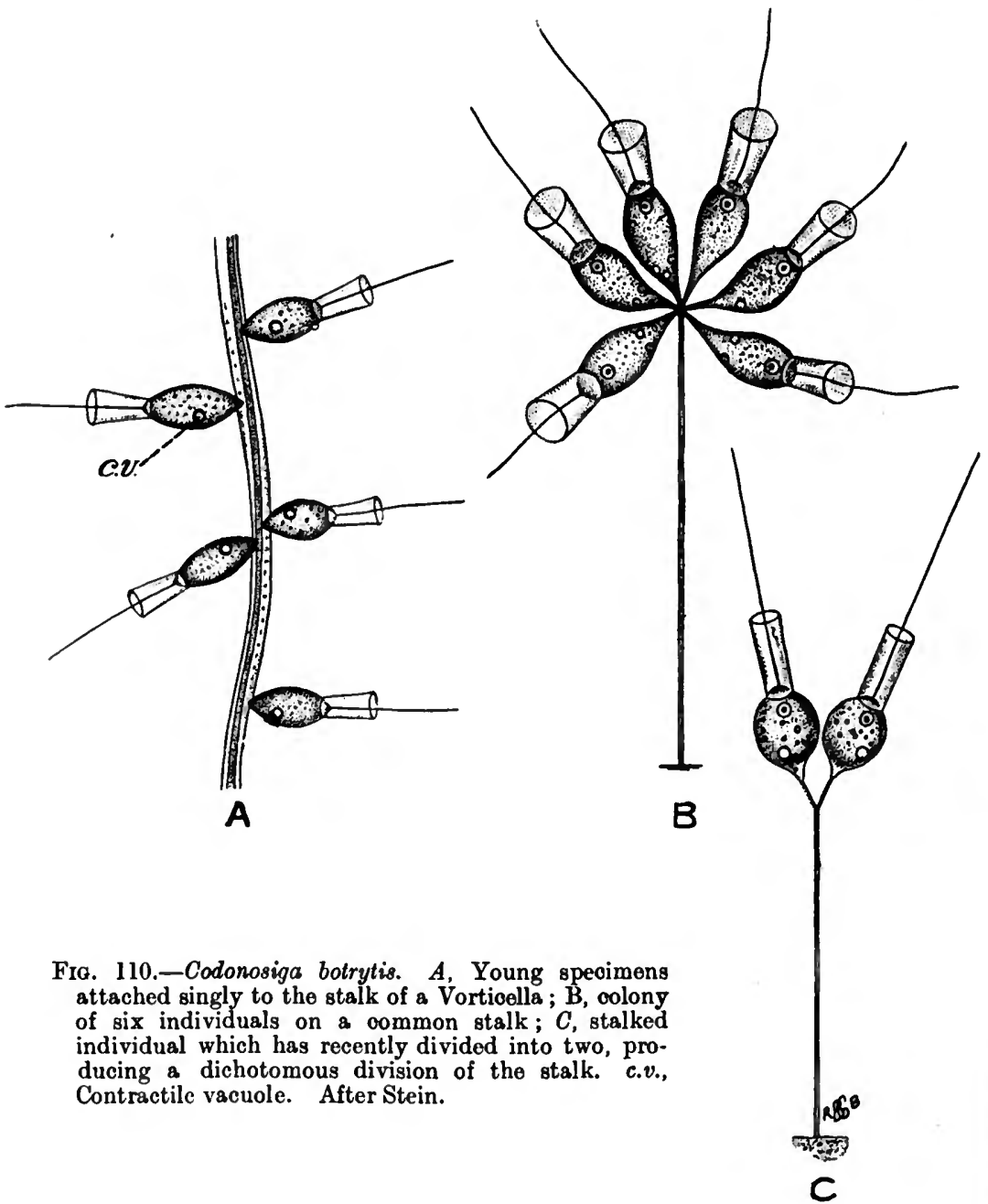

FIG. 110.-Codonosiga botrytis. A, Young speoimens attached singly to the stalk of a Vorticella; $\mathrm{B}$, colony of six individuals on a common stalk; $C$, stalked individual which has recently divided into two, producing a dichotomous division of the stalk. c.v., Contractile vacuole. After Stein.

trailing flagellum, free from the body, in the one form is represented by the marginal flagellum of an undulating membrane in the other - as, for exayple, Trichomastix and Trichomonas (Fig. 5), Prowazekia (Fig. 141), and Trypanoplasma (Fig. 36).

In one group of flagellates-hence known as the Choanoflagellata 
or Craspedomonads (Fig. 110)-a peculiar structure occurs, known as the "collar," a delicate protoplasmic tube or funnel which arises along a circular base-line of which the insertion of the flagellum is the centre, and so forms a cup, sleeve, or collar-like structure surrounding the flagellum for about a third or a half of its length. It is stated, both for Choanoflagellates and for the very similar collar-cells of sponges, that the collar is a membrane folded in a spiral manner, its insertion running along the body and round the base of the flagellum; but the spiral structure is not easy to make out. The Choanoflagellates are sedentary forms which, if set free temporarily from their attachment, swim with the flagellum directed backwards, doubtless the mechanical result of the presence of the collar. The function of the collar is probably connected with the capture and absorption of food-particles wafted towards the body by the flagellum. The collar is retractile, but is not capable of active movements such as are seen in an undulating membrane.

The organs of nutrition must be considered in connection with the four modes of life already mentioned.

(a) In holozoic forms the organism captures and ingests other organisms of various kinds. In some forms the ingestion of foodparticles may take place at any point on the body-surface ; examples of this are the amœboid forms, such as Mastigamoba, which capture their food by means of their pseudopodia, like an amœba; the holomastigote genus Multicilia (Fig. 113); the parasitic Lophomonas (Fig. 45), and possibly others. But in most cases food-particles are ingested at the base of the flagellum, the spot towards which they are propelled by the activity of the flagellum itself. There may, however, be no special aperture for food-ingestion, particles which impinge upon the soft protoplasmic body being simply absorbed directly with formation of a food-vacuole. With a more advanced type of organization, a special aperture or cytostome for the ingestion of food-particles is found at the base of the flagellum. The cytostome may be a simple aperture leading through the cuticle directly, or by means of a funnel-shaped depression, into the protoplasmic body, or it may, in more highly organized forms, lead into a special tube, termed an " œsophagus" or "cytopharynx," which receives the evacuations of the contractile vacuoles, and serves for excretion is well as ingestion (Fig. 84). In any case the cosophagus ends blindly in the fluid endoplasm. There is no special anal aperture for expulsion of fæcal material, which is expelled at any point of the body-surface in primitive forms, or through the cosophagus and cytostome in those more highly organized.

(b) In holophytic forms the organs of nutrition are those of the plant-cell (p. 188)-namely, chromatophores, or corpuscles containing chlorophyll or allied pigments ; pyrenoids, small glistening bodies 
embedded in the chromatophores, the centres of the formation of amyloid substances; and grains of amyloid nature formed by the constructive metabolism of the organism. It is also common to find in the holophytic flagellates a peculiar red spot, or stigma, placed near the anterior end of the body, and probably sensitive to light (p. 205).

In general, two types of holophytic flagellates can be recognized : first, forms in which, in addition to the organs already mentioned, those pertaining to the holozoic mode of nutrition are also present; secondly those possessing only the holophytic apparatus. The first type may be regarded as more primitive forms in which the holophytic habit of life has not become so engrained as to exclude any other mode of nutrition; but a change is still possible, and the organism can combine or vary the holophytic with the holozoic or saprophytic method. In the second type the organism has become plant-like, to the complete exclusion of other methods of nutrition; the body is generally enclosed completely in a firm cellulose envelope, allowing diffusion of liquids and gases, but without apertures through which foreign bodies can pass into the interior. Such forms, if they lose their flagellum in the adult state, are classed as unicellular Algæ, and the young flagellated individuals are termed "zoospores." The transition from holophytic flagellates to plants is a gradual one, and the border-line is simply fixed by the characters of the "adult," and is therefore as arbitrary as that between Sarcodina and Mastigophora discussed in a previous chapter.

(c) In saprophytic and parasitic forms no special organs of nutrition are present, since the food is absorbed in a fluid condition from the surrounding medium.

Contractile vacuoles are commonly present in those flagellates which inhabit fresh water. In the more primitive forms the vacuoles empty themselves direct to the exterior. In more highly organized types the vacuoles open into the cesophagus. In Euglena the two contractile vacuoles open into a reservoir-vacuole, which, according to Wager (213), is in open communication with the cosophagus (Fig. 84).

The nuclear apparatus consists, as a rule, of a single nucleus of vesicular type, with a distinct karyosome. Chromidia are generally absent, but are found in a few cases (Rhizomastigina).

The relations of the nuclear apparatus and the flagella have been discussed above, and are briefly as follows:

1. There is a single nucleus with a single centriole, which functions at the same time as centrosome and blepharoplast. Then either $(a)$ the centriole is within, or connected intimately with, the nucleus, in which case the flagellum appears to ariso directly from the nucleus, as in Mastigina (Fig. 38); or (b) the centriole, and the flagellum it gives off, are quite independent of the nucleus, as in Mastigella (Fig. 40). 
2. There is a single nucleus with its centrosome, and in addition one or more blepharoplasts in relation to the flagellar apparatus. Then $(a)$ at division the old blepharoplasts and flagella are lost, and new blepharoplasts arise during or after nuclear division from the centrosomes ; or $(b)$ the blepharoplasts and flagella persist, and the former divide independently to form daughter-blepharoplasts from which new flagella arise (Fig. 43).

3. In a certain number of Flagellata, grouped provisionally as Hæmoflagellates or Binucleata (see next chapter), two nuclei, each probably possess. ing its own centrosome, are present : a principal or trophic nucleus and an accessory or kinetic nucleus.

In Type 2 the blepharoplast attains to a greater or less degree of independence of the centrosome, and divides independently of it for many generations of ordinary vegetative reproduction by fission. But there are probably in all cases periods in the life-cycle when the cntire nuclear apparatus is reduced to a single nucleus and centriole, from which the condition in the adult, whatever it may bo, arises. For the so-called fourth typo of Hartmann and Chagas (62), see below (p. 273).

Reproduction and Life-Cycle.-The commonest method of reproduction is simple or binary fission in the free state. The products of the fission are of equal size, and the division of the body is invariably longitudinal (Senn, 358) - that is to say, along an axis continuing the direction of the principal flagellum or flagella. In addition to this, the typical method of reproduction, other types of division occur. Multiple fission in the free active condition is known in some parasitic forms, such as Trypanosoma lewisi and Lophomonas blattarum (Janicki, 70). On the other hand, fission may sometimes take place in a resting, non-flagellated condition, or within a cyst; in the first case it is frequently, in the second perhaps always, of a multiple type.

The occurrence of syngamy in the life-cycle is a point which has been disputed, probably owing to the fact that in forms of simple structure it takes place only at long intervals in the lifecycle, or under special conditions. Moreover, the longitudinal division prevalent in this group makes it practically very difficult to decide, except by continuous observation, whether two conjoined flagellates are individuals about to fuse in syngamy or to separate after fission. In thie colonial Phytomonadina, where highly-differentiated gametes are found, the occurrence of syngamy has long been known, but the existence of sexual processes in other flagellates has been doubted by high authorities. In recent years, howcver, it has been observed in a number of forms, and there can be no doubt of the existence of sexual processes in flagellates generally. A summary of recent observations, with full references, is given by Dobell (335, pp. 109-111). The available data are as yet insufficient to make it possible to give a connected account of syngamic processes in Flagellata generally, and only a few typical cases can be dealt with here.

A simple type of syngamy has been described in Copromonas 
subtilis (Fig. 111) by Dobell (335). In this species the two gametes appear perfectly similar to each other, and are not, in fact, distinguishable in any way from ordinary individuals of the species. Two such individuals come together and unite by their anterior or flagellar

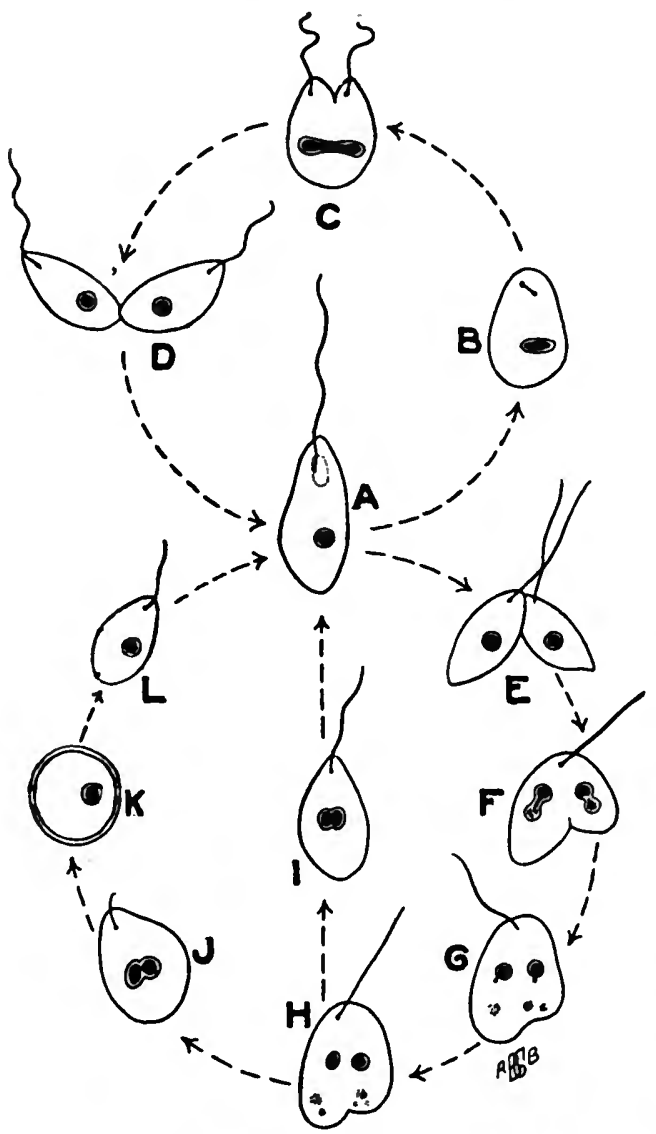

Fia. 111.-Life-cycle of Copromonas subtilis. $A$, Ordinary adult form; $B, C, D$, "vegetative" reproduction by binary fission; $E-J$, stages of reduction and syngamy: $F, G, H$, reduction; $I, J$, fusion of the two pronuclei; the zygote (I) may develop into an ordinary free-swimming individual, or $(J)$ may retract its flagcllum and become encysted; $K$, cyst ; $L$, liberation of an adult form from the oyst. After Dobell (335). extremities. In one gamete the flagellum is lost, and the coupleswims about by means of the remaining one; this is the only difference between the two gametes which could be interpreted as one of sex. While fusion of the bodies is still incomplete, the nucleus of each gamete divides by a simple type of promitosis (p. 109). One of each pair of sisternuclei thus produced is a reduction - nucleus, which degenerates; the other persists. The persistent nucleus of each gamete then divides a second time, but into two very unequal halves; the smaller nucleus in each case degenerates as a reduction-nucleus, while the larger persists as the pronucleus. The bodies of the gametes are now completely fused, and the fusion of the pronuclei follows. The zygote may become encysted at once, or may continue to live a free life. In the first case the fusion of the pronuclei takes place within the cyst, from which it is ultimately set free as an ordinary individual which feeds and multiplies vegetatively. In the second case the zygote becomes an ordinary free individual at once, the interlude of encystment being omitted. 
The syngamy of Copromonas is thus seen to be a case of perfect isogamy, and is probably to be regarded as representing a very primitive type, whence the more complex sexual processes of other Flagellata have been evolved-(1) by greater specialization and differentiation of the gametes in their relation to other phases of the life-cycle (gamete-formation) and to one another (sexual differentiation); (2) by correlation of the sexual phases with definite crises, to which they become restricted, in the general life-cycle.

In the Rhizomastigina sexual processes occur of a type resembling those found in the Sarcodina to such an extent as to indicate that the affinities of this group is rather closer to some of the primitive Rhizopods than to typical Flagellata. The life-cycle (Fig. 112) has been worked out in full detail in Mastigella vitrea by Goldschmidt (41). Vegetative reproduction in the free state is by binary fission of the ordinary type, and occurs when food is abundant ; a falling-off in the supply of nutriment leads to gamete-formation and syngamy. In the earliest stages of the sexual generation a differentiation of the individuals into macrogametocytes and microgametocytes is to be observed, though externally they are similar to ordinary individuals and continue their vegetative life during the early stages of gamete-formation. In the macrogametocyte, first a quantity of nucleolar substance, and then of chromatin, is set free from the nucleus; these two substances unite to form a chromidial mass from which a number of secondary nuclei are formed. The secondary nuclei become scattered through the cytoplasm, and each becomes surrounded by a protoplasmic body. The small cell thus formed is a macrogamete, which goes through reducing divisions. The still acrive macrogametocyte, which has its cytoplasm crammed with the small gametes, now becomes encysted. Within the cyst the gametes acquire flagella and become motile. At this stage the original nucleus of the gametocyte breaks up and disappears rather suddenly. Finally the cyst-wall is ruptured and the flagellated gametes escape.

The formation of the microgametes takes place in a manner essentially similar to that already described for the macrogametes, but with a few differences in detail. The microgametocytcs become encysted at the very beginning of the process; then formation of chromidia begins, and as soon as it is completed the primary nucleus degenerates; the microgametes have no flagella, and are shot out of the cyst when it bursts.

The free macrogametes measure on the average about $3.6 \mu$ diameter, and have a flagellum 15 to $18 \mu$ in length; the microgametes are $2.8 \mu$ in diameter, and have no flagellum. A macrogamete seeks out a microgamete and fuses with it, cytoplasm and nucleus. The zygote retains the flagellum of the macrogamete, and becomes a small, monad-like individual which multiplies by fission as such. After several generations the monads cease to multiply, and each grows up into an adult Mastigella. A development similar in the main is described by Goldschmidt for Mastigina, but some of the phases escaped his observation.

Comparing the sexual cycle of Mastigella (Fig. 112) with that of Copromonas (Fig. 111), the chief difference is seen to be that in the former an ordinary individual does not become a gamete directly but a gametocyte, which by a process of multiple fission gives rise to a generation of minute swarm-spores, the gametes. In the two sexes a slight differentiation of the gametes is seen. Further, in the life-cycle of Mastigella considered as a whole, there are two forms of individuals, each capable of multiplying vegetatively for many 
generations-namely, the monad form, product of syngamy, and the adult, mastigamœba-form, which ultimately produces the monad-like gametes. Hence the life-cycle in such a type is an alternation of generations (metagenesis), which, as in so many other

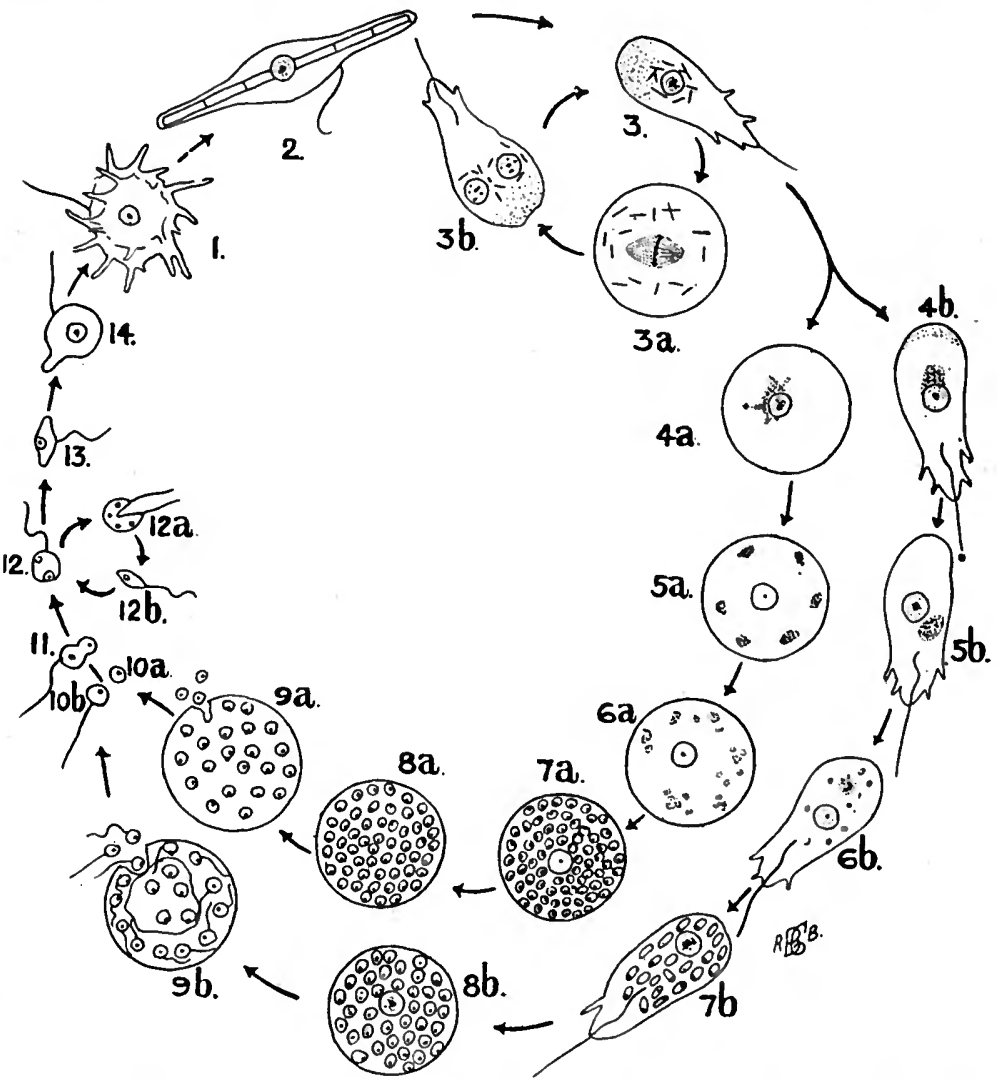

Fig. 112.-Life-cycle of Mastigella vitrea, diagrammatie. 1, 2, and 3, Different forms assumed by the adult "vegetative" type of individual ; $3 a, 3 b$, reproduction by binary fission; 4-10, gamete-formation; $a$ (in each case), miorogamete-formation, $b$, macrogamete-formation ; in the former the gamont becomes encysted, and the principal nueleus degenerates early in the process; in the latter the gamont remains motile and the principal nucleus persists to the last: 4-6, extrusion of chromidia from the nucleus and formation of secondary nuclei; 7,8 , formation of tho gametes round tho secondary nuclei ; 9 , extrusion of the gametes; $10 a$, the small, non-flagellated miorogametes; $10 b$, the larger, flagellated macrogametes; 11 , copulation of tho gametes; 12,12a, 12b, multiplication by binary fission of the monad-like zygote ; 13, 14, growth of the monad-form, after a period of multiplication, into the adult mastigam oba-form. After Goldschmidt (41).

aases in the animal kingdom, appears to have come ahout by multiplicative processes taking place in a larval type, phylogenetically older-namely, the monad form, the only form of individual that 
occurs in the life-cycle of Copromonas. In Mastigina, on the other hand, the monad form developed from the zygote apparently does not multiply by fission, but develops directly into the adult formperhaps a more primitive state of affairs.

A very instructive series is furnished by the colony-forming Phytomonads of the family Volvocida. At one end of the series are primitive types, such as Stephanosphora, where the colony is composed of eight monad individuals, all alike, which may be agamonts in one colony or gamonts in another. Each agamont multiplies by fission to form eight small cells, which remain connected together and grow into full-sized monads, thus giving rise directly to new colonies. In the gamont-colonies each gamont (gametocyte) gives rise by multiple fission to a large number of minute biflagellate swarm-spores, the gametes, which are set free and copulate. The syngamy is perfectly isogamous. The zygote grows in size, and finally multiplies to form the eight monads of a new colony.

At the other end of the series are the species of the genus Volvox, in which the colony is composed of a great number of individuals, which may be of three kinds, not necessarily all present in the same colony : (1) The ordinary "somatic" monads, locomotor and trophic in function, which do not reproduce themselves in any way ; (2) agamonts, so-called "parthenogonidia," which multiply by fission to form daughter-colonies; (3) gamonts or gametocytes, which are sexually differentiated as " microgonidia" and " macrogonidia." The microgonidia produce by multiple fission a swarm of small biflagellate microgametes, comparable to the gametes of Stephanosphara. In the macrogonidia, on the other hand, multiplicative processes are in abeyance, and each becomes a single, ovumlike macrogamete, which is fertilized by the relatively minuto microgamete. Thus, the syngamy in Volvox is anisogamous to the highest degree; and, as in other cases among Protozoa, this condition appears to have arisen from a primitive isogamy in which, in both sexes, the gametocytes sporulated to produce a swarm of minute gametes, by the process of sporulation becoming altogether suppressed in one sex-namely, the female-while retained in its primitive form in the other. The colonies of Volvox, with their differentiation of individuals, exhibit a condition transitional to that of the Metazoa. The trophic, non-reproductive individuals, taken as a whole, may be compared to the Metazoan soma, the reproductive individuals to the germen. In Pleodorina californica distinct male, female, or parthenogenetic colonies occur (Chatton), as is the case in some species of Volvox.

Classification.-The Flagellata are classified in different ways by differont authors, and in the present state of our knowledge of the group no system can be regarded as in any way final. As in other groups of Protozoa, thero 
are a certain number of well-defined orders and families characterized by the possession in common of certain features of organization which leave no doubt as to their taxonomic homogeneity. On the other hand, thero aro a large number of primitive forms whose characteristics are mainly of a negative order, and of which the affinities are in consequence vague and uncertain, the systematic position debatable. There is, moreover, frequently an element of uncertainty, in the case of many forms, as to whether they represent truly specific adult forms, or merely developmental stages of some other species of the Flagellata or Sarcodina. Finally there are a certain number of species and genera concerning which it is still debated whether they should be assigned to the Mastigophora or some other class of Protozoa.

Hartmann and Chagas (62) have proposed to utilize the relations of the flagellar to the nuclear a pparatus for systematic classification of the Flagellata, as suggetsted also by Prowazek (354). But, apart from the fact that these relations have as yet been investigated in very few flagellates, and that in such minute objects the details are very difficult to make out and liable to be a subject of dispute, it may be doubted whether these points of structure are sufficiently constant to be of classificatory value in this subclass, since they sppear to vary considerably in allied forms. Thus in Copromonas subtilis, according to Dobell (335), the blepharoplast persists through divisionphases. and divides independently of the nucleus; but in $C$. major, according to Berliner, the old blepharoplast and flagellum are lost at each division, and a new blepharoplast, from which the new flagellum grows out, is formed by division of the nuclear centriole in each daughter-individual. Again, the third type of flagellar insertion (p. 263) is found in the Trypanosomida, allied to the Cercomonadida, and in the trypanoplasms, which belong to the family Bodonido, as shown in the next chapter. Classification by these characters is, therefore, at least premature, if not fallacious. Compare also Senn (358).

The classification adopted here is in the main that of Doflein (7), with certain modifications. For convenience a number of forms are put together in the Pantastomina, without, however, claiming that this order is anything more than a cataloguer's makeshift for disposing of a number of forms of dubious position and uncertain affinities.

Order I. : Pantastomina.-Holozoic, with no definite mouth-opening ; food-particles ingested at any point on the surface of the body.

Suborder 1 : Rhizomastigina.-Body amœboid; food captured and ingested by means of pseudopodia.

Several genera, only known as yet from fresh water, are referred to this very interesting group; such are Mastigamaba, F. E. Schulze, Mastigina, Frenzel (Fig. 38), and Mastigella, Frenzel (Fig. 40), distinguished from one another by the nature of their amceboid movement and the characters of their pseudopodia. In appearance the species resemble amœbæ which possess a long and well-developed flagellum, or in Dimastigamoeba two, in Trimastig. amceba (Whitmore, 280) three flagella. Locomotion and food-capture aro carried on for the most part as in an amcba, and the flagellum appears to function chiefly as a taotile organ in the adult mastigamceba-phase; in the young monad-phase, on the other hand, the flagellum is the sole organ of locomotion and food-capture, as in an ordinary flagellate. The relation of the flagellum to the nucleus is of Type 1 described above (p. 263), a single centriole which functions both as centrosome and blepharoplast ; in Mastigina and Mastigamaba the flagellum arises from the nucleus (Type la); in Mastigella the origin of the flagellum is distinct from the nucleus (Type 1b). The life-cycle of Mastigella is described above (p. 265). In many points, especially in the formation of secondary gamete-nuclei from chromidia, the development resembles more that of the Sarcodina than that of the Flagellata, and by many authorities the affinities of the Rhizomastigina are considered to be rather with the first of these two classes. The mastigamœbæ certainly link the true flagellates with the Proteomyxa and Mycetozoa; and if the flagellum were lost in the adult phase, they would be classed in the Sarcodina without hesitation. 

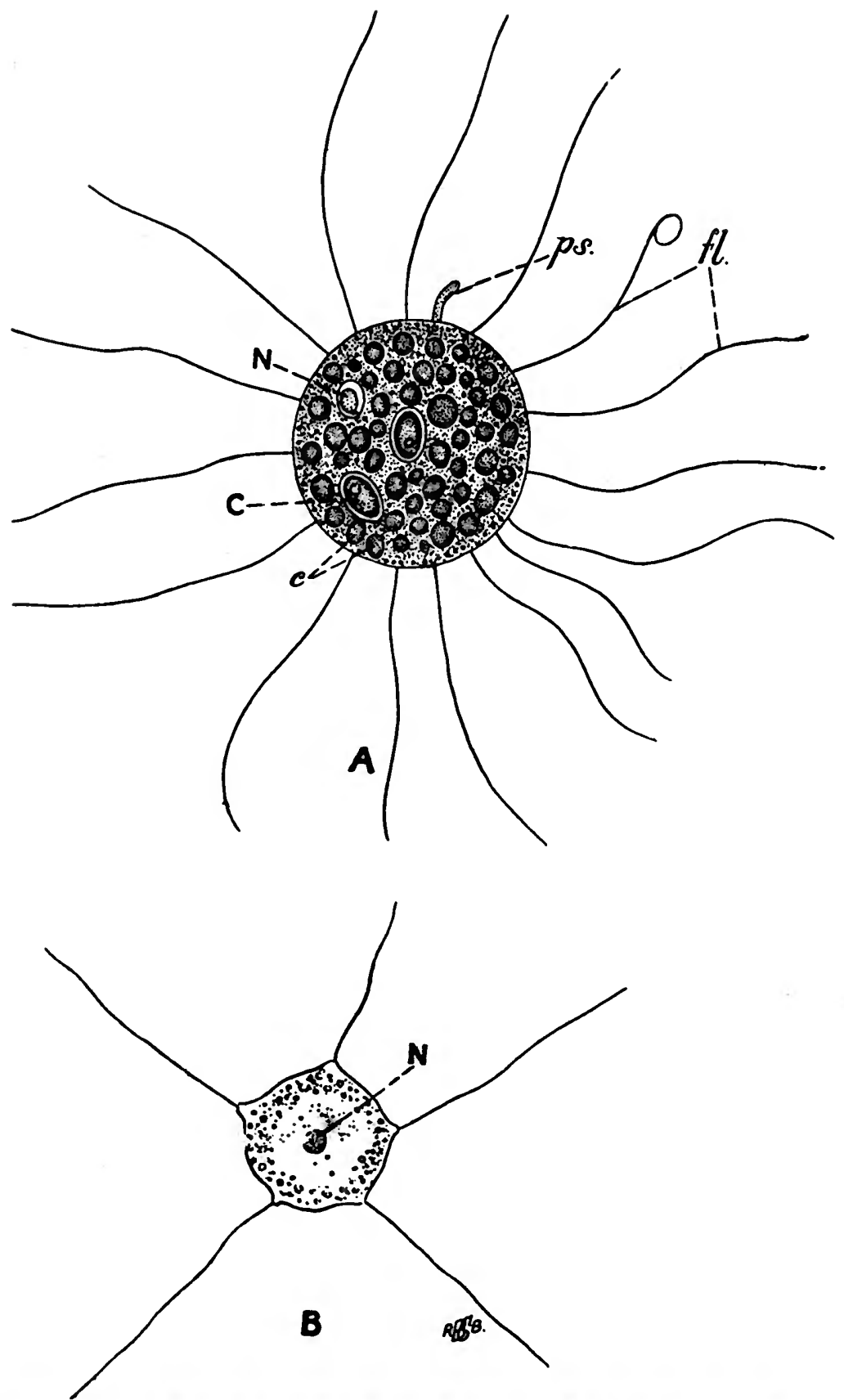

Fia. 113.-A, Multicitia lacustris, after Lauterborn. A., Flagella, one of which is curled up into loop; ps., pseudopodium-like process ; $N$., one of the nuclei (the others are hidden by the ingested food-masses); $C$., ingested Chlamydomonads ; $c$., ohlorophyll-bodies, the remains of other Chlamydo. monsds in process of digestion. B, Multicilia palustris, after Pénard. N., The single central nucleus. 
Suborder 2 : Holomastigina. - With numerous flagella radisting from a spherical or approximately spherical body.

This suborder contains the single genus Multicilia, Cienkowski, to which several species, some fresh-water, some marine, have been referred. The number of flagella varles in different species, and their precise relation to the nuclear apparatus remains to be made out. $M$. lacustris, Lauterborn (Fig. 113, $A$ ), is multinucleate ; $M$. palustris, Pénard (Fig. 113, B), has a single nucleus. The body is not covered by a cuticle, and may throw out pseudopodia, or even become amcboid (Lauterborn). Nothing is known of the life-cycle, but in $M$. lacustris Lauterborn observed reproduction by simple fission (plasmotomy ?). In the present state of our knowledge adequate data are lacking for discussion or the affinities of this genus. Doflein (7) regards it as a form lying at the root of the Infusorian stem, and derives the most primitive Ciliata from a form similar to Multicilia, in which the numerous flagella become specialized in structure and movement to give rise to an even coat of cilia ; Pénard (302), on the other hand, considers Multicilia allied to the Heliozos (p. 249). It is clear that the genus is one which would repay further study.

Order II.: Protomonadna.-Flagellates for the most part of small or minute size ; with a single flagellum ; or with a principal and one or two accessory flagella; or with two flagella, one directed anteriorly, the other posteriorly as a trailing flagellum. Nutrition holozoic, saprophytic, or parasitic ; in the first case the food-particles are ingested at the base of the flagellum, where a definite mouth-opening may be present or absent, but without a distinct cesophagus in any case. The contractile vacuole is generally single, if present, and empties itself direct to the exterior.

This order comp.ises a vast assemblage of genera and species, subdivided by Doflcin into eight families, one of which, the Trypanosomida, including the important parasitic genus Trypanosoma, is discussed in detail in the next chapter. The cuticle is generally thin, and the body is often capable of amcboid or metabolic movements; if amcobid, however, the flagellum is the organ of locomotion, so long as it is present, and not the pseudopodia. The relations of the flagellum to the nuclear apparatus are, in general, of the second type (p. 263), according to Hartmann and Chagas (62)-that is to say, with distinct centrosome and blepharoplast; but it is extremely probablo that in the simpler forms Typo 1 occurs also (compare Alexeieff, 327), and in the Trypanosomida the distinctive feature is the possession of Type 3, with trophonucleus and kinetonucleus, as also in some of the Bodonida (Prowa. zekia). The life-cycle of the free-living forms is probably in general of a simple type, similar to that described above in Copromonas (Fig. 111); but observations on the sexual processes are at present very scanty.

For a detailed description of the forms included in this order the reader must be referred to the larger treatises, especially Bütschli (2) and Senn (320); it must suffice here to mention some of the more typical forms. Cercomonas, type of the family Cercomonadidae (Fig. 114). has a single flagellum; the hinder end is frequently drawn out into a long tail-like process, and is capable of change of form. E Ecomonas (Oikomonas) differs in having the body rounded.

Monas, type of the family Monadidoe, has a principal flagellum and one or two accessory flagella. Cladomonas and Spongomonas (Figs. 41, 42) form arborescent colonies; the constituent monads have two flagella of equal sizo, both directed forwards. Alexeieff (327) considers that the Monadidoe should be placed in the suborder Chrysomonadina (see below).

Bodo (Fig. 115), type of the family Bodonidce, has two flagella, one directed forwards, the other backwards as a trailing flagellum; the species of this genus are free-swimming and do not form colonies; they occur both free: living and parasitic, for the most part in the digestive tracts of various animals. Bodo lacertos, from the cloaca of Lacerta spp., has been studied by Prowazek (354), who has described a process of autogamy, but doubt has been cast upon his observations by Dobell (336). Note also the occurrencs of Bodo-like forms in the development of Cryptodiffugia (p. 230, supra). The flagellato 
recently described by Wenyon (361) from a culture of human frecs, and referred by him to the genus Cercomonas, would appear rather to belong to the genus Bodo. To the family Bodonida must be referred also the genera Prowazekia and Trypanoplasma, dealt with in greater detail in the next chapter. Helcomastix, Senn (358), is to be referred to the Bodonidee or made the type of a distinct family; its two flagella of unequal length are both directed backwards in movement.

Finally, mention must be made of the group of flagellates characterized by the possession of a collar (see p. 261, supra), and hence commonly known as "choanoflagellates" or "craspedomonads." They are sedentary forms, attached by the end of the body opposite to the flagellum, and may remain single, but more usually form colonies often of considerable extent (Fig. 110). The flagellum is used mainly for food-capture, in which the collar also probably plays an importent part ; but an individual may become detached from its
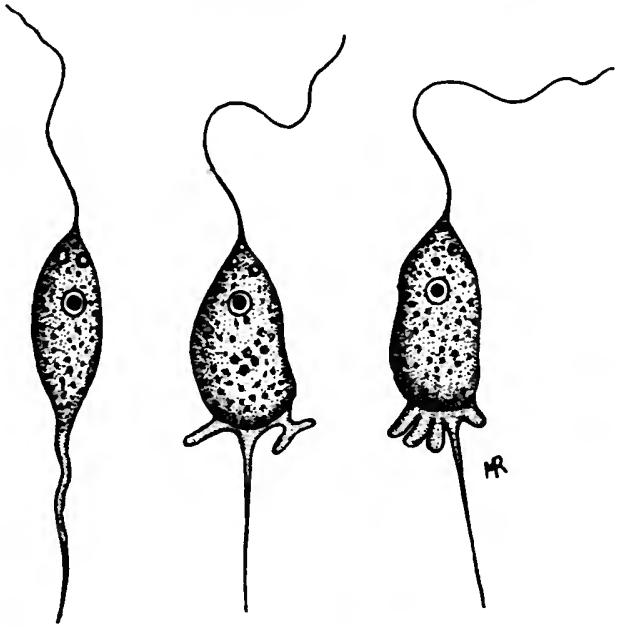

Fiu. 114.-Cercomonas crassicauda, Dujardin, showing amoboid changes of form. After Stein. support, and swim freely, the flagellum being then directed backwards. The systematic position of the choanoflagellates has been differently estimated by different suthors; by some they have been ranked as a primary subdivision of the Flagellata, which are then divided as a whole into Choanoflagcllata and Lissoflagellata, the second of these divisions being used to include all other flagellates. Since, however,

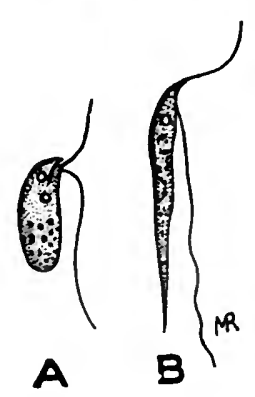

Fia. 115.-A Bodo saltans, Fin renberg. B. Bodo gracilis, Stein. After Stein. the choanoflagellates scarcely differ from ordinary monads except in the possession of the characteristic collar, a specialization of the food-capturing function related to a sedentary life, they are now generally ranked as a family of the Protomonadina, the Choanoflagellida.

Order III. : Polymastigina.-Flagella from three to eight in number, usually all more or less equal in size; in other points of structure similar to the last-mentioned order. Two families, which are sharply marked off from one snother, are referred to this order.

1. Tetramitida, with three or more flagella, which all arise at the anterior ond close together. The flagella may all be directed forwards, or one of them may be turned backwards as a trailing flagellum; in the latter case the trailing flagellum may or may not be united to the body by an undulating membrane.

The species referred to this family are for the most part parasitic. Endoparasitic forms of common occurrence, especially in the digestive tracts of vertebrates, are Trichomastix, with three anterior flagella and a free trailing flagellum, and Trichomonas (Fig. 5), with the same number and arrangement of the flagella, but having the trailing flagellum united to the body by an undulating membrane. These two forms occur frequently in the same host, and are porhaps to be interpreted as two developmental phases of the same 
organism rather than as distinct generio types. Trichomonas hominis is ontozoio in the human intestine, $T$. vaginalis in the human vagina; they appear to be harmless scavengers rather than parasites. The encystment of Trichomonas has been tho subject of some controversy. Acoording to Alexoieff (326), the supposed cysts of Trichomonas described by various authors are in reality independent vegetable organisms, of the nature

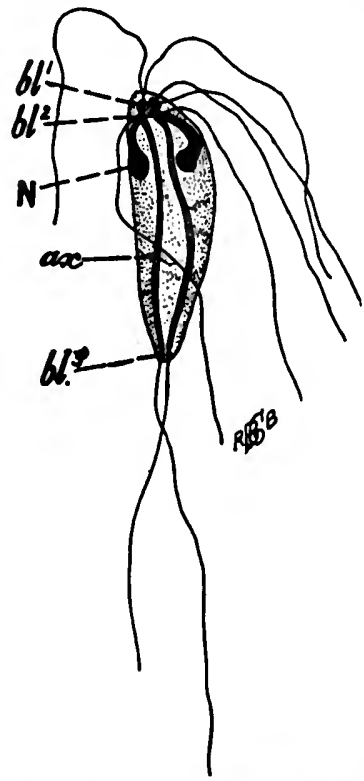

Fro. 116.-Octomitus dujardini. bl. 1 , Anterior blepharoplast, from which the first and second flagella of that side of the body arise; $b l .^{2}$, second blepharoplast, giving off the flagellum of the third pair; $N$., left-hand nucleus ; $a x$., left axostyle ; $b l .^{3}$, thind blepharoplast, at the extremity of the axostyle, giving off one of the flagella of the fourth pair. All the structures indicated are paired, and the letters indicate the member of each pair on the left side of the body. After Dobell (236). of yeasts. In some species of Trichomonas the anterior flagella are four in number (Alexeieff, 323) ; for such forms Parisi (A.P.K., xix., p. 232) has founded a subgenus Tetratrichomonas. The genus Macrostoma, socording to Wenyon (362), differs from Trichomonas in having the undulating membrane wedged in a deep groove; $M$. mesnili ocours in the human intestine. According to Alexeieff (324), Macrostoma is a synonym of Tetramitus. Monocercomonas, including a number of common intestinal parasites, has four anterior flagella of equal length, or two longer, two shorter (Alexeieff, 325).

Costia necatrix, also referred to this family, is ectoparasitic on the skin of fishes. According to Moroff, it has four flagella in two pairs, two larger and two smaller, all of which serve for locomotion; but the larger pair are used also for fixation, and the smaller pair for wafting into the mouth the food-particles, which consist chiefly of dead epithelial cells torn away from the epidermis (see also Neresheimer).

2. Octomitido.*-With six or eight flagella, arranged in pairs; the body is bilaterally symmetrical in structure. Entozoic forms, for the most part of intestinal habitat.

The remarkable bilateral symmetry of the species of this family is not merely an external characteristic of the body, but affects the internal structure as well, and the entire nuclear structure is doubled, with right and left halves. Octomitus (synonym, Hexamitus; see Dobell, 236), with four pairs of flagella (Fig. 116), includes a number of entozoic species-e.g., $O$. intestinalis, from the cloaca of the frog and other animals. Lamblia intestinalis (synonym, Megastoma entericum, Fig. 117) is a common inhabitant of the human intestine. It becomes encysted, and is probably disseminated in this form. Within the cyst it divides into two (Rodenwaldt). L. sanguinis, described by Gonder (A.P.K., xxi., p. 209) from the blood of a falcon, is probably an intestinal parasite gone astray (vide p. 258).

The order Polymastigina differs little from the Protomonadina except in the complication of the flagellar apparatus, correlated probably with the entozoic habit. Hartmann and Chagas propose to merge the Polymastigina

* Doflein terms this family the Polymastigida, but the name is clearly inadmissible, since the genus Polymastix belongs to the preceding family, and is closely allied to Trichomonas, but has six anterior flagella and no trailing flagellum (compare Alexeieff, 325). 
in the Protomonadina, and then to divide the order into two suborders; the first, entitled the Monozoa, would include the Protomonadina as constituted above, with the exception of the Trypanosomida ("Binucleata"), and with tho addition of the Tetramitida. The second suborder, Diplozoa, would include only the Octomitida. This arrangement certainly seems more natural than that which is usually adopted, so far as the Tetramitidas and Octomitida are concerned.

Order IV.: Eualenoidins.-Larger forms, with mouth-aperture and œsophagus; with a complex vacuole-system oponing into the cesophagus; often with holophytic apparatus, ohromatophores, stigma, etc.

This order represents, so far as struotural complication of the individual is concerned, the highest type of organization among Flagellata. The body. may be metabolic, or of definite contours, with thick cuticle. The free-living

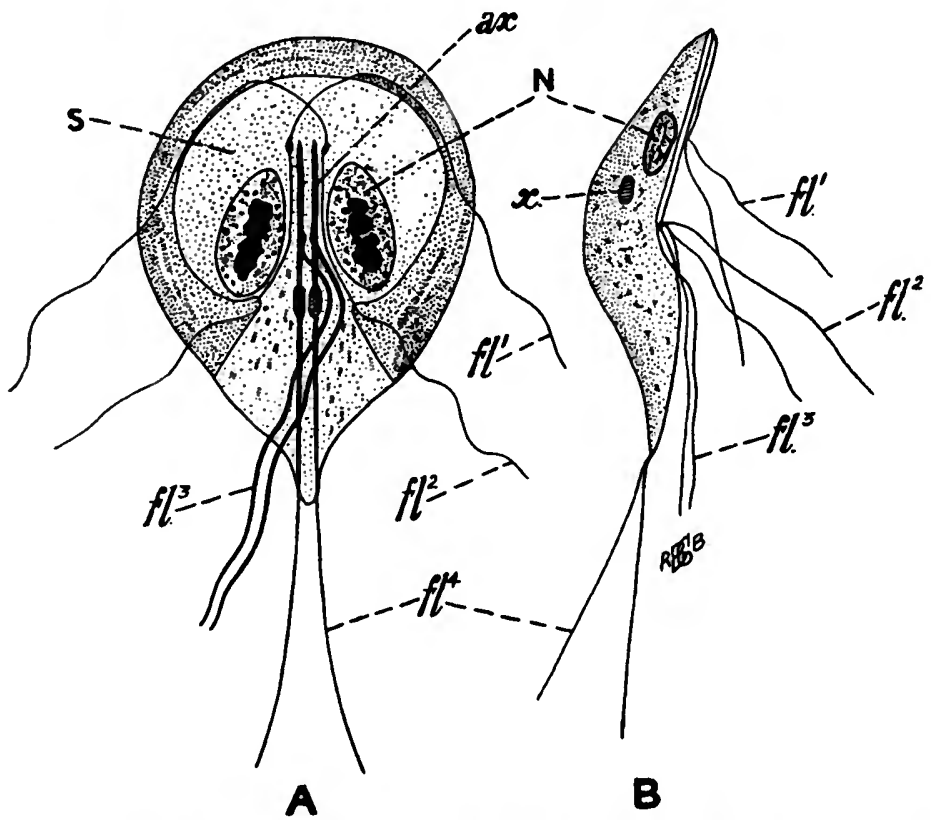

Frc. 117.-Lamblia intestinalis. $A$, Ventral view ; $B$, side view. $N$., One of the two nuclei ; $a x$., axostyles; $f .^{1}, f .^{2}, f .^{3}, f .^{4}$, the four pairs of flagella; 8. , suckerlike depressed area on the ventral surface; $x$, bodies of unknown function. After Wenyon (277).

forms are eithor holozoio or saprophytic, if colourless, or holophytio if provided with chromatophores, in which case they may be capable of ncurishing themselves by more than ono method. The flagellum may be single, or thero may be a second flagellum, usually smaller than the principal fagellum, and somotimes directed backwards as a trailing flagellum. The attachment of the flagellum is of the second typo (p. 263), with blepharoplast distinct from the centrosome. Aocording to Hartmann and Chagas (62), in Peranema trichophorum the centrosome first divides to furnish a blepharoplast, and the latter, having become completely independent of tho nucleus, divides into two, a distal blepharoplast or basal granule of the flagellum, connected by a rhizoplast (centrodesmose) with the proximal blepharoplast or anchoring granule. The authors consider that this should be regarded as a fourth type 
of flagellar insertion, characteristic of this order; but it is simplest to regard it merely as a sccondary complication of the second type, and ono which is not universal in this order, since in Copromonas subtilis the blepharoplast remains undivided, so that this species shows a flagellar attachment strictly of the second type. In Euglena, according to Wager (213), the flagellum paszes through the aesophagus and becomes attached to the wall of the reservoir-vacuole by a bifurcate base. On one of the branches is a distinct thickening in close contact with the stigma (p. 205). The thickening is probably the blepharoplast, and the two branches represent the rhizoplast.

The sexual processes of the Euglenoidina are but little known, and Copromonas is the only genus in which the complete life-cycle has been worked out; in this species it is of a simple type (p. 264, Fig. 111).

The order comprises three families. The first, Euglenida, contains forms provided with chromatophores, holophytic, saprophytic, and parasitic (Haswell) in habit. Examples : Euglena (Fig. 4), Phacus (Fig. 118). The second family, Astasiida, contains the genus Astasia (Fig. 15), colourless

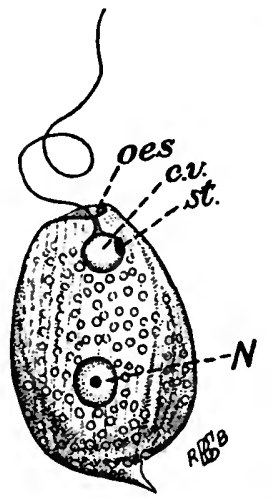

Fio. 118. - Phacus triqueter. œs., Esophagus; c.v., contractile vacuole; st., stigma ; $N$., nuclcus. After Stein. and saprophytic or parasitic. The third family, Peranemida, contains numerous genera without chromatophores, holozoic or saprophytic. Examples: Peranema, Copromonas (Fig. 111). The subfamilies Heteronemina and Anisonemina are hetoromastigote. Example : Anisonema (Fig. 25).

Order V.: Chromomonadina.-Small forms, without œesophagus or vacuole-system, with delicate cuticle and one or two flagella ; their characteristic feature is the possession, usually, of one or two conspicuous chromatophores, green, yellow, or brownish, in colour. The nutrition, for the most part holophytic, may be also holozoic or saprophytic. Divided into two suborders.

Suborder 1: Chrysomonadina.-With one or two flagella and one or two yellowish-brown chromatophores ; body of ten amcboid or metabolic ; colonyformation frequent; nutrition holozoic and holophytic. Three families. Examples : Chrysamoba, Chromulina, Dinobryon, etc. According to Scherffel, Chrysamceba is the amoeboid, non-flagellated phase of Chromulina ; compare also Lauterborn $(345 \cdot 5)$. To this suborder must be referred also tho Coccolithophoridce, marine flagellates which secrete the calcareous shells known as coccoliths (vide Lohmann).

Suborder 2 : Cryptomonadina.-Small forms with one or two flagella, colourless, or with chromatophores ranging in colour from yellowish-brown to olivegreen or blue-green. Holophytic or saprophytic, not holozoic. Examples : Chilomonas, colourless; Cryptomonas, some species of which are symbiotic in Sarcodina (p. 15). Doflein refers the Silicoflagellata to this order (p. 255).

Order VI.: Phytomonadina seu Phytorlagelilata.-Completely and exclusively holophytic, with cellulose envelope and without mouth-aperture.

This order comprises the most plant-like flagellates, to all intents and purposes unicellular algæ which retain throughout life their flagellar apparatus and their motility. The individual is generally small, and the body is, except in one family, of definite form and enveloped in a rigid cellulose envelope which may stand off from the body, and is perforated by pores through which the flagella pass out to the exterior. The flagella are usually two in number, sometimes four, of equal size. The cytoplasm generally contains a large green chromatophore and a red stigma. The flagellar insertion, according to Hartmann and Chagas, is of the second type, as in Protomonadina. The reproduction may take the form of multiple fission within tho body-envelope to form numerous swarm-spores, which when set free may be gametes or agamotes. Colony-formation is frequent in this order (p. 257). 
Three families are recognized. Tho first, reprosented by tho genus Pyra. mimonas, contains primitive forms in which the body is metaboiic and the cellulose envelope is absent. The second family, Chlamydomonadida, compriscs non-colonial forms such as Chlamydomonas, Hamatococcus, etc. Nephro-
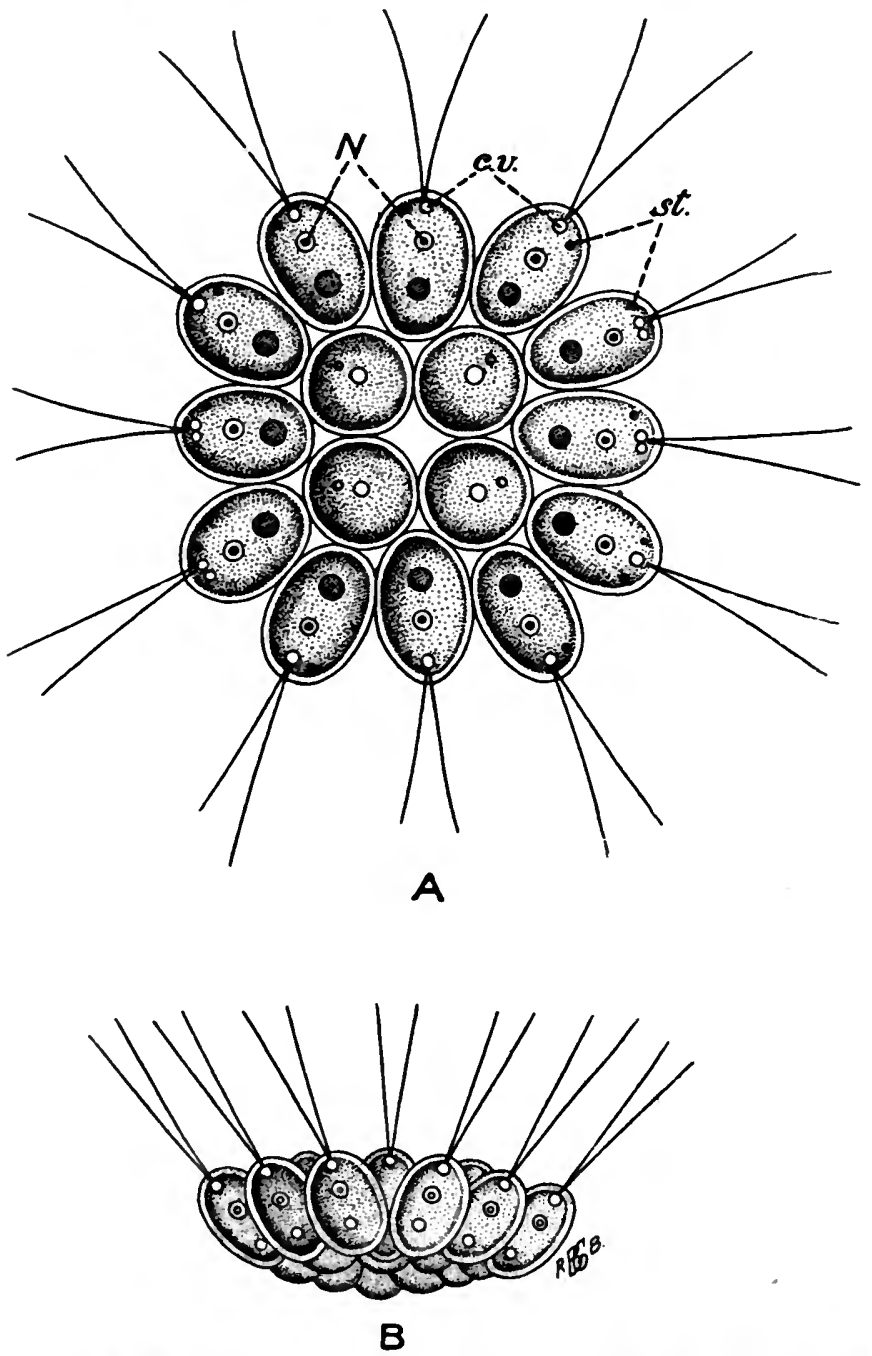

Fro. 119.-Gonium pectorale: colony of sixteen individuals, each with two flagella. $A$, In surface view; $B$, in side view. $N$., Nuclei ; $c . v$., contractile vacuoles; st., stigmata. After Stein.

selmis, referred by Senn (358) to this family, has two flagella, on which it creeps like a Bodo. The third family, Volvocida, comprises colony-forming species in which the individual is similar in structure to the Chlamydomonads, and the colony is composed of individuals ranging in number from four, eight, 
sixteen, or thirty-two, up to many thousands. Examples aro Gonium (Fig. 119), Stephanosphora, Volvox, etc.

In addition to the six orders of flagellates enumerated above, there remain some peculiar parasitic forms, the systematic position of which is extremely duubtful. Such are the family Lophomonadida, nepresented by Lophomonas blattaram, a common parasite of the end-gut of the cockroach and other Orthoptera, and the Trichonymphidee. including the genus Trichonympha and nllied forms, parasitic in the end-gut of termites of various species.

Lophomonas blattarum, which has recently been studied by Janicki (70), bears a tuft of flagella arising at the anterior pole of the body from a doublo ring, or rather horseshoe, of blepharoplasts, situated at the edge of a funnelshaped or cup-like structure, the calyx, which is prolonged into an axostyle (Fig. 45). The nucleus hes within the calyx, whioh is surrounded in its turn by a peculiar thickening or support, termed tho "collar," consisting of free, radially-disposed rods crowded together to form an aureole-like figure, approximately spherical. The nutrition is holozoic, and food-particles are ingested at any point on the body-surface, as in the Pantastomina. Multiplication takes place by binary or multiple fission in the free state ; and division of tho nucleus up to eight within a cyst has been observed, but the entire life-cycle has not been worked out. Associated with $L$. blattarum, another form, $L$. striata, occurs, but it is doubtful if this is a distinct species, or a phase or condition of $L$. blattarum.

The group or family Trichonymphidae comprises a number of peculiar parasites found in the digestive tract of various species of Termitida; such are the genera Joenia, Lophophora, Calonympha, Devescovina, etc., and finally the genus Trichonympha, from which the family takes its name. The chief peculiarity of these forms is the possession of numerous flagella, which may be disposed in tufts at the anterior end of the body, in a manner. similar to Lophomonas (which by some authorities is included in this family), or may be distributed over the whole body, like a coat of cilia, as in the genera Trichonympha, Dinenympha, etc.

According to Hartmann, Trichonympha hertwigi occurs under two forms, which he believes to represent male and female gamonts. They multiply by binary fission, and also by a process of sporulation to produce swarm. spores which are believed to be gametes. Dinenympha also exhibits sexual dimorphism, according to Comes (333).

From Janicki's investigations, there can be no doubt that Lophomonas is a true flagellate, possibly allied to Trichomonas, possibly, however, to the Pantastomina. The genus Joenia, parasitic in Calotermes flavicollis, was thought by its discoverer, Grassi, to connect Lophomonas and Trichonympha; the recently-described genus Lophophora (Comes, 332) also has points of resemblance to Lophomonas, but is remarkable for the presence of undulating membranes running the length of the body. By some authorities, however, the Trichonymphida have been placed with the Ciliata, while Hartmann considers that they should rank as an independent class of the Protozoa.

\section{Subolass II. : Dinoflagemlata seu Peridinlales.}

The characteristic feature of this subclass is the possession of two flagella, which arise close together about the middle of the body. One flagellum (Fig. 120, e) runs longitudinally backwards as a trailing flagellum; the other (Fig. 120, $d$ ) runs transversely round the body. It is further characteristic of this group for the cuticle to be greatly thickened, forming a tough cuirass, or lorica, investing the body. The two flagella are usually lodged in grooves in the cuirass, the longitudinal flagellum in a longitudinal groove or sulcus, the transverse flagellum in a circular groove, or annulus. 
The transverse flagellum executes undulating movements whis: were formerly mistaken for those of a ring of cilia ; hence the name Cilioflagellata formerly applied to this group.

The cuirass, composed of cellulose or an allied substance, is in its typical form a perfectly rigid structure, and is often prolonged into spikes and processes which cause the body as a whole to assume strange or even monstrous forms (Fig. 121). Detailed studies on the skeleton have been published by Kofoid in a series of memoirs (374-383). The nutrition is for the most part holophytic, out in some species ingestion of solid food has been observed. A great many parasitic forms have been made known of recent years (Chatton, 366-369 ; Caullery, 364) ; these are for the most part forms which, in the vegetative, parasitic phase are inert bodies with no sign of locomotor organs, often fixed and pedunculate when ectoparasitic; but in their reproductive phases they betray thcir affinities by the formation of numerous flagellated swarm-spores exhibiting the typical Dinoflagellate structure.

The pelagic species generally possess chroma-

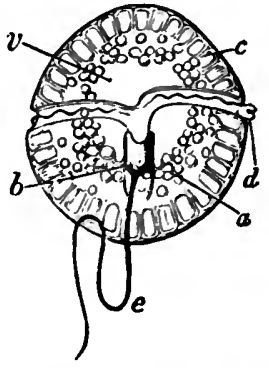

Fra. 120. - Glenodinium cinctum, Eh renberg. a, Amyloid granules; $b$, stigma ; $c$, chromatophores; $d$, flagellum of the transverse groove; $e$, flagellum of the vertical grcove; $v$., vacuole. From Länkester. tophores, and frequently a red stigma, which in some genera-Pouchetia (Fig. 31), Erythropsis-is modified into an eye-like organ. The deep-sea forms, on the other hand, are colourless.

In many Dinoflagellates a peculiar system of vacuoles is found (Fig. 122), consisting of two sacs containing watery fluid, each of which empties itself

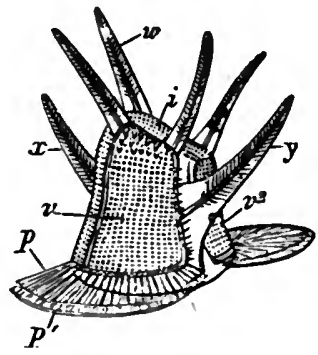

Fia. 121.-Ceratocorys horrida: cuirass. After Stein, from Lankester. to the exterior by its own duct. They differ from ordinary contractile vacuoles in possessing a distinct envelope and in not performing rhythmical contractions, and have hence been given the special name of "pusules" (Schütt). One of these organs, termed the " collecting-pusule." consists of a reservoir-vacuole surrounatd by a ring of smaller vacuoles which empty themselves into it; the other, termed the " sack-pusule," is a large cavity which takes up a great part of the interior of the cuirass. The function of these organs is probably hydrostatic.

The commonest method of reproduction is binary fission in the transverse plane of the body, in which each daughter-individual receives a half of the cuirass of the parent and regenerates the half that is wanting. Fission rapidly repoated may lead to the formation of chains of individuals. In other cases multiple fission within the cuirass has been observed, leading to the formation of swarm-spores which are possibly gametes; but little is known of the sexual processes of these organisms.

The Dinoflagellates are an exceedingly abundant and widespread group, 
highly differentiated as regards forms and species. The vast majority are pelagic in habit, and constitute an important element of the plancton fauna, both marine and fresh-water. A certain number of species are adapted to

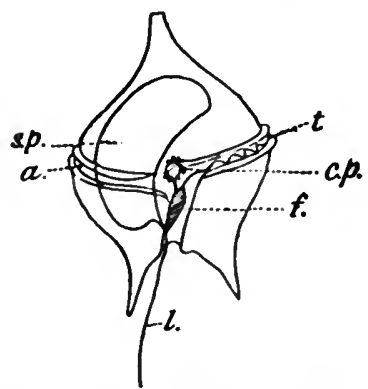

Fia. 122.-Peridinium divergens: ventral view showing the vacuble-system. c.p., The collecting-pusule surrounded by a rosette of still smaller pusules which open into it ; s.p., the large sac. pusule, or reservoir; both opening into the fundus $(f$.$) ,$ from which both the trans: verse flagellum $(t$.$) , lying in$ the annulus $(a$.$) , and the$ longitudinal flagellum (l.), arise. After Schütt, from Lankester. a family created by Chatton $(366,367)$ for certain parasitic forms; such are Blastodinium, an internal parasite of various copepods, and Apodinium mycetoides, an ectoparasite of appendicularians (Fritillaria). The parasitic vegetative form, without organs of locomotion, gives rise by periodic segmentation of mothercells to successive generations of swarm-spores, which in their structure resemble Gymnodinium.

\section{Bubchass III.: Cystoflagellata seu Rhynchoflagellata.}

This group comprises a small number of furms all marine and pelagic in habitat. Their chief peculiarity is that, like so marry other pelagic organisms of all classes, the body is inflated, as it were, with watery gelatinous substance, so that it attains to a size which far exceeds the actual bulk of the living substance contained in it. In censequence of the secondary increase in size, the powers of locomotion are feeble, and these organisms float more or less helplessly on the surface of the sea. 
The best known form is the common Noctiluca miliaris of our coasts. The adult Noctiluca is about the size of an ordinary pin's head (1 to 1.5 millimetres in diameter). The spherical body consists chiefly of jelly, with at one pole a superficial concentration of the protoplasm containing the nuclei and giving off the locomotor organs. From this central mass of protoplasm strands extend in an irregular network through the whole body, which is limited by a thin pellicle. The central protoplasm bears the so-called "perist ome," a deep groove containing the mouth-aperture near one end. The mouth is bordered by projections known as the "tooth" and the "lip," and near it arise two motile organs-a small flagellum, and a large tentacle-like process which shows a transversely striated structure and performs twisting and lashing movements. The tentacle is sometimes named the "flagellum," and the true flagellum the " cilium "; the former probably serves as the organ of locomotion, the latter for food-capture. The nutrition is holozoic.

Noctiluca reproduces itself by binary fission, and also by multiple fission producing a brood of small flagellate swarm-spores. The formation of the latter has been stated to be preceded by isogamous conjugation of the adults, but the matter is open to doubt, and it is possible that the swarm-spores themselves represent the gametes. Other genera of Cystoflagellata are Leptodiscus and Craspedotella (Kofoid, 373), both remarkable for their superficial resemblance to medusæ. No tentacle like that of Noctiluca is present in either of these forms, and locomotion is effected by rhythmic contractions of the disc-like body.

Bibliography.-For references see p. 486. 


\section{CHAPTER XIII}

\section{THE HAMOFLAGELLATES AND ALLIED FORMS}

General Characlers and Principal Types.-Under the term "Hæmoflagellates" are grouped together a number of forms of which the characteristic, though by no means invariable, habit is alternating parasitism in the blood of a vertebrate and in the digestive tract of a blood sucking invertebrate host. The group must be regarded, howcver, as one founded on practical convenience rather than on natural affinity-as a method of classification comparable to that of the gardener rather than of the botanist. The existence of a parasitic habit common to a number of different forms is in itself no proof of genetic affinity or community of descent, and it is highly probable that more than one line of ancestry has contributed, through divergent adaptation, to the composition of the group Hæmoflagellates. The name itself has, moreover, lost much of its significance, since closely allied to the forms parasitic in blood, and inseparable from them in a natural scheme of classification, aro other forms parasitic only in invertebrates, or cven free-living.

The chief morphological characteristic of the Hæmoflagellates is the possession of two nuclei, a trophonucleus and a kinetonucleus, and the relation of the locomotor to the nuclear apparatus is of the third type distinguished in the preceding chapter (p. 263); on this account they are ranked by Hartmann and Jollos (390) as a distinct order of the Flagellata termed the Binucleata.

The Hæmoflagellates as a group comprise a number of forms which represent in some cases distinct generic types, in others merely developmental phases alternating with other forms in the life-cycles of particular species. The following six generic names represent the more important of these types :

1. Trypanosoma (Fig. 126, etc.), with a single flagellum which arises near the kinetonucleus, at the extremity of the body which is posterior in progression, and runs forward as the marginal flagellum of an undulating membrane. At the anterior end of the body the flagellum is usually continued as a free flagellum, but in some cases it ends with the undulating membrane. A vast number of species parasitic in the blood of vertebrates and in the digestive tract of 
invertebrates alternately are comprised in this genus. Trypanosome-forms also occur as developmental phases in the life-cycle of species parasitic solely in the digestive tracts of insects.

2. Trypanoplasma (Figs. 36, 134), with two flagella arranged in a heteromastigote manner, and with the posterior trailing flagellum united to the body by an undulating membrane for the greater part of its length. A number of species are known, which by their distribution fall into three sections: (1) Species parasitic in the blood of fresh-water fishes, with alternating parasitism in the digestive tract of leeches ; (2) species parasitic in the digestive tract of marine fishes ; (3) species parasitic in various invertebrates.

3. Crithidia (Fig. 135), with a single flagellum which arises near the kinetonucleus, at about the middle of the body, in front of or close beside the trophonucleus, and runs along the pointed anterior end of the body to form the marginal flagellum of a relatively short, often rudimentary, undulating membrane, beyond which it is continued as a free flagellum. As an independent genus this type comprises species parasitic in the digestive tracts of various insects; but the majority of the so-called species of Crithidia aro merely phases in the developmental cycle of trypanosomes.

4. Leptomonas (Herpetomonas - Figs. 124, 136), with a single flagellum arising at the anterior end of the body, and with no trace of an undulating membrane. As an independent generic type this form occurs as a parasite of invertebrates, chiefly insects ; secondarily also in the latex of plants (Euphorbiaceæ). It occurs also as a developmental form of the next genus in the invertebrate host or in cultures.

5. Leishmania (Fig. 138), with an oval body containing a trophonucleus and kinetonucleus, but with no flagellum. As a generic type this form is an intracellular parasite of a vertebrate host, multiplying there by fission and developing into a typical Leptomonas-form. On the other hand, as a developmental phase this form represents simply a non-flagellated, resting stage which may occur in the life-cycle of either Trypanosoma, Crithidia, or Leptomonas.

6. Prowazekia (Fig. 141), with two flagella arranged in the heteromastigote manner, as in Trypanoplasma, but with the trailing flagellum quite free from the body, without an undulating membrane. Prowazekia is therefore quite similar in its morphology to Bodo, with which it was formerly confused, if, indeed, it is really distinct, and it differs from Bodo only in the possession of a kinetonucleus. Several species are described, free-living or intestinal in habitat.

Considering the above six types as a whole from a morphological standpoint, it is seen that there are two types of structure amongst them-the cercomonad or monomastigote type, represented by 
Trypanosoma, Crithidia, and Leplomonas, of which Leishmania may be regarded as the resting, non-flagellated phase ; and the bodonid or heteromastigote type with two flagella, seen in Trypanoplasma and Prowazekia. We shall return to this point in considering the affinities of the group as a whole and of its constituent genera.

The six types enumerated above are given with the nomenclaturo and definitions most commonly accepted, but it is necessary to state that the application and significance of the names Crithidia, Leptomonas, and Herpeto-

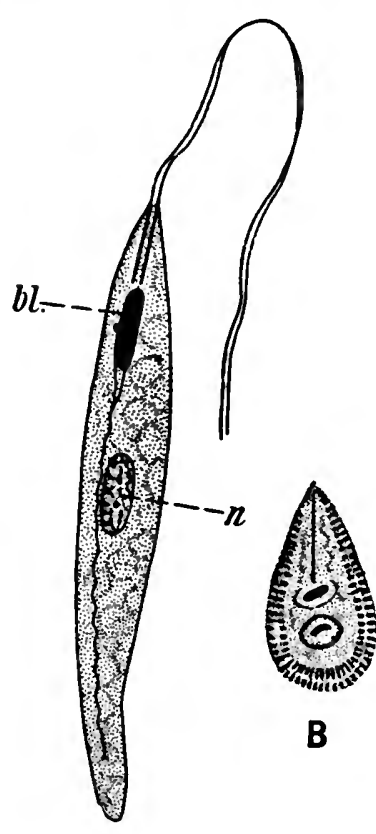

A

Fio. 124.-Herpetomonas musca-domestica (Burnett). $A$, Motile individual with two flagella; $B$, cyst : $n$., nucleus ; $b l$, kinetonucleus. After Prowazek. monas, are much disputed and are far from being settled. The type of the genus Herpetomonas of Saville Kent is a species found in the digestive tract of house-flies, $H$. musca. domestica (Fig. 124). According to Prowazek (557), this form possesses normally two flagella, which are connected together by a membrane; according to Patton (551) and many others, the biflagellate condition is due to precocious division of the normally single flagellum as a preparation for division of the body (compare Strickland, 558 ; Wenyon, 84). Those who follow Prowazek in regarding the biflagellate condition of Herpetomonas as its normal adult form employ the older genus Leptomonas of Saville Kent* for forms with.a single flagellum (Chatton, Roubaud, Prowazek). The main source of the confusion in the nomenclature arises from the uncertainty which still exists in many cases as to whether a given form or structural type is to be regarded as an independent specific or generic type, or as a developmental phase of another species. This applies especially to the genus Crithidia, founded by Léger (543) for a species, $C$. fasciculata, from the intestino of Anopheles maculipennis, and defined as a small uniflagellate form shaped like a grain of barley (Greek, кp $\theta \dot{\eta})$. Such forms, however, occur as developmental forms of trypanosomes or of leptomonads, and it is extremely probable that the species on which Léger founded his genus was simply a phase of this kind, which Wood. cock (527) has proposed to call the "trypanomonad" phase, in the development of a trypanosome. On this ground Dunkerly (535), who has recently discussed the wholo question, considers that the name Crithidia cannot be used as a generic name at all, but must be merged in Leptomonas, the name that should be used for all the uniflagellate parasites of insect-guts; while Herpetomonas should either become a synonym of Leptomonas, or should be used solely for Prowazek's biflagellato type, if that prove to be a distinet generic type. On the other hand, Léger and Duboscq $(646$, p. 232, footnote) consider that Crithidia should be retained, and Leptomonas ranked as a

* The genus Leptomonas was founded by Saville Kent, " Manual of Infusoria," vol. i., p. 243, for $L$. bïtschlii, parasite of the nematode worm Trilobus gracilis ; the genus Herpetomonas was founded on p. 245 of the same work for H. muscadomestica and H. lewisi (=Trypanosoma lewisi). Leptomonas is therefore technically the older genus. 
synonym of it. The question has given rise to a controversy which has been carried on by some of the participants in an acrimonious and even unseemly manner, and which it would be unprofitable to discuss further here, since the question is one which must be decided ultimately by facts, and not by personal opinions or tastes.

The various forms comprised in the Hæmoflagellates may now be considered in detail, beginning with the most important type.

\section{The Genus Trypanosoma.}

Occurrence. - Trypanosomes were first discovered as bloodparasites of cold-blooded vertebrates-fishes and batrachia; the type-species of the genus Trypanosoma is $T$. rotatorium (synonyms, $T$. sanguinis, Undulina ranarum) of the frog (Rana esculenta). Trypanosomes are now known, however, to occur commonly as blood-parasites in all classes of vertebrates. In a wild state many species of mammals, birds, and other vertebrate animals, are often found to harbour trypanosomes in their blood, though frequently in such scanty numbers as to render the detection of the parasites extremely difficult. It may be almost impossible in some cases to find trypanosomes in the blood of an animal by direct microscopic examination, owing to their great scarcity; but in such cases an artificial culture made from the blood may reveal the presence of the parasites, since in a few days the trypsnosomes originally present in small numbers in the blood multiply, under favourable conditions, to produce a swarm of flagellates. The cultural forms are quite different, as a rule, from the blood-forms which gave rise to them, and appear generally as crithidial or trypanomonad types; thus, cultures furnish evidence of the existence of a trypanosome in a given host, but give no indication whatever of the type of parasite actually present in the blood.

In some cases the trypanosomes appear to be present in the peripheral circulation of the vertebrate host only at certain periods, and at other times they are only to be found in the internal organs or tissues of the host, such as the spleen, bone-marrow, liver, lungs, etc. The trypanosome of Athene noctua-T. noctuae, for examplois to be found during the winter only in the bone-marrow of its host, and appears in the peripheral circulation during the summer months, and then most abundantly in the night-time (Minchin and Woodcock, 42). Hence, for various reasons, it may often be extremely difficult to decide whether a given animal is infected with trypanosomes or not; and in recent years trypanosomes have been discovered in animals in which their presence was previously quite unsuspected-for instance, in calves (Crawley, Carini, 423, Stockman; see also Bulletin of the Sleeping Sickness Bureau, No. 29, p. 320) and in sheep (Woodcock 527, p. 713, footnote). 


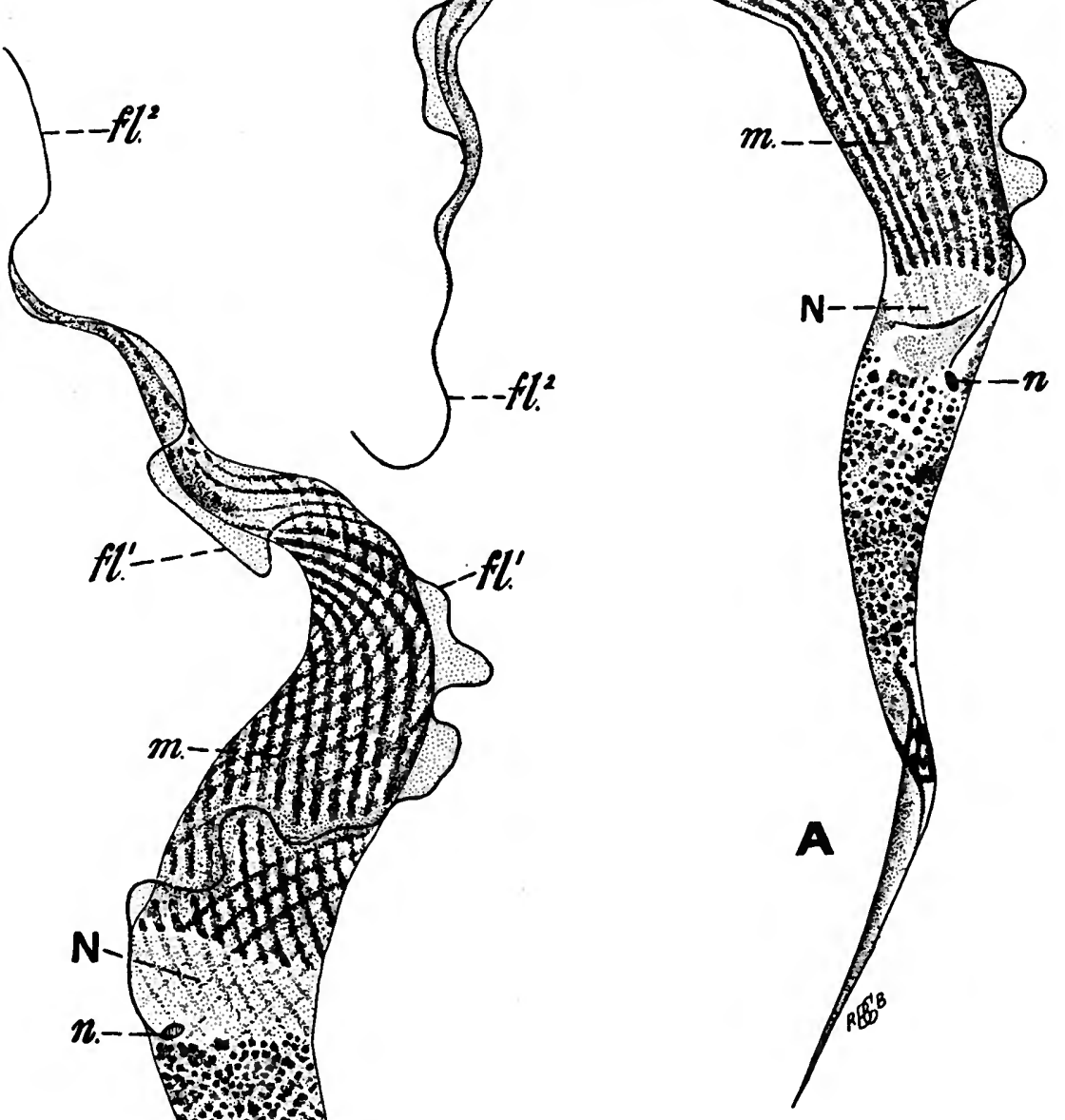

Fra. 125.-Trypanosoma mega, from the blood of African frogs. $f .{ }^{1}$, Marginal flagellum of the undulating membrane; $f .^{2}$, free flagellum; $m$., myoneme-striations (it is doubtful whether the granular streaks or the clear interspaces correspond exactly to the actual myonemes); $n$, kinetonucleus; $N$, space in which the trophonucleus lies, but, not being stained, it is not clearly defined in the preparation. After Minchin, magnified 2,000 ; compare Figs. 11 and 12 at the same magnification. 
Effects on the Host.-The trypanosomes found infesting wild animals in Nature are, as a rule, quite specific to a particular host, and, so far as can be observed, perfectly harmless to it. If the relations between host and parasite had always been of this type in all cases, our knowledge of trypanosomes would be in a much more backward state even than it is. Of recent years a vast amount of attention has been attracted to tinese parasites owing to the diseases of man and animals caused by certain species of trypanosomes, and hence termed comprehensively "trypanosomiases." The greater number of these pathogenic species belong, from the structural point of view, to a type which may be called the bruciitype (Fig. 12); such are T. brucii, cause of tsetse-fly disease; T. gambiense, of sleeping sickness ; $T$. evansi, of surra ; $T$. equiperdum, of dourine; and many others. The structural similarity of these species renders their identification a matter of extreme difficulty. Of a slightly different type is $T$. equinum, of "mal de caderas" in South America, with a very minute kinetonucleus ; but the recentlydescribed $T$. hippicum of "murrina" (Darling, 428) appears to be a typical member of the brucii-group. T. theileri, on the other hand, from cattle, is very distinct in size and appea:ance from the members of the brucii-group. Finally, $T$. cruzi, the cause of human trypanosomiasis in Brazil, stands apart from all the others in peculiarities of reproduction and development, which have led to its being ranked in a distinct subgenus, Schizotrypanum.

The problem of the pathogenic trypanosomes has been touched upon in Chapter II. From a survey of trypanosomes in general, it is clear that the normal type of these parasites is one which is specific to one or to a limited number of species of hosts, to which it is quite harmless. The pathogenic species are to be regarded as aberrant forms not yet adapted to their hosts, as an instance of a disharmony in Nature. They are species which have probably established themselves but recently in the hosts to whioh they are pathogenio. As contrasted with the natural, non-pathogenic forms, their most striking peculiarities are that they are not specific to one host, but can flourish in a great number of different species of hosts, and that in susceptible animals their power of multiplication has no limit. $T$. brucii, so deadly to many domestic animals, is known to occur also as a natural parasite of wild animals, to which it is harmless.

Structure.-The constitution of the trypanosome-body is of a very uniform type in its general traits, though subject to great variation in different cases as regards size, form, and minor details of structure. The body is typically long and sinuous, with the anterior end tapering gradually to a fine point, while the posterior extremity is usually broader, and tapers more abruptly, or ends bluntly; but in different forms, even of the same species, there may 
be great variation, from long, slender to short, stumpy types, and in some cases the posterior end is also greatly drawn out and attenuated. The principal nucleus or trophonucleus is usually situated near the middle of the body. The kinetonucleus is almost invariably behind the trophonucleus, ${ }^{*}$ sometimes close behind it, but more usually near the posterior extremity, separated from the trophonucleus by about half the length of the body.

The flagellum arises from a centriole (blepharoplast) which is in connection with the kinetonucleus. In the more primitive type of arrangement the blepharoplast is lodged within the kinetonucleus. itself, and then the flagellum appears to arise from the kinetonucleus directly (Wenyon, 84). In most cases, however, the blepharoplast is situated close beside, and usually in front of, the kinetonucleus, connected with it by a delicate rhizoplast. When the blepharoplast is distinct from the kinetonucleus, it is at present an open question whether the kinetonucleus contains a centriole of its own, in addition to the blepharoplast, or whether the blepharoplast represents a centriole which belongs to the kinetonucleus, but has migrated to the exterior of this body.

Passing from the blepharoplast to the surface of the body, the llagellum forms the free border of the undulating membrane, which runs forward from the vicinity of the kinetonucleus to the extreme anterior end of the body as a fin-like ridge or fold of the periplast, of variable width (cf. Fig. 126). The flagellum may in some cases end with the undulating membrane at the anterior end of the body, but more usually it is prolonged forward beyond this point, so that a free portion of variable length is to be distinguished from the marginal portion contained in the undulating membrane. The sinuous body, the undulating membrane, and the flagellum, are alike in a state of incessant movement during life, and in larger forms contractile myonemes are clearly visible in the periplast of the body (Fig. 28, p. 58) ; in the more minute individuals the presence of such elements must be inferred from their movements, but cannot always be demonstrated optically.

The movements of a trypanosome, speaking generally, are of two types: travelling movements, when it progresses with the free flagellum forwards, sometimes very fast, shooting across the field of the microscope in a straight line (mouvement en fleche), sometimes, on the other hand, pushing its way s lowly through the blood-corpuscles, with the flagellum directed either forwards or backwards in movement; and wriggling movements, when the animal writhes incessantly in serpentine contortions with little or no displacement

* The only known exceptions are furnished by eertain forms of the recently. described $T$. rhodesiense (vide Stephens and Fantham), and by some of the small forms seen during the multiplication of $T$. lewisi (Fig. 127, $L$ ). It is needless to point out that the statement made above applics to the typical trypanosome-form as found in the vertebrate blood, and not to the developmental forms through which they pass in the invertebrate host (orithidial and other types). 
from a given spot. Many trypanosomes, especielly the large stout forms, are very sluggish in their movements, and show but little power of progression. At the opposite oxtreme, in this respect, is the African parasite of cattle, well named by Ziemann $T$. vivax, which, according to Bruce and his collaborators (411, iii.), " dashes across the field of the microscope with such rapidity that it is impossible to followits movements, cyclone-like leaving a clear path, the corpuscles in its track having been flung on either sidc. If it remains at the same spot for a time, as it sometimes does, it has an appearance of great energy and power, throwing the surrounding red blood-corpuscles about in wild confusion."

In the foregoing paragraphs the terms "anterior" and "posterior," as applied to the trypanosome-body, have been used strictly with reference to its mode of progression. It is pointed out below, in the comparison with other types such as Trypanoplasma and Crithidia, that the extrenity
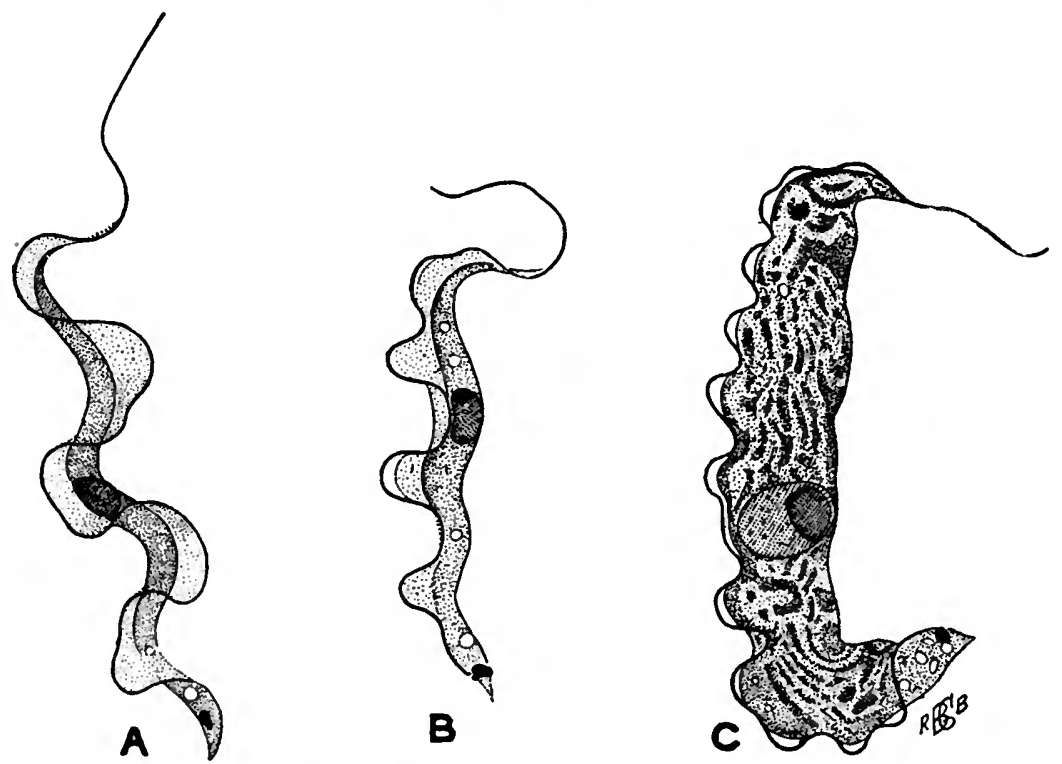

Fig. 126.-A, T'rypanosoma tince of the tench; note the very broad undulating membrano in this species; B, C, $T$. perces of the perch, slender and stout forms. After Minchin, $\times 2,000$.

of the body which is anterior, in the strictly morphological sense, in one species, may conceivably be posterior in another case. Hence some writers avoid the use of the words "anterior" and "posterior," and substitute for them "flagellar" and "aflagellar" respectively, to denote the two poles of the body. There is as yet, however, no concrete evidence for regarding the flagellar extremity as morphologically posterior in any known species of trypanosome.

The undulating membrane is to be regarded as a fold of the periplast or ectoplasm, into which the granular endoplasm may extend a short way in some cases; it arises from the body along a line which is sometimes spoken of as "dorsal," an unnecessary refinement of terms. The free edge of the membrane, with its marginal flagellum, can be shown by direct measurements to exceed considerably in length that portion of the body to which it is attached; consequently its free edge is thrown into folds or pleats more or less marked. In preparations, trypanosomes are seen to lie, speaking generally, 
in one of the three ways; a certain number show the body extended nearly in a straight line, with the free edge of the membrane much pleated, but as a rule the body is curved, and then either with one principal bend, like a $\mathrm{C}$, or with several S-like serpentine bends. In either case the undulating membrane is seen almost invariably to run on the convex side of each curve. In C-like forms (Fig. 125, $A$ ) the membrane runs evenly along the outside of the principal curve, and the myonemes parallel to it. In S-like forms (Fig. 125, B) the membrane is often seen distinctly to be spirally twisted round the body, the myonemes also exhibiting the same twist. In life the undulating membrane performs, as its name implies, movements like those of a sail flapping in tho wind. Wave-like undulations run along it from one end to the other, but not always in the same direction; it has been observed that reversals of the movements may take place, the waves first running in one direction for a time, and then suddenly undergoing a change and running in the opposite direction (Minchin and Woodcock, 42).

Much confusion exists in the nomenclature of the parts of the trypanosomebody, more especially with regard to the small body for which Woodcock's term "kinetonucleus" ("Geisselkern") is here used-a confusion due to differences of cytological interpretation. While it has never been doubted that the larger body $(N$.$) is a true nucleus, various views have been held$ with regard to the smaller body $(n$.$) , which, summarized briefly, are as$ follows: The older writers regarded it merely as an organ of the periplast from which the flagellum arose. Stassano and Bradford and Plimmer regarded n. as a body of nuclear nature, and termed it the "micronucleus," comparing it with the similarly-named body of Infusoria. Laveran and Mesnil $(464,391)$, on the other hand, regarded $n$. as the "centrosome," the name by which it is generally known in France. Schaudinn (132) emphasized strongly its nuclear nature, and stated that $n$. was not a centrosome, but nevertheless used for it the term " blepharoplast," by which it is still genorally known in Germany, although a true blepharoplast is a body of centrosomio nature. Moore and Breinl (484) reverted to the centrosonic view, and termed $n$. the "extranuclear centrosome," believing that it arose by division of the intranuclear controsome contained in the principal nucleus (N.). Hartmann and Prowazek (63), on the basis of their nuclear theory of the centrosome (see Chapter VI., p. 95), regarded $n$. as a body both of nuclear and centrosomic nature, using for it the term " blepharoplast "; so also Rosenbusch. Finally, Doflein (7), who is not convinced of its truly nuclear nature, continues to employ for $n$. the term " blepharoplast." With these many conflicting views with regard to the nature of $n$., the basal granule has been either ignored or overlooked, or considered as a mere "end-bead " of no particular importance, or ranked as a centriole, as it doubtless is. The nomenclature used here is based on the general theory that a centrosome, or its equivalent, a blepharoplast, is an achromatinic body of nuclear origin, but not equivalent to an entire nucleus, and on the conviction that $n$. is a true nucleus, and therefore is not to be regarded either as a centrosome or a blepharoplast. For a fuller discussion of these points, see Robertson and Minchin (80).

The trophonucleus of a trypanosome is typically a vesicular nucleus containing a karyosome in which is lodged a centriole. The karyosome varies in size in different species, and is sometimes double or multiple; in $T$. granulosum the smallest torms have a single karyosome which buds off others as the animal increases in size (Minchin, 478). By the method which is most in vogue, however, for making permanent preparations of trypanosomesnamely, the various modifications of the Romanowsky-stain-this structure is seldom to be made out, and the trophonucleus appears generally as an evenly-stained mass or as a dense clump of stained granules. It contains a centriole, difficult to make out in the resting condition, owing to its being embedded in the substance of the nucleus. The kinetonucleus consists mainly of a mass of plastin impregnated with chromatin, stoining very deeply, rounded, oval, or even rod-like in shape. According to Rosenbusch, the chromatinic mass of the kinetonucleus is to be regarded as representing 
a karyosome, and it is surrounded by a space, sometimes purely virtual, which represents the nuclear vacuole, bordered by a delicate nuclear mem. brane, on or close to which the basal granule of the flagellum is lodged.

In sume species of the brucii-group, an axial filament, apparentlyt a supporting structure of the nature of an axostyle, has been described (cf. Swellengrebel, 514). The system of fibrils, however, with which Prowazek decorates the trypanosome-body are probably artefacts ( $c f$. Minchin, 479).

Many trypanosomes contain granules in their cytoplasm which stain similarly to chromatin, so-called " chromatoid grains." According to Swellengrebel (514), they are of the nature of volutin (p. 68, supra).

The division of a trypanosome is initiated, as a rule, by the division of the blepharoplast or basal granule of the flagellum, and following close on this a reduplication of the flagellum takes place, the exact method of which is disputed. In some cases the old flagellum appears to split; in others the parent-flagellum remains unaltered, and a daughter-flagellum grows out from the daughter-blepharoplast. It is asserted by some that in all cases the new flagellum really arises as an independent outgrowth of a blepharoplast, and that the splitting of the old flagellum is only apparent, and due to the daughter-flagellum growing out at first in its sheath, from which it separates later (cf. Wenyon, 84). The division of the kinetonucleus follows hard on that of the blepharoplast, and next, as a rule, the trophonucleus divides. When the division of flagellum and nuclei is complete the body divides, beginning to do so at the flagellar end; the two sister-trypanosomes are often connected for a time by the posterior extremities.

The division of the kinetonucleus is a simple constriction into two; that of the trophonucleus is of a simple type, in which first the centriole and then the karyosome divides. The two daughter-karyosomes travel apart, and the nucleus follows suit. The two daughter-nuclei sometimes remain connected for a time by a long centrodesmose, which is finally severed. Such, at least, is the mode of division of the two nuclei as it has presented itself to the majority of investigators, and the nuclear division of trypanosomes is to be regarded as amitotic, or at least not further advanced towards mitosis than that of Coccidium described above (p. 106, Fig. 51). According to Rosonbusch, however, the division of the nuclei, both trophic and kinetic, takes place by true mitosis. This author is in advance of his contemporaries upon this point, and his statements require independent confirmation before they can be accepted unreservedly, since in objects of such minuteness, requiring delicate and elaborate technique, imagination may all too readily outrun perception.

Life-History.-The transmission of trypanosomes from the blood of one vertebrate host to another is effected, probably for every species of these parasites, by the agency of a blood-sucking invertebrate of some kind. When the host is a terrestrial vertebrate, the transmitting agent is generally an insect, such as a mosquito or some biting fly or bug, or an ectoparasite of the host, such as a flea, louse, or possibly a tick in some cases; the trypanosomes of aquatic vertebrates, on the other hand, are transmitted by leeches in all cases that have been investigated. In additiun to inoculative transmission (p. 24) of this kind, trypanosomes may pass directly from one vertebrate host to another during coitus; this is known to occur in the case of the parasite of "dourine" in horees ( $T$. equiperdum), and has been suspected, but not proved, to take place in other cases also. It is also possible for the vertebrate to become infected by devouring animals containing living trypanosomes, 
whether it be the blood-sucking invertebrate, or possibly the flesh or organs of another vertebrate infected with trypanosomes.

Two methods of inoculative transmission of trypanosomes have been distinguished ; in the one, known as the "direct" or " mechanical" method, the parasites merely become contained in or adhere to the proboscis of the blood-sucking intermediary when it sucks blood from an infected animal ; and when it feeds a second time the trypanosomes pass directly, and without having undergone any change or development, into the second host; in the other, known as the " indirect" or " cyclical " method, the trypanosomes, when taken up by the blood-sucking invertebrate, go through a developmental cycle in it, at the end of which, but not before, they are " ripe" for inoculation into a suitable vertebrate host. Comparing natural with artificial processes of infection, in the direct method the bloodsucking invertebrate may be said to play the rôle merely of an injection-syringe, but in the indirect method it acts also as a culturemedium, in which the parasite passes through various phases and assumes forms quite different from those occurring in vertebrate blood. Patton (393) has put forward the view that transmission is always by the. direct method, and that the crithidial and other forms found in the blood-sucking invertebrate are parasites of the invertebrate alone, and have no connection with the trypanosomes found in vertebrates; but the number of cases in which it has now been shown clearly that trypanosomes go through a definite cycle in the invertebrate host disproves Patton's contention, and renders it unnecessary to discuss it further. It is rather the direct method that stands in need of further demonstration; though undeniably possible as a laboratory-experiment, it may be doubted if it ever really occurs in Nature, and in any case it is probably to be regarded as a purely accidental rather than a normal occurrence.

It has been frequently asserted or assumed that trypanosomes can pass from parent to offspring, by so-called " hereditary transmission," in the invertebrate host, but convincing proof of this statement is as yet lacking entirely. Attempts to prove hereditary transmission by direct experiment have given, for the most part, negative results, and the observation so frequently made, that leeches, tsetseflies, fleas, mosquitoes, etc., bred from the egg and not exposed to infection, are entirely free from parasitic flagellates, affords cumulative evidence against the existence of any such method of transmission (cf. Kleine and Taute, 459). Brumpt. (419), however, asserts that $T$. inopinatum is transmitted hereditarily from parent to offspring of the leech Helobdella algira. According to Porter (554), "Crithidia" melophagia of the sheep-ked is also transmitted from parent to offspring in this insect; and if, as is extremely probable, the flagellate in question is the developmental phase of the trypano- 
some of the sheep, it would furnish another instance of hereditary transmission. Hence this mode of transmission must, apparently, be reckoned with in some instances, though it is evidently an extremely rare phenomenon in trypanosomes generally.

Just as a given species of trypanosome is, in Nature, capable of maintaining itself only in a particular species, or limited group of species, of vertebrate hosts, so it may be said, as a general rule, that in transmission by the cyclical method the parasites are specific in the same way to certain invertebrate hosts, in which alone they are able to go through their full natural cycle. Amongst the many blood-sucking invertebrates which may prey upon the vertebrate, we may distinguish "right" and "wrong" hosts; in the right host or hosts the parasite establishes itself more or less easily, and passes through a full and complete developmental cycle; in the wrong host it either dies out immediately or goes through only a part of its cycle. The distinction between right and wrong hosts must not, however, be taken in an absolute sense, but as implying only that, amongst many possible hosts, there is one at least to which the parasites have become better adapted than to any other; but the trypanosomes may sometimes succeed in maintaining themselves in other than the right host sufficiently long to pass back again into the vertebrate. Thus, in the case of the rat-trypanosome ( $T$. lewisi) the right host is a rat-flea (Ceratophyllus fasciatus, or possibly other species); but it may persist in the rat-louse (Homatopinus spinulosus), and even pass from it, though rarely, back into the rat again.

The following are a few well-established examples, in addition to that of $T$. lewisi already cited, of trypanosomes and their right hosts. Many pathogenic species of trypanosomes in Africa are transmitted by tsetse-flies-e.g., $T$. gambiense and $T$. vivax by Glossina palpalis, $T$. brucii by $G$. morsitans, ${ }^{*}$ etc. The recentlydescribed $T$. cruzi of Brazil was discovered in its invertebrate host, a blood-sucking hemipterous insect, Conorhinus megistus, before it was found in the blood of human beings. The trypanosomes of certain fresh-water fishes - namely, goldfish, perch, etc. - pass. through their developmental cycle in the leech Hemiclepsis marginata (Robertson, 503). T. raice of skates and rays develops in the leech Pontobdella muricata (Robertson, 500, 502). The trypanosome of African crocodiles, $T$. grayi, develops in the tsetse-fly Tlossina palpalis (Kleine, 458 ; Kleine and Taute, 459), and stages in its life-cycle have consequently been confused with those of $T$. gambiense in the same fly. The trypanosomes of birds are probably transmitted for the most part by mosquitoes, but the details of

- According to Tante, $G$. morsitans can act as a truo host for $T$. gambiense, and, conversely, according to Fischer, $G$. palpalis can do the ssme for $T$. brucii. 
their transmission have not yet been worked out in a satisfactory or conclusive manner.

It must be considered for the present an open question whether true trypanosomes occur as parasites of an invertebrate host exclusively; the answer to the question will depend on the significance given to the expression "true trypanosome." It is now practically certain that many leptomonads have a trypaniform phase in their development (see p. 314, infra), so-called "leptotrypanosomes." In Drosophila confusa, a non-biting, muscid fly, Chatton and Alilaire (compare also Chatton and Iéger) found in the Malpighian tubules a trypaniform type of flagcllate which they consider as a "eutrypanosome," as a species of Trypanosoma distinct from the Leptomonas occurring in the gut of the same fly (Fig. 137). Wenyon (84) also found similar forms in the Malpighian tubules of house-flies in Bagdad, and considered that they might belong to the cycle of the Leptomonas (Herpetomonas) in the same host. In both cases the phase in the Malpighian tubules is a little stumpy trypanosomelike form, very similar in its characters to $T$. nanum. The fact that these "eutrypanosomes" are so far known only to occur in flies which are infected also by a species of Leptomonas indicates that, like the "leptotrypanosomes," they are merely a phase in the cycle of the Leptomonas.

From the foregoing it is seen that the complete life-cycle of a trypanosome is an alternation of generations corresponding to an alternation of hosts. One part of the cycle is passed in the blood of a vertebrate, in which the predominant form is the trypanosometype of flagellate; the second part is passed in the digestive tract of an invertebrate, and here the predominant form is the crithidial or trypanomonad type. We may consider the life-history, therefore, under these two principal phases :

1. As a type of the life-cycle in the vertebrate host, that of the common rat-trypanosome may be taken. After infection, natural or artificial, of the rat, the trypanosomes make their appearance in the blood about the fifth, sixth, or seventh day. What the parasites have been doing during this time, the so-called "incubationperiod" in the rat, cannot as yet be stated definitely; it may be that the relatively few trypanosomes inoculated by the flea or syringe have merely been multiplying steadily, in the manner presently to be described, until they become sufficiently numerous in the blood to be detected by microscopic examination ; there may, on the other hand, be phases of the parasite as yet unknown during this period, and, according to recent statements (Carini, 422), a process of schizogony takes place in the lung similar to that discovered by Chagas in Schizotrypanum cruzi (see below).

When the trypanosomes first appear in the blood, their most striking peculiarity is the extraordinary diversity in type which they exihibit. Besides " ordinary" individuals of the normal dimensions of the " adult" form, there are others smaller or larger, the extremes of size being relatively huge in one direction, very minute in the other. These differences of size are due to the fact that the trypanosomes arc multiplying actively, the large forms being those 


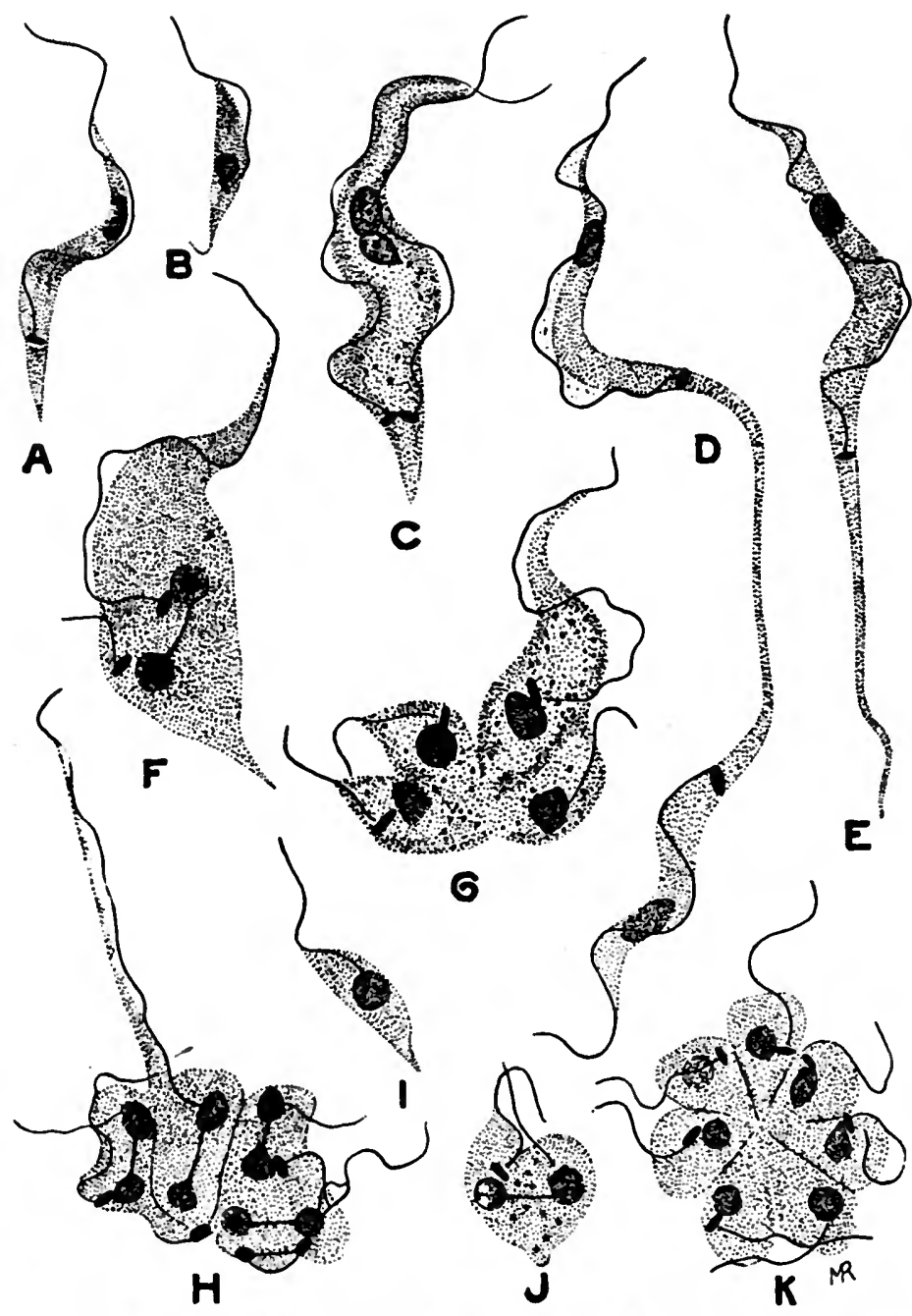

Fia. 127.-Various forms of multiplication in Trypanosoma lewisi from the blood of the rat. $A$, Trypanosome of the ordinary type; $B$, small form resulting from division; $C$, stago in cyual binary fission; the nuclei havo divided and two flagella arc present, but division of the body is beginning, and is indicated by a lighter streak down the middlo of the budy; $\mathrm{D}$, final stage of binary fission, which is complete except for a bridgo of protoplasm, much drawu out, connecting the hinder ends of the two sister-trypanosomes; $E$, form with hinder end drawn out (longocaudense type), the result of binary fission as seen in the last figuro; $F$, unequal binary fission of a large trypanosome; $G, H$, continued fission of the same type ; in $G$ a parent and three daughterindividuals, in $H$ a parent and seven daughter-individuals, can be distinguished; the parent-individual in each case is marked by the possession of a flagellum of the full normal length, while the daughter-individuals, formed by successive divisions, have flagella varying in length; $I$, a small form, similar to $B$, but with the kinetonucleus in front of tho trophonucleus; $J$, binary fission of a form similar to $I ; K$, further division of a similar form producing a rosette of seven individuals still connected together. From preparations made by Dr. J. D. Thomson; magnificd 2,000 diameters. 
which are about to reproduce themselves ly some form of fission, while the small forms are those which have resulted from a recent act of reproduction.

The multiplication of $T$. lewisi in the rat's blood takes various forms (Fig. 127). In some cases a trypanosome divides by equal binary fission $(C, D)$, but this is comparatively rare. More usually tho fission is markedly unequal, and of a multiple type. Small daughter-forms are'split off from large parent-individuals, and usually many at a time; the nucleus of the parentform divides several times, and subsequently the body divides into as many portions as there are nuclei, thus producing rosette-like forms (Fig. 127, $F, G, H)$ in which the original parent can usually be distinguished by its long flagellum from the small daughter-individuals with their flagolla growing out. The small forms are sometimes set free with a crithidial type of structure, the kinetonucleus in front of the trophonucleus (Fig. 127,L), and theso immature forms may proceed to reproduce themselves rapidly again by either binary or multiple fission, in tho latter case forming rosettes in which no large parent-form can be distinguished (Fig. 127. K).

A curious type of trypanosome found during the multiplication-period of $T$. lewisi is a form with the posterior end prolonged to a great length, so that it almost resembles a second flagellum (Fig. 127, E), and has sometimes been mistaken for such. This form has been described by Lingard as a distinct species under the name $T$. longocaudense. These forms appear to arise by binary fission (Fig. 127, D); they are of constant occurrence and very numerous at a certain stage of the multiplication-period.

The multiplication of $T$. lewisi in the rat's blood is most active from the eighth to the tenth day after infection, after which it is on the decline and gradually ceases. The relative number of forms of ordinary size increases steadily, while those of unusual dimensions, whether great or small, become continually scarcer, until about the twelfth or thirteenth day the tiypanosomes, now usually present in vast numbers in the blood, are of uniform size and appearance, exhibiting, apart from occasional abnormalities, individual variations only of a comparatively slight character; and all multiplication has ceased entirely, never to recommence in the same host. The trypanosomes swarm in the blood of the rat for a certain time, which varies in different cases, but is usually one or twa months. The infection of the rat is sometimes spoken of as " acute " when the trypanosomes are multiplying, and as " chronic" when multiplication has ceased, not, however, very well-chosen terms, since the trypanosomes soon begin to diminish in number, and finally disappear altogether; sometimes the diminution is very gradual and slow, sometimes it takes place with great rapidity. In either case the rat gets rid of its infection entirely sooner or later, without having suffered, apparently, any marked inconvenience from it,* and is then immune against a fresh infection with this species of trypanosome.

* Instances are on record of lethal epizootios of rats ascribed to $T$. lewisi; but the proof that this parasite was really the cause of the discase is lacking. Under normal oiroumstances rats show no perceptible pathological symptoms whatever 
A type of development in the vertebrate host contrasting in many points with that described in the foregoing paragraphs is seen in $T$. cruzi (Fig. 128), the cause of human trypanosomiasis in Brazil. In this case the ordinary or adult forms of the trypanosome found in the general circulation do not multiply there; but the investigations of Chagas and of Hartmann have made known two types of multiplication which take place in the internal organs of the body.

The first type of multiplication proceeds in the capillaries of the lung (Fig. 128, $b-e$ ). An adult trypanosome loses its flagellum, and in some cases its kinetonucleus also ; its body then becomes rounded off into an oval mass ; the trophonucious, and also the kinetonucleus, if present, multiply by suc cessive divisions to form eight nuclei of each kind; and finally the body divides within its own periplast into eight minute daughter-individuals, socalled "merozoites." The merozoites are stated to exhibit a dimorphism

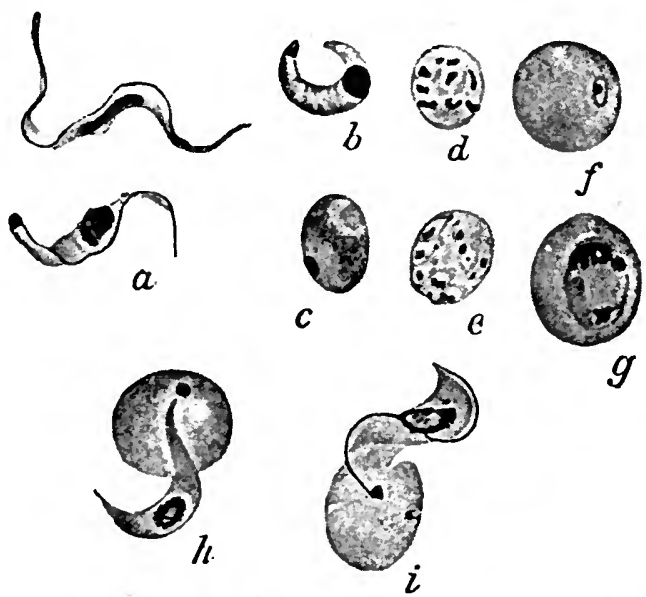

Fio. 128.-Phases of T. (Schizotrypanum) cruzi in vertebrate blood. a, The two forms of the adult trypanosome, " male " (upper) and " female " (lower), from human blood ; $b$, preparations for schizogony ; $c$, schizont; $d$, division of the nucleus of tho schizont; $e$, division of the schizont into eight merozoites; $f$, merozoite in a blood-corpuscle; $g$, intracorpuscular phase in late stage of growth; $h$, similar phase escaping from a corpuscle, the flagellum not yet formed; $i$, similar phase, the flagellum in process of formation. Stages $b-e$ are found in the lung, the others in the peripheral blood. After Chagas (425).

which Chagas regards as sexual; those produced by trypanosomes which retained their kinetonucleus have both trophic and kinetic nuolei and a rudiment of a flagellum (male forms); those derived from trypanosomes which lost both flagellum and kinetonucleus have only a trophonucleus (fcmale forms); in the latter case the single nucleus divides into two unequal parts, of which the smaller becomes the new kinetonucleus, and a flagellum is formed subsequently. - In either case the merozoites penetrate into blood-

frnm cven the most swarming infection with $T$. lewisi (for the action of the " renfurcés" strains seo p. 28). Those who study habitually the lethal species of trypanosomes often display a natural bias, not in the least justified, to assumo that a similar virulence is an inseparable attribute of all other species of these parasites. If that were so, it would be necessary to consider practically every specimen of pike, bream, perch, or tench, in the Norfolk Broads, for instence, to be in a diseased condition. 
corpuscles, and so pass into the general circulation. Within the corpuscle they grow into the adult form, which is finally set free from the corpuscle as a trypanosome of normal structure. The adult trypanosome (Fig. 128, a), swimming freely in the blood-plasma, may either be taken up by the invertebrate host in which it develops, or may repeat the process of multiplication by schizogony.

The second type of multiplication was first described by Hartmann from hypertrophied endothelial cells of the lung; Chagas (426) has aince found it in the tissues of the body, more especially in the cardiac muscle, central nervous system, and striped muscle. In this type the parasite is intracellular, and has the appearance and structure of a Leishmania (cf. Fig. 138), a rounded body containing a trophonucleus and a kinotonucleus, but no flagellum or undulating membrane.

On account of its power of multiplication by schizogony, Chagas has made $T$. cruzi the type of a special genus, Schizotrypanum; the type of multiplication observed in the lung-capillaries is not essentially different, however, from that of $T$. lewisi in the blood, except for its alleged sexual dimorphism; and, accord. ing to Carini (424), similar processes of schizogony occur in other trypanosomes The intracellular multiplication in the tissues, however, recalls strongly that of the parasite of kala-azar (see p. 316, infra). Schizotrypanum thus forms an important link between a typical blood-trypanosome, such as $T$. lewisi, and a tissue-parasite, such as the species of Leishmania, in which the freo trypanosome-phase no longer exists, apparently.

Chagas considers the multiplication of Schizotrypanum cruzi in the tissues as non-sexual, and serving to increase the number of parasites in the host, but that which takes place in the lung-capillaries as a process of gametogony whereby the sexually differentiated adult forms are produced. His grounds for this interpretation are, first, that in human blood the adult trypanosomes exhibit a dimorphism rarely found in guinca-pigs infected artificially, in which also schizogony in the lung is seldom observed; secondly, that the invertebrate host, Conorhinus, is always rendered infective if fed directly on infected human blood, but very rarely becomes infective if fed on guinca-pigs, even when these animals show an intense infection. He suggests that the greater resistance of the human organism to the parasite stimulates the production of sexual forms which the trypanosome may ccase to produce in a less resistant host.

In the more familiar pathogenic species, such as T. brucii, T. gambiense. etc., the development in the vertebrate host takes the form mainly of continued multiplication by binary fission simply. Reproduction of this kind may proceed until the trypanosomes swarm in the blood; or, on the contrary, the trypanosomes may be at all times relatively few in number, oven when fatal to their host. T. brucii, for example, may produco in different hosts an acute or a chronic form of disease; in the latter case the infected animal may livo a long time, and the parasite exhibits very limited powers of multiplication. The behaviour of the parasite in the natural hosts to which it is harmless has not been studied.

In many pathogenic species; periods of multiplicative activity, during which the trypanosomes are abundant, alternate with periods during which the parasites pass into a resting condition in the internal organs, and becomo scarce or disa ppear in the general circulation. In this phase they are alleged wo lose their flagellum, diminish in size, and become small, rounded "latent. borlies," which, according to Moore and Breinl (484), havo only a single nucleus; but according to Fantham they are Leishmania-like, with distinct trophonucleus and kinetonucleus. From resting stages of this kind the activs trypanosomes are developed again. Laveran (462), however, denies that there is a non-flagellated stage of development in the vertebrate host, and considers that the elements described as "latent bodies" represent involution-stages of the parasites-that is to say, forms which have become deformed in structure owing to unfavourable conditions, but not to such an extent as to be incapable of recovery if the conditions improve. 
In the vast majority of trypanosomes in their natural hosts, such as birds, fishes, etc., the mode of multiplication and the developmental cycle remains a mystery, although the sizes of the individual trypanosomes and their numbers are observed to vary at different times in the same host. Considerable light has been thrown upon this question by the recent investigations of Machado upon the multiplication of Trypanosoma rotatorium of frogs, a species re. markable for the polymorphism it exhibits. The results obtained by Machado may be summarized briefly as follows: Trypanosomes of any size may divido by binary fission when free in tho blood (supposed "non-sexual" reproduction). On the other hand, trypanosomes of large size may become rounded, flattened, leaf-like forms, losing rtheir flagellum; such forms undergo a process of schizogony in the internal organs, chiefly in the liver or kidneys, sometimes in the spleen, sometimes even in the circulating blood. The kinetonucleus approaches the trophonucleus, and may (1) remain distinct from it, 80-called "male" type ; or (2) may pass into the trophonucleus, in which the karyosome breaks up to form a small secondary karyosome; the kinetonuclear karyosome then fuses with, or becomes closely adherent to, the secondary trophonuclear karyosome-so-called "female" type. A multiplication of the nuclei then takes place : in the "male" type by independent divisions of the kinetonucleus and trophonucleus; in the "female" type by divisions of the single mass formed by fusion of the kinetonuclear and trophonuclear karyosomes, followed by budding off of small nuclei from the originally single nucleus. Thus the body of the rounded-off trypanosome becomes filled, within its periplast, with nuclei varying in number from five to seventeen; then round each nucleus ("female") or each pair of dissimilar nuclei ("male") the protoplasm becomes condensed to form as many merozoites, which are finally set free by rupture of the periplast. The merozoites of "male" type develop a flagellum; in those of "female" type the single nucleus divides into two nuclei of unequal size, a larger trophonucleus and a smaller kinetonucleus, and from the latter a basal granule is budded off from which the flagellum grows out (Fig. 30, G). In either case the merozoites (which may divide further after being liberated from the parent body) become transformed finally into the smallest forms of trypanosomes, which then grow up into the larger forms found in the blood. Machado's observations of fact, apart from his theoretical interpretations, explain the many different forms found in the frog's blood, which have recently been studied in detail by Lobedew ; compare also Mathis and Leger.

In other cases there may be three well-marked types of form-long and slender, short and stumpy, and intermediate or indifierent forms, as in T. gambiense (Fig. 12 ; cf. Minchin, 477, Hindle, 450, Bruce, 405); or there may be every gradation in size from small to large forms, as in $T$. granulosum of the cel (Fig. 129); or, finally, the trypanosomes may be practically uniform in size and structure, as in $T$. lewisi after the multiplication-period, $T$. vivax, etc. A satisfactory explanation of the polymorphism has not been found in all cases; the various forms may be in some instances stages of growth related to multiplication, as in T. lewisi during the multiplication-period ; in other cases the polymorphism-for example, of $T$. gambiense-may be sexual differentiation which is related to tho subsequent development in the invertebrate host; a third possibility is that in some cases the propagative forms, destined for multiplication in the invertebrate host, are differentiated from the other forms found in the vertebrate host, as in T. noctua (Minchin and Woodcock, 42). Different explanations must probably be sought in differenit cases.

2. The cycle in the invertebrate host always takes place entirely or mainly in the digestive tract, though the extent to which this region is invaded varies greatly. In the development of $T$. lewisi in the flea the parasites pass down as far as the rectum, and there 
undergo the principal phase of their cycle. In the development of the trypanosomes of fresh-water fish in the leech Hemiclepsis, the parasites do not pass farther back than the crop (Robertson, 503). Finally, in the many species of pathogenic trypanosomes which are transmitted by tsetse-flies of various species, two types of developmental cycle can be distinguished : in the one, the parasite invades

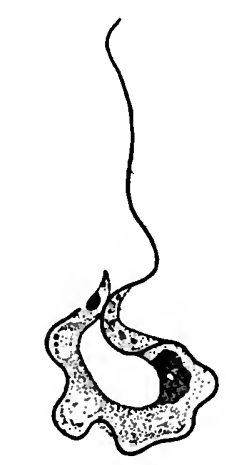

A

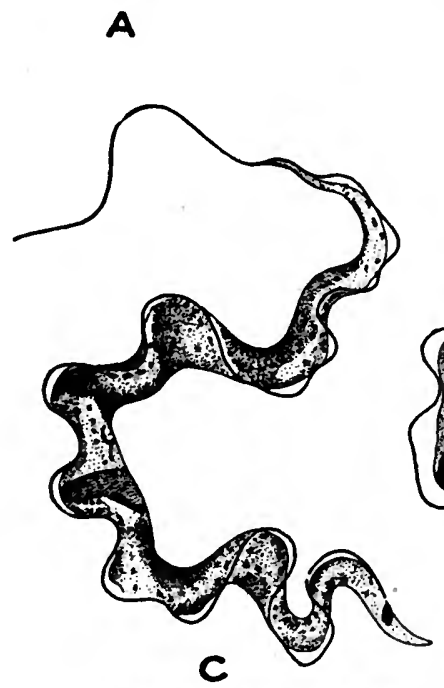

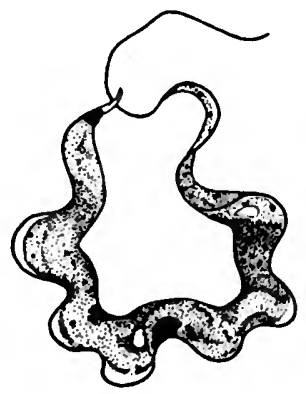

B
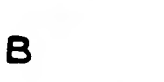
senting the principal multiplicative phase in the invertebrate host ; it is a form in which the kinetonucleus is placed in front of, or close beside, the trophonucleus, and in which, consequently, the undulating membrane is confined to the anterior region of the body, and may be quite rudimentary. As a rule the body of the trypanomonad is shorter, stiffer, more pear-shaped, than in the typical trypanosome-form; no longer sinuous and flexible, it is held straight and rigid in progression, which is effected almost entirely by the flagellum. In many cases, however, the free flagellum is very short, and used to attach the organism to the lining of the digestive tract. Besides the trypanomonad form, the developmental cycle may also include many other tyces of form, and often exhibits a degree of polymorphism which is most bewildering, and compared to which the diversity of form seen in the vertebrate host is but slight.

Taking the development of $T$. lewisi in the rat-flea as a typical cxample, the parasites when taken up by the flea pass with the ingested blood into the stomach (mid-gut) of the insect. In this part they multiply actively in a peculiar manner, not as yet described in the case of any other trypanosome in its invertebrate host (Fig. 130) ; they penetrate into the cells of the epithelium, and in that situation they grow to a very large size, retaining their flagellum and undulating membrane, and exhibiting active metabolic changes in the form of the body, which in early stages of the growth is doubled on itself in the hinder region, thus becoming pear-shaped or like a tadpole in form, but later is more block-like or rounded. During growth the nuclei multiply, and the body when full-grown approaches a spherical form, and becomes divided up within its own periplast into a number of daughter-individuals, which writhe and twist over each other like a bunch of eels within the thin envelope enclosing them. When this stage is reached, the flagellum, which hitherto had been performing active movements and causing the organism to rotate irregularly within the cell, disappears altogether, and the metabolic movements cease; the body becomes almost perfectly spherical, and consists of the periplast-envelopo within which a number of daughter-trypanosomes are wriggling very actively ; the envelope becomes more and more tense, and finally bursts with explosive suddenness, setting free the flagellates, usually about eight in number, within the host-cell. The products of this method of multiplication are full-sized trypanosomes, complete in their structure, and differing but slightly in their characters from those found in the blood of the rat. They escape from the host-cell into the lumen of the stomach.

To the intracellular multiplicative phase in the stomach a crithidial phase in the rectum succeeds (Fig. 131 . In the fullyestablished condition the rectal phase consists of small pear-shaped 
forms with the flagellum very short, in some cases projecting scarcely at all from the body at its pointed end. These forms are found attached by means of their flagella, often in vast numbers, to the wall of the rectum, sometimes also in the intestinal or pyloric region; they multiply by binary fission, and form a stock, as it were, of the parasites, which persists for a long time in the flea-probably, under favourable conditions, for the whole life of the insect. Experiments have shown that a flea once rendered infective to rats can

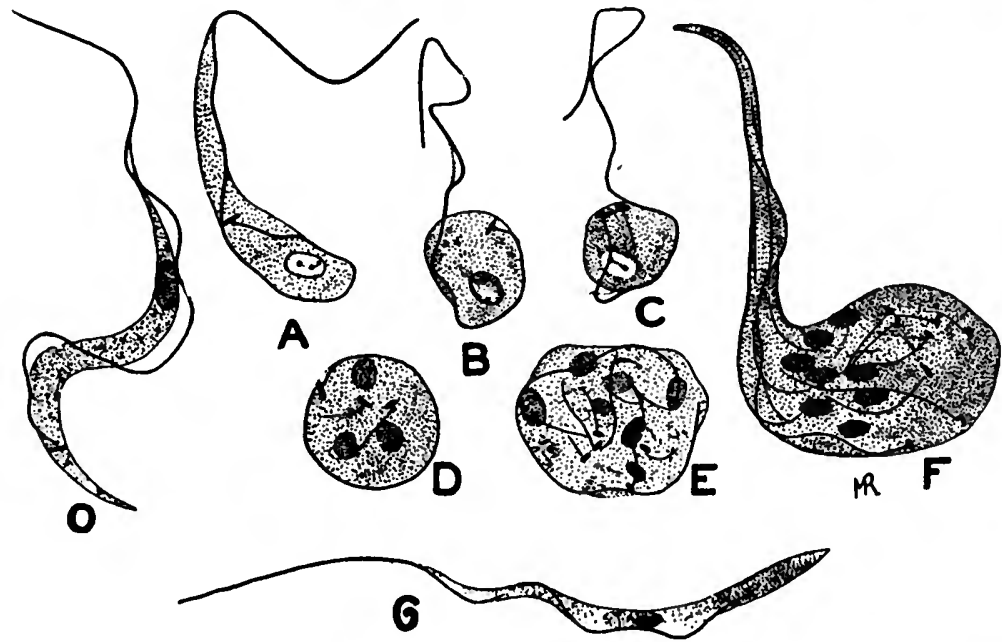

Fic. 130.-Trypanosoma lewisi: developmental phases from the stomuon of the rat-flea. $O$, Ordinary form from the blood of the rat; $A-F$, intraccllular stages : $A$, a trypanoso me eurled on itself ; $B$, similar form in which tho body has become rounded; $C$, multiplication beginning, division of kinetonueleus and trophonucleus, daughter-flagellum growing out ; $D$, further stage-three nuclei of each kind, two short daughter-flagella, and a long parent-flagellum wrapped round the body ; $E$, six nuclei of each kind, five daughter-flagella, parent-flagellum wrapped round the body ; $F$, eight nuclei of each kind, the daughtor-flagella running parallel with the parent-flagellum; $G$, the type of trypanosome resulting from the proeess of multiplication seen in the foregoing figures; this is the form which passcs down the intestine into the rectum. Magnified 2,000.

N.B.-The drawings in this figure and in Fig. 131 are made from preparations fixed wet with Schaudinn's fluid and stained with iron-hæmatorylin; in such preparations the trypanosomes always appear appreciably smallor than in films stained with the Romanowsky-stain (seo Minohin, 479); consequently these figures, though drawn to the same magnification as Figs. 11, 127, etc., are on a slightly smaller seale; compare the trypanosome drawn in $O$ with those in Figs. 11, $A$, and 127, $A$.

remain so for at least three months, without being reinfected. From the rectal stock trypaniform individuals arise by a process of modification of the crithidial forms, in which the flagellum grows in length, the anterior portion of the body becomes more drawn out, the kinetonucleus migrates backwards behind the trophonucleus, taking with it the origin of the flagellum, and an undulating mem- 
brane running the length of the body is established. The trypaniform individuals thus formed are of small size and broad, stumpy form ; they represent the propagative phase which passes from the flea back into the rat. From the rectum they pass forwards into the stomach, and from the stomach they appear to be regurgitated into the rat's blood when the flea feeds.

Experiments show that the flea becomes infective to the rat in about six days after it first took up the trypanosomes from an infected rat. The intracellular phase is at the height of its development about twenty-four hours after the flea takes up the trypanosomes; the rectal phase begins to be established towards the end of
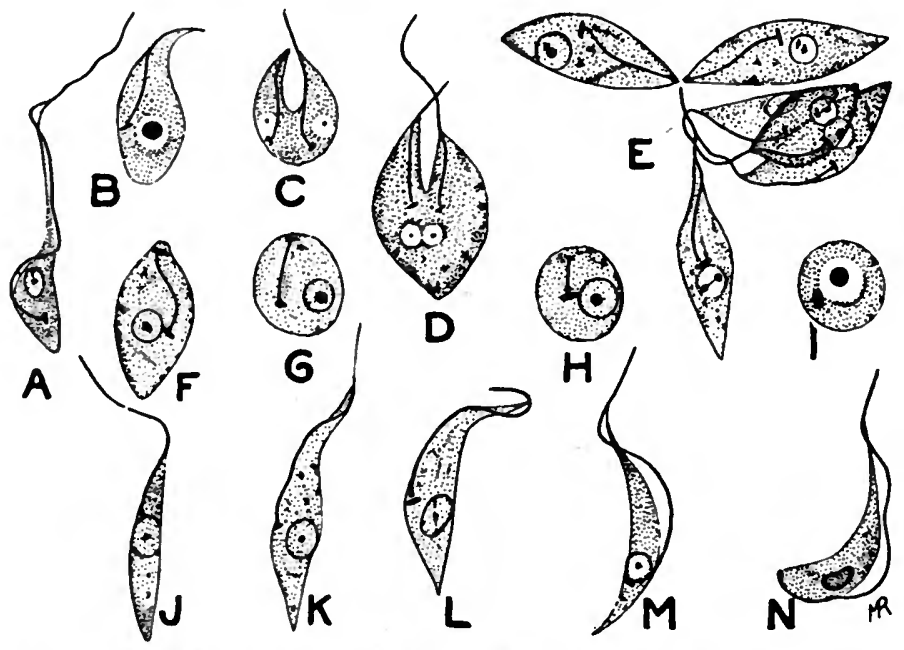

Fia. 131.-Trypanosoma lewisi: developmental phases from the rectum of the ratflea. $A$, Early rectal form; $B$, orithidial form attached to wall of rectum; $C, D$, division of crithidial form ; $E$, clump of crithidial forms detached from wall of reotum, hanging together by their flagella, one of them beginning to divide; $F, G, H$, crithidial forms without free flagells; $I$, rounded form without flagellum; $J, K, L, M$, series of forms transitional from the crithidial to the final trypaniform type; $N$, the last stage in the flea. Magnified 2,000.

the first or beginning of the second day; and the stumpy, trypaniform, propagative fhase is developed in the rectum towards the end of the fifth day.

The account of the development of $T$. lewisi in the flea given in the foregoing paragraphs is based upon investigations, some of them as yet unpublished, carried on in conjunction with Dr. J. D. Thomson by the author (480-482). Some of the phases of the parasite have also been dcscribed by Swellengrebel and Strickland.(517). A number of investigators-namely, Prowazek (497), Breinl and Hindle, Baldrey (396), Rodenwaldt, and othershave studied the devclopment of this trypanosome in the rat-louse (Hamatopinus spinulosus). Experiments have shown that this insect is also capable of transmitting the trypanosomo from rat to rat, but only, to judge from the 
published results, in rare instances, in striking contrast with the ease with which the transmission is effected by the rat-flea. The rat-louse may be regarded, therefore, as a host in which the trypanosome establishes itself only exceptionally, and by no means as the host to which it is best adapted. Crithidial and other forms have been seen in the louse, but the intracellular phase has not been observed, and it is probable that most of the forms described from this host are degenerating forms maintaining a feeble and precarious existence under adverse conditions, and destined to die off and disappear sooner or later.

The developmental cycle of Schizotrypanum cruzi in the bug Conorhinus megistus has been described by Chagas,* and is briefly as follows (Fig. 132):
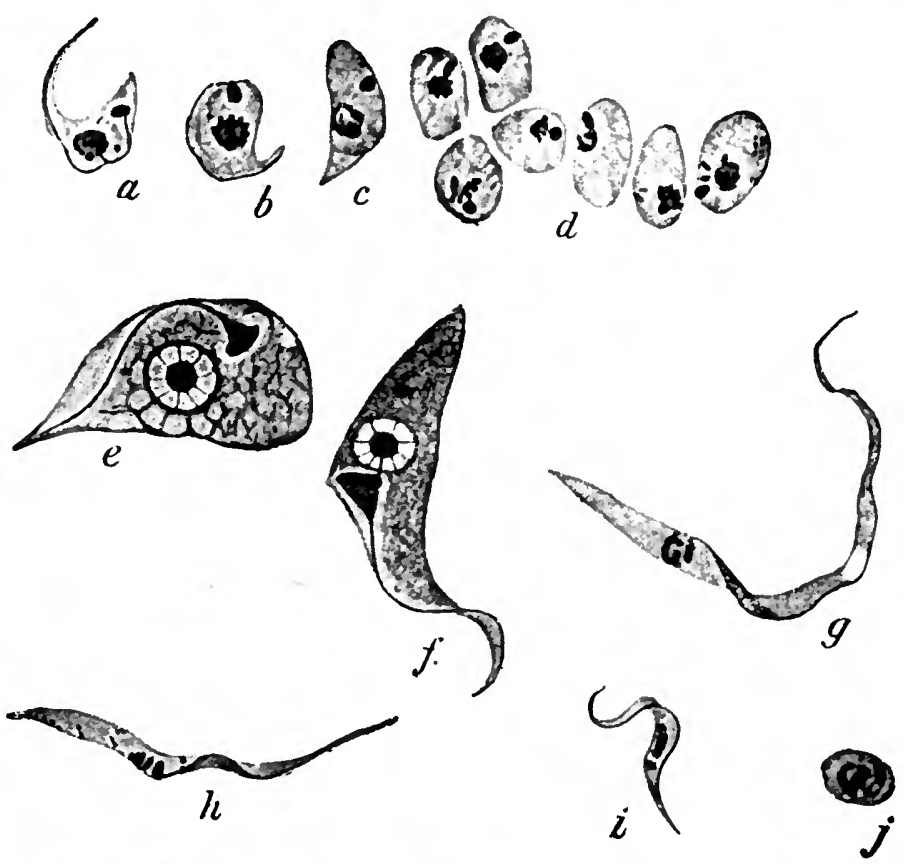

Fra. 132. - Phases of Schizotrypanum cruzi in the bug Conorhinus megisius. $a, b$, and $c$, Forms transitional from the ordinary trypanosomes to the rounded forms; $d$, clump of rounded forms; $e$ and $f$, change of rounded into orithidial forms; $g$ and $h$, crithidial forms; $i$, trypaniform type from the salivary glands $; j$, encapsuled form from the intestine. After Chagas (425).

The trypanosomes taken up by the bug into its stomach change in about six hours; they lose their flagellum and contract into rounded, Leishmanialike forms, which multiply actively by fission. After a time multiplication ceases, and the rounded forms become pear-shaped, develop a flagellum at the pointed end, and change into typical crithidial forms which pass on into the intestine, and there multiply by fission. In this way the characteristio condition of the infected bug is produced, with the intestine containing a swarm of trypanomonad individuals multiplying actively. The final stage in the insect is a small trypaniform type which is found in the body-cavity and salivary glands, whence it doubtless passes into a vertebrate host again.

* A critical summary and review of the memoir of Chagas is given by Minchin in Nature, vol Ixxriv., pp. 142-144 (August 10, 1910), with three text-figures. 
The three principal phases in the development of $T$. cruzi in the bug may be compared, without difficulty, with those of $T$. lewisi in the flea, though differing in minor details; in both cases an early multiplicative phase in the stomach is followed by a crithidial phase, also multiplicative and constituting the principal stock of the parasite, in the hinder part of the digestive tract; to this succeeds a propagative trypaniform phase, which in the case of $T$. lewisi passcs forwards to the stomach, but which in the case of $T$. cruzi appears to pass through the wall of the alimentary canal into the body-cavity, and so into the salivary glands. Other developmental forms have been described by Chagas, but their relation to the cycle of the parasite, if indeed they really belong to it, is not clear.

The developmental cycle of the trypanosomes of fresh-water fishes in the leech Hemiclepsis marginata (Robertson, 503) begins also by active multiplication in the crop about six to nine hours after the flagellates have been ingested. The trypanosomes divide by repeated binary fission of unequal type, budding off small individuals which are crithidial in type and multiply in their turn. In a few days the crop is populated by a swarm of trypanomonad forms of various sizes, multiplying actively. Towards the end of the digestion, the propagative phase begins to appear in the form of long, slender trypaniform individuals which arise directly from the crithidial forms, and pass forwards in great numbers from the crop into the proboscis-sheath, whence they are inoculated by the leech into a fresh host. A certain number of the crithidial forms remain behind in the crop, however, where during hunger-periods they may pass into a resting Leishmania-form; when the crop is again filled with fresh blood, these forms begin to multiply again, repopulating the crop with crithidial forms, from which a fresh batch of trypaniform propagative individuals arise towards the end of digestion again.

In the development of $T$. raia in the leech Pontobdella muricata (Robertson, $500,502)$, the ingested trypanosomes multiply in the crop in a similar manner by unequal binary fission, budding off small individuals which, however, are rounded and leishmanial in type, and which pass down from the crop into the intestine, where they develop a flagellum, become crithidial in type, and multiply actively. During hunger-periods they become leishmanial, resting forms which persist when all other forms have succumbed and died out, becoming crithidial again when the supply of food is renewed. From the crithidial forms arise the long, slender trypaniform individuals of the propagative phase, which pass forwand into the proboscis to be inoculated into the fish. The development of $T$. vittatce, from the blood of the Ceylon tortoise, Emyda vittata, in the leech Glossiphonia sp., is of a similar type, but takes place almost entirely in the crop (Robertson, 501).

The development of $T$. gambiense in the tsetse-fly, Glossina palpalis, so far as it has been described by Kleine (457), Kleine and Taute (459), and Bruce and his collaborators (415), presents some peculiar features not quite intelligible at present. The whole development takes a long time, about eighteen to twenty-five days or more, a fact which, together with the low percentage of flies which become infected, accounts for the existence of a developmental cycle having been missed by so many investigators, until it was first discovered by Kleine. From five to seven days after the infection of the fly the trypanosomes disappear or become scarce in its digestive tract, indicating, possibly, an intracellular stage yet to be discovered. Later, in a small percentage of the flies, the trypanosomes reappear in the digestive tract in enormous numbers. The flagellates at this stage vary greatly in size, form, and appearance, but crithidial forms are stated to be very rare, a feature in which the development contrasts with the usual type seen in other trypanosomes. Finally an invasion of the salivary glands takes place, though in what way it is brought about is not clear; short, stumpy trypaniform individuals are found in the glands, which represent the ripe, propagativa phase destincd to be inooulated into the vertebrate host. These ripe forms first make their appearance, according to Kleine, in the intestine. 
In many species of trypanosomes transmitted by tsetse-flies, a peculiar mode of development occurs, as already stated, in the proboscis, termed by Roubaud, who discovered it, a culture d'attente. The trypanosomes taken up from the vertebrate change very rapidly into trypanomonad ("leptomonad," Roubaud) forms, with the kinetonucleus far forward, and attach themselves by the tip of the flagellum to the wall of the proboscis tube. In this situation they multiply in the salivary fluid by binary fission, until great numbers are present. In some cases this culture in the proboscis appears to be the sole form of developmental cycle in the fly, as, for example in T. cazalboui (Roubaud, 506, Bouffard), T. vivax (Bruce, 411, iii.); this typo is termed by Roubaud évolution par fixation directe. In other species (T. dimorphon, T. pecaudi) the parasite multiplies first in the digestive tract of the fly, and then spreads forward into the proboscis-évolution par fixation indirecte of Roubaud; in this case, however, the possibility does not seem to be excluded that the forms seen in the digestive tract may have belonged to the developmental cycle of a distinct trypanosome. Development of this kind has only been observed in tsetse-flies.

According to Bouffard, $T$. cazalboui can be transmitted mechanically by Stomoxys, but goes through its developmental cycle only in the proboscis of Glossina palpalis; Stomoxys may therefore cause epidemics of the disease (" souma"), but endemic areas are always in regions where G. palpalis occurs. The tsetse-fly is not infective until six days after first feeding on an infected animal, and it then remains infective permanently, or at least for the greater part of its existence. Hence the proboscis-cycle is a rapid development, comparable, as regards the time it requires, to that of $T$. lewisi in the flea rather than to that of other trypanosomes in the digestive tract of the tsetise.

Finally,* mention must be made of the cysts of $T$. grayi, described by Minchin (476), occurring in the hind-gut of Glossina palpalis. The cysts result from the encystment of a crithidial form, and are very similar to the cysts of Herpetomonas, described by Prowazek (Fig. 124), from the hind-gut of the house-fly ; their mode of formation indicates that they are destined to pass out of the rectum to the exterior with the fæces, and Minchin has suggested that a contaminative method of spreading the infection may occur in addition to the usual inoculative method. The possibility must be reckoned with, however, that the cysts in question may be part of the cycle of a distinct flagellate parasite, perhaps peculiar to the fly alone, and may not belong at all to the life-cycle of $T$. grayi, which has now been shown to be the developmental form of the trypanosome of the crocodile (cf. Cystotrypanosoma, Roubaud, $557 \cdot 5)$. According to Kleine and Taute, trypanosomes, not encysted, may be found in the freces of infected tsetses.

Apart from the somewhat aberrant development of the members of the brucii-group, which require further elucidation, the cycle of a trypanosome in the invertebrate host appears to consist typically of three principal phases : (1) An initial multiplicative phase, which may be trypaniform, as in T. lewisi, or Leishmania-like, as in $T$. cruzi, or may take the form of unequal division of large trypaniform individuals to produce either small crithidial forms directly, as in fish-trypanosomes in the leech Hemiclepsis, or rounded Leishmania-forms which later become crithidial, as in $T$. raice and $T$. vittacet; to this initial phase succeeds (2) a crithidial phase, which may pass farther down the alimentary canal. and which in any case multiplies by fission and constitutes the principal stock of the

* The development described by Schaudinn (132) for $T$. noctua is dealt with in a subsequent chapter (p. 390). 
parasite, keeping up the infection of the invertebrate host. In hunger-periods the flagellates may persist as simple, rounded, Leishmania-like forms. Sooner or later many, it may be the greater numher, but not all, of the crithidial forms become modified into the trypaniform individuals, which represent (3) the propagative phase of the parasite, and pass forwards to be inoculated into the vertebrate host. Those crithidial forms which do not become transformed into the propagative individuals remain to multiply and replenish the stock.

A very much debated question in this development is that relating to the occurrence of sexual phases and syngamy, which, purely on the analogy of the malarial parasites, are assumed almost universally to occur in the invertebrate host. Not in a single instance as yet, however, has the sexual act been proved satisfactorily to take place in the development of trypano. somes. T'he fertilization described by Schaudinn (132) in " $T$. noctuce" is the well-known conjugation of Halteridium, which can be observed without difficulty ; and though Schaudinn described so-called "male" and "female" types of trypanosomes in the mosquito, he expressly stated that they did not and could not conjugate. The process of syngamy described by Prowazek (497) for T. lewisi in the rat-louse, though " confirmed " by Baldrey (396), Gonder ${ }^{\prime}(445 \cdot 5)$, and Rodenwaldt, is almost certainly the agglomeration of degenerating forms (Swellengrebel, 516; compare Reichenow, 78, p. 268). Less biassed investigators, who have studied the developmental cycle of trypanosomes with great care, such as Chagas, Robertson, and others, have been quite unable to observe sexual processes of any kind. The liability to error in the interpretation of observations is greatly increased, first by the fact that trypanosomes divide longitudinally and often unequally, secondly by the phenomena of agglomeration (p. 128), which occur readily under un. favourable conditions. Consequently the adhesion together of two trypanosomes may be due to quite other causes than sexual affinity. In some cases the alleged occurrence of syngamy has been based merely on the fact that non-flagellated forms have been seen, which, on the analogy of the malarial parasites (p. 362), are termed "oökinetes" and interpreted as zygotes.

It is certainly remarkable, in view of the paucity of data, that so many investigators, following Schaudinn's lead, should persist in ascribing all formdifferentiation in trypanosomes to sex, and should be unable, apparently, to conceive of any other cause of polymorphism in parasites which have to adapt themselves, in the course of their life-cycle, to a great diversity of conditions (compare also Doflein, 430). It must be emphasized that the only true criterion of sexual polymorphism is sexual behaviour, and until that has been established it is premature to speak of sexual differentiation.

Some investigators have upheld the unfashionable view that the syngamy of trypanosomes occurs in the vertebrate host; so Bradford and Plimmer, and more recently Ottolenghi, who has described in $T$. brucii, $T$. equinum, $T$. gambiense, and T. equiperdum, the following process of sexurl conjugation: Two trypanosomes of very different size and appearance attach themselves to one another by their hinder ends. One, regarded as tho microgamete, is more slender, and contains one trophonucleus or a larger nucleus of this kind and two smaller (reduction-nuclei); the other, the macrogamete, is much larger, and contains also a larger nucleus near the kinetonucleus and two or more other nuclei in process of degeneration. The macrogamete also has usually three, sometimes two or four, flagella and undulating membranes. After the two gametes have united by their hinder ends, a small nucleus is budded off from the principal nucleus of the microgamete, passes over into the body of the macrogamete, and fuses with its principal nucleus. Subsequently the microgamete appears to degenerate, and the fertilized macrogamete to 
divide up into trypanosomes of the ordinary type. Those who consider that syngamy can only occur in the invertebrate host will doubtless regard the process described by Ottolenghi as phenomena of agglomeration and degeneration. In the present state of our knowledge, however, it is best to keop an open mind on this question, and to await further investigations.

In T. gambiense, Moore and Breinl (484) have described a process of fusion between the kinetonucleus and trophonucleus in the formation of the "latent bodies," and have interpreted this as a sexual process, a suggestion hardly to be taken seriously. A similar process alleged to occur in the multiplicationforms of $T$. lewisi has been interpreted by Schilling as the inevitable autogamy.

All that can be said at present, with regard to sexual processes in trypanosomes, is that, on the analogy of other Protozoa generally, syngamy may be expected to occur in some part of the lifo-cycle. It remains, however, for further research to establish definitely the conditions under which syngamy takes place, and the nature of the process in these organisms; nor can it be considered as sound reasoning, in the absenco of concrete observations, to attempt to limit the possible occurrence of syngamy, or to infer the exact form it takes, either by analogies more or less far-fetched with one or another group of Protozoa, or by tho mere existence of form-differentiation, and still loss by the arbitrary interpretation of certain forms as zygotes or oökinetes.

A very variable feature in the development of trypanosomes is the suscoptibility of the invertebrate host. In the case of $T$. lewisi, only about 20 per cent., approximately, of the fleas fod experimentally on infected rats become infective in their turn, and in the case of tsetse-flies and pathogenic trypanosomes the percentage is much smallor. There are also grounds for suspecting that a certain condition or phase of the trypanosome in the blood of the vertebrato is sometimes necessary for establishing the developmental cycle in the invertebrate; compare the observations and conclusions of Chagas with regard to Schizotrypanum cruzi, mentioned above (p. 296). In Trypanosoma noctuce the summer form which appears in tho blood is of a type distinct from the winter forms found in the bonc-marrow (Minchin and Woodcock, 42). On the other hand, in the case of the trypanosomes of fresh-water fishes, Robertson (503) found that evcry leech became infected that was fed on an infected fish; so that the simplest method of determining whether a fish was infected was to feed a nowly-hatched Hemiclepsis on it.

A question often discussed is whether trypanosomes in any part of their development may pass through " ultramicroscopic" stages. Schaudinn (132) expressed the opinion that some stages of trypanosomes investigated by him were small enough to pass through bacterial filters; though he did not put this suggestion to an experimental test, it is often quoted as a proved fact.* Moore and Breinl (484) also asserted, without experimental data, that infected blood remained infective after filtration. On the other hand, attempts by Bruce and Bateman to obtain experimental verification of these statements gave negative results (compare also Report XI., p. 122, of the Sleeping Sickness Commission).

Recently it has been asserted by Fry that $T$. brucii can throw off granules which, when liberated, possess a certain motility of their own in the blood; this process is regarded as "essentially of a vital and not a degenerative nature." That a trypanosome or any other living cell might excrete grains which when set free could exhibit movements due to molccular or other causes is highly probable; but that such grains represent a stage in the lifehistory of a trypanosome is far from being so ; nor can analogy with spirochætes be considered as a legitimate argument in favour of any such conclusion.

There remains for consideration the development which trypanosomes undergo in artificial cultures, in which they exhibit a series of forms quite different from those seen in the blood of the vertebrate, and so far resembling the cycle in the invertebrate host in that the predominant phase is a crithidial

* It is doubtful whether the forms of which Schaudinn made this statement were really trypanosomes or spirochætes. 
or trypanomonad type of flagellate. Until the cultural development of a trypanosome has been compared in detail with its natural development in the invertebrate host, it is impossible to estimate precisely the bearing of the cultural series of stages from the point of view of the physiology and morphology of the parasite. The only investigator who has attempted this is Chagas (425), who found in cultures of Schizotrypanum cruzi the same thre principal phases-namely, rounded, crithidial, and trypaniform-that occu in the natural cycle, and in the same order of sequence. At present, therefore, it would be unprofitable to discuss in detail the series of forms occurring in artificial cultures, and it must suffice to refer the reader for further information to the principal works on the subject, namely, those of Novy and McNeal (489), Bouet, Franca $(438,443)$, Rosenbusch, Thomson (525), Woodcock (527), Lebedew, and Doflein (431). As already pointed out above, the cultural method is often of the greatest practical value in determining whether, in a given case, an animal is infected with trypanosomes or not.

Lebedew has described what he believes to be syngamy in the cultural phases of $T$. rotatorium; compare also the account of Leishmania below (p. 319).

The genus Trypanosoma comprises a vast number of species, parasitic in the blood of animals throughout the vertebrate series; and several attempts have been made to subdivide and classify
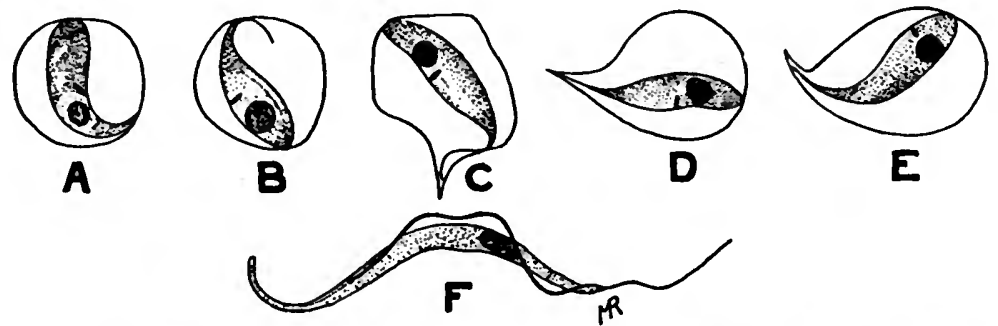

Fra. 133.-Endotrypanum schaudinni from the blood of Cholcpus didactylus. $A-E$, Various forms of the intracorpuseular parasite; $F$, trypanosome from the blood of tho same host. After Mesnil and Brimont, magnified about 1,500 diameters.

this comprehensive genus into smaller groups. Such attempts have either taken the course of splitting off particular forms, characterized by some special peculiarity, from the main group, or of subdividing the group as a whole on some principle of morphology or development. An example of the first method is tne foundation by Chagas (425) of the genus Schizotrypanum, as already mentioned, for $T$. cruzi, on the ground that it multiplies by schizogony and possesses intracorpuscular phases. The genus Endotrypanum was proposed by Mesnil and Brimont for a peculiar form which was diseovered by them within the red blood-corpuscles of a sloth (Chulapus didactylus), and which is very probably an intracorpuscular phase of a trypanosome found free in the blood-plasma of the same host. The life-cycle of Endotrypanum is not yet known. Chagas considers it not improbable that it should be placed in the 
same genus as $T$. cruzi, in which case the name Endotrypanum has the priority over Schizotrypanum. In the present state of knowledge, data are lacking for deciding how far it is possible to employ either multiplication by schizogony or an intracorpuscular habitat as characters for defining genera of trypanosomes. An intracorpuscular habitat is probably commoner in trypanosomes than has usually been supposed. It has been described quite recently by Buchanan in T. brucii.

Attempts to subdivide the genus Trypanosoma as a whole have bsen based on the possibility that the trypanosome-type of structure may have had two distinct phylogenetic origins, one through Leptomonas and Crithidia from a cercomonad ancestor, the other through Trypanoplasma from a heteromastigote or Bodonid type. The trypanosome-form might be imagined to have arisen from either of these two types. It could be derived from a form like Trypanoplasma by loss of the free anterior flagellum, in which case the flagelluir: of a trypanosome is to be regarded as posterior ; on the other hand, if, in a form like Leptomonas, the kinetonucleus and with it the origin of the flagellum, be shifted backwards to the neighbourhood of the trophonucleus, and if at the same time the flagellum runs forwards along the body connected to it by an undulating membrane, a Crithidia-like form results, from which, by still further displacement backwards of the kinetonucleus and flagellum to near the posterior end of the body, a trypanosomeform is produced in which the single flagellum is to be regarded as anterior. It is therefore conceivable that the trypanosome-form may comprise two morphological types, structurally indistinguishable, but entirely different in origin, and opposite in morphological orientation of the body.

From this point of view, Woodcock (395) subdivided trypanosomes into two genera : Trypanomorpha, with cercomonad ancestry and flagellum morphologically anterior; and Trypanosoma, in a restricted sense, with heteromastigote ancestry and flagellum morphologically posterior. The genus Trypanomorpha included only one species, $T$. noctuce of Athene noctua; all other species of trypanosomes were left in the genus Trypanosoma sens. strict. Liihe put forward a classification based on similar conceptions with different interpretations, and proposed three genera of trypanosomes : Hamatomonas (Mitrophanow) for the trypanosomes of freshwater fishes believed to have a heteromastigote ancestry ; Trypanozoon for the trypanosomes of mammals, such as T. lewisi, T. brucii, etc., regarded as having a cercomonad ancestry and an anterior flagellum; and Trypanosoma sens. strict. for the trypanosomes of frogs and reptiles. T. noctuce, on the other hand, he regarded, in agreement with Schaudinn (soe p. 390, infra), merely as a develop- 
mental stage of Homoproteus. Although, however, it is quite possible that some trypanosomes may have a lieteromastigote ancestry, all the developmental facts hitherto discovered indicate a cercomonad ancestry with a single anterior flagellum, and there is no concrete evidence of a heteromastigote origin for any species that has been studied up to the present. Trypanoplasms, so far as they have been studied, preserve their biflagellate, heteromastigote type of structure throughout their development in all active phases, and never pass through a trypaniform or crithidial phase. Trypanosomes, on the other hand, show constantly a crithidial phase in the invertebrate host, but havo not been observed in any case to be heteromastigote or even biflagellate, except temporarily during division, in any phase of the life-history. Consequently, attempts to subdivide trypanosomes on a morphological or phylogenetic basis must be regarded at present as premature (compare also Laveran, 461).

\section{The Gends Trypanoplasma.}

The peculiar distribution and occurrence of the species of this genus has been pointed out above. Originally founded for forms parasitic in the blood of fishes, it now comprises a somewhat heterogeneous collection of species, some of which were formerly referred to other genera of Flagellates. Of recent years, the number of species known to be parasitic in invertebrate hosts has increased, and is increasing rapidly. Such are $T$. ("Trypanophis") grobbeni, found in the gastrovascular system of Siphonophora (Keysselitz, 453) ; T. (" Borlo ") helicis, from tho receptaculum seminis of Helix pomatia and other snails (Friedrich); T.dendrocoeli, from the digestive tract of Dendrocoelum lacteum (Fantham and Porter, P.Z.S., 1910; p. 670); T. vaginalis, from the female genital organs of leeches (Hesse, C.R.A.S., cli., p. 504) ; and T. gryllotalpa, from the end-gut of Gryllotalpa vulgaris (Hamburger). These examples show that the genus, as at present defined, is of widespread occurrence. It may be doubted, however, if the various species described should all be placed together.

The species of Trypanoplasma parasitic in blood are only known as yet from fresh-water fishes ;* they have an alternation of hosts, being transmitted by leeches. The life-history of tho intestinal trypanoplasms has not been investigated, but in all probability they have but a single host, which acquiros the infection by swallowing accidentally their cysts or other resting stages passed out from a

* The "Trypanoplasina" stated by Bruce and his colleagues (412, pp. 495, 496) to occur in the blood of birds and in the digestive traet of tsetse-fies was in reality
a Lewcocytozoon. 
former host. T. helicis, according to Friedrich, passes from one snail to another mechanically in the spermatophores during coitus. The following account refers mainly to the blood-inhabiting species:

The body of a trypanoplasm is relatively broader and shorter, less sinuous and serpentine, than that of a trypanosome, and is at the same time softer and more plastic, being limited by an extremely thin periplast. The contractile, often slightly metabolic, body yields readily to pressure, and exhibits in consequence passive form-changes when moving among blood-corpuscles or
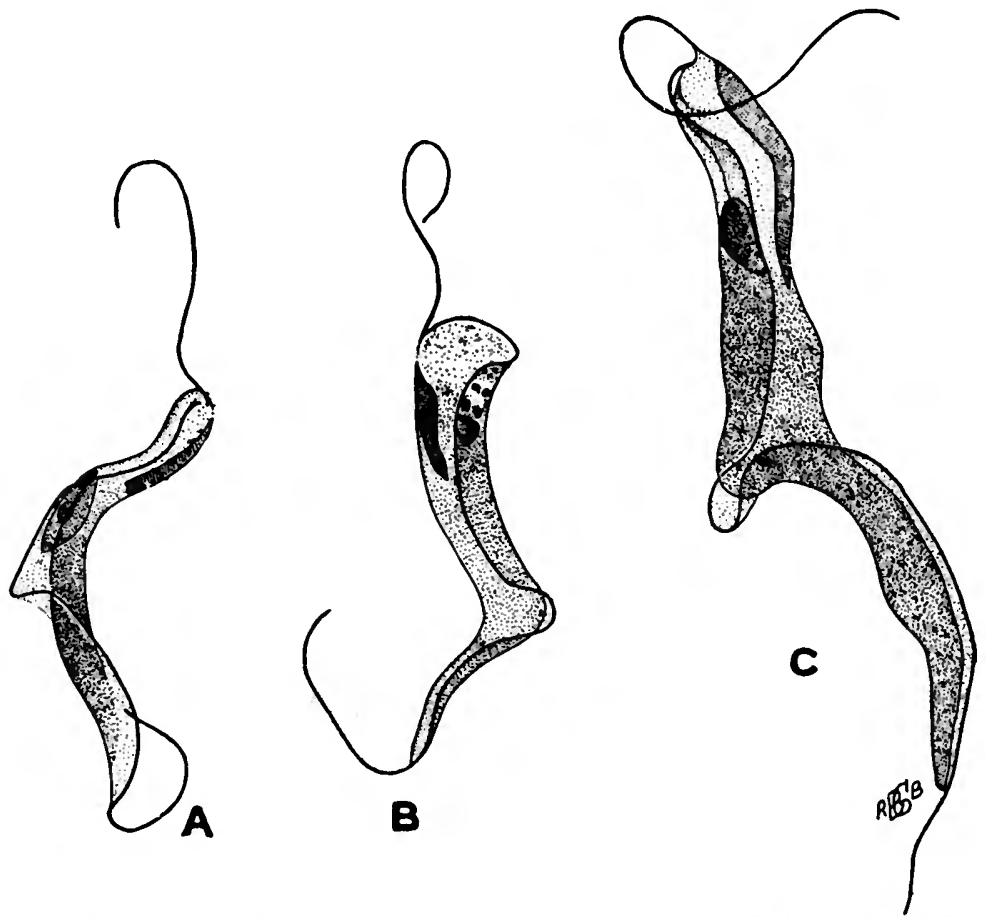

FrG. 134.-A, Trypanoplasma abramidis from the blood of tho bream; $B$ and $C$, $T$. keysselitzi from the blood of the tench : $B$, small ordinary form ; $C$, large form. After Mincnin, magnified 2,000.

other solid particles. The principal structural feature is the possession of two fiagella, which arise close together at the anterior extremity from a pair of blepharoplasts or diplosome, or from a single basal granule (Martin). One flagellum proiects freely forwards ; the other turns more or less abruptly backwards, and passes down the side of the body at the edge of an undulating membrane to the hinder end, beyond which it projects freely backwards to a variable extent in different species. In $T$. gryllotalpoe the un- 
dulating membrane only extends along two-thirds of the length of the body, after which the posterior flagellum becomes free. The kinetonucleus, situated at the extreme anterior end of the body, is relatively very large, usually exceeding the trophonucleus in size, and is sometimes constricted into two or three portions, but is generally a compact mass which stains deeply in preparations. In T. helicis, according to Jollos, it is prolonged backwards into fibrils, usually two in number, which extend some way down the body, and are probably comparable to an axostyle. The trophonucleus has a vesicular structure with a conspicuous karyosome. Its position in the body varies, being in some species close behind the kinetonucleus, in others near the middle of the body. It often appears to be lodged completely in the undulating membrane, which in this genus is often very broad and less sharply defined than in a trypanosome, appearing as the border of a flattened body. The cytoplasm frequently contains numerous " chromatoid grains."

Trypanoplasms in the blood of fishes often exhibit marked polymorphism, with two extremes of size, small and large (Fig. 134, $B, C)$. According to Keysselitz (454), the large forms are the gametes which conjugate in the leech, and are distinguishable as male and female forms, but the statement requires confirmation. From the investigations of Robertson (503) on the development, it appears more probable that the large forms are simply full-grown individuals, ripe for multiplication by fission. Unfortunately, next to nothing is known of the reproduction of the parasites in the vertebrate host, though it has been observed that their numbers are subject to considerable fluctuations, and that a fish showing at one time a very scanty infection of the blood may have a "relapse," and appear later well infected. Keysselitz accuses these parasites of pathogenic properties, but this charge is founded on observations on fish in captivity, in which weakened powers of resistance may lead to abnormal activities on the part of the parasite (compare also Neresheimer).

The development of blood -trypanoplasms in the invertebrate host, which is in all known cases some species of leech, appears to be of a comparatively simple type as compared with that of trypanosomes, and consists of little more than rapid multiplication by binary fission to produce a swarm of relatively small trypanoplasms, some of which, more slender and elongate in form, pass forwards into the proboscis, and are inoculated by the leech into a fish. Conspicuous in this developmont, as compared with that of trypanosomes, is the entire absence of any uniflagellate forms, crithidial or other. So long as a trypanoplasm is in an active state, it is invariably biflagellate. Resting forms without a locomotor apparatus may occur. In $T$. helicis, Friedrich describes winter 
forms with a single nucleus, which is in some cases the trophonucleus, in others the kinetonucleus.

The accounts given of the process of division are gomewhat conflicting. According to Martin, division of $T$. congeri is initiated by the division of the singie basal granule of the flagella, followed by splitting of each flagellum longitudinally. Next the trophonucleus divides amitotically, the karyosome becoming first drawn out into a band, after which the nucleus as a whole is constricted into two. Lastly the kinetonucleus becomes elongated, and divides simply by a transverse constriction into two pieces. Jollos, however, following Rosenbusch's statements for trypanosomes, affirms that the division of both nuclei is mitotic in $T$. helicis. Alexeieff, on the other hand, denies that the kinetonucleus of Trypanoplasma is a nicleus at all. This author also describes a series of chromatinic blocks at the base of the undulating membrane of $T$. intestinalis, similar to those seen in Trichomonas (compare Fig. 5).

Keysselitz (454) has described syngamy in the development of $T$. "borreli" in the leech Piscicola, but the description and figures are unconvincing, and the matter requires reinvestigation. No other investigators have found sexual processes of any kind in trypanoplasms.

\section{The Genus Crithidia.}

The distinctive structural feature of Crithidia (Fig. 135, $A$ ) is the relatively short undulating membrane which, with the single flagellum, arises in the middle of the body from the vicinity of a

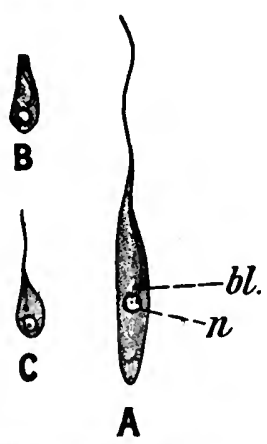

Fra. 135, - Crithidia minuta, Iéger, from the gut of Tabanus tergestinus. $A, \mathrm{Or}$ dinary motile individual; $B, C$, young forms, with flagellum short or rudimentary. After Léger. kinetonucleus situated beside, or in front of, the trophonucleus. The form of the body varies from a relatively long, slender type to the short, "barley-grain" form from which the name of the genus is derived.

As already pointed out, the application of the name Crithidia as the denomination of a genus is involved in considerable confusion and perplexity-partly because the distinctive morphological characters shade off by imperceptible gradations into those of trypanosomes on the one hand, and leptomonads on the other, but still more because a certain number of the "species of Crithidia" are unquestionably developmental stâges either of trypanosomes or leptomonads, and others are justly suspected of being so. In the present state of knowledge, it is safest to presume that any "Crithidia" from the digestive tract of a blood-sucking insect is a stage of a trypanosome from the blood of a vertebrate, until the contrary has been clearly established. At the same time the possibility must always be taken into account that a blood-sucking invertebrate may harbour flagellate parasites peculiar to itself in addition to those 
which it takes up in vertebrate blood, and that in this way stages of the life-cycle of two or more distinct parasites may be confused together. Up to the present, however, no blood-sucking insect has been proved satisfactorily to harbour flagellate parasites not derived from vertebrate blood.

After deducting doubtful species of Crithidia, there remains a residue which appears to comprise genuine, independent species, parasites of the digestive tract of insects. As examples of such species may be cited $C$. campanulata, recorded from the digestive tract of Chironomus plumosus (Léger, A.P.K., ii. 1903, p. 180), from that of the larva of Ptychoptera (Léger and Duboscq) and of caddis-worms (Mackinnon, 547) ; $C$. gerridis, from Gerris spp. (Patton, 550 ; Porter, 555); and possibly others. The life-cycle of $C$. gerridis has been investigated by Patton and Porter. The parasite appears under two principal phases : an active, flagellate phase, which grows to a large size, and multiplies by fission, sometimes very actively, forming rosettes; and a resting, nonflagellate Leishmania - form. The flagellate forms may be free in the digestive tract, or may attach themselves to the lining epithelium of the gut by their flagella. The non-flagellate forms are found in the crop, where they grow into the adult phase, and in the rectum, where they become encysted. The flagellate phase is found throughout the digestive tract and in the ovaries, but has not been observed to pass into the ova The encysted forms pass out of the rectum, and infect new hosts by the contaminative method.

\section{The Gends Leptomonas (Herpetomonas).}

The genus Leptomonas comprises typical intestinal parasites of insects, especially Diptora and, above all, Muscida. Several species are also known in Hemiptera. They are in most cases parasites of the insect alone, having no alternate host, and infection is brought about by the contaminative method, so far as is known, cysts dropped by one host being accidentally devoured by another. But some species are found as parasites of the latex of Euphorbiaceæ, and in this case an alternation of hosts occurs. The parasites are taken up from the plants by bugs (Hemiptera) which suck their juices, and by the agency of the bugs the flagellates are inoculated into other plants again (Lafont; Bouet and Roubaud, 530 ; França, 537, 538). There can be little doubt that in this case the bug is the primary, the plant the secondary host. The plants, or the parts of them that are infected by the Leptomonas, suffer considerablv. The term "flagellosis" has been proposed for the disease. 
The distinctive structural features of this genus are the possession of a single flagellum, arising from close beside a kinetonucleus which is placed far forwards in the body, and the entire absence of an undulating membrane (Fig. 136, $B$; Fig. 137, d). As already stated above, however, the application of the names Leptomonas and Herpetomonas is much disputed, and the morphological definition of the genera in question is attended with considerable difficulties, chiefly owing to the foct that in one and the same host a great variety of forms may occur, with regard to which it is not possible. in the present condition of knowledge, to state with certainty whether they represent distinct species of flagellates, referable even to distinct genera, occurring fortuitously in the same host, or whether they are all merely developmental phases of the same species. The following are the principal forms which may
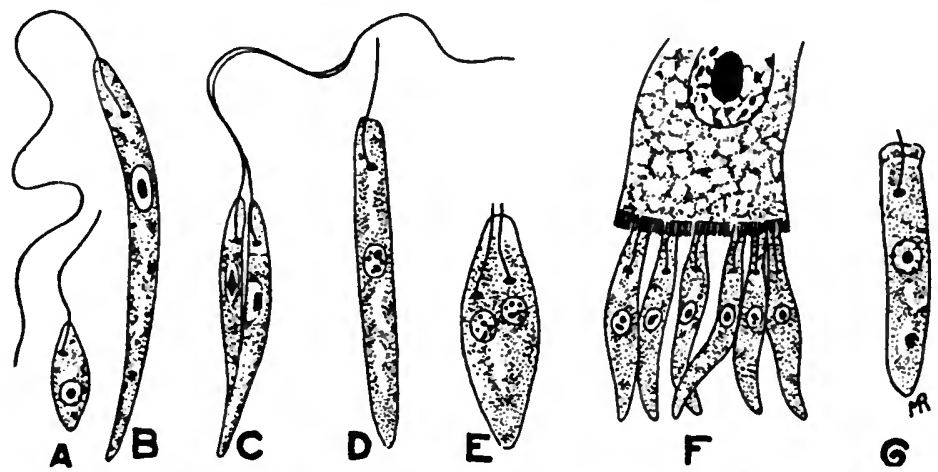

Fia. 136.-Leptomonas jaculum, Léger, from the intestine of Nepa cinerea. $A, B$, Monad forms ; $C$, division of a monad form ; $D$, monad form with short flagellum; $E, F, G$, gregarine-like forms: $E$, in division, $F$, attached to an epithelial cell by the rudimentary flagella, which resemble the rostra of gre. garine sporozoites. After Léger.

occur together in the same host: (1) Large, biflagellate individuals (Fig. 124, $A$ ), often with a distinct pair of rhizoplasts connected with the two flagella, the type to which, according to one set of opinions, the name Herpetomonas should be restricted, but which on another view represents merely an early stage in binary fission, with a daughter-flagellum precociously formed; (2) smaller flagellates with a single flagellum (Fig. 136, $B ;$ Fig. 137, d). the type for which the name Leptomonas is employed by those who regard the true Herpetomonas as typically biflagellate, while by those who hold the contrary view the two genera are ranked as syrionyms ; (3) crithidial forms (Fig. 137, g) ; (4) trypaniform flagellates (Fig. 137, $j, k$ ), with the kinetonucleus at the posterior end, and the flagellum running the whole length of the body with a more or less distinct undulating membrane-the "leptotrypanosomes" of Chatton. In 
addition to these four types of active flagellates, there may occur also non-flagellated individuals or with the flagellum rudimentarynamely, (5) long "gregariniform" individuals (Fig. 136, $E-G$; Fig. 137, $q$ ) and (6) oval or rounded Leishmania-forms. The latter may become encysted and function as the propagative stages. If the four active forms are all distinct species, one and the same host may have intestinal flagellates belonging to four different genera; if they are all phases in the development of one species, it becomes a subtle point for discussion which of the four forms is to be regarded as the "adult" generic type.
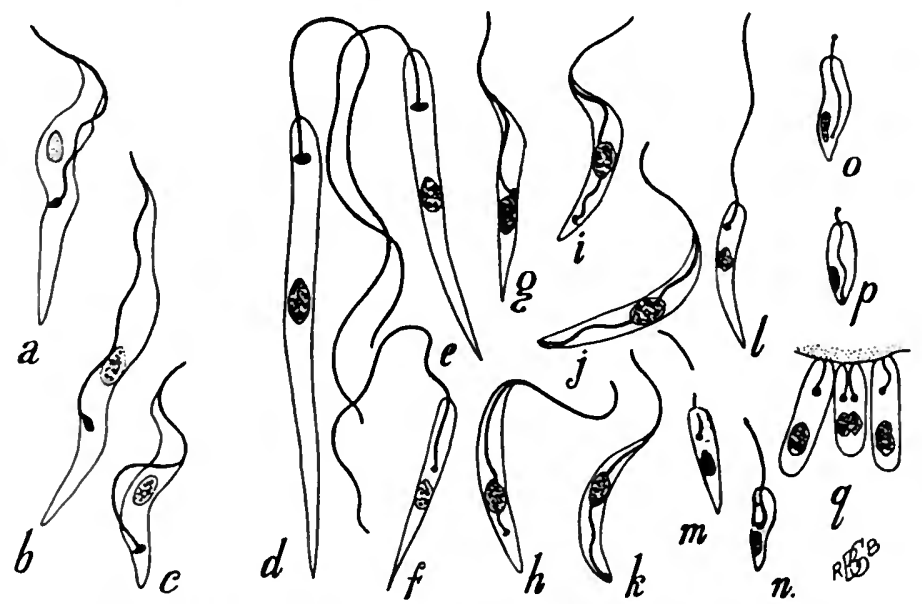

Fio. 137.-Flagellates from the digestive tract and Malpighian tubules of Dro.

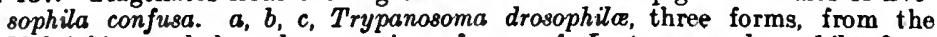
Malpighian tubules; $d-q$, various forms of Leptomonas drosophila from the intestine : $d, e, f$, leptomonad forms; $g$, crithidial form; $h, i$, transitional forms from the preceding to $j, k$, the leptotrypanosome-forms; $m, n$, small erithidial (" barley-grain ") forms; $o, p$, forms transitionsl from the preceding to $q$, gregariniform individuals attached to the epithelium by a rudimentary flagellum, the middle one of the three in process of division. After Chatton and Léger (533).

Not in every case, however, does such complexity of form occur in the same host. The development of a typical leptomonad, such as $L$. (H.) jaculum of Nepa cinerea, as described by Porter (556), is of a comparatively simple type, like that of Crithidia gerridis described above. Non-flagellated Leish. mania.like individuals give rise to flagellates of the true leptomonad type, which multiply by fission; these in their turn pass into a non-flagellated condition in the hind-gut, there becoming encysted and being cast out with tho faces to infect new hosts. Prowazek (557) has described in $H$. musca.domes tick an extraordinary complication of male and female types-autogamy. parthenogenesis of femalc forms, and "etheogenesis" of male forms ; none of these statements can be accepted as even probable until the weighty super. structure of theoretical interpretation is supported by a more substantial foundation of observed facts. Many of the stages described by Prowazek, espeeially his so-called "etheogenesis," represent stages in the development of distinct parasitic organisms not belonging to the group Flagellata; compare Flu, Dunkerly. 


\section{The Genus Leishmania.}

This genus was founded by Ross to include two human parasites : the so-called Leishman-Donovan bodies, cause of the disease known in India as "Kala-azar" ; and Wright's bodies (L. tropica), cause of boils known by various local names, but terined comprehensively "Oriental sore." To these a third species-namely, $L$. infantum-has been added by Nicolle (570), causing a disease in children in Tunis, Algeria, and Italy, and found also in dogs, which are regarded by Nicolle as the primary host of the parasite and the source of the infection in human beings. In all cases the type of parasite found in the vertebratc host is very uniform (Fig. 138)small bodies, usually rounded or oval, contained within cells and

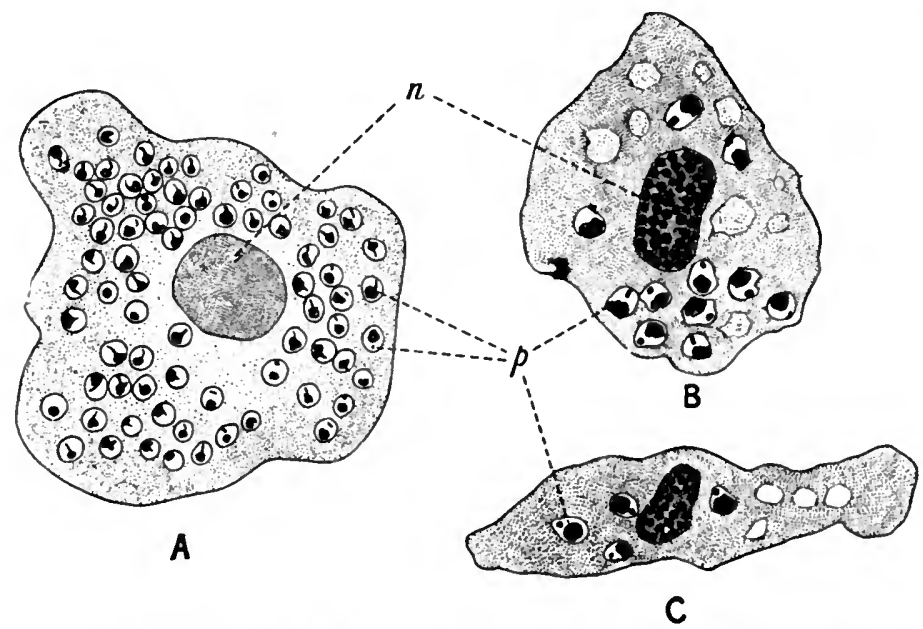

Fra. 138.-Leishmania donovani in cells. $A, A$ macrophage ; $B$ and $C$, endothelial cclls containing the parasites $(p) ;$.$n ., nucleus of the infected cell. After$ Christophers.

multiplying by fission (Fig. 139). Each parasite possesses two distinct nuclear bodies, which the development shows to be a trophonucleus and a kinetonucleus respectively. The cells which harbour the parasite are mainly, if not exclusively, of two classes-namely, leucocytes and endothelial cells; the latter become greatly hypertrophied, forming the so-called "macrophages" (Fig. 138, $A$ ), which may contain 150 to 200 parasites at a time. $L$. donovani was believed originally to occur also in red blood-corpuscles, and was first described as a species of the genus Piroplasma (p. 379). The balance of evidence, however, is against their occurrence in the hæmatids. If set free by the disintegration of their host-cell, they 
are probably taken up by leucocytes, and in them they may be carried into the general circulation.

Although the diseases caused by these parasites are termed comprehensively "leishmanioses," they are not all of one type. $L$. donovani produces a systemic disease, very deadly in its effects, and the parasite is found in immense numbers in the spleen, bonemarrow, liver, etc. L. infantum is similar in its effects. L. tropica, on the other hand, produces a purely local infection, manifested in the form of one or more boils on the skin, each of which, according to Wenyon (84), represents either a single infection by the insect, as yet not known with certainty, which transmits the disease, or a secondary infection by a house-fly or by the individual himself from another boil on the skin. The infection by $L$. tropica has an incubation-period of about two months. The disease lasts from twelve to eighteen months, and one attack, after it is healed spontancously, confers absolute immunity for the rest of the patient's life. Corresponding with these differences in

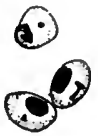

A

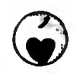

B
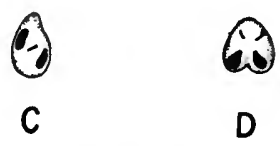

D

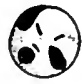

E

Fic. 139.-Leishmania donovani. $A$, Three parasites in the ordinary condition each showing a larger trophonucleus and a smaller kinetonucleus ; $B, C, D$, stages of binary fission; $E$, multiple fission into three parts. After Christophers.

the effects produced by the parasites, there are also slight structural differences to be made out in them. L. donovani (Fig. 138) is very uniform in shape, being rounded or ovoid ; L. tropica (Fig. 140), on the other hand, shows morv variety of form, with every transition from elongated, narrow forms with one end pointed to the typical oval body (Row, Wenyon).

No other stage than that described above is known from the human body; but it was first discovered by Rogers (576) for $L$. donovani, and subsequently confirmed by other investigators for this and other species, that in artificial cultures the parasite develops into a typical leptomonad form (Fig. 140). The Leishmania-forms in the cultures grow considerably in si $e$, and at the same time multiply by fission. The relatively large rounded forms become pear-shaped, and a flagellum is developed at the blunt end of the body ; finally the organism assumes the typical elongated form of a leptomonad, with a long flagellum arising close beside the kinetonucleus, which is situated near the anterior end of the body. Leishman and Statham have described a further stage in the 
development in which slender, so-called "spirillar" forms are split off from the large leptomonad forms.

There can be little doubt but that the cultural development observed in all the species of Leishmania represents the natural development which the parasite goes through in some invertebrate host. As regards, first, L. donovani, arguments have been brought forward incriminating tho bed-bug as the

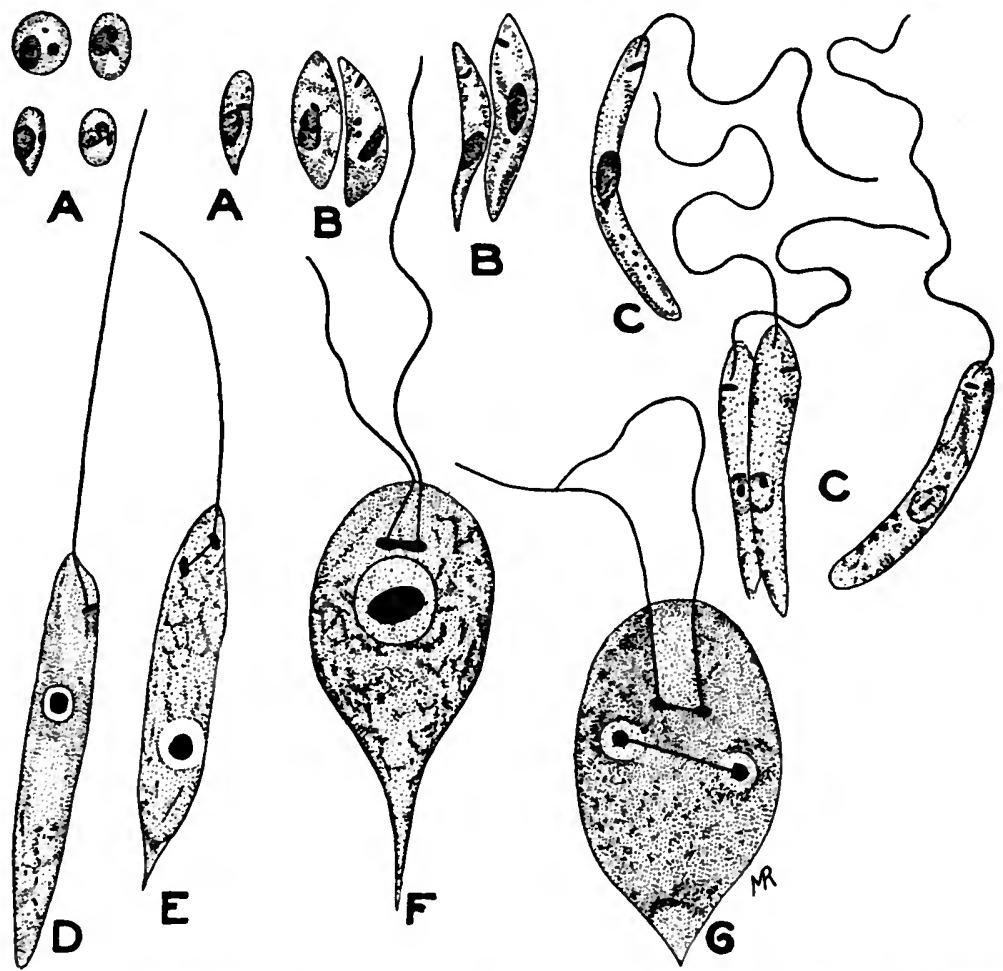

Fra. 140.-Leishmania tropica. A, Parasites from the sore, showing different forms ; $B$ and $C$, development in eultures: $B$, parasites growing and multiplying prior to the formation of the flagellum; $C$, adult flagellated leptomonad forms, with a couple probably the result of binary fission; $D$, adult leptomonad form; $E$, similar form with the kinetonucleus dividing; $F, G$, stont forms, two stages of division; note the flagella arising direct from the kinetonuclei, which are connected by a centrodesmose, indicating that the centriole is contained within the kinetonucleus (compare p. 87). $A-C$, after $R O w$, from preparations stained by the Romanowsky method, magnified 2,$000 ; D-G$, after Wenyon (84), from preparations stained with iron-hæmatoxylin after wet fixation.

transmitting agent, and Patton (573) has found that the parasite goes through the same stages of development in the digestive tract of the bug (Cimex rotundatus) as in the artificial cultures; but Donovan believes the true intermediate host in Madras to be another species of bug, Conorhinus rubrifasciatus, and Wenyon (84) considers that the development in the bug obtained by Patton is, like the development in artificial cultures, only an imitation of the development in the true host, and not a proof of transmission by the bug. Basile 
claims to have transmitted $L$. infantum by fleas. In the case of $L$. tropica, Wenyon points out that the sores occur almost invariably on parts of the person not covered or protected by clothing, a fact which is strongly against the infection being effected by fleas, bugs, or ticks, and indicates that the invertebrate host is some biting fly, probably either a species of mosquito or a sand-fly (Phlebotomus). Experimental evidence of transmission, however, is as yot lacking.

It is clear from the development that the species of Leishmania are nonflagellated phases of a true leptomonad, and it has been proposed by Rogers to abolish the genus Leishmania, and to place the parasites in question in the genus Herpetomonas. The life-cycle of a Leishmania is, however, so different from that of a typical Herpetomonas (Leptomonas), which is parasitic solely in the digestive tract of an insect, that the genus Leishmania may well be allowed to stand.

So long as the development is only known from artificial cultures, the significance of the "spirillar" forms of Leishman cannot be determined. Assuming that they are not merely degenerative forms, they may possibly represent the propagative stage in which the invertebrate host inoculates the parasite back into the vertebrate; the fact that $L$. donovani causes a systemic disease rather suggests that the initial phase in the vertebrate may be a flagellated form which is carried all over the body in the circulation, and from which the typical Leishmania-phase is developed. Another possible explanation of the spirillar forms is that they may be gametes, perhaps of male sex; but there is no evidence in support of this interpretation either from observation or analogy, since sexual phenomena in leptomonads have not been observed. Marzinowsky claims, however, to have observed copulation of male and female gametes in cultures of $L$. tropica.

Darling has described under the name Histoplasma capsulatum an organism causing a disease in human beings, and believed to be allied to Leishmania. It is stated to develop flagellated phases in lung-smears. For Toxoplasma, referred by some to a position near Leishmania, see p. 387. "Leucocytozoon" piroplasmoides, found in epizootic lymphangitis of horses in Senegal (Thiroux and Teppaz), is possibly a Leishmania, but only a single mass of chromatin appears to be present in the body, and no flagellated forms were obtained in cultures; possibly, therefore, its proper systematic position is near Toxoplasma.

\section{The Genus Prowazekia.}

This genus was created by Hartmann and Chagas (62) for $P$. cruzi, a species discovered in a culture from human fæces on an agar-plate in Brazil. Two other species have also been described from human fæces-namely, $P$. weinbergi, Mathis and Léger (Fig. 141, $A$ and $B$ ), and $P$. asiatica, Whitmore. It would appear, therefore, that several species (or possibly a singlo species) of this genus occur in various parts of the world in human fæces. Martini considers $P$. cruzi to be a cause of human diarrhcea and intestinal catarrh in China. Nägler has described a species $P$. parva (Fig. 141, $C$ ), which is free-living, and Dunkerly has found a Prowazekia in the gut of the house-fly. The form which Walker has described under the name "Trypanoplasma rana" very possibly should be referred to Prowazekia; it was obtained from cultures of the intestinal contents of the frog.

In its structure, Prowazekia resembles the genus Bodo in the heteromastigote arrangement of the flagella, and in its nuclear apparatus it resembles Try panoplasma, with trophonucleus and kinetonucleus. It differs structurally from Trypanoplasma only in the fact that the backwardly-directed flagellum is free from tho body, not united to it by an undulating membrane ; it bears, in fact, the same structural relation to this genus that $T$ richomastix has to $T$ richomonas. Alexeieff (388) denies that the genus is distinct from Bodo, and considers that the name Prowazekia should be cancelled; he identifies $P$. cruzi with $B$. edax, Klebs, and $P$. parva with $B$. saltans, Ehrenberg. 
Affinities and Phylogeny of the Homofagellates.-Two opposite views have been held with regard to the origin of the Flagellates parasitic in blood and their allies: First, that they have a double origin along two distinct lines of descent, some being derived from heteromastigote, the others from cercomonad ancestors (Woodcock, Doflein, Senn); secondly, that the Hæmoflagellates are closely allied to certain forms hitherto classed as Hæmosporidia (p. 388), and form with them a homogeneous group or order of the Flagellata, the so-called Binucleata (Hartmann).

The question of the Hæmosporidia will be discussed below (p. 389). It is sufficient here to deal with the Hæmoflagellates alone. From the general survey of the "forms" or "genera" dealt with in this chapter, it is very evident that Trypanosoma, Crithidia, Leptomonas (Herpetomonas), and Leishmania, are very closely

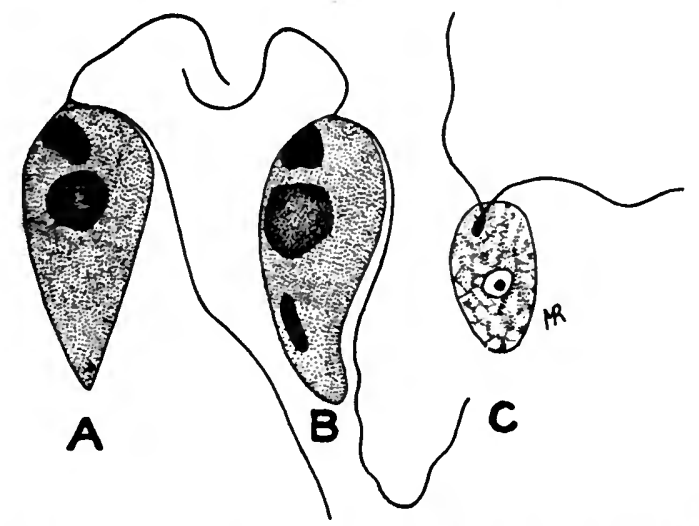

Fia. 141. $-A$ and $B$, Prowazekia weinbergi, Mathis and Léger (473), magnified 2,$400 ; C$, Prowazekia parva, Nägler (585), magnified about 2,250.

related to one another. Structurally the first three types shade off insensibly into one another, the dividing line between Trypanosoma and Crithidia, or between Crithidia and Leptomonas, being quite arbitrary, and far loss definite in reality than it appears when reduced to words; while Leishmania is a resting non-flagellated phase of any of the three. Developmentally the four types, or any two of them, may occur as phases in the life-history of a single species, so that the selection of a given form as the "adult" individual, by means oi which the generic name is to be determined, is also, in many cases, quite arbitrary. Phylogenetically an evolutionary series can be recognized beginning with Leptomonas, and passing through Crithidia to Trypanosoma, of which the central feature is the gradual development of an undulating membrane which finally runs the whole length of a more or less sinuous and 
serpentine body, probably as an adaptation to life and movement in a broth-like medium, containing numerous suspended bodies, such as occurs in the gut of an insect, especially a blood-sucking insect, or in the blood-fluid of a vertebrate. Leishmania, on the other hand, represents an offshoot from the main stem in which the resting, non-flagellated phase has become the most prominent stage in at least one part of the life-cycle.

On the other hand, the Hæmoflagellates of the biflagellate type, Trypanoplasma and Prowazekia, stand sharply apart from the uniflagellate genera. The orientation of the body, and of the undulating membrane, when present, in particular, is entirely different in the two types. The development in the in vertebrate host of Trypanoplasma and Trypanosoma, respectively, are quite distinet in type, neither form passing through any stages which suggest the slightest affinity with the other. The only feature common to the two types is the possession of a kinetonucleus in addition to the principal nucleus, and it is questionable to what extent this structure can be relied upon to indicate affinity. The large kinetonucleus of Trypanoplasma is very different in appearance from that of the uniflagellate genera ; and, according to Alexeieff (324), it is a structure of quite a different order from the cytological point of view. Finally it should be remarked that it is only in the biflagellate genera that parasitism in the gut of vertebrates is known to occur.

With regard to the origin of the forms parasitic in blood, two theories have been put forward. Léger (545) and Brumpt (389) have upheld the view that they were originally parasites of the digestive tracts of invertebrates, as many allied forms still are; that in many cases their invertebrate hosts acquired the bloodsucking habit, whereby the intestinal flagellates became accustomed and adapted to life in blood; and that, finally, forms so adapted passed from the invertebrate host into the blood of the vertebrate itself. Minchin (476), on the other hand, suggested that possibly the ancestral forms may have been parasites of the digestive tract of vertebrate hosts, and may have passed from the digestive tract into the blood, whence they were taken up by bloodsucking invertebrates and transmitted to fresh hosts, acquiring finally the power of being parasitic upon, and establishing themselves in, the in vertebrate host.

It must be admitted that all evidence which has accumulated of recent years is in favour of the view of Léger and Brumpt, so far as the uniflagellate forms are concerned. The types denoted by the generic names Leptomonas, Crithidia, and Trypanosoma, form a perfect evolutionary series, beginning with monogenetic parasites of invertebrates and culminating in digenetic bloodparasites. From the same stem other forms of parasitism are seen 
to arise in other directions, as in the digenetic flagellate parasites of Fuphorbiaceæ.

The biflagellate genus Trypanoplasma, on the other hand, comprises species which, like those of Prowazekia, appear to have been primarily parasites of the vertebrate digestive tract, and which in some cases have established themselves in the blood and have acquired an alternation of hosts (they can hardly be said to have an alternation of generations), having become parasitic in an intermediate host, always, so far as is known, a leech, in which they pass through a simple type of development, consisting of little more than simple multiplication by fission. Their structure indicates affinities with heteromastigote types such as Bodo and Trichomonas, common intestina] parasites, rather than with uniflagellate forms.

The suggestion is, therefore, that the flagellates parasitic in the blood of vertebrates have two distinct lines of ancestry : the one from heteromastigote forms such as Bodo and Trichomonas, originally parasitic in the gut of the vertebrate and culminating in the genus Trypanoplasma; the other derived from uniflagellate cercomonad ancestors originally parasitic in the digestive tracts of invertebrates, and culminating in the genus Trypanosoma (compare also Senn, 358). It must be emphasized strongly, however, that any such conclusions are of a tentative nature, and can have no finality, but are liable to modification with every increase of knowledge concerning these organisms.

Bibliography.-For references see p. 488. 


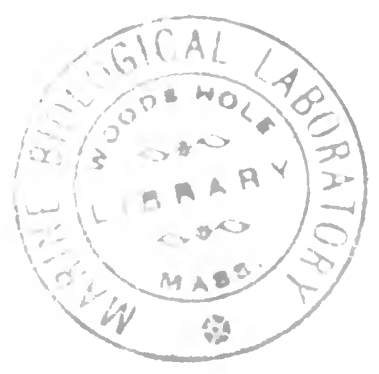

\section{CHAPTER XIV}

\section{THE SPOROZOA : I. THE GREGARINES AND COCCIDIA}

UNDER the common denomination Sporozoa are grouped together a great number of parasitic organisms extremely varied in form, structure, habitat, and life-history, but of which the most general though not invariable characteristic is that the propagation of the parasite from one host to another is effected by means of spores, in the primary sense of the word (see p. 165, footnote)-that is to say, resistant seed-likc bodies within which one or more parasitic germs are protected by a firm envelope or capsule, whereby they are enabled to resist the vicissitudes of the outer world until they pass, in one way or another, into the body of a suitable host; when this end is attained, the spore germinates - that is to say, the contained organisms are set free and a fresh infection is started.

It is very obvious that propagation by means of resistant spores is a character very inadequate for diagnosing an extensive group of Protozoa. In the first place, many organisms, parasitic or free-living, which are not included in the class Sporozoa, are propagated by means of resistant spores. In the second place, many forms included in the Sporozoa do not produce resistant spores, being propagated by methods which render any such phase unnecessary. The class therefore ceases to be amenable to strict verbal definitions, and it is not surprising that the limits assigned to it have varied at different times, and are even now debated. The class Sporozoa was originally founded by Leuckart to comprise two closely allied orders--the Gregarines and the Coccidia. To this nucleus other groups were added, in particular the various forms termed vaguely "psorosperms"*-a word coined originally by Johannes Müller to denote the spores of the Myxosporidia, but soon extended to other parasitic organisms. Thus "Sporozoa" and "psorosperms" became practically synonymous terms, and the class to which these names were applied became a most heterogeneous assemblage of organisms

* From the Greek $\psi \dot{\omega} \rho a$, mange, and $\sigma \pi t \rho \mu \alpha$, a seod, on account of the sores and uleers of the skin of fishes produced by My rosporidia, and the resemblance of their spores to little seeds. 
having nothing in common except the parasitic habit and the adaptations arising from it, more especially the propagation by spores.

The modern tendency is rather to split up this vast assemblage into smaller groups, and to abolish the Sporozoa as a primary subdivision of the Protozoa. It is practically certain, at least, that the two main subclasses into which it is always divided are perfectly distinct in their origin. The class Sporozoa is retained here solely in deference to custom and convenience, and without prejudice to the affinities and systematic position of its constituents, a question which will be discussed when the group as a whole has been surveyed.

The life-cycle of a Sporozoön may be started conveniently from the minute germ or sporozoite which escapes from the spore, or from the corresponding stage when spores are not formed. The sporozoite may have one of two forms: it may be an amœbula, a minute amœboid organism ; or it may be of definite form, a little rod-like or sickle-shaped animalcule ("falciform body," "Sichelkeim ") which is capable of twisting or bending movements, but retains its body-form, and progresses by gliding forwards; for this second type of sporozoite the term " gregarinula " has been proposed in a previous chapter (p. 169).

The sporozoite, whatever its form, is liberated in the body of the new host, and begins at once its parasitic career; it nourishes itself and grows, often to a relatively huge size, at the expense of the host. This phase of the life-history is termed the "trophic phase," and the parasite itself during this phase a trophozoite, by which term is understood a parasite that is actually absorbing nourishment from the host. The trophozoite may be lodged within cells (cytozoic), or in tissues of the body amongst the cells (histozoic), or in some cavity of the body in which it either lies free or is attached to the wall (cœlozoic). Whatever their habitat, the trophozoites of Sporozoa never exhibit any organs or mechanisms for the ingestion or digestion of food, but absorb their nutriment in all cases in the fluid state, by osmosis through the surface of the body, from the substance of the host ; if pseudopodia or flagella are possessed by these parasites, they are never used for foodcapture, except in so far as pseudopodia, by increasing the surface of the body, may augment its absorptive powers.

The parasite may exhibit multiplicative phases in which it reproduces itself actively, so that there may be many generations of trophozoites within one and the same host, which may thus be quite overrun by swarms of the parasites. Multiplication of this kind, which is non-sexual, is known as schizogony; the trophozoites which multiply in this manner are termed schizonts; and the minuto 
daughter-individuals, products of schizogony, are termed merozoites, to distinguish them from sporozoites which they may resemble closely. Sooner or later, however, the propagative phase, destined to infect new hosts, makes its appearance; so-called sporonts (see p. 330, infra) multiply by sporogony, which is combined with sexual phases, to produce the sporozoites. The life-cycle of the parasite may be passed entirely in one host, or there may be an alternation of hosts of different species, with a distinct series of phases of the parasite in each. When there is but a single species of host, the method of infection of new hosts is usually contaminative (p. 24), by means of resistant spores and cysts ; when there is an alternation of hosts, the infection may be inoculative (p. 23), without resistant phases, as in malarial parasites, or contaminative, with resistant phases, as in Aggregata (p. 353).

Whether the life-cycle be of simple or complex type, it ends with the production of sporozoites, bringing it back to the startingpoint again ; and in the vast majority of cases the sporozoites are enclosed, one or more together, in tough sporocysts to form the characteristic resistant spores. As a rule each spore arises from a single spore-mother-cell or sporoblast.

The Sporozoa fall naturally into two subclasses, which have received various names, according as one or another of their characteristic features has been considered diagnostic. It is best to define each subclass by a number of characteristics, since none by itself is sufficiently distinctive.

In the first subclass the trophic and reproductive phases are typically distinct-that is to say, the animal becomes full-grown, and ceases to grow further, before reproduction begins, hence Telosporidia (Schaudinn); reproduction takes place usually by a process of multiple fission in which the daughter-individuals are budded or split off on the outer surface of the parent-body, hence Ectosporea (Metchnikoff); and the germs or sporozoites produced are gregarinulæ, hence Rhabdogeniæ (Delage and Herouard).

In the second subclass the trophic and reproductive phases usually overlap-that is to say, the still-growing or even quite young trophozoite may begin to form spores, hence Neosporidia (Schaudinn); the spore-mother-cells are formed by a process of internal gemmation, being cut off within the cytoplasm of the parent, hence Endosporea (Metchnikoff); and the sporozoites produced are amœbulæ, hence Amœbogeniæ (Delage and Hérouard).

Of the three contrasted characters by which the two subclasses are distinguished, the most absolutely diagnostic is probably the form of the sporozoite. The names Telosporidia and Neosporidia 
are, however, in more common use than the other names of the subclasses given above.*

The subclass Telosporidia, as mentioned above, includes the three orders Gregarinoidea, Coccidiidea, and Hæmosporidia.

\section{ORder I.-GRegarinoidea.}

The chief characteristics of this order are-First, that the trophozoites are parasites of epithelial cells in the earlier stages of their growth, but in later stages they become entirely free from the cells, and lie in cavities of the body; their most frequent habitat is the digestive tract, but sometimes they are found in the body-cavity or the hæmocœle. The full-grown trophozoite is of relatively large size and definite form, with a thick cuticle as a rule. In addition to these characters, the reproduction and spore-formation, presently to be described, are quite distinctive in type, the most diagnostic feature being that each spore is the product of a single zygote.

The Gregarines are an extremely abundant order of the Sporozoa, highly differentiated in structure, and comprising a great number of species classified into genera and families. They occur most commonly as parasites of the digestive tract ar body-cavity of insects, but also as parasites of other classes, such as Echinoderms and Annelids; in Molluscs they are comparatively rare, and, though they occur commonly in Prochordata (Ascidians), they are not known from any class of Vertebrata in the strict sense of the word.

In the early phases of development, during which the trophozoite is a cell-parasite, it may be entirely enclosed in the cell, or only attached to it by one extremity of the more or less elongated body. In the latter case the sporozoite may have the anterior end of the body modified into a definite rostrum, by which it attaches itself to the host-cell, and from which is developed a definite organ of attachment, termed an epimerite (Fig. 142, ep.), often of complicated structure, and provided with hooks and other appendages. When the cytozoic phase is past and the host-cell is exhausted, the parasite drops off, shedding its epimerite as a rule. In the earlier phase, in which an epimerite is present, the parasite was termed by Aime Schneider a cephalont ("cephalin"), and in the later phase a sporont ("sporadin"), the original use of this term, now applied in a wider sense to denote in this and other orders of Sporozoa those individuals about to proceed to spore-formation. The body of the Gregarine-sporont always contains a single nucleus, but may be divided into partitions or septa formed as ingrowths of the ectoplasm, and is then said to be "septate" or "polycystid."

* The subclass Rhabdogeniæ, as instituted by Delage and Hérouard, included the Sarcosporidia, which, however, are almost certainly true Amcobogeniæ. 
As a rule, in such cases there is but a single septum, which divides the body into two parts termed respectively protomerite and deutomerite (Figs. 7, 142); but in the curious genus Treniocystis (Léger, 616) there are a number of septa, giving the parasite a superficial resemblance to a segmented worm.

The body of a gregarine consists typically of distinct ectoplasm and endoplasm. The ectoplasm may be further differentiated into three layers: an external cuticle or epicyte, a middle layer or sarcocyte, and a deeper contractilo layer or myocyte containing myonemes (Fig. 29, p. 58). The epimerite, with its hooks and processes, is derived from the epicyte; the septa, if present, from the sarcocyte. The endoplasm is usually extremely granular, and contains great quantities of stored-up food material in reserve for the reproductive processes; chief amongst these substances are paraglycogen - spherules, extremely characteristic of these parasites.

A remarkable feature of gregarines is the power possessed, by many species, of gliding forward, often at a great pace, without any visible organs of locomotion. Two explanations have been given of these movements: (1) by Schewiakoff, that they are due to extrusion of gelatinous fibres from the hinder end of the body, secreted between the epicyte and sarcocyte ; (2) by Crawley, that tho movements are produced by contractions of the myonemes which are only present in motile forms. In motionless forms the ectoplasm is very thin, and consists of epicyte alone.

The nucleus of a gregarine is usually very large, spherical, and vesicular in type, with one or more distinct karyosomes. It is typically single, except in the cases of precocious association mentioncd below-exceptions, however, which are only apparent, since in such cases the gregarine represents in reality two individuals fused into one. In the

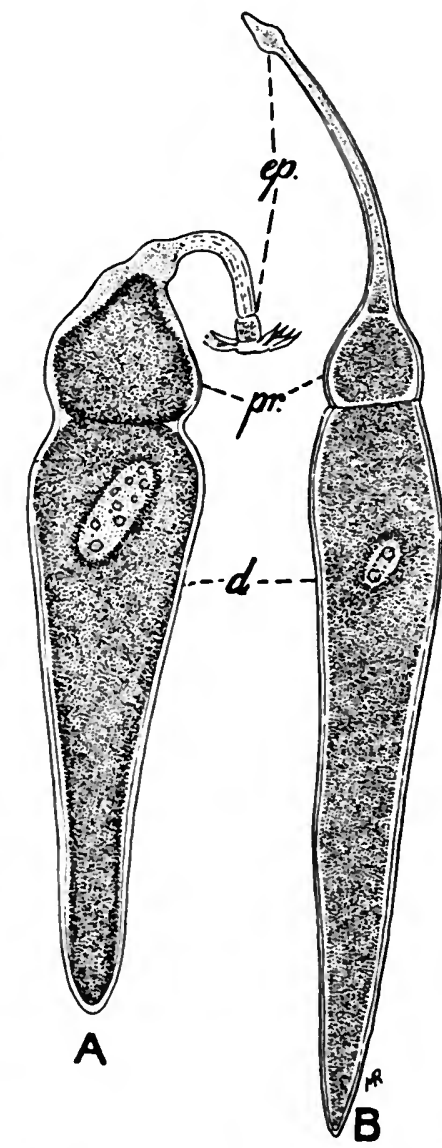

Fra. 142.-Examples of gregarines in the "cephalont" condition. A, Actinocephalus digacanthus; B, Stylorhynchus longicollis. ep., Epimorite ; $p r$. , protomerite ; d., deutomerite. After Sohneider. septate forms the nucleus lies in the deutomerite normally. In Pterocephalus ( $N$ ina), however, a second nucleus, which appears to be of transitory nature and to take no share in the reproductive processes, has been discovered in the protomerite (Léger and Duboscq, 621). The nucleus-like body observed by Siedlecki in Lankesteria ascidice. and by Wenyon (84) in $L$. culicis, occurring at the point of contact of the two associated sporonts in the cyst, is perhaps a body of similar nature. The nucleus of Callyntrochlamys phronimoe is remarkable for being surrounded by a halo composed of radiating processes, each a thin tubular evagination 
of the nuclear membrane (Dogiel, 605); as a rulo the surface of the nucleus is perfectly smooth.

Chromidia are stated to occur in the cytoplasm of some gregarines (comparo Kuschakewitsch). According to Comes, they are scarco in normal individuals, but becomo abundant with over-nutrition; since he states, however, that they arise in the cytoplasm, it is possible that they represent grains of the nature of volutin rather than true chromidia. According to Drzewecki, however, the nucleus of Monocystids may, during the early growth of the trophozoite, break up into chromidia and be re-formed again, or may throw out vegetative chromidia which are absorbed in the cytoplasm; Kuschakewitsch, however, regards this as a degenerative process.

Drzewecki affirms that Stomatophora (Monocystis) coronata, from the vesiculæ seminales of Pheretima sp., possesses a mouth-opening in a peristome, and an anal aperture, and takes up solid food in the form of the spermatozoa of its host. If so it is quite unique, not only among gregarines, but among Sporozoa generally. The ingested spermatozoa are stated to be taken up and digested by the nucleolus (karyosome). According to Hesse, the supposed mouth and peristome are parts of a sucker-like organ of attachment. The alleged nucleolar digestion is perhaps a misinterpretation of the extrusion of chroma. tinic particlos from the karyosome.

The Gregarines are subdivided at the present time into two suborders characterized by differences in the life-cycle. In the first suborder, known as the Eugregarinæ, the parasite has no multiplicative phase, but the trophozoites proceed always as sporonts to the propagative phase by a method of reproduction (sporogony) which is combined with sexual processes, and leads to the formation of resistant spores. In the second suborder, the Schizogregarinæ, the trophozoites which arise from the sporozoites become schizonts which multiply for several generations non-sexually, by schizogony, before a generation of sporonts (gamonts or gametocytes) is produced which proceed to reproduce themselves by sexual sporogony. Stated briefly, the Eugregarinæ have only a propagative phase, sporogony, in their life-cycle; the Schizogregarinæ have both a multiplicative phase, schizogony, and sporogony. The sporogony is of essentially the same type in both orders. It is simplest, therefore, first to describe the life-cycle of a eugregarine, and then to deal with the multiplicative phases of the schizogregarine. The complete life-cycle of a eugregarine may be divided into eight phases.

1. The sporozoites are liberated from the spores in the digestive tract of the host in all cases known, and usually proceed at once to attach themselves to, or penetrate into, the cells of the lining epithelium of the gut; but in a few cases the sporozoites pass through the wall of the gut into other organs, as does, for example, the common Monocystis of the earthworm, which penetrates into the vesicula seminalis, and finally into sperm-cells.

2. In the early cytozoic phase the trophozoite may be contained completely within a cell (Fig. 143, $A, B$,) or merely attached to it; the former condition, speaking generally, is characteristic 
of the Acephalina, the latter of the Cephalina. In either case, the first effect of the parasite is to produce a hypertrophy, often very great, of the cell attacked (Fig. $143, B$ ); later, however, the cell atrophies, dies, and shrivels up (Fig. 143, $C$ ).

(a) In the Acephalina the intracellular parasite is set free from the cell by its dissolution, and, if lodged in the epithelium of the gut, may pass out of the epithelium either on its inner side, into the lumen of the gut again, or on its outer side, into the bloodvessels or body-cavity.

(b) In the Cephalina the relation of the parasite to the host-

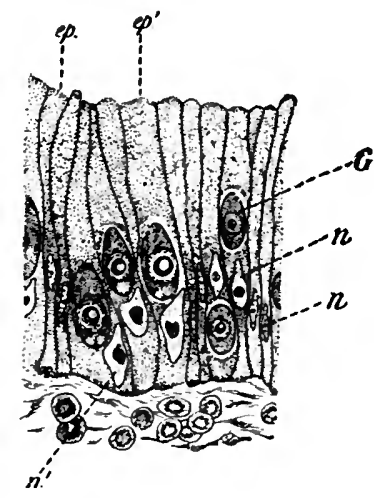

A

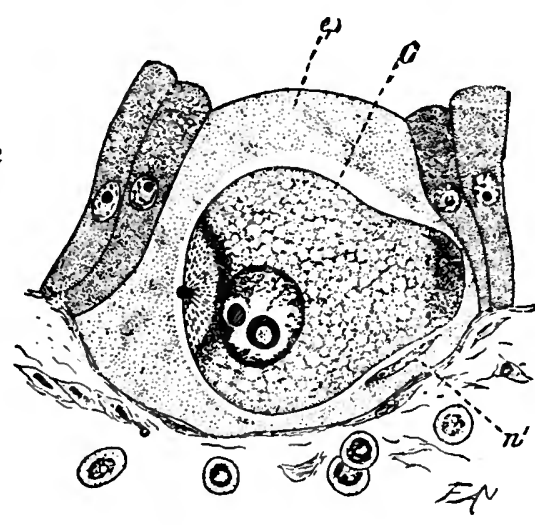

B

FIG. 143.-Lankesteria ascidice, parasite of Ciona intestinalis. $A$, Young intracellular stages in the intestinal epithelium; $B$, older intracellular stage ; $C$, extracellular trophozoite a.tached by a process of the anterior end of the body to a withered epithelial cell. ep., Normal epithelial cell; $e p$. , hypertrophied epithelial cell containing $(G$. $)$ the young grega.' rine; $n$., nueleus of normal eell; $n .^{\prime}$, nucleus of infected cell. After Siedleeki, magnified 750.

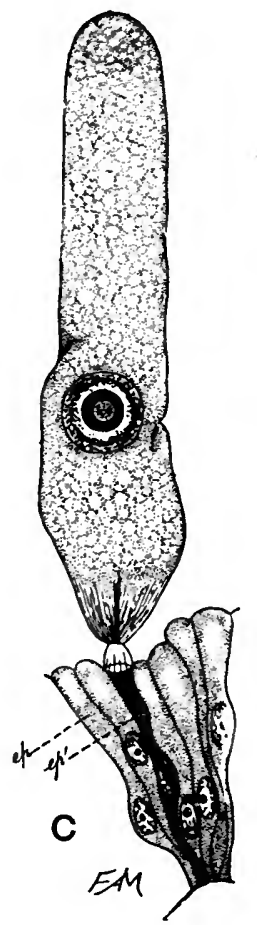

cell varies greatly, and has been studied in detail by Léger and Duboseq (618 and 620). The sporozoite may merely prick the surface of an epithelial cell with its rostrum (e.g., Pterocephalus), or may dip a short stretch of its anterior end into the cell (e.g., Pyxinia), or may penetrate so far that the nuclear region of the parasite is within the cell (e.g., Stylorhynchus), or, finally, may become completely intracellular (e.g., Stenophora). Ultimately, in all cases, the chief mass of the body of the gregarine projects from, or grows out of, the host-cell into the cavity of the digestive tract, and becomes the protomerite and deutomerite in septate forms; the attached 
portion of the body develops into an epimerite which may acquire a large size and a complicated structure. Originally attached to one cell, which it destroys, the epimerite may acquire a secondary attachment to other cells of the epithelium, which in this case are not injured by it, as in Pterocephalus. Ultimately the epimerite breaks off, and the body of the sporont drops into the cavity of the digestive tract. In some cases (Pyxinia) the early attached stages may free themselves from the epithelium several times, and attach themselves again.

3. When liberated from the host-cell, the trophozoite grows into the adult sporont, which, as its future history shows, is a gamont or gametocyte. A remarkable feature of gregarines at this stage is the tendency to associate together (Fig. 7), a habit from which the name Gregarina is itself derived. In some cases quite a number of individuals may adhere to one another in strings ; such associations, known as "syzygies," are, however, of a temporary nature, passing flirtations, as it were, which have no significance for the life-cycle or development. On the other hand, a true association of individuals destined to form gametes always, apparently, occurs at one time or another in the life of the sporont. In the majority of cases, however, the sexual association does not take place till the end of the trophic phase, when the sporont is full-grown and ripe for reproduction. But in a number of instances the association takes place early in the trophic phase, between quite young free trophozoites ; and "neogamous" association of this kind may lead to almost complete fusion of the bodies of the two individuals, only their nuclei remaining separate, thus producing the appearance of a binucleate trophozoite (Fig. 70, p. 128).

In general, the two trophozoites which associate are perfectly similar in appearance, and exhibit no differentiation; this is so in all cases where they pair side by side. In some cases where there is an early association end to end-that is to say, where one sporont attaches itself by its protomerite to the deutomerite of another (Fig. 7, p. 9), as is common in polycystid forms-the two sporonts may be differentiated one from the other. In Didymophyes, for instance, the protomerite of the posterior individual disappears; in Ganymedes the two sporonts are held together by a ball-and-socket joint (Huxley). It is not known whether these differences stand in any constant relation to the sex of the sporonts. In Stylorhynchus the two partners attach themselves to one another by their anterior extremities (Léger, 614).

4. As soon as growth is completed, the reproductive phases are in iated by the formation of a common cyst round the two associated sporonts, which together form a spherical mass (Fig. 144, a). The parasite is now quite independent of its host; it is, in fact, a 
parasite no longer, and may now be ejected with the fæces. The nucleus of each sporont then divides by repeated binary fission (Fig. 144, $b$ ) into a large number of nuclei, which place themselves at the surface of the body (Fig. 144,c).

A question much debated with regard to the life-history of gregarines is whether a single sporont can encyst by itself, without association with another, and then proceed to the formation of spores. It has been asserted frequently that this can occur, and the suggestion has been put forward that the differences in the size of the spore observed in some species may be correlated with double or solitary encystment. Schellack (630) has discussed the question in detail, and is of opinion that in septate cugregarines solitary encystment either does

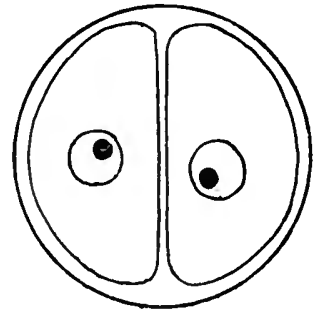

a.

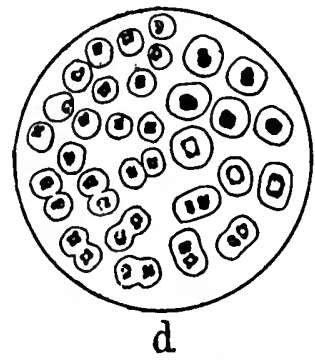

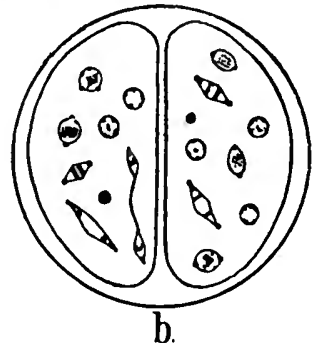

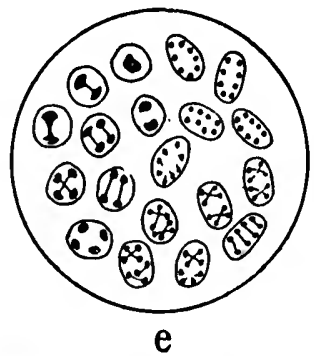

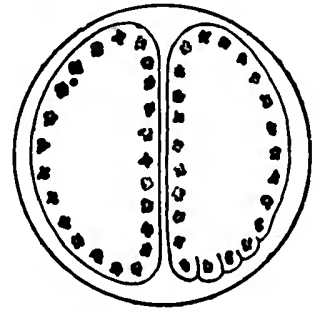

c.

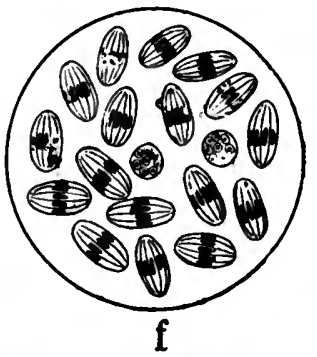

Fra. 144.-Schematic figures of syngamy and spore-formation in gregarines. $a$, Union of two sporonts in a common cyst; $b$, various stages of nuclear division in each sporont ; $c$, formation of gametids beginning (" pearl-stage "); $d$, stages in the copulation of the gametes: in the left upper quadrant of the figure, separate gametes are seen; in the left lower quadrant the gametes aro uniting in pairs; the right lower quadrant shows fusion of the pronuclei ; and in the right upper quadrant complete zygotes (sporoblasts) are secn; $e$, stages in the division of the nuclei of the sporoblasts, which assume an oval form; a different stage is seen in each quadrant, eight nuclei being present in the final stage; $f$, cyst with ripo spores, each containing eight sporozoites; two spores are seen in oross-section. Modified after Calkins and Siedlecki.

not occur, or leads to nothing if it does, but that amongst the Acephalina and schizogregarines it can take place; a clear case has been described by Léger in Lithocystis schneideri, parasite of Echinocardium; and in Monocystis pareudrili solitary encystment leading to sporc-formation is described by Cognetti de Martiis. In some species cysts containing three sporonts have been seen; Woodcock also found a specimen of Cystobia irregularis with three nuclei. With regard to the differences in the size of the spores, the possibility has to be taken into account that in some cases they may be developed parthenogenetically - that is to say, the gametids may each become a sporo. blast directly, without copulation with another. 
The first division of the nucleus of the sporont has given rise to considerable discussion and has been the object of much study. In the resting state tho sporont-nucleus is a body of relatively huge size, but the first spindle formed in the sporont is, like all the subsequent mitoses, a minute structure. Some authors have believed that the sporont contains two nuclei, comparable to those of Infusoria-namely, a very large macronucicus of purely vegetative nature, which takes no part in the subsequent development; and a minute micronucleus of generative nature, from which the first and subsequent
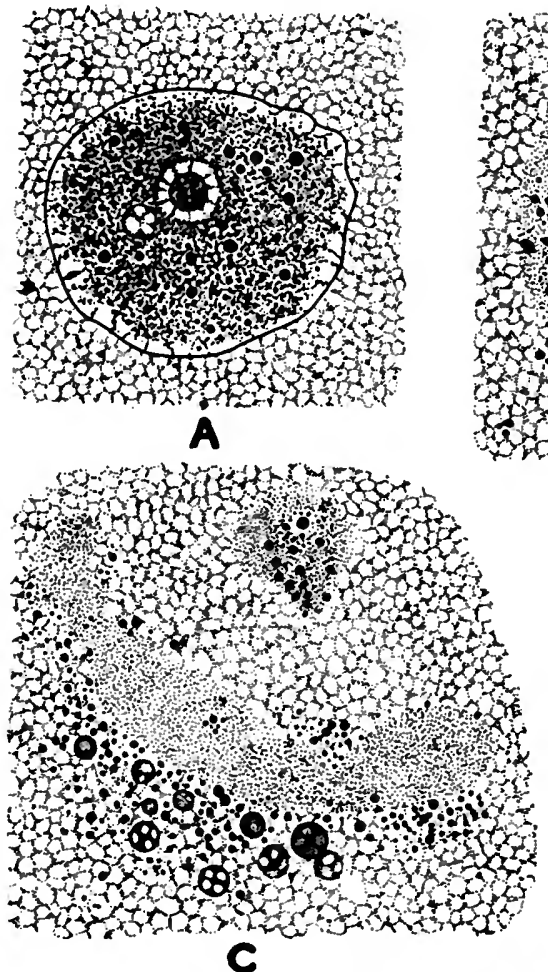

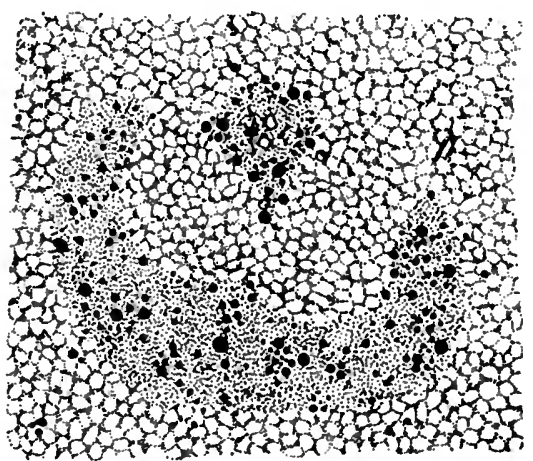

B

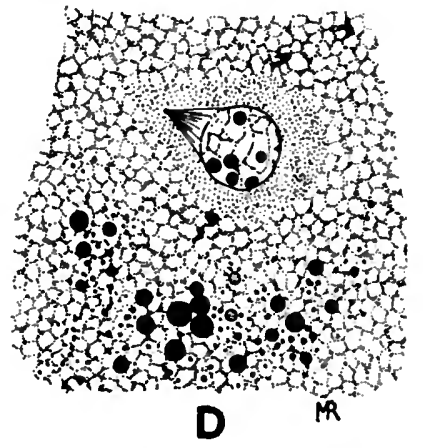

Fro. 145.-Stages in the formation of a generative nucleus (" micronucleas") from the primary nucleus of Pterocephalus (Nina) gracilis. A, Primary nucleus showing the first appearance of the micronucleus in a clear space; $B$, disruption of the primary nucleus; appearance of the micronucleus in the form of a few chromosomes in the centre of a little island of nuclear substance ; $C$, further stage in the formation of the micronucleus; $D$, micronucleus complete with the tirst centrosome ; the remainder of the primary nucleus in process of absorption. After Léger und Duboscq (621); $A$ magnitied 800, $B, C, D$, 1,000 , diameters.

mitoses arise. Recent researches, however-more especially those of Schnitzler on Gregarina ovata, Schellack (629) on Echinomera hispida, Léger and Duboscq (621) on Pterocephalus, Robinson on Kalpidorhynchus, Duke on Metamera, and cspecially Mulsow (123) on Monocystis rostrata-leave no doubt but that the sporont contains a single large nuclous, which consists chiefly of vegetative chromatin and other substances, but contains also the generative chromatin, relatively minute in quantity in proportion to the whole bulk of tho nucleus. 
The generative chromatin may organize itself into a definite secondary nucleus ("micronucleus") during the break-up of the sporont-nucleus, as in Pterocephalus (Fig. 145); or the first spindle arises within the sporont-nucleus before it breaks up, as in $G$. ovata (Fig. 146) ; or a number of distinct chromosomes are formed in the sporont-nucleus during the process of its disintegration, which pass to the exterior of the nucleus and form the equatorial plate of a spindle of which the achromatinic elements appear to arise chiefly outside the nucleus, as in Monocystis rastrata. In either case the first spindle consists only of the generative chromatin ; the remainder of the original sporont-nucleus is disintegrated and absorbed, or is left over in the residual protoplasm of the cyst. The statement of Kuschakewitsch, to the effect that the primary nucleus of the sporont may break up into a mass of chromidia, from which a number of secondary (generative) nuclei are re-formed, has not received confirmation in any quarter.

The mitoses in the sporont are remarkable, in most cases, for the very distinct centrosomes (Fig. 147), which appear at the side of the nucleus before
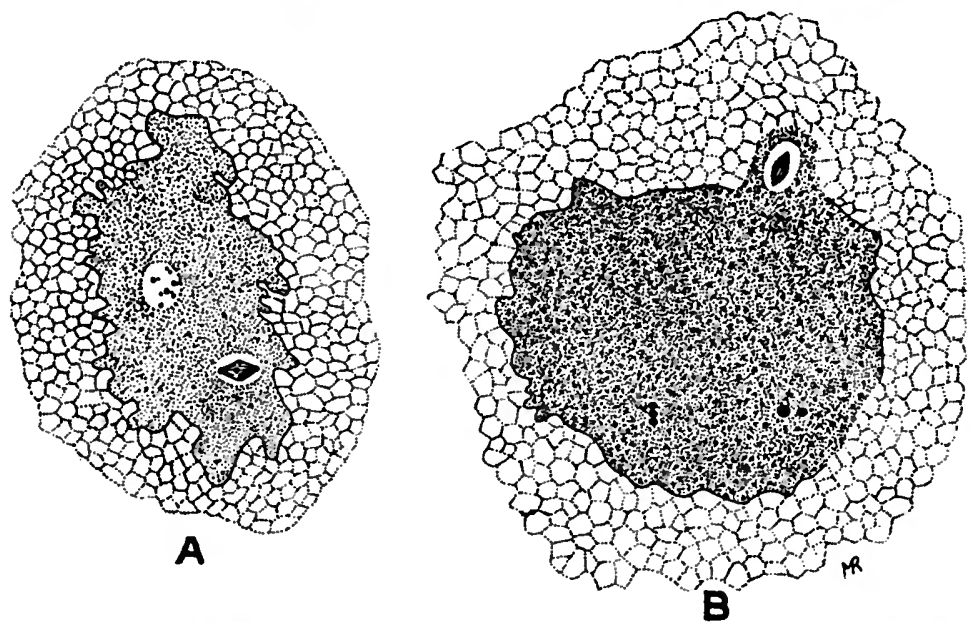

FIc. 146.-Two stages in the formation of the first division-spindle of Gregarina ovata, showing its origin from a very small part of the primary nucleus. In $A$ the spindlc is scen within the primary nucleus; in $B$ the spindle is becoming free from it at one point, after which the remainder of the primary nucleus degenerates. After Schnitzler; magnification 850 diameters.

division begins as a grain or a pair of grains placed at the apex of a "cone of attraction" ; in Monocystis rostrata, however, centrosomes appear to be absent. The number of chromosomes in the equatorial plate is usually four ; but in Monocystis rostrata the number appears to be cight, and in Pterocephalus and the allied genus Echinomera there are five chromosomes, frur of ordinary size and one large unpaired chromosome. Unlike the unpaired chromosome of Metazoa, that of the gregarines is present in both sexes; it gives rise, during the reconstitution of the daughter nuclcus, to the karyosome; and the karyosome is eliminated from the nuclear spindle at the subsequent mitosis. The significance of the unpaired chromosome is far from clear, and requires further elucidation.

5. Each of the nuclei of the preceding stage grows out from the surface of the body surrounded by a small quantity of protoplasm, and thus a great number of small cells are budded off over the 
whole body of each sporont. The small clear cells produced stud the opaque body of the sporont like pearls; hence this stage is often spokon of as the "pearl-stage" (perlage, etc.). The remainder of the body of the sporont is left over as residual protoplasm, which may contain nuclei, but which takes no further direct share in the development. The cells that are produced are known as the "primary

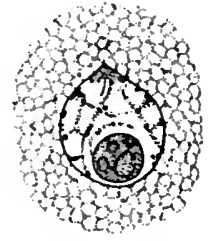

A

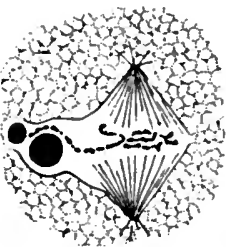

E

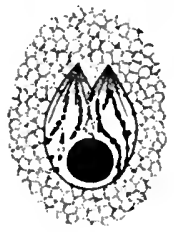

B

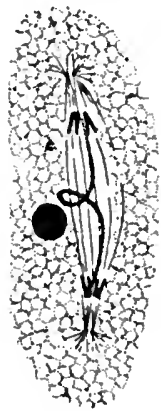

$\mathbf{F}$

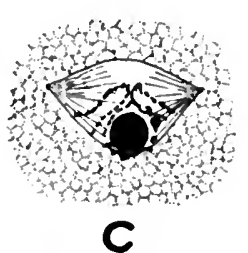

C

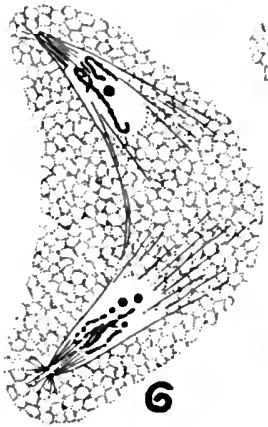

6

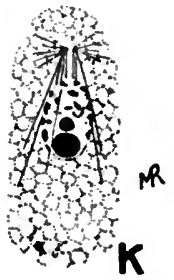

FIG. 147.- Stages of nuclear division in the cyst of Pterocephalus (Nina) gracilis. $A$, Resting nucleus with a centrosome at one pofe ; $B$, division of the centrosome ; $C, D$, formation of the nuclear spindle and equatorial plate; ejection of the karyosome; $E$, nuclear spindle, with the unpaired chromosome on the left, also the remains of the karyosome; $F$, diaster-stage, with the unpaired chromosome stretching across, the karyosome on the left; the centrosomes have each divided again ; $G, H$, later atages of division ; $I, J, K$, roconstruction of the daughter-nucleus; the unpaired chromosomo forms the karyosome. After Léger and Duboseq (621); magnification of tho figures, 1,200 diameters.

sporoblasts," but a better name for them is the gametids, since each one is destined to become a gamete The amount of transformation which a gametid undergoes in becoming a gamete may be very considerable, or it may be practically nil. In some cases the male gamete develops a special structure, while the female remains 
unmodified; in other cases both male and female remain in the undifferentiated condition of the gametid. For an account of the gametes of gregarines, see above (Fig. 79, p. 174).

Reduction has been described in several cases in the formation of the gametids. In the genus Gregarina the nucleus of the gametid divides twice to form two reduction-nuclei (Léger and Duboseq, 621); Paehler and Schnitzler have also described a reduction-division in the gametids of Gregarinx ovata. In Monocystis rostrata, on the other hand, the reduction takes place, according to Mulsow (123), in the last nuclear division in the sporontbody, prior to the budding off of the gametids. In this case the ordinary number of chromosomes is eight, as seen in all the divisions of the nuclei; in the final division the eight chromosomes associate to form four pairs, those of each pair being in close contact, but not fused ; in the mitosis that follows one chromosome of each pair goes to each pole of the spindle, thus reducing the number of chromosomes in each gametid-nucleus from eight to four.

6. When the gametes are ripe, they copulate in pairs, and probably in every case the gametes of each pair are of distinct parentage. This is certainly the case when the gametes show any trace of sexual differentiation, since those of one sex can be seen to arise from one sporont, and of the other sex from the other. In many cases the two sporonts are separated from one another by a partition dividing the cyst into two chambers, in one of which the male gametes are formed, in the other the female; when the gametes are ripe, the partition breaks down and pairing of the sexes takes place.

7. The zygote becomes oval or spindle-shaped, and a membrane is secreted at its surface to form the sporocyst, which becomes an exceedingly tough and impervious envelope, and is generally composed of two layers - epispore and endospore. Within the sporocyst the nucleus (synkaryon) divides usually three times to form eight nuclei, and then the protoplasm of the sporoblast divides up into as many slender, sickle-shaped sporozoites, leaving over a small quantity of residual protoplasm. The sporozoites are usually arranged longitudinally in the spore, with the residual protoplasm at the centre. The number of sporozoites in the spore is almost invariably eight; exceptions to this rule are only known amongst the schizogregarines.

The spores of gregarines differ enormously in different species in form and appearance, and often have the sporocyst prolonged into tails, spines, or processes of various kinds. Various mechanisms may be developed for liberating the spores from the cyst; for instance, in the genus Gregarina (Clepsydrina) the cyst is provided with sporoducts, and the residual protoplasin derived from the sporonts swells up when the spores are ripe, and forces them out through the sporoducts in long strings.

8. The ripe spore with its contained sporozoites passes out of 
the body to the exterior. Usually it passes out per anum with the fæces, but when the spores are formed in some internal organ of the body, as in the Monocystis of the earthworm, it may be necessary for the host to be eaten by some other animal, which then scatters the spores broadcast in its fæces. In all cases, so far as is known, the new host is infected by the casual or contaminative method, and in its digestive tract the spores germinate and liberate the sporozoites. In the case of Cystobia minchinii, parasite of Cucumaria, it is extremely probable that the host acquires the infection by taking up the spores per anum into its respiratory trees, where the spores germinate (Woodcock).

The schizogony characteristic of the schizogregarines takes place during either the second or third of the phases described in the foregoing paragraphs, in trophozoites derived from the sporozoites by growth, and it takes various forms which cannot be described in general terms; a few examples must suffice.

1. Selenidium caulleryi (Fig. 148): The sporozoite penetrates into a cell of the intestinal epithelium, and grows to a large size, remaining uninucleate. When full-grown, the intracellular parasite gives rise by a process of multiple fission to a great number of motile merozoites which penetrate into epithelial cells, grow, and finally become free sporonts. The schizogony of Merogregarina amaroucii (Porter) is of a similar type, but fewer merozoites are prodaced by the schizont.

2. In Schizocystis gregarinoides (Fig. 149) the sporozoite attaches itself by its rostrum to an epithelial ceil, and as it grows in size its nuclei multiply; it finally becomes a multinucleate schizont of very large size, which may be either vermiform, and is then attachod by an anterior sucker-like organ to the epithelium, or massive in form, and quite free. When full-grown, its body divides up into as many small merozoites as there are nuclei. The merozoites may probably repeat this development and multiply by schizogony again ; or a merozoite may grow, without multiplication of its nucleus, into a sporont, which procoeds to sporogony of a typical kind. In Schizocystis sipunculi (Dogiel, 603) the schizont has a principal nucleus near its anterior end, and forms a number of secondary nuclei near the hinder end of the body, apparently from chromidia given oft from the principal nucleus, which loses its chromatin. Reund the secondary nuclei protoplasm aggregates, and finally about 150 to 200 merozoites are formed, lodged in a cavity in the cytoplasm of the schizont. The principal nucleus and the maternal body of the schizont now degenerate, and the merozoites are set free.

3. In Porospora gigantea of the lobster, the largest gregarine known, the full-grown individuals round themselves off, become encysted singly, and divide up to form an immense number of so-called "gymnospores" (Fig. 150), each of which consists of a cluster of merozoites grouped round a central mass of residual protoplasm. The subsequent development and the sporogony are unknown; the schizogony was formerly mistaken for the sporogony (Léger and Duboscq, 621).

In the species Porospora legeri, recently described by Beauchamp (592) from the crab Eriphia spinifrons, a similar process of schizogony is recorded ; but in this case an associated couple or syzygy of two trophozoites becomes encysted together, to undergo a similar process of non sexual multiplication. The association is one of two septate trophozoites closely attached, with loss of the protomerite in the posterior individual, as in Didymophyes; the subse. quent development and sporogony are unknown. Léger and Duboseq (622) 
have described recently a number of new species of Porospora from various Crustacea ; they suggest that the genus Porospora represents the schizogony, the genus Cephaloidophora the sporogony, of the same sycle.

4. In the peculiar genus Ophryocystis (Fig. 151), parasitic in the Malpighian
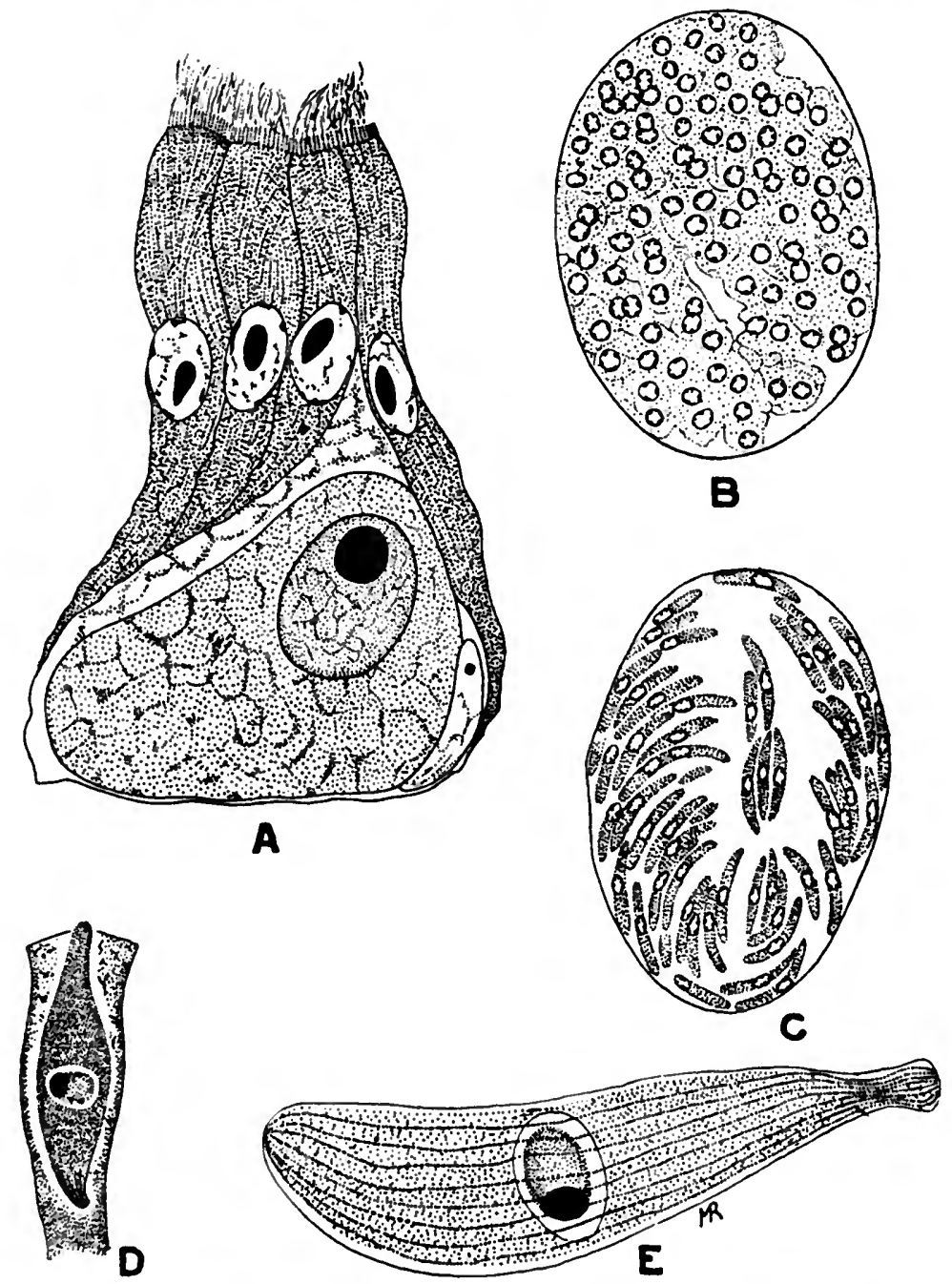

Fro. 148.-Selenidium caulleryi. A, Full-grown intracellular schizont, $\times 850$; $B$, stage in the multiplication of the nuclei of the schizont, $\times 1,200 ; O$, sohizogony complete, showing the merozoites, $\times 1,000 ; D$, young sporont embedded in an epithelial cell, $\times 700 ; E$, free, adult sporont, $\times 700$. After Brasil (596).

tubules of certain beetles (Tenebrionidoe, Curculionida, etc.), and formerly regarded as a distinct order of Sporozoa, the Amcebosporidia, a double schizogony takes place ; there are first of all multinucleate schizonts which can 
reproduce their like for many generations, but which finally produce merozoites which grow up into paucinucleate schizonts, and these produce merozoites which grow up into sporonts. The sporogony of this genus is aleo peculiar. Two sporonts associate, and the nucleus of each sporont divides into three; the body of each sporont then divides into a smaller cell with one nuclcus and a larger cell with two nuclei; the small cell is a gamete, which is
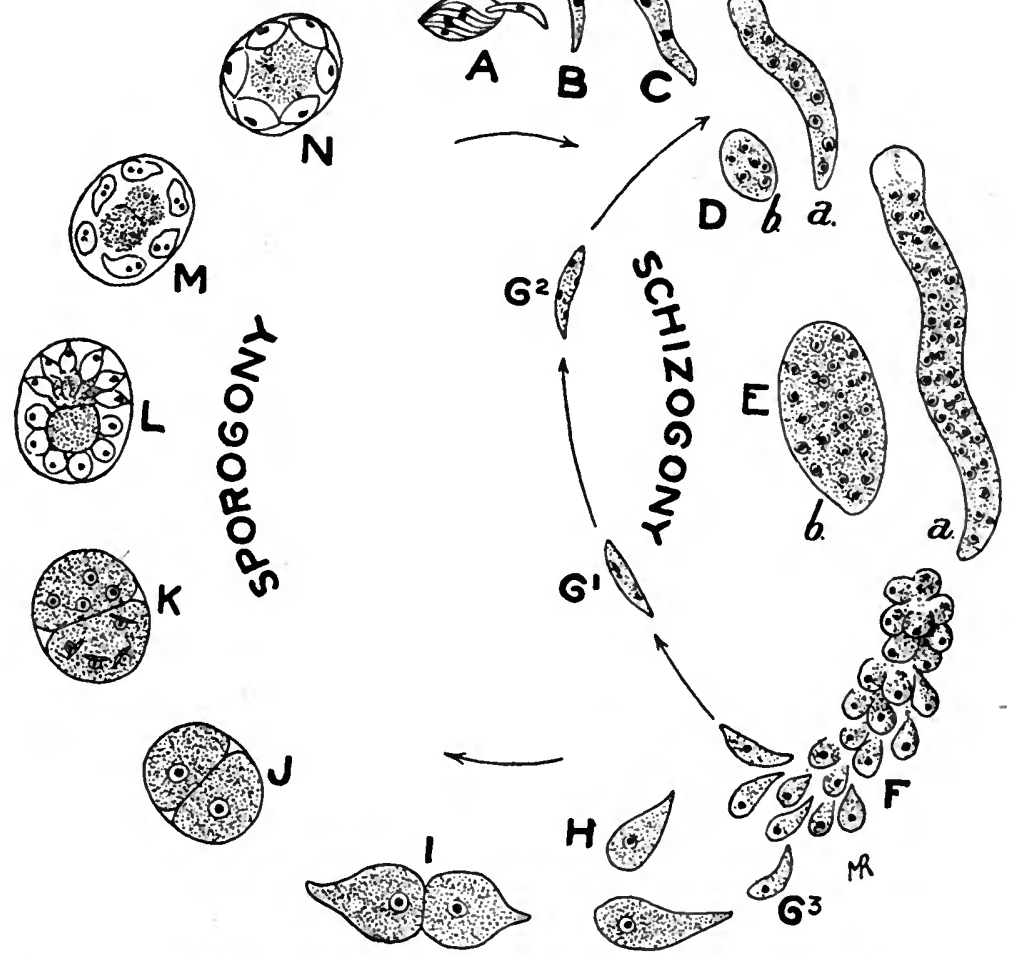

F10. 149.-General diagram of the life-cycle of Schizocystis gregarionides, after Léger $(617$, ii.). $A$, Sporozoite escaping from the spore; $B, C, D, E$, growth of the sporozoite into the multinucleste schizont, of which there are two types: the vermiform schizont $(a)$, which attaches itself to the epithelium by its anterior end, and the massive schizont $(b)$, which lies free in the gut of the host: $F$, division of the schizont into a number of merozoites, which may either grow into schizonts again $\left(G^{1}, G^{2}\right)$, or may grow into sporonts $\left(G^{3}\right)$; $H$, young sporonts; $I$, sssociation of two full-grown sporonts; $J$, formation of a common cyst by two associated sporonts; $K$, division of the nuclei in the sporonts ; $L$, formation of the gametes by the sporonts; $M$, copulation of the gametes; $N$, each zygote becomes a sporoblast and forms a spore.

enveloped by the larger binucleate cell. The two gametes copulate, and the zygote becomes a single spore with the usual eight sporozvites; the two binucleate envelope-cells form a protective envelupe to the spore during its development, and die off when it is ripe (Léger, 617, i.).

(For Schaudinnella see p. 355.) 
The Gregarinoidea are classified as follows :

\section{Suborder I.-Eugregarince (without Schizogony).}

Tribe 1: Acephalina.-Without an epimerite and non-septate; typically, though by no means invariably, "cœlomic" parasites. Example : Monocystis, with several species parasitic in the vesiculæ seminales of earthworms, and many allied genera and species; see especially Hesse. Also many other genera parasitic in various hosts-echinoderms, ascidians, arthropods, etc.

Tribe 2 : Cephalina.-With an epimerite in the early stages, at least, of the trophic phase ; in one family, Doliocystidae, non-septate, but all others septate, with protomerite and deutomerite, or with many segments (Treniocystis, Metamera). Typically parasites of the digestive tract, most common in insects.

This tribe comprises a great number of families, genera, and species; see Minchin (589). The type-genus Gregarina (Clepsydrina) comprises many common species, such as $G$. ovata of the earwig, $G$. blattarum of the cockroach, $G$. polymorpha of the mealworm (Fig. 7, p. 9), etc. Other well-known species are-Pterocephalus (Nina) nobilis, from the centipede (Scolopendra spp.); Stylo-

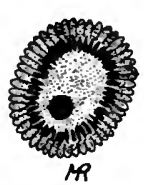

Fra. 150. - "Gymnuspore" of Porospora gigantea, consisting of a number of sporo zoites arranged radially round a central residual mass which contains a chromatinic body. After Léger and Duboseq (621). rhynchus longicollis (Fig. 142), from the cellar-beetle, Blaps mortisaga, and many others. The family Doliocystidoe contains species parasitic in marine Arnelids.

\section{Suborder II.-Schizogregarince (with Schizogony).}

Various methods of classifying the. Schizogregarines have been proposed. Léger and Duboseq (645) divide them into Monospora, which produce a single spore in the sporogonic cycle (example: Ophryocystis); and Polyspora, which produce many spores. Fantham proposes to divide them into Endoschiza, in which the schizogony take place in the intracellular phase, as in Selenidium and Ectoschiza, in which the schizont is a free trophozoite, as in Ophryocystis and Schizocystis; the aberrant genus Siedleckia is probably to be referred here also (see Dogiel, 606). The present state of knowledge is hardly ripe, however, for a comprehensive classification of the schizogregarines, and it may well be doubted whether they are to be considered as a homogeneous and natural suborder ; some of the families of the Schizogregarinæ: appear to be more closely allied to particular families of Eugregarina than to one another. Léger $(617$, ii.) points out that the family Schizocystidae shows close affinities with the eugrcgarine family Adinocephalidoe. 
Pfeffer asserts that the young intracellular stages of the mealwormgregarine multiply by fission. Porospora, with its remarkable schizogony, is apparently a septate cephaline gregarine of the

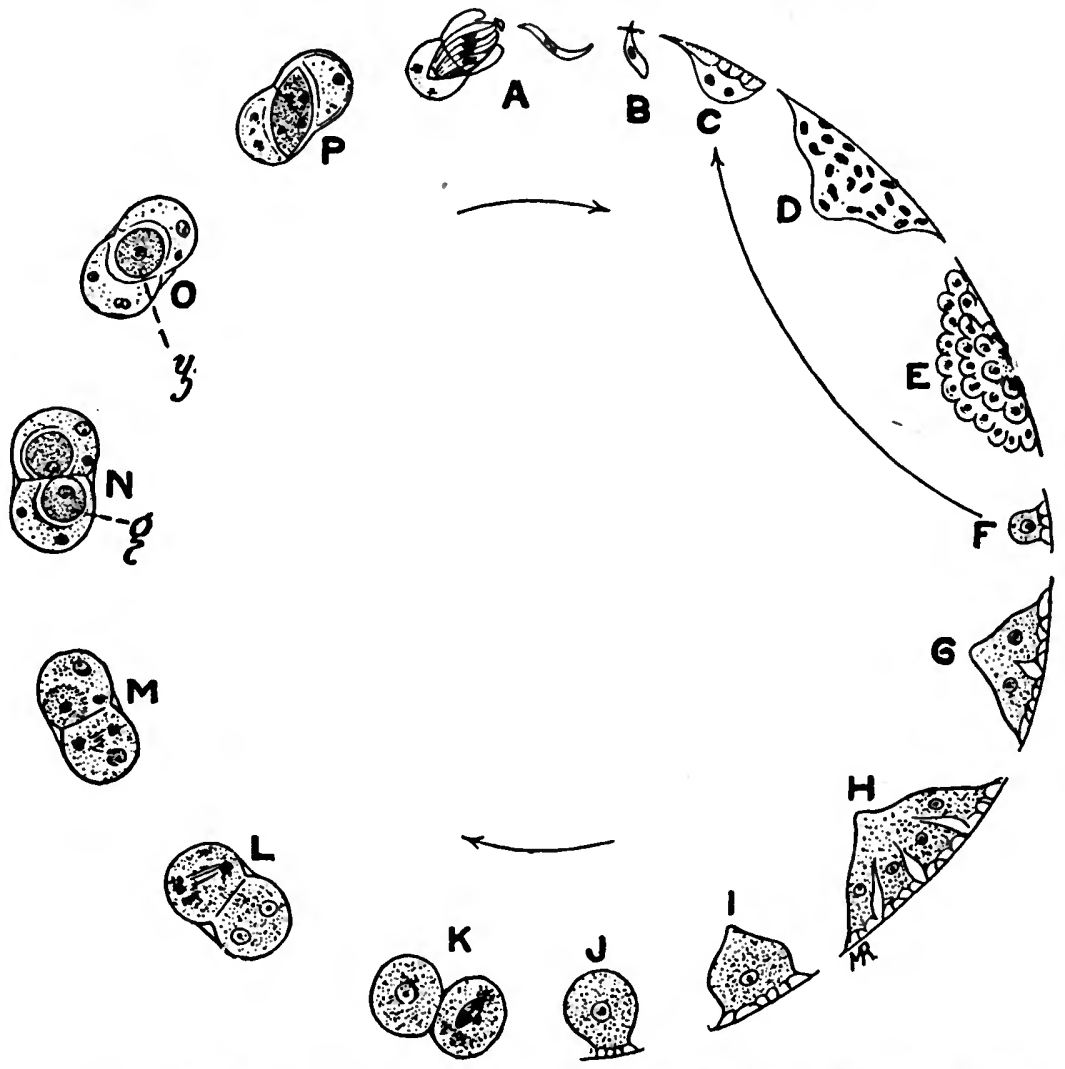

Fia. 151.-Diagram of the life-oycle of Ophryocystis, after Léger $(617, \mathrm{i}.) . \quad A$, The spore setting free sporozoites; $B$, the sporozoite attached by its rostrum to the epithelium of the Malpighian tubule; $C$, multiplioation of the nuoleus of the sporozoite, and growth to form $D$, the multinucleste or "myoetoid" schizont ; $E$, division of the multinucleste schizont into a number of mero. zoites $(F)$, each of whioh may become a multinucleste schizont again, or $(G, H)$ may become a paucinucleate or " gregarinoid " schizont; $H$, division of the paucinucleate schizont to form young sporonts $(I, J) ; K$, association of two sporonts; $L$, formation of a common cyst round the associated sporonts, and division of their nuclei; $M$, formation of three nuclei in each sporont; $N$, separation of a gamete $(g$.) within the body of each sporont, while the rest of the body, with two nuclei, becomes an envelope-cell; $O$, the two gametes have fused to form the zygote $(z$ ) or sporoblast ; $P$, the sporoblast has assumed the form of the spore, and its nuclei have divided into four; ultimately eight nuclei and as many sporozoites are formed.

ordinary type. A character such as the possession of the power of multiplication by schizogony is clearly one of great adaptive importance in the life-history of a parasitic organism, and therefore 
not likely to be of classificatory value. The classification of the future will probably be one which divides all gregarines into Ceplalina and Acephalina, and distributes the ochizogregarines amongst these two divisions.

At present the following families of schizogregarines are recognized : Ophryocystida, Schizocystida, Selenidiida, Merogregarinida, and Porosporida. For the family Aggregatida see p. 353.

\section{ORDER II.-Coccidia.}

The chief characteristics of the Coccidia are that, with very few exceptions, the parasites are of intracellular habitat during the trophic phase, and that a number of spores or sporozoites are produced within a cyst, all of which are the offspring of a single zygote. Further, there is always an alternation of generations, non-sexual multiplicative schizogony alternating with sexual propagative sporogony. As a general rule the entire life-cycle is confined to a single host, but in one family (Aggregatida) an alternation of hosts occurs, corresponding with the alternation of generations; that is to say, the schizogony takes place in one host, the sporogony in another.

Coccidia are found as parasites of various groups of the animal kingdom. In contrast to gregarines, they are found sparingly in Insects, and, indeed, in Arthropods generally with the exception of Myriopods ; but they occur commonly in Molluses, and especially in Vertebrates of all classes. They are found also in Annelids, but not abundantly, and in Flat-Worms (Turbellaria) and Nemertines. A parasite of the gregarine Cystobia chiridotce has been identified by Dogicl (602) as a coccidian, and given the name Hyalosphara gregarinicola.

The intracellular trophozoite is typically a motionless body, spherical, ovoid, or bean-shaped, often with a considerable resemblance to an ovum; hence these parasites were formerly spoken of as egg-like psorosperms ("eiförmige Psorospermien"), and the same idea is expressed in such a name as Coccidium ovi/orme, given by Leuckart to the familiar parasite of the rabbit now generally known as $C$. cuniculi (or $C$. stiedce). The same deceptive resemblance extends to the propagative phases, and the eggs of parasitic worms have before now been mistaken for coccidian cysts, or vice versâ.

The infection of the host takes place in every case, so far as is known at present, by the casual or contaminative method. Resistant spores or cysts of the parasite are swallowed accidentally with the food, and germinate in the digestive tract. The sporozoites escape and are actively motile ; in the majority of cases they penetrate into cells of the intestinal epithelium, but they may under- 

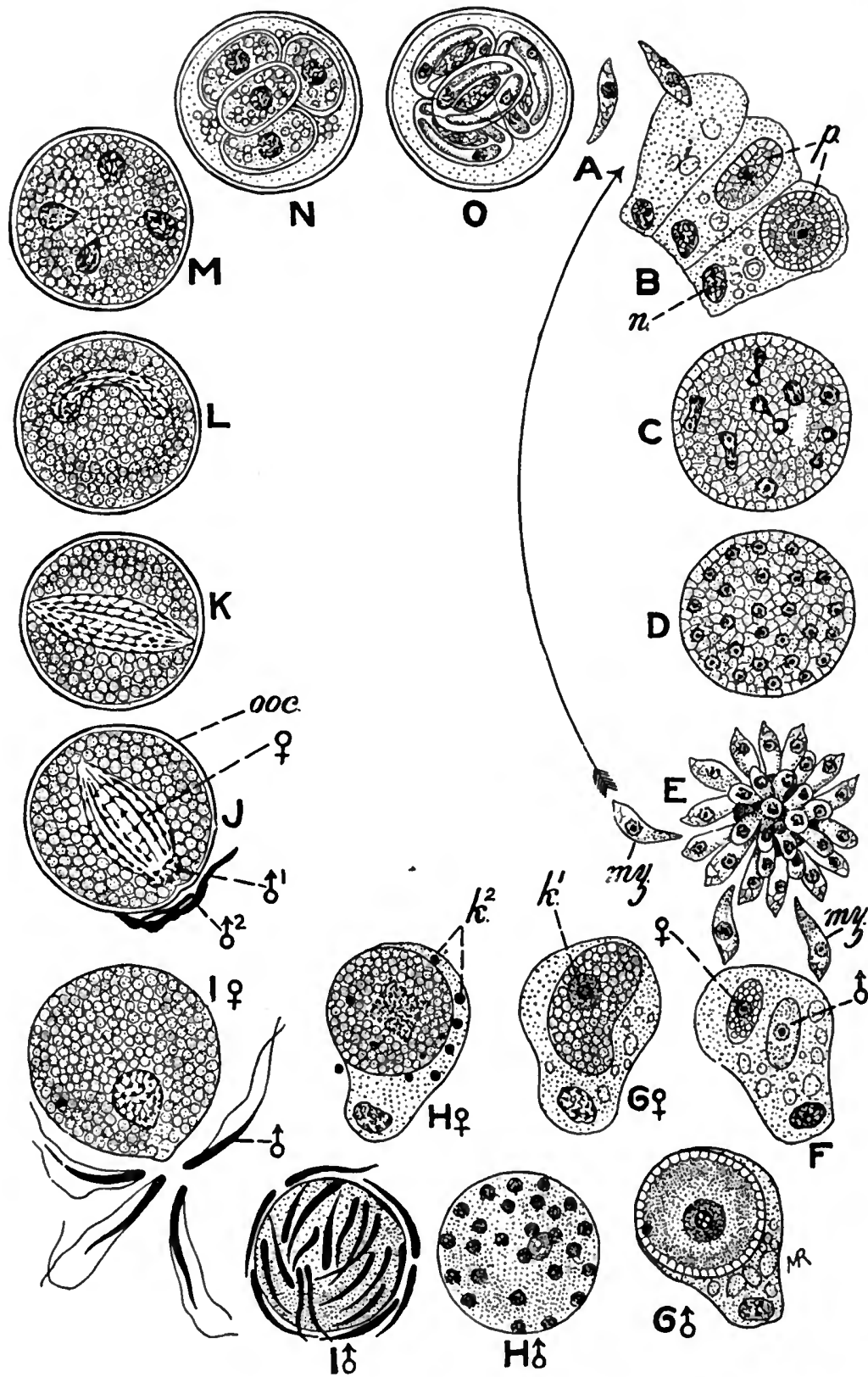

Fic. 152.-Life-cycle of Coccidium schubergi. $A-E$, Schizogony ; $F-I$, gametogony ; $K, L$, syngamy ; $L-O$, sporogony. $A$, Sporozoite liberated from the spore; $B$, three epithelial cells to show thrce stages of the parasite ; in the first (to the left) a sporozoite (or merozoite) is seen in the act of pene- 
take more extensive migrations, and find their way into some other organ of the body, of which they are specific parasites, such as the liver, fat-body of insects, genital organs, kidneys, and so forth. When they have reached the cell, of whatever tissue it may be, which is their destination, they penetrate as a rule into the cytoplasm, and come to rest there, but in some cases they are intranuclear parasites. The trophozoite grows slowly at the expense of the host-cell, which is at first greatly hypertrophied as a rule, but is ultimately destroyed; and when full-sized the parasite enters upon the multiplicative phase as a schizont. After several generations of schizogony, a generation of trophozoites is produced ultimately, which become sexually-differentiated sporonts and proceed to sporogony.

The great power of endogenous multiplication possessed by these parasites renders them often pathogenic, or even lethal, to their hosts, in contrast to the usually quite harmless gregarines. As a rule, however, the production of a pathological condition in the host reacts on the parasite, and stimulates, apparently, the development of propagative phases, which, by passing out of the host, purge it of the infection. In this way the disease- "coccidiosis," as it is termed generally-may cure itself, and the host recuperates its health, but without acquiring immunity against reinfection.

As a typical coccidian life-cycle may be taken that of Coccidium schubergi (Fig. 152), from the common centipede, Lithobius forficalus, described by Schaudinn (99) in a classical memoir. The complete life-history may be divided into eight phases, which are described

\section{Fia. 52 continued:}

trating the cell ; the other two oells contain parasites $(p$.$) in different stages$ of growth (schizonts): $n$., nucleus of the host-cell ; $C, D$, multipiication of the nuclei of the full-grown schizont; $E$, the schizont has divided into a number of merozoites $(m z$. $)$ implanted on a mass of residual protoplasm ; tho merozoites, when set free, may oither penetrate into epithelial cells and becomo schizonts again, as indicated by the long arrow, or may develop into sporonts (gametocytes); $F$, epithelial cell containing two young sporonts, the one male $(\delta)$, with fine granules, the other fomale ( $q$ ), with coarse plastinoid granules in its oytoplasm : $G \delta$. full-grown male sporont ;

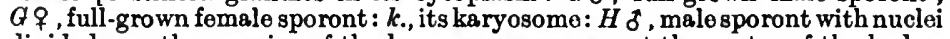
divided up ; the remains of the karyosome are seen at the contre of the body ; $H$, female sporont which has expelled tho karyosome $: k^{2}$, fragments of the karyosome in the host-cell ; $I \delta$, ripo male gametes round the residual mass of the body of the sporont; $I+$, female gameto ripe for fertilization, throwing out on one side a cone of reception towards the male gametes $\left(\sigma^{*}\right)$ swarming round it ; $J$, fertilized zygote which has surrounded itself by an oöoyst (ooc.); inside the body the female pronucleus ( $q$ ) has taken the form of a spindle, st one pole of which is seen the chromatin of the male pronucleus $\left(\sigma^{1}\right)$; outside the oöeyst is seen a clump of degenerating male gametes $\left(\delta^{2}\right) ; K$, the fertilization-spindle complete, with malo and female chromatin spread over it ; $L$, synkaryon dividing ; $M$, the synkaryon has divided into four ; $N$, four sporoblasts are formed, each of which has surrounded itself with a sporocyst, lying in a mass of residual protoplasm (aystal residuum); $O$, ripe oöoyst oontsining four spores, each onclosing two sporozoites and a small quantity of resldual protoplasm (sporal residuum). After Schaudinn (99). 
in the sequel, together with a brief summary of the chief variations which each phase may exhibit in other coccidia.

1. The sparozoites, liberated in the digestive tract, are small gregarinulæ which move by gliding movements, and penetrate into epithelial cells by means of their pointed anterior end (Fig. 152, $A, B$ ).

2. In the cytoplasm of the cell they grow into the large rounded schizonts, distinguished by the absence of reserve food-materials in their cytoplasm, and by the large vesicular nucleus with a karyosome (Fig. 152, B).

In a few rare instances-namely, Coccidium mitrarium, Lav. et Mesn., Cryptosporidium muris, Tyzzer, and the recently-described Selenococcidium intermedium (see p. 351, infra), the trophozoite is free as in gregarines. In Barroussia spiralis, from Cerebratulus sp., the schizont during its early phases of growth is vermiform and spirally twisted, but becomes rounded off when full grown (Awerinzew, 47). In the intranuclear parasite of the mole, Cyclospora caryolytica, the schizonts ane stated by Schaudinn (147) to be sexually differentiated, as also the merozoites to which they give rise. In the case of Adelea ovala, however, a sexual differentiation of the schizonts alleged by Siedlecki (Fig. 153) is stated by Schellack and Reichenow to be due to a confusion of two distinct species; the supposed microschizonts, giving rise to microgametocytes, of Siedlecki, are stated to be in reality the schizonts of Barroussia alpina, Léger, while Siedlecki's macroschizonts alone represent the true schizonts of Adelea ovata; compare also Debaisieux. Chagas, however, describes in Adelea hartmanni (Chagasia hartmanni, Léger, 644) distinct male and female generations, microschizonts and macroschizonts, multiplying by microschizogony and macroschizogony respectively.

3. In the full - grown schizont (agamont) the nucleus divides repeatedly by binary fission (Fig. 51, p. 106 ; Fig. 152, $C, D, E$ ) until a variable number of nuclei, about thirty or forty as a rule, are produced. The body of the schizont then divides into as many segments as there arc nuclei, leaving a certain quantity of residual protoplasm, and each segment becomes a merozoite (" schizozoite," Leger).

The schizogony takes place without any formation of resistant membranes by the parasite, but the remains of the host-cell may furnish an envelope or cytocyst within which the multiplication of the parasite proceeds. As a general rule the merozoites produced are arranged like a barrel round the residual protoplasm (Fig. 153, F), forming a so-called corps en barillet. In Caryotropha a double process of schizogony occurs, recalling somewhat that of Porospora; the schizont divides into a nunaber of cells, "schizontocytes" or "cytomeres," each of which divides in its turn into a cluster of merozoites arranged in a corps en barillet.

The nuclear multiplication in the schizont is not always effected by simple binary fission, as in Coccidium schubergi. In Adelea ovata binary or multiple fission of the nucleus occurs (Jollos). First the centriole contained in the karyosome, and then the karyosome itself, divides into two ; the whole nucleus may then divide into two also, or the division of the karyosome may be repeated several times, until the nucleus contains a numter of karyosomes. In the later nuclear divisions the karyosome becomes very small, consisting of little more than the bare centriole, while the peripheral chromatin increases 
greatly in amount, forming the characteristic star-shaped figures that have so often been depicted. According to Schellack and Reichenow, however, Jollos' observations relate to Barroussia alpina, and not to Adelea ovata, and his statements with regard to cytological details are criticized, and contradicted in part, by these authors and also by Debaisieux. In Caryotropha the nucleus
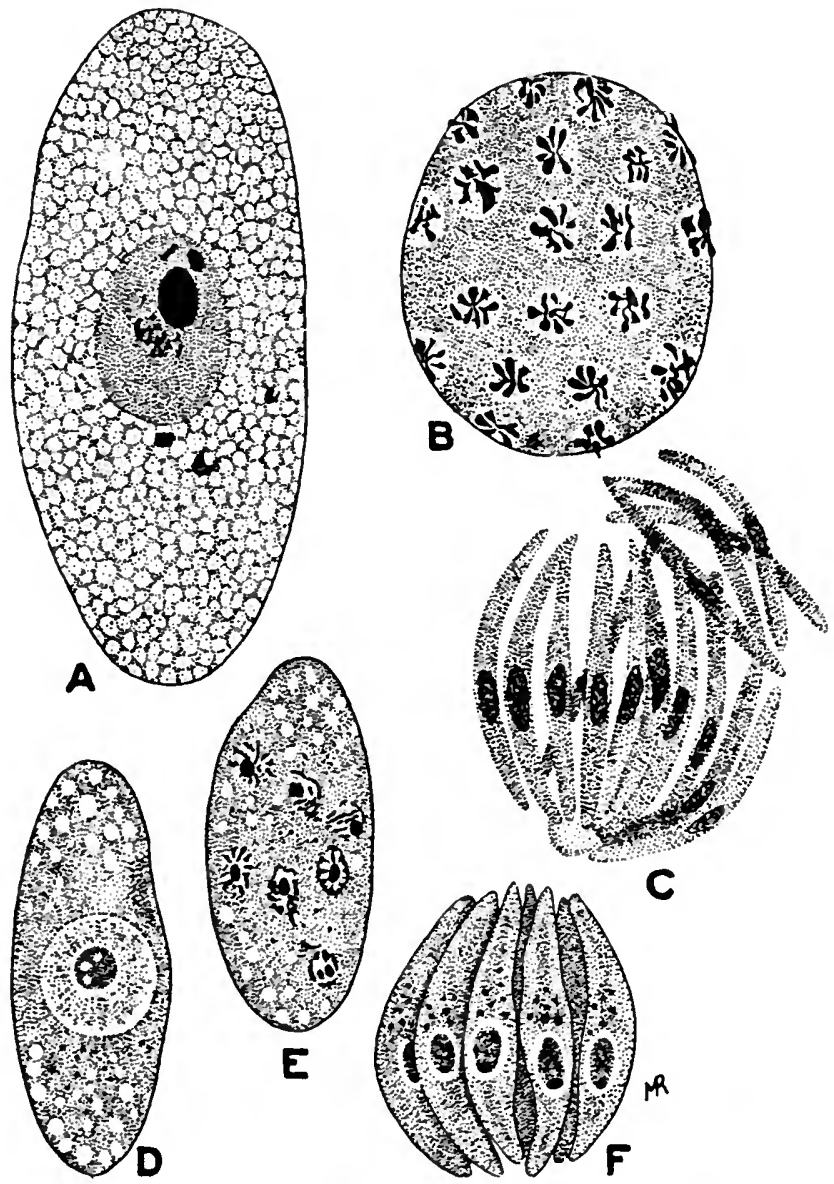

F1a. 153.- Schizogony of Adelea ovata. $A-C$, Multiplication of a female schizont to produce a cluster of merozoites $(C)$ in which the nucleus has no karyosome ; $D-F$. multiplication of male schizont to produce a corfs en barillet $(F)$ of merozoites, in each of which the nucleus has a conspicuous karyosome placed at one end of the nucleus. (According to Schellack and Reichenow, however, the figures $A-C$ alone represent the schizogony of Adelea ovata, and the figures $D-F$ represent that of a distinct species, Barroussic alpina.) After Siedlecki.

of the schizont resolves itself into a mass of chromidia, which is then constricted simply into two masses, then again into two, and so on (Siedlecki, 653).

Non-sexual multiplication has long been known to occur in Coccidia, but the schizogonous generation was regarded formerly as a distinct genus and 
species from the propagative, spore-producing phase, and was givon tho generic name Eimeria, with type $E$. falciformis of the mouse. When the true connection between the two forms was discovered, Eimeria became a synonym of Coccidium, or of whatever the generic name of the sporont might bo (e.g., Eimeria nepa, from Nepa cinerea, =Barroussia ornata). The nomenclaturepurists have, however, sought to abolish the generic name Coccidium, and to replace it by Eimeria, on the ground of priority-a procedure which, in my opinion, is contrary to public policy, and should not be followed, anything in the law of priority notwithstanding.

4. The merozoites (agametes), the daughter-individuals produced by schizogony, are set free from the remains of the host-cell (cytocyst). Each merozoite is very similar to a sporozoite in form, structure, and movements, differing only in minor points of detail ; for instance, in C. schubergi the nucleus of the merozoite has a distinct karyosome, wanting in that of the sporozoite. The merozoites penetrate into epithelial cells, and become trophozoites which may develop in one or the other of two ways-(1) into schizonts again, repeating the schizogony already described; (2) into sporonts (gamonts), destined to produce gametes and resistant propagative phases.

5. The growth of the sporonts is slower than that of the schizonts, and differs in the two sexes; in the male sporont (Fig. 152, $G \sigma^{*}$ ) the cytoplasm remains clear, free from enclosures, but in the female (Fig. 152, $G$ \&) it becomes crowded with reserve nutriment, stored up as a provision for the reproductive phases, in the form chiefly of so-called " plastinoid spherules."

In C. schubergi the female sporonts differ also from the spherical male form in being bean-shaped, but this is a specific peculiarity. In some species the female sporonts are very much larger than the male, as in Adelea ovata (Fig. 154), Orcheobius herpobdelloe, etc. In the last-named species. parasitic in the testis of the leech Herpobdella alomaria, the trophozoites which become schizonts are parasitic in the cytophores; but the merozoites destined to become sporonts are quite motionless, and lie free in the lymph, whence they are taken up passively by the lymphocytes, often several by one such cell. In the lymphocytes they associate in couples, a male and a female sporont together, and the female sporonts grow into long, monocystid-like bodies (Kunze).

6. When full-grown, the sporonts proceed to gamete-formation:

(a) In the male sporonts (Fig. 50, p 102 ; Fig. 152, $H \delta, I$ ठ) the nucleus gives off chromidia into the cytoplasm, and the chromidia collect at the surface of the body ; the old nucleus, now much poorer in chromatin, and with its karyosome still distinct, remains in the centre of the body. The chromidia become condensed and concentrated into patches to form secondary nuclei, which finally take shape as elongated compact bodies consisting of dense chromatin ; each such nucleus, together with an almost imperceptible quantity of cytoplasm, forms the body of a male gamete (microgamete), and is set free, while the greater part of the body of the sporont, together with its old nucleus, degenerates and dies off as residual protoplasm. 
(b) The body of the female sporont rounds itself off and bursts the host-cell. At the same time the karyosome is expelled from the nucleus (Fig. 75, p. 146 ; Fig. 152, $G$ o, $H$ q). It is then ripe for fertilization as a complete and mature macrogamete.

The process of gamete-formation varies considerably in its details in other coccidia, though similar in all essential points to that of $C$. 8 chubergi. The most important difference is that in many coccidia-as, for instance, in Adelea
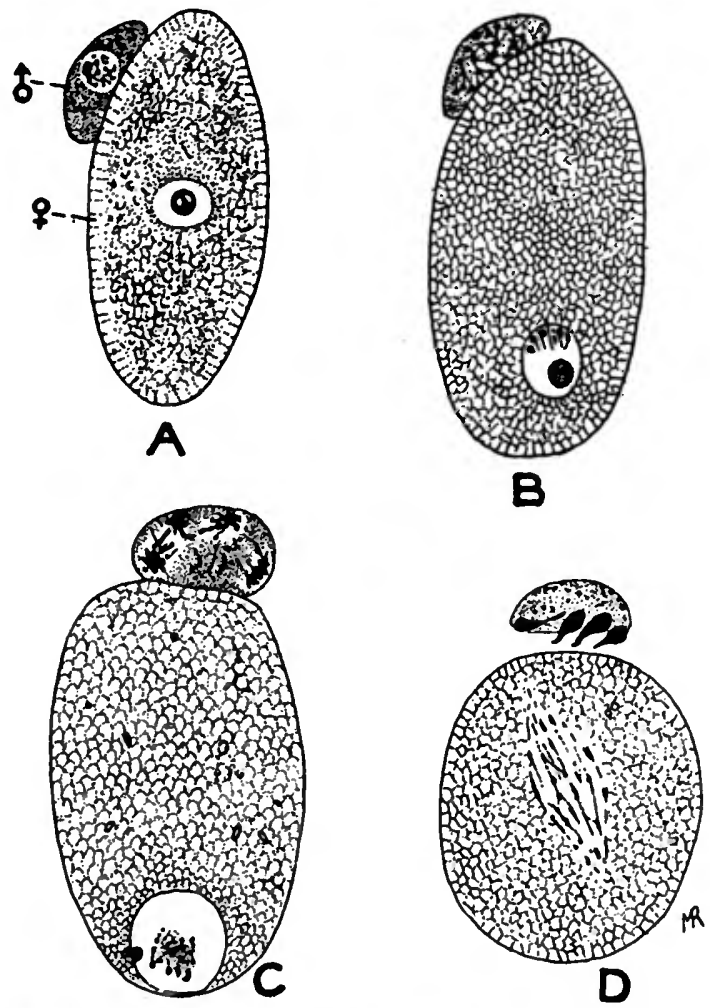

Frg. 154.-Adelea ovata: association of sporonts and gamete-formation. $A$, The two sporonts assoeiated; in the malo ( $\bar{b}$ ) the nuoleus beginning to break up into chromidia; $B$ the nucleus of the male sporont resolved into chromidia ; $C$, formation of four secondary nuolei from the chromidia ; $D$, in the male gametocyte four microgamotes are formed from the four secondary nuclei of the previous stage; in the female gamete the nuclcus has taken the form of a fertilization-spindle. After Dobell.

ovata (Fig. 154) - the two sporonts do not remain separate, as in C. schubergi, but associate in pairs; a small male sporont (gametocyte) attaches itself to the larger female form, and the gametes are then produced. In correlation with this habit, a great reduction in the number of the male gametes takes place, four only being produced. In Adelea (Chagasia) hartmanni, Chagas states that two or even four microgametocytes attach themselves to the female gametocyte; Dobell also figures attachment of two male sporonts in $A$. ovata. 
The maturation of the female gamcte does not necessarily take the form of expulsion of the karyosome; on the contrary, the karyosome may be retained throughout the development. In the macrogametc of Cyclospora caryolytica the nucleus divides twice to form two reduction-nuclei, which are cast off, and a third nucleus which persists as the pronucleus. A similar reductionprocess has been described by Chagas in Adelea hartmanni. In Adelea ovata, according to Jollos, a reducing division occurs in the female gametocyte before association with the male takes place; this is denicd, however, by Schellack and Reichenow and by Debaisieux.

In Caryotropha the malo sporont does not divide at once into microgametes, but first into a number of microgametocytes, each of which then produces microgametes. The process of gamete-formation is thus seen to be exactly parallel to the schizogony, in which the schizont first divides into cytomeres, which in their turn produce merozoites. It is obvious that in coccidia, as in Protozoa generally, schizogony and gamotogenesis are strictly homologous processes; the only difference, primarily, is in the nature and destiny of the swarm-spores produccd in each case, merozoites or gametes. This comparison accentuates the fact, which will be discussed further below, that in the coccidia multiple reproduction to produce gametes is entircly in abeyance in the female sex.

7. The fully-formed microgamete (Fig. 50, p. 102) is a minute, slender, serpentine organism, the body of which consists almost entirely of chromatin; the cytoplasm is represented by the two flagella, which arise close together at one end of the body. One flagellum is entirely free, the other runs along the body to the hinder end, from which it is continued freely; thus the structure of the male gamete recalls that of a trypanoplasm in the heteromastigote arrangement of the flagella.

The male gametes swarm round the inert female gamete, and one of them penetrates into it and fertilizes it. As soon as the entrance of a microgamete is effected, the macrogamete secretes a tough membrane, the oöcyst, at the surface of the body, preventing the penetration of any other microgametes. A fertilization-spindle (Fig. 69, p. 127 ; Fig. 152, $J, K$ ) is then formed in the zygote. The female pronucleus becomes spread out into a fusiform figure consisting of grains of chromatin on an achromatinic framework. When the spindle is complete, the male pronucleus breaks up into granules of chromatin which spread over the spindle, and are thus commingled intimately with the chromatin of the female pronucleus. When this has taken place, the spindle contracts to form the rounded synkaryon, and the syngamy is complete.

The structure of the microgamete varies in different species. In some cases (Adelea, Klossia, Legerella, Barroussia spiralis) flagella are wanting, and the microgamete is a slender, spirochæte-like organism, consisting entirely, so far as can be seen, of chromatin, but actively motile. When flagella are present, they are usually two in number. In Orcheobins herpobdella, Barroussia (Minchinia) -caudata, and some other species, the microgamete terminates anteriorly in a point or rostrum, close behind which two flagella are given off, and are directed obliquely backwards, quite free from the body. In Aggregata, Moroff (94) describes the microgametes as long and slender, with a nucleus of peculiar form, sometimes greatly drawn out, and with 
two flagella, both arising at the anterior end and directed forwards. In Coccidium roux, Elmassian describes two forms of microgametes differing greatly in size.

In forms in which the sporonts associate, as in Adelea and Orcheobrus, one of the four microgametes produced penetrates the macrogamete; the other three die off. In some species-e.g., Coccidium proprium of the newt-the oöcyst is formed prior to fertilization, and the male gamete enters through a minute aperture or micropyle, which is closed as soon as one has entered. In Cyclospora caryolytica, however, numerous microgametes penetrate into the macrogamete, but only one of them furnishes a male pronucleus, which copulates with the female pronucleus; the remaining male nuclei are absorbed.

A fertilization-spindle appears always to be formed in the process of syngamy, but may differ considerably in appearance from that seen in C. schubergi ; compare Kunze's description of the fertilization of Orcheobius herpobdella.

8. The zygote is enclosed, as stated above, in an oöcyst (Fig. 152, $J$ ) secreted at its surface as a membrane delicate at first, but very soon becoming thickened to a tough impervious capsule, in which the parasite can pass out of the body of the host and brave the vicissitudes of the outer world. The synkaryon divides in the genus Coccidium into four nuclei (Fig. 52, p. 106), and the body of the zygote then divides into as many sporoblasts, each with a single nucleus, leaving over a certain amount of residual protoplasm ("cystal residuum"). Each sporoblast secretes a sporocyst at its surface (Fig. 152, $N$ ), and within the envelope the sporoblastnucleus divides into two, after which the cytoplasm segments round each nucleus to form two sporozoites (Fig. 152, O), leaving a small amount of residual protoplasm ("sporal residuum"). These residua are slowly absorbed. When sporogony is complete, therefore, the tough oöcyst contains four spores, each consisting of a tough sporocyst containing two sporozoites. In order to develop further, the cyst must be swallowed by a new host, in the digestive tract of which the oöcyst dissolves, and the spores split open, liberating the sporozoites.

In other species of coccidia the details of the spore-formation may vary enorinously as compared with the example described. The contents of the oöcyst may divide into only two or into a very large number of sporoblasts. In the genus Caryospora (Léger, 644) and Cryptosporıdıum (Tyzzer) the oöcyst does not divide into sporoblasts, but gives rise to a single spore, contain. ing eight sporozoites in Caryospora, four in Cryptosportdıum. In Paracoccidıum prevot sporocysts are formed in the oöcyst, but absorbed again, so that the sporozoites finally lie free in the oöcyst, as in the genus Legerella, in which no sporocysts are formed at all, but the body of the zygote divides directly into sporozoites. With these exceptions, resistant spores are always formed, in numbers varying from two to some thirty or so in different genera.

The spore may contain one. two, three, four, or $n$ sporozoites, and is then said to be monozoic, dizoic, trizoic, tetrazoic, or polyzoic ; it is rarely octozoic, as in gregarines, but Caryospora is so. In Caryotropha mesmili the spore contains twelvo, in Angerocystis audounnee about thirty sporozoites (Brasil, 597). In contrast also with gregarines, the spores of coccidia are generally smooth, round, or ovoid bodies, but in a few cases (e.g., Minchinia chitonis) bear tails or spikes. 

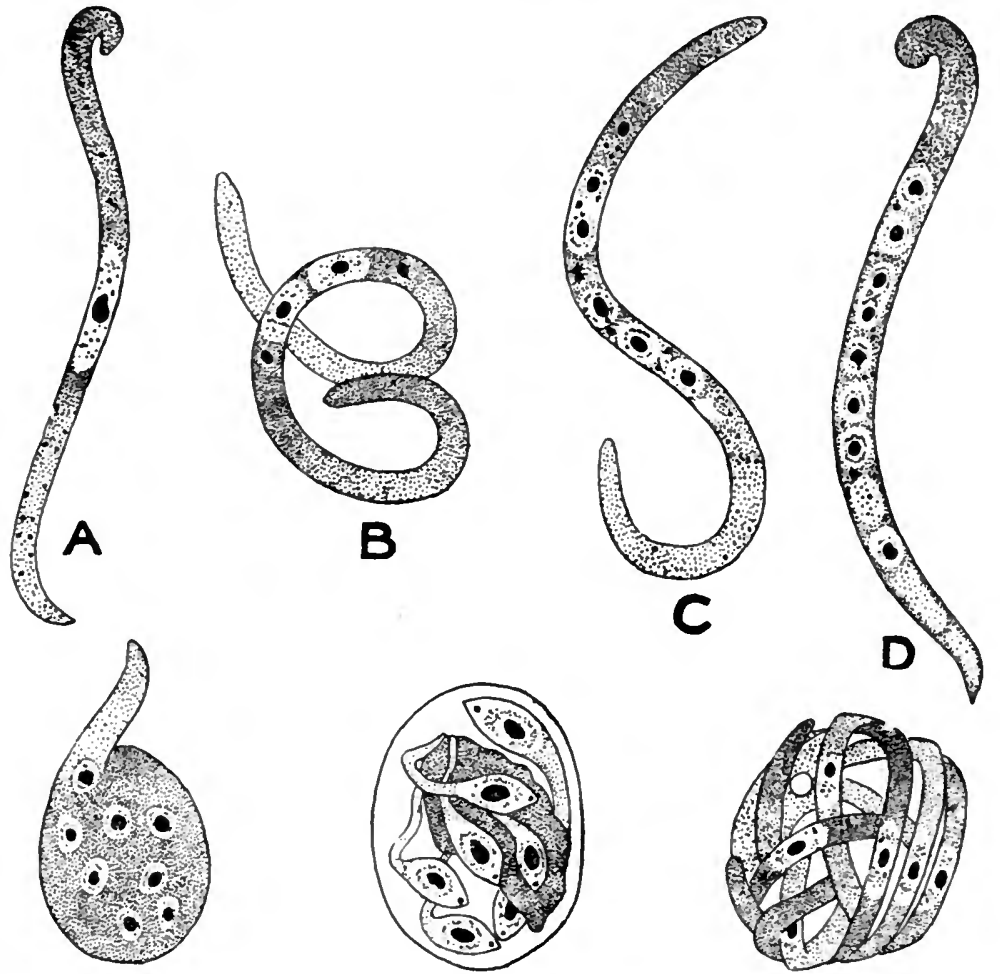

E
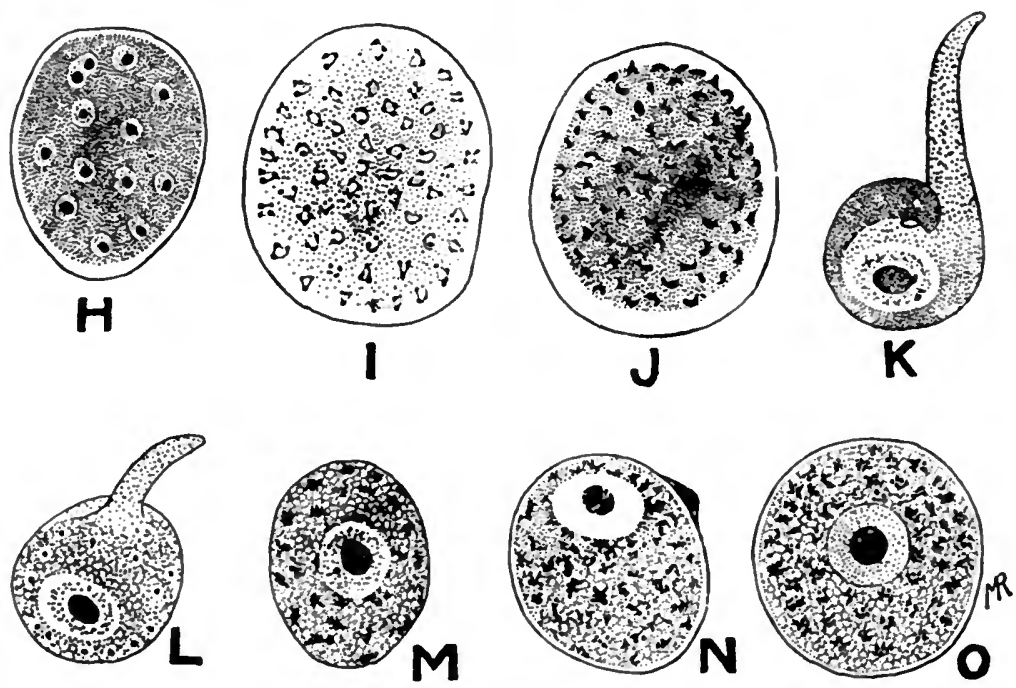

Fro. 155.-For descriplion see foot of opposite page. 
The germination of the spore takes place always, apparently, in the digestive tract of the specific host, and there alone; it may be in some special part of it, as in C. cuniculi, the spores of which, according to Metzner, germinate in pancreatic but not in gastric, juice.

The remarkable form Selenococcidium intermedium (Fig. 155), parasitic in the intestine of the lobster, described by Léger and Duboscq (646), differs from all other known coccidia in the character of its trophozoites and its schizogony. The trophozoites are vermiform, nematode-like organisms, extremely active in their movements, and frequently coiling themselves up and wriggling like worms (Fig. 155, $A-D$ ). The anterior end of the body is blunt, the posterior pointed; the surface of the body contains myonemes running spirally, visible in the living state at the anterior end as oblique striations. The youngest trophozoites have a single nucleus, but as they grow the nuclei multiply, until in the full-grown organism there are eight. The trophozoite is now a schizont, and penetrates into an epithelial cell of the intestine in order to multiply by schizogony. The vermiform body rolls up within the cell into a compact oval mass (Fig. 155, E), and then each of its eight nuclei grows out into a tongue-like cytoplasmic process. In this way eight merozoites are formed round a central residual mass. The merozoites are set free as the uninucleate trophozoites (Fig. 155, $F, G$ ). This "indifferent" type of schizogony may continue for several generations, until a final generation appears in which the schizonts are sexually differentiated; smaller, slender trophozoites with eight nuclei give rise to eight merozoites which grow into male sporonts, and larger, stouter forms with four nuclei produce four merozoites which become female sporonts.

The male sporonts (gametocytes) arise from vermicules with clear cytoplasm, which penetrate into an epithelial cell and roll up into an ovoid mass (Fig. 155, $H$ ); they may do this when they have but a single nucleus, but usually not until the nuclei have increased to eight. In the compact, intra. cellular gametocyte the nuclei multiply rapidly in a manner similar to that described above for the schizont of Adelea, by binary or multiple fission following division of the karyosome. In later stages of multiplication the karyosomes become very small, and tho peripheral chromatin of the nuclei increases greatly, so that they have the appearance of patches of granules (Fig. 155, I). When the multiplication is complete, each such patch of granules forms the dense, comma-shaped nucleus of a microgamete (Fig. 155, $J$ ). An enormous number of microgametes arise from each gametocyte, hut the structure of the free microgametes has not been made out.

The female gametocytes arise from stout vermicules which penetrate into a cell and become rounded off, the nacleus remaining single all the time (Fig. 155, $K, L, M$ ). The oval gametocyto grows, and its cytoplasm becomes full of chromatoid grains. When full-grown it appears to go through a process of maturation, in which, as in Adelea, the karyosome divides into two, and one half is expelled. The full details of the fertilization have not been made out, but macrogametes have been seen with the nucleus placed superficially, and with a small corpuscle, apparently a microgamete, adherent to the body (Fig. 155, N). After fertilizstion the zygote becomes spherical and surrounds

F1o. 155.-Selenococcidium intermedium: various phsses in the life-cycle. $A, B$, $C, D$, Vermicules with one, two, five, and eight nuclei respectively ; $E$, vermicule rolling up prior to schizogony; $F$, schizogony nesrly complete; eight pesr-shaped merozoites, each with a singlo nucleus, budded off from a mass of residual protoplasm to which they are still attached by long stalks; $G$, schizogony complete; eight uninucleate vermicules rolled up together; $H, l, J$, formation of microgametes: $H$, the nuclei of the miorogametocyte at an early stage of division ; $Y$, later stage; $J$, formation of a great number of $00 \mathrm{mms}$. shaped microgametes; $K, L$, vermicule rolling up to become a macrogametocyte ; $M$, fully-formed macrogametocyte, its oytoplasm full of patches of chromatoid granules that stain deeply with iron-hrematoxylin; $N$, macrogamete at the moment of fertilization ; $O$, oöoyst with very numerous ohroms. toid grains. After Léger and Duboscq (646). 
itself by a tough oöcyst (Fig. 155, O); in this stage it is expelled from the body with the faces. The subsequent development of the oöcyst, spore. formation, etc., are not known.

From these data it is sufficiently clear that Selenococcidium is perfectly gregarine-like in its trophic phase and in its schizogony; the trophozoites are froe vermicules which multiply just as in Schizocystis. The parasite only penetrates into a cell when it enters upon reproductive phases. On the other hand, the sporogony, so far as it is known, and especially the sexual processes, are entirely coccidian in type. Selenococcidium links the gregarines and coccidia in a striking and convincing manner, as will be discussed further below.

Classification. -The Coccidia have beer classified in various ways at different times, as increased knowledge of these organisms has shown older schemes to be artificial or unnatural. The following classification is in the main that of Lühe (392), with certain modifications. Some genera have not, however. been investigated sufficiently to make their systematio position certain.

\section{Suborder I. : Prococcidia.}

Trophozoites freo, vermiform, motile; schizogony similar in type to that of Schizogregarines. The only genus known at present is Selenococcidium. The genus Siedleckia should perhaps bo placed here, perhaps in the Schizogregarines near Schizocystis ; its sporogony is as yet unknown.

\section{Suborder II. : Eucoccidia.}

Trophozoites typically intracellular, motionless, oviform, rarely free or vermiform ; schizogony of coccidian type.

Section A.-Forms in which the sporonts do not associate prior to gameteformation, and numerous microgametes are produced :

Family 1 : Coccidiida (Eimerida). -The schizogony is of a simple typo, as described in C. schubergi. Examples: Coccidium (Eimeria) and allied genera; Barroussia (Barrouxia), with type B. ornata, from the gut of Nepa cinerea; Cyclospora, including $C$. caryolytica, from the intestine of the mole; and other genera. Cryptosporidium muris, from the gastric glands of the mouse, has free trophozoites and produces a single tetrazoic spore.

Family 2 : Caruotrophida.--With double multiple fission in the schizogony. Example : Caryotropha mesnilii, parasite of the Annelid Polymnia nebulosa (Siedlecki, 653). Klossiella muris, * from the kidney of the mouse, should perliaps be referred to this family, possibly also Merocystis kathoe (Dakin).

SE to gamete-formation, and the number of male gametes is reduced to four:

Family 3: Adeleida.-With sporocysts. Examples: Adelea, with seversl species, of which the best known is $A$. ovata, parasite of the intestine of Lithobius ; Klossia, with type K. helicina, from the kidneys of Helix spp.; Orcheobius herpobdella, from the testis of the leech Herpobdella (Kunze); and Caryospora simplex, from the intestine of Vipera aspis, in which the contents of the oöcyst form a single octozoic spore (Léger, 644). Minchinia chitonis, from the liver of Chiton and Patella spp., should perhaps be reforred to this family, but the gamete-formation is not yet known.

Family 4: Legerellida.-Without sporocyst8. Example: Legerella nova, parasite of the Malpighian tubes of Glomeris.

A classification similar in the main to the above has been put forward by Léger (644), who terms Section A the Eimeridea, Section B the Adeleidea.

* My friend Dr. A. C. Stevenson, of the Pathological Department, University College, who has studied Klossiella, informs me that he considers it possible that it may represent a stage of Hamogregarina musculi (p.'377). 
Léger proceeds to divide the two sections furthor by the number of sporozoites produced in the oöcyst, but we venture to doubt if this is a method of classification which is natural. In the section Adeleidea, Léger includes the hæmogregarines as a family, Hamogregarinido, characterized by producing one octozoic spore; but this is true only of two species, so far as is known at present, and certainly not of many others (see p. 378, infra).

There remains for mention the family Aggregatida, comprising certain organisms, gonerally regarded as coccidia, parasitic upon Cephalopods of various genera (Sepia, Eledone, Octopus, etc.). These parasites fall into numerous species, of which Moroff (94) enumerates twenty-one, but they are comprised in a single genus which has gone through many vicissitudes of nomenclature, having figured at different times under the names Benedenia, Legeria, Legerina, and Eucoccidium; but when it had, apparently, settled down under the last of these names, it was discovered that the schizogony, formerly supposed to be absent in this genus of parasites, occurs in a distinct host-namely, a crab-where it had been seen by Frenzel and named by him Aggregata ; this name stands, thereforo, as the "correct" name of this genus of parasites.

Not less debatable than the name of these parasites is their systematic position. While, up to a comparatively recent time, their schizogonous phases in crabs had been regarded as those of cclomic gregarines, their sporogonous cycle in Cephalopods was accepted as that of a coccidian. Siedlecki (652) investigated the sexual phases, and found a type of sporogony quite in accordance with that of coccidia-namely, sporonts (gametocytes) separated from one another, the male gametocyte producing a great number of microgametes, one of which fertilized a macrogamete, with subsequent division of the zygote to form a number of sporoblasts and spores.

Recently, however, Moroff published a note in which he maintained that the fortilization was of a type quite different from that described by Siedlecki. Ho asserted that the macrogamotocyte gave rise before, not after, fertilization to a number of sporoblasts, and that the sporoblasts in question were tbe truo macrogametes, each of which, after being fertilized, gave rise to a single spore. In other words, Moroff described the fertilization as being of the gregarine-type, and not that charactoristic of coccidia. Consoquontly these organisms have been classified by Fantham and by Léger and Duboseq (645) amongst the schizogregarinos.

In his latest work, however, Moroff (94) 3cknowledges that the proofs of the process of fertilization alleged by him are inadequate to establish the point at issue, and that further investigations are necessary; he is no longer prepared to insist on the gregarine-nature of these organisms. Until, therefore, the question has been settled by fresh observations, the account of the sporogony and sexual phases given by Siedlecki must stand. These parasites may be regarded as a distinct family of the coccidia, the Aggregatida, characterized by an alternation of hosta corresponding to an alternation of generations. The life-cycle in its general outline is as follows: The spores are produced in the bodies of Cephalopods ; the dead bodies of the Cephalopods, killed in various ways (by porpoises, for example), are eaten by crabs, which thus infect themselves; the spores germinate in the intestine of the crab and liberate the sporozoites, which traverse the wall of the intestine and come to rest in the subepithelial connoctive-tissue layer. There the parasite grows to a large size, forming a cyst which bulges into the body-cavity, and reproduces itself by sohizogony, a process which has heen studied exhanstively by Leger and Duboscq (645). The final result is a vast number of merozoites. If now the crab be eaten and digested by a Cephalopod, the merozoites resist the digestive juices and establish themselves in their new host.

The cycle in the Cephalopods has been studied by Moroff. The merozoites grow into sporonts or gametocytes which are not sexually differentiated, but when their growth is complete sexual differences aro seen in the mode of gamete-formation. Whatever the mothod of fertilization, a number of sporoblasts are formed from which the spores arise; each spore has a tough 
sporocyst, and contains, in different species, from three to twenty-four eporozoites. The various species of Aggregata appear to be specific to particular hosts, whether crabs or cephalopods.

If the Aggregatidae are coccidia; they differ from other coccidia in having an alternation of hosts, and in the absence of an oöcyst formed round the zygote. If, on the other hand, they are gregarines, they differ from all other known gregarines (with the exception of the doubtful form Schaudinnella, 800 p. 355, infra), not only in the alternation of hosts, but also in the fact that the gametocytes remain separate and produco gametes without previous association. If the view put forward by Moroff is the true one, they are to be regarded rather as forms derived from the ancestral form of gregarines and coccidia (see below), before the habit of association of gametocytes, so characteristic of gregarines, had been acquired.

Comparison of the Life-Cycles of Coccidia and Gregarines.-It is seen that a typical coccidian, such as Coccidium schubergi, differs from a typical gregarino mainly in the following points: (1) The trophozoites are intracellular; (2) the gametocytes aro more or less widely separated from one another at the time they produce gametes; (3) the female gametocyte does not divide into a number of gametes, but remains undivided to form a single macrogamete, disproportionately large as compared with the male gametes; (4) the zygote undergoes a process of division, with the result that all the spores produced within the cyst are the offspring of a single zygote, while in gregarines the cyst contains many zygotes and each zygote gives rise to a single spore.

When, however, the coccidia are considered as a whole, it is seen at once that the first two points do not furnish absolute distinctions; in Selenococ. cidium the trophozoites are motile and extracellular, and in Adeleida the gametocytes associate together. There remains only the sporogony which stands out as the distinctive feature of each group. It is by no means difficult to understand, however, the manner in which the two types of sporogony, different as they may appear, could have arisen from a common source.

The common ancestral form, from which the two groups arose by divergent ovolution and adaptation to different modes of parasitism, may be supposed to have been a parasitic organism in which the trophozoites that grew into gametocytes were separated from one another, as in coccidia, and consequently, when full-grown, produced their gametes separately; and each gametocyto produced a number of gametes which differed only slightly from one another, as in gregarines.

From such a form the coccidia arose by the acquisition of an intracellular habitat on the part of the trophozoites, whereby the gametocytes remained more or less widely separated when they produced gametes. As a result of this condition the gametes have to seek each other out, and may easily miss one another ; consequently there was a tendency to greater specialization of the gametes. The male gametes became very small and very motile, and were produced in large numbers. The female gametocyte, on the other hand, no longer divided up into a number of gametes, but became a single large macro. gamete. As soon, however, as fertilization is effected, the suppressed divisions of the female gametocyte take place in the zygote, which divides into the sporoblasts produced formerly by the division of the gametocyte.

The gregarine-type, on the other hand, arose from the ancestral form by the trophozoites which grow into sporonts being free and motile in the later stages of their growtt; consequently, gametocytes of different sexes were able to come together and produce their gametes in close proximity, and finally to associate intimately and produce their gametes within a common cyst. In such a condition it was impossible that the gametes should miss one another; consequently there was no tendency to increased specialization of the gametea, but, on the contrary, a tendency for the gametes to lose even the slight degree of specialization inherited from the ancestral form, with the result that a more or less perfect isogamy was developed; and instead of the microgametes being produced in excess, the numbers of each kind of gamete produced are approximately equal. 
It follows, from the course of evolution sketched in the foregoing paragraphs, that in both gregarines and coccidia the cyst is to be regarded as a secondary acquisition. In the ancestral form there were simply scattered zygotes from which the spore with its contained sporozoites arose; the spore may, in fact, be regarded as representing the primary form of the encysted parasite, comparable to an encysted zygote of the Flagellata. It is indeed obvious that the cyst of gregarines and coccidia respectively are quite different things. In gregarines the cyst is formed round the two associated gametocytes-it is a "copularium," as Léger has termed it ; in coccidia the cyst is a protective membrane formed round the zygote, immediately after fertilization. In the genus Legerella among coccidia, however, the cyst is the sole protective membrane formed to enclose the sporozoites, no sporocysts being produced, a condition which is of interest, since it leads on to that found in the Hæmosporidia.

In both coccidia and gregarines secondary departures from the primary type of the life-cycle occur. In coccidia the gametocytes of certain forms (Adeleidce) have acquired the habit of association prior to gamete-formation; this has not led, however, to a development in the direction of isogamy, as in gregarines, but merely to a reduction in the number of male gametes formed. In some gregarines, on the other hand, notably in those forms of "cœlomic" habitat, or parasitic in the hæmoccle, the sporonts in the later stages of growth are inert and motionless; this condition has led to neogamous association of young sporonts while still motile and capable of coming together proprio motu.

Hero mention must be made of the remarkable form Schaudinnella described by Nusbaum (624), parasitic in the gut of an oligochete worm. Tho full-grown trophozoites of Schaudinnella are gregarine-like, and may be either free in the lumen of the gut or attached to the epithelium by an epimerite; the body is non-septate. Temporary associations (syzygies) may be formed which have nothing to do with sexual conjugation, since the associates part again and produce gametes separately and independently. The full-grown sporonts are distinguishable as male and female forms. 'T'he female sporonts divide up into eight or ten spherical cells, the macrogametes. The malo sporonts divide up into a great number of minute spindle-shaped elements, the microgametes. Copulation takes place between a microgamete and a macrogamete. The zygote may become encysted and cast out with tho fæces, or may peuetrate into the wall of the intestine. In the first manner infection of new hosts is brought about; in the second, multiplication of tho parasite in the same host. The zygotes in the wall of the intestine grow in size, and divide each into a number of sporozoites.

Some doubt may be felt as to whether the life-history of Schaudinnella has been interpreted correctly throughout; it is unusual for endogenous mult. plication to be preceded by sexual processes, and the development requires further examination. If, however, the account of the gamete-formation be correct, Schaudinnella is a form which in this respect stands very near to tho hypothetical ancestral form of gregarines and coccidia.

There can be no doubt that the gregarines and coccidia are closely allied in every respect, and that the two groups are distinguished by points of difference which can be referred quite simply to adaptation to slightly different habits in their parasitic life.

Bibliography.-For references see p. 494. 


\section{CHAPTER XV}

\section{THE SPOROZOA : II. THE HEMOSPORIDIA}

IN the order Hæmosporidia are comprised a number of organisms characterized by the following peculiarities: They are parasites of the blood-corpuscles, red or white, of vertebrates during a part of the life-cycle; like the Coccidia, they exhibit an alternation of generations, non-sexual schizogony and sexual sporogony ; and, in all cases thoroughly investigated up to the present, the altemation of generations corresponds to an alternation of hosts, the schizogony taking place in the blood or internal organs of a vertebrate, the sporogony in the digestive tract or other organs of an invertebrate ; lastly, resistant spores are not, as a rule, produced in this order, being rendered unnecessary by the fact that the parasite is never, so to speak, in the open, but always sheltered within the body of one or the other of its two hosts during its entire life-cycle.

The Hremosporidia, as the name is generally understood, are a group which comprises a number of forms differing considerably amongst themselves. Some of the types referred at present to this order will, perhaps, when thoroughly investigated, be removed from the order altogether. The existence of these dubious forms renders the precise limits of the group uncertain and ill-defined. All that can be said at present is that the order contains a nucleus of true Hæmosporidia presenting very obvious and close affinities with the Coccidia, and, in addition to such forms, certain others, the true affinities of which remain to be determined, but which can be ranked provisionally in the group.

Under these circumstances, the occasion is not yet ripe for treating the group in a comprehensive manner, as has been done with Gregarines and Coccidia. The difficulty of dealing with these blood-parasites is enhanced by the fact that there is perhaps no group in the animal kingdom in which the nomenclature-purist has wrought such havoc as in the Hæmosporidia. Matters have reached such a pitch that in some cases the popular names of certain forms are more distinctive than their strictly scientific appellations, so that the very raison d'être of a scientific terminology has been stultified. 
In the sequel, therefore, the Hæmosporidia will be discussed under five principal types, each of which comprises several forms. So far as possible, the "correct" names of these forms will be stated. Finally an attempt will be made to discuss the position and affinities of the group as a whole. The following is a summary of the distinctive characters of the types in question :

1. The Hamamoba-Type.-The trophozoites of the schizogonous cycle occur within red blood-corpuscles, and are amœboid; they produce a characteristic pigment, termed "melanin." When the blood is drawn and cooled down on a slide, the male sporonts, if present, form filamentous male gametes resembling flagella, and are consequently said to "exflagellate." The invertebrate host, so far as is known, is a mosquito.

2. The Halteridium-Type.-The intracorpuscular trophozoite is a characteristic halter-shaped parasite of red blood-corpuscles, which is amoboid, and which, like the last, produces melanin-pigment, and "exflagellates" on the slide. Only known from the blood of birds ; the in vertebrate host, so far as is known, is a Hippoboscid fly.

3. The Leucocytozoon-Type.-The full-grown sporonts are found within white blood-cells, which are greatly altered by the parasite. They are not amoboid, and do not produce pigment, but they "exflagellate" when the blood is drawn. Onlv known in birds; the invertebrate host is unknown.

4. The Hamogregarine-Type.-Parasites usually of red blood-corpuscles, sometimes of white ; they are not.amoeboid, do not produce pigment, and do not "exflagellate." They occur throughout the whole vertebrate series, but are most abundant in cold-blooded vertebrates. Those of fishes, amphibia, and reptiles, are transmitted generally by leeches; those of mammals and some reptiles apparently by ectoparasitic Arthropods.

5. The Piroplasma-Type.-Parasites of red blood-corpuscles, amchoid or of definite form; they do not produce pigment and do not "exflagellate"; generally very minute. They are known only in mummals, and the invertebrate host is always a tick.

These five types will now be considered in more detail.

1. The Hoemamoebre.-The characteristic form of parasite in this section is a minute, amœba-like organım contained within a rod blood-corpuscle; as it grows it gradually exhausts and destroys the corpuscle, and at the same time produces the characteristic melaninpigment. Such are the well known malarial parasites of mammals and birds. Unfortunately, the accepted rules of nomenclature render it obligatory to use the generic name Plasmodium for these parasites, a most unsuitable name, since they are not plasmodia in any phase except very temporarily, when they are sporulating. They may, however, be termed familiarly "hæmamœbæ," pro- 
vided the word be not written in italics or with an initial capital letter ; anything is better than to speak of them as "plasmodia."

In human beings three distinct species at least of hæmamœbæ are recognized-namely, the parasites of tertian, quartan, and pernicious or tropical malaria, now generally named Plasmodium vivax, $P$. malarice, and $P$. falciparum, respectively ; the last-named is distinguished from the other two by the sporonts being crescentshaped, and was put formerly in a distinct genus, Laverania, which has been abolished. Hæmamœbæ similar to those causing malaria in man have been described from other mammals-for example, monkeys, several species bats; and squirrels. The human malarial parasites go through their sporogony in mosquitoes of the subfamily Anophelinæ; the life-cycle of those of other mammals has not been yet fully investigated.

In birds hæmamœbæ are of very common occurrence. For these Labbé created the genus Proteosoma, a name still in use unofficially as a distinctive appellation; but the correct name of the avian malarial parasites, commonly assumed to belong all to one species, is variously stated to be Plasmodium procex or $P$. relictum. In contrast with the human malarial parasites, those of birds are transmitted by mosquitoes of the subfamily Culicinæ.

Lastly, parasites are known, from certain reptiles, which are intracorpuscular in habitat, amœboid in form, and produce pigment. Hence they appear to be genuine hæmamœbæ, but they do not exflagellate when the blood is drawn,* and very little is known of their life-cycle. By. some authorities these reptilian forms are referred also to the genus Plasmodium, but it is best for the present to maintain the genus Homocystidium, Castellani and Willey, for these reptilian forms. Examples are $H$. metschnikovi (Simond), from an Indian tortoise, Trionyx indicus; $H$. simondi, Castellani and Willey, from a Ceylon gecko, Hemidactylus leschenaulti; and various other species.

Since the transmission of the malarial parasites by mosquitoes was first discovered by Ross in his experiments on the Proteasomaparasite of birds, the development of human malarial parasites has been studied in full detail by numerous investigators, amongst whom Grassi and Schaudinn (130) must be specially mentioned. Consequently the life-cycle of these parasites is better known than that of almost any other Protozoa, and is now to be found described in every textbook. It will be sufficient, therefore, to describe the life-cycle of the species parasitic in human beings in brief outline, as typical of this class of parasites (Fig. 156).

- Aragao and Neiva have observed in Plasmodium (Hamocystidium) diploglossi that, in the male gametocytes on the slide, violent streaming movements occur. such as are the prelude, in other hæmamœbæ, to exflagellation; but formation of gametes was not seen. 
The sporozoites introduced into the blood by the proboscis of a mosquito are minute active organisms of slender form (Fig. 156, XIX.). Each sporozoite attacks a red blood-corpuscle and penetrates into it. Within the corpuscle it becomes a small, amœboid trophozoite, which grows at the expense of the corpuscle (Fig. 156, I.-V.). A characteristic feature of the young tiophozoite is the possession of a large space-probably a vacuole-in the body, which gives he parasite an appearance which has been compared to a signet-ring. As the parasite grows, this space disappears and the body becomes compact. The characteristic pigment is formed within the body of the parasite at an early stage of its growth, and as it increases in size the pigment-grains become more numerous. When the parasite is full-grown it is a schizont, and proceeds to multiply by schizogony (Fig. 156, 6-10). The body becomes rounded by cessation of the amcboid movement, and the nucleus, hitherto single, multiplies by repeated division. Then as many small daughter-individuals (merozoites) as there are nuclei are budded off round the whole periphery of the schizont, leaving at the centre a small quantity of residual protoplasm containing the pigment-grains; this is the characteristic rosette-stage, or corps en rosace. The corpuscle now disintegrates, setting free the merozoites.

Th three species of human malarial parasites are distinguished by differences in their amoeboid activity, their effects on the corpuscles, the number of merozoites produced, and other points, but more especially by the time required for a complete schizogonous generation. Thus, in Plasmodium vivax the growth and multiplication of the schizont requires about forty-eight hours; in $P$. malarice, seventy-two hours; in $P$. falciparum, twenty-four hours or an irregular time. The attacks of fever produced by the parasites occur when the rosettes are breaking up and setting free the merozoites, probably because the disintegration of the body of the parasite sets free toxic substances contained in it. Hence in the tertian ague caused by $P$. vivax the fever returns every third day; in quartan ague of $P$. malarice, every fourth day ; while $P$. falciparum causes irregular or quotidian fevers, more or less continuous.

The schizogony of the tertian and quartan parasites proceeds in the peripheral blood, but that of the pernicious parasite takes place more generally in the internal organs. The amoboid trophozoites present them. selves under the most varied forms in the corpuscles; especialy noteworthy in the quartan parasite is the occurrence of hæmogregarine-like forms (Billet, 664).

There is some doubt as to whether the trophozoites are in all cases within, or merely attached to, the corpuseles. Schaudinn (130) held at first the view that in all cases the parasites were intracellular, and that appearances tending to prove the contrary were the result of alterations due to manipulation in making preparations. It is nevertheless maintained by many authors that some stages, at least, of the parasites are attached to the corpuscles; Halberstaedter and Prowazek, for example, believe that in $P$. pitheci the trophozoites which develop into female sporonts are extracellular, whilst those which become schizonts are irtracellular.

Different species of hæmamœbæ differ also in the effects they produce on 


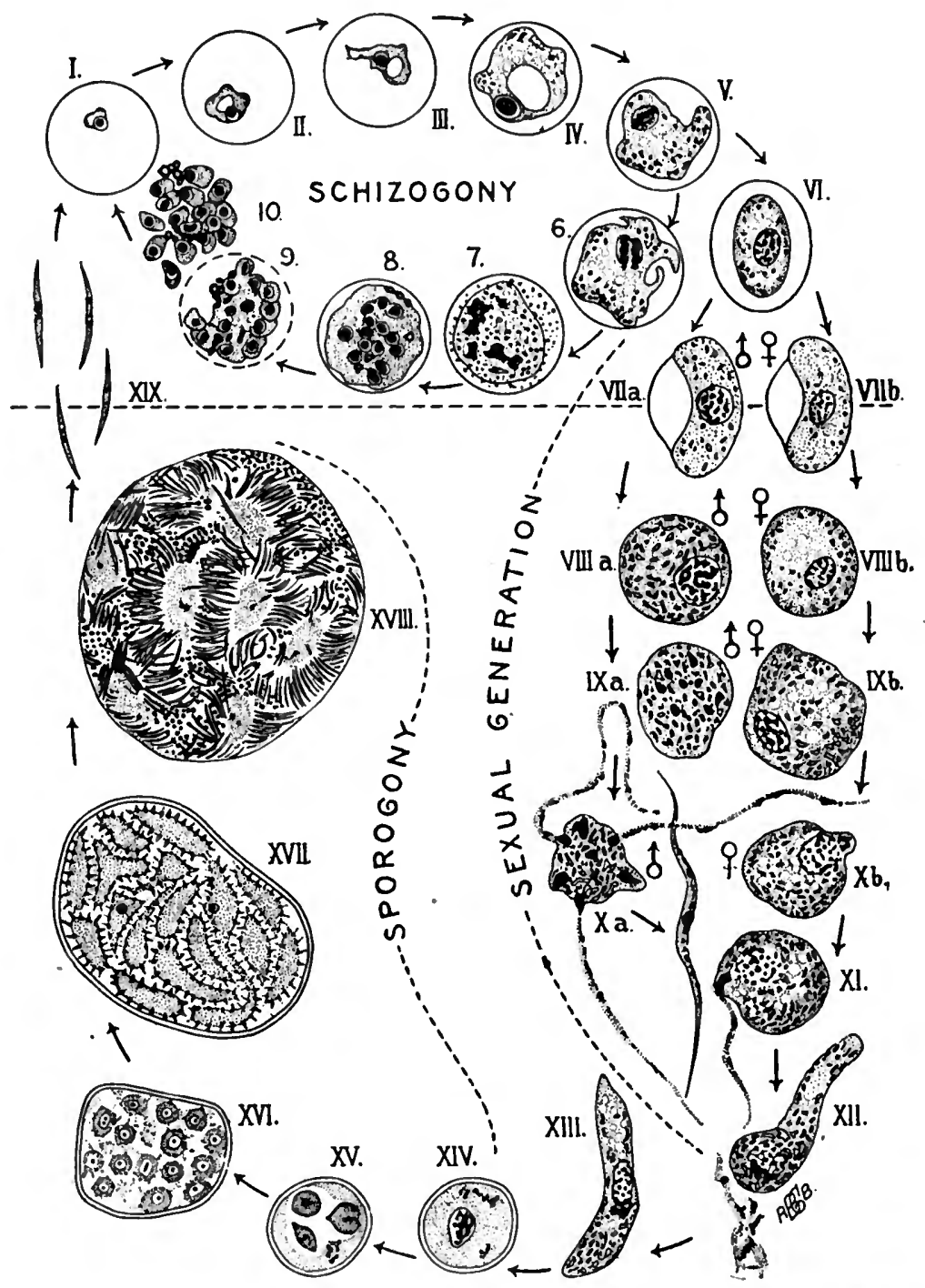

Fra. 156.-Life-cycle of a malarial parasite: combined diagram (the figures are not in all cases from the same species, and some of them are schematic). All the figures above the dotted line represent stages passed in human blood; those below are the stages that are found in the mosquito.

I.-V. and 6-10, Schizogony of the tertian parasite, Plasmodium vivax, after Schaudinn (130), magnified about 1,500 diameters. I., Youngest intracorpuscular stage, which has arisen either from a sporozoitc (XIX.) or a merozoite (10) that has penetrated into, or is attached to, the corpuscle (represented by a circular outline). II.-IV., Further stages of the growth of the parasite : a vacuole is formed in its body which gives it the characteristic " signcte ring" appearance (IV.). V. and 6, Later stages of growth; the vacuol- 
the corpuscles. An effect commonly seen is the so-called "stippling" (Tüpfelung) of the corpuscles, which exhibit a dotted appearance (Schüffner's dots).

'The merozoites, when set free, penetrate into other corpuscles, and become in their turn trophozoites, which may either grow into schizonts again and repeat the process of multiplication by schizogony, or may grow into sporonts. As in Coccidia, a number of generations of schizogony. succeed each other before sporonts are produced. At first the parasites are not sufficiently numerous to bc perceptible in the blood or to evoke febrile symptoms, and during this, the so-called "incubation-period," schizogony alone occurs, in all probability; but when the numbers of the parasite are sufficient to affect the health of the host, the reaction of the host against the parasite probably stimulates the production of the propagative phases. The trophozoites which grow into sporonts

Fra. 156 continued:

disappears; in 6 the parasite is full-grown and its nucleus is beginning to divide. 7,8 , Progress of the nuclear divisions, complete in 8.9 , Division of the body of the parasite to form the merozoites; the blood-corpusclo beginning to degenerate. 10, The parasite has divided up intp sixteen merozoites, leaving the pigment-grains in a small quantity of residual protoplasm; the corpuscle has completely disappeared and the merozoites are set free.

VI., VIIa., VIIb., Formation of the gametocytes of pernicious malaria (Plasmodium falciparum); the gametocytes arise from the intracorpuscular parasites by a series of stages similar to those represented in II.-V., but without a vacuole in the body. In $P$. falciparum the ripe gametocytes have the form of crescents, as shown, but in the tertian and quartan parasite the gametocytes are simply rounded, as VIII $a$. and VIIIb. VII $a$., Male crescent with larger nucleus and scattered pigment; VII $b$. , female crescent, with a smaller nucleus and the pigment more concentrated round it. (N.B.-VIIa. and VIIb. are drawn on too small a scale; the crescent should be as largo as XIII.)

VIII.-XIII., Stages of the sexual generation of the tertian parasite in the stomach of the mosquito, after Schaudinn. $a$, Male forms; $b$, female forms. (In pernicious malaria the crescents round themselves off, become free from the corpuscle, and assume forms similar to VIII. $a$ and $b$.) VIII., Rounded-off parasites free from the corpuscle. IX., Gamete-formation; in $a$ the nucleus is divided into eight; in $b$ the nucleus has passed to the surface of the body. X., Further stage; in $a$ the body of the gametocyte is throwing off the long slender microgametes; one of which is represented free; in $b$ the nucleus is dividing to throw off a reduction-nucleus. XI., Process of syngamy; a male gamete is seen penetrating the body of a fomale gamete. XII., Zygote shortly after fertilization; the body is growing out and becoming vermiform, with the synkaryon at the hinder end; male and female chromatin still distinct ; near the zygote is seen a clump of degenerating microgametes. XIII., Motile oökineto formed from the zygote; the synkaryon, with male and female chromatin still distinct, is seen near the midule of the body; the pigment-grains are at the hinder end of the body, whenco they are soon rejected.

XIV.-XVIII., Sporogony : diagrammatic. The oökinete (XIII.) penetrates the stomach-wall and becomes encysted (XIV.); its nuclei multiply (XV.), and it forms a number of sporoblasts so called (XVI.); in each sporoblast the nucleus divides to form a great number of small nuclei, which grow out in tongue-like processes from the eurface to form the sporozoites (XVII.); the ripe cyst contains great numbers of sporozoites with a certain amount of residual protoplasm; the sporozoites when set free (XIX.) pass into the salivary glands, and thence through the proboscis into the blood of the vertebrate again. 
have, according to Schaudinn (130), no signet-ring stage in their development, but are of compact form, and grow more slowly than the trophozoites which become schizonts. The sporonts are of two types, male and female (Fig. 156, VIIIa., VIIIb.); the male forms have a large nucleus and lightly-staining, clearer cytoplasm ; the female forms have a smaller nucleus and more deeply staining cytoplasm. In the tertian and quartan parasites the sporonts are distinguishable from the schizonts by their greater size and more abundant pigment in larger grains. In the parasite of pernicious malaria, the sporonts are further characterized by their sausagelike form (Fig. 156, VII a., VIIb.), and are thereby easily distinguishable from the rounded schizonts.

The sporonts only undergo further change if taken up by a mosquito of a species capable of acting as the specific host of the parasite. When human blood containing various stages of the parasite is ingested by a culicine mosquito, all stages of the parasite are digested with the blood; but if taken up by an anopheline, the ripe sporonts resist the action of the digestive juices of the mosquito, and develop further in its stomach, while all other stages succumb. The sporonts burst the corpuscle in which they are contained, and round themselves off. In the male sporont the nucleus undergoes rapid fragmentation into some four or six nuclei (Fig. 156, IXa.), leaving a residual karyosome at the centre of the body, as in Coccidium (Schaudinn, 99). The daughter-nuclei place themselves at the surface of the body, and grow out with explosive suddenness into fine filaments of chromatin, ensheathed in a scarcely perceptible layer of cytoplasm (Fig. 156, $\mathrm{Xa}$.). Each such filament is a microgamete, of slender, spirochæte-like form, without flagella, but endowed with powers of active movement. The microgametes lash about violently, often dragging the body of the sporont after them, and presenting a superficial resemblance to flagella, which, indeed, they were formerly thought to be; hence the process of microgamete-formation, which can be observed without difficulty in freshly-drawn blood, was thought to represent a flagellated "Polymitus" stage of the parasite, and was termed "exflagellation." The microgametes by their movements finally become detached, and swim away from the body of the sporont, which perishes as residual protoplasm.

In the female sporont the nucleus divides to give off a reductionnucleus (Fig. 156, $\mathrm{Xb}$.) ; it is then ripe for fertilization by a microgamete (Fig. 156, XI.), which penetrates the body and fuses with the female pronucleus. The zygoto then changes from a rounded form into an elongated vermicule, termed an "oökinete" (Fig. 156, XII., XIII.), which moves by gliding movements, like a gregarine. The oökinete bores its way through the lining epithelium of the 
gnat's stomach, and comes to rest in the subepithelial tissue ; here it rounds itself off and forms an oöcyst (Fig. 156, XIV.), becoming surrounded by a delicate membrane, which is not, however, of a tough and impervious nature like a coccidian oöcyst, since the parasite continues to absorb nutriment and to grow in size, bulging out the stomach-wall towards the body-cavity. As it grows, the originally single nucleus of the zygote multiplies by binary fission, and the cytoplasm becomes concentrated round each nucleus to form a "sporoblast," so called (Fig. 156, XV., XVI.). In each sporoblast the nucleus divides repeatedly, and then the surface of the sporoblast grows out into slender tongue-like processes, each carrying out one of the nuclei in it (Fig. 156 XVII.). Thus a vast number of minute sporozoites are formed by a process of multiplication recalling that seen in the schizogony of Aggregata or Porospora. Finally the cyst contains some hundreds, or even thousands, of sporozoites, together with a certain amount of residual protoplasm, in which the melanin-pigment of the macrogamete is contained (Fig. 156, XVIII.). The ripe cysts burst and scatter their contents in the body-cavity (hæmocœle) of the mosquito; the sporozoites pass by means of the blood-currents to the salivary glands, in which they collect in vast numbers. The mosquito is now infective ; at its next feed, which is usually the fourth, counting as the first that by which it first took up the parasites in the infected blood, the tiny sporozoites pass with the salivary secretion down the proboscis into the blood of the man on whom the mosquite feeds, and so produce a new infection.

A disputed point in the life-cycle is the manner in which relapses are brought about in malarious persons; as is well known, persons who have had malaria may have fresh attacks of the disease under conditions which preclude infection by mosquitoes, and leave no doubt but that the parasite has been present in the body in a latent or inconspicuous condition, and has for some reason reacquired the power of multiplication until its presence becomes perceptible again. Two views have been put forward to explain relapses. According to Schaudinn (130), in the healthy intervals all forms of the parasite have died off except the female sporonts, which are the most resistant forms of the parasite, and maintain their existence in a resting state; when, however, the conditions occur, whatever they may be, which favour a relapse, the female sporonts multiply parthenogenetically (Fig. 72 , p. 137), and produce a brood of merozoites which are the starting-point of a fresh series of schizogonous generations. Ross, on the other hand, belicves that in the healthy intervals the number of parasites in the blood merely falls below that sufficient to produce febrilc symptoms, and that a relapse is brought about simply by an increase in the numbers of the parasites present.

The number of cysts formed in the stomach of the mosquito may be very large, 500 or more; and the systs themselves vary in size considerably, some developing only a few hundreds of sporozoites, whilo in others they are to be counted in thousands. Even in mosquitoes of a species susceptible generally to a particular species of malarial parasite, however, the sporonts do not succeed in every case in passing through their sexual stages and developing normally (compare Darling, 669). In many cases also the cysts degenerate 
and form masses of pigment, the so-called "black spores" of Ross. Similar degeneration-phenomena have been observed by Schaudinn (147) in the oöcysts of Cyclospora caryolytica, and may be compared to the transformation of chromidia into pigment in the degeneration of Actinospharium in cultures (p. 209).

The "exflagellation," or formation of microgametes, which takes place, under normal circumstances, in the stomach of the mosquito, can be seen also in blood freshly drawn and cxamined on a slidc, if ripe sporonts are present. The process is greatly furthered by lowering the density of the blood-for example, by adding to it not more than one-fifth of its volume of ordinary water, or by simply breathing on the blood when drawn (compare Neumann, 677).

It is curious that, whilc so many experimenters have established absolutely beyond all doubt the transmission of hæmamœbæ by mosquitoes, those of man by anophelines, and those of birds by culicines, no experiments seem to have been performed to determine how long a mosquito, once infected, remains infective without bcing reinfected. In other cases of similar transmission, such as that of trypanosomes, yellow fever, etc., it is known that the invertebrate host, once rendered infective, remains so for a very long time, probably for the rest of its life. In the case of malarial parasites this point remains to be tested experimentally.

The hæmam œbæ of Primates have been studied by a number of investigators, and several species distinguished : Plasmodium kochi (Laveran) from the ehimpanzee and various African monkeys; $P$. pitheci from the orang-outang, and $P$. inui from Macacus spp. (Halberstaedter and Prowazek, Mathis and Léger, 473) ; P. cynomolgi from Macacus cynomolgus (Mayer, A.P.K., xii., p. 314); and $P$. brasilianum from the ouakari, Brachyurus calvus (Berenbèrg-Gossler). The schizogony appears to be generally similar to that of the species parasitic in man; ring-stages occur, and the multiplication is in some cases similar to the tertian, in other cases to the quartan parasite. Binucleate trophozoites are of common occurrence, and binary fission also occurs (Flu, A.P.K., xii., p. 323). A striking feature of monkey-malaria is the comparative rarity of multiplicative phases, which may be in relation to the fact that these parasites cause no appreciable symptoms of disease in their hosts; in both respects they are comparable to non-pathogenic trypanosomes. Transmission is probably effected by anopheline mosquitoes (Mayer).

In bats two distinct forms of intracorpuscular parasites have been described under distinct generic names : Polychromophilus, from Vespertilio and Miniopterus spp., and Achromaticus, from Vesperugo spp. These two genera aro distinguished by the fact that Polychromophilus produces melanin-pigment, and Achromaticus does not. Polychromophilus is apparently an ordinary hæmamceba which should be included in the genus Plasmodium. Achro. maticus, on the other hand, appears, from the recent investigation of Yakimoff and others (753), to be a true piroplasm (see below).

Plasmodium vassali from squirrels has ring-like young trophozoites, and its schizogony takes place by binary or multiple fission, more commonly the former (Vassal) ; some forms of the parasite figured resemble Piroplasma.

The life-history of the Proteosuma-parasite of birds has been studied in detail by Neumann ; the principal phases of the parasite are essentially similar to those of the hæmamœbæ parasitic in man. Experimenting with canaries, Neumann transmitted the infection by means of Stegomyia fasciata, but this mosquito was found to be less efficient as a host for Proteosoma than the species of Culex. Of Stegomyia only 11.4 per cent. developed ripe cysts, as against 85 per cent. of Culex; the development of the parasite is accomplished in nine to eleven days in Culex, in thirteen to fifteen days in Stegomyia - and a far smaller number of the parasites succeed in developing in Stegomy ca, in which the maximum number of cysts scen in the stomach of any mosquito was thirty-six, whilc in Culex much larger numbers, 500 to 1,000, are recordod.

But littlo is known of the life-cycle of the reptilian hrmamœbæ of the genus Hamocystidium. Aragao and Nciva have described schizogony of the 
ordinary multiple type, taking place in the blood-corpuscles, in $H$. tropiduri and $H$. diploglossi. According to Dobell, however, the schizogony of $H$. simondi consists simply of binary fission as a rule, sometimes of division into four. The male and female gametocytes, sharply differentiated by their staining properties in this as in other species, are stated also to have the nucleus divided into two when mature ; Woodcock (687), howcver, disputes the correotness of Dobell's interpretations. In no case as yet is the invertebrate host of any Hamocystidium known.

2. The Halteridia.-The characteristic form of parasite in this section, only known to occur in the blood of birds, is an organism which is found within the nucleated red corpuscle, and which does not displace the nucleus of the corpuscle, but grows round it into a halter-like form, whence the name Halteridium given to it by Labbé. Hence the parasite is easily distinguished from Proteosoma, which is more compact in form, and which displaces the nucleus of the corpuscle. Halteridium is amœboid, but the form-changes are generally slight; it produces the characteristic melanin-pigment in abundance; and when the blood is drawn, "exflagellation" of the ripe male sporonts takes place very readily. Not merely the gamete-formation, but the subsequent fertilization and the format on of the oökinete, can be observed on the slide. It is in this form that Macallum first followed out the whole process, and so made clear the true significance of the "Polymitus" stage in the malarial parasites.

The correct generic name for the Halteridium-parasite is believed to be Hamoproteus. Labbé considered the halteridia of different birds to be all one species, to which he restricted the specific name danilewskyi (Grassi and Feletti). By other naturalists several species have been distinguished and named after the birds in which they occur, as $H$. noctua of the little owl, $H$. columba of pigeons, etc. The halteridia of different birds show considerable differences in form, structure, and appcarance, and there can be no doubt that there are many species of these parasites; but it by no means follows that a given species is restricted to a particular host. It is probable that in some cases one and the same species may be capable of infecting several species of avian hosts. The Sergent brothers were unable, however, to infect canaries with $H$. columboe of pigeons.

The life-cycle of these parasites has been the subject of conflicting statements. We shall consider first the type of development made known by the Sergent brothers $(680)$ in part, and more fully by Aragao (Fig. 157). The development described by Schaudinn (132), to which the utmost doubt attaches, will be dealt with later (p. 390).

The invertebrate host of $H$. columba is a biting fly of the genus Lynchia, of the dipterous family Hippoboscide. These flies, though 


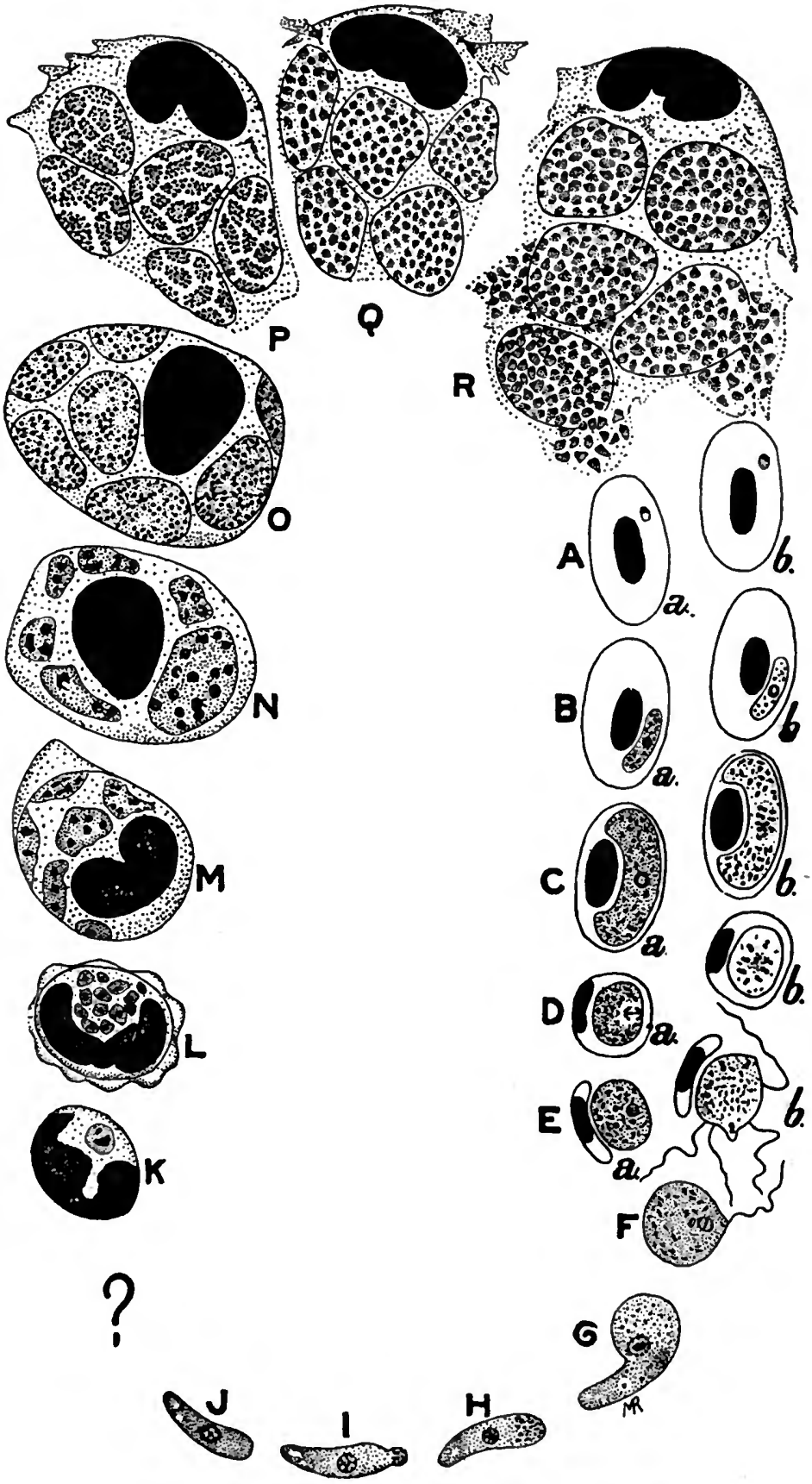

FIG. 157.-For description see foot of opposite page. 
provided with wings (some genera of this family, such as Melophagus, the common sheep-ked, are wingless), are extremely louse-like in appearance, and creep in the plumage of birds; they attack nestlings as well as adults-a fact which explains the appearance of the infection in pigeons before they have left the nest.

When blood containing the parasites is taken up by a Lynchia, the ripe gametocytes burst the corpuscles in which they are con tained, round thomselves off, and form gametes, in the manner already described for hæmamobæ, in the stomach of the fly (Fig. 157, D, E). Fertilization then takes place, and oökinetes are formed (Fig. 157, $F-J$ ). Practically the only difference from the hæmamœbæ is that the oökinetes get rid of their melanin-pigment, which is cast off in a small bead of protoplasm at the hinder end.

The oökinete grows considerably larger than the full-grown halteridia of the blood. The development of the parasite does not proceed further, apparently, than this stage in the fly, and it is the oökinete which is inoculated back into the bird's blood by the Lynchia.

At this point there is a gap in the development which it remains for further observations to fill up. Thirteen or fourteen days after the actual infection by the fly the parasite makes its first recorded appearance in the pigeon, within a leucocyte which is adherent to the wall of a blood-capillary, so that possibly the previous development of the parasite has taken place in an endothelial cell (Aragao). The parasite has the form of a small round body contained in the cytoplasm of a leucocyte (Fig. 156, $K$ ); it has a single nucleus of

Fia. 157.-Developmental eycle of Hamoproteus columbc8, after Aragao (683). $A$, Youngest halteridia in the blood-corpusoles: $a$, female; $b$, male; $B, C$, growth of the gametocytes, female $(a)$ and male $(b) ; D$, gamete-formation : $a$, reducing division in the female gametouyte; $b$, division of the nucleus of the male; $E$, ripe gametes: $a$, female; $b$, male ("Polymitus" stage) ; $F$, copulation of male and female gametes; $G$, the zygote beginning to assume the oökinete-form; $H$, the oökinete with pigment in the body; $I$, the pigment passing to the hinder end of the body ; $J$, the oökinete after it has got rid of the pigment.

$K$, Youngest stage in the leucocyte in the lung of the pigeon; $L$, the preceding stage has divided into a number of small individuals, each with ono nucleus, and the leucocyte has increased in size : $M$, the individuals of the last stage have grown in size and become multinucleate; the leucocyte still further enlarged ; $N$, further advance on the last : $O$, the greatly hypertrophied leucocyte contains a number of multinucleate masses; $P, Q$, further multiplication of the nuelei ; the leucocyte beginning to break down; $R$, the multinucleate masses beoome divided into a vast number of small uninucleate individuals, which are set free from the leucocyte by its disintegration, and which penetrate into blood-corpuscles and there become the youngest halteridia, as in $A$.

The stages $D-J$ are passed through in the fly (Lynchia), the stages $K-C$ in the pigeon. Between $J$, the last stage seen in the fly, and $K$, the earliest stage yet found in the pigeon, is a gap which it remains for further investigation to fill.

The stages $H-J$ are drawn rathor too small in proportion to those preceding. 
irregular form, often seen in process of division. The parasite grows, its nucleus multiplies, and it divides into a number of small bodies, twelve to fifteen in number, each with one nucleus (Fig. 156, L). During this process the leucccyte also increases in size. Each of the small bodies produced by division grows rapidly in its turn, and its nucleus divides repeatedly to produce a very large number of nuclei, which become arranged in clumps resembling the sporoblasts of a malarial parasite (Fig. 156, M-O). Finally each mass becomes divided up into a great number of minute "merozoites" of irregular form (Fig. 156, $P-R$ ). During this process the leucocyte first becomes greatly hypertrophied, and finally breaks down altogether, setting free the merozoites, which pass into the blood and attack the blood-corpuscles, into which they penetrate and become the young halteridia (Fig. 156, $A$ ). The development in the lung that has been described takes about twelve days, so that the youngest parasites make their appearance in the circulating blood about the twenty-sixth day after infection by the fly.

In the blood-corpuscles the youngest halteridia are minute bodies with a single nucleus, which grow into the adult form, and become male or female gametocytes, readily distinguishable by the characters of the cytoplasm, which is darker in the female, and of the nucleus, which is larger in the male (Fig. 156, $B, C$ ). No multiplication takes place in the red corpuscle; the sole multiplicative stage known with certainty is that in the lung. Consequently, in the pigeon the infection dies out after a time, unless re-infections take place, and the degree to which parasites abound in the blood is related directly to the number of infected flies fed on the bird. This may not be equally true, however, of other species of these parasites.

From Aragao's account it would appear that in $H$. columbae only male and female halteridia (sporonts) occur. In other species, however, indifferent forms occur also, which, it may be supposed, are destined as schizonts to repeat the process of schizogony, and so to maintain the infection in the bird, like the schizonts of the malarial parasites. Anschiitz has described in $H$. oryzivora (of Padda oryzivora) a process of schizogony taking place in the circulating blood.

The development of the halteridia in the leucocytes may be considered, probably, as equivalent to the schizogony of the malarial parasites. On this interpretation the missing part of the development is that which corresponds to the sporogony of the malarial parasite, and which in this case is either suppressed entirely ("aposporogony," Aragao), or takes place in the vertebrate host, in some manner yet to be described, instead of in the invertebrate. The absence of sporogony, and of any but the sexual phases, in the Lynchia, doubltess explains the short duration of the infectivity of the fly; according to Aragao, if the flies are fed for three days on clean pigeons, they cease to be infective. Some of the stages in the lung show a certain resemblance to the sporogony of the malarial parasites, especially the formation of sporoblastlike masses, which, however, are probably more comparable to the schizontocytes of Caryotropha than to true sporoblasts. 
Labbé described for halteridium a process of multiplication in the red corpuscle which has never been confirmed. He stated that the nucleus of the parasite divided into a number of small nuclei placed at the two ends of the halter-shaped body, which then divided up into two bunches of small merozoites. It is, of course, possible that the development may differ in different species. But it is more probable that the supposed nuclei at the ends of the body are merely metachromatinic grains, possibly the "alkaliphilous" granules described by Mayer (685, p. 234).

3. The Leucocylozoa.-The true leucocytozoa-that is to say, the species of the genus Lencocylozoon of Danilewsky-are only known to occur in the blood of birds, as stated above; they must be

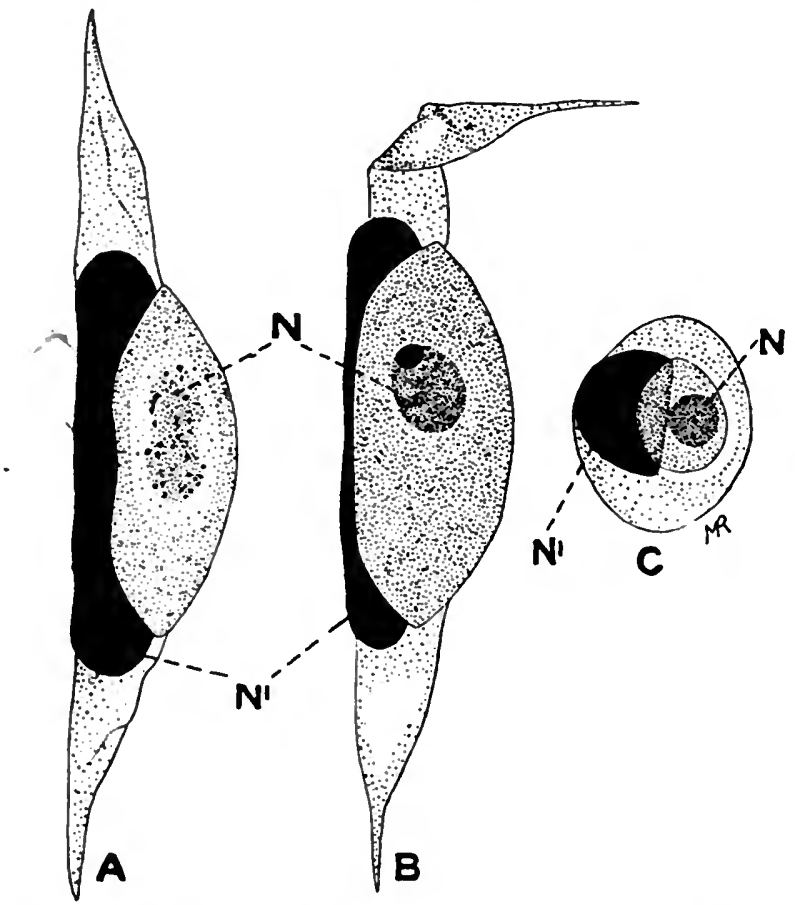

Fra. 158.-Leucocytozoon ziemanni from the blood of the Little Owl, Athene noctua. $A$, Male, $B$, female, $C$, young form. $N$., $N$., nucleus of the parasite ; $N^{1}, N^{1}$, nucleus of the host-cell. Original ; magnification 2,000 .

distinguished clearly from the pseudo-leucocytozoa of mammals, which are in reality hæmogregarines, and will be dealt with as such below. The leucocytozoa of birds are found in the blood as bodies usually elongated and spindle-shaped, sometimes, however, rounded in form, which represent each a gametocyte, male or female, contained in its host-cell (Fig. 158). The exact composition of these bodies is, however, a little doubtful ; it is not quite certain where the host-cell ends and the parasite begins. The centre of the body 
is occupied by an oval, compact mass of cytoplasm containing a nucleus. By some this mass is regarded as the whole parasite, by others as its endoplasmic region alone. In the female forms the cytoplasm is dense and stains deeply, and the nucleus is relatively small, with a distinct karyosome sometimes placed eccentrically. In the male forms the cytoplasm is paler, and the much larger nucleus stains feebly, with a diffuse granular structure and without a conspicuous karyosome. Stretched along one side of the body of the parasite is the nucleus of the host-cell compressed, usually more or less drawn out, and staining deeply. The surface of the body is covered by a thin membrane, which is prolonged usually into two horn-like processes at the two poles of the body. It is doubtful whether these two processes consist solely of the substance of the host-cell, or whether they contain ectoplasmic extensions of the parasite also. In any case it is certain that the parasite modifies the host-cell in a singular manner. It is also disputed whether the host-cell itself is an erythroblast or a mononuclear leucocyte. Most recent investigators, however, incline to the latter view ; but Keysselitz and Mayer (A.P.K., xvi., p. 237) state that the host-cell is an erythroblast. No melanin-pigment is formed.

The young forms of the parasite are compact, rounded, or hæmogregarine-like, contained in white cells with a large nucleus, and without the horn-like processes characteristic of the adult. Fantham (689) has described in $L$. lovati of the grouse multiplication by schizogony taking place in the spleen. The schizonts produce a number of merozoites which escape into the blood, and doubtless give rise to the young forms of the leucocytozoa. The periodicity of the sexual forms in the blood observed by Mathis and Léger (473) depends, probably, on successive schizogonous generations occurring in the internal organs, such as Fantham has described.

The method of transmission and the invertebrate host are as yet unknown. If blood containing the parasites in the condition of ripe gametocytes be drawn, the sexual phases and fertilization can be studied without difficulty on the slide. The female gametocytes round themselves off, losing their spindle-like form, and burst their envelope. The male gametocytes contract themselves into two or three rounded masses, which give off about eight thread-like microgametes altogether, in a manner similar to the "exflagellation" of the malarial parasites. The microgametes become detached and fertilize a female.

Schaudinn. (132) gave an account of the development of these parasites which cannot be accepted as correct. According to him, L. ziemanni of Athene noctun is in reality the resting stage of a large trypanosome, which 
when full-grown attaches itself to an erythroblast and develops into the leucocytozoon, losing its locomotor apparatus. The large trypanosomes in question were supposed to be the sexual, propagative phases, male and female, of a very minute spirochæte-like trypanosome, which represented the indifferent, multiplicative form of the parasite. The existence, however, of young forms of the leucocytozoon, no less than the schizogony discovered by Fantham, disprove entirely any such origin from trypanosomes.

In correspondence with his ideas upon the nature and orgin of leucocytozoa, Schaudinn regarded the nucleus of the female forms (Fig. 158, B) as con. sisting of a trophonucleus with a kinetonucleus (" blepharoplast") close beside it ; while the nucleus of the male leucocytozoon (Fig. 158, $A$ ) was supposed to consist of a cluster of amall trophonuclei, each with a small kinetonucleus beside it, precocious division of the two nuclei of the "male trypanosome " being supposed to have produced a number of couples of nuclei in readiness for gamete-formation. These cytological interpretations cannot be upheld. There is nothing in the structure of the nucleus of the male leucocytozoon to support the notion that it is not a single large nucleus, and the "blepharoplast" of the female form appears to be simply the karyosome, eccentric in position.

Schaudinn also described what he believed to be the development of Leucocytozoon (or, as he named it, Spirochata) ziemanni in Calex pipiens. According to his account, the oökinete became an elongated, worm-like body which divided up to produce an immense. number of spirochætes, or very slender trypanosomes. The spirochætes were stated to tind their way into the Malpighian tubules, where they multiplied and occurred in vast numbers. The spirochrtes, inoculated by the mosquito into the blood of the owl, there became the " indifferent form of the leucocytozoon."

The statements of Schaudinn with regard to the development of Leucocytozoon have received no confirmation, in spite of the efforts of the Sergent brothers to find experimental proof for them. These investigators wero unable to obtain any development of the leucocytozoon in Culex, or to transmit the parasite from owl to owl by the agency of mosquitoes. They found, however, that mosquitoes were commonly infected with spirochætes in the Malpighian tubules, but injection of these spirochetes into the owl produced no infection with Leucocytozoon, and there can be no doubt that the spirochætes in question were true spirochætes, not connected in any way with either trypanosomes or leucocytozoa. Mayer (685) obtained only ökinetes, apparently similar to those of halteridium, but non-pigmented and slightly larger, in mosquitoes fed on owls infected with leucocytozoa, and observed no sign whatever of nuclear multiplication in the oökinetes; Woodcock's unpublished results were practically the same as those of Mayer. Mathis and Léger (473) obtained no development of $L$. sabrazesi in mosquitoes, bugs, and leeches, fed on well-infected fowls, nor could they bring about transmission by means of mosquitoes.

\section{The Hamogregarines.-Parasites of this type have been} found in the blood of all classes of vertebrates, and are especially common in cold-blooded animals, such as fishes and reptiles. Until quite recently, hæmogregarines were not known to occur in birds; but Aragao (692) has described a number of species parasitic in the leucocytes of various species of birds in Brazil. It is a cuicus anomaly of the distribution of these parasites that, while common in marine fishes, they are not known in freshwater fish, with the sole exception of the eel. While in other classes they are parasitic in the red corpuscles, in mammals they are parasitic in either the red or the white corpuscles, but more com- 
monly in the latter as the so-called "leucocytozoa," not to be confused with the true leucocytozoa dealt with in the last section.

Hæmogregarines present themselves usually as more or less elongated parasites of quite definite form, sausage-shaped or wormlike, not amoboid, lying within the blood-corpuscle. The middle of the body is occupied by a conspicuous nucleus, and there are often numerous metachromatinic grains in addition, but no melaninpigment is produced. The parasite may be liberated from the corpuscle as a free vermicule, the resemblance of which to a small gregarine is accentuated by its active gliding movements ; liberation of the vermicules may often be seen when the blood is drawn, but no "exflagellation" ever occurs, since, as will be seen when the development is described, the microgametes are formed in a manner totally different from that characteristic of the hæmamœbæ.

In many hæmogregarines the body of the parasite, when lodged within the blood-corpuscle, is enclosed in a distinct capsule or membrane, which may be of considerable thickness, and often stains deeply. When the parasite is liberated from the corpuscle, the capsule may be left behind as a conspicuous enclosure of the corpuscle which has puzzled some observers, and has even been described as a distinct form of parasite (compare Sambon and Seligmann). In $H$. bicapsulata the capsule is thickened at the two extremities of the sausage-shaped body to form two caps, plainly visible in the living condition, and staining a bright red colour in preparations made with the Romanowsky-stain (Franca, 712).

Different species of hæmogregarines differ considerably in their appearance and size relatively to the blood-corpuscle in which they are lodged, and distinct genera have been founded on these differences; but as yet the complete life-cycle is known in so few cases that it is not possible at present to draw up a classification of these parasites that can have any pretence to be natural.

The following are the principal genera that have been suggested for these parasites. Lankesterella (Drepanidium) is of very small size, the full-grown vermicule being not more than two-thirds of the length of the blood-corpuscle; type, $L$. ranarum (minima), parasitic in the blood-corpuscles of the frog. In Karyolysus the parasite is about the same length as the corpuscle, or slightly shorter; the generic name is derived from the action of the parasite on the nucleus of the host-cell, which is often broken up and "karyolysed," though not invariably. This form of parasite is especially common in Reptilia Squamata, lizards and snakes; type, K. lacertarum. In the genus Hamogregarina (sens. strict.) the full-grown vermicule is much longer than the corpuscle, within which it is doubled on itself in the form of the letter $U$, with the nucleus situated at the bend; type, $H$. stepanowi of European watertortoises, Emys lutaria and Cistudo europoea. Finally there are the "leucocytozoa" of mammals, for which the generic names Hepatozoon, Miller, and Leucocytogregarina, Porter, have bcen proposed; if it becomes necessary to separate them from the genus Homogregarina, Miller's name has the priority, as Wenyon (690) has pointed out. The fact, however, of parasitism in a white 
corpuscle, instead of a red, does not of itself supply adequate grounds for a generic, or even for a specific, distinction, since in some species-for example, H. agama - the parasites may occur either in white or red corpuscles (Laveran and Pettit). For the present, therefore, these leucocytozoa, so called, may remain in the genus Hamogregarina, until greater knowledge of the life-histories of hæmogregarines makes possible a natural classification of these organisms. A hæmogregarine of leucocytic habitat has been described also from a frog by Carini (Rev. Soc. Sci., São Paulo, 1907, p. 121).

As a type of the life-cycle of the hæmogregarines may be taken H. stepanowi (Fig. 159), which has been studied by Reichenow (78). The chief points in this author's account of the life-history are confirmed in essential details, but with specific variations, by that given by Robertson (725) for the life-cycle of $H$. nicoria.* In both cases the developmental cycle in the tortoise comprises two forms of schizogony, the one producing schizonts, the other sporonts; and the invertebrate host is a leech.

(1) The sporozoite penetrates into a blood-corpuscle, and grows into a long vermicule, which is at first doubled on itself (Fig. 159, $F^{\prime}$ ). The two limbs of the U-shaped body within the corpuscle fuse together to produce a bean-shaped parasite-the macroschizont.

(2) The macroschizont of $H$. stepanowi, remaining within the blood-corpuscle, goes through its schizogony in the bone-marrow of the tortoise, producing some thirteen to twenty-four macromerozoites (Fig. 159, $B, C$ ). The number produced is larger in the earlier stages of the infection than in older infections (Fig. 159, $D-H$ ). In $H$. nicorice, however, the macroschizont is set free in a capillary of the Iung, and there produces about seventy macromerozoites.

In the account of the schizogony given by Reichenow, the significance of the rccurved vermicules is not clear. In drawn blood they can be observed to be set free from the blood-corpuscles, and then, as free vermicules, to exhibit active powers of movement, which indicate the existence of some sort of locomotor apparatus, probably of myonemes. According to Reichenow, however, liberation from the corpuscle never occurs normally within the body of the tortoise, but the recurved vermicule remains within the bloodcorpuscle in which it has grown up, and its two limbs fuse to form the body of the bean-shaped macroschizont. If that is so, it is difficult to understand why the motile vcrmicule is ever developed. One is inclined to suspect that it becomes free from the corpuscle in which it has developed, and as a "schizokincte" (Minchin and Woodcock, 483) finds it way as a motile vermicule to the bone-marrow (or lung in $H$. nicorice), where it penetrates another corpuscle (or remains free in a capillary vessel, $H$. nicorice) and becomes the macroschizont.

(3) The macromerozoites produced penetrate into blood-corpuscles, and may $(a)$ repeat the development already described, and become macroschizonts again ; or they may $(b)$ develop into microschizonts, which produce micromerozoites in small numbers,

* Nothing in the work of these authors confirms in any way the peculiar account of the life-history of $H$. stepanowi given by Hahn, whose work is eritioized by Reichenow. 

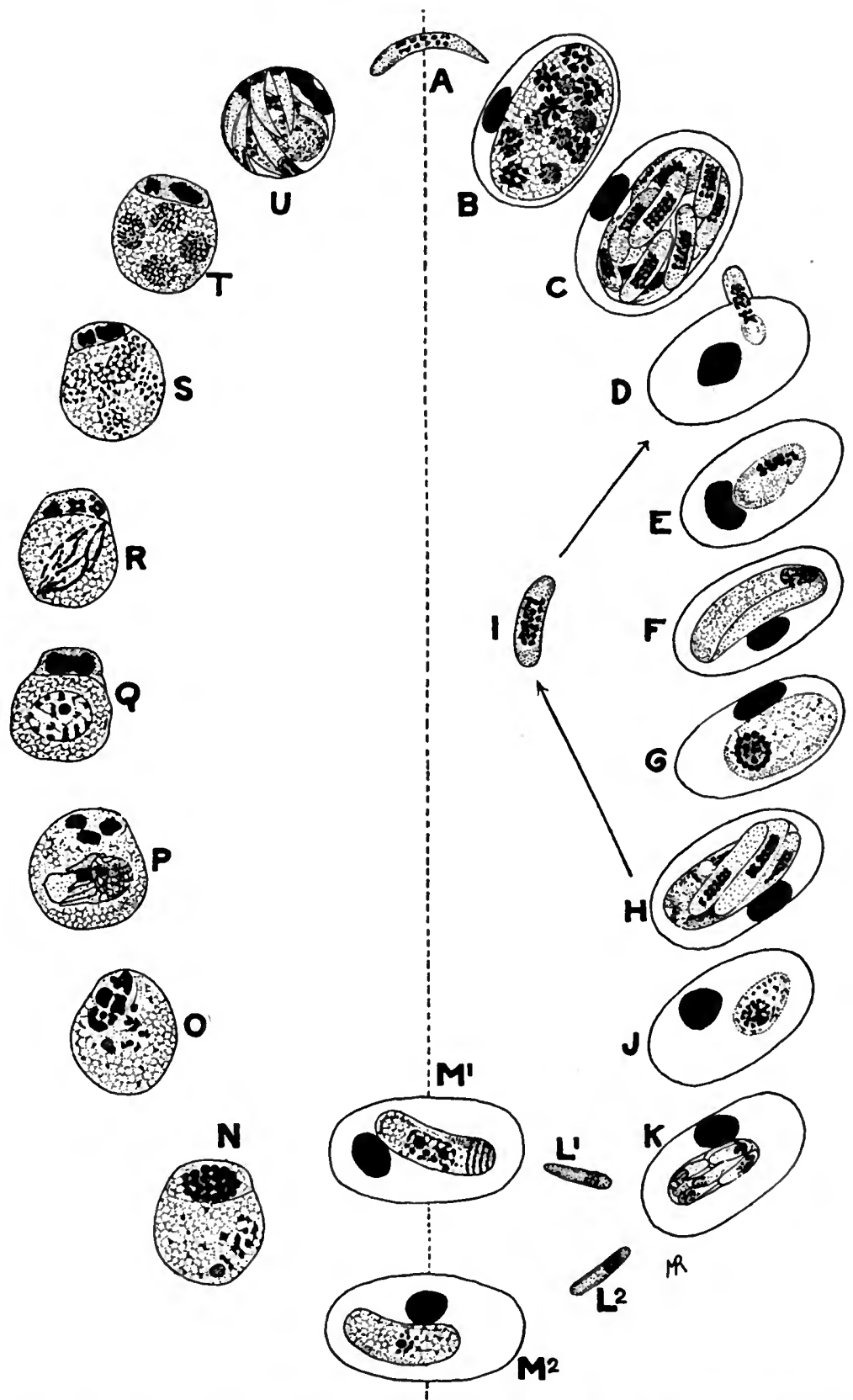

Fia. 159.-Life-cycle of Hamogrcgarina stepanowi. The figures to the right of the dotted line represent the phases in the blood of the tortoise; those to tho left. the phases in the leech. 
destined to grow into gametocytes (Fig. 159, $J-L$ ). In H. stepanowi the microschizont sporulates in the bone-marrow or in the circulating blood, and produces six micromerozoites. In $H$. nicorice it sporulates only in the circulating blood, and produces six to eight micromerozoites.

(4) The micromerozoites penetrate into a blood-corpuscle, and may $(a)$ repeat the microschizogony, or $(b)$ develop into sporonts (gametocytes).

(5) The sporonts (Fig. 159, $M^{1}, M^{2}$ ) are sexually differentiated. They represent the end of the development in the tortoise, and can only develop further in a leech. H. stepanowi develops in Placobdella catenigera (=Hamenteria costata), H. nicorioe in Ozobranchus shipleyi.

(6) When the leech sucks the blood of an infected tortoise, it may take up every stage of the parasite into its stomach, where, however, all stages are digested except the sporonts, which resist digestion and pass on into the intestine. There they associate in couples, male and female together. The male sporont produces four microgametes, and one of the four penetrates the macrogamete and fertilizes it (Fig. 159, N-R).

(7) The zygote forms an oöcyst with a thin membrane, and divides within it into eight sporozoites (Fig. 159, $s-U$ ), which pass into the blood-spaces and collect in the dorsal blood-vessel of the leech. How they pass from thence into the tortoise is uncertain.

The existence of two types of schizogony-macrocysts producing macromerozoites, and microcysts producing micromerozoites-in the cycle of the same species of hæmogregarine, has long been known, but without the significance of this fact being understood.

While the life-history described above is very probably typical of the hæmogregarines of aquatic cold-blooded vertebrates, where the intermediate host is a leech, that of terrestrial animals, so far as it is known, is of a somewhat

Fra. 159 continued :

$A$, Sporozoite ; $B, C$, early schizogony, in which a large number of mero. zoites are produced; $D$, merozoite penetrating a blood-corpuscle; $E-H$, later schizogony, in which fow merozoites are produced; in $f$ the reer.rved vermicule within the corpuscle is seen; $I$, free merozoite about to penetrate a corpuscle and recapitulate the stages $D-H$, or to initiate the next phase ; $J, K$, the stages of the final schizogonous generation which produces the gametocytes; $L^{1}, L^{2}$, sexually-differentiated meruzoites of the final generation, which grow up into male $\left(M^{1}\right)$ or female $\left(M^{2}\right)$ gametocytes respectively; these are the stages which devclop in the leech when taken up by it.

$N$, Association of male and female gametocytes in the gut of the ieech; $O$, formation of four male gametes by the male gametocyto; $P$, one of the male gametes has penetrated into the body of the female gamete, and the two pronuclei are undergoing fusion, with formation of a fertilization-spindle ; $Q$, zygote with synkaryon and the degenerating remains of tho male gametocyte attached to it, which is scen also in the next four stages; $R, S, T$, succossive divisions of the synkaryon; $U$, ripe cyst containing eight sporozoites, residual protoplasm, and the remains of the male gametes.

After Reichenow (78), modified in arrangement. 
different type. In these cases the invertebrate host appears to be always an ectoparasitic arthropod. The only life-cycle of such forms which has been described completely is that of the parasite of the leucocytes of rats, which has been described by Miller under the name Hepatozoon perniciosum. This parasite appears to be identical with that named by Balfour (694) Leucocytozoon muris and by Adie L. ratti; its correct name, therefore, is Homogregarina (Hepatozoon) muris. According to Miller, this parasite causes lethal epidemies amongst tame rats, but in London it occurs commonly in the blood of wild sewer-rats, and appears to be quite harmless to them. It is a parasite of worldwide distribution, apparently, having been recorded from rats in the Punjaub (Adie), Khartoum (Balfour), North America (Miller), Brazil (Carini), and various other parts of the world (sce Franca and Pinto, A.I.B.C.P., iii., p. 207).

The life-cycle of $H$. muris, according to Miller, is in the main as follows: The sporozoites are liberated in the intestine of the rat, and pass through the wall of the gut into the blood-stream; they may be found in the circulation twenty-four hours after infection. Ultimately the sporozoites reach the liver and penetrate into liver-cells ; in this situation they grow into schizonts, which when full-grown sporulate to produce some twelve to twenty, usually about sixteen, merozoites. The merozoites may penetrate into liver-cells again and repeat the schizogony, or they may pass out into the capillaries of the liver; in the latter event they are taken up by leucocytes, doubtless as an act of phagocytosis. The merozoites are able, however, to resist any digestive action of the leucocytes; they become encapsuled in the leucocytes, and in this state they are carried into the general circulation. They do not increase in size in the leucocytes, and their further development, so far as the rat is concerned, is at an end. Hence the "leucocytozoon" of the rat is an encapsuled merozoite of a hæmogregarine which, strictly speaking, is a parasite of the rat's liver, and not of the blood at all; in the leucocytes its rôle is one merely of passive resistance. These merozoites represent at the same time the sporonts, the propagative phase which develops further in the invertebrate host, in this case a rat-mite, Lalaps echidninus, which sucks the rat's blood, and so takes up the parasite into its stomich.

In the stomach of the mite the hæmogregarines are set free as motile vermicules which associate in couples. According to Miller, this association is a true copulation of two gametes which fuse into a zygote; from the analogy of the life-cycle described above, it is more likely that some stages have been overlooked, and that the vermicules are gametocytes which associate, with subsequent production of gametes by the male and fertilization of the female by a microgamete.

The zygote, however formed, becomes a motile oökinete which passes through the wall of the gut into the body-cavity of the mite, and there forms an oöcyst which, like that of the malarial parasites, has a thin wall, permitting the parasite to absorb nourishment from the surrounding tissues and to grow to a large size. When full-grown, the contents of the oöcyst divide up into a large number of sporoblasts, each of which becomes surrounded by a delicate sporocyst. The contents of the spore divide up into some twelve to twenty sporozoites, and then the development of the parasite is at an end so far as the mite is concerned. The cyst and spores are the propagative phase, and in order that they may develop the mite must be eaten by a rat ; if this occurs, the sporozoites are liberated in the stomach and the cycle is complete.

In the case of other mammalian hæmogregarines, fragments of the development are known which indicate a life-cycle similar in the main to that of $H$. muris, allowing for specific differences. Forms parasitic in the red blood. corpuscles are $H$. gerbilli of Gerbillus indicus (Christophers, 699); H. balfouri (jaculi) of the jerboa (Balfour, 693); and the three species recently described by Welsh and others (Journ. Path. Bact., xiv.) from marsupials, one of which (H. peramelis) is remarkable for having been found only in the free, extracorpuscular condition. The schizogony of $H$. gerbilli has not been described, but that of $H$. jaculi takes place in the liver, and is of two types, producing in the one case a large number of small merozoites, in the other a small 
number of large merozoites (compare $H$. canis, below). In both $H$. gerbilli and $H$. jaculi free vermicules occur, and are set free readily in vitro; those of $H$. gerbilli are recurved when contained in the blood-corpuscle. Stages of the development of $H$. gerbilli were found in a louse, Hamatopinus stephensi; first free vermicules in the stomach and intestine, later large cysts in the body-cavity containing a great number of spores, each of which encloses six to eight sporozoites. It seems impossible that the parasites encysted in the body-cavity of the louse should get back into the gerbille in any other way than that of being eaten by the gerbille. Christophers found that, though the sporozoites were liberated in the intestinal juice of the gerbille, they soon died in it, but that in the blood-plasma of the gerbille they became extremely active; this observation may perhaps be interpreted as indicating that the spores germinate in the intestine, and the sporozoites, when liberated, pass at once through the wall of the intestine into the blood-cireulation.

The crithidial forms seen by Balfour in Pulex cleopatroe ean have no connection whatever with the hæmogregarine of the jerboa; the flea is probably not the right host for this parasite.

A number of leucocytic gregarines have been described from various mammals, amongst which may be mentioned $H$. canis (Christophers, 700), $H$. funambuli (Patton, 721), and $H$. musculi (Porter). The life-cycle of $H$. canis has been described by Wenyon (84). The schizogony takes place in the bonemarrow and the spleen of the dog, and is of two distinct types. In the one case the schizont divides into a small number of merozoites, usually three, of large size. In the second case the schizogony rosults in the production cf a large number of small merozoites. The larger merozoites grow up into schizonts again; the small merozoites pass into the blood, are taken up by the leucocytes, and become the gametocytes, as in $H$. muris. The sporogony takes place in the tick, Rhipicephalus sanguineus, and is similar throughout to that of $H$. muris. The sexual phases were not observed by Wenyon, but aecording to Christophers (701) the vermicules become free in the stomach, and penetrate the epithelial cells, in which they multiply by fission to form gametes; probably this applies to the male sex alono. The next stage is an oöcyst in the tissues of the tick. The oöcyst grows in size, its nuclei multiply, some thirty to fifty uninucleate sporoblasts are formed, and each secretes a sporocyst and becomes a spore containing on the average sixteen sporozoites. The oöcyst-wall dissolves, and the ripe spores are set free in the body of the tick. Wenyon considers it possible that the dog acquires the infection by eating infected ticks.

Free vermicules of $H$. funambuli werc seen in a louse by Patton, and a similar observation was made for $H$. musculi by Porter. H. musculi also reproduces by schizogony in the bone-marrow of its host.

The hæmogregarines of birds described by Aragao (692) appear to be very similar to those parasitic in the leucocytes of mammals. The schizogony takes place in the epithelial cells of the gut or in the cells of the liver, lung, or bone-marrow, it results in the formation of a number of small, commashaped merozoites, which escape from the cell and are taken up by the mononuclear leucocytes. They do not, however, remairr in a resting phase in the leueoeytes, but grow within them to a fair size. When set free from the leueocyte, they perform active movements. The intermediate host and the mode of transmission remain, however, to be discovered.

The schizogony of hæmogregarines parasitic in snakes has been studied by Sambon and Seligmann, Hartmann and Chagas (89), and Laveran and P'ettit (716). It takes place in the capillaries of the liver and lung or in the bone-marrow. The parasite becomes free from the corpuscle in the capillary, and grows to a large size. In $H$. sebai the number of merozoites formed varies from two or four to over thirty, but is more often from four to eight. The merozoites are larger when a smaller number is produced. Possibly the variation is related to the age of the infection, as in $H$. stepanowi, or to the destiny of the merozoites, whether to become schizonts or gametocytes, as in $H$. canis.

The sporogony of the hæmogregarines of terrestrial reptiles is practically 
unknown in its details, but the transmission appears to be effected by ticks; so Karyolysus lacertarum by Ixodes ricinus (Schaudinn, A.P.K., ii., p. 339, footnote), $H$. mauritanica by Hyalomma agyptium (Laveran and Pettit, 718), and the hæmogregarines of snakes (Flu, 707).

The minute "drepanidia" of frogs and newts appear to stand rather apart from the true hrmogregarines; beyond the fact that they multiply by schizogony in the red blood-corpuscles, but little is known of their development. According to Hintze, Lankesterella ranarum has no invertebrate host, but passes from the blood into the wall of the intestine, where it forms resistant cysts like a coccidian parasite. The cysts were believed to pass out of the frog with the fæces and infect other frogs by the direct contaminative method. It is, however, very doubtful if the cysts described by Hintze really belong to the cycle of the Lankesterella; from other observations it is possible that the drepanidia are not hæmogregarines at all, but stages in the life-cycle of a trypanosome (compare Billet, 696). According to Franca (709), "Dacty. losoma" splendens of the frog produces. Leishmania-like merozoites, with distinct kinetonuclei (compare also Seitz). Until further researches have been undertaken, the position of the drepanidia must remain uncertain.

Neresheimer (720) has described the penetration of the red blood-corpuscles of frogs by Lankesterella sp., a process in which remarkable phenomena are oxhibited. When a Lankesterella, in approaching a blood-corpuscle, is within a distance from the corpuscle about equal to the length of the parasite, the edge of the corpuscle turned towards the parasite shows distinct amoboid movements. As the parasite comes still nearer, two long processes are thrown out by the corpuscle, forming a deep bay, into which the parasite enters; as soon as it does 80, the two processes approsch each other, fuse and engalf the parasite, just as an amcba ingests its prey. The parasite, after this point is reached, appears to be drawn into the corpuscle without further exertion on its part; the protoplasm of the corpuscle closes up behind it, and the corpuscle regains its normal smooth contour, with the parasite lying within it. The whole process of penetration takes one or two minutes. Neresheimer compares the activity of the corpuscle to the "cone of reception" formed by an ovum when approached by a spermatozoon.

From the foregoing account of the life-cycles of næmogregarines, it is seen that the sporogony varies greatly, from the production of eight sporozoites in the oocyst of $H$. stepanowi and $H$. nicorice, to the condition of $H$. canis, $H$. muris, and $\boldsymbol{H}$. gerbilli, in which a large number of spores are formed with a variable number of sporozoites. It is impossible, therefore, to accept as adequate the diagnosis given by Léger (644) of the "Homogregarinida" as producing a single octozoic spore (see p. 353, supra).

5. The Piroplasms.-The parasites of this type are minute organisms, capable of amœboid movement, but generally of a definite form, which is usually pear-shaped or rod-like. They are contained, sometimes as many as a dozen or more together, within a mammalian red blood-corpuscle. They produce no pigment, but destroy the corpuscle in which they are contained, and set free the hæmoglobin, which is then excreted by the kidneys of the host. In consequence of this, the diseases produced by these parasites, termed generally " piroplasmoses" (or " babesioses "), are of a very characteristic type, the most striking symptoms being an enormous destruction of blood-corpuscles and a red coloration of the urine by hæmoglobin (hæmoglobinuria). From this peculiarity are derived popular names, such as "redwater," etc., applied to diseases caused by piroplasms. 
The best-known member of this group of organisms is a parasite of the blood of cattle (Fig. 160), which has been most unfortunate in its nomenclature, and has appeared under a variety of generic names (Hamatococcus, Pyrosoma, A piosoma, Piroplasma), but of which the correct name is probably Babesia bovis (or bigemina). The typical form of this parasite is a pear-shaped body within the blood-corpuscle. It multiplies by binary fission, and is often double in consequence-whence the specific name bigemina.

Many other species are now known, parasites of domestic animals in various parts of the world, and of recent years a number of species have been made known from wild animals, but our knowledge of piroplasms in a natural state is not very extensive. No species is known with certainty to be parasitic upon human beings, but a disease known as "spotted fever of the Rocky Mountains" has been stated to be caused by Piroplasma hominis, and it is possible that the organisms

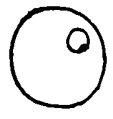

a.

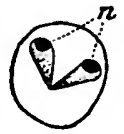

8.

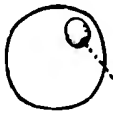

b

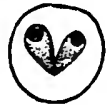

h.

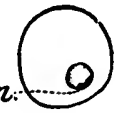

c.

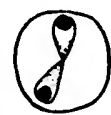

1

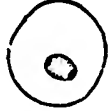

d.

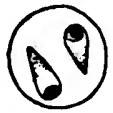

i.

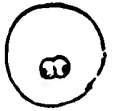

e.

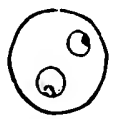

k.
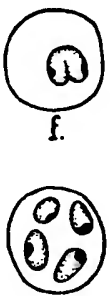

1.

Fia. 160 -.Piroplasma bigeminum (Babesia bovis) in the blood-corpuscles of the ox. $a, b$, Youngest forms ; $c-f$, binary fission; $g-j$, various forms of the twin parasites; $k, l$, doubly-infected oorpuscles. After Laveran and Nicolle.

described from the blood of yellow fever patients by Seidelin (757), and named by him Paraplasma flavigenum, may be allied to the piroplasms.

The investigations upon these organisms carried on during the last few years have led to their being divided up into a number of genera based on differences of form and structure. The following enumeration of the genera of "Piroplasmidæ" may serve at the same time to indicate the structural varieties exhibited by these parasites (compare Fr, ,nça, 736).

(1) Piroplasma, Patton (Babesia, Starcovici).-Pear-shaped forms, dividing by a process of gemmation-hence commonly found in pairs in the corpuscle. Species are known from oxen, shoep, horses ( $P$. caballi of "biliary fever"), dogs, monkeys, rats, and various wild animals.

(2) Theileria, Bettencourt, Franca and Borges.-Bacilliform or rod-shaped parasites arranged in a characteristic figure of a 
cross.* T. parva is the parasite of "East Coast fever" of cattle in Africa. Other species have been described from the fallow-deer and from Cephalolophus grimmi.

(3) Nicollia, Nuttall.-Oval or pear-shaped parasites with peculiar nuclear structure (see below), and with quadruple division, producing a figure at first like a fan, then like a four-leaved clover. One species, N. quadrigemina from the gondi, Ctenodactylus (Nicolle, 746).

(4) Nuttallia, França.-Parasites oval or pear-shaped (not rodshaped); multiplication-forms like a cross. $N$. equi, of equine piroplasmosis ; $N$. herpestidis, of a mongoose (Herpestes ichneumon).

(5) Smithia, França.-Pear-shaped forms, occupying the whole diameter of the corpuscle, not in pairs; quadruple multiplication in the figure of a cross. S. microti from Microtus arvalis.

Future research will, no doubt, determine the value of these generic distinctions, some of which seem to rest upon a somewhat slender foundation.

As is evident from the foregoing classification, the form of the parasite varies considerably in different species, and even in the same species. In many cases the body may show amœboid changes of shape, and may throw out long pseudopodial processes. The two principal types of form of the full-grown parasite are the pearshaped and the bacillary forms; but the smaller parasites may be ring-like, with the nucleus excentric, and placed near the margin of the body in some cases. The relation of these forms to one another, and their significance in the life-cycle, are not clear, but the annular forms appear to be young stages of either the pear-shaped or bacillary forms. Kinoshita claims to be able to distinguish indifferent (schizonts) from sexually-differentiated forms (sporonts) (compare Theileria, p. 382, infra).

The minute structure of the body is very simple, since the cytoplasm has as a rule no enclosures except the nucleus, which is single. In some cases, however, the cytoplasm may be vacuolated to some extent, and in the ring-like forms has a large central vacuole. The nucleus itself appears to be of a simple type of

* A confusion has arisen between two parasites very similar as regards the appearances they present in the blood, but differing in every other respect-namely. Theileria parva, the true parasite of "East Coast fever" of eattle, and Babesia (Piroplasma) mutans, also found in cattle. In both parasites alike the charac. teristic cross-forms appear in the blood. In Theileria parva, however, the crossforms aro an aggregation of four distinct gametocytes (sce p. 382, infra) whieh have invaded the same corpuscles, while in Babesia mutans the cross-forms aro pmoduced by quadruple fission of an ordinary multiplicative individual; this difference has the consequenee that, sinee the gametoeytes of $T$. parva are not capable of further development in the blood of the ox, direct inoculation of blood from an infected to a healthy ox does not produce an infection in the latter, as happens always when a healthy ox is inoculated with blood containing Babesia mutans. The diagnosis of the genus Theileria given by França would appear to apply to $B$. mutans rather than to $T$. parva. Seo especially Gonder (739). 
structure, a compact mass of chromatin or karyosome contained in a vacuole-like space-in other words, a protokaryon of the simplest type (compare BreinJ and Hindle, 730). The remarkable form Nicollia quadrigemina has an oval nucleus at the blunt end of the body, with two karyosomes, a larger one placed close to the surface, and a smaller one nearly at the centre of the pear-shaped body (Nicolle, 746).

With the unreliable method so much in vogue until quite recently, of making preparations by drying blood-smears and staining them with the Romanowsky stain, the nucleus may show various appearances about which much has been written, and which eannot be interpreted with certainty until they have been examined by better cytological methods. In such preparations the appearance is usually presented of a deeply-stained karyosome lying at the edge of, or near to, a diffuse, more or less irregular chromatinmass; or the nucleus as a whole may appear as an evenly-stained mass lying usually at one end of the body in bacillary forms, or near the rounded extremity in the pear-shaped forms. In other cases, in addition to the principal chromatinic mass, some specimens may exhibit a grain or dot, which from its staining reactions appears to be chromatin. Many efforts have been made to establish on this slender basis a theory of nuclear dimorphism for piroplasms, and to interpret the second grain as a kinetonucleus; but it bears no resemblance to any such body in its struotural and eytological relations, and is inconstant in its oceurrence, being entirely absent as a general rule.

A question much discussed is that of the occurrence of flagellated forms of piroplasms in the blood of the vertebrate host. In a few rare cases, in parasites preserved by the defective method mentioned in the last paragraph, irregular streaks of substance similar to chromatin in its staining properties have been seen extending from the karyosome even some way beyond the body of the parasite (Fantham, 735; Kinoshita, 741), and these appearances have been interpreted as flagella; but the published figures of these structures do not in the least favour any such interpretation. Kinoshita suggests that the "flagella" figured by him may represent formation of mierogametes. Of more value are the observations of Nuttall and Graham-Smith (748) on the living parasites. They observed that a pear-shaped parasite, when free in the blood-plasma, is capable of moving very rapidly, with the blunt end forwards, while the posterior pointed end exhibits active vibrations which they compare to those of a fish's tail. In some cases the hinder end was observed to be prolonged into a flagellum-like process. The authors cited explain the absence of flagellated forms in permanent blood-preparations by supposing that the flagellum becomes retracted when preserved; if so, it is a structure of a very different kind to a true flagellum, such as that of a trypanosome, and its relations to the progression of the parasite also differ.

Breinl and Hindle (730) have figured biflagellate organisms from the blood of dogs dying irom piroplasmosis. The flagellates in question were of transitory appearance, and were only found in the blood of the dog the day before its death. The authors interpret these forms as a phase of the piroplasm; but a consideration of the figures given, and of the eircumstances under which the flagellates were found, leave hardly any doubt but that the forms seen were intestinal flagellates, Bodo or Prowazekia sp., which, in the pathological condition of the host, had passed into the blood (s9e p. 258).

The development of the parasite in the vertebrate host appears to consist solely of multiplication by fission (Figs. 160, 161), usually either binary or quadruple, within the corpuscle; though the presence of the annular forms, apparently representing young 
individuals, would seem to indicate the existence of some form of schizogony, yet to be discovered, in the tissues or internal organs of the body. When the parasite or parasites have destroyed the corpuscle in which they are lodged, they are set free in the bloodplasma and penetrate other red corpuscles.

Theileria parva stands apart from other piroplasms in its developmental cycle in the vertebrate host. According to Gondcr $(738,740)$, the minute sporozoites injected by the tick collect in the spleen and lymphatic glands, where they penctrate into lymphocytes, in which they grow rapidly. The originally single nucleus divides repeatedly, and large multinucleate plasmodial masses are formed which finally divide up into as many minute mero. zoites, "agamonts," as there are nuclei; the process recalls strongly the schizogony of Homoproteus columbae (Fig. 157, $K-R$ ), and leads to the breakup of the lymphocyte. The first schizogonous generation may be repeated several times, but at last a generation of "gamonts" is produced, which are distinguished from the agamonts by characteristio differences in the nuclear structure. The gamonts multiply by a process of schizogony, the final or "gamogenous" generation, ending in the production of gametocytes, minute parasites which do not multiply further, but penetrate into the red blood-corpuscles, where they grow into adult gametocytes of two kinds-male gametocytes, which are long, slender, " bacillary" forms; and female gametocytes, which are plump, rounded, or pear-shaped forms. The gametocytes can only develop further in the tick Rhipicephalus (see below).

The forms found in the red corpuscles in the peripheral blood are either gamonts or gametoeytes, incapable of developing beyond the latter stage except in the tick; this explains a peculiarity of this parasite, namely, that inoculation of infected blood into a healthy animal does not produce an infection.

The position of the genus Achromaticus, founded by Dionisi for $A$. vesperuginis, parasitic in the blood of bats of the genus Vesperugo, is still doubtful. It oeeurs under a number of different forms, some free in the blood-plasma; others, more common, within the corpuseles. The free forms are rounded or spindle-shaped; the intracorpuscular parasites may be also of these two forms, but are more often pear-shaped. Within the corpuscles the rounded and pear-shaped forms divide into two or four by a process of schizogony. According to Gonder (737), the parasite has a double nuclous in all stages, but this is not confirmed by Yakimoff and Co. (753), who regard the parasite as a true Piroplasma. Neumann (745) states that in the bat-mite (Pteroplus vespertilionis) the parasites undergo a transformation into flagellated organisms, and considers Achromaticus allied to trypanosomes. It is not improbable that stages of Achromaticus, both in the vertebrate and invertebrate hosts, have been confused with stages of the trypanosome found in the blood of the same vertebrate hosts.

The process of division in Piroplasma canis (Fig. 161) has been studied in great detail by Nuttall and Graham-Smith (748), and by Christophers (732). The small rounded forms divide by simple binary fission of the ordinary type. In the larger forms the division takes place in a peculiar manner, more akin to gemmation than to ordinary fission. Before division the parasites become amceboid and irregular in form, and the nucleus has the form of a compact mass. The nucleus then sends out two buds which grow towards the surface of the body, and at this point two protoplasmic buds grow out into which the nuclear buds pass. The buds increase in size until they become two pear. shaped piroplasms, joined at their pointed ends by the continually-diminishing remains of the body of the original parent-individual The connecting mass dwindles to a mere point, and finally the two daughter-individuals separate. A modification of this method leads to the quadruple fission producing four buds and four daughter-individuals, as in Babesia mutans. 
The piriform parasites escape from the corpuscle when it is exhausted, and approach other corpuscles, moving with considerable rapidity. The parasite attacks the corpuscle with its blunt extremity foremost, and "rapidly indents its surface. Then violent movement of the thin end of the parasite occurs, and the side of the corpuscle becomes greatly distorted... . Gradually the parasite sinks more deeply into the corpuscle, ard finally disappears within it, when the movements of the corpuscle cease and it resumes its rounded shape " (Nuttall and Graham-Smith, 748, vi., p. 235 ; comparo the penetra. tion of blood-corpuscles by Lankesterella described above). Only piriform or long parasites enter corpuscles, never the round forms - but immcdiately after its entry into the corpuscle the parasite becomes rounded. If rounded parasites are set frce from a corpuscle by its rupture, they die off, as do also the pear-shaped forms if they do not succeed in penetrating into a corpuscle.
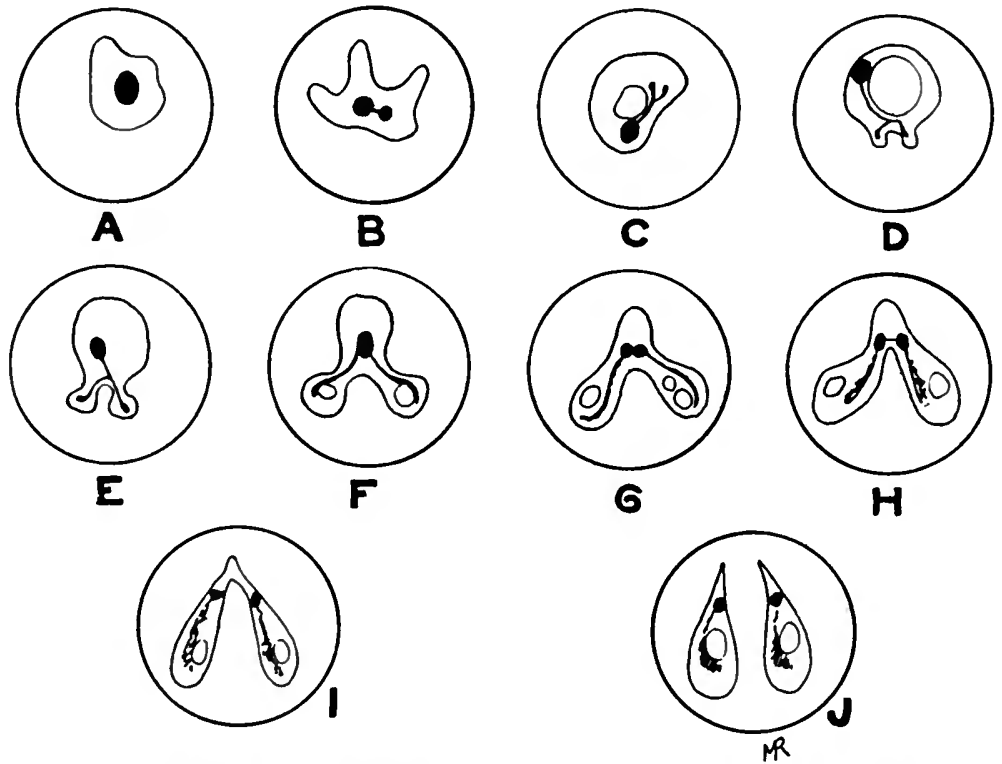

Fia. 161.-Diagrams showing the mode of division of Piroplasma canis in the blood-corpuscle. $A$, Parasite about to divide; $B$, tho nucleus budding off a smaller mass ; $C$, the nuclear bud has grown out into a forked strand; $D$, the forked ends of the strand are growing out into protoplasmic buds; $E, F, G$, growth of the buds at the expense of the main body $H, I, J$, final stages of the division of the body. After Nuttall and Graham-Smith.

A peculiar parasite, perhaps allied to the true piropasms, is Anaplasma marginale, which occurs in the blood of cattle, and causes a discase characterized by destruction of the red corpuscles and production of high fever, leading to a degeneration of the large parenchymatous organs. The parasite occurs within the red corpuscles, and is described as consisting solely of chromatinic substance, without a cytoplasmic body; hence the parasites were formerly described as " marginal points." The parasite has the form of a round or oval coccus-like body which multiplies by simple fission. It is transmitted by a tick, Rhipicephalus decoloratus. See especially Theiler (752).

The transmission of piroplasms was first discovered by the American investigators Smith and Kilborne, who in a classical 
memoir showed that the parasite of Texas cattle-fever (Babesia bous or bigemina) was transmitted from sick to healthy oxen by the agency of ticks. The method of transmission is of a peculiar type, which finds its explanation in the habits and life-history of ticks. These arachnids have typically three stages in their lifehistory-(1) the minute six-legged larva hatched from the egg, which, after growing to its full size, sheds its skin and appears as (2) the nymph, eight-legged, but sexually immature ; the nymph after another moult becomes (3) the adult tick, sexually mature and with four pairs of legs. In each of these three stages of the lifehistory the tick feeds, as a rule, but once. Consequently, if the parasites are taken up by the tick at one stage of its existence, they cannot be reinoculated into another host until a later stage of the tick. Smith and Kilborne found that the parasites taken up by the adult female ticks passed through their ova into the next generation of the ectoparasites, so that the minute larval ticks, progeny of an infected mother, were the infective agents which spread the disease amongst the cattle.

Subsequent investigations have confirmed and extended the diseovery made by Smith and Kilborne, and in every case the invertebrate host of any species of piroplasm appears to be a tick. In $P$. bovis (bigeminum) the parasites develop only if taken up by an adult female tick (Koeh), but this is not so in other cases. The parasites may be taken up by the tick at various stages, and returned to the vertebrate host at a later one; for instance, by the larva and returned by the nymph, or by the nymph and returned by the adult, or by the adult and returned by the larva of the next generation.

Although the transmission of piroplasms by ticks is well established, the developmental cycle of the parasite in the tick is known only in a fragmentary and incomplete manner. The most complete accounts are those given by Christophers (732) for Piroplasma canis, and Koch (743) for P. bovis, whose observations supplement each other, since Koch studied chiefly the earlier stages, while Christophers' investigations appear to be more complete for later phases of development. Stages in the tick are also described by Dschunkowsky and Luhs (734), but in a disconnected manner, and observations on the development in cultures have been published by Kleine (742) and by Nuttall and Graham-Smith (750). Accounts differ chiefly as to the events at the beginning of the development. So far as it is possible to make a connected story out of the published observations, the development in the tick appears to comprise six principal phases:

1. The piroplasms taken up in the blood pass into the stomach of the tick, and there the pear-shaped forms are set, free from the corpuscles, these forms alone being capable of further development. After about twelve to eighteen hours they become amœboid, sending out in all directions slender, stiff, sharply-pointed pseudopodia which are slowly retracted and emitted again. Usually the pseudopodia are given off chlefly from the thicker end of the pear-shaped body, but in some cases the form is spherical and the appearance of the parasite strikingly Heliozoon-like (Fig. 162, $A-C)$. The nucleus of the parasite divides into two parts $-a$ larger mass, 
staining more deeply; on which the pseudopodia are centred; and a smaller, paler body placed more excentrically. In the pear-shaped forms the large, dark nucleus is placed at the blunt end, the small, pale body near the pointed end. Forms similar to these have been obtained in cultures, and evidently

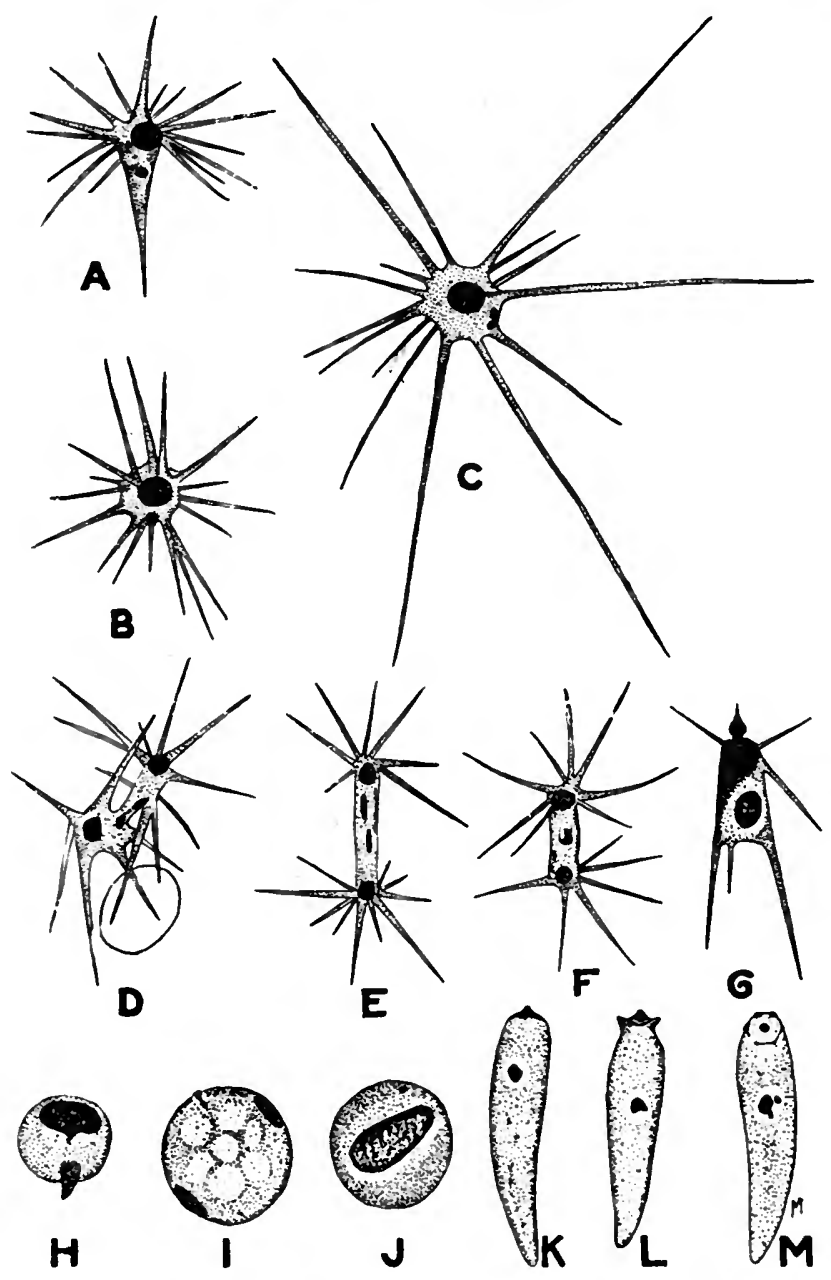

Fra. 162.-Stages in the development of Piroplasma in the tick $A-C$, Amœboid forms (gametes ?): $A$, pear-shaped, with the pseudopodia given off at the thicker end of the body ; $B, C$, spherical or Heliozoon-like, with the pseudopodia radiating out on all sides: $D-F$, fusion of the gametes (?); $G$, result of fusion (?); $I-J$, globular bodies (zygotes ?); $K-M$, motile vermicules (oökinetes?). $A-J$ after Koch ( $P$. bovis); $K-M$, after Christophers (P. canis).

represent the first stages of the development; but they appear to have been missed by Christophers, unless it is to be assumed that these forms occur in $P$. bovis, and not in $P$. canis.

The star-like forms would appear to represent the gametes; they congregato 
in clusters, and according to Koch they fuse in pairs (Fig. 162, D-G) ; cytological details of the syngamy, if such it be, are lacking (but compare Theileria, infra).

2. The stellate stage is succoeded by a spherical stage, very possibly repre. senting the zygote. This body grows in size, but its development, as described by Koch, is difficult to understand, and requires further elucidation. The final result is a globular mass with a single nucleus, found in great numbers on the third day, according to Koch (Fig. 162,J). Whether these bodies have arisen by division of the zygote, or represent simply the zygotes, is not clear, but the latter alternative seems the more probable.

3. The globular stage is succeeded by a club-like or retort-shaped stage. According to Christophers, whose account of tho life-cycle appears to begir at this stage, a split appears in the globular body, whereby a portion contain. ing the nucleus is divided off incompletely from a portion which has no nucleus. The non nucleated portion then swings round and forms the tailpiece of the complete club-shaped body, which has a single nucleus at the swollen extremity. The club-shaped bodies appear to represent the oökjnetes (Fig. 162, $K-M)$. They are motile and gregarine-like, and in some cases have an organ resembling an epimerite, regarded by Christophers as a boring organ, at the anterior extremity. Their size is about four times that of the piroplasems in the blood.

4. The club-shaped bodies pass from the gut of the tick into the ovary and oviduct, and penetrate into the ova. There they become again globular in form, and are found in the yolk of the egg, and later in the cells of the embryo developed from the egg. When, however, the parasites have been taken up by a nymph, as may happen in $P$. canis, the globular bodies are found in the tissue-cells of the body. This globular stage, termed "zygote" by Christophers, very probably corresponds to the oöcyst of the bæmamœbæ.

5. The globular body of the previous stage divides up by multiple fission into a number of "sporoblasts," which do not remain aggregated together, but scatter themselves through the tissues of the tick, larva, nymph, or adult, as the case may be.

6. The sporoblasts divide in their turn into a great number of sporozoites, small bodies with a single nucleus similar in appearance to the piroplasms in the blood. The sporozoites collect in vast numbers in the salivary glands of the tick, and pass into the vertebrate when next the tick feeds. According to Gonder, ticks infected with Theileria parva purge their salivary glands com. pletely of the parasites when they feod, and are only infective for a single meal.

The development of Theileria parva in the tick has been described by Gonder (740). Within an hour after passing into the stomach of the tick the parasites become free from the corpuscles. The immature gametocytes die off, but the adult forms proceed to gamete-formation. The free parasites are at first rounded off, but soon send out processes and become amoboid. The male gametooytes send out a single process, and creep about actively like a limax-amœba; their nucleus goes through an unequal division, after which the gametocyte becomes a gamete. The female gametes, which are inactive; go through a similar reduction-process. Pairing of two gametes and fusion of the cytoplasmic bodies takes place, but before the nuclei fuse esch nucleus goes through a second reduction-division. After copulation of the nuclei the zygote becomes an active oökinete, first retort-shaped and then gregariniform, which penetrates into the salivary glands, and there goes through a multiplicative process, very similar to that of Halteridium in the lung of the pigeon (cf. Fig. 157), producing a swarm of sporozoites which are inocu ated into the vertebrate host by the tick. Thus in Theileria also there is no flagellated stage at any part of the life-cycle-a fact which does not, however, prevent Gonder from seeing " blepharoplasts," and even crithidial forms on every possible occasion; he seems to consider nuclear reduction and blepharoplast-formation as the same thing. It is a pity that the effect of such excellent work should be marred by so much theoretical bias. Aber wie die Alten sungen...! 
From the foregoing it is seen that the development of piroplasms appears to be of a type essentially similar to that of the hæm. amœbæ and hæmogregarines. In the present fragmentary state of our knowledge, however, it would be premature to generalize concerning the development of thesc forms. The most noteworthy feature of the development is the entire absence of flagellated forms from the life-cycle. The alleged flagellate forms of $P$. canis in the dog's blood described by Breinl and Hindle have been dealt with above; it only remains to be mentioned that Miyajima obtained trypanosomes in cultures of the blood of calves suffering from piroplasmosis, an observation which led to the discovery of a trypanosome in calves not previously known to exist (see p. 283).

Doubtjul Genera of Homosporidia.-A certain number of bloodparasites have been described which at present are not sufficiently well known to make it possible to assign to them a definite systematic position. When more thoroughly investigated, many of them may turn out to belong to other groups than the Hæmosporidia; it is even possible that some of these bodies are not parasites at all, but merely some forms of cell-enclosures.

The genus Toxoplasma was founded by Nicolle and Manceaux (754) for $T$. gondii, a parasite of the gondi (Ctenodactylus gondii); other species have since been described-namely, T. cuniculi, Carini, from the rabbit, T. canis, Mello, from the dog, and T. talpee, Prowazek, from the mole. The organisms in question are parasites of the white blood-corpuscles, and occur most abundantly in the spleen or liver, causing a disease which is frequently fatal. The parasite is a crescent-shaped body, with one end thicker than the other, and containing a single nucleus; they multiply by binary or multiple fission. Nicolle and Manceaux regarded them as allied to Leishmania, but their resemblance to this genus appears to be purely superficial, since in Toxoplasma no kinetonucleus is present, and in cultures no flagellated stage is developed.

Elleipsisoma thomsoni is the name given by França (441) to a parasite of the blood of moles discovered by Thomson (524). It occurs as an amœboid intracorpuscular parasito with a single nucleus situated at the margin of the body, which contains no melanin-pigment. Multiplication takes place exelusively in the lung, and is by binary or multiple fission, according to França ; the young forms are either vermiform, with the nucleus drawn out, or oval, with a compact nucleus; they penetrate into the corpuscles and grow there. França considers this form to be allied to Toxoplasma.

The name Toddia bufonis is given by França (440) to certain bodies in the red blood-corpuscles of batrachia, first described by Todd. The earliest stage in the corpuscle is a small globule of chromatin; Franç believes that the parasite when it penetrates the corpuscle is reduced to its nucleus alone, and that it gradually forms a cytoplasmic body which becomes substituted for that of the corpuscle. As the cytoplasmic body is formed, crystals appear in it, one large crystal or as many as three smaller ones. Finally the corpuscle is seen with a slightly hypert rophied nucleus pushed to one side, and its contents consisting chiefly of substance which stains intensely blue with the Romanowsky stain, in which are the crystals and the nucleus of the parasite, now 3 to $3.5 \mu$ in diameter. No multiplication-stages have been observed.

Globidium multifidum is the name given by Neumann (488) to a parasite of the red blood-corpuscles of Gobius minutus and Arnoglossus grohmanni. It was met with in the form of a cluster of some thirty to sixty merozoite-like bodies, each $2.5 \mu$ in length by $1.5 \mu$ in breadth; similar bodies wero seen in 
blood-corpuscles singly, but their growth and multiplication were not ob. served. The parasite appears to develop in red corpuscles, which it finally fills completely, breaking up the nucleus ; no pigment is formed. Tho youngest forms show sometimes a grain near the nucleus, possibly a kinotonucleus. With the bodies described by Neumann may be compared those observed by Mathis and Iéger (473, pp. 417-419, Plate XIII., Figs. 12-16) in a fish, Clarias macrocephalus; possibly they have some connection with the trypanosome found in the same host.

Immanoplasma scyllii, Neumann (488), is a parasite of the red bloodcorpuscles of Scyllium canicula. It grows to a size of 30 by 20 , and in life is feebly amoboid. Its protoplasm stains very deep blue by the Romanowsky stain, and its nucleus appears usually as if separate from the rest of the body of the parasite, lying apparently free from it in the blood-corpuscle. Some forms of the parasite have paler protoplasm with a larger nucleus, others darker protoplasm with a smaller nucleus; the two forms are possibly male and female. No pigment is produced. The development of the parasito remains at present unknown.

Finally mention must be made of the so-called "Kurloff-Demel bodies," found in the Jeucocytes of the guinea-pig. According to Patella (755) they are true "leucocytozoa," but according to Mathis and Léger (473) they are not of parasitic nature. A memoir will be published shortly by Dr. E. H. Ross, however, in which it will be shown that the Kurloff-bodies are true parasites, representing, apparently, a stage of a motile organism, probably a spirochæte, found free in the blood. The author proposes for this parasite the name Lymphocytozoon cobayce.

Affinities of the Hamosporidia.-Two opposed and conflicting theories with regard to the systematic position of the Hæmosporidia hold the field at the present time.

1. The older and more generally accepted view is that the Hæmusporidia are closely allied to the Coccidia, sufficiently so, in fact, to be classed with them in a single order. Thus, Doflein divides the Telosporidia into two orders, the Gregarinoidea and the Coccidiomorpha, the latter comprising two subdivisions, Coccidia and Hæmosporidia ; while Mesnil placed the Hæmosporidia, together with the genus Legerella, amongst the Coccidia in an order Asporocystea, characterized by the absence of sporocysts in the oöcyst, a character that cannot be utilized in this manner now that some hæmogregarines have been shown to form sporocysts.

2. Hartmann and others (e.g., Awerinzew) maintain that the Hæmosporidia should be removed altogether from the Sporozoa, and should be classed, together with the Hæmoflagellates, as an order of the Flagellata, for which the name Binucleata is proposed, since the chief structural feature common to all members of the order is supposed to be the possession of two differentiated nuclei, a kinetonucleus and a trophonucleus, distinct from each other.

It must be clcarly understood that the theory of the Binucleata, as propounded by Hartmann and his school, is not merely one of a general relationship between Hæmosporidia and Flagellata. This wider point of view will be discussed when the affinities of the Telosporidia as a whole are considered. The question at present under discussion is whether the Hæmosporidia, more 
than the other Telosporidia, are allied specially to the Hæmoflagellates, more so than to other Flagellata; whether, in short, the Hæmosporidia should be removed from the Telosporidia altogether, and should be classified, together with the Hæmoflagellates, in one natural order, family, or other systematic category. In dealing with the Hæmoflagellates in a previous chapter, cause was shown for believing them to have two distinct lines of ancestry, the one from a Cercomonad, the other from a Bodonid type of Flagellate; in that case it is the Cercomonad section-that is to say, the trypanosomes and their allies-to which the Hæmosporidia must be considered to bo specially related on the theory now to be discussed.

Léger and Duboscq (646), recognizing distinct Bodonid and Cercomonad stems in the Iæmoflagellates, derive the Gregarines, Coccidia, and Hæmogregarines, from the Bodonid stem (trypanoplasms), the Hæmamobæ and Piroplasms from the Cercomonad (trypa nosome) type.

The close relationship of the Hæmosporidia and the Coccidia seems at first sight so obvious, from a general consideration of the life-histories of typical members of each group, that any theory to the contrary must justify itself by convincing and cogent arguments. The chief grounds upon which affinities between Hæmosporidia and Hæmoflagellates are alleged are found, when analyzed, to be of three kinds-namely : first, developmental data ; secondly, structural-that is to say, mainly cytological-peculiarities; thirdly, resemblances between certain forms which appear to be sufficiently close to link the two groups together by a series of gradual transitions. The evidences of affinity between Hromosporidia and Hæmoflagellates based on these three classes of facts must be considered separately.

1. Developmental Data.-Beginning with the first of the five types of Hæmosporidia which have been recognized above-namely, the hæmamœbæ or malarial parasites, it is very evident, as Schaudinn (658) first pointed out. that their life-cycle resembles in the closest manner that of the Coccidia. With one exception, every phase in the life-cycle of a malarial parasite has a corresponding phase in that of a coccidian, and the same terminology can be used throughout for describing the stages of the development; the one exception to this statement-the only phase that requires a special name-is the oökinete-stage of the malarial parasites, which is not known to occur in any coccidian. It is clear, however, that the points in which the lifecycles differ from one another in the two cases are such as can be correlated with the differences in the mode of parasitism - that is to say, with the fact that in Coccidia, speaking generally, there is a singlo host, and the mode of infection is contaminative, while in the hæmsmobæ there are two hosts, and the vertebrate is infected by the inoculative method. Corresponding with this difference, the zygote in the Coccidia prepares at once for leaving the body of the host and passing out into the open, and protects itself by a firm envelope; while that of the hæmamœbæ, produced in the body of an intermediate host, does not encyst itself, but is actively parasitic, continuing to absorb nourishment from the host and to grow. Further, in the hremamcbe the parasite is always in the body of one or the other of its two hosts, and consequently tough, impervious cysts and spores like those of Coccidia aro superfluous and are never formed; the oöcyst is a thin membranc through which soluble foodstufis can diffuse and sporocysts are not secreted, as is the case also in some Coccidia. The adaptive significance of these differences is so obvious that it docs not require further elucidation or discussion.

The development of the halteridium-type, as described by Aragao, can be 
derived without difficulty from that of the hæmamœbæ; and, in spite of the hiatus in what is known of the life-cycle, there is no difficulty in comparing and homologizing the phases of Hamoproteus columba with thoso of a malarial parasite, and consequently with thoso of a coccidian. The development of Leucocytozoon requires investigation, but tho littlo that is known-namely, the schizogony, sexual phases, and oökinete-formation-is entirely of the hæmamœba-type.

More striking than in any other type of the Hæmosporidia are tho coccidian features of the hremogregarincs. In such a form as $H$. stepanowi the life-cycle is seen to exhibit not merely a general similarity to that of the Coccidia, but even a special resemblance to particular forms. The mode of gamete-forma. tion is that which characterizes the family Adeleidae among Coccidia, and the many developmental similarities between $H$. stepanowi and the only known coccidian parasite of a leech, Orcheobius herpobdella, have led Reichenow to derive them from a common form. In many hæmogregarines, apparently, the parasite obtains an entry into the vertebrate host, not by the inoculative method, but by the contaminative, through the vertebrate devouring the invertebrate host. In such cases $(H$. muris, $H$. gerbilli) the characteristic coccidian sporocysts reappear in the sporogony. It is not necessary, however, to dilate further on the coccidian affinities of the hæmogregarines, since they are recognized by Hartmann and his school, and the latest revisions of tho order Binucleata do not comprise the hæmogregarines, which are left in the Telosporidia.

As regards the piroplasms, it is perhaps unsafe to generalize in the present fragmentary state of our knowledge of the life-cycle, and in particular of the sexual phases; but so far as it is known, the phases of the development appear to correspond closely with those of the typical Hæmosporidia. But at least it can be said that the development of piroplasms does not afford the slightest support to the view that they are in any way allied to Hæmoflagellates; indeed, it can be affirmed, on the contrary, that, of all the forms included in the Hæmosporidia, the piroplasms exhibit the least indications of, flagellate affinity.

From a gencral consideration of the life-cycles of the typical Hæmosporidia, such as the hæmamobre and hæmogregarines, and omitting doubtful forms, it is very clear that what may be called the nucleus of the group bears a close and unmistakable resemblance to the Coccidia. One section, comprising the hæmamœbæ, halteridia, and leucocytozoa of birds, are to be derived from an ancestor which formed gametes after the manner of Coccidium, and in these types the phenomena of "exflagellation" can be observed readily. In the other section, comprising at least the hæmogregarines, gamete-formation is of the type of that scen in Adeleida, and docs not tako place until the gameto. cytes have associated; consequently cxflagellation in vitro does not occur, but coupling of the sporonts, as in gregarines, has often been described, but wrongly interpretcd as copulation ( $c f$. Sambon and Seligmann).

In the face of such profound homologies with Coccidia, what are the arguments from the developmental cycle in favour of a contrary opinion? The case for the alleged Hæmoflagellate affinities of the Hæmosporidia rests on the famous memoir of Schaudinn (132) on the blood-parasites of the Little Owl, a work which must now be considered briefly.

The Little 0 wl (Athene noctua) harbours the full number of known avian blood-parasites-namely : (1) a proteosoma; (2) a halteridium; (3) a small form of trypanosome; (4) a large form of trypanosome; (5) a leucocytozoon : (6) a spirochrte.

According to Schaudinn, these six forms belong to the life-cycle of three species of parasites. First, the proteosoma (1) is a distinct form, not related to any of tho othors. Secondly, tho halteridium (2) and the small trypanosome (3) are alleged to bo two phases of the same parasito. Thirdly, the large trypanosome (4), the leucocytozoon (5), and the spirochætc (6), are supposed to represent different phases of ono and the samc life-cycle.

The halteridium (Hamoproteus noctuœ) was stated by Schaudinn to be the 
resting intracorpuscular diurnal phase of a trypanosome which at night developed a locomotor apparatus, became free from the blood-corpuscle, and swam freely in the plasma; in the morning the trypanosome penetrated into a corpuscle, lost its locomotor apparatus again, and became a halteridium. Male, female and indifferent forms were distinguished. The smallest indifferent forms went through a six-day development and growth, in the corpusclc as a halteridium by day, free in the plasma, as a trypanosome by night, until full grown; then they multiplied rapidly by repeated fission to produce trypanosomes of the smallest size. These young forms might grow up into indifferent forms in their turn, or might become male or female forms ; in the latter event their development was slower, and in its later stages the parasite lost the power of forming a locomotor apparatus or of leaving the corpuscle. Thus arose the adult male and female halteridia, which, in order to continue their development, required to be taken up by a gnat, Culex pipiens. In the stomach of the gnat the parasites formed gametes which copulated and produced zygotes in the well-known manner. Each oökinete, according to Schaudinn, formed a locomotor apparatus (see Fig. 30, p. 59) and either became a trypanosome which might be of female or indifferent type, or gave rise to several trypanosomes in the male sex. The trypanosomes of each type multiplied in the digestive tract of the gnat to produce a swarm. of trimorphic individuals, but no further copulation of the male and female forms occurred or could occur (Schaudinn, 132, p. 401). Ultimately, after complicated migrations, the trypanosomes were inoculated by the gnat into the owl ogain ; the male and indifferent forms passed through the proboscis, but the female forms were too bulky to do so, and, as the male forms were stated to die off in the blood, there was effective inoculation of indifferent forms only, which start on the cycle of development already described.

These remarkable statements, the origin and significance of which have leen, for the last seven years, a veritable riddle of the sphinx, have met with general scepticism except from a few devoted partisans, who have been striving continually to find corroborative evidence for Schaudinn's theories, in spite of the mass of evidence to the contrary that has been steadily accumulating. Recently Mayer (685) has affirmed that in owl's blood containing only halteridia, kept under observation in hanging drops under the microscope, trypanosomes make their appearance which could only have come there by transformation of halteridia. These experiments are supposed to prove conclusively one part, at least, of Schaudinn's statements-namely, that the halteridia are merely intracorpuscular stages of trypanosomes.

Against Schaudinn's views, on the other hand, two principal objections, out of many, may be urged:

First, that the development of Hamoproteus columbo, as made known by the Sergent brothers and by Aragao, is of a totally different type to that described by Schaudinn; it comprises no trypanosome-phases at any point of the life-cycle, and the invertebrate host is not a gnat, but a biting fly of an altogether different kind. To meet this objection, Mayer proposes to restrict the name $H$ cemoproteus to forms which develop after the manner of $H$. columbe, and to revive the name Halteridium (in italics and with an initial capital letter) for parasites that, on the Schaudinnian theory, are really trypanosomes.

Secondly, that the small trypanosomes of Athene noctua are connected by every possible transitional form with the fargest found in the same bird, and there is every reason to suppose that in this case, as in other birds or vertebrates of all classes, they are all merely forms of one polymorphic try. panosome (Minchin and Woodcock, 42).

It may be added that the whole mystery receives a cornpleto solution on a simple supposition - namely, that the trypanosome of the Little $0 \mathrm{wl}$, like other known species of trypanosomes (see p. 308), has intracorpuscular forms which have been confused with the true halterida; on such an assumption, so eminent an investigator as Schaudinn can be acquitted of having made what would appear at first sight to be a gross error of observation, and Maycr's observations are easily explainod. Mayer seems, in fact, to have figured 
such forms on his Plate XXII., Figs. 2-4-8mall intracorpuscular forms, more or less Leishmania-like, without pigment, and with, apparently, distinct tro. phonucleus and kinetonucleus.

It is not necessary to deal with Schaudinn's statements concefning Leucocytozoon further than has been done above (p. 370). It is now as certain as anything can ever be in such matters that Leucocytozoon has nothing whatever to do with either trypanosomes or spirochætes. The six forms of bloodparasites of the Little $\mathrm{Owl}$ may be regarded as belonging to five species, namely: A proteosoma (1), a halteridium (2), a trypanosome (3 and 4), a leucocytozoon (5), and a spirochrte (6). Of these five, it is probable that only the proteosoma, the trypanosome, and possibly the spirochete, can develop in, and be transmitted by, a gnat; the halteridium and the leucocytozoon require, probably, quite different intermediate hosts. If, therefore, a Culex were fed on an owl containing in its blood halteridia and leucocytozos abundontly, and trypanosomes and spirochetes in scanty numbers, the first two parasites might be expected to die out after the oökinete-stage, while the trypanosomes, and possibly the spirochres, would multiply, and thus produce very easily the impression that they were derived from the intracorpuscular parasites.

Even less cogent for the theory of Hæmoflagellate affinities than the arguments deduced from the development of Hæmosporidia are those based on the development of Hæmoflagellates. Thus the schizogony of Schizotrypanum discovered by Chagas has been compared to that of a malarial parasite, and has been adduced seriously as an additional proof of the alleged affinities between trypanosomes and hæmamœbæ. But "schizogony" - that is, reproduction by simple or multiple fission without concomitant sexual phenomens, -occurs throughout the whole range of the Protozoa, and affords no proof whatever of genetic affinities. Those who bring forward such an argument must surely have forgotten that the word "schizogony" was originally coined by Sehaudinn for the non-sexual multiplication of Trichospharium sieboldi, a marine Rhizopod (p. 181).

2. Cytological Data.-The theory of the Hæmoflagellate affinities of the Hæmosporidia has led to the most laborious and painstaking efforts to discover in the body of each and every Hrmosporidian parasite, in at least some of its phases, a second nucleus, the homologue of the kinetonucleus; and any little granule, however minute, that can be coloured like chromatin is proclaimed triumphantly to be the inevitable kinetonucleus, or any streak of similar staining properties to be a flagellum.

Consider first by itself the case of a cell in which, in addition to the nucleus, there is seen a grain which, by some particular dye, is stained in a manner similar, or nearly so, to the chromatin of the nucleus. This is not by itself a decisive proof that the grain in question is chromatin, since, as pointed out above, other grains may take up so-called "chromatin-stains"; the body in question may therefore be chromatin or some other substance. If it be chromatin, it may be a chromidial granule extruded from the nucleus; or it may be a body of the nature of a karyosome, situated close to the edge of the nucleus, or possibly, in some cases, where the nucleus has no limiting membrane, a littlo way from the main mass of the nucleus; or it may be a true kinetonucleus. If it be not chromatin, it may be a centrosome or blepharoplast ; or a grain of metachromatinic substance, such as volutin ; or, lastly, some other kind of metaplastic body. There are therefore many possible alternatives before a grain that stains like chromatin can be identified definitely as being a kinetonucleus and nothing else.

What are/the criteria by which a grain that stains like chromatin can be identified as a kinetonucleus, to the exclusion of other possible interpretations of its nature? In the first place, according to modern views (see p. 288, supra, and compare especially Rosenbusch, 505), a kinetonucleus is not a simple granule, mass or lump of chromatin, but it is a true nucleus with centriole, karyosome, and a nuclear cavity, actual or virtual, containing nuclear sap at least, if not peripheral chromatin also. Secondly, a kineto- 
nucleus when present is a permanent cell-element which, like the principal nucleus, divides when the cell divides, and is propagated by fission equally with the cell itself. Thirdly, and this is the most important criterion of all, the kinetonucleus is in relation with a flagellum during at least some phases of the development, though for a time the locomotor apparatus may be temporarily absent, its existence indicated only by the kinetonucleus during resting phases.

The smaller chromatinic body of Leishmania may be cited as an example of a body which fulfils these conditions, and which can be identified unhesitatingly as a true kinetonucleus, homologous in every way with that of a trypanosome. But with the alleged kinetonuclei of Hæmosporidia the matter stands quite otherwise. It is not. possible to discuss fully here every separate instance, but a few typical examples of snoh bodies may be dealt with briefly.

In female halteridia and leucocytozos (Fig. 158), a large grain is seen by the side of the nucleus, and often interpreted as a kinetonucleus. Until this body has been shown conclusively to be related in some phase of the lifehistory to a flagellum, it is far simpler to regard it as a karyosome which, like that of the merozoites of Adelea (Fig. 153, $F$ ), is excentric, or possibly extranuclear in position; assuming, that is, that the body in question is a true chromatinic nuclear element.

In the merozoites of Proteosoma, Hartmann (675) has discovered a flagellumlike, process at the anterior end, arising from a grain which he regards as a kinetonucleus ("blepharoplast" in the German use of the term), thus confirming certain obiter dicta of Schaudinn (132, p. 436) with regard to the merozoites and/sporozoites of the tertian parasite. It may be pointed out that the rostrum of the sporozoites of Gregarines appears to be a perfectly similar structure, which very possibly represents a rudimentary flagellum arising from a true blepharoplast of centrosomic nature. Hartmann's discovery is therefore more proof. of the affinities of proteosoma with other Telosporidia than with Hæmoflagellates.

The supposed kinetonuclei of piroplasms have been mentioned above; the entire absence (pace Hartmann) of flagellated stages throughout the lifecycle make it impossible to accept any such interpretation of the nature of these granules so highly inconstant in théir occurrence.

Lastly it should be mentioned that Schaudinn, and recently Hartmann, have maintained that the microgametes of halteridia and other Hæmosporidia have the structure of a trypanosome. Inasmuch as Schaudinn also pointed out the great structural similarity between trypanosomes and spermatozoa, this point might not count for much, even if it were true; unless the Metazoa also are to be classified amongst the Binucleata, a conclusion which, indeed, seems to follow from the nuclear theory of Hartmann and Prowazek (63). In objects of such extreme minuteuess, however, statements ascribing to them complicated details of structure must be regarded with great scepticism until thoroughly substantiated. It is a sufficient warning of the need of caution to bear in mind the controversy that has raged over the question of the minute structure of spirochætes, with regard to which Schaudinn was obliged to retire from the position he took up at first-namely, that their structure was similar to that of a trypanosome.

3. Possible Transitional Forms.-The parasite of kala-azar was originally described by Laveran under the name Piroplasma donovani,* in the belief that it was a true piroplasm; and many writers have been struck by the external similarity of the two parasites, in spite of the difficulty in finding in Piroplasma a satisfactory representative of the constant and definite kinotonucleus of Leishmania. In fact these two genera are often cited as the connecting link between Hæmoflagellates and Hæmosporidia, and are supposed to indicate the course of evolution whereby serum-parasites of the first type became

* On the other hand, the parasite of Oriental Sore was first described by Wright under the name Helcosoma tropicum, and referred to the Microsporidia. 
cell-parasites of the second (compare Léger and Duboscq, 646). However enticing such a view may seem when only the forms parasitic in the vertebrate hosts are taken into consideration, the facts of the development in the invertebrate hosts must dispel completely any notion of affinity between the two types. Nothing could be imagined more different thon the development of Leishmania, with its typical leptomonad forms (Fig. 140), and that of Piroplasma (Fig. 162), with no flagellated stages at all in its life-cycle. It becomes evident at once that any apparent resemblance between the two genera is due to convergent adaptation induced by a similar mode of parasitism, and that the two forms are in reality poles apart, with no more real affinity than porpoises and fishes, or bats and birds. It is certainly not at this point that any transition from one group to the other is to be sought.*

In the foregoing paragraphs an attempt has been made to sum up the arguments for and against the theory tnat the Hæmosporidia are to be removed from the vicinity of the Coccidia, and classified with the trypanosomes and allied forms in an order of the Flagellata. When the evidence on each side is weighed in the balance, in one scale must be placed the complete similarity of the life-cycles of typical Coccidia and Hæmosporidia, a similarity seen in every phase of the life-cycle, and extending even to minor developmental details; and in the other scale certain cell-granules of doubtful significance. It is almost inconceivable that more importance should be attached to cytological details, the genetic and classificatory value of which is at present quite uncertain, than to the homologies of the life-cycle as a whole, in estimating the affinities of the orders of Protozoa; the more so since even in the Hæmoflagellates themselves the possession of the binucleatc type of structure does not, apparently, indicate a common ancestry for all members of the group.

The conclusion reached is, then, that the Hæmosporidia as a group, excluding doubtful forms insufficiently investigated at present, are closely allied to the Coccidia. It is, indeed, probable that there are two lines of evolution in the group-the one represented by the hæmamœbæ, halteridia, and truc leucocytozoa, descended from a Coccidium-like ancestor; the other represented by the hæmogregarines, from an ancestral form similar to Adelea or Orcheobius. Léger (644) has classified the hæmogregarines in the section Adeleidea of the Coccidia, and one may regret that the distinguished French naturalist did not go one step farther and place the hæmamœbæ in his section Eimeridea (see p. 352, supra).

On the other hand, any resemblances which the Hæmosporidia exhibit to trypanosomes and allied forms are due to convergent adaptation on the part of the Flagellates themselves, and more especially to the secondary acquisition by the latter of intracellular

* Leger and Duboseq (646), who derive Leishmania and Babesia directly from Crithidia as a oommon ancestor, do not seem to have taken the development of Babesia (Piroplasma) into consideration at all; they neither refer to it in their text nor cite any of the relevant memoirs in their bibliography. 
parasitism, and consequent temporary loss of the locomotor apparatus. It may well be, therefore, that some forms now generally included amongst the Hæmosporidia (e.g., possibly the drepanidia) may prove, when better known, to be stages of Hæmoflagellates, and to have in reality nothing to do with the true Hæmosporidia.

Affinities of the Telosporidia.-From the foregoing discussion, the conclusion has been drawn that the Coccidia and the typical Hæmosporidia are closely allied, sufficiently so to be grouped together in a single order, for which the name "Coccidiomorpha" may be used. In a former chapter (p. 354) the relationship of the Gregarines and Coccidia was discussed, and it was pointed out that there was no difficulty in assuming a common ancestral origin for the two groups-a conclusion which, indeed, has never been called in question. The Telosporidia, taken as a whole, may be regarded, therefore, as a homogeneous and natural group, in which the close affinity existing between its constituent members may be regarded as indicating a common phylogenetic origin. If this conclusion be accepted, it remains to discuss the affinities of the Telosporidia as a whole to other groups of Protozoa. It is not unreasonable to suppose that a parasitic group of this kind has been evolved from free-living non-parasitic ancestors, and the question to be discussed is to which of the groups of Protozoa the ancestral form of the Telosporidia belonged. Of the three great classes of the Protozoa, the Infusoria may almost certainly be excluded from consideration in regard to this question, since, in view of the very specialized and definite features of this group, there are no grounds whatever for connecting them with the Telosporidia. There remain, therefore, only the Sarcodina and Mastigophora to be considered.

At different times two opposed theories have been put forward with regard to the affinities and ancestry of the Sporozoa. One view sees in them the descendants of typical forms of Sarcodina, such as $A m c b a$ (Awerinzew, 890); the other derives them from flagellate ancestors such as are represented at the present day by Euglena or Astasia. It is no longer possible, however, to regard the Sporozoa as a whole as a homogeneous group, ald the two so-called "subclasses," Telosporidia and Neosporidia, must be considered separately, each on its own merits. The Nedsporidia are considered at the end of the next chapter. The question here is of the Telosporidia alone. For this group opinion is practically unanimous at the present day in favour of a flagellate ancestry, a theory which must be considered critically.

One of the main arguments generally put forward for the theory of the flagellate origin of the Telosporidia is the existence of flagel- 
lated stages in the life-history. In the first place, the microgametes are very often flagellated, as has been stated frequently in the two foregoing chapters. In the second place, the youngest stages in the development-the merozoites or sporozoites-exbibit structural features which are either those of a flagellate swarmspore (Hartmann, 675 ; Schaudinn, 132), or can readily be derived from a flagellula in which the flagellar apparatus has become rudimentary, as in the sporozoites of gregarines, where the rostrum may be interpreted, with a high degree of probability, as representing a rudimentary flagellum. The existence of flagellated stages of the kinds mentioned in the development of the Telosporidia is by no means, however, a cogent argument for a flagellate ancestry for the group, since quite typical Sarcodina of all orders exhibit flagellate swarm-spores and gametes. It may be urged that in the case of these types of Sarcodina, also, the existence of flagellate stages indicates a flagellate ancestry; but such an argument merely evades the question at issue, which is not whether the Telosporidia are derived from Flagellata indirectly through Sarcodine ancestors, but whether or not they are descended directly from ancestors that were typical Flagellata. The existence of flagellated swarmspores and of gametes representing a modification of such swarmspores is not sufficient of itself to prove a flagellate ancestry for the Telosporidia.

Far more cogent arguments for the flagellate affinities of the Telosporidia may be drawn from the characters of the adult forms, especially from the gregarine-type of body, elongated and vermicular in character, and perfectly definite and constant in form, which occurs in every group of the Telosporidia at one point or another in the life-history. Such a type of body can be readily derived, as Butschli (2) pointed out, from an organism similar to Astasia or Euglena, in which the flagellar apparatus has been lost, and all special organs of nutrition, whether holozoic or holophytic, have disappeared in.relation with the parasitic mode of life. On the other hand, the gregarine-type of body cannot be derived from the adult forms of the Sarcodina, which are typically amoboid, and without any definite body-form other than that imposed by the physical nature of their body-substance.

We may therefore consider the ancestral form of the Telosporidia to have been a flagellate organism with an elongated form of body, with a definite form, owing to the presence of a cuticle of a certain degree of thickness and toughness, and with a flagellar apparatus at the anterior end. Suich a form would have been not unlike the leptomonads now found commonly as parasites of insect-guts; but there is no reason to suppose the ancestral form to have had a kinetonucleus and the third type of flagellar insertion. Such a 
form probably used its flagellum for the purpose of attaching itself to the epithelium of the digestive tract, as leptomonads do now (compare Figs. 136, 137); and from this primitive type of attachment the epimerite of the gregarines may have been derived by secretion of chitin round the attaching flagellum, just as the primitive tuft of fixing cilia, the "scopula," of the primitive Vorticellids appears to become converted into the chitinous stalks of such forms as Epistylis (p. 441).

The conclusion drawn from these various considerations is, therefore, that the Telosporidia may be regarded as a group descended from flagellate ancestors modified in adaptation to a parasitic mode of life; not, however, specially from flagellates of the "binucleate" type of structure.

Bibliography.-For references see p. 496. 


\section{CHAPTER XVI}

\section{THE SPOROZOA : III. THE NEOSPORIDIA}

A TYPICAL member of the subclass Neosporidia is a parasite of which the life-cycle is initiated by the liberation from the spore of one or more amœbulæ within the body of the host, in the digestive tract in all known cases. For this initial amcbula-phase Stempell's term, planont (i.e., "wanderer"), may be employed conveniently, since in no case does it remain in the lumen of the digestive tract, but penetrates into the wall of the gut, and in most cases migrates thence into some organ or tissue of the host, where it lives and multiplies activery, being usually at this stage an intracellular parasite, in some cases, however, occurring free in the blood or lymph.

The planont-phase is succeeded typically by a plasmodial phase, which arises in some cases by simple growth of the amobula (probably then a zygote), accompanied by multiplication of its nuclei; in other cases by association together and cytoplasmic fusion of at least two distinct amœbulæ, of which the nuclei remain separate. The plasmodial stage is very characteristic of this subclass ; it represents the principal or "adult" trophic phase of the parasite, and is also the spore-forming phase; and, as the name Neosporidia implies, the production of spores begins, as a rule, when the plasmodium is still young, and continues during its growth.

In some cases, however, no plasmodium is formed, but the planont-phase is succeeded by uninucleate " meronts " or schizonts, which multiply by fission and give rise ultimately to sporonts in which spore-farmation sets a limit to the growth. In such forms the general course of the life-cycle is not essentially different in any way from that of a member of the Telosporidia, such as Coccidium. The tendency, therefore, of many Neosporidia to form spores during the trophic phase cannot be used to frame a rigorouslyexact definition of the group. A more distinctive characteristic of the subclass is the complete absence of flagellated phases in any part of the life-cycle, and more especially the fact that 
the sporozoites are always, apparently, amœbulæ, and never gregarinulæ.*

The Neosporidia are divisible into two sections, known respectively as the Cnidosporidia and the Haplosporidia. The Cnidosporidia are distinguished by the possession in the spore of peculiar structures termed polar capsules, which are lacking in the Haplosporidia.

A polar capsule (Fig. 163) is a hollow, pear-shaped body, with a tough envelope, probably chitinoid in nature. It is situated at one pole of the spore, with its pointed end immediately below the surface, in continuity with a minite pore in the sporocyst. Coiled up within the capsule is a delicate filament, often of great length, probably of the same nature as the capsule, and continuous with it. Under suitable stimulation the polar filament is shot out through the pore in the sporocyst. In their structure the polar capsules resemble the nemetocysts of the Colentera. Each polar capsule is formed within a capulogenous cell.

The Cnidosporidia comprise four ordersthe Myxosporidia, Actinomyxidia, Microsporidia, and Sarcosporidia. The Haplosporidia constitute an order apart.

Order I.: Myxosporidia. - This order is characterized chiefly by the following points : The principal trophic phase is a multinucleate plasmodium of relatively large size, resembling an amceba in its appearance and movements. The spores are also relatively large, and exhibit typically a binary symmetry, having a sporocyst composed of two valves and usually two polar capsules, sometimes increased in number to four, rarely reduced to one.

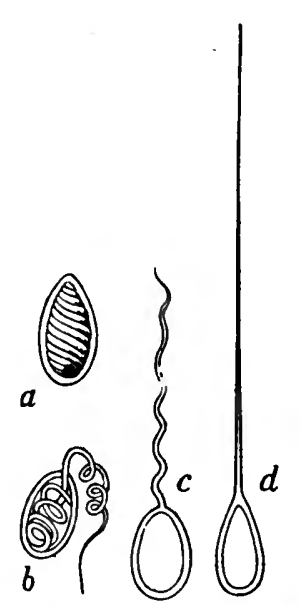

Fro. 163. - Polar capsules of the spores of Myxosporidia. $a$, Polar capsule with the filament coiled within it ; $b$, with the filament partly extruded; $c, d$, with the filament completely extruded. After Balbiani.

The My xosporidia comprise a great number of genera and species, parasitic for the most part in cold-blooded vertebrates, especially fishes, in which they are found very commonly. They are not as yet known as parasites of birds or mammals, but a few species are known from invertebrate hosts.

Myxosporidia are typically tissue-parasites, occurring in various tissues of the body, by preference muscular or connective, but also

* A possible exception to this statement is furnished by the family Coelosporidida of the Haplosporidia (p. 424). But the position of all the forms in this order is more or less questionable, and their attachment to the typical Neosporidia is
still probstionary. 
other classes of tissue. A few species are known to attack the nervous system-for instance, Lentospora (Myxobolus) cerebralis, cause of "Drehkrankheit" in Salmonida (Plehn), and Myxobolus neurobius of trout (Schuberg and Schröder). In the tissue attacked the parasite may be concentrated at one spot, so as to form a distinct cyst visible to the naked eye ; or parasite and tissue may be mixed up together in a state of "diffuse infiltration" such that microscopic examination is required to detect the parasite, and as its body becomes used up, to form spores, the tissue becomes infiltrated with vast numbers of spores lying singly or in groups between the cells.

In many species of Myxosporidia, on the other hand, the spore-

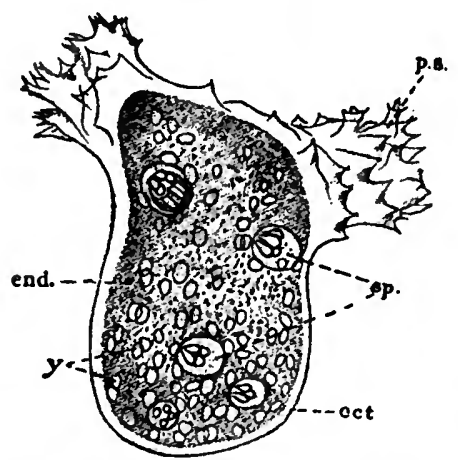

Fro. 164. - Chloromyxum leydigi, parasite of the gall-bladder of the dogfish, skate, etc. ; trophozoite (plasmodium) in an active state. ect., Eotoplasm ; end., endoplasm; $y$., yellow globules in tho endoplasm ; sp., spores, each with four polar capsules. After Thélohan, from Minohin, magnified 525. forming plasmodial phase is found in cavities of the body-not in any known instance in the lumen of the digestive tract, but frequently in the gall-bladderor urinary bladder of the host. In such cases the parasite may lie quite freely in the cavity it inhabits, or may be attached by its pseudopodia to the lining epithelium; in the latter caso the attachment is purely mechanical, and does not involve injury to the epithelial cells.

As might be expected, the Myxosporidia parasitic in tissues are often very deadly to their hosts, and are sometimes the cause of severe epidemics among fishes. Those species, on the other hand, which inhabit cavities with natural means of exit from the body appear to be as harmless to their hosts as are the majority of parasitic Protozoa in nature.

The adult trophic phase is usually a large amoeba-like organism with a distinct ectoplasm and endoplasm. In some species the ectoplasm, which appears to be purely protective in function, exhibits vertical striations, or is covered by a fur of short, bristle-like processes, the nature and significance of which are uncertain-as, for example, Myxidium lieberkiihni, the common parasite of the urinary bladder of the pike (Esox lucius). The form of the body changes constantly, with extrusion of pseudopodia, which are used for locomotion to a limited extent, more often for fixation, but never for food-capture. They may, however, by increasing the body-surface, increase also the power of absorption of food- 
stuffs by diffusion, the method by which the organism, like other sporozoan parasites, obtains the required nourishment. The pseudopodia vary in form in different species, from coarsely lobose and blunt to fine filaments ending in sharp points. In some species the formation of pseudopodia is localized at one pole of the body, termed " anterior," and in such cases a peculiar propulsive pseudopodium ("Stemm-pseudopodium") may be developed at the posterior pole like a tail, which by its elongation pushes the body forward.*

The endoplasm is distinguished from the ectoplasm by its coarsely granular appearance. In addition to numerous nuclei and stages of spore-formation, the endoplasm may contain various metaplastic products, such as crystals, pigment-grains, fat-globules, etc. ; but never food-vacuoles or solid ingested food-particles.

The plasmodial trophozoite forms spores in its endoplasm, as a rule, during the whole period of growth, but may also multiply by plasmotomy. In Myxidium lieberkühni, for example, plasmotomy proceeds actively during the summer months, and leads to the wall of the pike's bladder being carpeted with the slimy, orange-coloured plasmodia, the presence of which can generally be detected at a glance; sporeformation, on the other hand,

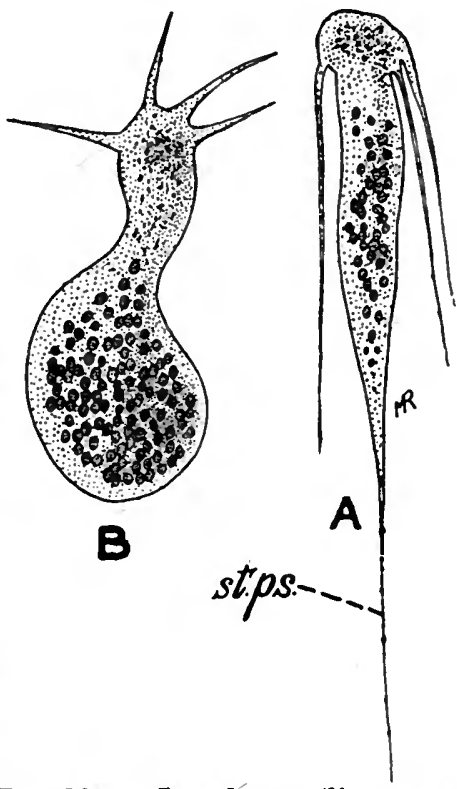

Fia. 165. - Leptotheca agilis: young plasmodial trophozoites in which the spore-formation has not begun. $A$, Individual moving forward by meins of the "Stemm-psendopodium" (st. ps.) ; $B$, individual in whioh only the anterior psendopodia aro developed. After Doflein. takes place almost exclusively during the colder months of the year.

Spore-formation in the Myxosporidia is a somewhat complicated process, and is accompanied by sexual phenomena, which axe commonly stated to be autogamous, but which are probably nothing of the sort. There is a slight difference between the mode of spore-formation in the Disporea, in which each trophozoite produces but two spores, and the Polysporea, which produce many.

* Auerbaoh (758, p. 11) seems to have mistaken altogether the significance of Doflein's "Stemm-pseudopodium," and applies the term to the anterior pseudopodia, which appear to be rather tactile in function in such cases. 


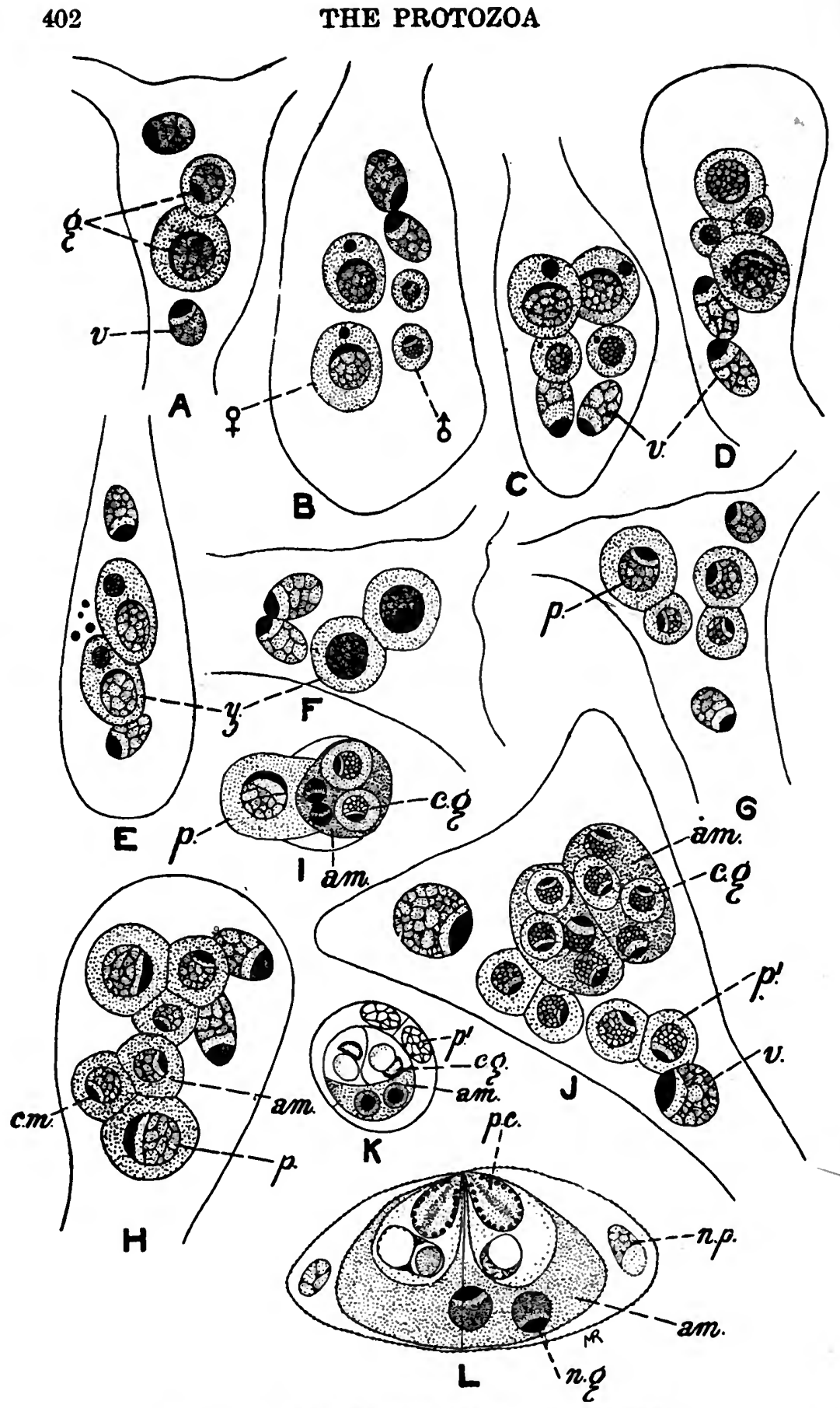

FIG 166.-For description see foot of opposite page. 
An example of the Disporea is Ceratomyxa drepanopsetto, of which the spore-formation is described by Awerinzew (759). The trophozoite has at first only two nuclei, which are considered by Awerinzew to be derived, " beyond all doubt," from division of a single nucleus; it seems far more probsble, on the contrary, that the binucleate trophozoite is to be derived from the association and fusion of two distinct planonts. In the binucleate trophozoite each nucleus divides by karyokinesis into two nuclei, a larger and a smaller (Fig. 166, $A$ ). The two smaller nuclei are vegetative, the two larger generative, in function. Round each of the two generative nuclei the protoplasm becomes concentrated so as to form two cells which lie embedded in the endoplasm of the trophozoite. These two cells are usually of distinctly different sizes, and represent a microgametocyte and a macrogametocyte respectively. Each gametocyte next divides into two gametes (Fig. $166, B, C, D$ ), and in each gamete a certain amount of chromatin is extruded from the nucleus, first into the cytoplasm of the gamete, and then into the endoplasm of the mother-trophozoite. Then each microgameto copulates with one of the two macrogametes (Fig. 166, $E, F^{\prime}$ ). The two zygotes thus formed represent the sporoblasts, each of which forms a spore independently of the other.

Each sporoblast divides into two cells, a larger and a smaller (Fig. 166, $G$ ), and the smaller divides again into two (Fig. 166, $H$ ); the result is an aggregate of three cells: a larger, which gives rise ultimately to the two parietal cells which form the valves of the sporocyst, and may be termed the " sporocystmother-cell"; and two smaller cells, one of which is the mother-cell of the two capsulogenous cells, the other the future sporozoite or amcbula. The sporocyst-mother-cell may become temporarily separate from the capsule. mother-cell and the amœbula.

The three cells of the previous stage build up the spore in the following manner: The sporozoite grows in size, and its nucleus divides into two. The capsule-mother-cell divides into two capsulogenous cells, each of which forms a polar capsule in its interior. The amœbula and the two capsulogenous cells are placed close together and arranged in a definite manncr (Fig. 166, $I$ ). The sporocyst-mother-cell divides into two parietal cells (Fig. 166, $J$ ), which place themselves on either side of the cell-complex composed of the associated amcebula and capsulogenous cells (Fig. 166, K), and each secretes one valve of the sporocyst enveloping the whole complex.

Thus the trophozoite consists finally of an amceboid body containing two

Fra. 166.-Spore-formation in Ceratomyxa drepanopsettce. A, Trophozoite sketched in outline, showing in the plasmodium two generative nuclei (g.), each surrounded by a cell-body, and two vegetative nuclei $(v$.$) which lie in the pro.$ toplasm of the body (left blank); note that the generative cells are of different sizes; $B, C$, the two generative cells have divided each into two, so that there are now two macrogametes $(q)$ and two microgametes $(\sigma)$; chromatin is being given off from the nuclei of the gametes into the cytoplasm; $D$, cach microgamete is apposed to a macrogamete; the chromatin-bodies given off by the gamete-nuclei are now extruded into the plasmodial body; $E, F$. stages in the syngamy between the gametes; in $E$ the cell-bodies are fuscd, in $F$ the zygotes $(z$.$) are complete; G$, each zygote (sporoblast) has divided into a larger $(p)$ and a smaller cell ; $H$, the smaller cell in each sporoblast has divided into two, the capsule-mother-cell (c.m.) and the sporozoite $(a m)$; the larger cell $(p$.$) is the mother-cell of the parietal cells; I$, a single sporoblast showing the parietsl mother-cell $(p$.$) still undivided, the sporozoitc (\mathrm{am})$ with its nucleus divided into two, and two capsulogenous cells $(c . g$.) derived from division of c.m. in $H ; J$, a trophozoite showing the two parietal mothercells of $H$ each divided into two parietal cells $(p$. ) but still separate from the cell-complex consisting of tho binucleate sporozoite $(\mathrm{am})$ and the two capsulogenous cells (c.g.); $K$, union of all the cell-elements of the spore; the two parietal cells $\left(p .^{\prime}\right)$ surround the cell-complex (drawn on a smaller scale than the last figure); $L$, spore ncarly fully formed: n.p., nucleus of a parietal cell; am, sporozoite with two nuclci (n.g.); p.c., polar capsules. After Awerinzew (759). 

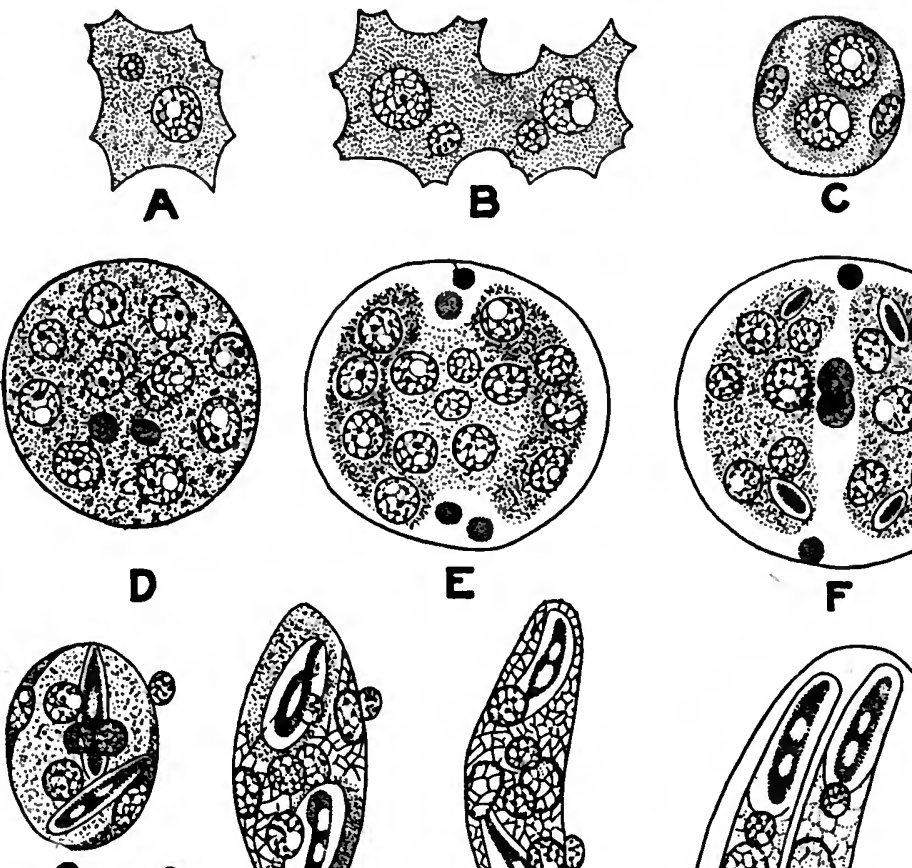

6

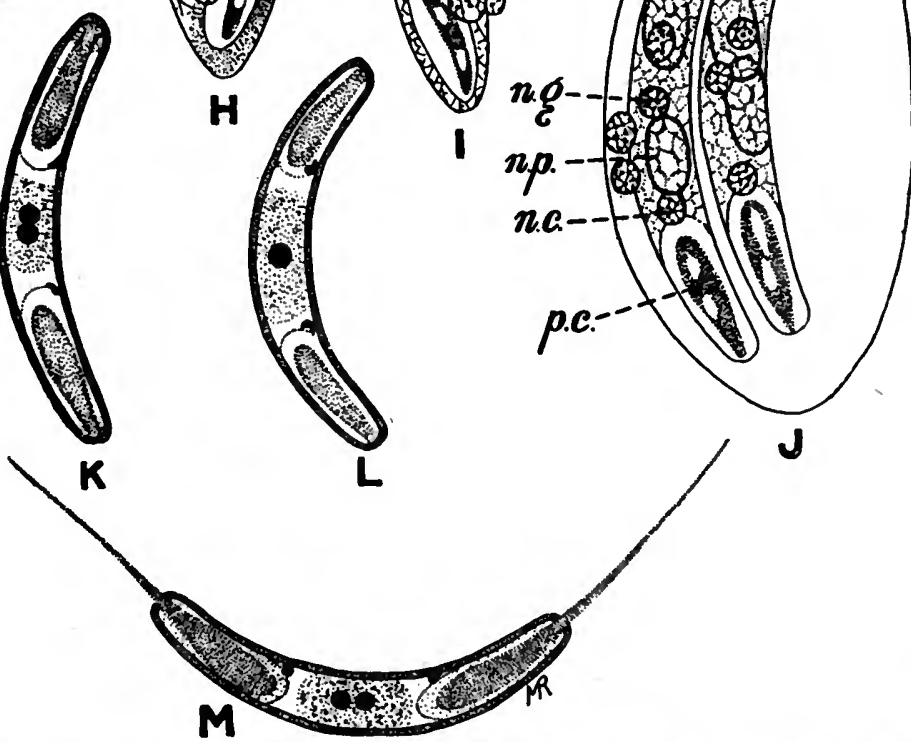

FIG. 167.-Spore-formation in Spharomyxa sabrazesi. A, Propagative cell with two nuclei of different sizes; $B$, two such propagative cells undergoing fusion to form $C$, a pansporoblast with four nuclei, two snialler, peripheral, the nuclei of the two envelope-cells, and two larger, central, the nuolei of the spore-forming elements; $D$, pansporoblast with fourteen nuolei, eight peripheral, six central ; two of the latter, smaller than the others, represent redno- 
vegetative nuvlei and two spores. Each spore (Fig. 166, L) consists of (1) a sporocyst compused of two valves, each secroted by a parietal cell ; (2) two polar capsules, each secreted by a capsulogenous cell ; (3) a binucleate amœbula, the sporozoite or sporoplasm. When the spore is fully formed, the cells which form the valves and polar capsules are used up and degenerate, remnants only of their nuclei being visible. The spores represent the propagative phase, and the trophozoite with its vegetative nuclei degenerates.

The Myxosporidia Polysporea differ from the Disporea in that the plasmo. dium contains a great number of nuclei, some of which are purely vegetative in function, others gencrative; and the plasmodium produces in its interior numerous spores, which are always formed in couples. This peculiarity is due to the fa $t$ that the two sporoblasts arise from a cell-complex which is termed a "pansporoblast," producing two sporoblasts and two spores.

Spore-formation in Polysporea has been studied in Spharamyxa sabrazesi (not labrazesi), from the gall-bladder of Hippocampus, by Schröder (767 and 768), and in Myxobolus pfeifferi, a deadly tissue-parasite of the barbel, by Keysselitz. Their results are almost identical in each case, except for minor details, and are in the main as follows (Figs. 167, 168).

The generative nuclei of the plasmodium become the centres of a condensed patch of protoplasm (Fig. 168, A), forming a " propagative cell " (Keysselitz). These colls may multiply with mitosis of the nuclei for a while, but finally proceed to sporo-formation. The nucleus of a propagative cell divides into two, a larger and a smaller, and division of the cell follows sooner or later (Fig. 167, $A$; Fig. $168, B, C$ ). Two such couples of cells become associated, and the two smaller cells form an envelope surrounding the two larger cells, which by their association form the pansporoblast (Fig. 167, $B, C ; \mathrm{Fig}$. 168, D). Hence the pansporoblast is from the first a complex of two distinct colls, and not, as was formerly supposed, a single cell. The two cells of the pansporoblast may be termed gamonts, since they give rise ultimately to gametes, but not to gametes alone, like tho gametocytes of Ceratomyxa. The cytoplasm of the two gamonts may fuse into orfe mass, but the nuclei remain separate and undergo repeated divisions, until the pansporoblast within its envelope contains twelve nuclei, and may consist of as many separate cells (Fig. 167, $D$; Fig. 168, $E$ ). The nuclei or cells then become arranged in a definite manner; eight of them take up a peripheral position, four of them place themselves more centrally (Fig. 167, E). The four central cells aro the gametcs; their nuclei undergo reducing divisions, and the four cells then pair off into two couples; in each couple the cytoplasmic bodies of the two cells fuse together, but their nuclei remain distinct. It is probable that in each couplc one nucleus is descended from that of one of the two original propagative cells, the other nucleus from that of the other.

At this stage the pansporoblast divides into two masses, the sporoblasts

Fra. 167 continued:

tion-nuclei beginning to degenerate; the envelopc-nuclei are not represented;

$E$, the pansporoblast beginning to divide into two sporoblasts; within the envelope aro seen also some small bodies of doubtful nature; $F$, the two sporoblasts completely separated, between them two residual nuclei; each sporoblast has six nuclei, four peripheral, two central ; at tho two extremities of the sporoblast the polar eapsules are beginning to bo formed; $G$, one of the two sporoblasts at a later stage, showing two parietal cells, situated superfioially; two nuclei of the ospsulogenous eclls, each near a polar capsule; the two germinal nuclei close together at the centre; and a residual nucleus attached to the surface; $H, I$, further stages in the development of the sporoblast; $J$, pansporoblast with two spores almost fully formed, and the two residual nuclei ; each spore has a polar capsule (p.c.) at each end, and near it a nueleus of the eapsulogenous cell (n.c.); two largo parietal nuelei (n.p.), in process of degeneration; and two germinal nuolei (n.g.); $K, L, \mathcal{H}$, fullyformed spores; in $K$ and $M$ tho two germinal nuclei aro still separate, in $L$ they have undergone fusion; in $M$ tho two polar filaments are extruded. After Schröder (767 and 768). 
each containing six nuclei-namely, four peripheral and two central (Fig. 167, $F$; Fig. 168, $F$ ). The reduction-nuclei are left out in the cold, and die off. From each sporoblast a spore is formed in the following way (Fig. 167, $G \longrightarrow J ;$ Fig. 168, $G$ ) : Of the four peripheral cells, two aro parietal cells which give rise to the two valves of the sporocyst, the other two as capsulogenous cells prodnce the two polar capsules; the two central nuclei with the mass of protoplasm in which they lie become the amcebula or sporozoite of the spore. The sporozoite is thus at first binucleate, but when the spore is fully formed its two nuclei fuse into one. There can be no doubt that this fusion represents a karyogamy, and that the single nucleus is a synkaryon. The cells which form the valves of the sporocyst and polar capsules degenerate when the spore is completely formed.

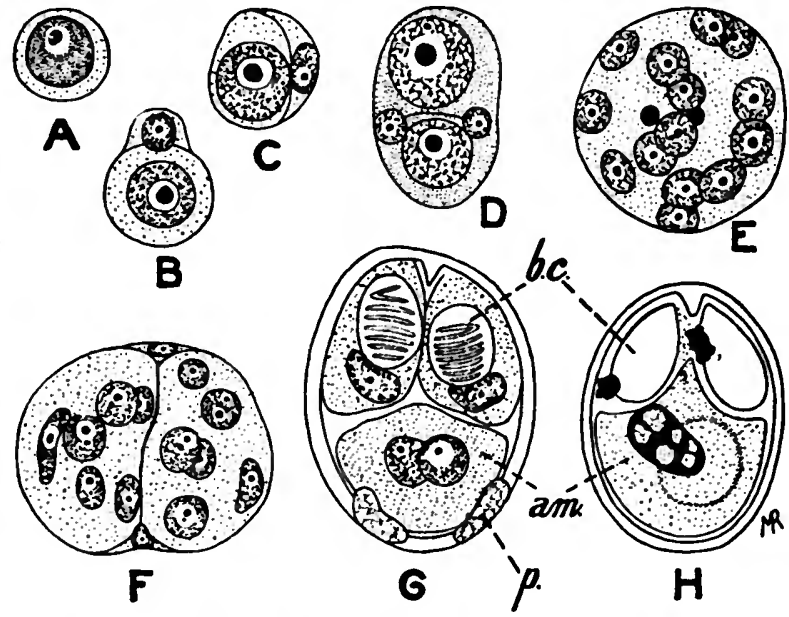

Fig. 168.-Spore-formation in Myxobolus pfeifferi. A, Propagative cell from the plasmodium ; $B$, division of the propagative cell into two uncqual halves; $C$, the smaller cell of the preceding stage applies itself as a fattened envelopecell to the larger ; $D$, a mass formed by union of two couples of cells similar to those of the preceding stage-the pansporoblast; $E$, pansporoblast with fourteen nuclei, two representing the envelope-cells, and twelvo arisen by division of the two larger cells of the preceding atage; $F$, the pansporoblast divided into tho two envelope-cells and two masses, sporoblasts, each containing six nuclei, which aro arranging themselves so that two nuclei are more central, four more peripheral in position ; $G$, spore in process of formation; the sporoeyst is formed by two parietal cells $(p$.$) ; at the npper pole are$ the two polar capsules (p.c.) in their capsulogenous cells; and the lower part of the spore is occupied by the binucleste smcbula $\left(a m_{.}\right) ; H$, ripe spore; in the amobula the two nuclei have fused into one; lettering as in the last. After Keysselitz.

Mercier $\left(7655^{\circ}\right)$, on the other hand, who has also studied tho spore.formation of Myxobolus pfeifferi, differs from Keysselitz in his account, more especially with regard to the sexual processes. Ho affirms that the plasmodium contains four zones: (1) A peripheral zone, without nuclei or spores; (2) a zone of nuclei, all similar amongst themselves, which multiply by mitosis ; (3) a zone containing nuclei of different sizes and early stages of spore-formation; and (4) a central rogion containing ripe spores. In the third zone difforentiation of microgametes and macrogametes takes place. The gametes copulate, and the zygote is a pansporoblast. Its nucleus (synkaryon) divides to form fourtcen nuoldi, two of which are rejected, while from the remaining twelve arise two sporoblasts, each with six nuclei, and finally two spores. 
According to Awerinzew (760), in Myxidium sp. a propagative cell may give rise sometimes to a single spore, as in Ceratomyxa, in other cases to three spores; this must doubtless be interpreted to mean that a propagative cell may become a sporoblast without entering into association with another propagative cell, and that in other cases three propagativo cells may form an association; these variations present an analogy with the solitary encystment or triple associations of gregarines (p. 331).

The process of syngamy in these parasites has been described as being a process of autogamy, but whether it is so or not depends entirely upon the manner in which the plasmodium arises; if a single, uninucleate amœbula becomes a plasmodium by growth accompanier by nuclear multiplication, then the sexual process is a case of autogamy ; but if, as is more likely, two or more distinct amœbulæ become associated to form a plasmodium, then the two nuclei of the gametocytes in Disporea, of the "pansporoblast" of Polysporea, may well be of distinct parentage, and in that case the sexual process is not autogamous.

Comparing the different modes of spore-formation, it is seen that in all cases alike the spore arises from a sporoblast which divides into several cells : two to form the sporocyst, which consists of two distinct valves meeting in a suture, and thus defining a sutural plane in the spore; two (or four in Chloromyxida) to form the polar capsules; and a fifth to furnish the binucleate sporozoite. The spores of Myxosporidia have, as has been seen, a complex structure, and are highly characteristic bodies-the original psorosperms of Johannes Müller. In minor details of form and structure they vary enormously in different species. The greatest diameter of the spore may lie in the sutural plane, as in Polysporea generally, or in a plane at right angles to it, as in Disporea (Fig. 169). The sporocyst may be prolonged into tails and processes of various kinds ; the polar capsules may be close together at one pole of the spore, or at opposite poles. In all cases, so far as is known, the spores germinate in the intestine of the new host, which becomes infected casually by taking in the spores with its food. Other methods of infection have been imagined, but have never been demonstrated experimentally.

The most complete account of the germination of the spore and of the early development of the parasite in its now host is that given by Auerbach (758) for Myxidium bergense, parasite of the gall-bladder of Gadus virens. The spores from the gall-bladder pass through the rectum to the exterior. To develop further, they must be taken up by the new host, in the stomach of which, however, the spores undergo very little change; the sporozoite rounds itself off, and in some cases its nuclei copulate, in others they remain apart. From the stomach the spores pass into the duodenum, and as soon as they are acted upon by the bile the polar filaments are extruded, the valves of the sporocyst split apart, and the amcboid sporozoite creeps out. When the amcebula becomes free, its two nuclei fuse into one if they have not done so already.

The free amcbula wanders actively up the bile-duct, and penetrates into a cell of the lining epithelium. Within the cell the nucleus of the parasite undergoes a change, becoming looser in texture. The amobula leaves the cell and becomes free in the bile again, where it multiplies by fission, producing in this way very numerous amoebulx, which may occur singly or in clumps. 
The amœbulæ next associate in couples. In each couple the cytoplasm of the associates undergoes partial fusion. In one associate the loosely-textured nucleus remains unaltered; in the other the nucleus divides by mitosis, and one of the two daughter-nuclei, with a small quantity of the cytoplasm, is cast off. The bodies of the two associates now fuse completely into a single mass containing two nuclei, a large and a small. The larger nucleus represents the unaltered nucleus of one of the two original associates; the smaller nucleus is the reduced nucleus of the other associate.

The binucleate stage formed by the association of two amœbulæ (planonts) is the foundation of the plasmodium. The two nuclei remain separate and multiply independently, maintaining their difference in size. Hence the young plasmodia consist of nuclei of two sizes, small and large; and this difference in size, which has often been noted in the plasmodia of other species, is due, according to Auerbach, to the fact that the smallor nuclei are derived from a nucleus which has undergone reduction, while the larger nuclei are derived from one which has not done şo.

So far as it is possible to generalize from the few recorded observations and experiments, the germination of the Myxosporidian spore probably takes place always in the digestive tract of the new host. The first act in the process is the rounding off of the amooboid sporozoite; next the polar capsules are discharged, the function of these organs being, apparently, that of fixing the spore to the wall of the gut. Then the two valves of the sporocyst separate, and the amobula creeps out; its two nuclei copulate to form the synkaryon, if they have not done so already. In this way the planontphase arises from the sporozoite, and is set free in the digestive tract, whence it migrates to the organ or tissue of which it is a specific parasite. When its destination is an organ which, like the gall-bladder, is in open communication with the gut, the migration may be comparatively simple and direct; but in the majority of cases the journey to be accomplished is a complicated one. It is probably safe to assume that in most cases the planont passes through the wall of the gut into the channels of the blood or lymph, and by this route arrives ultimately at its destination. In the organ or tissue which the parasite attacks, the planont probably passes through a period of cell-parasitism and proliferation by binary fission; ultimately the plasmodial phase is initiated by association of two planonts, of which the bodies fuse, but the nuclei remain separate. Growth of the body with independent multiplication of its nuclei to four (Disporea) or many (Polysporea) produces the spore-forming plasmodium characteristic of the order.

The Myxosporidia are classified as follows :

SUborder I.: Dispores.-Only two spores formed in the plasmodium. The greatest diameter of the spore is at right angles to the sutural plane (Fig. 169).

One family, Ceratomyxida, with two genera: Ceratomyxa, with several species parasitic in the gall-bladders of fishes; Leptotheca (Fig. 165), including several species from gall-bladders of fishes but $L$. ranarum occurs in the kidneys of frogs (Rana spp.). 
SUbORder II. : Polysporea.-Numerous spores formed in the plasmodium. The greatest diameter of the spore lies in the sutural plane.

Three families : (1) Myxobolida, with two polar capsules (sometimes reduced to one), and with a peculiar vacuole, which stsins with iodine, in the amœbula. Typically tissue-parasites of fishes; principal genera Myrobolus, with round or oval spores, and Henneguya, with tail-like processes to the spore.

(2) Myxidiida ; spores with two polar capsules, no iodinophilous vacuole ; typically "free" (i.e., cœlozoic) parasites. Principal genus Myxidium, with the polar capsules at opposite poles of the spore; M. lieberkühni, the common parasite of the pike.

(3) Chloromyxida, with four polar capsules; the best known species is Chloromyxum leydigi (Fig. 164), from the gall-bladder of various Elasmobranch fishes.

To the typical Myxosporidia enumerated in the above summary must be added two genera recently described:

Coccomyxa morovi (Léger and Hesse, 765), from the gall-bladder of the sardine : the plasmodium has only two nuclei, a large and a small, and forms a single spore with two valves and two parietal cells, one polar capsule, and an amcbula with two nuclei. This form seems to be transitional between Mysosporidia and Microsporidia, and should perhaps form the type of a third suborder, the Monosporea.

Paramyxa paradoxa (Chatton, 761), a parasite of the intestine of a pelagic Amelid larva ; the multiplicative amcbula stage is succeoded by a plasmodial

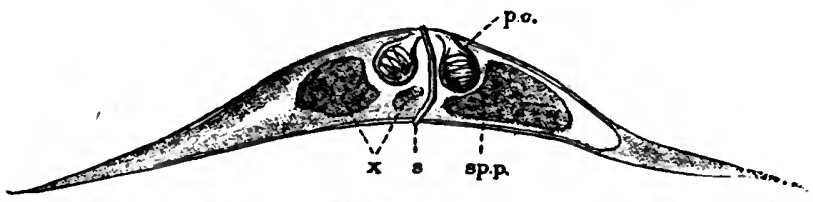

Fic. 169.-Spore of Ceratomyxa spharulosa. p.c., Polar capsules ; sp.p., sporoplasm ; s., suture of the sporocyst ; $x$, "irregular, pale masses of undetermined origin." After Thélohan, magnified $\mathbf{7 5 0}$.

stage with two nnclei of unequal size, which multiply by fission. Finally the plasmodium produces four spores, each with a single parietal cell and no polar capsule. Chatton is of opinion that this species is the type of a new order of Cnidosporidia, to be named Paramyxidia.

Order II.: Actinomyxidia.-The members of this group are only known, up to the present, as parasites of oligochæte worms, fresh-water or marine. They were discovered originally by Stolc, who found, in the intestinal epithelium of different species of Tubificido, the spores of three genera of these parasites, named by him Synactinomyxon, Hexactinomyxon, and Triactinomyxon, respectively. The nature and affinities of these organisms remained for some time doubtful ; but the investigations of Caullery and Mesnil (769) on Spharactinomyxon stolci, a species found by them in the coelome of several species of Tubificida, established indisputably the position of these parasites amongst the Cnidosporidia. Their distinctive features are-first, that the plasmodial stage is represented only by a binucleate amobula, which is the spore-forming phase ; secondly, that the spore is of very large size and exhibits a ternary symmetry, with three valves and three polar capsules. 
The development of Spharactinomyxon (Fig. 170) begins with a uninucleate amœbula (Fig. 170, $A$ ), which represents the planont phase, and doubtless multiplies by fission, since these parasites, though rare generally, occur abun-

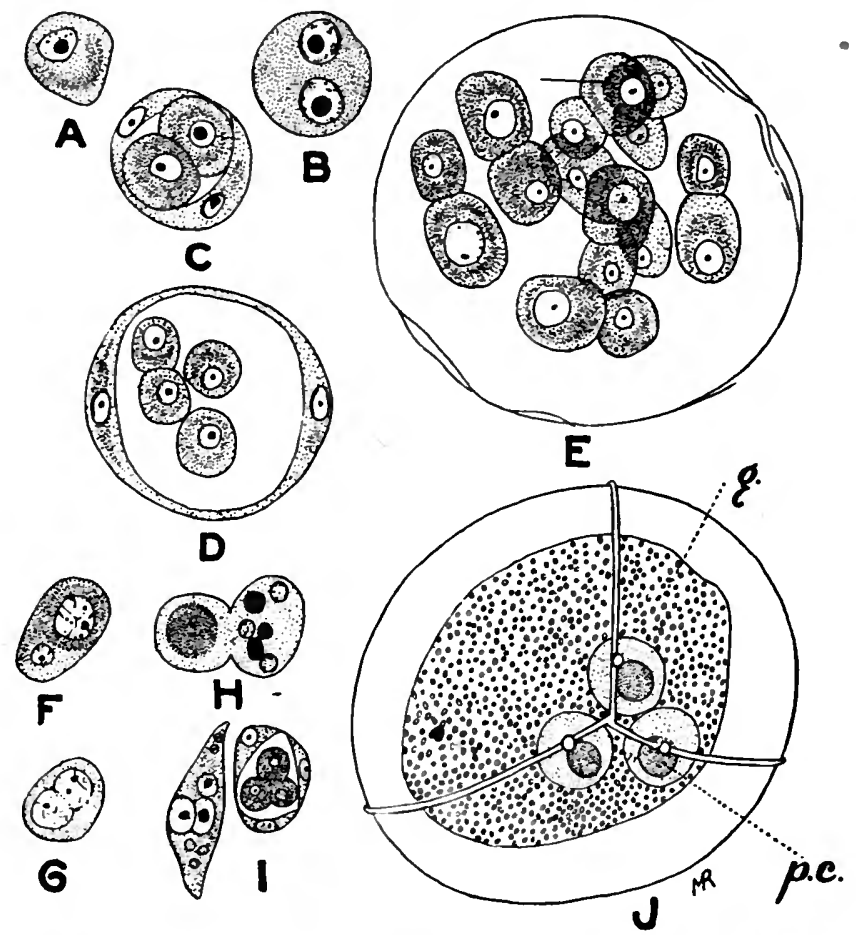

Fic. 170.-Stages in the development of Spharactinomyxon stolci. A, Amabula with a single nucleus; $B$, binucleate amœbula; $C$, the two nuclei of the preceding stage have each divided, and the body is divided into four cclls, two peripheral envelope-eclls and two central germinal cells; $D$, the space enclosed by the envelope-cells has become greatly enlarged, and the two germinal cells have divided into four; $E$, the germinal cells have increased in number by repeated division into sixteen, which as gametes are copulating in eight pairs ; $F, G$, two stages in the fusion of two gametes; $H$, the zygote has divided into two cells ; in one of these (on the left), which will form the germinal mass of the spore, the nucleus is at this stage undivided; in the other (on the right), which will form the accessory structures of the spore, the nucleus has divided to form six nuclci; $I$, further stage of the preceding : in the germinal cell (on the left) the nucleus has divided into several nuolei of unequal sizes; the sporal tissue (on the right) is represented by six cells, three peripheral, the parietal cells, and three central, the capsulogenous cells; $J$, ripe spore, enclosed by a sporocyst composed of three valres meeting in sutures; the germinal mass $(g$.$) , separate in the preceding stage, has migrated$ into the interior of the spore, and contains an immense number of nuelei ; at the upper pole of the spore are seen the three polar capsules (p.c.). After Caullery and Mesnil (769).

dantly in the infected animals. The planont phase is succeeded by a binucleate stage (Fig. 170, B), the origin of which is uncertain, but which most probably arises from an association and plastogamic fusion of two planonts, and which represents the spore-forming phase. 
The binucleate amcbula is sucreeded by a stage with four cells (Fig. 170,C), the result of the division of each nucleus, with subsequent division of the cytoplasm of the amœbula. Two of the cells take up a superficial position and form an envelope for the other two, which are the gametocytes. Tht two enveloping cells do not develop further, but the two internal cells proceed to multiply by repeated division to form sixteen gametes (Fig. 170, $D, E$ ), eight derived from each of the gametocytes. The gametes now copulate in pairs, those of each couple being slightly different from one another, chiefly as regards the size of their nuclei. It is very probable that in each couple one gamete is descended from one of the two original gametocytes, the other from the other (Fig. 170, $F, G$ ). In this way eight zygotes are formed, each of which represents a sporoblast and proceeds to form a spore.

Each sporoblast now divides into two cells, which may be distinguished as Cell $A$ and Cell $B$ respectively. Cell $A$ is the mother-cell of all the accessory elements of the spore-namely, parietal cells and capsulogenous cells. Cell B is the mother-cell of the germinal elements. The development of these two sets of elements proceeds at first quite independently. Cell A divides into six cells (Fig. 170, H, I, right), three parietal cells which secrete the three valves of the sporocyst, and three capsulogenous cells which produce the three polar capsules. Cell $B$ is at first a cell with a single large nucleus, which now begins to divide, and when it does so Cell $B$ separates from the six cells derived from Cell A (Fig. 170, $H, I$, left). As a result of the nuclear division in Cell $\mathrm{B}$, it becomes a large multinucleate plasmodium, the germinal mass, containing larger central nuclei, and smaller towards the periphery. The larger nuclei are perhaps trophic in function, the smaller germinal.

As a result of these changes, the body now consists of two envelope-cells, destined to degenerate, containing sixteen cell-masses; eight, consisting each of the six sporo-forming cells, which take up a more central position, and eight multinucleate germinal masses, which lie at the periphery of the body. Each central mass forms the sporocyst and polar capsules of the spore, and when these parts are completely formed the germinal masses migrate bodily into the spores, each germinal mass occupying the cavity of one of the spores (Fig. 170,J). Within the spore the germinal mass remains for a time in the condition of a multinucleate plasmodium, but divides ultimaely into a vast number of uninucleate sporozoites. The spore germinates, doubtless, in the digestive tract of a new host, setting free a swarm of amcobulæ which as planonts pass through the intestinal epithelium and initiate a fresh developmental cycle.

The spore-formation in Actinomyxidia is seen to agree in all essential details with that of the Myxosporidia, and inasmuch as each zygote becomes a sporoblast, and gives rise to an entire spore, with all its accessories, the process is similar to that of the Disporea. The chief points in which the Actinomyxidia differ from the Myxosporidia are the absence of the large trophic plasmodial stage, the ternary symmetry of the spore, and the enormous number of sporozoites contained in the relatively huge spore.

Order III: : Microsporidia.-The characteristic feature of this order is furnished by the spores, which are minute oval refringent bodies in which no polar capsule is visible in the fresh condition; but when treated with reagents the spores are seen to contain, with one exception, a single polar capsule, from which, after suitable stimulation, a polar filament of very great length is extruded. The existence of the polar capsule in the Microsporidian spore was discovered by Thélohan, who in consequence of this discovery 
united the Microsporidia with the Myxosporidia into a single order - the Myxosporidia (sensu latiori) - which was divided by him into two suborders : Phænocystes (=Myxosporidia sensu strictiori) and Cryptocystes (=Microsporidia). This classification is found in many textbooks; but in view of the possession of polar capsules by other orders of the Cnidosporidia, it is more convenient to maintain the old order Microsporidia of Balbiani.

The Microsporidia first attained an unenviable notoriety through the ravages caused by Nosema bombycis, the cause of " pébrine," or silkworm-disease ; hence the spores are often spoken of as " pébrinecorpuscles." The silkworm-disease was investigated by Pasteur, who found that the silkworms acquired the infection in two ways: first, by the contaminative method, by eating leaves contaminated by the faces of other infected caterpillars, and thus infecting.themselves with tne spores of the parasite by way of the digestive tract; secondly, by the so-called "hereditary" method-that is to say, through the parasite penetrating into the ovaries and eggs of the female silkworm-moth, and, in the form of spores, remaining dormant in the egg through its embryonic development until the hatching of the caterpillar, which in this way is born infected with the disease.

In contrast with the Myxosporidia, the Microsporidia are chiefly parasites of arthropods, especially insects, and are comparatively scarce in fishes, from which, however, a few species are knowne.g., Glugea stephani of the flounder, $G$. anomala of the stickleback, etc. Two species are known which are parasitic in gregarines. No species of Microsporidia are known as yet from warm-blooded vertebrates, though their occurrence in such hosts has often been alleged erroneously ; for instance, Leishmania tropica (p. 316), parasite of Oriental Sore, was referred originally to the Microsporidia by Wright (581) under the name Helcosoma tropicum. In addition to Nosema bombycis already mentioned, other highly pathogenic species are known-Thelohania contejeani, cause of destructive epidemics amongst river-crayfishes in parts of France ; and Nosema apis, cause of the recent destructive epidemic among hive-bees in England (vide Fantham and Porter, P.Z.S., 1911, p. 625).

As a general rule the parasites of this order are cell-parasites, which multiply and form their spores within cells, and the trophic phase of the parasite is typically mintute and microscopic in size. Usually some particular tissue is attacked, but the pathogenic species owe their lethal powers to the fact that they infest all the tissues of the body. In a few cases, however, the parasites produce cysts of relatively large sizc, visible to the naked eye in the tissues of the host-as, for example, tho species of the genus Glugea.

The most marked effect of the parasites is to produce, in many cases, an extraordinary hypertrophy of the host-cell, and in par- 
ticular of its nucleus, which becomes of gigantic size, and multiplies by division, usually in a direct and irregular manner (Fig. 171). Hypertrophied nuclei may also come into contact with one another, and fuse into irregular masses; and the nuclear hypertrophy affects not only the infected host-cell, but also neighbouring cells (Schuberg). According to Mercier, a species of Pleistophora parasitic in the cells of the fat-body of the cockroach stimulates the cells to multiply, with mitosis of the nuclei, and so form neoplasial growths. These facts are of considerable interest from the point of view of the growth of tumours.

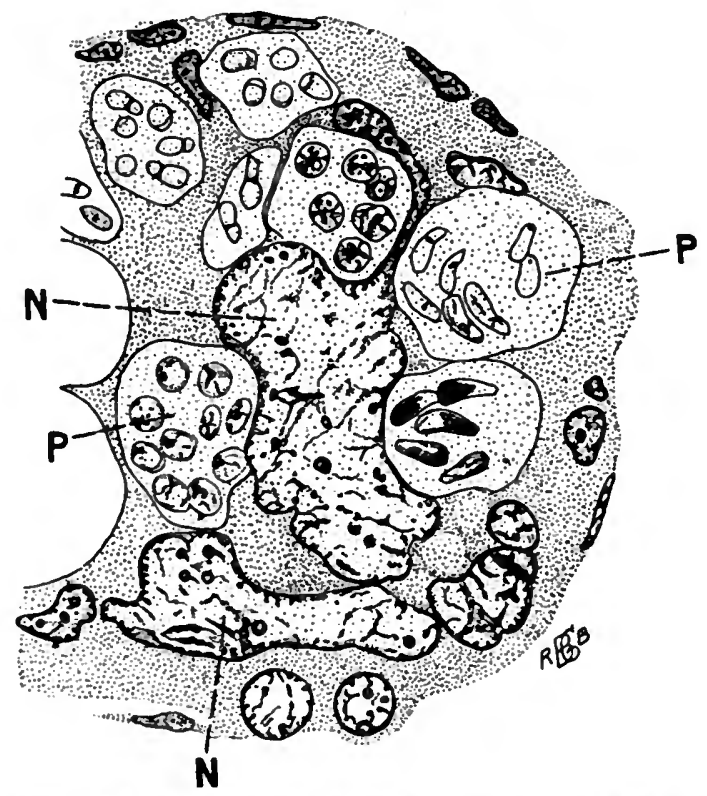

Fro. 171. - Section of a testis-tubule of a barbel infected by Pleistophora longiflis, showing the cysts of the parasite $(P ., P$.$) and the greatly hypertrophied tissue-$ nuclei $(N ., N$.$) . After Schuberg.$

Corresponding with the two types of the parasite mentioned above, there are two methods of development to be distinguished in the trophic phase. As an example of the first, Nosema bombycis, of which the development has recently been described in full detail by Stempell (785), may be selected; while Glugea anomala (Stempell, 784) is an example of the second type.

The development of Nosema bombycis in the silkworm (Fig. 172) begins with small uninucleate amobulæ, which are found first free in the digestive tract, and later in the lymph-channels (Fig. 172, 5-8); they multiply by simple fission and wander all over the body, and are hence termed planonts (i.e., wanderers) by Stempell. After a time the planonts penetrate into cells, and there grow larger, assume a definite oval or spherical form, and become " meronts" or schizonts, which multiply by binary or multiple fission until they have filled and exhausted the host-cell (Fig. 172, 9.13); but they do not pass into other cells. The multiplication of the meronts may 

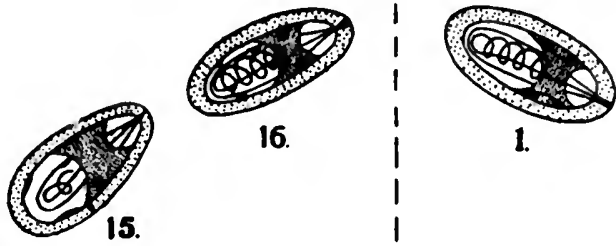

1.
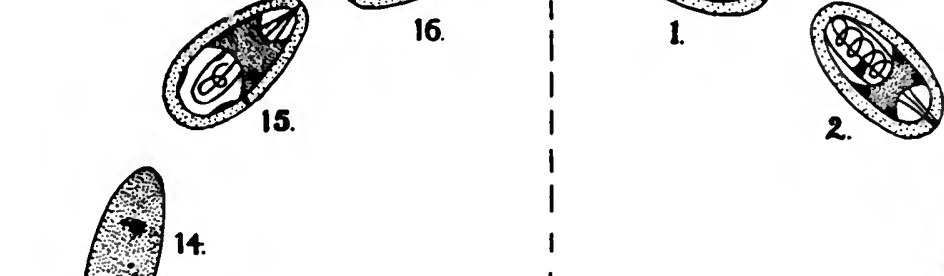

16.
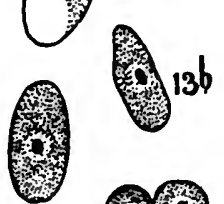

128
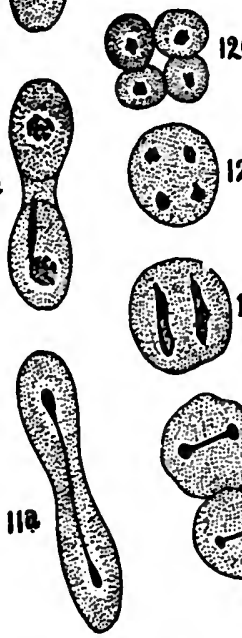

$12 c$

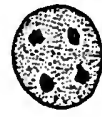

$12 \pi$

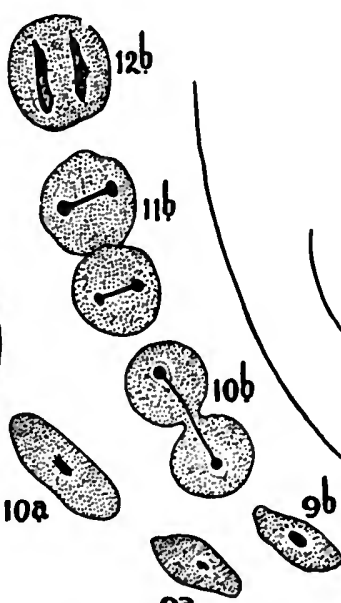

$9 a$

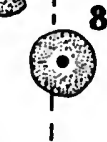

3.

3.

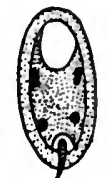

4.

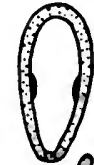

5. 3

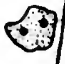

Fra. 172.-Diagram of the life-cycle of Nosema bombycis. All the stages to the left of the dotted line are passed within a single oell, in which the parasite goes through its sporogony after active multiplioation by schizogony. 1, 2, Spores showing the division of the two nuclei of the sporozoite; 3,4 , germination of the spore, showing first the extrusion of tho polar filament, and then tho escape of the sporozoite, which leaves two nuclei behind in the empty sporocyst and comes out with two nuclei ; 5 , uninucleate planont ; 6 , multiplication of the planont by fission; 7,8 , planonts, the latter entering the host-cell ! 
be very similar in its general appearance to that of yeast-organisms, and may result in the formation of chains of cells. When the host-cell is used up, the meronts do not multiply further, but produce a final generation of uninucleate cells which, as sporonts (Fig. 172, 13), give rise in this genus each to a single spore.

In the development of a spore, the nucleus of the sporont (sporoblast) buds off three small nuclei (Fig. 172, 14), two of which, as parietal nuclei, form the sporocyst, while the third is concerned with the formation of the single polar capsule, and the fourth or principal nucleus remains as the nucleus of the amœbula (Fig. 172, 15). Doubtless there are divisions of the protoplasm corresponding to the divisions of the nuclei, but in such minute bodies they cannot be made out clearly. The sporocyst, when formed, is a tough capsule, which, though produced by two cells, does not show any indications of a double composition, but appears to be cast in one piece. In some species only a single parietal cell has been seen. The spore as a whole (Fig. 172, 16, $1,2,3$ ) is egg-shaped, with one end, commonly termed " anterior," narrower than the other. It contains two vacuoles, one near the anterior, the other near the posterior end. The single polar capsule is of relatively large size; situated axially in the spore, it occupies its whole length, and contains a polar filament of immense length, wound spirally in its interior. In Glugea anomala a spore $6 \mu$ in length may eject a polar filament $150 \mu$ long (Stempell, 784); while in Pleistophora longifilis the filament may measure as much as $510 \mu$, more than forty-one times the length of the spore (Schuberg). The existence of a polar capsule is denied by Schuberg, who maintains that the filament is coiled up within the posterior vacuole of the spore.

The amcobula occupies the middle region of the spore, between the two vacuoles, and apparently separating them; but in reality it has the form of a ring or girdle, wrapping round the axial polar capsule and filament, and placed slightly nearer the anterior pole of the spore. The amcebula containg at first a single nucleus, which, according to Stempell, divides into two and then into four. Schuberg, however, maintains that the amœbula, and indeed the entire spore, contains but a single nucleus; ho denies the existence of parietal and capsulogenous nuclei, and in his opinion the bodies that have been interpreted as such are in reality metachromatinic grains. Stempell's description of the development of the spore is, however, in accordance with that given by other investigators.

When the spore germinates in the intestine of a new host, the polar filament is shot out, and the amcobula creeps out through a pore at the anterior end; there would appear to be at this point a small cap which closes the spore, and which is blown off by the explosion of the polar capsule (Fig. 172, 4). According to Stempell, the amœbula emerges from the spore with two nuclei, leaving the other two behind in the sporocyst as reduction-nuclei ; then the two nuclei of the amcebula copulate, in an autogamous manner, to form a synkaryon, and the uninucleate amcebula that results initiates the generation of planonts. It seems, however, not improbable that some process of copulation with other amcbulæ, liberated from other spores, may occur at this stage, and romains to be described.

A life-oycle similar in the main to that described for Nosema bombycis, with planonts, meronts, and sporonts, as successive phases, is probably

Fia. 172 continued:

9-13, multiplication of the meront (schizont) in the cell, in two different ways, the one shown in the series $9 a, 10 a, 11 a, 12 a, 13 a$, the other in the series $9 b, 10 b, 11 b, 12 b, c 12 c, 12 d, 13 b: 13, a$ and $b$, young sporonts ; 14, divisions of the nucleus of the sporont; two small nuclei which have been budded off at the lower end are the future parietal nnolei ; from the principal nucleus the nucleus of the polar capsule is being budded off; 15,16 , formation of the spore, with two parietal nnclei, one capsulogenous nucleus applied to the polar capsule, and the nuoleus of the sporozoite, at first single (15), later double (16). After Stempell (785), slightly modified. 
oharacteristic of Microsporidia generally. Its most important variations are exhibited in the mode of spore-formation and in the vegetative or multiplicative stages. Only in the genus Nosema does the sporont give rise to a single spore. In Thelohania chatogastris, studied by Schröder (781), for example, the sporonts are distinguished from the meronts by being enclosed in a delicate cyst, within which the sporont multiplies by successive divisions into eight uninucleate sporoblasts (Fig. 173), connected at first by a central mass of protoplasm like a rosette; but as soon as the sporocyst is formed the sporoblasts become separate. The nucleus of each sporoblast divides until there are five, two for the amœbula, one for the polar capsule, and two for the sporocyst, and the development is similar to that of the spore of Nosema bombycis already described.

A noteworthy feature of many Microsporidia is that the spores formed are of two sizes, microspores and macrospores, which may differ considerably in their dimensions. In Pleistophora longifilis the macrospores are $12 \mu$ in length by $6 \mu$ in breadth, while the microspores are 2 or $3 \mu$ in length and broad in proportion (Sehuberg). It is very probable that these differences are related to differences in sex of the contained amcebulæ, and that the two kinds of spores produce macrogametes and microgametes respectively.

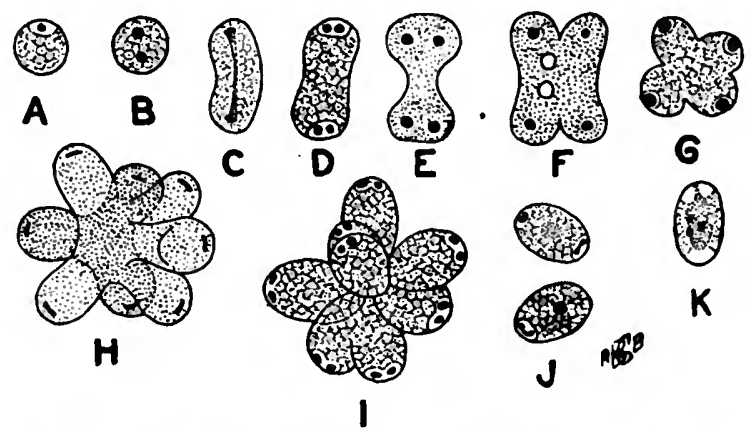

FrG. 173.-Stages in the spore-formation of Thelohania chretogastris. A, Uninucleate sporont ; $B, C$, division of its nucleus into two ; $D, E, F, G$, division of the nucleus and body into four; $H$, division into eight sporoblasts; $I$, eight sporoblasts, each with the nucleus dividing again; $J$, two sporoblasts from a clump, showing further divisions of the nuclei; $K$, young spore showing two parietal and three central nuclei (nucleus of the capsulogenous cell and two nuclei of the amoebula). After Sohröder (781).

In Pleistophora periplaneto, according to Shiwago, several planonts ("amoeboids") fuse into a plasmodium; their nucloi become resolved into chromidia which become mixed together $\rightarrow$ a process interpreted by Shiwago as chromidiogamy. From the chromidia secondary nuclei aro formed, which become the nuclei of the sporonts ("daughter-amœeboids"). The sporonts become free from the plasmodium and form spores. If this account be confirmed, it is clear that the alleged autogamy of the Microsporidia, if it occurs, is not necessarily an autogamy without amphimixis. In Thelohania monadis, according to Perez (778), the nucleus of the sporont becomes resolved into a cloud of chromidia, from which the eight nuclei of the sporoblasts are reconstructed.

The greatest difference in the vegetative phase from the condition described for Nosema bombycis is seen in the genus Qlugea, where the multiplication of the meront leads to.the formation of a multinucleate plasmodium -a result easily explained on the supposition that the nucleus of the meront divides repeatedly, but the body as a whole does not do so. In this way a relatively large plasmodial trophozoite, comparable to that of the Myxosporidia, is pro- 
duced, which may form a eonspicuous cyst. From the plasmodial stage sporonts arise by separation of a mass of protoplasm round a nucheus within the body of the parasite, and thus distinct cells are formed lying in vacuoles in the plasmodium. Such cells are commonly tcrmed "pansporoblasts," but the use of this term is best avoided, since the cells in question are in no way equivalent to the pansporoblasts of Myxospuridia, which are associations of two gamonts; but they correspond exactly to the sporonts of Nosema and other genera, and proceed to the formation of spores in the manner that has been described already, dividing first into several sporoblasts.

The plasmodia of the Glugea-type lead, as already stated, to the forma. tion of conspicuous cysts, visible to the naked eyo, in the tissues of the host; but the composition and nature of these cysts are at present a matter of dispute. According to Stempell (784), in Glugea anomala, the body of the parasite is sharply defined and marked off from the tissues of the host by a thick membrane or autocyst ("Eigencyst") formed by the parasite itself (Fig. 174,e). Within the autocyst is contained the plasmodjum, consisting of

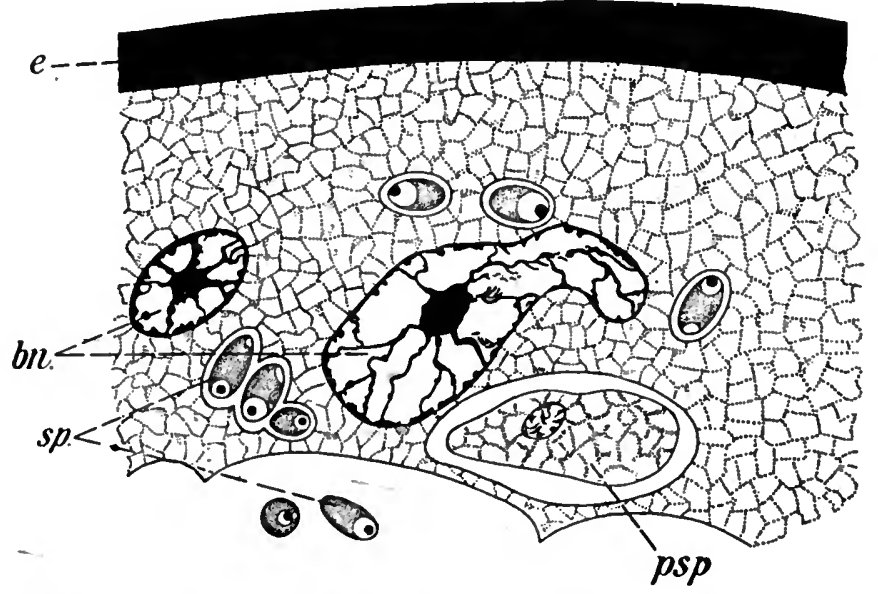

Fig. 174.-Glugea anomala, Moniez: part of a section of a cyst. c., Envelope (autoeyst); $b n$, vegetative nuclei ; $s p$., spores; $p s p$, sporont lying in a space in the protoplasm. After Stempell.

protoplasm containing many nuclei, amongst which the most conspicuous are largo-indecd, relatively gigantic-vegetative nuclei, which multiply by direct division. From the vegetative nuclei the minute nuclei of the sporonts are stated to arise, while in other case vegetative nuclei break up and degenerate.

Schröder (781) and Schuberg, on the other hand, maintain that the largo vegetative nuclei of Stempell are in reality tissue-nuclei of the host, greatly hypertrophied and mixed up with the plasmodium of the parasite. Schuberg found that Pleistophora longifilis, from the testis of the barbel, causes a hypertrophy, not only of the host-cell in which it is contained, but also of neighbouring cells, the effect of which is to produce a sort of host-plasmodium, as it were, containing gigantic host-nucloi of irrogular form (Fig. 171), amongst which the sporonts and spores of the parasite are scattered. Mrazek also interprets the supposed vegetative nuclei of Myxocystis as hypertrophied host-nuclei (see below). This interpretation of the composition of the plasmodium greatly diminishes, or even abolishes, the principal distinction between Ghugea and the other genera of Microsporidia. In opposition to this view, 
Stomroll (786) brings forward a number of arguments, the most cogent of which is the existenco of the autocyst separating the plasmodium of the parusite, containing the nuclei of disputed nature, from the tissues of the host.

The most recent investigations of Awerinzew and Fermor confirm com. plctely Stempell's interpretation of the cysts of Glugea anumala ; compare also Weissenberg. These authors find nuclei of various sizes in the protoplasm of the cyst, larger or smaller. The larger nuclei are found in the outer, nonvacuolated protoplasmic layer of the Glugea ; they grow in length and become sausage-shaped, and are ultimately segmented into smaller nuolei, whioh may form chains at thoir first origin, like the meronts of Nosema and other forms. In this way arise tho smaller nuclei, which oither become sporonts, or remain as vegetative nuclei in the protoplasmic walls of the vacuoles containing the spores, where they ultimately degenerate and break up. Tho sporonts are stated to arise in toto from nuclei, without visiblo participation of the protoplasm of the cyst; they become enclosed separately in vacuoles, within which each sporont forms a cluster of spores. Thus in $_{\text {in }}$ older cysts the contral part of the body becomes divided by fine protoplasmic partitions into a mass of separate chambers or vacuoles, each containing ripe spores. Glugea anomala is to be regarded, therefore, as a colonial organism in which meronts and sporonts, homologous with those of Nosema, etc., lie embedded in the protoplasm of their own cyst-the meronts in the peripheral zone of growth, the sporonts and spores in the central protoplasmic region of the cyst.

Classification.-The two types of the trophic phase that have been doscribed in the foregoing paragraphs have becn utilized by Perez (779) to subdivide the Microsporidia into two subordcrs, as given below. Stempell (785), on the other hand, divides the group into three families; the uncertainty that prevails at present with regard to the exact structure of the trophic phases in some forms is a hindrance to finality in the classification of this ordor.

Suborder I. : Schizogenea (seu Oligosporea).-The principal trophic phase is a uninucleate meront which multiplies by fission, and from which the sporont finally arises. Several genera, characterized by tho number of spores produced by the sporont: One spore, Nosema ; two spores, Perezia ; four spores, Gurleya ; eight, spores, Thelohania; sixteen spores, Duboscqia (see below); $n$ spores, Pleistophora; but Stempellia (Léger and Hesso, 775), for S. mutabilis, parasite of the fat-body of Ephomerid larvæ, produces spores to the number of eight, four, two, or one indifferently ; Octosporea, the species of which aro parasitic in Muscida, produces eight spores in one species, one in another. These anomalies indicate that the classification by the number of spores producod by the sporont is purely artificial (Chatton and Krempf). Telomyxa glugeifurmis (Léger and Hesse), also from the fat-body of Ephemerid larvæ, produces eight, sixtcen, or $n$ spores, and stands apart from all other known Microsporidia in possessing two polar capsules in the spore.

Soborder II. i Blastog Enea (seu Polysporea). - The principal trophic phase is a multinucleate plasmodium producing sporonts by internal cleavage; example: Glugea. To this section, also, the peculiar form Myxocystis has been referred, which was discovered by Mrazek in the body-cavity of Oligochætes. Myxocystis occurs in the form of large masses floating frecly in the body-cavity, each mass remarkablc for an envclope composed of a fur of vert:cal filaments, not unlike stiff cilia, and enclosing nuclei and spores in various stages of developnicnt. According to the most recent investigations of Mrazek, however, each of these masses represents in reality a lymphocyto containing numcrous parasites, which multiply and form spores, and provoke a great hypertrophy of the host-cell, accompanicd by multiplication of its nucleus. Hence the true Myxocystis is an intracellular parasite referable, apparently, to the order Schizogenea, and characterized chiefly by the peculiar form of its spores. Duboscqia legeri, Perez (780), from the body-cavity of Termes lucifugus, is perhaps an organism of similar naturo; it is described 
as a floating plasmodium in which sporonts arise, each of which produces sixteen spores; it has, however, been referred by its discoverer to the Blastogenea.

Order IV.: Sarcosporidia.-The parasites of this order are considered at present to constitute a single genus, Sarcocystis, with numerous species. In contrast to the three orders of Cnidosporidia dealt with in the foregoing pages, the Sarcosporidia are pre-eminently parasites of the higher vertebrates, more especially of mammals, occurring occasionally, though rarely, in man (see Darling); but they are known also to occur in avian and reptilian hosts, though sparingly. On the other hand, no Sarcosporidia are known to be parasitic in invertebrate hosts of any kind. In their hosts the Sarcosporidia are tissue-parasites, occurring principally in the striped muscles, but occasionally in unstriped. In a few cases they are found in connective tissue, but this appears to be a secondary condition in which a parasite living first in the muscle-fibres becomes free from them at a later period. As a general rule the Sarcosporidia appear to be harmless parasites, which do not make their presence known by any symptoms of discase, and can only be detected by post-mortem examination. Some species, however, are an exception to this rule, and are extremely pathogenic to their host-for example, Sarcocystis muris of the mouse. The extent to which the health of the host is impaired appears to be directly proportional to the numbers of the parasite in the body, and consequently to the power which a given species may possess of multiplying and overrunning the host. In most species the capacity for endogenous multiplication appears to be extremely limited.

In spite of the fact that Sarcosporidia are very common parasites of domestic animals, and have been found frequently in man, our knowledge of their structure and life-history is in a very backward state. As a rule Sarcosporidia present themselves as opaque, whitish bodies, usually elongated and cylindrical in form, encysted in the muscle-fibres of the infected animal, and known commonly as "Miescher's tubes." They are distinctly visible to the naked eye, and often very large. Sarcocystis tenella of the sheep reaches a length of 16 millimetres, while in the roebuck (Cervus capreolus) cysts of 50 millimetres in length are recorded. The Miescher's tube, when examined microscopically, is seen to be a body of complex structure, and consists chiefly of vast numbers of sickleshaped spores-“"Rainey's corpuscles "-lying in clumps or bunches contained in chambers separated off from one anotber by partitions. The whole organism is enclosed by a distinct envelope, often exhibiting vertical striations, and the partitions between the chambers containing the sporos are continuations of the envelope. The exact 
structure of the spores is still a matter of dispute, and it is possible that there is more than one kind of spore even in the same species of parasite. A remarkable feature of the sporesin some species, at least-is that they are motile when set free: for example, in $S$. muris. They are also extremely delicate structures, easily injured by external media, in marked contrast to the spores of the other orders of Cnidosporidia. The spores of S. muris, S. bertrami (of the horse), and S. tenella, reproduce themselves by division (Negri, Fiebiger, Teichmann). Finally it must be mentioned that the spores of Sarcosporidia contain a true toxin, which was named by Laveran and Mesnil "sarcocystine." Its properties have been investigated recently by Teichmann (25) and Teichmann and Braun (26).

The natural mode of transmission of the Sarcosporidia remains to be discovered. It was found by Theobald Smith that mice could be infected experimentally with $\mathcal{S}$. muris by feeding them with the flesh of other infected mice; but it is extremely unlikely that cannibalism is the method whereby sheep and other ruminants become infected with these parasites. All experiments indicate that the spores germinate in the digestive tract of the new host; but the delicate nature of the spores seems to preclude any possibility of the occurrence of ordinary contaminative infection, as in other Cnidosporidia. In this connection attention should be drawn to the statement of Watson, that the spores are to be found in the circulating blood, indicating the possibility of transmission by an intermediate host.

In spite of several recent investigations upon the structure and development of the Sarcosporidia, the subject is in a confused state, even the structure of the spores being still disputed. It is therefore difficult to obtain a clear notion of the course of the life-cycle in these organisms.

According to Laveran and Mesnil, the spores of S. tenella (Fig. 175) are sausage-shaped bodies, curved, with one end more pointed than the other. At the pointed ond is a striated structure representing a polar capsule, and at the blunt end is a nucleus, while the middle of the body is occupied by coarse, deeply-staining, metachromatinic grains. Watson also figures a large nucleus near the blunt end of the spore, and places the polar capsule at the pointed end. Negri also describes the spores of $S$. muris and S. bertrami as having the nucleus near the blunt end, while the opposite extremity appears hyaline and homogeneous for a certain distance. Betegh, again, describes a nucleus at the blunt end of the spore, and one or two "centrosomes" in the middle region. Erdmann (790), on the other hand, places the nucleus in the middle of the body amongst the metachromatinic grains, and describes it as consisting of a large dense karyosome lodged in a small vacuole; she does not seem to be decided, however, whether the polar capsule is at the pointed or the blunt end of the spore. Teichmann describes a large nucleus at the blunt end of the body, and is doubtful as to the existence of a polar capsule. So far as it is possible to draw any conclusions from so many contradictory statements, the clear description given by Laveran and Mesnil seems to be, on the whole, confirmed. But' according to Crawley, the spores of S. rileyi are binucleate; compare those of Gastrocystis (Fig. 179, p. 428). It is not 
clear which part of the spore contains the amœbula which is liberated from it, as presently to be described.

In addition to spores having the complicated structure described for those of $S$. tenella, there appear to be also spores of much simpler structure, as, for example, in S. muris. Apparently the more complicated spore is propagative in function, serving to infect new hosts, while the simpler form, which should perhaps be regarded rather as a sporoblast, as a simple cell not differentiated as a spore, eerves for spreading the infection in the same host. The occurrence of the simpler type of spore in $S$. muris would account for the manner in which this parasite overruns its host, and is usually lethal to it, whlle $S$. tenella, which appears to produce chiefly propagative spores, is a harmless parasite. How far these suggestions are true must be determined by future investigations.

The discovery made by Smith, mentioned above, that mice could be infected with $S$. muris by feeding them with the flesh of other infected mice, has been confirmed and extended by other observers. According to Nègre, the fæces of mice which have been fed with infected muscular tissue are infective to other mice if ingested by them; they possess this power about fifteen to sixty days after the mouse was fed with muscle containing Sarcosporidia, and retain their infectivity oven if kept dry in an open bottle for a month, or heated to $65^{\circ} \mathrm{C}$. for fifteen minutes. Negri was able to infect gunnea-pigs with $S$. muris by feeding them with the flesh of infected mice, and found that in the guineapig the parasite appeared with quite different characters from those which it presents in the mouse, so that it might be taken easily for a distinct species. Darling also infected guinea-pigs with $S$. muris in the same way, and points out the resemblance between the experimental sarcosporidiosis of the guinea-pig and a case of human sarcosporidiosis observed by him; it is suggested that the sarcosporidia occasionally observed in the human subject are those of some domestic snimal undergoing a modified or abortive development in a host that is not their usual one. Erdmann also infected mice with $S$. tenella in a simijar manner. It is remarkable that parasites generally so harmless should be so little specific to particular hosts, and the results of Negri render the value of the characters used for distinguishing spocies of Sarcosporidia as doubtful in

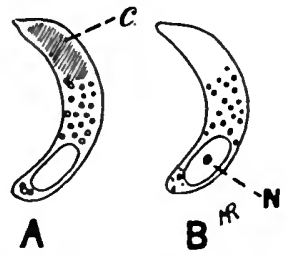

Fio. 175. - Spores of Sarcocystis tenella, $A$, In the fresh condition ; $B$, after staining with ironhæmatoxylin : $N$., nucleus ; $c$, striated body (polar capsule ?). After Laveran and Mesnil. their validity as the distinctions fonnded on their occurrence in certain hosts.

According to Erdmann (791), the spore germinates in the intestine of the new host, and the first act in the process is the liberation from the spore of its toxin, sarcocystine, which causes the adjacent epithelium of the intestino to be thrown off. At the same time an amcbula is set free from the spore; and, owing to the intestine boing denuded of its lining epithelium, the amcebula is able to penetrate into the lymph-spaces of the submucous coat and establish itself there. Before this happens, however, the metachromatinic grains of the spore disappear, and it is suggested that this disappearance is related to the secretion of the sarcocystine, and that the toxin is contained in the metachromatinic grains. If, however, a polar capsule be discharged during the germination of the spore, as in other Cnidosporidia, it might well be that the toxin is contained in the polar capsule, and is set free by its discharge, like the poison in the nematocysts of the Coelentera. However that may be, it would appear as if the sarcocystine were a weapon, as it were, the function of which is to facilitate the invasion of the germ, the amcbula, by destroying the lining epithelium of the gut.

The liberation of the amcebula from the spore initiates the first period of the development, which is passed in the lymph-spaces of the intestine, and which lasts, according to Erdmann, some twenty-eight to thirty days. 
Analogy with other Noosporidia would lead us to identify this with the planont-phase, initiated, possibly, by sexual processes between different amœbulæ and subsequent active multiplication. The socond period of the development begins with the penetration of the amobula into a muscle-fibre, in which the parasite grows into a Miescher's tube and forms spores.

The intramuscular development of the parasito begins by multiplication of the nuclei to about twelvo, forming a plasmodium (Fig. 176, A). This next becomes divided up, in parasites about thirty-three days old, into separate cells,

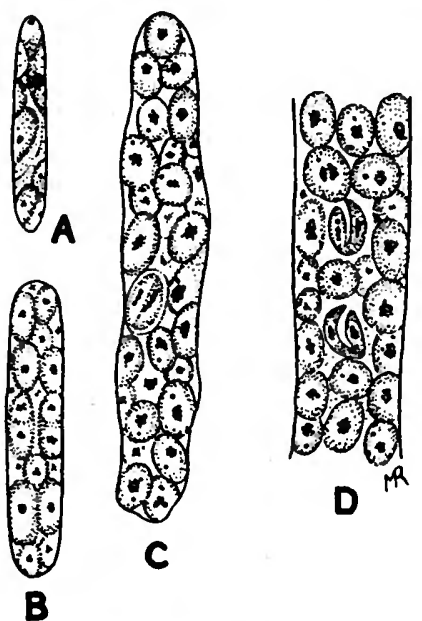

Fig. 176.-Four stages in the development of a "Miescher's tube" of Sarcocystis muris in the pectoral museles of white rats infected ex. perimentally. $A$, Parasite $25 \mu$ in length, fifty days after infection; the contents of the body beginning to divide into separate cells ; $\mathcal{B}$, parasite of the same age, $35 \mu$ in length, division of the eontents further advanced; $C$, parasite of the same age, $60 \mu$ in length, containing separate cells; at the centre the division of a sporont into two sickle-shaped bodies is seen to be taking place; $D$, middle portion of a tube about $450 \mu$ in length, seventy days after infection, showing. two couples of sickle-shaped bodies formed by division of a sporont. After Negri.

centre of the body may consist of granular débris, derived from the disintegration of spores which are past their prime and have degenerated.

So far as it is possible to draw conclusions in the present state of knowledge, the Sarcosporidia would appear to be true Cnidosporidia, with spores which contain each a single polar capsule, 
and from which an amobula is liberated, as in other Neosporidia (Amœbogeniæ).

Order V.: Haplosporidia.-The distinctive features of this order are for the most part of negative character, and, as the name implies, the tendency is towards simplicity in structure and development. The spores are without the polar capsules which are so marked a peculiarity in the four previous orders, and have the form of simplt cells, each with a single nucleus, and with or without a sporocyst, which, however, when present, is not formed by distinct parietal cells.

In organisms of such simple structure, the absence of distinctive peculiarities renders the limits of the group indefinite, and the affinities of its members vague and undecided, and it is possible that the order Haplosporidia, as generally understood, is a heterogeneous assemblage, many members of which present only developmental analogies to the true Neosporidia-that is to say, a similarity in the life-history which is an adaptation to a similar mode of life, and not a true indication of genetic affinity. Léger and Duboseq (646) point out that the characters- " peu limitatifs "of the Haplosporidia would suit Protista of the most diverse affinities, and scarcely mark them off from yeasts or Chytridineæ. With the exception of the family Haplosporidiida, they regard the group Haplosporidia as purely provisional, and comprising heterogeneous forms with undecided affinities.

The life-cycle of a typical Haplosporidian parasite is very simple. The initial phase is an amobula or planont, which multiplies by fission, division of the nucleus being followed by division of the body to form two planonts, which may continue to divide for many generations. From a planont arises ultimately a plasmodial phase, the result of divisions of the nucleus without corresponding divisions of the body, which grows to a relatively large size. The plasmodium is the principal trophic phase. It may multiply by plasmotomy or by schizogony, or may proceed to spore-formation, and then it divides into as many cells as there are nuclei. The cells formed in this way are either sporoblasts, each of which becomes a single spore (Oligosporulea), or they represent sporonts ("pansporoblasts "), which give rise each to a cluster of spores (Polysporulea). The spores are usually simple rounded bodies, invested by a more or less distinct protective membrane, which in rare instances becomes a definite sporocyst prolonged even into tails or spikes.

The Haplosporidia were divided by Caullery and Mesnil (802) into three families. In order to include forms more recently discovered, Ridewood and Fantham liave extended the classification, and recognize two suborders : 
Suborder I. : Ongosporulfa.-The plasmodium divides at once into sporoblasts, each of which becomes a single spore.

Family Haplosporidinda.-Spores with a double envelope, the outer sometimes prolonged into tails or processes. Genera: Haplosporidium, Urosporidium, and Anurosporidium; all the known species are parasitic in Annelids.

Family Bertramiida.-Spores with a simple envelope, or with none. Bertramia, with several species: $B$. capitella, parasite of the cclome of Capitella capitata; $B$. asperospora, a common parasite of the body-cavity of Rotifers. B. kirkmanni, described by Warren from Rotifers in Natal, is stated to have several nuclei and a vacuole in the spore, and appears to belong to a distinct genus.

In this family the genus Iclithyosporidium is ranked provisionally, as the mode of spore-formation is unknown as yet. Ichthyosporidium is a common paraeite of fishes, often lethal to an extreme degree. It occurs in the form of plasmodia, sometimes irregular, sometimes more or less spherical in form, scattered in various organs, but usually in the muscles or the connective tissue; the plasmodium contains numerous vesicular nuclei with distinct karyosomes, and may be naked at the surface, or narked off from the surrounding tissues by a membrane or envelope, often of considerable thickness. The plasmodia multiply actively by plasmotomy, and an intenso infection is produced. Parasites with a single nucleus are also found, which may either represent the planont stage, or may be derived from the division of a plasmodium; from them the plasmodial stage arises by multiplication of the nuclei. No other stage of the parasite is known, and the method of transmission remains to be discovered.

Bertramia bufonis, described by King (Proc. Acad. Sci. Philad., 59, p. 273), is possibly a species of Ichthyosporidium or allied to this genus.

Family Colosporidiida, for the genera Colosporidium, Mesnil and Marchoux and Polycaryum, Stempell : All the species known aro parasites of Crustacea (Phyllopoda and Cladocera). The plasmodium forms globules of fatty substance in the interior; it becomes encysted as a whole, and breaks up into sporozoite-liko bodies within the cyst.

Caullerya mesnili, Chatton (803), parasite of the epithelium of the mid-gut of Daphnia spp., produces, by fragmentation of the plasmodium, spores with resistant envelopes containing each about thirty nuclei. Chatton considers it to be intermediate between the Haplosporidiida and Colosporidiida ; possibly it should be referred to the next suborder.

Blastulidium padophthorum, Perez, referred to this family, js, according to Chatton (804), a Chytridinian. Colosporidium blatella, Crawley, is referred by Léger (C.R.A.S., cxlix., p. 239) to the genus Peltomyces (Myce. tozoa, p. 243).

Suborder II. : Polysporules.-The plasmodium divides into sporonts, each of which produces a cluster of spores.

Two genera, each with a single species : Neurosporidium cephalodisci, from the nervous system of Cephalodiscus nigrescens (Ridewood and Fantham); and Rhinosporidium kinealyi, from the septum nasi of human beings in India (Minchin and Fantham; Beattie); a case has also been observed in America (Wright).

Rhinosporidium causes vascular pedunculated growths or tumours, resembling raspberries, in the septum nasi or floor of the nose. In sections of the growth, great numbers of the parasite are found embedded in the connective tissue, while the mature cysts may be in the stratified epithelium (Wright). The youngest parasites are rounded cells with a single nucleus and a distinct envelope (Beattio). By division of the nucleus the parasite becomes a multinucleate plasmodium, the so-called "granular stage," often of irregular form, but this may be due to the action of the preserving reagents. Older parasites are spherical, with the onvelope thickenod to form a thick transparent cyst, external to which a nucleated envelope is formed by cells of thê connective tissue (Beattie). The contents of the cyst (Fig. 177, A) become divided up into numerous 
uninucleate sporonts ("pansporoblasts") towards the centre or at one pole, while the peripheral zone or the opposite pole remains in the plasmodial condition. The sporonts grow in size, and at the same time multiply by repeated fission to form a cluster of about sixteen spores, a "spore-morula" (Fig. 177, B), enclosed by a membrane. Between the spore-morulx an indefinite framework is formed by the residual protoplasm in which the sporonts have developed (Beattie). Hence the full-grown parasite exhibits three zones, which may be concentric or polar in arrangcment : a plasmodial region, peripheral or polar; an intermediato zone of spore-formation; and a central or polar region containing ripe spore-morulæ. The process of spore-formation continues until the whole cyst is full of spore-morulæ. The ripe cysts burst and scatter their contents in the tissues. It is possible that spores set free in this way may germinate in the tissues and give rise to fresh cysts; but it is more probable that the spores, if they escape the phagocytes, are dis. charged from the surface of the epithelium. From the analogy of other Neosporidia, it is reasonable to suppose that the youngest uninucleate forms of the parasite are the multiplicative phase in the tissues, and that the spore-morulæ represent the propagative phase. Nothing is known, however, of the mode of transmission of the parasite or of the manner in which the infection is acquired.

A parasite is described by Laveran and Pettit from Salmo irideus, which in the opinion of the authors presents affinities with Rhinosporidium and Neurosporidium. It causes a disesse in the fish, termed in German "Taumelkrankheit."

In addition to the more or less typical genera of Haplosporidia mentioned in the foregoing paragraphs, a number of other forms have been described, of which the

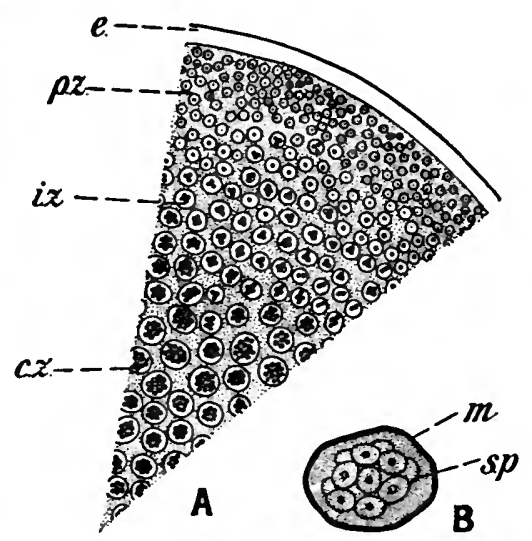

Fro. 177.-Rhinosporidium kinealyi. $A$, Segment of a section through a cyst: e., hyaline envelope; p.z., peripheral zone of pansporoblasts ; i.z., intermediate zone of pansporoblasts containing a few spores; c.z., central zone of ripe spore-morulø; $B$, ripe spore-morula ; m., membrane; sp., spores. After Minchin end Fantham. affinities and systematic position remain for the present uncertain. Such are the "Serumsporidia" of Pfeiffer, and other forms, for a review of which the reader must be referred to the comprehensive memoir of Caullery and Mesnil (802) or to the original descriptions. The remarkable form, Schewiakovella schmeili, however, presents peculiarities which deserve special mention. It is a parasite of the body-cavity of Cyclops spp., and was the subject of detailed study by Schewiakoff. In the active condition it occurs as an amœba with a single nucleus and a contractile vacuole, or as a plasmodium formed by fusion of such amœbæ. Encystment of either the amcbæ or the plasmodia occurs, and within the cyst a number of simple, uninucleate spores are formed, which, although possessing a distinct envelope, multiply further by fission, with mitosis of the nucleus. Germination of the spores sets free small amœbulæ. In many points this form is unique amongst the Sporozoa, and should perhaps be classed rather with the parasitic amœbæ.

Incerte Sedis. - In conclusion a number of forms must be mentioned which have been referred to the Neosporidia, but of which the position and affinities are quite doubtful. 
Under the generic namo Microklossia, Krassiltschik has described certain cell-parasites of caterpillars, which appear to belong to the Neosporidia, though it is not possible to assign the genus to a definite position, since the structure of the spores has not been made out, and the account given of the life-cycle requires revision. According to Krassiltschik, the cycle begins with non-sexual schizogony; tho nucleus of the schizont divides into four or eight nuclei, and as many merozoites are produced within the body of the schizont. Schizogony is succeeded by formation of "macronts" and "micronts" which give rise to gametes; the macront by a process of fission similar to the schizogony produces four to cight macrogametes, while the micront produces in a similar way two, four, or eight, microgametes. The nucleus of the macrogamete divides to form two reduction-nuclei. Tho microgamete attaches itself to one pole of the macrogamete, and its nucleus passes over into the cytoplasm of the latter and fuses with the female pronucleus. In the zygote the synkaryon buds off daughter-nuclei, round which the cytoplasm of the zygote is condensed to form internal buds-" protoblasts." The protoblasts are set free, and produce in their turn "deutoblasts," which are set free, become amoba-like, multiply in the blood of the insect, and infect the tissues and organs of the host, especially the fat-body and the wall of the digestive tract. In the fat-body the deutoblasts produce a generation of "tritoblasts" which multiply actively and spread amongst the tissue. From the tritoblasts arise finally a generation of "teloblasts," which divide each into a rosette of small cells, the definitive sporoblasts, round a central residual mass. Each sporoblast produces a spore, a smooth, strongly refractile body, ellipsoidal or egg-shaped, in which no details of structure could be made out. The spores appear to be produced in the wall of the digestive tract, whence they are set free with the fæces. The concluding phases suggest a Nosema-type, but the earlier part of the life-cycle, if correctly described, appears to be a type sui generis.

Under the name Lymphocystis johnstonei, Woodcock (824) described a parasite of plaice and flounders, which forms conspicuous cysts in the lymph. spaces of the skin and mesentery. Each cyst (Fig. 178) contains a single parasite, which may attain 1.5 millimetres in diameter, and shows a remarkablo structure. The body is enclosed by a thick, structureless membrane, and contains at the centre a very large nucleus, irregular in shape, staining feebly, and containing a number of karyosomes in a faintly-staining reticulum. Surrounding the nucleus is a chromidial network forming a ring or zone of considerable thickness, filling the greater part of the cytoplasm between the nucleus and tho envelope. The outermost zone of the chromidial net may contain a series of small, clear " spherules."

According to Awerinzew (815 and 816), the youngest stages of Lymphocystis are minute cells with a single nucleus which grow very rapidly, and as they do so the chromatin passes out of the nucleus to form the chromidial ring. The spherules are massee of plastin which separate from the chromidial net. From the chromidia secondary nuclei are formed, round which a portion of the cytoplasm is cut off to form small cells, termed by Awerinzew " secondary amoboids," and compared by him to the sporonts of Alugea. Within the secondary amoboids spores are formed, of which, however, the structure has not been made out clearly. In teased-up preparations of Lymphocystis, Awerinzew found spores similar to those of Henneguya, and proposed to place the parasite in that genus. He has now become doubtful, however, whether the Henneguya-spores belong to the Iymphocystis or to a distinct parasite, since he was unable to demonstrate a similar structure in the spores found in the secondary amcoboids. Awerinzew is of opinion, nevertheless, that Iymphocystis should be referred to the Cnidosporidia, but this form requires further investigation.

Toxocystis homari, Léger and Duboseq (646), is a parasite of the posterior intestinal cæcum of lobsters. In appearanco it resembles a hæmogregarine, motionless, with granular cytoplasm and a small karyosomatic nucleus at tho middle of the body; there are also usually two, sometimes one, "para- 
nuclear bodies," round masses larger than the nucleus, and staining very intensely with nuclear stains. The parasite occurs between the basal membrane and the epithelium, or in the epithelial cells, or occasionally free in the lumen of the cæcum. Multiplication appears to take place by longitudinal fission. No other stages are known.

Gastrocystis gilruthi, Chatton (819), is a parasite of sheep and goats discovered by Gilruth in Australia, but of common occurrence in Europe. The parasite appears as a cyst, visible to the naked eye, in tho mucous membrane of the stomach. The cyst has an envelope formed by a single cell with a large nuclous ; the envelope is concentrically striated, and bears externally a fur of short, stiff, bristle-like processes, recalling the covering of Myxidium lieberkihni, Myxocystis, and Sarcosporidia. The younger cysts contain a plasmodium with a vast number of nuclei, some of them in groups of two, three, four, and so on up to a large number, which are then arranged in a single layer cnclosing a blastula.like sphere or blastophore. The blastophore becomes separated off from the interstitial protoplasm of the plasmodium, and each nucleus grows out from the surface in a tongue-like process to form a cluster of sporozoite-like bodies or germs in a manner very similar to the sporulation of a malarial parasite or of Porospora or Aggregata. The ripe cyst is full of an enormous number of these germs (Fig. 179), each of which is a fusiform body, about $10 \mu$ in length, with one end pointed and terminating in a rostrum, the other blunter. Near the blunt end is a largo nucleus, and at about the middle of the body is a deeply-staining mass resembling

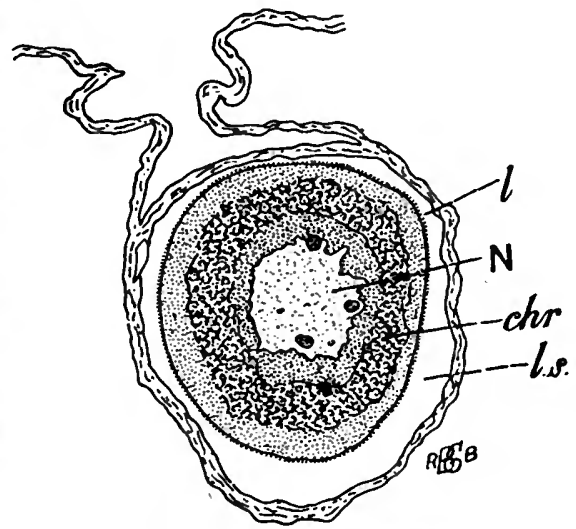

Fra. 178.-Lymphocystis johnstonei : section though one of the parasites lying in the mesentery. $N$., The large nucleus of the parasite; chr., the ring of chromidia; l.s., lymph-space; $l$., layer of lymphocytes adherent to the parasite. After Woodcock (824), magnified 45 diameters. a separate karyosome or a kinetonucleus. The surface of the germ is clothed by a delicate pellicle. The germs are set free from the cyst by dehiscence.

The affinities of Gastrocystis remain for the present quite uncertain. Negre reports the occurrence of a similar cyst in the duodenum of a mouse of which the freces infected other mice with sarcosporidiosis (see p. 421), and suggests that Gastrocystis may be a stage in the development of Sarcosporidia.

Pansporclla perplexa, Chatton (818), is a parasite of the intestine of Daphnia spp., occurring in the form of amoboid bodies, reaching $80 \mu$ in diameter, adherent, but not permanently attached, to the epithelium of the intestinal wall. The amœboid movement may be active, but does not serve for foodcapture, since nutrition is effected by the osmotic method. The cytoplasm is divided into hyaline ectoplasm and granular endoplasm containing a single large nucleus in which the karyosome has the form of one or two caps adherent to the nuclear membrane. The amcboid phase does not multiply by fission, but becomes encysted, and then the nucleus divides repeatedly until a large number of small nuclei are present. The body then bccomes divided into a number of spores, each containing eight nuclei, of which six degenerate, so that the ripo spore is binucleate. Germination of the spore sets free a binucleate amœbula which divides, apparently, into two, each of which has 
a single nucleus and grows up into the adult amœba-like phase, Sexual phenomena have not been observed, though their occurrence is indicated by the development described. Chatton considers tliat the parasite has resemblances to Amcebæa, Mycetozos; and Sporozoa.

Chytridiopsis, Schneider.--Léger and Duboseq (823) describe several species parasitic in the intestine of insects, and have followed out the development of $C$. socius. The youngest form is a minute amcbula which penetrates into án opithelial cell, and grows, with multiplication of its nuclei, to form a plasmodium or schizont, which then divides up to form a mass of uninucleate "schizozoites," each one at first crescent-shaped, then amoboid. The schizozoites are set free in the intestine, and penetrate other cells; they either grow into schizonts, which repeat the process of schizogony, or into gametocytes. Certain schizozoites grow within the cells without multiplication of the nuclei till they attain a diameter of about $10 \mu$; then the nucleus divides rapidly, and a number of mierogametes are formed. Other scnizozoites become macrogametes, which aro about $8 \mu$ in diameter and appear to be fertilized each by a microgamete. The nucleus of the zygoto divides into a great

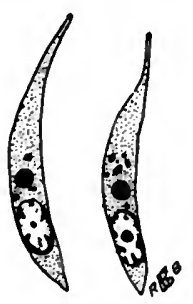

Fic. 179.-Spores of Gastrocystis gilruthi. After Chatton (819). number of nuclei, three or four of which travel to the surface of the spherical body and form a cystenvelope; the remaining nuclei retain their central position, and the body of the zygote divides into uninucleate spores. In this way resistant cysts are formed containing a large number of spores, each containing a single nucleus and a vacuole. The oysts are cast out of the body and infect new hosts.

Léger and Duboscq consider that Chytridiopsis may be allied to the Microsporidia; but having found no polar filament in the spore, they prefer to regard it as having affinities with Mycetozoa.*

The genera Amoebidium and Siedleckia were held formerly to constitute a distinct order of the Sporozoa, which was named the Exosporidia. Amoebidium has been shown clearly by Chatton (817) to be an organisin of the nature of a fungus; while Siedleckia is now gencrally referred to tho schizogregarines, as suggested by Minchin (589); see Dogiel (606). Compare also Capillus intestinalis, Granata, parasito of the intestine of Millepedes.

Affinities of the Neosporidia.-It is sufficiently apparent; from the structure and development of typical examples of any order of this subclass, that their affinities are wholly with the Sarcodina. In the case of many of the more primitive forms, it is an open question whether they should be classed in the Neosporidia or in one of the orders of the Sarcodina. Comparing them with the Telosparidia, it is seen that the two characteristics of that subclass which indicate affinities with the Flagellata are absent altogether in the Neosporidia-namely, the possession of flagellated swarm-spores or gametes, and the definite, gregarine-like body-form of the adults. No flagellated stages are known to occur at any period of the lifehistory in any member of the Neosporidia, and the body-form of the adult in this group is typically that of an amœba. Many of the Myxosporidia might almost be regarded as parasitic amobæ with a peculiar type of reproduction. Even more remarkable is the

* It is not clear on what grounds Schepotieff $(269$, p. 516) considers Chytridiopsis to be a Flagellate. 
regularity with which the sporozoite in the Neosporidia has the form of an amœbula, as contrasted with the equally-constant gregarinulaform of the Telosporidian sporozoite. The characters implied in the terms Amobogeniæ and Rhabdogeniæ appear to be more diagnostic of the two groups than any other. There can be little doubt, therefore, that the union of the Telosporidia and Neosporidia in one class-the Sporozoa-is a quite artificial arrangement, and that the two subclasses in question show distinct affinities, and are descended from distinct ancestral forms-the Telosporidia from Flagellata, the Neosporidia from Sarcodina.

Bibliography.-For references see p. 449. 


\section{CHAPTER XVII}

\section{THE INFUSORIA}

THE term Infusoria had originally a much wider application than at present, being used to denote the various microscopic animalcules which make their appearance in infusions exposed to the air. Hence the Infusoria included any Protozoa, and even organisms distinct from them, such as Rotifers. Just as the word " insect" has been restricted in its zoological application to a single class-the Insecta Hexapoda-so the term Infusoria hàs become narrowed down to denote the Infusoria Ciliata and Suctoria, which constitute, taken together, one of the most definite and sharply-marked classes of the Protozoa, characterized by two principal structural features : first, the possession of cilia during the whole or a part of their active life ; secondly, the differentiation of the nuclear apparatus into a vegetative macronucleus and a generative micronucleus (p. 153).

The Infusoria fall naturally into two subclasses: the Ciliata proper, in which the cilia are retained throughout life; and the Acinetaria or Suctoria, in which cilia are present only during the early or larval phases of the life-history, and are lost in the adult organism, which is of sedentary habit, and in which foodcapture is effected by special organs-suctorial tentacles.

\section{Subclass I.-Ciliata.}

The Ciliata, the most abundant and familiar of microscopic forms of life, may be considered in a sense the highest of the Protozoa, since in no other class does the cell-body attain to so great a complication of parts and organs or to so high a degree of structural differentiation. Not even in the Metazoa are single cells to be found of such visibly complicated structure, since in the Metazoa the cell is specialized usually for one particular function of a living body, while in the Ciliata the single cell performs all the functions of life. Moreover, the differentiation of the nuclear apparatus into generative and vegetative portions may be considered analogous with, and parallel to, the differentiation of germen and soma in the Metazoa ; and Lewin (172) regards the micronuclcus as living inde- 
pendently during the asexual cyele, with the cell as its environment. In contrast to the extreme elaboration in the structure of the individual, the life-cycle as a whole is generally of a simple type, and the majority of the freeliving species are practically monomorphic; but some of the parasitic forms show a succession of form-changes in their life-cycle.

Habits, Mode of Life.-The majority of Ciliata are free-living aquatic forms, marine or fresh-water, probably without exception holozoic in the mode of nutrition; but a great number of parasitic forms are known. A ciliate, whatever its mode of life, may be free or sedentary. The free forms may be of swimming or creeping habit, using their cilia in the one case to move freely through the water or to ghide along firm surfaces, in the other to creep over solid objects or on the surface film of the water. The sedentary forms may be attached temporarily or more or less permanently to some object, which is often the body of some larger animal. Parasitic forms may be, as in other cases, epizoic or entozoic; but the word " parasitic" must be taken in a wide sense, sinee many Ciliata living in or upon other organisms are not parasitic in the strict sense of the word, though many truly parasitic forms oecur.

Body-Form.-Correlated with the diversity in the habit of life, the body-form and external structure show many variations. The primitive type of ciliate may be considered to be an ovoid, gooseberry-shaped organism with a principal axis parallel to the direction of movement, consequently with an

FIG. 180.-Sptrostomum ambiguum, one of the largest free-living Ciliata, reaching a length of 3 millimetres, consequently a favourable object for physiological experiments. $N$, Macronucleus, greatly elongated, in shape like a string of beads or sausages (so-called "moniliform" type); $o$, mouth at the hinder end of the elongated peristome; c.2., contractile vacuole, supplied by a very long feeding-canal (f.c.); the micronucleus is not shown. After Stein.

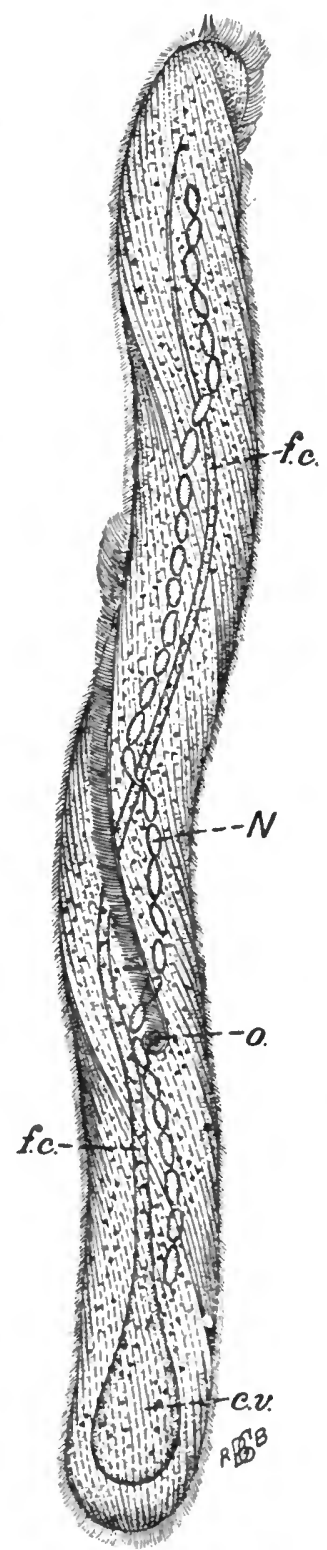

anterior and a posterior pole (Fig. 14, p. 32) The mouth is terminal at the anterior pole. The cilia clothe the whole body evenly, being 
arranged in meridional rows running from the anterior to the posterior pole, and are of equal length in all parts of the body. An ideally-simple type of this kind is very nearly realized in some of the primitive forms, but as a rule is modified in various ways.

In the first place, the mouth does not remain anterior, but is shifted to the side of the body, as far as, or even farther than, halfway to the posterior pole (Fig. 181); consequently the rows of cilia become displaced from their primitively meridional arrangement, and tend to run obliquely round the body. Secondly, a differentiation is set up between the general coat of cilia clothing the body and locomotor in function, and special cilia near or around the mouth, which are usually much longer than the others, and modified in various ways in connection with the function of foodcapture. The mouth itself becomes surrounded by a special area

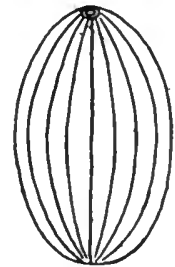

A

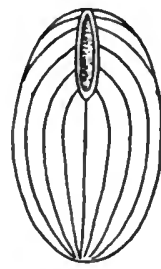

B

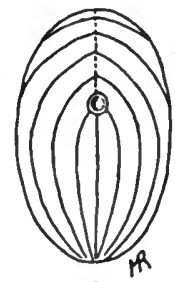

C
Fic. 181.-Diagram illustrating the shifting of the mouth, and the consequent displacement of the rows of cilia, in Cilista, from a form in which the mouth is at the anterior pole and the rows of cilia run a meridional course $(A)$, to a form in which the mouth is shifted to the side of the body $(C)$. After Delage and Hérouard. termed the "peristome," in which are found the special food-capturing cilià.

In forms of creeping habit the form becomes still more modified. The body becomes flattened, and a ventral surface, turned towards the substratum and bearing the mouth and peristome, is distinguished from the opposite or dorsal surface. Even more marked are the adaptations of the coat of cilia to this mode of life (Fig. 182). The locomotor cilia become restricted to the ventral surface, and those on the dorsal side either tend to disappear altogether or persist with a purely tactile function. The cilia of the ventral surface tend to form tufts which fuse into cirri (p. 55), with which the animal creeps as if on legs.

Sedentary forms may be attached temporarily by means of special cilia or adhesive organs, or more or less permanently by a portion of the body-surface on the side opposite to the mouth. In such forms (Fig. 183) the general coating of cilia may be retained, or may disappear entirely, only the peristomial cilia persisting; but locomotor cilia may be developed temporarily, enabling the animal to become detached from one spot, and to swim away and attach itself again elsewhere. In sedentary forms the point of attachment may be drawn out into a stalk, which may be of great length relatively, and may be a secreted structure or a portion of the body drawn out. In the second case the stalk may contain highly-per- 
fected contractile mechanisms, enabling the animal to stretch out a long way from the base of attachment, or to retract itself close to it. Sedentary forms may also secrete round themselves a proteotive sheath or tube.

Structure of the Body.-The mouth, or cytostome, is an aperture leading into a longer or shorter œsophagus, or cytopharynx, which ends blindly in the endoplasm. The indigestible remains of the food are cast out through a pore in the cuticle-a cell-anus, or cytopyge, which, though a permanent structure, is usually only visible at the moment of defæcation; but in some cases there is a distinct anal tube leading to an anal pore, visible at all times. In the Gymnostomata (see p. 439, infra) the mouth can be closed or opened by a system of rods contained in the wall of the osophagus (Fig. 184), which contains no vibratile apparatus; but in all other

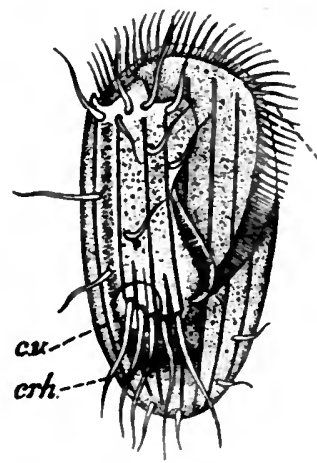

A

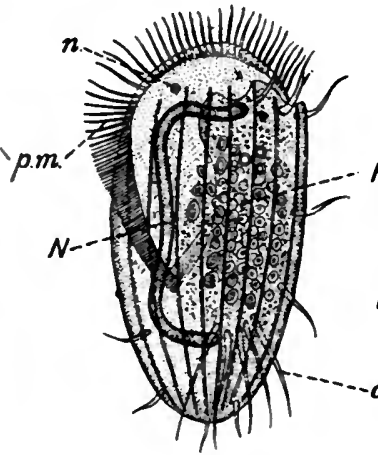

B

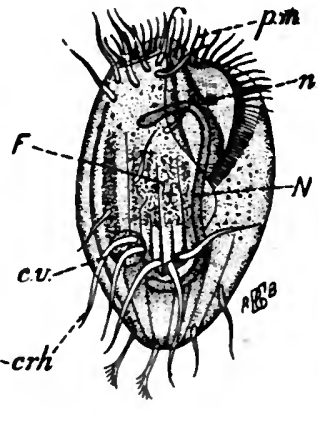

C

Fia. 182.-A and $B$, Euplotes patella: $A$, ventral view ; $B$, dorsal viow ; $C, E$ uplotes harper. In all the figures : $N$, macronucleus ; $n$, micronucleus; $c . v$, contractile vacuole; $c r h$, oirri ; p.m., peristomial membranella ; $F$, area containing foodvacuoles enclosed by the macronucleus. After Stein, the mioronuoleus added from original prepsrations.

Ciliata the mouth (if present) is permanently open, and the œsophagus has no rod-apparatus, but contains one or more undulating membranes. In the orders Heterotricha, Hypotricha, and Peritricha (see pp. 439, 440, infra), the peristome contains a spiral zone of cilis modified in various ways, leading to the mouth, and continuous with the undulating membrane in the cesophagus. In the two first of these orders the cilia in the adoral zone are generally fused in transverse rows to form membranellæ. In the Peritricha the adoral zone is composed of two parallel undulating membranes, and in this order the mouth, together with the anus and the contractile vacuoles, are sunk into a funnel-shaped or tubular depression called the "vestibule" (Fig. 183, V.). The two undulating membranes, after describing a spiral which varies from one and a quarter to five com- 


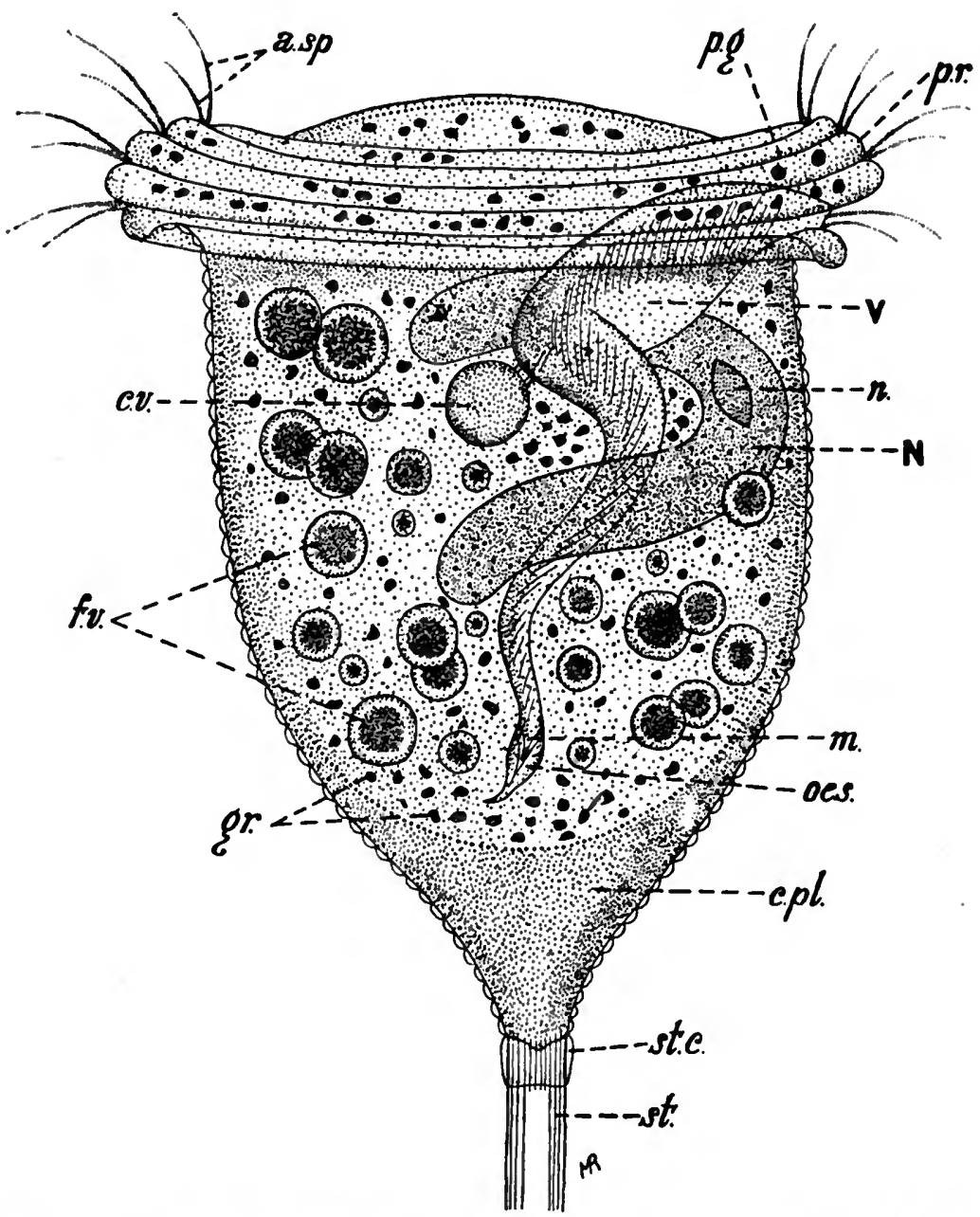

Fio. 183.-Campanella umbellaria. p.g., Peristomial groove in whioh runs the adoral spiral zone of oilia, which in this species takes $4 \frac{1}{2}$ turns; p.r., peristomial ridges between tho peristomial gnooves; a.s.p., the two undulating membranes, each made up of three rows of cilia fused, which compose the adoral spiral, scen in optical section; the two undulating membrancs pass down into the vestibule $(V$.$) , and run down inside it spirally as far as its$ termination at $m$., which represents the true mouth, leading into the short eytopharynx or œsophagus (œes.); $n$, mioronuoleus; $N$, macronucleus ; c.pl., cortical ectoplasm, thick at the base of tho body, thin at the sides; st.c., "collar" of the stalk ; st., stalk ; gr., granules in tho endoplasm which stain red with neutral red in the living condition; $f . v$., food-vacuoles; $c . v$, contractile vacuole opening by two canals into the restibule. After Schröder (864). 
plete turns, pass down into the vestibule, at the bottom of which is the mouth, leading into a short œsophagus (Fig. 183, $m$, œes.). The vestibule, into which the fæces and the excretions of the contractile vacuoles are evacuated, forms a sort of cloaca, combining, as it were, the functions of a stomodæum and a proctodæum.

The body of a ciliate Infusorian is composed of ectoplasm and endoplasm, the first of these two regions being highly differentiated and complex in structure. The surface of the entire body is clothed by a pellicle (Fig. 185, $p$.) - the most superficial differentiation of the ectoplasm-usually in the form of a thin, delicate membrane, which is sometimes, however, greatly thickened to form a cuirass or lorica. In addition to the mouth and anal pore already mentioned, the pellicle is perforated by the openings of the contractile vacuoles, one or more in number. The cilia also pass through the pellicle.

Beneath the pellicle the ectoplasm, in its full development, may be differentiated into four layers, which, however, are not all of them invariably present. The most external layer of the ectoplasm is the so-called alveolar layer (Fig. 185, al.), consisting of the outermost stratum of the alveoli of the protoplasmic framework, which take a regular arrangement, the walls between contiguous alveoli being disposed vertically to the pellicle, thus giving the appearance of a radially-striated layer. Within the alveolar layer

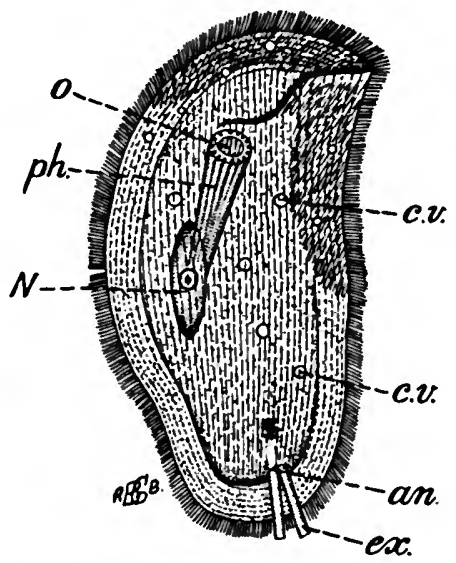

Fia. 184.-Chilodon cucullulus. 0 , Mouth ; ph., pharynx surrounded by a supporting apparatus of rods; $N$, macronucleus; c.v., c.v., contractile vacuoles; an., anus, temporarily visible during the extrusion of fæcal matter (ex.). After Stein. is found commonly a protoplasmic zone containing small, spindleshaped bodies-the so-called trichacysts (Fig. 185, tm.)-from which a long, stiff thread is discharged upon suitable stimulation. Within the trichocyst-layer comes a contractile layer, containing myonemes which run primitively beneath, or parallel to, the rows of cilia at the surface. The cilia themselves take origin from basal granules placed externally to, or between, the myonemes, and pass to the exterior between the alveoli of the alveolar layer. The most internal stratum of the ectoplasm is a spongy protoplasmic zone traversed by irregular spaces and channels containing fluid, and representing an excretory layer. The liquid from this region drains into the contractile vacuole or vacuoles. The smaller channels 


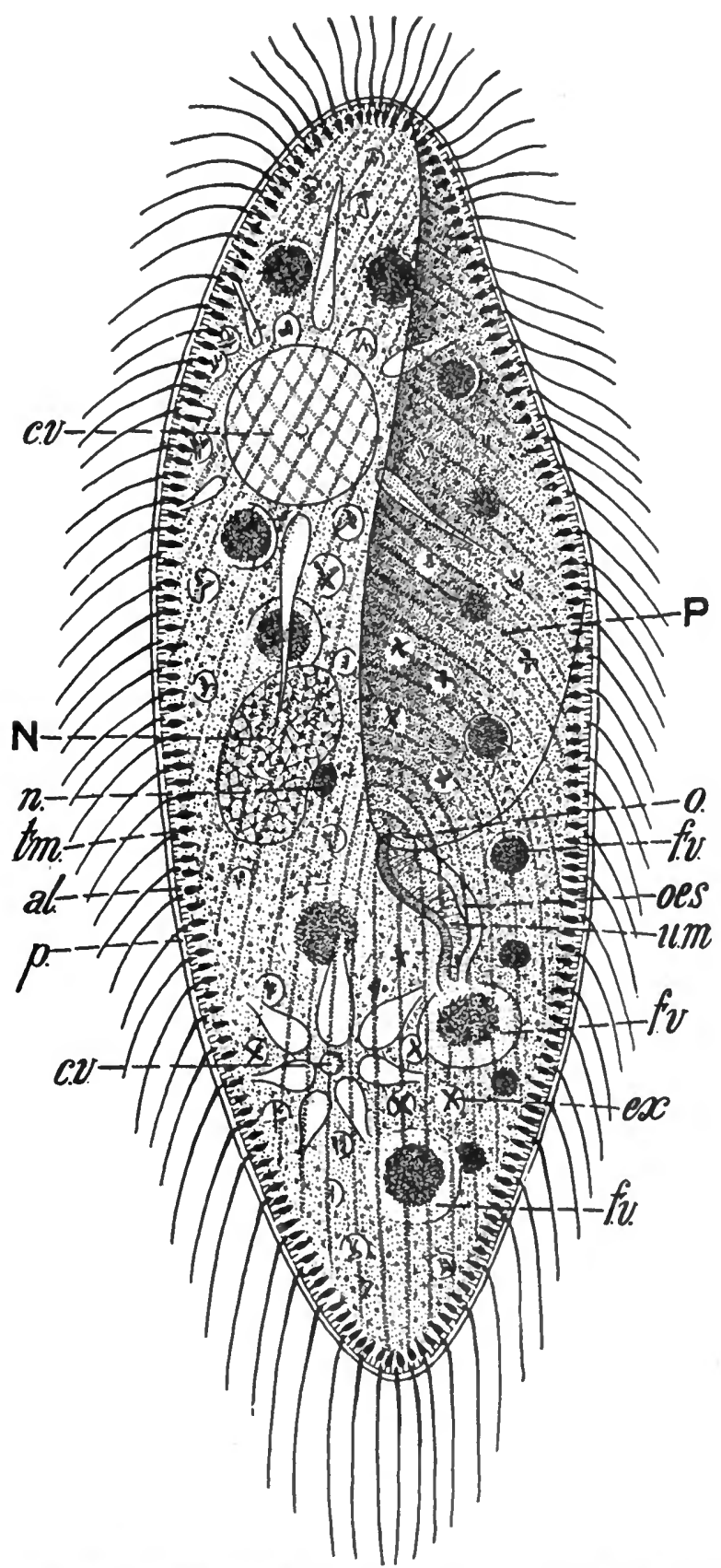

Fro. 185.-Paramecium caudatum: scmi-diagrammatic figure to show the structure. $P .$, peristomial groovo ; $o$, mouth ; as., œsophagus, containing an undulating membrane (u.m.) ; f.v.', food-vaouole forming at the base of the cesophagus; $f . v ., f . v$. , other food-vacuoles circulating in the endoplasm; c.v., c.v., tho two contractile vaouoles, showing a different condition in each, the upper one full and ready to empty itself, the lower one beginning to fill after a contraction; $e x$., excretory crystals in tho endoplasm ; $N$, maoronucleus ; $n$, mioronucleus ; $t m$., triohooysts; al., alveolar layer; $p$., pelliolo. After Lang (10), slightly modified. 
unite usually into more or less conspicuous main ducts-so-called "feeding-canals"-which empty themselves into a contractile vacuole.

The arrangement of the contractile vacuoles and canals varies considerably in different species. Thus, in Stentor (Fig. 8) there is a single contractile vacuole, with a feeding-canal running the length of the body ; so also in Spirostomum (Fig. 180, f.c.). In Paramecium (Fig. 185) there are two contractile vacuoles near each end of the body. The vacuole contracts suddenly, diminishing to a tiny globule, and then some six or eight feeding-canals make their appearance, arranged round the vacuole in a star-like figure, but at first distinct from the central vacuole. The inner ends of the feeding-canals gradually swell, and, after reaching a certain size, burst through and empty themselves into the central vacuole, which grows slowly to its full size, and as it does so the feeding-canals disappear by degrees from view. When the vacuole has reached its full size, it empties itself to the exterior, and the process begins again. The contractile vacuole itself may be considered as a centralized portion of the canal-system, and though when full it bulges into the endoplasm, it belongs strictly to the ectoplasm.

The endoplasm is the seat of nutrition, and also, as containing the nuclear apparatus, of reproductive processes. It is of fluid consistence, and exhibits streaming movements, termed "cyclosis" - that is to say, currents of protoplasm which flow round constantly in one direction, as if the endoplasm was being stirred round and round. The endoplasm contains enclosures of various kinds, chief amongst which are the food-vacuoles, containing ingested foodparticles in process of digestion. The food-vacuoles are formed at the base of the cesophagus, down which food-particles are wafted by the action of the adoral cilia and membranes. When full-sized, the food-vacuole becomes detached from the end of the cesophagus, like a soap-bubble from a pipe, and passes round the body in the currents of the endoplasm, the indigestible frcal residue being expelled finally from the anal pore (p. 433, supra). In addition to food-vacuoles, the endoplasm contains various metaplastrc grains, excretory granules, "spheroplasts" (see p. 448), and sometimes symbiotic algæ.

The nuclei are typically two in number-a large, conspicuous macronucleus, staining deeply ; and a micronucleus of much smaller size, often very inconspicuous, and difficult to stain. In primitive forms the macronucleus is a compact body, and the micronucleus appears as a small refringent globule close beside it, often lodged in a depression of the surface of the macronucleus'(Fig. 185, $N, n$ ). But the nuclei show very great variation in form, number, and appearance. The macronucleus may be drawn out into the shape 
of a sausage or of a horseshoe, as in Vorticellids (Fig. 183, $N$ ), or exhibit the form of a string of beads, as in Stentor (Fig. 8) and Spirostomum (Fig. 180); or there may be two macronuclei connected by a delicate filament, with a micronucleus beside each, as in Stylonychia; or, finally, the macronucleus may be broken up to form a diffuse network or a great number of small nuclei. The micronucleus may be single or multiple, but does not vary in form to any marked extent.

Life-History.-Reproduction takes the form of binary fission, usually in the free state; but some species become encysted prior to division, and then divide into two, four, eight, os a large number of small individuals within the cyst. Binary fission in the free state is, with few exceptions, transverse to the long axis of the body ; but in the fixed, sedentary forms the fission is usually in the vertical plane, or slightly oblique to it, and often takes the form of very unequal fission or budding. In some of the entozoic species of Astomata (p. 439), repeated transverse division of the body without complete separation of the daughter-individuals from one another leads to the formation of chains of individuals, of which the most anterior may be larger than the others.

As in other Protozoa, colonies may be formed in Ciliata as the result of imperfect separation of sister-individuals produced by fission. This is especially common in the sedentary Peritricha, leading usually to the formation of arborescent growths ; but sometimes the colony takes other forms, as, for example, in Ophrydium, where it consists of a great number of individuals embedded in a common mass of jelly which floats freely.

Encystment is related in various ways to the life-conditions of the Ciliata. Most frequently it appears to take place as a protection against desiccation in free-living forms, or as an adaptation to a change of hosts in parasitic forms. But in some cases it is related to the digestion of food, in others to reproductive processes. In some species it is stated to take place if the supply of food fails, and it can be induced artificially in various ways.

The process of syngamy has been described above (p. 152, Fig. 77). Summarized, it consists essentially of the following processes; some exceptions are described below :

1. Degeneration and ultimate absorption of the macronucleus of each conjugant.

2. Reducing divisions of the micronucleus to form four micronuclei, three of which are absorbed.

3. Division of the single remaining micronucleus into two pronuclei, one stationary, the other migratory.

4. Passage of the migratory pronucleus of each conjugant across into the body of the other conjugant, where it fuses with the stationary pronucleus. 
5. Separation of the conjugants; division of the synkaryon to form a new micronucleus and macronucleus.

Classification.-The Ciliata are divisible into two sections, which comprise in all four orders :

\section{Section A.-Aspirigera.}

Without a spiral zone of adoral cilia or membranellæ.

ORDER I. : HoLOTRICHA. - Cilia of approximately even length all over the body, forming a continuous, evenly-distributed coat in more primitive forms, arranged in bands or restricted to special regions in more specialized forms.

Suborder 1: Astomata.-Mouthless forms of parasitic habit. Opalina, Anoplophrya, Discophrya, eto. (soo p. 451).

Suborder 2: Oymnostomata.-Mouth a simplo pore, near or at the anterior pole of the body, leading into a simple, usually straight oesophagus without cilia or undulating membranes, often with a rod-apparatus by which the mouth is closed and opened for food-ingestion.

Classified in various ways; throo familics recognized by Doflein (7): (1) Enchelidae, including Holophrya, Prorodon (Fig. 14), Coleps, Didinium, etc. ; Buetschlia, parasitic in the rumen of ruminants. To the family Enchelida must be referred, apparently, the remarkable form described by Meunier sunder the name Aymnozoum viviparum, which is stated to have the following characteristics: The surface of the body bears no cilia, which appear to be wanting altogether in this form; the mouth-opening is at one extremity of the ovoid body, and contains an extrusible proboscis, used for the capture of prey (see p. 442); the micronuclcus is contained within tho macronucleus ; reproduction is by transverse fission, and also by internal budding, producing embryos which may produce in their turn other ombryos in a similar manner before boing liberated from the parent body, from which they are set free by dehisconce. (2) Trachclida, including Trachelius, Trachelocerca, Amphileptus, Lionotus, Loxoles, Dileptus, etc. (3) Chlamydodontida, including Chilodon (Fig. 184), Nassula, otc. (4) Foettingeriuda (Chatton, 831·5): Fettingeria, Perikaryon.

Suborder $3:$ Hymenostomata.-Mouth usually at tho side of the body and at tho bottom of a peristomial depression, leading into a short cesophagus never supportod by a rod-apparatus, but containing an undulating membrane; consequently not capable of being closed, but permanently open.

Families: (1) Chiliferida: Leucophrys, Glaucoma, Frontonia, Colpoda, etc. (2) Paramecidae: Paramecium (Fig. 185), etc. (3) Pleuronemida: Pleuronema (Fig. 27), etc. (4) Isotrichidoe! Isotricha, parasitic in the rumen of ruminants; and other families. (5) Microthoracida: Microthorax, Conchophrys (Chatton, 831·5).

\section{Section B.-Spirigera.}

With a conspicuous spiral zone of larger cilia or vibratile mombranes leading to the mouth; œesophagus as in Hymenostomata.

ORDER II.: HETERotricha.-Generally of swimming habit, sometimes sedontary.

Suborder 1 : Polytricha.-Body covered with an even coat of cilia.

Principal familics: (1) Plagiotomidar; example: Spirostomum (Fig. 180). (2) Bursarida, examples: Bursaria; Nyctotherus (Fig. 9), with species entozoic in various animals; Balantidium, also entozoic. (3) Stentorida; example: Stentor (Fig. 8). (4) Tintinnide (compare Entz, 53); examples: Tintinnus, etc.

Suborder 2: Oligotricha.-Body-cilia greatly reduced or absent.

Familios: (1) Halteridce; example: Halteria. (2) Ophryoscolecida, with numerous genera parasitic in the stomachs of ruminants; examples: Ento. dinium, Ophryoscolex, Cycloposthium. 
OrDer U. : HYPotricha. - Ciliata typically of creeping habit; the body flattened, with dorsal and ventral surfaces, the ciliation highly modified and specialized, usually with cirri on the ventral surface.

Principal families: (1) Peritromida, with cilia on the ventral surface; example: Peritromus. (2) Oxytrichida, with cirri ; examples: Oxytricha, Urostyla, Stylonychia. (3) Euplotidae; example: Euplotes (Fig. 182).

ORDer IV.: Preriricha.-Typically of sedentary habit, the locomotor cilia reduced to a single ring or absent temporarily or permanently; the adoral spiral runs down into a deep depression, the vestibule, into which open the anus and contractile vacuoles, and at the base of which is the mouth, lesding into the cesophagus.

Suborder 1 : Scaiotricha.-The adoral zone describes a left-handed spiral.

Two families: (1) Spirochonida : Spirochona, ectozoic on the gill-plates of Cammarus pulex, has a non-contractile body which bears at the upper extremity a spirally-folded membranous funnel, on the inner side of which is a zone of cilia. Allied genera are Kentrochona and Kentrochonopsis, both ectozoic on the gill-plates of Nebalia. (2) Licnophorida ; example : Licnophora, ectozoic on various marine animals (one species entozoic in the respiratory trees of Holothurians) ; attachment by a sucker-like dnsc.

Suborder 2: Dexiotricha. -The adoral zone describes a right-handed spiral.

Family : Vorticellida, with three subfamilies: (a) Urceolarince, unstalked, attached temporarily by a sucker or disc, surrounded by a persistent zone of locomotor cilia; examples: Trichodina, Cyclochoeta. (b) Lagenophryina; example: Lagenophrys. (c) Vorticellina, with numerous genera: Vorticella, Carchesium, Zoothamnium, etc., with contractile stalks; Epistylis, Opercularia, Campanella (Fig. 183), Ophrydium, etc., with non-contractile stalks ; Cothurnia, Vaginicola, with sheaths ; Scyphidia, freo-swimming.

The entozoio Ciliate exhibit two different methods of nutrition : first, the holozoic method, in which the animals ingest solid food-particles, like the free-living species, and possess in consequence a distinct mouth and contain food-vacuoles in their interior; secondly, the osmotic method, seen in the astomatous forms, which absorb fluid nutriment by diffusion from their host, and in which a mouth is rudimentary or absent and food-vacuoles are not found. The Ciliata of the astomatous type represent the truly parasitic forms, a familiar example of which is the genus Opalina, with species parasitic in the common frog and other vertebrates. Common entuzoic genera of the holozoic type are Balantidium and $N$ yctotherus, found in the digestive tracts of various animals; such forms are perhaps for the most part scavengers; according to Comes (A.P.K., xv., p. 54), Balantidium nourishcs itself exclusively on red blood-corpuscles, which are set free in the intestine from wounds caused by other parasites, especially Trematodes. Species which inhabit the human intestine are Balantidium coli, B. minutum, and Nyctotherus faba.

On the other hand, ciliates may crop up in cultures of human fæces, which, like the amœbre and flagellates found there, are not to be regarded as inhabitants of the human intestine, but as free-living forms which have passed through the digestive tract in an encysted condition without being destroyed, and germinate when set free from the gut. An example is Chilodon dentatus (uncinatus), described by Guiart from human faces; possibly also the ciliates described by Martini (850) in a case of dysentery.

The freo-living Ciliats exhibit, as a rule, great uniformity of character in the active state, occurring constantly in one specific form which only varies slightly in size under natural conditions; they are, in fact, as nearly as possible monomorphic. Some of the parasitic forms exhibit, however, a well-marked recurring cycle of forms in relation to the special necessities of their mode of life, as is described below (p. 450). In some free-living forms also different forms occur in the same species. Tho small free-swimming conjugants (gametes, so called) of the sedentary Vorticellids have been noted above (p. 172). In Leucophrys patula, a free-swimming species, large and small individuals occur; but, according to Prowazek (861), 
this dimorphism has no relation to sex, but only to differences in the surrounding medium; he states that by the addition of quinine $(1: 80,000)$ to a culture of the small forms he was able to bring about the appearance of the large forms.

The body of a ciliate is often prolonged into processes, spikes, etc., giving the animal a curious appearance. The most bizarre forms are found amongst the species entozoic in the digestive tracts of ruminants, such as Ophryoscoler, Entodinium, etc.; but some free-living species also exhibit peculiarities of external form. Actinobolus radians (Holotricha) has the body covered with tentacle-like processes, each bearing a trichocyst at the extremity. Legendrea loyeza (Fauré-Fremiet), allied to Prorodon, bears on the left side of the body about twenty digitiform processes of variable length, flexible but not motile ; each process is composed of clear protoplasm enveloped by the pellicle, and at its slightly dilated extremity is lodged a bundle of trichocysts. Hastatella radians is a free-swimming Vorticellid which bears two circlets of pointed "fulcra," or spines, one circlet on the external border of the peristome, the other about the middle of the body (Collin).

The pellicle may be greatly thickened to form a lorica, as in Coleps, where it is composed of a series of plates; or may be decorated with warts or sculpturings of various kinds, formed as local thickenings, as in some species of Vorticella, etc.

The body is often enveloped in a protective sheath or envelope secreted by the animal, especially in sedentary forms. The animal may then be capable of protruding its body from the sheath, and retracting itself back again into it, and when retracted the aperture of the sheath may be closed by a special lid, or operculum. In the Tintinnida, some species of which are free-swimming, others sedentary, the body secretes a shell or house, to which foreign bodies may bo added, derived for the most part from the fæcal pellets of the animal itself ; the structure of these shells has been studied in detail by Entz (53).

The sedentary habit of life occurs in species of all orders, though especially characteristic of Peritricha. The mechanism of fixation varies greatly in different cases. Stentor attaches itself by cilia, and also by pseudopodial processes thrown out from the point of fixation, and from this type is to be derived that of the Tintinnida (Faure-Fremiet, 836).

The hypotrichous genus Ancystropodium (Oxytrichida) swims freely or attaches itself by its posterior cirri ; the body is then drawn out at the hinder end into a long stalk (Fauré-Fremiet, 837). Trachelius ovrum possesses a conspicuous, suckor-like organ by means of which it attaches itself to the stalks of Epistylis-colonies, in order to devour the members of the colony (Hamburger, 841).

In the Peritricha the attachment may be permanent or temporary; in the latter case the animal fixes itself by a sucker-like organ of the aboral pole. In Trichodina the adhesive organ is surrounded by a ring of cilia ; in Cyclochata there is an additional circlet of stiff bristles; in Licnophora the disc of attachment is in the form of a cup surrounded by four concentric ciliary membranes (Stevens, 872); these three genera, and others with similar modes of attachment, are ectozoic forms, attaching themselves to the skin of various aquatic animals. Fauré-Fremiet (834) has traced the evolution of the permanently fixed Vorticellid type from temporary fixation by an aboral sucker. As a starting-point is taken Hemispeira asteria, which attaches itself to the gills of Asterias by a bundle of fixative cilia. In Scyphidia fixation is by a cup. like sucker containing a circular brush of rod-like processes, equivalent, apparently, to the fixative cilia of Hemispeira. For this brush-like organ the term "scopula" is proposed; the cilia in it have lost their motility and secrete a terminal chitinous knob. Epistylis fixes itself in a similar way by means of a scopula, of which each rod forms a secretion of albuminoid naturo (Schröder, 865), which grows continually, forming a bundle of delicate tubes composing the stem, and ensheathed by an outer covering secreted by a rim round the scopula; the stem that results is a non-contractile structure representing a secretion of the body, and not a prolongation of the body itself. The contractile stalk of Vorticella, Carchesium, etc., arises by an out- 
growth of the central part of the scopula on a prolongation of the bodysubstance, leaving a peripheral ring of scopular rods surrounding a central protoplasmic cord, which furnishes the contractile muscular stalk.

The mouth and cytopharynx, whether capable of being closed, as in the Gymnostomata, or permanently open, as in other forms, constitute together a conspicuous organ in the holozoic Ciliata, sometimes showing remarakble adaptations to special modes of feeding. In the gymnostomatous genus Didinium the eytopharynx contains a peculiar tongue-like organ, a prolongation of the endoplasm, which shows a longitudinal striation due to the presence of fine rods- " trichites." The tongue of Didinium is used for capturing prey, consisting chiefly of Paramecium and other Ciliata, and the manner.in which it is used recalls the tongue of a chameleon. If the Didinium comes into contact with its prey, the tongue is shot.out by a violent contraction of the pharynx, and adheres to the victim, which, aceording to Thon, is killed instantly ; but according to Mast larger Ciliata sometimes escape, and in doing so may break off and earry away the tongue of tho Didinium. A Paramecium when thus attacked emits a cloud of trichocysts, but none are discharged by the Didinium. Tho prey when mastered is drawn into the endoplasm by the retraction of the tongue. The recently-described genera Proboscidium and Gymnozoum (Meunier) possess similar organs. The hymenostomatous genus Pleuronema and allied forms are remarkable for the huge size of the un. dulating membrane (Fig. 27). The animal, after swimming freely for a time, comes to rest, with its body-cilia sticking out stiff and straight; the undulating membrane is then protruded from the mouth, and by its active movements serves to waft food-particles into the pharynx.

The peristome, or region round the mouth, exhibits a wide range of special adaptations in relation to the function of food-capture, as is apparent from the classification given above. Absent or scarcely developed, as a rule, in the Gymnostomata, in the Hymenostomata it has the form, usually, of a simple groove leading to the mouth (Fig. 185, P.); in the Spirigera, on the other hand, it is generally disc-like, bearing the adoral zone which terminates in the mouth ; the extreme type of complication is seen in the Peritricha, where the peristomial disc can be contracted completely over the mouth by means of circular myonemes situated in the margin of the disc like a sphineter (see below), while a central funnel-shaped portion is prolonged inwards, with the mouth at its extremity to form the vestibule, in a manner analogous to the stomodæum of the Metazoa. The adoral ciliary spiral may consist simply of longer cilia, moro poworful than those of the general body-covering, the inost primitive condition; or of transversely-planted, comb-like membranellæ or "pectinellæ," the usual arrangement in Heterotricha and Hypotricha ; or of a pair of undulating membranes running parallel to one another in the spiral, as in Peritricha. These various structures, seen in optical section in the living state, have often produced erroneous impressions of bristles, cirri, etc. The adoral spiral varies greatly in extent, and the peristomial region shows numerous modifications which cannot be described or summarized briefly; the reader must be referred to the beautiful descriptions of Schröder (864-867), amongst recent witers. In the remarkable peritrichous form Opercularia (Cochlearia) faurei, the adoral spiral takes five complete turns, running like a screw round a sort of retractile proboscis (Collin, 832).

The ciliary apparatus and its modifications (Fig. 186)-have been the subject of much minute and detailed study; among recent investigators must be mentioned especially Maier (73) and Sehuberg (44). The body-cilia run in rows with a meridional, spiral, or other arrangement; they arise in depressions of the body-surface which have usually the form of furrows, but in some cases (Paramecium, Frontonia) each eilium arises from the centre of a small depressed area of the surface. In Paramecium the areas are for the most part hexagonal in form, but in places they are rhombic ( $c f$. Khainsky, 170.5). The points of the trichocysts are situated in the angles of the polygons, and also in the broader edges between the areas in each row.

Each cilium takes origin from a basal granule situated at the level of the 
myonemes, or just external to this level, below the alveolar layer (Fig. 186, $B, D)$; the cilium passes outwards in the edges of the alveoli-that is to say, along the lines in which the walls of contiguious alveoli touch at their cornersand pierces the pellicle to pass to the exterior. In Anoplophrya, Collin (50) describes root-like fibrils which pass inwards through the endoplasm, and are inserted on the membrane of the macronucleus. Khainsky (170.5) also describes fibrils passing inwards from the basal granules of the cilia of Paramecium. In the remarkable form Pycnothrix monocystoides, which possesses an ectoplasm of great thickness and distinctness, there are, according to Schubotz (868), two layers of basal granules, one more superficial, the other deeper. Each granule of the outer layer gives off a cilium on its outer side, and on its inner side a fibril connecting it to a granule of the deeper layer, from which, again, a fibril passes inwards and becomes directly continuous with a myoneme.

The free cilium shows, according to Schuberg (44), a distal "end-piece," which stains more lightly and is of finer calibre, and a basal, thicker, and darkly-staining portion (Fig. 186, $A$ ) ; the basal portion is of eveh thickness, and is about double the length of the distal end-piece, which tapers to a fine point. Motile cilia are not stiff, but change their form by bending in a helicoid spiral, or in a portion of such a spiral, like the flagella of the Flagellata and of spermatozoa. Cast-off cilia often coil up at their proximal end into a fine loop.

A cilium is composed of two different parts-an elastic axial filament of firm consistence covered by a sheath of more fluid contractile substance. According to Khainsky (170.5), the sheath of the cilium is in continuity with the substance of the pellicle. The end-piece represents the axis exposed and continued beyond the sheath. The axis is compared by Schuberg to that of the axopodia (p. 48), and is the form-determining element for the fluid sheath. Cilia perform active movements even when separated from their basal granules, which are not to be regarded, in Schuberg's opinion, as kinetic centres; the movements caused by the fluid envelope are probably due to alterations in surface-tension (p. 200, supra).

The basal granules of the cllia aro not regarded by Maier or Schuberg as centrosomic in nature. Maier considers that they probably arise as cytoplasmic bodies at the surface of the cell, and arc to be interpreted as special thickenings at the roots of the cilia; Khainsky $(170.5)$ takes a similar view. In this connection, however, attention should be drawn to the observations of Entz (53), who finds that in the division of Tintinnida the new peristomo arises in the interior of the cytoplasmic body as a split or cavity, and that the basal granules appear first, the pectinellæ later; the basal granules are stated to be formed in connection with the nuclear apparatus, and their substance to be formed either from the macronucleus or micronucleus. Tho connection between the basal granules and the macronucleus described by Collin (50) would scem also to indicate a nuclear origin for them. The question of their centrosomic nature must remain, therefore, open for the present. According to Schuberg, the basal granules of each row of cilia aro connected with one another by a fine longitudinal fibril.

The typical motile cilia described above become modified in various ways, chiefly by fusion of separate cilia to form more complex structures. The stiff, tactile bristles have precisely the same structure and mode of insertion as the ordinary cilia (Fig. 186, $H, t . c$.), and in this case the change is purely one of function or substance rather than of perceptible cytological structure. The undulating membrancs found in the pharynx of the Hymenostomata are formed by fusion or adhesion of a single row of cilia, of which the basal granules are ranged in a series to form a "basal rim " (Basalsaum) from wbich the membrane takes origin (Fig. 186, E, u.m.). According to Schuberg, the fibrils of which the membranes, membranella, etc., are made up correspond, not to a whole cilium, but to its axial portion alone. Sumetimes, however, more than one row of cilia contribute to the formation of an undulating membrano; the two mombranes which compose the adoral 


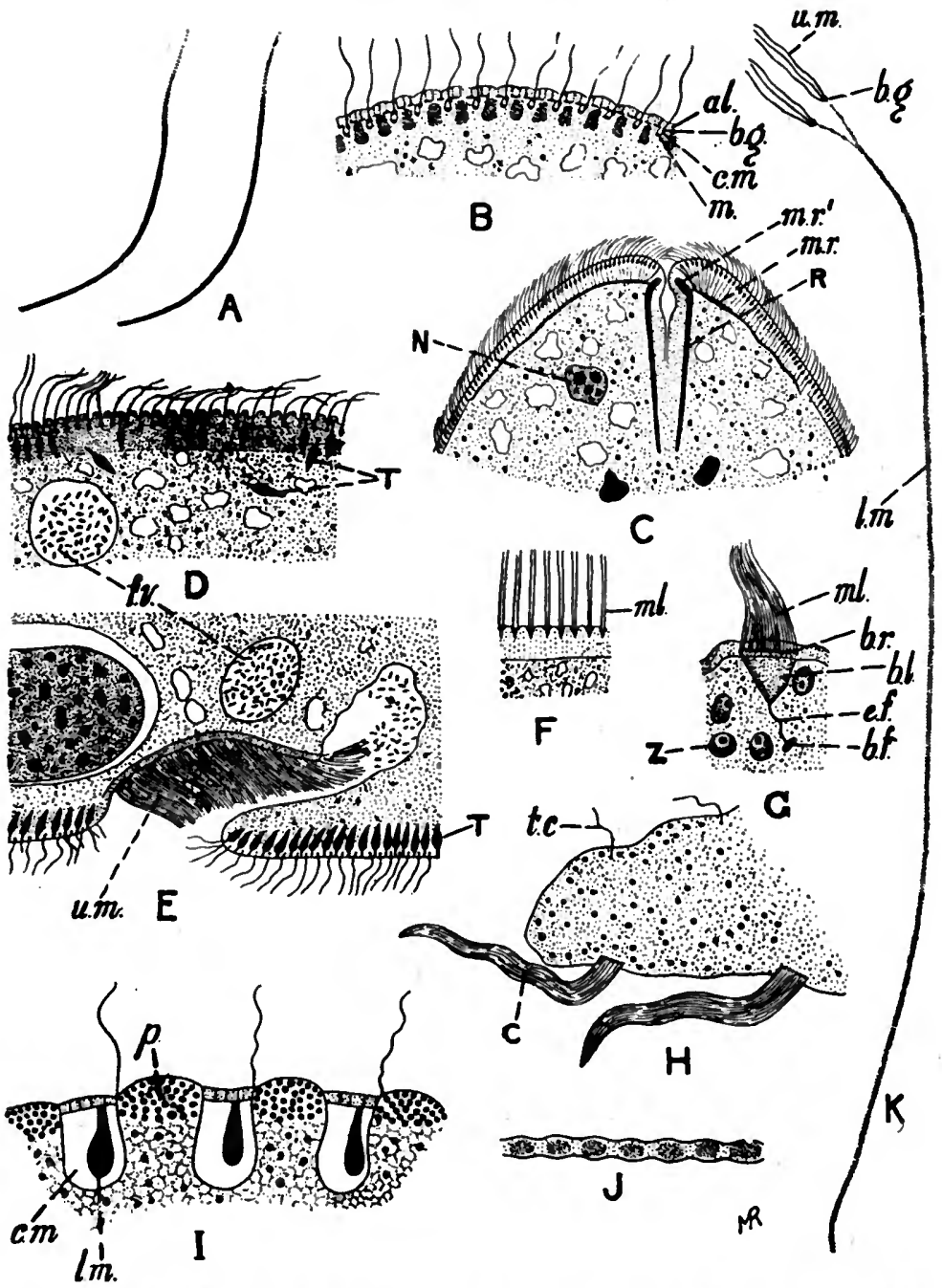

Fia. 186. -Details of the structure of the ciliary and contractile apparatus of Ciliats. A, Two isolated cilis of Stentor'coeruleus, showing the deoply-stained proximal portion, of even thickness thronghout, and the lighter distal portion, tapering to a point ; magnified 2,250 diameters; $B$, section through the surface of the body of Prorodon teres, showing the cilis arising from basal granules (b.g.), situated above canals $(c, m$. ), at the hase of which run the myonemes $(m$.$) ,$ seen in transverse seotion; $C$, section through the mouth of Prorodon teres (Fig. 14), showing the rod-apparatus $\left(R_{\text {. }}\right)$, each rod with two myonemes (m.r., m.r. ${ }^{1}$ ) ; $N$., nucleus ; $D$, section of the body-surface of Paramecium caudatum, showing the cilia arising from basal grains: T., triohooysts; $f . v$, food-vacuole; $E$, section through the mouth and cesophagus of Paramecium caudatum, showing the undulating membrane (u.m.) in the casophagus: other letters as in $D ; F$, scotion through the adoral zono of $N y$ ctotherus cordiformis, showing the membranella ( $\mathrm{ml}$.) out across, each composed of two cilia arising from a pair of basal granules; $G$, section of the adoral zone of Stentor niger, showing a membranella $(\mathrm{ml}$.$) , composed of fused oilia arising$ 
spiral of Vorticellids are formed each by the concrescence of three rows of cilia (Fig. 186, K, u.m.) ; in Glaucoma scintillans there is a " peroral " mem. brane built up of five rows, an "endoral " membrane of ten rows of fused cilia (Maier, 73).

The membranellæ of the adoral zone are formed each by the concrescence of two transverse rows of cilia (Fig. 186, $G, \mathrm{ml}$.). In some genera (Stentor, Spirostomum) the basal rim of each membranella is continued down into the endoplasm in the form of a fibrillar plate, triangular in form, with the apex continued into a terminal filament, which is attached at its proximal extremity to a fibril running longitudinally, parallel to the row of membranella. The nature of this basal fibril has been much discussed; it has been regarded as a nervous element, co-cordinating the movements of the membranellæ; Maier, on the other hand, regards the basal lamella and its terminal filament as serving for the firmer attachment of the membranellæ, and considers the basal fibril to be a contractile element; Schröder states that the basal fibril is really a broad band, and believes its function to be purely mechanical ; Schuberg rejects the nervous theory of the basal system of the adoral zone of Stentor, but comes only to negative conclusions with regard to its function.

The cirri of the Hypotricha are formed by concrescence of a tuft of cilia arising from a number of basal granules which are arranged to form a basal plate (Fig. 186, H, C.). The posterior ciliary ring of Vorticellids is composed of " membranulæ" (Maier), each formed by concrescence of a single row of cilia, three in each row. The two circlets of Didinium are also membranula (Thon).

Closely connected with the bases of the cilia in position, and with the ciliary apparatus in their general arrangement, are the myonemes. The most superficial study of the Ciliata suffices to convince the observer that these animals have in many cases an extremely cfficient contractile system. Such forms as Stentor, Vorticella, etc., contract with such lightning rapidity that it is almost impossible to kill and preserve them expanded; the spasmodio action of their contractile organs contrasts sharply with the slow contractility of lower Metazoa, such as polyps. Trachelocerca, according to Lebedew (93), contracts in an instant to one-twelfth of its length when expanded.

In their primitive arrangement the myonemes run parallel to the rows of cilia, immediately beneath the basal granules or close beside them (Fig. 186, $B, m$.). In Stentor the myonemes are broad and band-like, and composed of alternating light and dark portions (Fig. 186, J); they are lodged in canals below the alveolar layer, running in the intervals between the "ribs" or pigmented strips of the body-surface; the rows of cilia run above each myoneme-canal, slightly to the side of it (Fig. 186, I). The myonemes run the length of the body, from the foot to the adoral zone of membranella. At the extremity of the foot they bend inwards and form a cone or "foot.

Fig. 186 continued:

from a number of basal granules in a row forming the basal rim (b.r.); below the basal rim is the basal lamella (b.l.), continued at its apex into the endfibril (e.f.), which passes down to the basal fibril (b.f.), seen cut in transverse section : Z, zooohlorella; $H$, part of a section of the body of Stylonychin histrio, showing two tactile cilia (t.c.) on the dorsal surface, and on the ventral surface $t$ wo cirri $(C$.$) , each composed of a fused tuft of cilia arising from a basal$ plate of granules; $I$, section of the body-surface of Stentor carvleus, showing the longitudinal myonemes (l.m.) lodged in cansls (c.m.) between the pigmented " ribs" $(p$.) of the outer surface; $J$, one of the longitndinal myonemes of Stentor in surface view. showing the alternating light and dark portions; $K$, detail from a longitudinal section of Epistylis plicatilis, showing the two undulating membranes (u.m.) of the peristome in transverse section, osoh composed of three fused cilia a rising from three basal granules (b.g.) fused together ; from each basal plate arises a fibril ; the two fibrils join and become continuons with one of the longitudinal myoncmes running down the body to the stalk.

$A$ after Schuberg (44); $B-H$ after Maier (73); $I, J$, after Schröder (867); $K$ after Sohröder (865). 
plate." At the upper end of the body fine continuations of the longitudinal myonemes can be traced to the adoral zone, ending in the basal rims of the membranellæ (Schröder, 867).

Stentor may be taken as a type showing the contractile system highly developed in functional efficiency, but more or less primitive in arrangement. Canals lodging the myonemes are not present universally, oven in highly contractile forms; they are absent, according to Lebedew (93), in Trachelocerca, but they are figured by Maier (73) in Prorodon teres (Fig. 186, B). In their general form the myonemes are simple fibrillæ, often beaded when contracted.

In the more specialized forms the contractile system acquires a more oomplicated arrangement. In Campanella, Schröder (864) describes five systems of myonemes: (1) Alinular myonemes of the basal part of the body; (2) longitudinal myonemes of the outer body-wall, doubtless representing the primitive system (Fig. 186, $K$ ); (3) annular myonemes forming the sphincter-like muscle of the margin of the peristome; (4) a spiral myoneme running under the adoral spiral, and continued down the wall of the vestibule; (5) a series of retractor-myonemes of the peristomial disc. In Epistylis plicatilis, on the other hand, Schröder (865) found only three systems: The longitudinal myomenes (2), the annular peristomial myonemes (3), and the vestibular myoneme (4). To these systems found in the Vorticellids with non-contractile stalks must be added, in the genera Vorticella, Carchesium, etc., the powerful stalk-muscle ("spasmoneme") formed by the union of the longitudinal myonemes (Schröder, 866). In Vorticella monilata fine connections run from the hinder ciliary ring upwards and downwards to the longitudinal myonemes when the cilia are developed, but disappear when these cilia disappear. In Licnophora, according to Stevens, the fibril that runs under the adoral spiral is continued down to the disc or cup of attachment and ramifies in its walls.

In the aberrant form Pycnothrix monocystoides, Schubotz describes a remarkable development of the myonemes in the form of a dense plexus of fibrils at the inner limit of the ectoplasm. The fibrils are connected with the basal granules of the cilia, and run in two directions, forming a deeper layer of circular myonemes and a more superficial layer of longitudinal myonemes.

The question has been much discussed whether the contractile system, often so highly developed, is accompanied by any conducting elements of nervous nature. That many ciliates react with extreme rapidity to stimuli has been noted above, and that their movements are co-ordinated is sufficiently apparent. Neresheimer (856) describes in Stentor filaments believed to be of nervous nature, neuronemes which take origin from the foot and run about halfway up the body, at which point each neuroneme either ends in a bulbous swelling or becomes thinner and disappears. The neuronemes are situated externally to the myonemes, and run parallel to them. By experiments with various drugs, Neresheimer tried to prove the existence in Stentor of true nervous elements, as compared with Paramecium and other forms in which neuronemes were not found, and concluded that the elements described by him were truly nervous in nature. Schröder (867) casts doubt on the existence of neuronemes and criticizes Neresheimer's technique. Lebedew (93), however, describes fibrils, possibly nervous in nature, running parallel to the myonemes in Trachelocerca.

For the present the existence of nervous clement.s in Ciliata must remain doubtful. But of the sensory function of the cilia there can be hardly any doubt, and the fact that their basal granules are always in close proximity to the myonemes is extremely significant. Such a direct contact between the sensory and contractile mechanisms may render conducting elements of nervous nature unnecessary, except for purposes of co-ordination of movements. In somo cilia, as alrcady stated, the motile function is lost, and only the sensory function remains. The genus Mycterothrix (Trichorhynchus) is characterized by a rostrum bearing a number of stiff, tactile cilia (FaureFremiet, 839). In some cases, however, sensory organs occur which appear not to be derived from cilia, as, for example, the tentacle-like or club-shaped 
organs, probably tactile, between the membranellæ of the adoral spiral of Tintinnidae (Schweyer).

The nature and mechanism of the peculiar trichocysts remains to be explained. The trichocyst in the unexploded state is a spindle-shaped body with a fine, hair-like process at its outer end which reaches to tho pellicle (Fig. 186, D, T.). The exploded trichocyst tapers gradually to a sharp point at its proximal end; distally jt shows a cap-like swelling (Fig. 187, $D-G$ ). According to Khainsky $(170.5)$ the trichocyst consists of two portions: a distal or outer part which stains deeply, and a proximal or inner part which stains a lighter colour (Fig. 187, $A-C$ ).

The unexploded trichocyst consists entirely or almost entirely of the darker substance; in the process of explosion the dark substance is converted into the light, so that in the exploded trichocyst only a small portion of the dark substance remains to form the distal cap. The notion, recently upheld by Mitrophanow (855), that the trichocyst consists of viscid fluid contained in a cavity in the ectoplasm, whence it is expelled by a sudden contraction of the ectoplasm, and stiffens to a solid thread under the action of the watery medium, cannot be maintained (Schuberg, 44); nor does there seem to be any ground for comparing it to a Colenterate nematocyst or to a polar capsule of a Cnidosporidian spore. According to Mitrophanow, the substance of the trichocysts appears first near the nucleus in the endoplasm as small grains which pass out into the ectoplasm. Trichocysts do not occur in any Peritricha, but in one species, Eprstylis umbellaria, large oval nemstocysts occur, arranged in pairs-a phenomenon unique amongst the Ciliata.

The contractile vacuoles open to the exterior as a general rule, but in the Peritricha, \& s already stated, they open into the vestibule; in this order there is usue lly a roservoir.

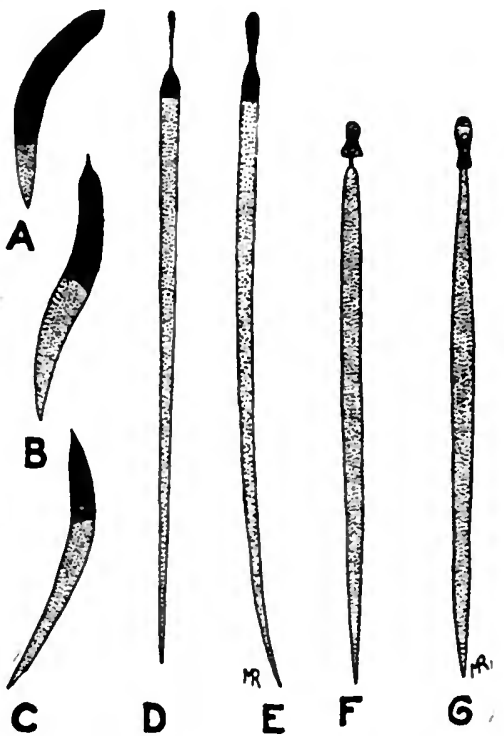

Fia. 187.-Trichooysts of Infusoria. $A-E$, Stages in the explosion of the trichocysts of Paramecium caudatum, showing the manner in which the trichocyst grows in length, with conversion of a darkly-staining substance in to a lighter material ; the fully-exploded trichocysts are seen in $D$ and $E$. After Khainsky (170.5). $F, G$, Exploded trichocysts of Frontonia leucas. After Schuberg. magnified 1,500 diameters. vacuole into which one or two con. tractile vacuoles ompty themselves, and which in its turn voids its contents into the restibule. In Campanella, however, there is no reservoirvacuole, and the single contractile vacuole opens by bwo canals into the vestibulo (Schröder, 864). In Opulina there are no contractile vacuolcs, and in some species (e.g., O. ranarum) no excretory organs are to be found; but in other species the endoplasm contains an axial series of more or less irregular vacuoles, opening one into the other and to the exterior by a pore at the posterior end of the body. These vacuoles are sometimes in close relation with the nuclei, often enveloping them to form a perinuclear space (Metcalf, 852). In Pycnathrix monocystoides the endoplasm is traversed by a branched system of excretory canals, which unite into a single efferent duct opening at the surface of the body near the posterior end by a pore; the duct is ciliated, and is homologized by Schubotz with the cytopyge, which in Nyctotherus is 
also ciliated. These excretory systems of Opalina and Pycnothrix differ in being endoplasmic from the ordinary contractile vacuoles, which are always formed in the ectoplasm.

The endoplasm of the Ciliata may contain enclosures of various kinds: food-vacuoles; metaplastio bodies in the form of excretory grains, crystals, pigment-grains, etc. ; zoochlorellæ, and occasionally parasites of one kind or another, etc. Special attention has been drawn by Fauré-Fremiet (38.5 and 835) to the bodies termed by him spheroplasts, and considered by him to be homologous with the mitochondria (p. 41). The bodies in question are small spherules, which multiply by fission when the cell-body divides; they are permanent cell-organs to the same extent as the nuclear apparatus, of which, however, they are entirely independent.

As pointed out above, the form of the macronucleus and the number of nuclei vary greatly in different species. The cases will be considered below in which the micronucleus appears to be wanting (Opalina), or is contained in the macronucleus in the ordinary condition of the body (Trachelocerca, Ichthyophthirius). As a rale the macronucleus has a finely granular appearance, with the chromatin distributed evenly over the nuclear framework; but in a fow cases it. has a distinctly vesicular structure, with a large karyosome, as in Loxodes (Joseph, Kasanzeff), Chilodon (Nägler, 96), eto. The macronucleus divides by binary fission of a simple and direct type (Fig. 54) The micronucleus, on the other hand, divides by mitosis (Fig. 61). In Trachelocerca, a form which may possess one or many nuclei (but no separate micronuclei), Lebedew (93) describes a peculiar mode of multiplication of the nuclei, which divide by multiple fission to form a morula-like body consisting of a mass of small nuclei which separate from one another (Fig. 66). In Loxodes, another form in which the number of nuclei varies greatly in different specimens, the macronuclei do not divide, but only the micronuclei do so, and the macronuclei arise by growth and modification of the micronuclei (Kasanzeff). In many cases in which the macronucleus is of the elongated moniliform type, or in which the body in the ordinary state contains two or more macronuclei, they come together to form a single compact macronucleus prior to division; but in other similar cases this does not occur, and when the body divides the nuclei are distributed irregularly between the two daughter-individuals, as in Trachelocerca, Opalina, etc. The distributed form of nucleus is especially characteristic of the astomatous parasitic forms, and in the opinion of Pierantoni (A.P.K., xvi., p. 99) is correlated with nutrition by the osmotic method.

The micronucleus is less variable in form or number, as a general rule, than the maaronucleus, but is not infrequently multiple, especially when there is more thsn one macronucleus; but in Trachelius ovum a single large macronucleus is combined with thirteen micronuclei (Hamburger, 841).

The conjugation of the Ciliata conforms, as a general rule, in its main outlines to the scheme sketched out above (Fig. 77), but some important variations must be noted. In the first place, the conjugation is often preceded by active division of the animals, so that the conjugants* are much smaller than the ordinary individuals of the species. When the two conjugants come together, the micronucleus of each usually divides into four, but sometimes into eight, as in both conjugants of Euplotes and the microconjugant of Peritricha ; in either case, however, only one micronucleus persists, and furnishes the two pronuclei.

The Peritricha exhibit in their conjugation certain peculiarities which are clearly of a secondary nature and correlated with their sedentary habit. Certain individuals divide two or three times successively to produce four or eight microconjugants (" microgametes") which acquire a ring of locomotor

* It is preferable not to speak of two conjugating Infusoria as gametes, since it is very doubtful if they correspond to the gametes in the other classes of Protozos. It is on the whole more probable that the conjugants correspond rather with gamonts or gametocytes, which originally produced a number of gametes, reduced now to two, represented in each conjugant by the two pronuclei. 
cilia and swim off. Each microconjugant attaches itself to a macroconjugant -that is to say, to an ordinary sedentary individual ; each of the nnjugants has a single micronucleus and macronucleus, but as soon as they hecome associated the changes preparatory to syngamy begin. In the microcols igant the micronucleus divides three times to produce eight micronucle in Carchesium the first of these divisions is an equating division; the second reduces the number of chromosomes from sixteen to eight; and the third division is again an equating division (Popoff, 125). Meanwhile the macronucleus is in process of degeneration, and is breaking up into fragments. Of the eight micronuclei, seven degenerate, one persists and divides into two pronuclei. In the macroconjugant, meanwhile, similar events are taking place, but the micronucleus only divides twice, first by a reducing, then by an equating division, to produce four micronuclei, of which three degenerate, while the fourth persists and divides into the two pronuclei.

of the two pronuclei now present in each conjugant, one degenerates in each case; the persistent pronucleus of the microconjugant passes over into the macroconjugant and copulates with its persistent pronucleus. The fragments of the masronucleus also pass over into the macroconjugant, but are there absorbed slowly. The body of the microconjugant then falls off and dies; only the macroconjugant is fertilized.

Variations of minor importance are seen in the behaviour of the synkaryon of the exconjugant after fusion of the pronuclei has taken place. For example, in Paramecium bursaria the synkaryon divides to form four nuclei, two of which become macronuclei, whereupon the exconjugant divides into two ordinary individuals (Hamburger, 842); in Licnophora the synkaryon divides into eight, which become a micronucleus and a macronuclear chain of seven segments (Stevens, 872) ; in Carchesium the synkaryon divides also into eight to furnish a micronucleus and seven separate macronnclei, but the micro. nucleus then divides six times, with subsequent divisions of the body and sorting out of the macronuclei, until seven individuals, each with a single micronucleus and macronucleus, aro produced (Popoff, 125); in Anoplophrya the synkaryon divides into four nuclei, two of which degenerate, the remaining two becoming a micronucleus and a macronucleus respectively. The method of nuclear reconstruction may vary even in the same species, as shown by Prandtl (126) in the case of Didinium.

The most important deviations from the usual scheme of conjugation are seen in those forms in which there is no separate micronucleus in the ordinary condition. The cases of Opalina and Ichthyophthirius, parasitic forms and therefore open to the charge of degeneration, are dealt with below. In Trachelocerca phonicopterus, a freo-living species, conjugation has been described by Lebedew (93) between individuals containing many nuclei all similar in appearance, each with a large karyosome. Prior to conjugation the chromatin passes out of the karyosome into the nuclear cavity of each nucleus (Fig. 188, $A, B$ ), which then divides into four. The chromatin forms a compact mass at one pole of each nucleus. During conjugation these masses of chromatin pass out of the nuclei, and lie free in the cytoplasm between them (Fig. 188, $C-G$ ) ; each such mass is now to be regarded as a micronucleus and lies in a clear area, finally becoming a vesicular nucleus with a distinct alveolar structure; the old nuclei can now be considered as macronuclei. All the nuclei now collect in a mass near the middle of the body. The macronuclei ultimately degenerate; the micronuclei multiply by fission, but ultimately, according to Lebedew, they all degenerate with the exception of one in each conjugant ; the persistent micronucleus divides into two pronuclei which conjugate in the usual way ; unfortunately, the author's observations contain so many gaps that this statement cannot be considerod established so decisively as could be desired. The exconjugants contain each a single synkaryon which divides by successive divisions into a number of nucloi not differentiated into micronuclei and macronuclei.

The case of Trachelocerca, as it is described, furnishes an important clue to understanding the nrigin of the heterokaryote condition of Infusoria from 
that found in other Protozoa. In this case, during the ordinary vegetativo condition, the gencrative chromatin representing the micronucleus of other Infusoria, and the vegetative chromatin representing the macronucleus, aro contained in one and the same nucleus, and become separate only when syngamy is about to take place. The first sign of the separation is the formation of chromidia from the karyosome within the nucleus, resulting in the forniation of a secondary nucleus which becomes separate and which behaves exactly as an ordinary micronucleus ; thus indicating a clear homology between the micronuclei of Infusoria and the secondary generative nuclei of Sarcodina.

The production of numerous micronuclei in the conjugation of Trachelocerca

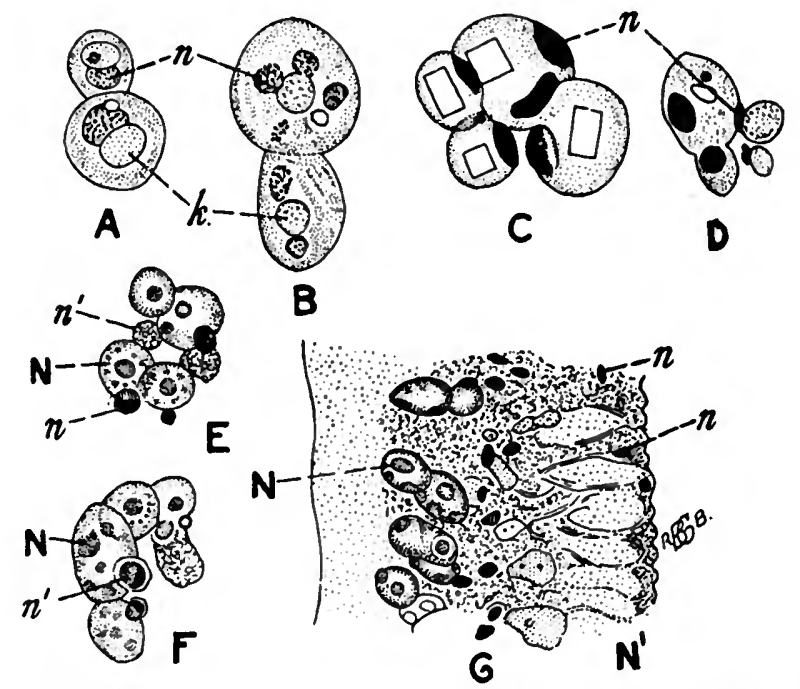

Fia. 188.-Formation of micronuclei in Trachelocerca phœnicopterus. $A, B, \mathrm{~A}$ nuelcus has divided into two, and from the karyosome $(k$.) of each daughternucleus masses of chromatin are being given off into the nuclear cavity; $C, D$, the two nuelei of the preceding stages have divided again, to form a group of four, and the chromatin-masses $(n)$ have acquired a compact structure and are passing out of the nuelei to form the micronuolei; in $C$ crystals are seen in the cavities of the old nuclei, probably a sign of degeneration; $E, F$, two groups of nuclei, both from the same specimen; the micronuclei given off from the old nuclei become surrounded by a vacuole $(n$ in $F$ ), and then acquire an alveolar structure $\left(n^{\prime}\right.$ in $\left.E\right) ; G$, portion of a preparation of the body of a eonjugant, the wavy contour on the right being the surface of the body which is in contact with the other conjugant ; numerous micronuclei $(n)$ are seen, and also macionuclei, some of which still appear normal $(N)$, others degenerating $\left(N^{\prime}\right)$. After Lebedew (93).

is noteworthy, and would appcar to favour tho theory (see p. 154) that primitively numerous gametes (swarm-spores) were produced in the conjugation of Infusoria.

Examples of a complicated life cycle are to be found in Ciliata chiefly, perhaps solely, among parasitic forms. As an oxample may be taken Ichthyophthirius multifilits, a parasite of the skin of various species of fiesh-water fishes. In aquaria, where, owing to the limited space, the parasites, if present, find their way to the fish very easily, and where, consequently, a fish hecomes infected with vast numbers of the ciliates, the parasites are usually lethal to the host, and cause its death, according to Buschkiel, in about fourteen days. In Nature, on the other hand, "ichthyophthiriasis"' is seldom observed, prob- 
ably owing to the fact that under natural conditions only a very small proportion of the young prrasites succeed in establishing themselves on a fish, and consequently the infections produced are so slight that they are overlooked, and the fish is unharmed.

The life-cycle of Icluthyophthirius is as follows: The youngest parasites hatched out from a cyst are very small, and have a macronucleus and a micronucleus. They seek out a fish and boro into its epidermis, attaching themselves by one end of the club-shaped bocty and rotating actively, with the result that epithelial cells are displaced, and either cast off into the water or form a ring-like wall round the parasite. In this way the infusorian works its way gradually into the deeper layers of the epidermis. which closes over it, so that the parasite lies finally in a closed hollow space in the epidermis. In this position it grows in size, and at a certain point the micronucleus disappears, passing into the macronucleus to form a nucleolus.like body within it. The parasite appears to the naked eye as a little white spot on the skin, occurring on any part of the body-surface or on the gills. It retains its cilia, and can be seen rotating within tho cavity in which it lies.

The full-grown Ichthyophthirius may reach 1 millimetre in diameter, but is usually less, about 0.75 millimetre. When full-grown the ciliate breaks out of the cavity in the epidermis and sinks to the bottom, attaching itself to the ground or to water-weeds, and becomes encysted. Within the cyst it multiplies by binary fission repeated eight times, producing 256 small ciliates; sometimes this multiplication takes place without encystment. During this process of multiplication the micronucleus reappears, boing extruded from the macronucleus of each individual when not less than four are present in the cyst; but the exact period at which the micronuclei appear varies in different cases. In addition to the micronucleus, one or two other extrusions from the macronucleus take place (Buschkiel); but whether these represent other micronuclei or expelled vegetative chromatin is not clear; in any case they degenerate and disappear. When the micronuclous makes its appearance, it divides by mitosis at each division of the cell-body, as in ordinary Ciliata, while the macronucleus divides in the usual way by direct division.

When the full number of tiny ciliates is formed, each with a macronucleus and micronucleus, sexual phenumena occur, but the events that take place are described differently by different investigators. According to Neresheimer (858), in each individual the micronucleus divides twice, and three of the four micronuclei produced degenerate; the fourth then divides again. The reduction-process is, therefore, according to this account, similar to that of other Ciliata, and the organism appears to be ready for conjugation, with two pronuclei ; but Neresheimer was unable to observe conjugation taking place cither in the cyst or after the organisms have become free; he observed, however, sometimes two micronuclei, sometimes one, both in free forms and in those attached to the fish, and from this it was inferred that the two pronuclei fuse autogamously, leaving the possibility open, however, that heterogamous conjugation might sometimes occur. According to Buschkiel, on the other hand, the micronucleus of each individual divides twice, and, of the four thus produced, two degenerate, and the remaining two fuse autogamously while still within the cyst.

The little ciliates are set free from the cyst, and seek out a new host in their turn. From the time that the full-grown parasite leaves the fish to the time that the brood is liberated from the cyst is, according to Buschkiel, about twenty hours, more or less. If an infected aquarium be kept empty of fish for sixty hours, it becomes disinfected, since the parasites all die off if they cannot attach themselves to a fish very soon after they are hatched out.

The entozoic Ciliata, in which adaptation to a purely parasitic life has led to the degeneration of the apparatus of a holozoic mode of nutrition-that is to say, of the mouth, peristome, and accessory cilia-are sometimes classified as an order, Astomata, of the Holotricha; but there can be little doubt this group, like others founded on negative characters, is a heterogeneous collection of forms in which the characters they possess in common are due to convergent 
adaptation to their mode of life (cf. Léger and Duboscq, 848). The bestknown genera are Anoplophrya, a typical ciliate with micronucleus and macronucleus and with a rudimentary cytostome, constituting with Hoplitophrya, Herpetophrya Discophrya, ote., the group Anoplophryina; Chromidina and Opalinopsis, parasitic in Cephalopods, are probably allied to the foregoing (cf. Dobell, 833). Tho species of Opalina, eonstituting the group Opalinince, are parasitic in frogs and various cold-blooded vertebrates; their nuclei vary in number in different specios from two to an indefinitely large number, but are all similar and without differentiation into micronuclei and macronuclci at any period of the life-cycle. Cépède has monographod the section Anoplophryina, and has described a number of new genera and species, distributed amongst eleven families. The Astomata are internal parasites of their hosts, ospecially of the digestivo tract. Protophrya ovicola occurs in the brood-sae of the molluse Littrina rudis, and is parasitic upon its eggs, causing their disintegration (Kofoid).

The remarkable form Pycnothrix monocystoides, from the gut of Hyrax capensis, described by Schubotz, deserves special mention. It reaches a length of 3.2 millimetres, and contains parasitic nematodes. The animal itself has a great superficial resemblance to a nematode or to a monocystid gregarine; it has a very thick and distinct ectoplasm, covered by an even coat of short cilia, and with two longitudinal grooves which Schubotz regards as equivalent to the peristomial grooves of other Ciliata. Each groove contains a series of pouch-like depressions, which open down into the endoplasm, and are provided with special tracts of myonemes. Schubotz regards these pouches as a series of cytostomes, but no food-particles or vacuoles are found in the endoplasm; the interpretation, therefore, of these openings as eytostomes can only be taken in a phylogenotic senso; actually they appear to represent perforations of the tough ectoplasm whichmay focilitate absorption of food by the osmotic method. For the cilia myonemes and excrctory system of this form see above (pp. 443, 446 447); the micronucleus and macronucleus are each single and of the ordinary type. Pycnothrix stands at present quite isolated.

The species of the genus Opalina differ in certain peculiarities of structurc and life-history from all other ciliates. The life-history of the common species of Opalina parasitic in the rectum of the frog has been studied by Metcalf (853) and Neresheimer (857), whose accounts agree as regards the general life-cycle, but differ in some cytological details.

Opalina ranarum multiplies in a vegetative manner during the summer and autumn months, but in the spring a special propagative cyelc occurs in relation to change of hosts and is followed by sexual processes.

The vegetative reproduction increases the numbers of the parasite in the host; it consists of two processes, multiplication of the nucloi and division of the body, which go on independently. The animal contains a great many nuclei, and when it reaches a certain size the body divides either longitudinally or transversely to produce two daughter-individuals, each of which grows again to the full size. The multiplication of the nuclci is effected by a simple mitosis, similar to that of the mieronucleus of other Infusoria, and without centrosomes.

In the spring the parasites divide rapidly and repeatedly, without growing to full size betwecn the divisions, so that they become continually smaller in size. A fow individuals, however, do not undergo this process of rapid fission, but remain of the ordinary type, forming a stoek which persists and carrics on the infection in the frog, while those which divide up are destined to pars out of it. At the beginning of the process of rapid division, the nuelei extrude chronidia, some of whieh are absorbed, while from the remainder secondary nuclei are formed (Neresheimer). Finally the old nuclei are atworbed. The secondary nuclei also multiply by mitosis ; and, according to Metcalf, in the later mitoses preceding encystment the number of chromosomes is reduced to one-half the ordinary number (in $O$. intestinalis from eight to four, in $U$. caudata from six to three). The result of the repeated division 
is to produco snall individuals containing, as a rule, from three to six secondary nuclci. Such individuals become encysted (infection-cysts), and pass out of the frog in the frees. The animal at first fills the cyst complotely and shows no cilia. but after a time the body shrinks within the cyst, and the animal is then soen to have a ciliary covering.

The fæces of the frogs are readily devoured by tadpoles, which thus become infected with cysts. In tho gut of the tadpole the Opalina emerges from its cyst. It at once divides up into uninucleate individuals, the gameies, elongated club-shaped forms with a sparse coat of cilia over the flattened body. Under unfavourable circumstances the gametes undergo a.gglomeration in rosettes, adhering by their pointed ends (Neresheimer). Under uormal circumstances they copulato in pairs as isogametes, according to Neresheimer, in $O$. ranarum; but Metcalf describes smaller uninucleate microgametcs and larger macrogametes with one or two nuclei, in other specics; the male pronucleus then fuses with one of the two nnclei of the macrogamete. The zygote of $O$. ranarum, with the two pronuclei still separate, rounds itself off and becomes encysted (copulation-cyst) ; within the cyst the two pronuctei, which have passed into a spindle-stage, undcrgo fusion. The zygote emerges from the cyst with a synkaryon, and it becomes an adult $O$ palina.

Neresheimer considers that the life-cycle of Opalina proves that its affinities are with Flagellata rather than with Infusoria. In deciding this question. it must be considered, in the first place, whether in such a form the life-cycle. or the structural features of the body, are most likely to indicate affinitythat is to say, least likely to exhibit seccndary peculiarities duc to adautation. Opalina is a parasitic form, and its life-cycle shows very obviously a direct daptation, of a type very common in parasitic Protozoa, to its mode of life; multiplicative reproducticn incrcasing its numbers within the host, and propagative reproduction, combined with sexual phenomena, leading to the infection of new hosts. On the other hand, its minute structuro is that typical of Ciliata, a character hardly likely to be due to the influence of parasitism, as Copoff (125) has well pointed out.

The chief difference between Opalina and other Ciliata, which requires special consideration, is the fact that the animal contains but one kind of nucleus. This, however, is a character known in other genera of Ciliata also--e.g., Trachelocerca, Ichthyophthirius. There can bo but littlo doubt that the " heterokaryote" condition of the Infusoria, with distinct generative and vegetative nuclei. must have bcen derived phylogonetically from a condi. tion in which, as in other Protozoa, the two kinds of chromatin were contained in one and the same nucleus; and to find this condition still retaincd in somo Infusoria would not bo remarkable. In such forms it is to be oxpected that prior to gamete-formation the vegetative chromatin, equivalont to the macronucleus, would be expelled, and the pronuclei would be formed irom generative chromatin.

There is nothing, therefore, to be said against the view of Popoff, that Opalina shows the most primitive type of gamete-formation known at present amongst the Ciliata. Its nuclei contain gencrative and vegctative chromatin combined, and in preparation for syngamy nuclei are formcd which are purely generative, out of chromidia expelled from the primary nuclei. The forras. tion of uninuclcate gametes which copulate (lotal karyogamy) has beon re. garded by almost all those who nave theorized on the subject as being probably the most primitive type of syngany from which the conjugation (partial karyogamy) of the ('iliata has been dorived (p. 15 $t$ !.

In Trachelocerca (1, 150) the gamont produces in a similar manner a number of generative nuclei (micronuclci) prior to the syngamic process; but hero, as in Ciliata gencrally, the gimont no longer divides into a number of gametes ; only one micronucleus in each gamont persists to form the two pronuclei, and the usual process of partial karyogamy takes place. These curnsiderations indicate that the monomorphic character of the Infusorian life-cycle is a secondary feature ; as the structural complication of the body has increased, so the tendency to divide up into relatively minute swarm-spores has been 
suppressed, and has been replaced by the peculiar type of syngamy characteristic of the group.

The question of the exact systematic position of Opalina cannot be decided until more is known of the lifo-cycles of other parasitic Ciliata ; but at present there do not seem to be any cogent reasons for removing this genus from the Ciliata.

Affinities of the Ciliata.-A typical ciliate, such as Paramecium, with its even coat of fine cilia, its heterokaryoto nuclear apparatus, and its peculiar type of syngamy with partial karyogamy, stands apart and apparently isolated from the typical members of other classes of the Protozoa. Never-

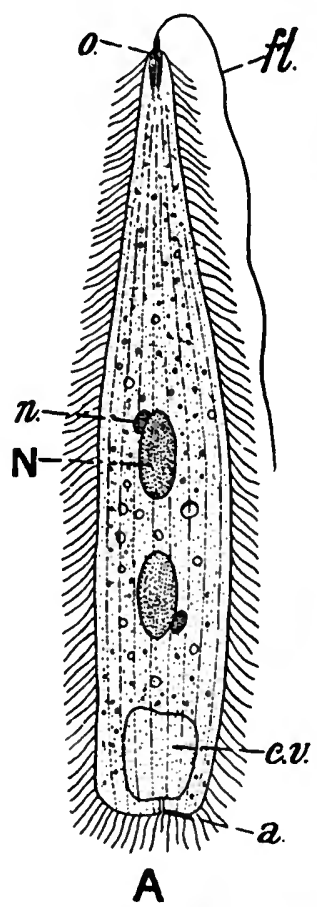
theless, even within the limits of the class Ciliata, examples are to be found in which the heterokaryote condition is not developed, or only appears prior to syngamy in the form of a separation of generative from vegetative chromatin (Trachelocerca, Opalina), and in which the syngamy takes the form of total karyogamy between minute gametes, swarm-spores (Opalina). Such cases, while they minimize the gap between Ciliata and other Protozoa, do not bring the ciliates noarer to any particular class, since a similar type of syngamy and of preparations for it may occur either in Sarcodina or Mastigophora.

As the most distinctive feature of the Ciliata there

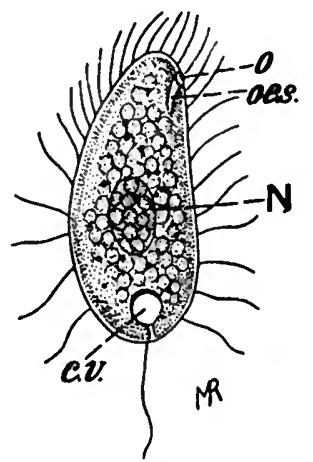

B

Fra. 189.-A, Monomastix ciliatus : $f$., flagellum ; $o$, mouth; $N$, macronucleus ; $n$, micronucleus ; $c . v$, contractile vacuole; $a$., anus, near which opens the efferent canal of the contractile vacuole. After Roux, magnified 1,000. B, Maupasia paradoxa: œes., œsophagus; other letters as in A. After Schewiakoff, magnified 1,300. through endoparasitic forms; and it is on the whole moro likely that freeliving forms, such as the holomastigote genus Multicilia, are the nearest representatives of the earlier ancestral forms of the Ciliata.

Two interesting forms have been described which combine in some respects the characters of both Flagellata and Ciliata.

Maupasia paradoxa (Fig. 189, B) is described by its discoverer, Schewiakoff (863), as having the body metabolic, with cilia in the anterior part of the body, and the remainder covered with long flagella. At tho hinder end of the body is a longor flagellum implanted elose beside the aporture of the efferent duet of the contractile vacuole. The mouth-opening, on the ventral side of 
the body, leads into a short cesophagus. The nucleus is single, without a micronucleus. Schewiakoff makes Maupasia the type of a distinct order of tho Ciliata-the Mastigotricha.

Monomastix ciliatus (Fig. 189, A), described by Roux (862), and referrod by him also to the Mastigotricha, has an even coat of cilia all over the body, and possesses two macronuclei, near each of which is a micronucleus; its most remarkable feature is the possession of a long flagellum implanted at the anterior end of the body close to the mouth.

From these various considerations, it seems highly probable that the Ciliata are descended from flagellate ancestors; but it is not possiblo at present to indicate with any approach to exactncss the line of descent.

\section{Subolass II.-Acnnetaria (Suctoria, Tentaculifera).}

The Acinetaria are distinguished from the Ciliata by the possession of the following characters in combination : The adult organism is of sedentary habit, and has no cilia, though the youngest stage in the life-history is typically a free-swimming ciliated organism; there is no mouth, but both the capture and ingestion of food is effected by means of special organs peculiar to this subclass, and known as tentacles.

An acinetan may be attached to various objects, and is frequently epizoic. Some species attach themselves indifferently to a living or a lifeless object ; others are constantly epizoic, and occur always attached to some particular animal, frequently to a particular organ of it. Very few species, however, are truly parasitic in the adult condition; on the other hand, many species are parasitic in the early larval stages of their life-history, and frequently so within the bodies of Ciliata (Fig. 192). The marine genus Ophryodendron, however, is a true ectoparasite of hydroids, according to Martin, and contains nematocysts derived from its hosts. In this case the parasitism is correlated with a peculiar dimorphism of " proboscidiform " and "vermiform" individuals, the former possessing a tuft of tentacles on a proboscis-like process, the latter being without tentacles altogether. The vermiform individuals are budded from the proboscidiform, and either form can produce ciliated buds, which develop into proboscidiform individuals again; but the vermiform type does not grow into the proboscidiform. In Dendrosomides paguri, however, Collin (881) finds that similar vermiform individuals become transformod into the tentaclo-bearing form. In Rhabdophrya trimorpha, ectozoic on a Copepod (Cletodes longicaudatus), there are three forms of individuals-namely, in addition to tentaculated and vermiform specimens, peculiar " unciform" individuals, which are also without tentacles (Chatton and Collin, 876).

The form of the body varies greatly, but may be said to be typically vase-like, with or without a stalk or peduncle. In sessile forms the body is attached by a broad base to the substratum. In 
stalked forms the body is raised up from the point of attachment on a straight, non-contractile stalk of secreted substance; similar to that of many Vorticellids, and the animal as a whole may resemble in its general contours an Epistylis or other Vorticellid (Figs. 10, 190). Collin (877) finds that the stalk consists of a sheath, a cortical layer thickest at the base, and a medullory substance stratified longitudinally to the longitudinal axis. The base of the stalk rests on a cushion of secreted substance-the portion which is first formed, and which is produced by a special organ of the larva comparable to the scopula (p. 441) of the Vorticellids.

The body is often protected by a secreted house or theca, con-

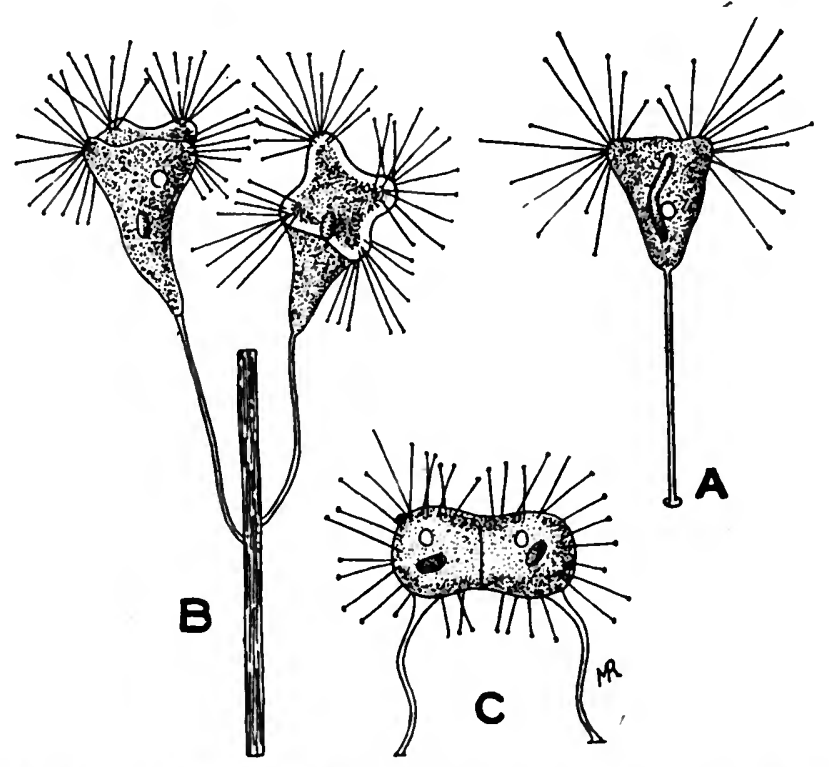

Fia. 190.-A, Podophrya mollis ; B, Tocophrya quadripartita, two specimens attached to the stalk of Epistylis plicatilis ; C, Podophrya fixa, two specimens conjugating. After Saville Kent.

tinuous with the stalk in the pedunculate forms. In Astrophrya arenaria the house is built up of foreign particles of various sizes (Awerinzew). As in the attached ciliates, colonies may be formed of considerable size and extent, and of various forms. The nonpedunculate genus Dendrosoma produces spreading colonies, which bear a considerable resemblance to a polyp-colony.

The characteristic tentacles are stiff protoplasmic processes consisting of a parietal layer of ectoplasm in the form of a tube enclosing a canal containing fluid. The apex of the tentacle usually torminates in a sucker-like knob; suctorial tentacles ("Saugten- 
takel ") of this type are always present. In the genus Ephelota there are present in addition prehensile tentacles ("Greiftentakel "), which end in a fine point. The exterior of the tentacle is clothed by a delicate pellicle, continuous with that of the body, and forming in the suctorial tentacles a sheath or tube, from the end of which the sucker protrudes. The tentacles are slowly retractile. When expanded they appear homogeneous; bat in the process of retraction they exhibit a spiral marking, due apparently to creases and folds in the pellicle, and not to be interpreted as indicating the presence of myonemes. The tentacles are used for the capture of prey, which consists chiefly of ciliates. As soon as the sucker-like extremity of a tentacle touches a ciliate it is held fast ; the substance of the prey is then slowly absorbed by the tentacle, and passes as a stream of granules down the axis of the tentacle. During this process the ciliate remains alive, with cilia movin gand contractile vacuoles pulsating, until about half its substance is absorbed (Filipjev).

In the genus Rhyncheta there is but a single tentacle of great length ; in Urnula (Fig. 191), one or two. Other genera bear usually many tentacles, which may be distributed evenly over the body-

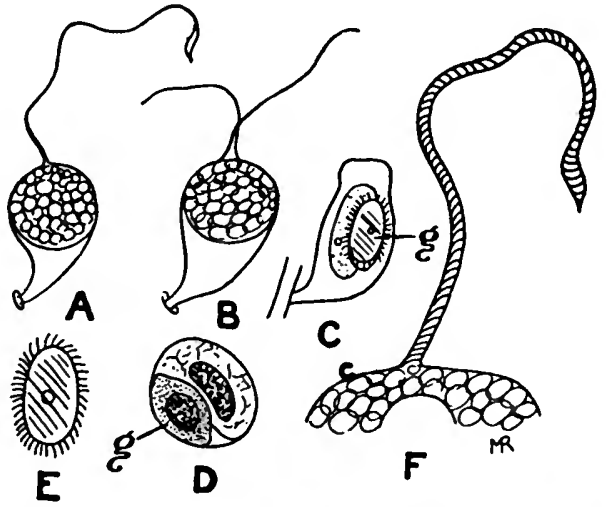

FIa. 191.-Urnula epistylidis, epizoio on Dendro soma radians. $A, B$, Individuals with one or two tentacles respectively ; $C$, formation of a bud $(g) ; D$, the same scen in transverse section passing through the bud and the macronucleus of the parent ; $E$, free-swimming larva ; $F$, enlarged view of the single tentacle, showing the spiral striation. After Hickson and Wads. worth (886).

surface, or, more commonly, occur in special regions of the body or are distributed in tufts and patches. In Dendrocometes the tentacles occur in bunches borne on branches or arm-like processes of the body-wall.

Ishika wa describes in the larger prehensile tentacles of Ephelota buetschliana a system of filaments, consisting of fine threads running parallel to one another in pairs and continued into the body as far as its base. The filaments stain deeply with iron-hæmatoxylin. According to Collin (877), each such pair of filaments is in reality the optical section of a fine tube. A suctorial tentacle, according to Collin, represents a dcep invagination of the ectoplasm, opening at its innermost end into the endoplasm like the cytopharynx of a ciliate. The prehensile tentacles, on the other hand, are special formations of a different kind, simple evaginations of the body-wall, pseudopodial in nature, and containing from one to three axial filanents, the number increasing with the age of the tentacle. 
When a ciliate-for oxample, a Paramecium-is captured by tho tentacle, its protoplasm stroams down tho tentacle to form a mass in tho endoplasm of the acinetan. Before the process of suction is complete the mass breaks up into smaller masses, and these again into still smaller oncs, which aro carricd away by the cyclosis of the endoplasm, and other masses of small size continue to be formed at the base of the tentacle. Round each of theso food-masses a fluid vacuole is formed, in which the ingested protoplasm is for the mcat part dissolved, becoming reduced to a fow granulations. The vacuole then gives off fluid and diminishes in size, and the contents are concentrated to form a refractile body. Three kinds of such refractile bodies are formed : so-called "colourless bodies" which stain fcebly with nuclear stains, and are derived from thesprotoplasm of the prey ; "tinctin-bodies," staining dceply, and originating, as described by Martin, from the chromatin of tho proy ; and .others, found in some acinotans, derived from the chlorophyll of green ciliates and algal spores devoured by the animal. If a Tocophrya be starved, the refringent bodies are slowly absorbed, and the protoplasm becomes quite clear (Filipjev). Hence the refringent bodies that arise from the diges. tive vacuoles represent reservo-material; thero appears to be no defæcation of indigestible residues.

The nature and origin of the tentacles of aoinetans have been much discussed, and some authors have sought to derive them from cirri or cilia. Schuberg (44) points out, however, that the structure of the tentacles is quite the opposite of that of the cilia ; in a cilium the axial portion is of firm con. sistence, the superficial layer is fluid, while in a tentacle the axis is fluid and the superficial sheath is of firm texture. Collin (877) considers that the pre. hensile tentacles are modifications or adaptations of a pseudopodium-like precess; on the other hand, he regards the suctorial tentacles as organs of quite a different kind, more like the cytostome of a ciliate than anything else ; they may be considered each as a cytostome which has grown out from the body on a slender process or stalk (compare also Hickson, 826).

In correlation with their sedentary habits, the organization of the Acinetaria is grcatly simplified as compared with the Ciliata, and the remarkable structural and functional differentiation of the ectoplasm seen in the Ciliata is wanting altogether in Acinetaria, in which the ectoplasm is relatively a feebly-developed layer. Contractile vacuoles are usually present, one or more in number. As in Ciliata, the macronucleus exhibits a great variety of forms. One of the most remarkable is seen in the colonial form Dendrosoma, where the macronucleus is branched to the same degree as the colony, throughout which it extends continuously.

The methods of reproduction are more varied, and exhibit a greater specialization, in the Acinetaria than in the Ciliata. Simple binary fission in the adult condition is rare in acinetans. Collin (881), however, has observed division into two or four within a cyst in Podophrya fixa. The fission usually takes the form of bud-formation. The buds may be formed either on the exterior of the body or in the interior in special brood-cavities, and they may be produced in either case singly and successively or in batches or relays of several at a time. The bud is usually a simple outgrowth of the cytoplasm containing a prolongation budded off from the macronucleus, and one of the daughter-nuclei derived from a division of the micronucleus. At first a simple cell without structural differ- 
entiation, the bud is set free with a complete or partial coat of cilia as a free-swimming " embryo," "larva," "swarm-spore" (Schwärmer), or "gemmula." The larva often becomes parasitic within the body of another Infusorian (Fig. 192), multiplying there by binary fission. Finally it becomes free again, swims away, attaches itself in a suitable locality, and develops into the adult form.

The ciliated larvæ of acinetans exhibit various types of ciliation, commonly classed as peritrichous (Fig. 193, $A, B$ ), holotrichous (Fig. 191. $E$ ), and hypotrichous. Collin (882) has studied recently the morphology of the different types of larvæ.

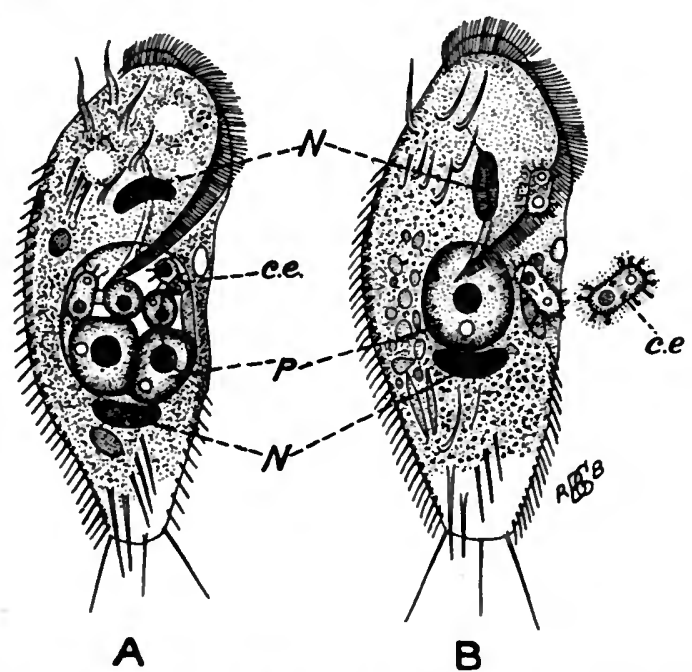

Fra. 192.-A and B, Slylonychia mytilus infested by parasitic Aoinetans. $N, N$, Macronuclei of the Stylonychia; $P$, parasitio Acinetan embryo from which arise small ciliated larva (c.e) which swim off and develop into the adult free-living Acinetan. After Stein.

The most primitive and commonest larval type of larva amongst the Acinetaria is very similar to a free-swimming stage of a Vorticellid. It has a principal axis round which the body is radially symmetrical, with an upper polc (posterior in movement) bearing a rudimentary adoral zone, and a lower (anterior) pole bearing a mass of secretion or a sucker, indicating the future point of fixation and representing the scopula of the Vorticellid; the body is surrounded by several rings of cilia forming a zone more or less equatorial in position. Such a form, while retaining its radiate symmetry, may become either lengthened or shortened to a remarkable degree in the direction of the principal axis; in the elongated forms the rings of cilia may increase in number until they cover the whole body, thus producing the holotrichous type. On the other hand, the body may become elongated in the morphologically transverse plane, and acquire a bilateral symmetry, with a dorsal surface representing the primitive upper polo and bearing the rudimentary adoral zone, and a ventral surface, with the sucker in the middle of it, repre. senting the primitive lower pole; zecundary anterior and posterior extremities are now distinguishable in relation to progression, but representing opposite 
points of the primitive transverse planc. In such a type tho zones of cilia run obliquely along the sides of the body, or may bo confined to the ventral surface, where they run a more or less elliptical course round the sucker, thus producing the hypotrichous type seen in Ephelota gemmipara and in tho persistent larval form Hypocoma acinetarum. Examples of holotrichous larvæ are seen in Tocophrya limbata and Urnula epistylidis (Hickson and Wadsworth, 886). In all cases the principal or dorsiventral axis of tho larva becomes the principal axis of the adult; in the process of budding, howover, tho principal axis of the bud arises at right angles to that of the parent, according to Collin; Filipjev, however, docs not confirm this for Tocophrya quadripartita.

The remarkable form Tachyblaston described by Martin lives in the adult condition attached to the stalk of Ephelota; it produces buds each with a single tentaclo, which creep up the stalk of the Ephelota and penetrate into the body, becoming parasitic in it and multiplying by fission to produce ciliated larvæ, which in their turn swim out, attach themselves to tho stalk of the Ephelota, and kccome adult forms.

The conjugation of the Acinetaria conforms in general to the type of the process scen in Ciliata, as regards cytological details. Conjugation may take place between two individuals fixed near
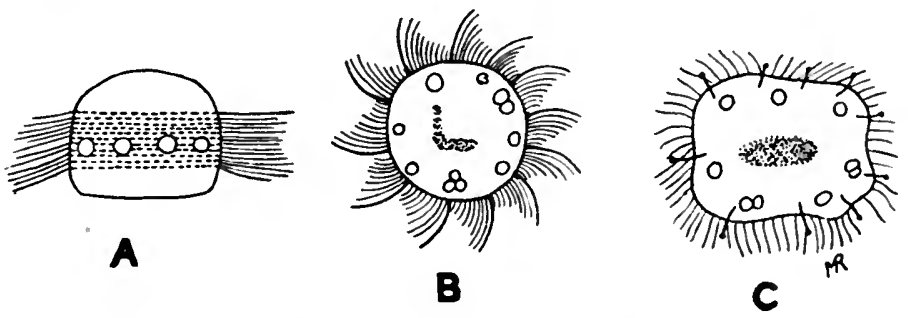

F1g. 193.-Free-swimming larva of Dendrosonic rulians. $A$, Side veiw ; $B$, viewed from above ; $C$, older larva with the first rudiments of the tentacles beginning to appear. After Hickson and Wadsworth (886).

together (Fig. 190,C); then a lobe or outgrowth may be formed from one individual, which meets a similar outgıowtl from the other, thus establishing contact. On the other hand, as in Peritricha, conjugation may take place between a fixed, ordinary individual and a free-swimming bud or larva liberated from another adult individual (Martin; Collin, 879). In Dendrocometes the macronuclei come into contact during conjugation, but separate again (Hickson and Wadsworth).

Classification.-The Acinetaria are divisiblo into eight families ( $c f$. Doflin, 7).

1. Hypocomida, for the single, somewhat aberrant genus Hypocoma, which is free-swimming. ciliated on one surface, and with a single suctorial tentaclepossibly a persistent larval form (see Collin, 877).

2. Urnulida.-Witl or without a house, with one or few tentacles. Rhyncheta, Urnula (Fig. 191).

3. Metacinetida. - With a stalked house opening at the upper end for tho exit of the tentacles. Metacineta. 
4. Podophryidae.-Stalked or sessile, with no house and with numerous tentacles. Sphacrophrya, Podophrya (Fig. 190), Ephelota.

5. Acinetide. - Stalked or sessile, with a house of simple form and wide aperture, and with numerous tentacles, all knobbed. Tocophrya (Fig. 190), Acineta (Fig. 10).

6. Dendrosomida (Trichophryida).-Sessile, without a house; tentacles knobbed, arranged in tufts or branches. Trichophrya, Dendrosoma, Lernaeophrya (Perez), Rhabdophrya (Chatton and Collin), Astrophrya (Awerinzew).

7. Dendrocometida. - Flat forms with numerous branched arms on the ends of which the suckers occur. Dendrocometes.

8. Ophryodendrida. - Marine stalked forms with numerous short tentacles concentrated on proboscis-like processes. Vermiform individuals also occur (p. 455). Ophryodendron.

Affinities of the Acinetaria. - The presence of cilia in the young stages, the possession of distinct vegetative macronuclei and generative micronuclei, and the process of conjugation, similar in all essential details to that of the Ciliata, can leave no doubt as to the position of the Acinetaria in the class Infusoria, and their affinities with the Ciliata. Collin, in a series of interesting studies, has drawn attention to many points indicating a close relationship between Acinetaria and Vorticellids, more especially the structural homologies between the larve of the one and the free-swimming stages of the other group; for example, the peritrichous arrangement of the cilia, the rudimentary adoral zone at the posterior pole, and the fixation by means of a scopula.like organ at the anterior polo, points especially well seen in the larva of Tocophyra cyclopum.

Bibliography.-For references see p. 502. 


\section{CHAPTER XVIII}

\section{AFFINITIES AND CLASSIFICATION OF THE MAIN SUBDIVISIONS-DOUBTFUL GROUPS}

IN the foregoing chapters the Protozoa have been dealt with systematically, grouped in a somewhat conservative manner under the four old-established and generally-recognized classes. At the same time it has been pointed out that one class at least-namely, the exclusively-parasitic Sporozoa-comprises two subclasses which are quite distinct from one another, and are descended, in all probability, from ancestors differing, greatly in characters and affinities. And in the case of the three remaining classes, consisting mainly of free-living, non-parasitic forms, two which exhibit more primitive characters-namely, the Sarcodina and Mastigophora -are connected with one another by transitional forms which render the distinction between them very arbitrary (p. 213); while the third, the highly-specialized Infusoria, are linked closely by structural characters and by transitional forms to the Mastigophora.

Many authorities on the Protozoa have put forward schemes of classification which are intended to express the affinities and interrelationships of the chief groups in a clearer and more satisfactory manner than the fourfold classification generally recognized. The systems proposed have taken the form either of subdividing the Protozoa into more than four classes or of uniting the recognized subdivisions into a smaller number of categories.

Rolleston and Jackson (15) divide the Protozoa as a whole into three groups: (1) the Rhizopoda (=Sarcodina); (2) the Endoparasita (=Sporozoa); and (3) the Plegepoda, "referring to their mode of progression by means of a rapidly-repeated stroke $\left(\pi \lambda_{\eta} \gamma \dot{\eta}\right)$ of vibratile processes," to comprise the Mastigophora and Infusoria.

Doflein (891) recognizes two principal stems in the Protozoan phylum: (1) the Plasmodroma, to include the Sarcodina, Mastigophora, and Sporozoa, organisms that make use of locomotor organs which represent true psendopodia, or their derivatives or modifications; and (2) the Ciliophora, comprising the Ciliata and Suctoria, in whioh the locomotor organellæ are cilia. The obvious criticism of this scheme is that, whatever opinion may be held as to the desirability of drawing a line between the Infusoria, so highly specialized in. many respects, and other Protozoa, the distinctive character chosen is not a happy one, since whatever may be predicated of flagella as derivatives of pseu. dopodia applies, apparently, with equal force to cilia. 
Hartmann (892) recognizes six classes of the Protozoa : Class I., the Sarcodina, including four subclasses-namely, Rlizopoda, Heliozoa, Radiolaria, and Mycetozoa; Class II., the Cnidosporidia, including Microsporidia, Sarcosporidia, Myxosporidia, and Actinomyxidia ; Class III., the Mastigophora, including the Rhizomastigina, Protomonadina, Binucleata, Chromomonadina, Euglenoidea, and Phytomonadina, the order Binucleata including the Hæmoflagellates and the Hæmosporidia with the exception of the hæmogregarines; Class IV., the Telosporidia. including the grogarines, coceidia, and hæmogregarines; Class V., the Trichonymphida ; Class VI., the Infusoria. With regard to this classification, the order Binucleata has bcen dcalt with at length above; it only remains to say that the isolated position given to the Trichonymphida appears to express the defective stato of knowledge con. cerning the affinities of these peculiar parasites, rather than their true taxo. nomic importance.

A number of radical changes in tho classification of the Protozoa are proposed by Awerinzew (890). With Hartmann he unites the Hæmoflagcllates and Hæmosporidia in an order Binucleata to be placed in the Flagellata. The class Sporozoa is to be ontirely abolished. Tho order Amcbina (Amœbæa) is removed by him entirely from the Sarcodina, which will then comprise only the Foraminifera and some Heliozoa. The Amœbina are to be put with the Flagellata as the Amœboflagellata, a group from which all other Protozoa aro supposed to have arisen, and from which the Amœbina branch off in one direction, the Flagellata and Dinoflagehata in inother. The gregarines are believed by Awerinzew to bo connected on the one side with tho Amœbina, on the other with the Coccidia. In tho Neosporidia, the Sarcosporidia are regarded as allied to Flagellata; the Myxosporidia, Microsporidia, and Actinomyxidia, are considered not to be Protozoa at all ; the. Haplosporidia are to be placed provisionally as an independent group taking origin from Amœbina. For the Infusoria, it is suggested that they take origin from amoboflagellate ancestors rather than from true Flagellata.

The object of what is termed a natural as opposed to an artificial system of classification is to endeavour to express by the arrangement of the groups the affinities of the living organisms concerned, and more especially the genetic relationships of one to another on the theory of evolution-that is to say, on the assumption or belief that forms now cxisting are descended from older ancestral forms, and that any two existing forms are deseended from a common ancestral form more or less remote, according as the two existing forms in question have diverged more or less widely from one another. The foundation of a natural classifieation is therefore the phylogeny of the groups dealt with-that is to say, their pedigrees and lines of descent, so far as they can be traced. Phylogeny must, however, always be a matter of speculation, and to a large extent of personal opinion, rather than of direct observation. It is only possible to infer from the study of existing species what the ancestral forms may have been like, since it is unnecessary to point out that no form can be the ancestor of another species existing at the same time. The most that can be said of two co-existing species is that one of them may be believed to have diverged much less in its eharacters from the common ancestral form than the other. When, therefore, a given form is said to have an amœbic or a cercomonad ancestry, it is not intended to imply by that statement 
that the ancestor was Amœba proteus or Cercomonas crassicauda, but only that it was a form such that, if it existed at the present day, it would be referred by its characters to the genus $A m c b a$ or Cercomonas, as the case might be.

The data for drawing phylogenetic conclusions in Protozoa consist entirely of comparisons between the structure and life-history of the various existing forms. Palæontology gives no assistance, since only skeletons are preserved as fossils. All that can be learned from the geological record is that the differentiation of the main groups must have taken place at an immeasurably remote period of the earth's history, since skeletons of Foraminifera and Radiolaria-groups of which the structure and life-history indicate a long pedigree - are found in the earliest fossiliferous strata. It is little wonder, therefore, that the phylogeny of the Protozoa is a subject on which the most opposite opinions are held, as is apparent from the classificatory systems cited above. There can be no finality in a phylogenetic theory, nor, consequently, in any scheme of classification put forward. Both the one and the other express merely the state of current knowledge, and may be expected to undergo modification as knowledge advances.

It is impossible to discuss here at length the phylogeny and classification of the Protozoa, and only a few guiding principles can be put forward. From a general survey of the phylum, it may be claimed first of all that the Protozoa constitute a compact group with definite characters, not a mere receptacle into which can be put anything and everything of microscopic dimensions which is not a bacterium, a fungus, or a parasitic worm, as some writers seem to think. Common to all Protozoa in at least the principal stages of the life-cycle is the differentiation of the body into distinct nucleus and cytoplasm-that is to say, the possession of that type of organization to which $I$ have proposed to restrict the application of the term cell. Doubtless there are, or have been, transitions from this type to the simpler grade of organization characteristic of the bacteria and allied organisms, but such transitions must be sought for outside the phylum Protozoa.

The essential unity and homogeneity underlying the innumerable differentiations of form and structure in the Protozoa may be taken to mean that the phylum as a whole is descended from a common ancestral form, and the first problem is, then, to attempt to form some notion of what the ancestor was like. In dealing with the more specialized forms, such as those constituting the Infusoria or the two principal subdivisions of the Sporozoa, it has been pointed out that each group appears to be derived either from flagellate or sarcodine ancestors. In reviewing the Mastigophora and Sarcodina, it was further pointed out that, greatly as the typical representa- 
tives of the two classes may differ, there are forms of which the systematic position is quite arbitrary. In such a form as Pseudospora, it becomes almost purely a matter of opinion or taste which phase of the life-cycle is to be regarded as the "adult" form determining the class in which the genus is to be placed.

Thus, all paths of evolution in Protozoa appear to lead baokwards to one or the other of the two forms that occur so frequently in the actual development as the earliest phases-the amobula and the flagellula. Most of those who have speculated on the phylogeny of the Protozoa have, consequently regarded the ancestral form of the phylum as one combining amœboid and flagellate characters. Bütschli (2) considered that the Rhizomastigina represent more nearly than any other existing group the primitive type of Protozoon. Since then, however, the life-cycle of the mastigamœbæ has been studied, and it is seen that the adult amceboid form is preceded in development by a simpler monad form (p. 266, Fig. 112), which makes it very doubtful if the mastigamceba itself can be taken as a prinitive type. Awerinzew (890) also regards an "amœboflagellate" type as the primitive stock of Protozoa, which gave rise to all existing groups, and became differentiated into the Amobina on the one hand, the Flagellata on the other.

If an organism possesses two kinds of locomotor organs-pseudopodia and flagella-it is reasonable to suppose that a still more primitive and ancestral form would have possessed only one of these two kinds of organs. It has been seen that there is a gradual transition from pseudopodia to flagella, the intermediate type of organ being a pseudopodium (axopodium) with a firm, rigid, or elastic secreted axis. The question then arises, Which end of the series is to be put first, the flagellum or the pseudopodium? Inasmuch as flagella are found commonly in bacteria, it might be argued that they represent the most primitive type of locomotor organella, and that a simple flagellate monad would represent most nearly the ancestral type of organization in Protozoa. Then it must be supposed that the formation of pseudopodia is a secondary character, acquired by the ancestral form, and the pseudopodia themselves would represent either simple outgrowths of the naked body (lobopodia) or modifications of flagella (axopodia).

Having regard, however, to the manner in which flagella themselves arise-as simple outgrowths from the body-and to the fact that their structure and mode of action are apparently of a much more specialized type than those of pseudopodia, the conclusion seems irresistible that pseudopodia preceded flagella in evolution. We may, then, regard as the most ancestral type in the Protozoa a minute amobula-form, in structure a true cell, with nucleus and 
cytoplasm distinct, which movod by means of pseudopodia; but it must be supposed that some of the pseudopodia very soon underwent modifications which resulted in the acquisition of true flagella, and thus arose at a very early stage of evolution the flagellula or monad-form. In all probability these earliest monads were forms with an amœboid body, most nearly represented at the present day by such forms as Cercomonas (Fig. 114) or the flagellulæo of Mycetozo (Fig. 98). From such forms arose the Sarcodina and their derivatives (Neosporidia) by loss of flagella and specialization of the amceboid form in the adult, and the Mastigophora and their derivatives (Telosporidia, Infusoria) by specialization of the flagellar apparatus combined with the acquisition of a cortex and loss of amœboid movement.

If the foregoing phylogenetic speculations be accepted, it is clear that in a natural classification of the Protozoa the Sporozoa must be abolished as a class, and the two groups comprised in them must either be raised to the rank of independent classes or distributed amongst the others-the Telosporidia placed near the Mastigophora, the Neosporidia ncar the Sarcodina. The primary subdivision of the Protozoa, if it is to represent the first branching of the ancestral stem, should be one which places on one side the Mastigophora, Telosporidia (better Rhabdogeniæ), and Infusoria, oul the other the Sarcodina and Neosporidia (better Amœbogoniæ). Beyond this point it is scarcely profitavle at the present time to push phylogenetio speculations farther.

In conclusion, two groups of organisms require brief mentionthe Spirochrtes and the Chlamydozoa-since by many authorities they have been referred to a position in or near the Protozoa.

\section{THE SPIROCh}

Under the name "spirochætes" are grouped a number of organisms, free-living or parasitic, with flexible bodies of slender, thread-like form, concerning the nature and systematic position of which a great deal of confusion has existed of recent years, due chiefly to conflicting statements with regard to the facts of their structure and methods of reproduction. The group comprises five principal types, regarded each as of generic rank :

1. Spirochata sens. strict., a name given by Ehrenberg in 1833 to a relatively large, free-living form, $S$. plicatilis. Other species of the genus have been described. For a full account, see Zuelzer (904).

2. Cristispira, a name proposed by Gross (897) for a number of species parasitic in the digestive tract or crystalline style of Lamellibranch molluscs, and characterized by the possession of a crest or 
ridge, commonly but wrongly termed an " undulating membrane," running the length of the body. The type of the genus is $C$. balbianii, originally named by Certes Trypanosoma balbianii, from the crystalline style of the oyster.

3. Saprospira, Gross (898), for free-living, saprophytic forms similar in structure to Cristispira, but without the crest.

4. Spiroschaudinnia, the name proposed by Sambon for the many species of minute spirochætes parasitic in the blood of vertebrates and in various invertebrates. Such are S. recurrentis (=obermeieri), parasite of human relapsing fever; $S$. duttoni, parasite of African relapsing fever; $S$. gallinarum of fowls; $S$. anserina of geese; and numerous other species from various hosts. In structure the body of these species appears to be little, if anything, more than a flexible thread of chromatin ; but the development indicates rather that, as in the genus Cristispira, the interior of the body is divided into minute segments or chambers. The species parasitic in blood are transmitted by the agency of blood-sucking arthropods. S. duttoni, for example, is transmitted by a tickOrnithodoros moubata-which lives in the mud-floors of huts or in the soil in spots where caravans camp habitually. The spirochætes are taken up from human blood by the adult ticks, and pass through the egg into the next generation of nymphs, ${ }^{*}$ which transmit the infection to human beings.

5. Treponema, the name proposed by Schaudinn for T. pallidum, the spirochæte of syphilis discovered by him. A second species$T$. pertenue, the parasite of yaws (frambœsia)-is also recognized. Structurally this type is very similar to the last.

Some authors-for instance, Gross (899) and Dobell (895)-consider that there is " no valid reason for drawing a generic distinction between Treponema pallidum and such forms as "Spirochata' recurrentis, etc." Gross combines Types 4 and 5 under the name Spironema proposed by Vuillemin; but since this name is preoccupied, Dobell places them together in Schaudinn's genus Treponema.

The forms parasitic in the blood of human beings and other vertebrates were generally regarded as Bacteria of the genus Spirillum, or at least of the section Spirillacea, until quite recent years, and the diseases caused by them were spoken of as spirilloses. The chief points of difference between tho spirilla of relapsing fevers and those of the ordinary type were the flexibility of the body in the former and the failure to grow them in cultures. The confusion prevailing at present originated with Schaudinn's famous memoir on the blood-parasites of the Little Owl (132). While, on the one hand, it is to Schaudinn's credit to have recognized the affinities of the parasitio "spirilla" to Ehrenberg's free-living genus Spiroch ceta he was, on the other hand, misled by the superficial resemblance between spirochætes and certain small, slender forms of trypanosomes, which again he connected, quite erroneously, with the life-cycle of Leucocytozoon (see p. 370). Schaudinn therefore regarded the spirochrtes as Protozoa allied to trypanosomes, and endeavoured to prove a similar type of organization in both classey of organisms: a nuclear

* The six-legged larval stage is suppressed-that is to say, passed through in the egg-in this species of tick. 
apparatus with kinetonucleus and trophonucleus, and a locomotor apparatus with flagellum and undulating membrane. Schaudinn further constructed a hypothetical form of "Urhæmoflagellat" connccting the spirochæto and trypanosome type of organization; he put forward the suggestion (903) that "as the general structural plan of a trypanosome (nuclcar and locomotor apparatus) may be found realized in various groups of Protozoa as a transitory developmental condition (comparable somewhat to the gastrula.condition in the Metazoa), so also the spirochæte may crop up occasionally as a morpho. logical type in the development of Protozoa, and as a developmental stage may indicate to us phylogenetic relations."

Schaudinn lived long enough, fortunately, to retract many of his stato ments with regard to the structure of spirochætes, and acknowledged that the trypanosome-type of structure was not to be made out in the minute parasitic spirochrtes. Nevertheless, since his time the investigators of these organisms have been divided into two camps - those who hold fast to Schaudinn's theory of the spirochætes as Protozoa, and those who class them with Bacteria, respectively ; it being generally assumed, for some unknown reason, that it they are not Protozoa they must be Bacteria, or vice versa. A third set of authorities compromise by placing the spirochætes in an intermediate position between the two groups.

In considering the question of the affinities of the spirochretes, attention has been directed not only to their structure, but also to their life-history; and a hot controversy has raged with regard to their mode of fission, whether it takes place longitudinally, as in a trypanosome, or transversely, as in a bacterium. Investigators contradict each other flatly with regard to this point; but from the most recent investigations it soems probable, at least, that the division is always transverse, and that the appearance of longitudinal division is due to the peculiar method of "incurvation" described by Gross (Fig. 194). A spirochæte about to divide grows greatly in length, and one end of the body doubles back on itself, continuing to do so until the recurved limb of the body is of the same length as the remainder; the two halves twist round each other and produce an appearance which may be mistaken easily for longitudinal fission ; but the actual division of the body takes placo at the point where it is bent over, and is transverse.

With regard to the development, nothing has been found in the least confirmatory of Schaudinn's statements with regard to "Spirochota ziemanni," with the sole exception of the statements of Krzysztalowicz and Siedlecki (901), who profess to have seen trypanosome-stages in the development of Treponema pallidum; but their statements are entirely unconfirmed by other investigators. Of a very opposite type are the statcments of Leishman (902) with regard to the development of $S$. duttoni in the tick. The spirochæte appears to break up into minute masses of chromatin, " coccoid granules," in the ova and tissue-cells of the tick. The coccoid granules appear to devolop into spirochætes again.

The observations of Leishman have recently been fully confirmed by the investigations on the development of Spiroschaudinnia gallinarum published by Hindle (900) who gives a useful diagram of the entire life-history. Bosanquet (894) also observed the formation of coccoid bodies in Cristispira anodonta by the segmentation of the elongated body into a number of ceccoid bodies like a string of beads. A development of this type suggests very strongly affinities with bacteria, but none whatever with Protozoa of any class. The coccoid grains may bo compared with the spore-formation in bacteria, and with that described by Gross (898) in Saprospira grandis. In all cases, throughout the serics of living beings, wherever an organism exhibits in its fullydeveloped "adult" stage peculiarities of a special kind, it is above all to the early developmental forms that the naturalist turns for indications of the true affinities of the organism in question.

Recently the structure of spirochates has been studied carefully by Gross $(897,898)$, Zuelzer (904), and Dobell (895), by means of proper cytological methods of technique. The results show a complete difference in every 
respect between spirochætes and trypanosomes and other flagellates. In the words of Dobell, "the nuclear and cytoplasmic structures are wholly different ; a trypanosome has a flagellum, a spirochæte has none; the crista is not an undulating membrane; the cell-membranes are not similar; and, moreover, the method of division is quite different in the two organisms."

Doflein (7) places the spiroohætes as a group named the Proflagellata, supposed to be transitional from bacteria to flagellates. Zuelzer (904) takes a similar view, rejecting, however, any affinity between spirochætes and Hartmann's "Binucleata." Awerinzew (890) puts forward the remarkable suggestion that the Flagellata "pass on into different Binucleata, and end with the Spirochoeta

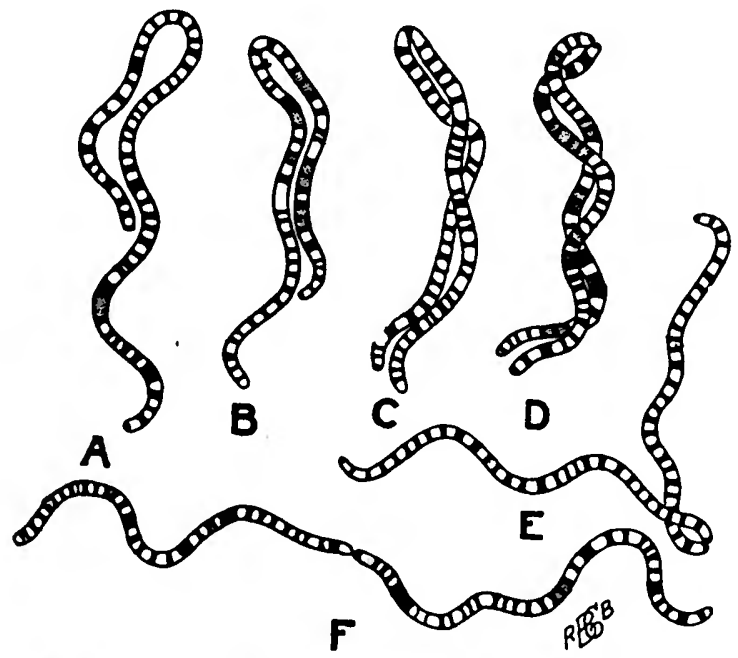

Fra. 194.-Stages in the division of Cristispira pectinis. $A, B$, Two successive stages of the incurvation; $C$, incurvation complete; $D$, division of the body at the point where it is bent back; $E, F$, scparation of the two daughter. spirochætes. After Gross (897).

(sic)," from which it would appear that he regards the spirochætes as the last product of the line of evolution that produced the trypanosomes and allied forms.

For the various reasons that have been set forth above, it appears impossible to include the spirochætes any longer in the Protozoa. Dobell regards them as "an independent group of unicellular organisms which show very little affinity to any other group." Gross, on the other hand, considers that the Spironemacea-i.e., the genera Cristispira, Saprospira, and Spironema, in the sense in which this genus is understood by him (see above)-form a family which can be ranked in the bacteria, but which is related to the Cyanophyceæ, especially the Oscillatoriæ. 


\section{The Chlamydozon.}

The name Chlamydozoa of Prowazek (Strongyloplasmata, Lipschütz) was proposed in order to include in the first place a class of highly problematic organisms believed to be the causes of certain diseases of man or animals. It is not yet certain exactly what diseases are to be referred to Chlamydozoa. According to Hartmann (909), undoubted chlamydozoal diseases are vaccinia and variola, trachoma, and niolluscum contagiosum, amougst human beings, and in birds epithelioms contagiosum and diphtheria. Further diseases probably attributable to Chlamydozoa are hydrophobia, scarlet fever, measles, foot-and-mouth disease of animals, and "Gelbsucht" of silkworms. In all these diseases the virus has certain common properties, while exhibiting specific peculiarities in each case. It can pass through ordinary bacterial filters without losing its virulence, and it produces characteristic reaction-products or cell-inclusions in the infected cell.

In order to understand why these organisms should be mentioned in a book dealing with Protozoa, the subject is best dealt with in an historical manner. The advances in the knowledge of the diseases mentioned may be summarized briefly in four principal stages :

1. Various investigators at different times have made known the existence of peculiar cell-inclusions in the infected cells in a certain class of diseases, inclusions which have been known by the names of their disco verers-for instance, in trachoma (Prowazek's bodies), vaccinia. (Guarnieri's bodies), scarlet fever (Mallory's bodies), hydrophobia (Negri's bodies), etc.

2. By many investigators the characteristic cell-inclusions were identified as the actual parasitic organisms causing the disease. They received zoological names, were referred to a definite position in the ranks of the Protozos, and attempts were made to work out and construct a developmental cycle for them. The supposed parasites of molluscum contagiosum were referred to the coccidia; those of vaccinia and variola were given the name Cytoryctes; of hydrophobia, Neuroryctes; of scarlet fever, Cyclasterium.

Calkins (908) studied in great detail the cell-inclusions of vaccine and smallpox, and described a complete developmental cycle, in its main outlines as follows: The primary infection is brought about, probably, at some spot on the mucous membrane of the respiratory or buccal passages by air-borne germs (spores). After active proliferation at the seat of the primary infection, the parasites are carricd to all parts of the body in the circulation, probably during the initial fever. These two early phases are hypothetical. The third phase is the appearance of the parasites in the cells of the 
stratified epithelium of the epidermis. In this situation they run through two cycles-the one cytoplasmic, the other intranuclear. The first is the vaccine-cycle, and is the only part of the development of which the harmless vaccine-organism is capable; the variola-organism, however, after passing through a vaccine-cycle, proceeds to the extremely pathogenic intranuclear cycle.

The vaccine-cycle, according to Calkins, begins with the appearance of "gemmules" in the cytoplasm of the oells affected. Each gemmule is a minute grain of chromatin without cytoplasm of its own at first, but as it grows a cytoplasmic body is formed. When full-grown, the parasite sporulates by fragmentation of its nucleus into a great number of grains, which, as gemmules, pass into other cells and repeat the development already described. Several generations of this type may succeed each other before giving rise to the next type.

The intranuclear variola-cycle begins in the same way with gemmules, which, however, penetrate into the nucleus, and develop a cytoplasmic body. According to Calkins, they become sexually differentiated, and produce gametes which conjugate. The final result is the production of numerous spores, which are probably the means of spreading the infection.

Calkins referred Cytoryctes to the Microsporidia. Now, however, he inclines to the opinion that the genus should be placed amongst the Rhizopods (4).

Negri (910) also describes a developmental cycle for Neuroryctes hydrophobice, which he regards as a true Protozoon, and which Calkins refers also to the Rhizopoda. Siegel (914) describes under the name Cytorhyctes organisms of a type perfectly different from those described by Calkins. He distinguishes four species-Cytorhyctes vaccinice of vaccine and smallpox, C. luis of syphilis, C. scarlatince of scarlet fever, and $C$. aphtharum of foot-and-mouth disease.

3. The parasitic life-cycles described by Calkins and others have been criticized by a number of investigators, who have maintained that the bodies in question are not Protozoa, nor even independent living organisms at all, but merely degeneration-products of the cell itself, provoked by a virus yet to be found. Thus, with regard to Guarnieri's bodies (Cytoryctes) of vaccine, it is maintained by Foa, Prowazek, and others, that they consist of nucleolar substance (plastin) extruded from the nucleus; that they have no definite developmental cycle; and that infection can be produced by lymph in which Guarnieri's bodies have been destroyed, or by tissue in which they are not present. With regard to the Negri bodies, Acton and Harvey (906) come to the same conclusions, and state that similar nucleolar extrusions can be brought about also by other stimuli than the rabies-virus. 
4. The foregoing sceptical phase has been succeded by the positive belief that the true parasitic organism in these diseases consists of certain minute bodies-the Chlamydozoa or strongyloplasms.*

The chief characteristics of the Chlamydozoa, according to Prowazek and Lipschütz (913) are, first, their minute size, smaller than any bacteria hitherto known, enabling them to pass the ordinary bacterial filters ; secondly, that they develop within cells, in the cytoplasm or nucleus, and produce characteristic reactionproducts and enclosures of the cell (their position within the cell is not the result of phagocytosis); thirdly, that they pass through a series of developmental stages, and are specially characterized by their mode of division, which is not a simple process of splitting, as in bacteria, but is effected with formation of a dumb-bell-shaped figure, as in the division of a centriole. Two dots are seen connected by a fine line like a centrodesmose, which becomes drawn out until it snaps across the middle, and its two halves are then retracted into the body. Chlamydozoa have not yet been grown successfully in cultures, but infections can be produced with pure colloid-filtrates, free from bacteria, but containing the minute bodies themselves. They are characteristically parasites of epiblastic cells and tissues.

As an example of the development of a chlamydozoon may be taken that of the vaccine-virus, which, according to Prowazek (913) and Hartmann (909), is briefly as follows :

1. The infection begins and ends with numerous " elementary corpuscles" (gemmules of Calkins?), which occur both within and amongst the cells. They are very minute, and can pass bacterial filters.

2. Within the cells the elementary corpuscles grow into the larger " initial bodies."

3. The infected cell extmides nucleolar substance-plastin-from its nucleus, which envelops the parasites as in a mantle (hence the name Chlamydozoa, from $\chi \lambda$ גaús, a mantle), thus producing in the case of vaccine the characteristic Guarnieri's bodies, in which the parasites multiply. It is this mantle of nucleolar substance, apparently, which represents the "cytoplasm" of Cytoryctes, as described by Calkins.

* The name Chlamydozoa, as denating a class of microscopic organisms, must on no account be confused with the names Cyluryctes, Neuroryctes, etc., which represent the generic names of the supposed parasites of variola and rabies respectively. To those who rugard Cytoryctes, etc., as true organisms, the Chlamydo. zOa are merely chromidia or dots of chromatin in the body of the parasito; to those who believe in the Chlamydozos as complete organisms, Cytoryctes, eto., are cell-inclusions or degeneration-products of the nucleus. The conceptions implied in the words Chlamydozos and Cytoryctes respectively are antagonistio and mutually destructive ; if the one is a reality, the other is non-existent. It is altogether incorrect to spcak of Cytoryctes, Neuroryctes, etc., as genera of Chlamydozoa. 
4. Finally, the Guarnieri's body breaks up, and the cell becomes full of initial corpuscles, which divide up in their turn into numerous elementary corpuscles, and the cycle is complete.

An interesting probrem, from both the medical and biological points of view, is that of the relation of the organism of vaccinia (cow-pox) to that of variola (small-poz). It is well known that an inoculation with vaccine-lymph (vaccination) produces a transitory local disturbance which confers partial immunity against infection with variola. It does not seem to be quite clear whether the organisms, of vaccinia and variola are to be regarded as two distinct species or as two phases or conditions of the same species of organism; the latter is the view of Calkins, as stated above. Manson has suggested (Brit. Med. Journ., 1905, ii., p. 1263) that the relationship between the organisms of vaccinia and variola may be similar to that between Leishmania tropica, of Oriental Sore, and L. donovani, of Kala-azar. No evidence has been brought forward as yet, however, to show that an infection with Oriental Sore confers any immunity against Kala-azar.

The Chlamydozoa have been most studied in those cases where their power of producing disease has forced them upon the attention of medical investigators, but it is not to be supposed that as a group of organisms they occur solely as parasites of higher animals. It is probable that they are of widespread occurrence, and that the peculiar nuclear parasite of Amoba known as Nucleophaga, Dangeard, for instance, should be referred to the Chlamydozoa (compare Schepotieff, 269), and perhaps also the similar parasite of Paramecium described by Calkins under the name Caryoryctes. No Chlamydozoa are known, however, to occur as free-living, nonparasitic organisms, but this circumstance may be due to their extreme minuteness; the species 'snown owe their detection to the disturbances they cause in their hosts. Finally, it must be mentioned that the parasitic theory of cancer, sometimes thought to bc long since defunct, has been revived recently by Awerinzew (907), who is of opinion that cancer is caused by intranuclear parasites of the nature of Chlamydozoa.

Such, briefly summarized, is the present position of the problem. Future research must decide the truth or falsity of one or the other of the solutions that have been advocated. It only remains to discuss briefly the nature of the Chlamydozoa, if the interpretation of Prowazek and his adherents be accepted. According to Prowazek and Lipschütz (913), the Chlamydozoa belong neither to the Bacteria nor to the Protozoa. Hartmann (909), however, seems to consider that their development and their characteristic mode of division are Protozoan characteristics. The "development," however, seems to consist of little, if anything, more than growth in size As "elementary corpuscles" they are smaller, as "initial bodies" larger. The dumb-bell-shaped figure seen in division may mean 
simply that their substance is of a viscid or semifluid nature, and that their bodies are not limited by a membrane; consequently, when the two halves travel apart in the process of division, the substance of the body is drawn out into a connecting thread until its surface tension overcomes its cohesion. On the other hand, they exhibit nothing of cell-structure or of any other characteristics which indicate any affinity to the Protozoa. Their type of organization seems to be the simplest possible in a living body-a mere grain of chromatin without cytoplasm, and without a membrane or envelope of any kind. In the latter respect they appear to be of a simpler type of organization than any bacterium, and perhaps represent more nearly than any other known organism the simplest possible form of living being.

Bibliography.-For references see p. 504

Iie domum, satura, venit Hesperus, ite capellse. 


\section{BIBLIOGRAPHY}

The references to literature are numbered consecutively, but are grouped according to the chapters.

An asterisk $\left(^{*}\right)$ attached to a reference indicates that the work in question contains full references to the previous litesature of the subject.

Memoirs in which only new species are described are not cited, unless there is some special reason for doing so. All new species are recorded in the "Zoological kecord." published annually by the Zoological Society of London ; the last volume published up to date is that for 1910 ; the volume for 1911 will appear towards the end of 1912.

The litles of the subject-matter of articles are in many cases not given verbatim, but in abbreviated form.

The abbreviations employed for the titles of periodicals are given beiow. (In other cases the titles of periodicals are abbreviated in a manner which does not require special explanation.)

A.I.C.P. Archivos do Instituto Bacteriologioo Camara Pestana (Lisbon).

A.I.P. Annales de l'Institut Pasteur (Paris).

A.K.G.A. Arbeiten aus dem kaiserlichen Gssundheitsamte (Berlin).

A.P.K. Archiv für Protistenkunde (Jena).

A.S.T.H. Archiv für Schiffs- und Tropenhygiene (Loipzig).

A.T.M.P. Annals of Tropical Medioine and Parasitology (Liverpool).

A.Z.E. Archives de Zoologie expérimentale et générale (Paris).

B.A.S.C. Bulletin Internationalo de l'Académio des Sciences à Cracovio.

B.B. Biological Bulletin (Woods Holl, Mass.).

B.C. Biologisches Centralblatt (Leipzig).

B.I.P. Bulletin de l'Institut Pasteur (Paris).

B.S.P.E. - de la Sooiété de Pathologie Exotique (Paris).

B.S.Z.F. - de la Société Zoologique de France (Paris).

C.B.B.P.K. Centralblatt für Bakteriologie, Parasitenkunde und Infectionskrankheiten (Jena).

C.R.A.S, Comptes-rendus hebdomadaires des Séances de l'Académie des Sciences (Paris).

C.R.S.B. - des Séances et Mémoires de la Société de Biologie (Paris).

J.E.M. Journal of Experimental Medicine (Baltimore).

J.E.Z. - of Experimental Zoology (Baltimore).

J.H. - of Hygiene (Cambridge).

J.L.S. - of the Linnean Society : Zoology (London).

M.I.O.C. Memorias do Instituto Oswaldo Cruz (Rio de Janeiro).

P.R.S. Proceedings of the Royal Society of London.

Py. Parasitology (Csmbridge).

P.Z.S. Proceedings of the Zoological Society of London.

Q.J.M.S. Quarterly Journal of Microscopical Science (London).

S.B.A.B. Sitzungsberichto der königlich-preussischen Akademie der Wissenschaften zu Berlin.

S.B.G.B. - der Gesellschaft naturforschender Freunde zu Berlin.

S.B.G.M.P. - der Gesellschaft für Morphologie und Physiologie in Münohen.

S.M.I. Scientific Memoirs by Officers of the Medical and Sanitary Depart. ments of the Government of India (Calcutta).

V.D.Z.G. Verhandlungen der deutsohen zoologischen Gesellsohsft (Leipzig).

Z.A. Zoologischer Anzeiger (Leipzig).

Z.a:P. Zeitachrift für allgemeine Physiologie (Jona).

Z.H. - für Hygiene und Infectionskrankheiten (Leipzig).

Z.w.Z. - für wissenschsftliche Zoologie (Leipzig). 


\section{CHAPTER I}

\section{General Work on Protozoa.}

(1) Brompt, E. (1910). Précis de Parasitologie. Paris : Masson et Cie.

*(2) BürschLI, 0. (1882-1889). Protozos. Bronn's Klassen und Ordnungen die Thier-Reichs, I.

(3) - (1910). Vorlesungen über vergleichende Anatomie, 1. Leipzig : W. Engelmann.

(4) Calknss, G. N. (1901). The Protozoa. New York : Macmillan and Co.

*(5) - (1909). Protozoology. New York and Philadelphia : Lea and Fiebiger.

*(6) Delage, Y., and Hérovard, E. (1896). Traité de Zoologie Conorète, I. Paris : Schleicher Frères.

*(7) Dofrern, F. (1911). Lehrbuch der Protozoenkunde. Thirdedition. Jena : Gustar Fischer.

(8) Hartoo, M. (1906). Protozoa. Cambridge Natural History, vol. i. London : Macmillan and Co.

(9) Kent, W. S. (1880-1882). A Manual of the Infusoria. London : David Bogue.

*(10) Lava, A. (1901). Lehrbach der vergleichenden Anatomie der wirbellosen Thiere, 2te Auflage. Jena : Gusta $\nabla$ Fisoher.

(11) LankestrR, E. R. (1891) Protozoa. Encyclopadia Britannica, ninth edition ; reprinted in Zoological Articles. London : A. and C. Black.

(12) - (1903 and 1909). A Treatise on Zoology. Part I., Fascs. 1 and 2. London : A. and C. Black.

(13) Mrnchis, E. A. (1907). Protozoa. Allbutt and Rolleston : A System of Medicine, vol. ii., part ii., p. 9.

(14) Prowazer S. V., and others (1911). Handbuch der Pathogenen Protozoen. Leipzig : J. A. Barth. Lief. 1 and 2.

(15) Rolleston, G., and Jackson, W. H. (1888). Forms of Animal Life. Second edition. Oxford : Clarendon Press.

\section{CHAPTER II}

In addition to the general works cited under the previous chapter, see especially :

(16) Goodey, T. (1911). A Contribution to our Knowledge of the Protozos of the Soil. P.R.S. (B.), Ixxxiv., p. 165.

(17) LAUTERBoRN, R. (1901). Die " sapropelische" Lebewelt. Z.A., xxiv., p. 50.

(18) Laveran, A., and Mesnir, F. (1899). De la Sarcocystine, toxine des Sarcosporidies. C.R.S.B., li., p. 311.

(19) - and Petrt, A. (1911). Les trypanotoxines. B.S.P.E. iv. p. 42.

(20) Mesnil, F. (1905). L'Hérédité dans les Maladies à Protozoaires. B.I.P., iii., p. 401.

(21) Mnchin, E. A. (1910). Phenomens of Parasitism amongst Protozoa. Journ. Quekett Microscop. Club (2), xi., p. 1.

(22) Roudsky, D. (1910). Lo Trypanosoma lewisi Kent renforcé. C.R.S.B., lxix., p. 384.

(23) - (1911). La possibilité de rendre le Trypanosoma lewisi virulent pour d'autres rongeurs que le rat. C.R.A.S., clii., p. 56. (See also Bulletin of the Sleeping Sickness Bureau, vol. iii., pp. 81 and 265 for further references on this subject.)

(24) Russerl, E. J., and Hotchinson, H. B. (1909). The Effect of Partial Sterilization of Soil on the Production of Plant Food. Journ. Agric. Sci., iii., p. 111.

(25) Teichmann, E. (1910). Das Gift der Sarcosporidien. A.P.K., xx., p. 97.

(26) - and BrAUN, H. (1911). Ein Protozoontoxin (Sarcosporidiotoxin). A.P.K., xxii., p. 351 .

(27) WENDELSTADT and Fellmer, T. (1910). Einwirkung von Kaltblüterpassagen auf Nagana- und Lowisi-Trypanosomen. Zeitschr. f. Immunitätsforschung, v., p. 337.

(28) WINTER, F. W. (1907). Untersuchung über Peneroplis pertusus (Forskal). A.P.K., х., p. 1 . 


\section{CHAPTER III}

In addition to the general works cited under Chapter I., see especially :

(29) Heron-Aulen, E., and Earuand, A. (1909). A New Species of Technitella. Journ. Quekett Microsc. Club (2), x., p. 403.

(30) Koltzoff, N. K. (1903). Formbestimmende elastische Gebilde in Zellen. B.C., xxiii., p. 680 .

(31) - (1906). Die Gestalt der Zelle. Arch. mikr. Anat., lxvii., p. 364.

(32) Prowazek, S. v. (1908). Biologie der Zellen, I. B.C., xxviii., p. 782.

(33) - (1909). Theorie der Cytomorphe. Z.A., xxxiv., p. 712.

(34) Rhumbler, L. (1898). Physikalische Analyse von Lebenserscheinungen der Zelle, I. Arch. Entwicklungsmech., vii., p. 103.

(35) - (1902). Die Doppelschalen von Orbitolites. A.P.K., i., p. 193.

(36) Verworn, M. (1888). Biologische Protisten-Studien. Z.w.Z., xlvi., p. 455.

\section{CHAPTER IV}

In addition to Nos. 34 and 35 , see :

*(37) BÜrschI, O. (1894). Microscopic Foams and Protoplasm. (Transiation by E. A. Minchin.) London : A. and C. Black.

(38) Fadré-Fremiet, E. (1908). La Structure des Matières Vivantes. B.S.Z.F., xxxiii., p. 104.

*(38.5) - (1910). Les Mitochondries des Protozoaires et des Cellules sexuelles. Arch. d'Anat. Microsc., xi., p. 457.

*(39) Fischer, A. (1899). Fixirung, Färbung und Bau des Protoplasmas. Jena : Gustav Fischer.

(40) Rhumbler, L. (1902). Der Aggregatzustand und die physikalischen Besonderheiten des lebenden Zellinhalts. Z.a.P., ii., p. 183.

\section{CHAPTER V}

In addition to the references cited above for Chapters I. and III., and those cited below for Chapter X., see :

(41) Goldschmid, R. (1907). Lebensgeschichte der Mastigamöben. A.P.K., Suppl. I., p. 83.

(42) Muschin, E. A., and Woodcock, H. M. (1911). The Trypanosome of the Little Owl (Athene noctua). Q.J.M.S., lvii., p. 141.

(43) SchaUdnN, F. (1894). Camptonema nutans. S.B.A.B., lii., p. 1227. Reprinted, Schaudinn's Arbeiten, 1911, p. 50.

(44) Schoberg, A. (1905). Cilien und Trichocysten einiger Infusorien. A.P.K., vi., p. 61 .

\section{CHAPTER VI}

In addition to the works cited here, see also the bibliographical references for Chapter VII:

(45) Aragao, H. DE B. (1910). Ueber Polytomella agilis. M.I.O.C., ii., p. 42.

(46) Aw erinzew, S. (1907). Struktur des Protoplasma und des Kerns von Amaba proteus (Pall.). Z.A., xxxii., p. 45.

(47) - (1909). Entwicklungsgeschichte von Coccidien aus dem Darme von Cerebratulus sp. (Barrouxia spiralis). A.P.K., xviii., p. 11.

(48) Calkins, G. N. (1903). The Protozoan Nucleus. A.P.K., ii., p. 213.

(48.5) Chagas, C. (1911). Die zyklischen Variationen des Caryosoms bei zwei Arten parasitischer Ciliaten. M.I.O.C., iii., p. 136.

*(49) Chatron, E. (1910). La structure du Noyau et la Mitose chez les Amœbiens. A.Z.E. (5), v., p. 267.

(50) Collin, B. (1909). La Conjugaison d'Anoplophrya branchiarum (Stein) (A. circulans, Balbiani). A.Z.E. (5), i., p. 345.

*(51) DoBkl, C. C. (1909). Chromidia and the Binuclesrity Hypothesia Q.J.M.S., liii., p. 279. 
*(52) Dosen, C. C. (1911). Contributions to the Cytology of the Bacteria. Q.J.M.S., Ivi., p. 395. "Autorreferat" in A.P.K., xxiv., p. 84.

(53) Extz, G. (1909). Organisation und Biologie der Tintinniden. A.P.K., xv., p. 93.

(54) Ermard, H. (1911). Die Henneguy-Lenhosseksche Theorie. Ergebn. Anat. Entwick., xix. (second half), p. 893.

(55) Faurḱ-Fremiet, E. (1910). Appareil nucléaire, Chromidies, Mitochondries. A.P.K., xxi., p. 186.

(56) França, C., and Atrias, M. (1907): Les Trypanosomes des Amphibiens, II. Le Trypanosoma rotatorium de Hyla arborea. A.I.C.P., i., p. 289.

(57) Goudschmmt, R. (1904). Die Chromidien der Protozoon. A.P.K., v.,p 126.

(58). - and Poporr, M. (1907). Die Karyokinese der Protozoen und der Chromidialapparat der Protozoen- und Metazoenzelle. A.P.K., viii., p. 321 .

(59) Gunlermond, A. (1910). Corpuscules metachromatiques ou Grains de Volu. tine. A.P.K., xix., p. 289.

(60) Hartmann, M. (1909). Polyenergido Keme. B.C., xxix., pp. 481 and 491.

(61) - (1911). Die Konstitution der Protistenkerne. Jena : Gustar Fischer.

(62) - and CHAGAS, C. (1910). Flagellatenstudien. M.I.O.C., ii., p. 64.

(63) - and Prow AzEk, S. v. (1907). Blepharoplast, Caryosom und Centrosom. A.P.K., x., p. 306.

(64) Hertwia, R. (1898). Kerntheilung, Richtungskörperbildung und Befruchtung von Actinospharium Eichhorni. Abhandl. bayer. Akad. (II. Cl.) xix., p. 631 .

(65) - (1899). Encystierung und Kernvermehrung bei Arcella vulgaris. Kupfier's Festschrift, p. 567.

(66) - (1902). Die Protozoen und die Zelltheorie. A.P.K., i., p. 1.

(67) - (1903). Das Wechselverhältnis von Kern und Protoplasma. S.B.G.M.P., xviii. $p$ \%7.

(68) - (1907). Der Chromidialapparat und der Dualismus der Kernsubstanzen. Ibid., xxiii., p. 19.

(69) JAus, E. (1904). Kernteilung und Geisselbildung bei den Schwärmern von Stemonitis flaccida. Ber. Deutsch. Bot. Ges., xxii., p. 84.

(70) J LNIOKI, C. (1910). Parasitische Flagellaten, I. Lophomonas blattarum, L. striata. Z.v.Z., xcv., p. 243.

(71) - (1911). Der Parabasalapparat bei parasitischen Flagellaten. B.C., xxxi., p. 321.

(71-5) - (1912). Parasitische Arten der Gattung Paramaba. Verh. Naturforsch. Ges. Basel, xxiii.

(72) LFoer, L, and DoboscQ, O. (1911). Deux Grégarines des Crustacés. A.Z.E. (5), vi., "Notes et Rovue," p. lix.

(73) MaIar, H. N. (1903). Der feinere Bau der Wimperapparate der Infusorien. A.P.K., ii., p. 73.

(74) Mrsnu, F. (1905). Chromidies et Questions connexes. B.I.P., iii., p. 313.

(75) Mrncrin, E. A. (1911). Some Problems of Evolution in the Simplest Forms of Life. Journ. Quekett Microsc. Club (2), xi., p. 165.

(76) Näolar, K. (1911). Protozoen aus einem Almtümpel, I. Amœba hartmanni, n. sp. Anhang : Zur Centriolfrage. A.P.K., xxii, p. 56.

77) Poporr, M. (1909). Die Zellgrösse, ihre Fixierung und Vererbung. Arch. Zellforschung, iii., p. 124.

(78) Reichenow, E. (1910). Homogregarita steparowi. Die Entwicklungsgeschichte einer Hämogregarine. A.P.K., xx., p. 251.

(79) Robrentson, M. (1911). The Division of the Collar-Cells of the Calcarea Heteroccela. Q.J.M.S., lvii., p. 129.

(80) - and MnrcHur, E. A. (1910). The Division of the Collar-Cells of Clathrina coriacea. Q.J.M.S., Iv., p. 611 .

(81) SchadinN, F. (1896). Der Zeugungskreis von Paramaba eilhardi. S.B.A.B., p. 31. Reprinted, Schaudinn's Arbeiten, 1911, p. 115.

(82) - (1896). Das Centralkorn der Heliozocn. V.D.Z.G., vi., p. 113. (With discnssion by Lauterborn and Bütschli.)

(83) StzDleCKI, M. (1905). Die Bedeutung des Karyosoms. B.A.S.C., .p. 559.

(84) Wenyon, C. M. (1911). Oriental Sore in Baghdad, together with Observations on a Gregarine in Stegomyia fasciata, the Hrmogregarines of Dogs, and the Flagellates of House Flies. $P y .$, iv., p. 273.

(85) ZuELzer, M. (1904). Diffugia urcedata. A.P.K., iv., p. 240.

(86) - (1909). Wagnerella borealis. A.P.K., xvii., p. 135. 


\section{CHAPTER VII}

In addition to the works cited here, see also Nos. $45,48,49,50,56,58,60,62$, 64. $66,69,70,71,71 \cdot 5,78,79,80,81,82$, and 80 above.

(87) Aragao, H. DE B. (1904). Amoeba diplomitotica. M.I.O.C., i., p. 33.

(88) Awerinzew, S. (1904). Teilung von Amoba proteus. Z.A., xxvii., p. 399.

(89) HartmanN, M., and Chagas, C. (1910). Schlangenhämogregarinen. A.P.K., xx., p. 351.

(90) - - (1910). Die Kernteilung von Amœba hyalina. M.I.O.C., ii., p. 159.

(91) Hertwig, R. (1903). Korrelation von Zell- und Kerngrösse. B.C., xxiii., pp. 49 and 108.

(92) - (1908). Neue Probleme der Zellenlehre. Arch. f. Zellforschung, i., p. 1.

(93) LEBEDEW, W. (1908). Trachelocerca phœnicopterus. A.P.K. xiii., p. 70.

(94) Mororf, T. (1908). Die bei den Cephalopoden vorkommenden AggregataArten. A.P.K., xi., p. 1.

(95) N $\triangle$ GLER, K. (1909). Entwicklungsgesohichtliohe Studien über Amöben. A.P.K., xv., p. $\mathrm{l}$.

(96) - (1911). Caryosom und Contriol beim Teilungsworgang von Chilodon uncinatus. A.P.K., xxiv., p. 142.

(97) Prowazer, S. v. (1903). Die Kernteilung des Entosiphon. A.P.K., ii., p. 325.

(97.5) Reichenow, E. (1909). Homatococcus pluvialis. A.K.G.A., xxxiii., p. 1.

(98) SchaUdiNs, F. (1894). Kerntheilung mit nachfolgender Körpertheilung bei Amaba crystalligera. S.B.A.B., 1894, p. 1029. Reprinted, Schaudinn's Arbeiten, 1911, p. 95.

(99) - (1900). Der Generationswechsel bei Coccidien. Zool. Jahrbücher (Abth. f. Anat.), xiii., p. 197. Schaudinn's Ar' eiten, 1911, p. 208.

(100) SchewIAKOFF, W. (1887). Dio karyokinetische Kerntheilung der Euglypha alvedata. Morph. Jahrbuch, riii., p. 193

(101) SwARCZIWSKY, B. (1908). Die Fortpflanzungsersoheinungen bei Arcella vulgaris. A.P.K., xii., p. 173.

\section{CHAPTER VIII}

In addition to the works cited here, see also Nos. 41, 47, 50, 51, 57, 64, 67, 68, $74,75,81,85,92,93,99$, and 101 .

(102) Bartserl, G. A. (1911). Conjugation of Closely Related Individuals of Stylonychio. Proc. Soc. Exper. Biol. Med., viii., p. 122.

(103) Вотт, M. (1907). Fortpllanzung von Pelomyxa. A.P.K., viii., p. 120.

(104) Calmins G. N. (1904). Studies on the Life-History of Protozoa, IV. J.E.Z., i., p. 423.

(105) - (1906). The Protozoan Life-Cycle. B.B., xi., p. 229.

(106) - and Coul, S. W. (1907). The Conjugation of Paramecium aurelia (caudatum). A.P.K., х., p. 375.

(107) Dargkard, P. A. (1911). La Conjugaison des Infusoires ciliés. C.R.A.S., clii., p. 1032.

(108) - (1911). La Féoondation des Infusoires ciliés. C.R.A.S., clii., p. 1703.

(109) Drhorne, A. (1911). Permutation nuoléaire dans la Conjugaison de Colpidium colpoda. C.R.A.S., clii., p. 1354.

(110) DовкцL, C. C. (1911). The Principles of Protistology. A.P.K., xxiii, p. 269.

(111) Dorlen, F. (1907). Die Konjugation der Infusorien. S.B.G.M.P.; xriii., p. 107.

(112) Exbiques, P. (1907). La Coniugazione e il Differenzismento sessuale negli Infusori. A.P.K., ix., p. 195.

(113) - (1908). Die Conjugation und sexuelle Difterenzierung der Infusorien. A.P.K., xii., p. 213.

(114) Gedpes, P., and Thomson J. A. (1901). The Evolutiou of Sex. Revised edition. London.

(115) HaMbUroER, C. (1908). Dle Conjugation von Stentor crerulcus. Z.w.Z., Xc., p. 423.

(116) Hartmann, M. (1909). Autogamie bei Protisten. A.P.K., xiv., p. 264. 
(117) Hartoo, M. (1910). Apropos of Dr. Hartmann's "Autogamie bei Protozoen." A.P.K., xviii., p. 111.

(118) Hertwia, R. (1902). Wesen und Bedeutung der Befruchtung. Silzber. $k$. Akad. Wiss. München., xxxii., p. 57.

(119) - (1905). Das Problem der sexuellen Differenzierung. V.D.Z.G., 1905, p. 186.

(120) Hickson, S. J. (1910). The Origin of Bex. Ann. Rep. Trans. Manchester Microsc. Soc., 1909 , p. 34.

(121) Jenninas, H. S. (1910). What Conditions induco Conjugation in Paramecium? J.E.Z., ix., p. 279.

(122) Maupas, E. (1889). Le Rajeunissement karyogamique ohez les Ciliés. A.Z.E., (2) vii., p. 149.

(123) MoLsow, K. (1911). Fortpflenzungserscieinungen bei Monocystis rostrata. A.P.K., xxii., p. 20.

(124) PearL, R. (1907). A Biometrical Study of Conjugation in Paramecium. Biometrika, v., p. 213.

(125) Poporf, M. (1908). Die Gametenbildung und die Conjugation von Carchesium polypinum. Z.w.Z., lxxxix., p. 478.

(126) Prandtl, H. (1906). Die Konjugation von Didinium nasutum. A.P.K., vii., p. 229.

(127) Prowazek, S. v. (1905). Der Erreger der Kohlhernie, Plasmodiophora brassica. A.K.G.A., xxii., p. 396.

(128). - (1907). Die Sexualität bei den Protisten. A.P.K., ix., p. 22.

(129) SchaUdNN, F. (1896). Copulation von Actinophrys. S.B.A.B., p. 83.

(130) - (1902). Krankheitserregende Protozoen, II. Plasmodium vivax. A.K.G.A., xix., p. 169 .

(131) - (1903). Die Fortpflanzung einiger Rhizopoden. A.K.G.A., xix., p. 547.

(132) - (1904). Generations- und Wirtswechsel bei Trypanosoma und Spirochoete. A.K.G.A., xx., p. 387. Reprinted, with "Nachtrag," in Fritz Schaudinn's Arbeiten, 1911.

(133) - (1905). Die Befruchtung bei Protozoen. V.D.Z.G., xv., p. 16.

(134) Schulina, C. (1910). Autogamie bei Trypanosoma lewisi. A.P.K., xix., p. 119.

(135) Strempex, W. (1906). Die neuere Protozoenforschung und dic Zellenlehre. S. B. Med.-naturwiss. Ges. Münster i. W., June 13.

(136) Stevens, N. M. (1910). The Chromosomes and Conjugation in Boveria subcylindrica, var. concharum. A.P.K., xx., p. 126.

(137) VersluYs, J. (1906). Die Konjugation der Infusorien. B.C., xxvi., p. 46.

(138) WoOdRUFT, L. L. (1905). Life-History of Hypotrichons Infusotia. J.E.Z., ii., p. 585 .

(139) - (1908). Life-Cycle of Paramecium. Amer. Nat., xlii., p. 520.

(140) - (1909). Further Studies on the Life-Cycle of Paramecium. B.B., xvii., p. 287.

(141) - (1911). Two Thousand Generstions of Paramecium. A.P.K., xxi., p. 263.

(142) - (1911). The Adaptation of Paramacia to Different Environments. B.B., xxii., p. 60.

(143) - and BarsserL, G. A. (1911). Rhythms in the Reproductive Activity of Infusoria. J.E.Z., xi., p. 339.

\section{CHAPTER IX}

In addition to the works cited here, see also Nos. $41,65,78,85,86,99,101$, 130 , and 131 .

(144) ElpatikwskY, W. (1907). Fortpflanzung von Arcella vulgaris. A.P.K.,

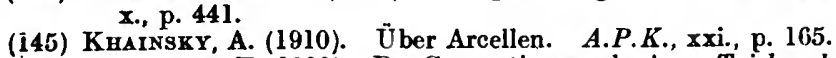

(146) Schaudin, F. (1899). Der Generationswechsel von Trichospharium sieboldi. Anhang. Abhandl. Preuss. Akad. Wiss.

(147) - (1902). Cyclospora caryolytrca. A.K.G.A., xviii., p. 378. Reprint in Fritz Sohaudinn's Arbeiten, 1911, p. 318. 


\section{CHAPTER $\mathbf{X}$}

In addition to the works cited below, see also $34,35,36,3740$, and 237.

(148) BarratT, J. O. W. (1905). Die Kohlensäureproduktiois von Paramecium aurelia. Z.a.P., v., p. 66.

(149) - (1905). Der Einfluss der Konzentration anf die Chemoteris. Z.a.P., v., p. 73.

(150) Bıss, C. C. (1911). A New Conception of Immunity : its Application to the cultivation of Protozos and Bacteris. Journ. Amer. Med. Assoc., lvii., p. 1534.

(151) bolssevan, M. (1908). Kernverhältnisse von Actinospharium eichhorn bei fortgesetzter Kultur. A.P.K., xiii., p. 167.

(152) BORGERT, A. (1909). Erscheinungen fettiger Degeneration bei tripyleen Radiolarien. A.P.K., xvi., p. l.

(152.5) BovarD, J. F. (1907). Structure and Movements of Condylostoma patens. Univ. California Publ., iii., p. 343.

(153) Btjtschi, O. (1906). Zur Kenntnis des Paramylons. A.P.K., vii., p. 197.

(154) Dease, A (1905). Die kontraktile Vacuole und die Wabenstruktur des Protoplasmas. Bot. Zeitung, lxiii., p. 163.

(155) DoвzL, C. C. (1907). Physiological Degeneration in Opalina. Q.J.M.S., li., p. 633 ,

(156) Erdunis, R. (1910). Depression und facultative Apogamio bei Amoba diploidea. Hertwig's Pestochrift, i., p. 323.

(157) GaRbowski, L. (1907). Gestaltsveränderung und Plasmoptyse. A.P.K., ix., p. 53.

(158) Gremss, G. (1911). Fixierung und Färbung der Protozoen. Vide Prowazek (14), p. 7.

(159) - and Prowazer, S. v. (1908). Wirkung des Chinins auf die Protistenzelle. A.S.T.H., zii., Beiheft 5, p. 188.

(160) Gremley, A. W. (1902). Artificial Production of Spores in Monas by a Reduction of the Temperature. Univ. Chicago Decennial Publ., x., p. 73.

(161) Greenwood, M. (1886-1887). Digestive Processes of some Rhizopods, I. Journ. Physiol., vii., p. 253. II., ibid., viii., p. 263.

(162) - (1894). Constitution and Formation of "Food-Vacuoles" in Infusoria, etc. Phil. Trans. (B), clxxxv., p. 355.

(163) - and SaUnders, E. R. (1894). The Rôle of Acid in Protozoan Digestion. Journ. Physid., xvi., p. 141.

(164) Hratwig, R. (1904). Pbysiologische Degeneration bei Actinospharium eichhorni. Haeckel' Festschrift (Jena, G. Fischer), p. 301.

*(165) Jennnas, H. S. (1904). The Behaviour of the Lower Organiams. Washington : Carnegie Institute.

(166) - (1904). The Behaviour of Paramecium. Journ. Comp. Neurology, xiv., p. 441; Contr. Zool. Lab. University of Philadelphia, xi., 1905.

(167) - (1904). The External Discharge of the Contractile Vacuole. Z.A., xxvii., p. 656.

(168) - (1904). Physical Imitations of the Activities of Amabe. Amer. Natural, xxxviii., p. 625 .

(169) Joseph, H., and Prowazer, S. v. (1902). Die Einwirkung von RöntgenStrahlen auf einige Organismen. Z.a.P., i., p. 142.

(170) Ksnitz, A. (1907). Der Einfluss der Temperatur auf pulsierenden Vakuolen der Infusorien. B.C., xxvii., p. 11 .

(170.5) Krainsky, A. (1910). Morphologie und Physiologie einiger Infusorien (Paramecium caudatum). A.P.K., xxi., p. 1.

(171) LewIN, K. R. (1910). Nuclear Relstions of Paramecium caudatum during the Asexual Period. Proc. Cambridge Phil. Soc., xvi., p. 39.

(172) - (1911). Behaviour of the Infusorian Mioronucleus in Regeneration. P.R.S., lxxxiv., p. 332.

(173) Lipska, I. (1910). L'Influence de l'Inanition ohez Paramecium caudatum. Rev. Suisse Zool., xviii., p. 591.

(174) MaCkINNon, D. (1908). Encystation of Actinosphosrium eichhorni under Different Temperatures. Q.J.M.S., lii., p. 407.

(175) McLendon, J. F. (1909). Protozosn Studies. J.E.Z., vi., p. 260 .

(176) Mast, S. O. (1910). Reactions of Amøba to Light. J.E.Z., ix. p. 265. 
(177) Mesnil, F., and Motton, H. (1903). Une Diastase Protéolytique Extraito des Infusoires Ciliés. C.R.S.B., lv., p. 1016.

(178) - - (1903). L'Aotion Antiprotéolytique Comparée des Diverses Sérums sur l'Amibodiastase eto. C.R.S.B., lv., p. 1018.

(179) Metalnikofr, S. (1903). Die intracelluläre Verdauung. Bull. Ac. St. Petersbourg, xix., p. 187.

(180) Metrchnikoff, E, (1889). La Digestion Intracellulaire. A.I.P., iii., p. 25.

(181) Nrrenstein, E. (1905). Ernährungephysiologio der Protisten. Z.a.P., v., p. 434.

(182) - (1910). Fettverdaung und Fettspeioherung bei Infusorien. Z.a.P., x., p. 137.

(183) NowIKOFr, M. (1908). Die Wirkung des Sohilddrüsenertrakts auf Ciliaton. A.P.K., xi., p. 309.

(184) Poporr, M. (1907). Depression der Protozoenzelle und der Geschlechtszellen der Metazoen. A.P.K., Suppl., i., p. 43.

(185) - (1909). Der Einfluss ohemischer Reagentien anf der Funktionszustand der Zelle. S.B.G.M.P., xxv., p. 55.

(186) - (1909). Einige Ursachen der physiologischen Depression der Zelle. Arch. Zellforschung, iv., p. 1.

(187) Prandte, H. (1907). Die physiologisohe Degeneration der Amoeba proteus. A.P.K., viii., p. 281.

(188) Prowazek, S. ₹. (1903). Studien zur Biologie der Zelle. Z.a.P., ii., p. 385.

(189) - (1903). Regeneration und Biologie der Protozoen. A.P.K., iii., p. 44.

(190) - (1903). Degenerative Hyperregeneration bei den Protozoen. A.P.K., iii., p. 60 .

(191) - (1908). Das Lecithin. B.C., xxviii., p. 382.

(192) - (1908). Einfluss von Säurelösungen niedrigster Konzentration auf die Zell- und Kernteilung. Arch. Entwicklungsmech., xxv., p. 643.

(193) - (1909). Biologie der Zellen. II. Zelltod und Struktiurspannung. B.C., xxix., p. 291.

(194) - (1910). Die Physiologie der Einzelligen. Leipzig : Teubner.

(195) - (1910). Giftwirkung und Protozoenplasma. A.P.K., xviii., p. 221.

(196) - (1910). Biologie der Protozoen, V. A.P.K., хx., p. 201.

(197) Pütrer, A. (1900). Thigmotaxis bei Protisten. Arch. Anat. Physiol., Physiol. Abt., Suppl. Band, p. 243.

(198) - (1903). Die Wirkung erhöhter Saverstoffspannung auf dio lebendige Substanz. Z.a.P., iii., p. 363.

(199) - (1903). Reizbeantwortung der ciliaten Infusorien. Z.a.P., iv., p. 406.

(200) - (1905). Leuchtende Organismen. Z.a.P., v., Referato, p. 17.

(201) - (1905). Die Atmung der Protozoen. Z.a.P., v., p. 566.

(202) - (1908). Vergleichende Physiologie des Stoff wechsels. Abh. k. Ges. Wiss. Göltingen (n.F.), vi., p. I.

*(202) - (1908). Erforsehung des Lebens der Protisten. Tigerstedt, Handbuch der physiologischen Methodik.

(203) Rhumbles, L. (1905). Die Oberflächenkräfte dor Amöben. Z.w.Z., lxxxiii., p. 1.

(204) - (1910). Die verschiedenen Nahrungsaufnahmon bei Amöben als Folge verschiedener Colloidalzustände ihror Oberflächen. Arch. Entwicklungsmech., xxx., p. 194.

(205) RoEsle, E. (1902). Die Reaction einiger Infusorien auf einzelue Induktionsschlage. Z.a.P., ii., p. 139.

(206) Screwlakofr, W. (1893). Dio Natur der sogennanten Excretkörner der Infusorion. Z.w.Z., lvii., p. 32.

(207) Sarte, G. (1903). Actinospharium eichhorni : A Biometrical Study in the Mass Relations of Nucleus and Cytoplasm. Biometrika, il., p. 241.

(208) Staniewicz, W. (1910). La Digestion de la Graisge dans les Infusoires Ciliés. B.A.S.C., p. 199.

(209) Statkewitsch, P. (1905). Galvanotropismus und Galvanotaxis der Ciliata. Z.a.P., v., p. 511 .

(210) - (1901). Zur Methodik der biologischen Untersuchungen über die Protisten. A.Y.K., v., p. 17.

(211) Verworn, M. (1904). 'Die Localisation der Atmung in der Zelle. Denkschr. Ges. Jena, xi., p. 561.

(212) - (1907). Algemeine Physiologie. Jena. Fourth edition. 
(213) WAGER, H. (1900). On the Eyespot and Flagellum of Euglena viridis. J.L.S., xxvii., p. 463 .

(214) W ALLENGREN, H (1902). Inanitionserscheinungen der Zelle. Z.a.P., i., p. 67.

(215) - (1002). Zur Kenntnis der Galvanotaxis. Z.a.P., ii., p. 341.

(216) Woudnorr, L. L. (1908). Effects of Alcohol on the Life-Cycle of Infusoria. B.B., xv., p. 85 .

(217) - (1911). Tho Effect of Exeretion Products of Paramecium on its Rato of Reproduction. J.E.Z., x, p. 559 .

(218) - and Bartseld, G. A. (1911). The Reproduction of Paranecium aurelia in " "Constant" Culture Medium of Bcef Extract. J.E.M., xi., p. 135.

(219) - - (1911). The Temperature Coefficient of the Rate of Reproduotion of Paramecium aurelia. Amer. Journ. Physiol., xxix., p. 147.

(220) - and BonzeL, H. H. (1909). The Relative Toxicity of Various Salts and Acids towards Paramecium. Amer. Journ. Physiol., xxv., p. 190.

(221) Zokizer, M. (1905). Die Einwirkung der Radiumstrahlen auf Protozoen. A.P.K., v., p. 358.

(222) - (1907). Der Einfluss des Meerwassers auf dio pulsierende Vaouole. S.B.G.B., p. 90.

(223) Zomstern, H. (1899). Morphologie und Physiologie der Euglena gracilis. Pringsheim's Jahrbücher f. wiss. Botanik, xxxiv., j). 419.

\section{CHAPTER XI}

\section{SARCODINA}

\section{(a) General Works.}

(224) Cash, J., and Hopkinson, J. (1905, 1909). The British Freshwater Rhizopoda and Heliozoa. London, Ray Sociely, vol. i. (1905) and ii. (1909).

(225) Hartog, M. (1910). Rhizopoda. Encyclop. Brit., eleventh edition, xxiii., p. 244.

(226) LIIDY, J. (1879). Freshwater Rhizopods of North America. Rep. U.S. Ged. Survey, xii.

\section{(b) Amøbæa.}

See also Nos. 32, 34, 36, 46, 49, 65, 71.5, 76, 81, 85, 87, 88, 90, 95, 98, 101, $103,131,144-146,156,161,168,176,178,187,203,204,222$.

(227) Alexeiefr, A. (1911). La Division nucléaire et l'Enkystement chez quelques Amibes, I.-III. C.R.S.B., lxx., pp. 455, 534, 588.

(228) Awmrinzew, S. (1907). Die Struktur des Protoplasma und des Kerns von Amoeba proteus. Z.A., xxxii., p. 45.

(229) - (1906). Die Struktur und die chemischo Zusammonsetzung der Gehäuse bei den Süsswasserrhizopoden. A.P.K., viii., p. 95.

(230) - (1906). Zur Kenntnis der Süsswasserrhizopoden. A.P.K., viii., p. 112

(231) Calkins, G. N. (1904). Evidences of a Sexual Cycle in Amaba proteas. A.P.K., v., p. 1.

(232) - (1907). Fertilization of Amobu proteus. B.B., xiii., p. 219.

(233) Casagrandi, O., and Barbagallo, P. (1897). Entameeba hominis, s. Amaba coli. Ann. Igiene Sperimental., vii., p. 103.

(234) Chatton, E. (1910). Protozoaires parøsites des Branchies des Labres : Amoeba mucicola, Trichodina labrorum. A.Z.E. (5), v., p. 239.

(235) Craig, C. F. (1908). The Amœbio in the Intestine of Man. Journ. Infect. Diseases, v., p. 324.

(236) Dobell, C. C. (1909). The Intestinal Protozoa of Frogs and Touds. Q.J.M.S., liii., p. 201.

(237) - (1909). Physiologieal Degeneration and Death in Entamaba ranarum. Q.J.M.S., liii., p. 711 .

(238) Doflein, F. (1907). Amöbenstudien. A.P.K., Suppl. i., p. 250.

(239) - (1907). Der Teilungsvorgang bei den Süsswasserthalamophoren. S.B.G.M.P., xxiii.

(240) Fantham, H. B. (1910). The Protozoa Parasitic in the Red Grouse, etc. P.Z.S., 1910, p. 692 . 
(241) Fantham, H. B. (1911). The Amœbo Parasitio in the Human Intestine. A.T.M.P., v., p. 111 .

(242) Gavdichrad, A. (1908). Formation de Corps spirillaire dans une Culture d'Amibe (Entamaba phagocytoides). C.R.S.B., lxiv., p. 493.

(243) Gooud, L. J. (1894). The Minute Structure of Pelomyxa palustris. Q.J.M.S. xxxvi., p. 295.

(244) Greig, E. D. W., and Weurs, R. T. (1911). Dysentery and Liver Abscess in Bombay. S.M.I., 47.

(245) Grosse-Allermand, W. (1909). Amaba terricola. A.P.K., xvii., p. 203.

(246) GruBER, K. (1911). Eigenartige Körperformen von Amœba proteus. A.P.K., xxiii., p. 253.

*(247) Hartmani, M. (1911). Die Dysenterie-Amöben. Vide Prowazer (14), p. 50 .

(248) Hrokson, S. J. (1909). The Proteomyxa. Lankester's Treatise on Zoology, i., faso. 1 .

(249) - (1909). The Loboss. Ibid.

(250) HoOgrnasd, H. R. (1907). Vampyrella lateritia. A.P.K., viii., p. 216.

(251) - (1907). Hyalodiscus rubicundus. A.P.K., ix., p. 84.

(252) JörakNS (1902). Die Darmamöben und die Amöbenenteritis Veröff. $M$ iltiär-Sanitätswesens, xx., p. 110.

(253) Luston, W. G., and Martin, C. H. (1911). Pathogenio Amøbø from Bombay. Q.J.M.S., Ivii., p. 107.

(254) McCarrison, R. (1909). Amabo in Intestines of Persons suffering from Goitre in Gilgit. Q.J.M.S., liii., p. 723.

(255) Martrs, C. H. (1911). Nuclesr Division of the Large Amøba from Liver Abscess. Q.J.M.S., lvii., p. 279.

(256) Mracikr, L. (1910). L'Amibe de la Blatte (Entamoba blatta). A.P.K., xx., p. 143.

(257) Metcalf, M. M. (1910). Studies upon Amœeba. J.E.Z., ix., p. 301.

(258) MrNchis, E. A. (1910). Parasites observed in the Rat-Fles (Ceratophyllus fasciatus). Hertwig's Festschrift, i., p. 289.

(259) Musqrave, W. E., and Clega, M. T. (1904). Amœbas : their Cultivation and Etiologio Significance. Manils, Dept. of the Interior, Bureau of Govt. Laboratories, Biol. Lab., xviii.

(260) Neresheimer, E. (1905). Vegetative Kernveränderungen bei Amaba dofleini. A.P.K., vi., p. 147 .

(261) Noc, F. (1909). La Dysenterie amibienne en Coobinohine. A.I.P., zaiii., p. 177.

(262) Penard, E. (1902). Faune Rhizopodique du Bassin du Léman. Geneva : Kundig.

(263) - (1905). Les Amibes à Pellicule. A.P.K., vi., p. 175.

(264) PopOrF, M. (1911). Der Entwicklungscyolus von Amoba minuta, etc. A.P.K., xxii., p. 197.

(265) PrandT, H. (1907). Der Entwioklungskreis von Allogromia sp. A.P.K., ix., p. 1.

(266) RoBertson, M. (1905). Pseudospora volvocis. Q.J.M.S., xlix., p. 213.

(267) SchaudinN, F. (1895). Teilung von Amoeba binucleata. S.B.G.B., 1895, p. 130. Keprinted, Schaudinn's Arbeiten, 1911, p. 101.

(268) Schees, C. (1899). Fortplanzung der Amöben. Kupffer's Festschrift, p. 569.

(269) SchepotierT, A. (1910). Amöbenstudien. Zool. Jahrbücher (Anat. u. Ontog.), xxix., p. 485.

(270) Schurotz, H. (1905). Amoba blatta und Amxba proteus. A.P.K., vi., p. 1.

(271) Stolo, A. (1906). Plasmodiogonie, eine Vermehrungsart der niedersten Protozoen. Arch. Entwicklungsmech., xx.., p. 111.

(272) Sun, A. (1910). Über einen Parasiten aus der Körperhöhle von Ptychodera minuta. A.P.K., xx., p. 132.

(274) Topsent, E. (1893). Pontomyxa flava. A.Z.E. (3), i., p. 385.

(275) Vamlkamps, E. (1905). Biologie und Entwicklungsgeschichto von Ameba limax. A.P.K., v., p. 167.

(276) Veley, L. J. (née Goold) (1905). Pelomyxa palustris. J.L.S., xxix., p. 374.

(276.5) W $\triangle$ LRer, E. L. (1911). Amœbæ in the Manila water-supply, etc. Philippine Journ. Sci., vi. (B), p. 259.

(277) Wenyon, C. M. (1907). Protozos in the Intestine of Mice. A.P.K., Suppl., i., p. 169. 
(278) Werker, H. (1911). Entamaba coli. Vide Prowazkx (14), p. 67.

(279) Whimore, E. (1911). Parasitäre und freilebende Amöben aus Manila und Saigon. A.P.K., xxiii., p. 7].

(280) - (1911). Kulturamöben aus Manila. Ibid., p. 81.

\section{(c) Foraminifera.}

See also Nos. 28, 29, 35, 100, and 131 .

(281) AWERINzEw, S. (1910). Gromia dujardini. ZsA., Xxxv., p. 425.

(282) Hickson, S. J. (1911). Polytrema and some Allied Genera. Trans. Linn. Soc. London (2), xiv., p. 443.

(283) LaUteraorn, R. (1895). Paulinella chromatophora. Z.w.Z., lix., p. 587.

(284) LEYdex, E. v., and SchuodnNw, F. (1896). Leydenia gemmipara. S.B.A.B., p. 951.

(285) Lister, J. J. (1895). Life-History of the Foraminifera. Phil. Trans. (B), olxxxvi., p. 401.

(286) - (1903). The Foraminifera. Lankester's Treatise on Zoology, i., fasc. 2, p. 47.

(287) - (1906). Life-History of the Foraminifera. Pres. Address Zool. Bec. Brit. Assoc., Fork, 1906.

(288) Rhombler, L. (1903). Systematische Zussmmenstellung der recenten Reticuloss. A.P.K., iii., p. 181.

(283.5) Swarczewsey, B. (1909). Allogromia ovoidea. A.P.K., xiv., p. 396.

\section{(d) Xenophjophora.}

(289) Aron. (1909). The Xenophyopboridø, F. E. Schultze (sic). Lankester's Treatise on Zoology, i., fasc. 1, p. 284.

(290) Schulze, F. E. (1905). Die Xenophyophoren. Wiss. Ergebn. Expedition "Valdivia," xi.

(291) - (1906). Die Xenophyophoren der Siboga-Expedition. Uitkomst. Siboga, iv. bis.

See also Nos. 69 and 127.

(e) Mycetozoa.

(292) Blomfres.d, J. E., and Schwartz, E. J. (1910). The Tumours on Veronica Chamadrys caused by Sorosphara Veronica. Ann. Botany, xxiv., p. 35.

(293) JAH, E. (1908). Myxomyoetenstudien. 7. Ceratiomyxa. Ber. Deutsch. Bot. Ges., xxvia., p. 342.

(294) - (1911). Myromycetenstudien. 8. Der Sexualakt. Ibid., xxix., p. 231. (295) LFGER, L. (1908). Sporomyxa scauri. A.P.K., xii., p. 109.

(296) - and Hesse, E. (1905). Un Parasite des Otiorhynques (Mycetosporidium). C.R.S.B., Iviii., p. 92.

(297) Listkr, J. J. (1909). The Mycetozos. Lankester's Treatise on Zoology, i., fasc. 1, p. 37.

(298) - (1909). Chlamydomyxa and Labyrinthula. Ibid., p. 274.

(299) MaRCHAND, E. F. L. (1910). Lo Plasmodiophora brassica. C.R.A.S., cl., p. 1348.

(f) Heliozos.

See also Nos. 43, 64, 66, 82, 86, 129, 151, 161, 164, 174, and 207.

(300) Ca ohlery, M. (1910). Un Protozoairo Marin du Genre Ciliophrys Cien. kowsky (C. marina, n. sp.). C.R. Assoc. Franc. Sci., Lille, 1909, p. 708.

(301) Hartog, M. (1910). Heliozos. Encyclop. Brit., eleventh edition, riii., p. 232.

(302) Prínard, E. (1903). Quelques Protistes Voisins des Héliozosires ou des Flagellés. A.P.K., ii., p. 283.

(303) - (1904). Les Héliozoaires d'Eau Douce. Geneva : Henry Kündig.

(304) Przmsmycki, A. M. (1901). Parasitisohe Protozoen aus dem inneren der Rotatorien. B.A.S.C., 1901, p. 358.

(305) SchavdnNN, F. (1896). Heliozoa. Das Tierreich., Berlin, 1896.

(306) SohoutkdEN, H. (1907). Quelques Flagellés. A.P.K., ix., p. 108.

(307) WrudoN, W. F. R., and HicksoN, S. J. (1909). The Heliozos. Lankester's Treatise on Zoology, i., faso. 1, p. 14. 
See also No. 152.

\section{(g) Radiolaria.}

(308) Boraert, A. (1911). Fremdkörperskelete bei tripyleen Radiolarien. A.P.K., xxiii., p. 125 .

(309) Brandt, K. (1902). Die Colliden. A.P.K., i., p. 59.

(310) Bütschir, O. (1906). Die chemische Natur der Skelctsubstanz der Acan tharia. Z.A., xxx., p. 784.

(311) Gambli, F. W. (1909). The Radiolaria. Lankester's Treatise on Zoology, i., fase. 1, p. 94.

(312) Hartmann, M., and Hammer, E. (1909). Die Fortpflanzung von Radiolarion. S.B.G.B., 1909 , p. 228.

(313) Haztog, M. (1910). Radiolaria. Encyclop. Brit., eleventh edition, xxii., p. 802.

(314) Hचтн, W. (1911). Fortpflanzung von Thalassicolla. S.B.G.B., 1911, p. 1.

(315) Mororr, T. (1910). Vegetative und reproduktive Erscheinungen bei Thalassicolla. Hertwig's Festschrift, i., p. 73.

(316) - and Stiassy, G. (1909). Bau und Entwicklung von Acanthometron pellucidum. A.P.K., xvi., p. 209.

(317) Scotr, R. (1911). On Traquairia. Ann. Botany, xxv., p. 459.

(318) Srrasxy, G. (1910). Die Beziehung der sog., "gelben Zellen" zu den kolonie-bildenden Radiolarien. A.P.K., xix., p. 144.

\section{CHAPTER XII}

\section{MASTIGOPHORA}

\section{(a) General Works.}

(319) HАRтоG, M. (1910). Flagellata. Encyclop. Brit., eleventh edition, x., p. 44. "(320) SeNs, G. (1900). Flagellata. Engler and Prantl, "Die natürlichen Pflanzenfomilien,"I. Teil, 1. Abth., a, p. 93.

(321) Wrilfy, A., and Hickson, S. J. (1909). The Mastigophora. Lankester's Treatise on Zoology, i., fasc. 1, p. 154.

\section{(b) Flagellata.}

See also Nos. 41, 45, 62, 70, 71, 97, 97.5, 153, 160, 213, 223, 236, and 277.

(322) Alexerert, A. (1909). Les Flagelles Parasites de l'Intestin des Batraciens Indigènes. C.R.S.B., lxvii., p. 199.

(323) - (1909). Trichomonas a Quatre Flagelles Antérieurs. C.R.S.B., lxvii., p. 712 .

(324) - (1910). Les Flagellés Intestinaux des Poissons Marins. A.Z.E. (5), vi., Notes et Revue, p. i.

(325) - (1911). Notes sur les Flagellés. Ibid., p. 491.

(326) - (1911). "Kystes de Trichomonas intestinalis." C.R.S.B., lxxi., p. 296.

(327) - (1911). . La Position des Monadides dans la Systematique des Flagellés, etc. B.S.Z.F., xxxvi., p. 96.

(328) Bressen, W. (1909). Trichomonas intestinalis und vaginalis des Menschen. A.P.K., xpiii., p. 115.

(329) Brrungr, E. (1909). Flagellaten-Studien. A.P.K., xv., p. 297.

(380) Borne, A., and Prowazer, S. v. (1908). Znr Frage der Flagellatendygen. terie. A.P.K., xii., p. 1 .

(331) Chatron, E. (1911). Pleodorina californica à Banyuls-sur-Mer. Bull. Sci. Franc. Belg. (7), xliv. p. 309.

(332) Comes, S. (1910). Lophophora vacuolata. Boll. Acc. Gioen. Catania (2), riii., p. 11.

(333) - (1910). A Proposito del Dimorfismo sessusle riscontrato in Dinenympha gracilis. Ibid., p. 20.

(334) Dakmewsigy, W. B. (1886). Une Monade (Hexamitus), Parasite du Sang. Arch. Slav. Biol., i., p. 85.

(335) DовеL, C. C. (1908). Structure and Life-History of Copromonas subtilis. Q.J.M.S., iii., p. 75. 
(336) Donend, C. C. (1908). The "Autogamy" of Bodolacerta. B.C., xxviii , p. 548.

(337) FoA, A. (1905). Due nuovi Flagellati parassiti (Calnnymphr grassii and Devescovina striata). Rend. Acc. Lincei, xiv. (2), p. 542.

(338) Grassi, B., and Foa, A. (1904). Processo di Divisione delle Joenie e Forme affini. Ibid., xiii. (ii.), p. 241.

(339) HАAsE, G. (1910). Euglena sanguinea. A.P.K., xx., p. 47.

(340) Hamburaer, C. (1905). Dunaliella salina und eine Amöbe aus Salinenwasiser von Cagliari. A.P.K., vi., p. 111.

(341) - (1911). Euglena ehrenbergii, insbesondere die Körperhülle. Sitz-ber. Heidelberg. Ak. Wiss., 1911.

(342) Hartmans, M. (1910). Bau und Entwicklung der Trichonymphiden (Trichonympha hertwigi). Hertwig's Festschrifi, i., p. 349.

(343) HasweLL, W. A. (1907). Parasitic Euglenæ. Z.A.. xxxi., p. 296.

(344) Krysseutiz, G. (1908). Studien żber Protozoen. A.P.K., хi., p. 334.

(345) LadterbokN, R. (1895). Eine Süsswasserart der Gattung Multicilia (M. lacustris). Z.w.Z., 1x., p. 236.

(345.5) - (1911). Pseudopodien bei Chrysopyxis. Z.A., xxxviii., p. 46.

(346) Lizberanz, E. (1910). Die parasitische Protozoen des Wiederkäuermagens. A.P.K., xix., p. 19.

(347) Lohmans, H. (1902), Dio Coccolithophoridæ. A.P.K., i., p. 89.

(348) Martin, C. H., and Robertson, M. (1911). Cæcal Parasites of Fowls, etc. Q.J.M.S., lvii., p. 53.

(349) Mozorr, T. (1903). Einige Flagellaten. A.P.K., iii., p. 59.

(350) Nereshermer, E. (1911). Costia necatrix. Vide Prowazer (14), p. 98.

(351) Noo, F. (1909). Lo Cyole évolutif de Lamblia intestinalis. B.S.P.E., ii., p. 93.

(352) PASCHER, A. (1910). Chrysomonaden aus dem Hirsohberger Grossteiohe. Leipzig : Werner Klinkhardt.

(353) Plimmer, H. G. (1909). Report on Deaths at the Zoological Gardens during 1908. P.Z.S., 1909, p. 125.

(354) Prowazer, S. v. (1903). Flagellatenstudien. A.P.K., ii., p. 195.

(355) - (1904). Einige parasitisohe Flagellaten. A.K.G.A., xxi., p. 1.

(356) Rodenwaldt, E. (1911). Trichomonas, Lamblia. Vide Protiaze (14), p. 78.

(357) Scherfter, A. (1911). Die Chrysomonadineen. A.P.K., xxii., p. 299.

(358) SenN, G. (1911). Oxyrrhis, Nephroselmis und einige Euflagellaten. Z.w.Z., xcvii., p. 605 .

(359) Stein, F. (1878, 1883). Der Organismus der Infusionsthiere. III. Lejpzig : Wiihelm Engelmann.

(360) Stevenson, A. C. (1911), The Protozon parasitic in Bufo regularis in Khartoum. Rep. Wellcome Lab. Khartoum, iv., p. 359.

(361) Wranyon, C. M. (1910). A Flagellate of the Genus Cercomonas. Q.J.M.S., 1v., p. 241.

(362) - (1910). Macrostoma mesnili from the Human Intestine. Py., iii., p. 210.

(c) Dinofiagellata and Cystofiagellata.

(363) Bgraert, A. (1910). Kern- und Zellteilung bei marinen Ceratium-Arten. A.P.K., xx., p. 1 .

(364) CaULLEY, M. (1910). Ellobiopsis chattoni, Parasite de Calanus helgolandicus Bull. Sci. Franc. Belg. (7), xliv., p. 201.

(365) Coutiker, H. (1911). Les Ellobiopsis des Crevettes bathypélagiques. C.R.A.S., clii., p. 409.

(366) Chatron, E. (1906). Les Blastodinidés. C.R.A.S., oxliii., p. 981.

(367) - (1907). Nouvel Aperçu sur les Blastodinidés (Apodinium mycetoides). C.R.A.S., cxliv., p. 282.

(368) - (1910). Sur l'Existence de Dinoflagellés parasites cœlomiques. Les Syndinium chez les Copépodes pélagiques. C.R.A.S., cli., p. 654 .

(369) - (1910). Paradinium poucheti, Fagellé parasite d'Acartia clausi. C.R.S.B., lxix., p. 341 .

(370) Doarel, V. (1906). Dio Peridinien. Mitth. Zool. Stat. Neapcl, xviii., p. I.

(371) Dubosce, O., and Corus, B. (1910). La Réproduction sexúe d'un Pro. tiste parasite des Tintinnides. C.R.A.S., cli., p. 340.

(372) Joluos, V. (1910). Dinoflagellatenstudien. A.P.K., xix., p. 178.

(373) Koford, C. A. (1905). Craspedotella, a New Genus of the Cystoflageilata. Bull. Mus. Harvard, xlvi., p. 163. 
(374) Korord, C. A. (1906). Asymmetry in Triposolenia. Univ, California Publ. Zool., iii., p. 127.

(375) - (1906). Structure of Gonyaulax triacantha. Z.A., xxx., p. 102.

(376) - (1907). Structure and Systematic Position of Polykrikos. Ibid., xxxi., p. 291.

(377) - (1907). The Plates of Ceratium. Itid., xxxii., p. 177.

(378) - (1908) Exuviation, Autotomy, and Regeneration, in Ceratium. Univ. Califoruia Publ. Zool., iv., p. 345.

(379) - (1909). On Peridiniur steini. A.P.K., xvi., p. 25.

(380) - (1909). Morphology of the Skeleton of Podolampas. Ibid. p. 48.

(381) - (1909). Mutations in Ceratium. Bull. Mus. Harvard, lii., p. 211.

(382) - (1910). A Revision of the Genus Ceratocorys. Univ. California Publ. Zool., vi., p. 177.

(383) - (1910). Forms of Asymmetry of tho Dinoflagellates. Proc. Internat. Congr. Zool., vii.

(384) Kṫster, E. (1908). Eine kultivierbare Peridinee. A.P.K., xi., p. 351.

(385) Plate, L. (1906). Pyrodinium bahamense. A.P.K., vii., p. 411.

(386) SchüTT, F. (1895). Die Peridineen der Plankton-Expedition. Ergebn. Plankion-Exped., iv.

(387) Stran, F. (1883). Der Organismus der Infusorien. III. (ii.) Die Naturgeschichte der Arthrodelen Flagellaten. Loipzig: W. Engelmann.

\section{CHAPTER XIII}

\section{H FMOFLAGELLATES}

\section{(a) General Works.}

See also the Bulletin of the Sleeping Sickness Burzau, London, for abstracts and reviews of literature.

(388) Alexeierf, A. (1911). La Structure des "Binucléates" de Hartmann. C.R.S.B., lxix., p. 532.

(389) Brumpr, E. (1908). L'Origine des Hémoflagellés du Sang des Vertébrés. C.R.S.B., lxiv., p. 1046.

(390) HartmanN, M., and Jollos, V. (1910). Die Flagellatenordnung " Binucleota." A.P.K., xix., p. 81.

*(391) Inaferax, A., Mesnil, F., and Nabarro, D. (1907). Trypanosomes and Trypanosomiases. London: Baillière, Tindall and Cox.

*(392) LÜHE, M. (1906). Die im Blute schmarotzenden Protozoen. Mense's Handbuch der Tropenkrankheilen, iii., p. 69.

*(393) Patron, W. S. (1909). Our Present Knowledge of the Hæmoflagellates and Allied Forms. Py., ii., p. 91.

*(394) ThImm, C. A. (1909). Bibliography of Trypanosomissis. London : Sleeping Sickness Bureau.

*(395) WooDCOCK, H. M. (1909). Tho Hæmoflagellates and Allied Forms. Lan. kester \& Treatise on Zoology, i., fasc. 1, p. 193.

\section{(b) Trypanosoma and Trypanoplasma.}

Seo also Nos. 19, 22, 23, 27, 42, 56, 132, 134, 686, and 696.

(396) BALDREY, F. S. H. (1909). Die Entwicklung von Trypanosoma lewisi in der Rattenlaus Hamatopinus spinulosus. A.P.K., xv., p. 326.

(397) - (1911 Evolution of T. evansi through the Fly: Tabanus and Stomoxys. Journ. Trop. Velerin. Sci., vi., p. 271.

(398) Boso, F. J. (1904). La Structure et l'Appareil Nucléaire des Trypanosomes. A.P.K., v., p. 40.

(399) Bodrt, G. (1906). Culture du Trypanosome de la Grenouille ( $T$, rotatorium). A.I.P., xx., p. 564 .

(400) - and RotbaUd, E. (1910). Transmission des Trypanosomes par les Glossines, I. and II. A.I.P., xxiv., p. 658. III., B.S.P.E., iii., p. 599. IV., Ibid., p. 722.

(401) BoUfBARD, (X. (1910). Glossina palpalis et T. Cazalboui. A.I.P., xxiv. p. 276. 
(402) Bradford, J. R., and Plimmer, H. G. (1902). The T. l.mucii found in Nagana, or Tse-tse Fly Disense. Q.J.M.S., xlv., p. 449.

(403) Breini, A., and Hindse, E. (1910). Life-History of T. lewisi in the RatLonse. A.T.M.P., iii., p. 553.

(404) BrdCE, D. (1911). Morphology of T. evansi. P.R.S. (B), lxxxiv., p. 181.

(405) - (1911). Morphology of T. gambiense. Ibid., p. 327.

(406) - and Bateman, H. R. (1908). Have Trypanosomes an Ultramioroscopical Stage in their Life-History ? (No /) P.R.S., (B), lxxx., p. 394.

(407) - Hamerton, A. E., Bateman, H. R., and Mackie, F. F. (1909). T. ingens, n. sp. P.R.S. (B), lxxxi., p. 323.

(408) - - (1909). Development of $T$. gambiense in Glossina palpalis. Ibid., p. 405.

(409) - - (1909). A Trypanosome in the African Elephant. Ibid., p. 414.

(410) - Development of Trypanosomes in Tsetse Flies. Ibid., Ixxxii., p. 368.

(411) - - Trypanosome Diseases of Domestio Animals in Uganda, I.V. Ibid., Ixxxii., p. 468; lxxxiii., pp. 1, 15, 176, and 180 .

(412) - - - (1910). The Natural Food of Qlossina palpalis. Ibid., 1xxxii., p. 490. (413) $-\frac{-}{\text { Tsetse Fly. Ibid., p. } 498 \text {. }}$ (1910). Mechanical Transmission of Sleeping Siokness by the

(414) - - - (1911). Experiments to Ascertain if $T$. gambiense during its Development within Glossina palpalis is Infective. Ibid., lrxxiii., p. 345 .

(415) - - - (1911). Further Researches on the Development of T. gambiense in Glossina palpalis. Ibid., p. 513.

(416) - - and BRUCE (LADY) (1911). T. gallinarum. Rep. Sleeping Sickness Comm., xi., No. 32, p. 170.

(417) - - - (1911). A Trypanosome found in the Blood of a Crocodile. Ibid., No. 36, p. 184

(418) Brumpt, E. (1906). Lo Mode de Transmission des Trypanosomes et des Trypanoplasmes par les Hirudinées. C.R.S.B., lxi., p. 77.

(419) - (1907). L'Hérédité des Infections à Trypanosomes et à Trypanoplasmes chez les Hôtes intermédiaires. Ibid., lxiii., p. 176.

(420) Buchanan, G. (1911). Developmental Forms of $T$. brucei (pecaudi) in the Internal Organs of the Gerbil. P.R.S. (B), Ixxxiv., p. 161 .

(421) Carini, A. (1910). Stades Endoglobulaires des Trypanosomes. A.I.P., xxiv., p. 143.

(422) - (1910). Formas de Eschizogonia do T. lewisi. Soc. de Med. e Cir. de Sao Paulo, August 16, 1910 (quoted from B.I.P., ix., p. 937).

(423) - (1911). Présence de Trypanosomes chez les bovidés, à Sao Paulo. B.S.P.E., iv., p. 191 .

(424) - (1911). "Schizogonien bei Trypanosomen. A.P.K., xxiv., p. 80.

(425) Chaqas, C. (1909). Eine neue Trypanosomiasis des Menschen. M.I.O C, i., p. 159.

(426) - (1911). Lo Cycle de "Schizotrypanum cruzi" ohez l'Homme et les Animaux de Laboratoire. B.S.P.E., iv., p. 467.

(427) Crawley, H. (1910). T. americanum from the Blood of American Cattle. Journ. Comp. Path. Therap., xxiii., p. 17.

(428) Darina, S. T. (1911). Murrina. Journ. Infect. Diseases, viii., p. 467.

(429) - (1911). Mode of Infeotion and Methods of Controlling an Outbreak of Equine Trypanosomiasis in the Panama Canal Zone. Py., iv., p. 83.

(430) Dorlein, F. (1909). Problem der Protistenkunde. I. Die Trypanosomen. Jens: G. Fischer.

(431) - (1910). Experimentelle Studien über die Trypanosomen des Frosches. A.P.K., xix., p. 207.

(432) Dutron, J. E., Todd, J. L., and Tobey, E. N. (1906, 1907). Certain Parasitio Protozos observed in Africa. Part I., Liverpool Trop. Med. Memoirs, xx., p. 87. Part II., A.T.M.P., i., p. 287.

(433) Elders, C. (1909). Trypanosomissis beim Menschen auf Sumatra. C.B.B.P.K. (I Abth. Orig.), liii., p. 42.

(434) Fantnam, H. B. (1911). I ife-History of $T$. gambiense and $T$. rhodesiense as seen in Rats and Guines-pigs. P.R.S. (B), lrxriii., p. 212. 
(435) Frscher, W. (1911). Zur Kenntnis der Trypanosomen. Z.H., 1xx., p. 93. (436) Franca, C. (1908). La Biologie des Trypenosomes. A.I.C.P., ii., p. 43.

(437) - (1908). Lo Cycle Évolutif des Trypanosomes de la Grenouille. Ibid., p. 89.

(438) - (1908). Lo Trypenosome de l'Anguille (T. granulosum). Ibid., p. 113.

(439) - (1910). Un Trypanosome du Lerot $(T$. elyomis). Ibid., iii., p. 41.

(440) - (1911). Hématozoaires de la Guinée Portuguaise. Ibid., pp. 201, 229.

(441) - (1911). Les Hématozosires des Taupes. Ibid., p. 271.

(442) - (1911). Relation autogenetique entre les grands et les potits Trypanosomes de la Grenouille. C.R.S.B., lxx., p. 978.

(443) - (1911). La Transformation " in vitro" des Formos crithidiennes de "T'. rotatorium" en Formes trypanosomiques. B.S.P.E., iv., p. 534.

(444) Frimdricr, L. (1909). Bau und Naturgesohiohte des Trypanoplasma helicis. A.P.K., xiv., p. 363.

(445) FRY, W. B. (19i1). The Extrasion of Granules by Trypanosomes. P.R.S. (B), lxxxiv., p. 79.

(445.5) GoNDER, R. (1911). Arzneifeste Mikroorganismen. I. T. lewisi. C.B.B.P.K. (I Abth. Orig.), lxi., p. 102.

(446) Намвurakr, C. (1911). Einige parasitische Flagellaten. Verh. Heidelberg. Naturhist.-Med. Ver. (n. F.), xi., p. 211.

(447) Hartmann, M.(1910). Eine weitera Art der Sohizogonie bei Schizotrypanum cruzi. A.P.K., xx., p. 361.

(448) Hrnde, E. (1909). Life-History of T. dimorphon. Univ. California Publ. Zool., vi., p. 127.

(449) - (1910). Degeneration Phenomena of T. gambiense. Py., iii., p. 423.

(450) - (1910). A Biometrio Study of T. gambiense. Ibid., p. 455.

(451) - (1911). The Passage of T. aambiense through Mucous Membranes and Skin. Ibid., iv., p. 25.

(452) Jollos, V. (1910). Bau und Vermehrung von Trypanoplasma helicis. A.P.K., xxi., p. 103.

(453) Krysseutz, G. (1904). Trypanophis grobbeni. A.P.K., iii., p. 367.

(454) - (1906). Generations- und Wirtswechsel von Trypanoplasma borreli. A.P.K., vii., p. 1.

(455) - (1907). Die undulierende Membran bei Trypanosomen und Spiroohäten. A.P.K., х., p. 127.

(456) - and MAYER, M. (1908). Die Entwicklung von T. brucei in Glassina fusca. A.S.T.H., xii.. p. 532.

(457) Krmswe, F. (1909). Positive Infectionsversuche mil T. brucei durch Glossina palpalis. Deutoch. Med. Wochenschr., xxxv., p. 469. Die Entwioklung von Trypanosomen in Glossinen. Ibid., p. 924. Die Aotiologie der Schlafkrankheit. Ibid., p. 1257. Tsotsefliegen und Trypanosomen. Ibid., p: 1956.

(458) - (1910). Trypanosomenbefunde am Tanganyika. Ibid., xxxvi., p. 1400.

(459) - and TAUTe, M. (1911). Ergänzungen zu unseren Trypanosomenstudien. A.K.G.A., xxxi., p. 321. Reprinted as " Trypansomenstudien."

(460) Koch, R., BECK, M., and KueINe, F. (1909). Die Tätigkeit der zur Erforschung der Schlafkrankheit im Jahre 1906-07 nach Ostafrika entasndten Kommission. A.K.G.A., xxxi., p. 1 .

(461) Laveran, A. (1911). Identification et Classification des Trypanosomes des Mammifères. A.I.P., xxv., p. 497.

(462) - (1911). Les Trypanosomes, ont-ils des Formes latentes chez leurs Hôtes vertébrés ? C.R.A.S., cliii., p. 649 .

(464) - and Mesnil, F. (1902). Des Trypanosomes des Poissons. A.P.K., i.. p. 475 .

(465) - and Peтtrt, A. (1910). Dea Trypanosomes du Mulot et du Campagno: (T. grosi et T. microti). C.R.S.B., Ixviii., p. 571.

(466) - - (1910). Lo Trypanosome du Lorot (Myoxus nitela) et la Puce qui parait le propager (T. blanchardi). Ibid., p. 950 .

(467) Lebatlly, C. (1906). Les Hématozoaires parasites des Téléostéens marins. Arch. Parasitol., x., p. 348.

(468) LeBedeFF, W. (1910). T. rotatorium, Gruby. Hertwig's Festschrift, i., p. 397.

(469) Machudo, A. (1911). Zytologisohe Untersuchungen über $T$. rotatorium, Gruby. M.I.O.C., iii., p. 108.

(470) Manteurke (1909). Studien über die Trypanosomiasis der Ratten. A.K.G.A., xxxiii., p. 46. 
(471) Martin, C. H. (1910). Trypanoplasma congeri-I. The Division of the Active Form. Q.J.M.S., Iv., p. 485.

(472) Martin, G., Jlabefof, A., and Rouraud, F. (1908). Transmission du "Nagana" par les Stomoxes et les Moustiques. B.S.P.E., i., p. 355.

(473) Mathis, C., and Legke, M. (1911). Parasitologie et Pathologie humaines et animales au Tunkin. Paris : Masson et Cie.

(474) Mesnn, F. (1910). L'Identification de quelques Trypanosomes pathogènes. B.S.P.E., iii., p. 376.

(475) - and Brimont, E. (1908). Un Hématozoaire nouveau (Endotrypanum) d'un Édenté de Guyane. C.R.S.B., lxv., p. 581.

(476) Mrnchin, E. A. (1908). The Derelopment of Trypanosomes in Tsetse-Flies and Other Diptera. Q.J.M.S., lii., p. 159.

(477) - (1908). Polymorphism of T. gambiense. Py., i., p. 236.

(478) - (1909). The Flagellates parasitio in the Blood of Freshwater Fishes. P.Z.S., 1909, p. 2.

(479) - (1909). Structure of T. levisi in Relation to Microscopical Technique. Q.J.M.S., liii., p. 755.

(480) - and Thomson, J. D. (1910). Transmission of $T$. lewisi by the Rat-Flea (Ceratophyllus fasciatus). P.R.S. (B.), Ixxxii., p. 273.

(481) - - (1911). Transmission of T. lewisi by the Rat-Fles. Brit.Med.Journ., 1911 , i., p. 1309.

(482) - - (1911). An Intracellular Stage in the Development of $T$. lewisi in the Rat-Flea. Ibid., ii. (August 19), pp. 361-364.

(483) - and Woodcock, H. M. (1910). Blood-Parasites of Fishes occurring at Rovigno. Q.J.M.S., 1v., p. 113.

(484) Moore, J. E. S., and BreinL, A. (1907). Cytology of the Trypanosomes, part i. A.T.M.P., i., p. 441.

(485) - - (1908). T. equiperdum. P.R.S. (B.), lxxx., p. 288.

(486) - - and HndLe, E. (1908). Life-History of T. lewisi. A.T.M.P., ii., p. 197.

*(487) Nereshemme, E. (1911). Die Gattung Trypanoplasma. Vide Prowazkx (14), p. 101.

(488) Nedmann, R. O. (1909). Protozoische Parasiten im Blut von Meeresfischen. Z.H., lxiv., p. 1 .

(489) Novy, F. G., and McNeal, W. J. (1905). Trypanosomes of Birds. Journ. Infect. Diseases, ii., p. 256.

(490) - - and Torrex, H. N. (1907). Trypanosomes of Mosquitoes and Other Insects. Ibid., iv., p. 223.

(491) OtTolenehi, D. (1908). T. Irucei und T. equinum. C.B.B.P.K. (I. Abth. Orig.), xlvii., p. 473.

(492) - (1909). Die Entwioklung einigor pathogenor Trypanosomen im Säugetierorganismus. A.P.K., xviii., p. 48.

(493) Patton, W. S., and Strickland, C. (1908). The Relation of Blood-sucking Invertebrates to the Life-Cycles of Trypanosomes. Py., i., p. 322.

(494) Prtrie, G. F. (1905). The Structure and Geographical Distribution of Certain Trvpanosomes. J.H., v., p. 191.

(495) - and Avami, C. R. (1909). On the Sessonal Prevalence of T. lewisi in Mus rattus and in Mus decumanus. Py., ii., p. 305.

(496) Pourcard, A. (1910). Sur la Coloration vitale des Trypanosomes. C.R.S.B., lxviii., p. 505.

(497) Prowazs , S. v. (1905). Studien über Säugetiertrypanosomen. A.K.G.A., xxii., p. 351 .

(498) - (1909). Kritische Bemerkungen zum Trypanosomenproblcm. A.S.T.H., xiii., p. 301.

(499) Robertson, M. (1906). Certain Blood-inbabiting Protozoa. Proc. $R$. Phys. Soc. Edinburgh., xvi.. p. 232.

(500) - (1907). A Trypanosome found in the Alimentary Canal of Pontobdello muricata. Ibid., xvii., p. 83.

(501) - (1909). Life-Cycle of T. vittatce. Q.J.M.S., liii., p. 665.

(502) - (1909). A Trypanosome found in the Alimentary Tract of Pontobdella muricata. Q.J.M.S., liv., p. 119.

(503) - (1911). Transmission of Flagellates living in the Blood of Fishes. Phil. Trans. (B.), ccii., p. 29.

(504) Rodenwald, E. (1909). T. lewisi in Homatopinus spinulosus. C.B.B.P.K. (I Abth. Orig.), lii., p. 30. 
(505) Rosen вusch, F. (1909). Trypanosomen-studien. A.P.K., xv., p. 263.

(506) RovBAUD, E. (1909). Les Trypanosomes pathogenes et la Glossina pal palis. Rapport de la Mission d'Études de la Maladie du Sommeil au Congo Français (Paris, Masson et Cie.), p. 511.

(507) - (1910). Phénoménes morphologiques du Dévoloppement des Trypanosomes chez les Glossines. G.R.A.S., cli., p. 1156.

(508) Stassano, H. (1901). La Fonction et Relation du petit Noyau des Trypanosomes. C.R.S.B., liii., p. 468.

(509) Stephens, J. W W., and Fantham, H. B. (1911). Peculiar Morphology of a Trypanosome from a Case of Sleeping Sickness (T. rhodesiense). P.R.S. (B.), lxxxiii., p. 28.

(510) Stockman, S. (1910). A Trypanosome of British Cattle. Journ. Comp. Pathol. Therapeut xxiii., p. 189.

(511) Strickiand, C. (1911). Mechanism of Transmission of $T$. lewisi by the RatFlea. Brit. Med. Journ., 1911, p. 1049.

(512) - and Swellengreber, N. H. (1910). On T. lewisi and its Relation to Certain Arthropoda. Py., iii., p. 436.

(513) Stuhlmann, F. (1907). Die Tsetsefliegen (Glossina fusca und Gl. tachinoides). A.K.G.A., $\mathrm{xxvi.,} \mathrm{p.} 301$.

(514) Swellunorebel, N. H. (1909). Bau und Zellteilung von T. gambiense und T. equinum. Tijdschr. Ned. Dierk. Ver. (2), xi., p. 80.

(515) - (1910). Fixation and Staining of T. lewisi. Py., iii., p. 226.

(516) - (1910). Normal and Abnormal Morphology of T. lewisi. Ibid, p. 459.

(517) - and Strickland, C. (1910). The Development of T, lewisi outside the Vertebrate Host. Ibid., p. 360.

(518) - - (1911). Remarks on Dr. Swingle's Paper, "The Transmission of T. lewisi by Rat-Fleas," etc. Ibid., iv., p. 105.

(519) Swrnale, L. D. (1907). On T. lewisi. "Trans. Amer. Micr. Soc., xxvii., p. 111 .

(520) - (1E11). Transmission of $T$. lewisi by Rat-Fleas. Three New Herpetomonads. Journ. Infect. Diseases, viii., p. 125.

(521) TAUTe, M. (1911). Die Beziehungen der Glossina. morsitans zur Schlafkrankheit. Z.H., lxix., p. 553.

(522) Thrrovx, A. (1905). T. padda. A.I.P., xix., p. 65.

(523) - (1905). T. duttoni. Ibid., p. 564.

(524) Thomson, J. D. (1906). Blood-Parasites of the Mole. J.H., vi., p. 574.

(525) - (1908). Cultivation of the Trypanosome found in the Blood of the Goldfish. Ibid., viii., p. 75.

(5-6) Werkitzki, F. W. (1910). Blepharoplastlose Trypanosomen. C.B.B.L.K. (I Abth. Orig.), liii., p. 303. (See also Bulletin of the Sleeping Sickness Bureau, vol. iii., pp. 221, 313, and 458, for further references.)

(527) Woodcock, H. M. (1910). On Certain Parasites of the Chaffinch (Fringilla coelebs) and the Redpoll (Linota rufescens). Q.J.M.S., lv., p. 641.

(528) YakimoFf, W. L., KoHL-YakimoFr, N., and Korssak, D. W. (1910). T. korssaki of Mus agrarius, Piroplasmoses of Mus agrarius, Reindeer, Yak, and Bears. C.B.B.P.K. (I Abth. Orig.), lv., p. 370.

(529) Zupitza, M. (1909). Die Vogel. und Fischtrypanosomen Kameruns. A.S.T.H., xiii., Beiheft 3, p. 101.

\section{(c) Crithidia, Leptomonas, Herpetomonas, etc.}

See also No. 84.

(530) Bodet, G., and Rodbadd, E. (1911). La Présence au Dahomey et Transmission du Leptomonas davidi. C.R.S.B., lxx., p. 55.

(531) Chatron, E. (1909). Un Trypanosomide nouveau d'une Nyctéribie, et les Relations des Formes Trypanosoma, Herpetomonas, Leptomonas ct Crithidia. C.R.S.B., lxvii., p. 42.

(532) - and AlrlaIre, E. (1908). Coexistence d'un Leptomonas et d'un Trypanosoma chez un Muscide non vulnérant, Drosophila confusa. C.R.S.B., lxiv., p. 1004.

(533) - and LEakr, A. (1911). Eutrypanosomes, Leptomonas et Leptotrypanosomes chez Drosophila confusa (Muscide). C.R.S.B., lxx., p. 34 .

(534) - - (1911). Quelques Leptomonas de Muscides et leurs Leptotrypano. somes. Ibid., p. 120.

(535) DonkerLy, J. S. (1911). Life-History of Lept. musca-domesticce. Q.J.M.S., Ivi., p. 645. 
(536) Fud, P. C. (1911). Die im Darm der Btubenfliege vorkommenden protozoären Gebilde. C.B.B.P.K. (I Abth. Orig.), I vii., p. 522.

(537) França, C. (1911). L'Eristence en Portugal de Lept. davidi dens le Latex do Euphorbia peplus et $E$. segetalis. B.S.P.E., iv., p. 532.

(538) - (1911). Notos sur Lept. davidi. Ibid., p. 669.

(539) Geona ewitcB, J. (1909). Le développement do Crithidia simulio. C.R.S.B., lxvii., p. 517.

(540) Lafont, A. (1910). La Présenco d'un Leptomonas . . . dans le Latex de Trois Euphorbiacées. A.I.P., xxiv., p. 205.

(541) - (1911). La Transmission du Lept. davidi des Enphorbes par un Hémiptere. C.R.S.B., lxx., p. 58.

(542) LEakR, L. (1902). Ia Structure et Multiplication des Flagellés du Genre Herpetomonas Kent. C.R.A.S., oxxxiv., p. 781. (543) - (1902). Un Flagellé Parasite de l'Anopheles maculipennis. C.R.S.B.,

(544) - (1904). Un nouveau Flagellé, Parasite des Tabanides. C.R.S.B., Ivii., p. 613 .

(545) - (1904). Les Affinités de l'Herpetomonas subulata et la Phylogónie des Trypanosomes. C.R.S.B., Ivii., p. 615 .

(546) - and Dubosce, O. (1909). Parasites de l'Intestin d'une Larve de Ptychop. tera. Bull. Acad. Belgique, No. 8, p. 885.

(547) Mackunnon, D. L. (1910). New Parasites from Triohoptera. Py., iii., p. 245.

(548) - (1910). Herpetomonads from Dung. Flies. Ibid., p. 255.

(549) - (1911). More Protozoan Parasites from Trichopters. Ibid., iv., p. 28.

(550) Patron, W. S. (1908). Life-Cycle of a Speoies of Crithidia parasitic in Gerris fossarum. A.P.K., xii., p. 131.

(551) - (1908). Herp. lygai. A.P.K., xiii., p. 1.

(552) - (1909). Life-Cycle of a Speoies of Crithidia parasitio in Tabanus hilarius and Tabanus sp. A.P.K., IV., p. 333.

(553) - (1910). Infection of the Madras Bazaar Fly with Herp. musca-domestica. B.S.P.E., iii., p. 264.

(554) PorTER, A. (1910). Crithidia melophagia. Q.J.M.S., Iv., p. 189.

(555) - (1909). Crithidia gerridis. Py., ii., p. 348.

(556) - (1909). Life-Cycle of Herp. jaculum. Ibid., p. 367.

(557) Prowazek, S. v. (1904). Die Entwioklung von Herpetomonas. A.K.G.A., xx., p. 440.

(557.5) ROUBAOD, E. (1911). Cystotrypanosoma intestinalis. C.R.S.B., Ixxi., p. 306.

(558) Strickland, C. (1911). A Herpetomonas parasitic in the common Greenbottle Fly, Lucilia sp. Py., iv., p. 222.

(559) SwkLlenarebe, N. H. (191i). Morphology of Herpetomonas and Crithidia, etc. Ibid., p. 108.

(560) WrRaskr, H. (1908). Eine eingeisselige Flagellatonform im Darm der Stubenfliege. A.P.K., xiii., p. 19.

\section{(d) Ioishmanis, oto.}

See also No. 84. For references to literature and critical summaries and reviews, see Kala Azar Bulletin (Royal Society, London).

(561) BasIre, C. (1910). Leishmaniosi del Cane e l'Ospite intermedio del KalaAzar infantile. Rend. Acc. Lincei (5), xix. (2), p. 523.

(562) - (1911). Trasmissione delle Leishmaniosi. Ibid. (5), xx. (1), p. 50.

(563) - (1911). Leishmaniosi e suo Modo di Trasmissione. Ibid. (5), ixx. (2), p. 72 .

(564) - LA Cava, F., and Visentini, A. (1911). L' Identita delle Leishmaniosi. Ibid., p. 150 .

(565) Daring, S. T. (1909). Histoplasma capsulatum and the Lesions of Histoplasmosis. J.E.M., xi., p. 515.

(566) Donovan, C. (1909). Kala-Azar in Madras. Bombay Medical Congress, February 24, 1909.

(567) LeishmaN, W. B., and Statham, J. C. B. (1905). Development of the Leishman Body in Cultivation. Journ. R. A. Med. Corps, iv., p. 321.

(568) Marshall, W. E. (1911). Pathological Report, Kala-Azar Commission. Rep. Wellcome Lab., iv., p. 157.

(569) MarzinowSKY, E. J. (1909). Cultures de Leishmania tropica. B.S.P.E., ii., p. 591 . 
(570) Nrcolte, C. (1909). Le Kala-Azar infantile. A.I.P., xxiii., p. 361.

(571) - and Comte, C. (1908). Origine osnine du Kala-Azar. C.R.A.S., oxlvi., p. 789.

(572) Novy, F. G. (1909). Leishmania infantum. B.S.P.E., ii., p. 385.

(573) Patron, W. S. (1908). The Leishman-Donovan Parasite in Cimex rolundatus. S.M.I., xoxi.

(574) - (1908). Inoculation of Dogs with the Parasite of Kala-Azar (Herpetomonas [Leishmania] donovani). Py., i., p. 311.

(575) - (1909). The Parasite of Kala-Azar and Allied Organisms. Trans. Soc. Trop. Med. Hygiene, ii., p. 113.

(576) Roarrs, L. (1904). Trypanosomes from the Spleen Pratozoic Parasites of Cachexial Fevers and Kala-Azar. Q.J.M.S., xlviii., p. 367.

(577) - (1907). The Milroy Lectures on Kala-Azar. Brit Med. Journ., February 23, Maroh 2 and 9.

(578) Row, R. (1909). Development of the Parasite of Oriental Sore in Cultures. Q.J.M.S., liii., p. 747 .

(579) Thurovx, A., and TEPPAz, L. (1909). La Lymphangite epizootique des Equidés au Sénégal. A.I.P., xxiii., p. 420.

(580) Visentini, A. (1910). La Morfologia ed il Ciolo di Bviluppo della Leishmania. Istituto d. Clin. Med. d. R. Univ. Roma.

(581) Wriont, J. H. (1903). Protozos in Tropical Ulcer ("Delhi Sore"). Journ. Med. Research, x. (n.s. v.), p. 472.

\section{(e) Prowazelia.}

(582) Alexkietr, A. (1911). La Morphologie et la Division de Bodo caudatus. C.R.S.B., lxx., p. 130 .

(582.5) Donkerly, J.S.(1912). Thelohania and Prowazekia in Anthomyid Flies. C.B.B.P.K. (I Abth. Orig.), Ixii., p. 136.

(583) Hartmans, M. (1911). Die Flaggellatenordnung Binucleata und die Gattung Prowazekia. A.P.K., xxii., p. 141.

(584) Martint, E. (1910). Pr. cruzi und ihro Beziohungen zur Ätiologie von ansteckenden Darmkrankheiten zu Tsingtan. Z.H., Irvii., p. 275.

(585) Nakglar, K. (1910). Pr. parva. A.P.K., xxi., p. '111.

(586) WALKKR, E. L. (1910). Trypanoplasma rana. Journ. Med. Research, xriii., (n.s. XVIII.), p. 391.

(587) Whтмову, E. R. (1911). Pr. asiatica. A.P.K., xxii., p. 370.

\section{CHAPTER XIV}

\section{SPOROZOA-TELOSPORIDIA}

(a) Ceneral Works. *(588) Haomengulkr (1899). Bibliotheca Sporozoologica. Ann. Mus. Nat.
Hist. Marseille (2), i.

*(589) Minchin, E. A. (1903). The Sporozos. A Treatise on Zoology (Lankester) (London, A. and C. Black), p. 150.

(590) Woodcock, H. M. (1910). "Sporozoa. Encyclop. Brit., eleventh edition, xxv., p. 734. Coccidia. Ibid., vi., p. 615. Gregarines. Ibid., rii., p. 555. Hæmosporidis. Ibid., xii., p. 806. Endospora. Ibid., ix. p. 383 .

See also Nos. 72, 84, and 123.

(b) Gregarines.

(591) Aw erinzew, S. (1909). Die Vorgänge der Schizogonie boi Gregarinen aus dem Darm von Amphiporus sp. A.P.K., Ivi., p. 71.

(592) Bеadchamp, P. do (1910). Une Grégarine nouvelle du Genre Porospora. C.R.A.S., cli., p. 997.

(693) BrRnd, A. (1902). Dic im Darme der Làryc von Tenebrio molitor lebenden Gregarinen. A.P.K., i., p. 375.

(504) BrasL, L. (1905). La Réproduction des Grégarines monooystidées. A.Z.E. (4), iii., p. 17. 
(595) Brasil, I (1905). La Réproduetion des Grégarines monocystidées. A.Z.E. (4), iv., p. 69.

(596) - (1907). La Sohizogonie et Is Croissance des Gamétocytes chez Selenidium caulleryi. A.P.K, viii., p. 370.

(597) - (1909). Documents sur quelques Sporozosires d'Annélides. A.P.K., Ivi., p. 107.

(598) Cognetit de Martus, L. (1911). Lo Monocistidee o loro Fenomeni ripro. dutrivi. A.P.K., xuii., p. 205.

(599) Comes, S. (1007). Der Chromidialspparat der Gregarinen. A.P.K., x., p. 416.

(600) CrAwLey, H. (1905). Movements of Gregarines. Proc. Acad. Philadelphia, lvii., p. 89.

(601) Conхпіонам, J. T. (1007). Kalpidorhynchus arenicolos. A.P.K., х., p. 199.

(602) Dogres, V. (1906). Cystobia chiridotos. A. P.K., vii., p. 106.

(603) - (1907). Schizocystis sipunculi. A.P.K., viii., p. 203.

(604) - (1909). Die Sporocysten der Cölom-Monocystideø. A.P.Z., rvi., p. 194.

(605) - (1910). Callynthrochlamy's phronima. A.P.K., 1x., p. 60.

(606) - (1910). Einige nene Catenats. Z.w.Z., xciv., p. 400.

(607) Drzewzcki, W. (1903, 1907). Vegetative Vorgänge im Kern und Plasma der Gregarinen des Regen wurmhodens. A.P.K., iii., p. 107. II. Stomatophora coronata. Ibid., x., p. 216.

(608) DUKE, H. L. (1910). Metamera schubergi. Q.J.M.S., lv., p. 261.

(609) Fanthaм, H. B. (1908). The Schizogregarines. Py., i., p. 369.

(610) HAL, M. C. (1907). A Study of some Gregarines, with espeoial Rererence to Hirmocyetis rigida. Stud. Zool. Lab. Univ. Nebraska, vii., p. 149.

(611) Hesse, E. (1909). Ies Monoeystidées des Oligochètes. A.Z.E. (5), iii., p. 27.

(611.5) HoFfmaxx, R. (1908). Fortpflanzungserscheinungen von Monocystideen des Lumbricus agricola. A.P.K., xiii., p. 139.

(612) HoxnEY, J. S. (1910). Ganymedes anaspidis, Q.J.M.S., Iv., p. 155.

(613) Koschakewrtson, S, (1907). Vorgänge bei den Gregarinen des Mehlwurm. darms. A.P.K., Suppl. I., p. 202.

(614) LEOER, L. (1904). La Réproduotion seruée chez les Stylorhynchus. A.P.K., iii., p. 303.

(615) - (1904). Sporozosires Parasites de l'Embia Salieri. Ibid., p. 358.

(616) - (1906). Treniocystis mira. A.P.K., vii., p. 307.

(617) - (1907, 1909). Les Schizogrégarines des Trachéates: I. Ophryocystis. A.P.K., viii., p. 159. II. Schizocystis. Ibid., xviii., p. 83.

(618) - and DúовcQ, 0 . (1902). Les Grégarines et l'Epithélium intestinal chez les Trachéates. Arch. Parasital., vi., p. 377.

(619) - - (1903). Lo Développement des Grégarines Stylorhynehides et Stenophorides. A.Z.E. (4), i., Notes et Revue, p. Ixurix.

(620) - - (1904). Les Grégarines et l'Epithélinm intestinal des Traohéates. A.P.K., iv., p. 335.

(621) - - (1909). ' La Sexualité ehez les Grégarines. A.P.K., xvii., p. 19.

(622) - - (1911). Denx nonvelles Espèces de Grégarines appartenant au Genre Porospora. Ann. Univ. Grenoble, xxiii.,p. 401.

*(623) LÜHr, M. (1904). Die Sporozoiten, die Wachstnmsperiode und die ausgebildeten Gregarinen. A.P.K., iv., p. 88.

(624) NusBaUM, J. (1903). Fortpflenzung einer Gregarino-Schaudinella henlea. Z.vo.Z., lxxv.,p. 281.

(625) ParmLer, F. (1904). Die Morphologie, Fortpflanzung und Entwicklung ron Gregarina ovata. A.P.K., iv., p. 64 .

(626) Prerrer, E. (1910). Dio Gregarinen im Darm der Larve von Tenebrio molitor. A.P.K., xix., p. 107.

(627) Porter, A. (1909). Merogregarina amaroucii. A.P.K., xv., p. 228.

(628) Robinson, M. (1910). On the Reproduction of Kalpidorhynchus arenicalas, Q.J.M.S., liv., p. 565 .

(629) Schellack, C. (1907). Die Entwicklung and Fortpflanzung von Echinomera hispida. A.P.K., ix., p. 297.

(630) - (1908). Die solitäre Eneystierung bei Gregarinen. Z.A., ixxii., p. 597.

(631) Schnitzler, H. (1905). Die Fortpflanzung von Clepsidrina ovata. A.P.K., vi., p. 309.

(632) WooDCock, H. M. (1906). Life-Cycle of "Cystobia" irregularis. Q.J.M.S. 1., p. 1. 


\section{(c) Coccldia.}

See also Nos, 47, 83, 94, 99, and 147 .

*(633) Blanchard, R. (1900). Les Coccidies et lour Rôle pathogene. Causeries Sci. Soc. Zool. France, p. 133.

(634) Chagas, C. (1910). Adelea hartmanni. M.I.O.C., ii., p. 168.

(635) DakIN, W. J. (1911). Merocystis katha. A.P.K., xxii., p. 145.

(635'5) Debaisievx, P. (1911). Recherches sur les Coccidies. La Cellule, xxvii., pp. 89 and 257.

(636) DoBEL, C. C. (1907). Life-History of Adelea ovata. P.R.S. (B.), Irxix., p. 155.

(637) Elamassian, M. (1909). Coccidium rouxi, Zoomyxa legeri. A.Z.E. (5), ii., p. 229.

(638) FАNтhaм, H. B. (1910). Eimeria (Coccidium) avium. P.Z.S., 1910, p. 672.

(639) - (1910). Avian Coccidiosis. Ibid., p. 708.

(640) HADLEY, P. B. (1911). Eimeria avium. A.P.K., xxii., p. 7.

(641) Jollos, V. (1909). Multiple Teilung und Reduktion bei Adelea ovata. A.P.K., xv., p. 249.

(642) Konze, W. (1907). Orcheobius herpobdella. A.P.K., ix., p. 382.

(643) Laveran, A., and Petrit, A. (1910). Une Coccidio de Agama colonorum. (Cocc. agama). C.R.S.B., lxviii., p. 161.

(644) Learr, Io (1911). Caryospora simplex, et la Classification des Cocoidies. A.P.K., xxii., p. 71.

(645) - and DuboscQ, O. (1908). L'Évolution schizogonique de l'Aggregata (Eucoccidium) eberthi. A.P.K., xii., p. 44.

(646) - - (1910). Selenococcidium intermedium. A.Z.E. (5), v., p. 187.

(647) Metzene, R. (1903). Coccidium cuniculi. A.P.K., ii., p. 13.

(648) Mororf, T. (1906). Adelea zonula. A.P.K., viii., p. 17.

(649) - and Finbiger, J. (1905). Eimeria subepithelialis. A.P.K., vi., p. 166.

(650) Prazz, C. (1903). Lo Cycle évolutif de l'Adelea mesnili. A.P.K., ii., p. 1.

(651) Soneluack, C., and ReIchenow, E. (1910). Lithobius-Coccidien. Z.A., xxxvi., p. 380.

(652) Sirdlecki, M. (1898). La Coccidie de la Seiohe. A.I.P., xii., p. 799.

(653) - (1907). Caryotropha mesnilii. B.A.S.C., 1907, p. 453.

(654) Stevenson, A. C. (1911). Coocidiosis of the Intestine of the Goat. Rep. Wellcome Lab. Khartoum, iv., p. 355.

(655) Tyzzer, E. E. (1910). Cryptosporidium muris of the Common Mouse. Journ. Med. Research, xxiii (n.s. XVIII.), p. 487.

(656) Woodcock, H. M. (1904). On Klossiella muris. Q.J.M.S., xlviii., p. 153.

\section{CHAPTER XV}

\section{H AMOSPORIDIA}

\section{(a) General Works.}

*(657) Laveran, A. (1905). Hæmocytozoa. B.I.P., iii., p. 809.

*(658) Scraddins, F. (1899). Der Generationswechsel der Cocoidien und Hæmo. sporidien. Zool. Centralbl., vi., p. 765.

(659) W ASIKLEWSKI (1908). Studien und Mikrophotogramme zur Kenntnisse der pathogenen Protozoen. II. Untersuchungen über Blutschmarotzer. Leipzig : Barth.

See also Nos. 130 and 686.

(b) Hæmamœbю.

(660) Aracao, H. DE B., and Nerva, A. (1909). Intraglobular Parasites of Lizards. Pl. diploglossi and Pl. tropiduri. M.I.O.C., i., p. 44.

(661) Beren bera-Gosslek, H. v. (1909). Naturgeschichto der Malariaplagmodien. A.P.K., xvi., p. 245.

(662) Bertrand, D. M. (1911). Les Parasites endoglobulaires pigmentés des Vertébrés. Paris : Jouve et Cic.

(663) Bruler, A. (1905). Une Forme particuliere de l'Hématozoaire du Paludisme decrite par MaI. Ed. et Ét. Sergent. C.R.S.B., Iviii., p. 720.

(664) - (1906). La Forme hémogregarinienne du Parasite de la Fíevre quarte. C.R.S.B., lx., p. 891. 
(665) Brunz, A. (1906). Diagnose différentielle des Formes annulaires des Hématozoaires du Paludisme. C.R.S.B., lxi., p. 754.

(666) - (1910). Evolution chez le même Sujet du Paludisme tierce primaire en Paludisme tieroe secondaire. B.S.P.E., iii., p. 187.

(667) Cardamatis, J. P. (1909). Le Paludisme des Oiseaux en Grèce. Étude du Parasite de Danilewsky. C.B.B.P.K. (I Abth. Orig.), lii., p. 351.

(668) Castemuni, A., and Wruey, A. (1904). Hæmatozos of Vertebrates in Ceylon. Spolia Zeylanica, ii., p. 78.

(669) Darfine, S. T. (1910). Transmission and Prevention of Malaria in the Panama Canal Zone. A.T.M.P., iv., p. 179.

(670) DoвkL, C. C. (1910). Life-History of Hamocystidium simondi. Hertwig's Festschrift, i., p. 123.

(671) FlU, P. C. (1908). Affenmalaris. A.P.K., xii., p. 323.

(672) Grirutr, J. J., Sweer, G., and Dodd, S. (1910). Proteosoma biziuras and Hamogregarina megalocystis. Proc. Roy. Soc. Victoria (n.8.), xxiii., p. 321.

(673) Grassi, B. (1901). Die Malaria, Studien eines Zoologen. Jena : Gustav Fischer.

(674) Halberstandter, L., and Prowazez, S. v. (1907). Die Malariaparasiten der Affen. A.K.G.A., xxvi., p. 37.

(675) Harmann, M. (1907). Das System der Protozoen. Zugleich vorläufigo Mitteilung über Proteosoma. AsP.K., x., p. 139.

(676) MaYer, M. (1908). Malariaparasiten bei Affen. A.P.K., xii., p. 314.

(677) Nrumans, R. O. (1908). Die Übertragung von Plasmodium pracox auf Kanarienvogel durch Stegomyia fasciata. A.P.K., xiii., p. 23.

(678) Ross, R. (1910). The Prevention of Malaria. London : John Murray.

(679) Skrarist, Ét, and Skraknt, Ed. (1910). L'Immunité dans le Paludismo des Oiseaux, etc. C.R.A.S., cli., p. 407.

(680) Thanoux, A. (1906). Des Relations de la Fievre tropioale avec la Quarte et la Tierce. A.I.P., xx., pp. 766 and 869 .

(681) VAsSaL, J. J. (1907). L'Hématozoaire de l'Ecureil (Hamamaeba vassali). A.I.P., xxi., p. 851 .

Soe also No. 132.

(c) Halteridia.

(682) Axscứ TZ, G. (1910). Ueberträgungsversuche von Hamoproteus oryzivora und Trypanosoma padda. C.B.B.P.K. (I Abth. Orig.), liv., p. 328.

(683) ARAG AO, H. DE B. (1908). Der Entwicklungsgang und die Übertragung von Hosmoproteus columba. A.P.K., xii., p. 154.

(684) MaYER, M. (1910). Die Entwicklung von Halteridium. A.S.T.H., xiv., p. 197.

(685) - (1911). Ein Halteridium und Leucocytozoon des Waldkauzes. A.P.K., xxi., p. 232.

$(685.5)$ Mrrchin, E. A. (1910). Report on Blood.Parasites collected by tho Commission. Rep. Sleeping Sickness Comm., x., p. 73.

(686) Shroent, Ed., and Skraent, Etr. (1907). Les Hématozosires d'Oiseaux. A.I.P., xxi., p. 251.

(687) Woodcock, H. Y. (1911). An Unusual Condition in Halleridium. Z.A., xxxviii., p. 465.

(d) Ioncocytozos (Vers).

See also Nos. 132, 473, and 686 .

(688) Bermstneft, N. (1904). Das Leucocytozoon Danilewakyi. A.P.K., iii., p. 376.

(689) Fantham, H. B. (1910). Parasitio Protozos of the Rod Grouse. P.Z.S., 1910 , p. 692.

(690) Wenyon, C. M. (1910). On the Genus Leucocytozoon. Py., iii, p. 63.

\section{(e) Hæmogregarines.}

Seo also Nos. 78, 84, and 89.

(691) Adre, J. R. (1906). "Leucocytozoon" ratti. Journ. Trop. Med., ix., p. 325

(692) Aragao, H. DE B. (1911). Hämogregarinen von Vögeln. M.I.O.C., iii. p. 64.

(693) BaLfour, A. (1906). H. balfouri. Rep. Wellcome Lab. Khartoum, ii., p. 96. (694) - (1906). "Leucocytozoon" muris. Ibid., p. 110. 
(695) Brrmstneft, N. (1903). Eine neue Blutparssiten der indischen Fröвche. A.P.K., ii., p. 343.

(696) BIIIET, A. (1904). Trypanosoma inopinatum et Drepanidium. C.R.S.B., lvii., p. 161.

(697) Boukr, G. (1909). Hómogregarines de l'Afrique occidentale française. C.R.S.B., lxvi., p. 741.

(698) Carini, A. (1910). "H. muris." Rev. Soc. Sci. São Paulo, v.

(699) Christophers, S. R. (1905). H. gerbilli. S.M.I., 18.

(700) - (1906). Leucocytozoon canis. S.M.I., 26.

(701) - (1907). Leucocytozoon canzs in the Tiok. S.M.I., 28.

(703) Danmewsky, B. (1886). Les Hématozosires des Lézards. Arch. Slav. Biol., i., p. 364.

(704) - (1887). Les Hématozoaires des Tortues. Ibid., iii., pp. 33 and 370.

(705) - (1889). La Parasitologie comparée du Sang. I. Nouvelles Reoherchea sur les Hématozosires du Sang des Oiseaux. Il. Recherches sur les Hématozoaires des Tortues. Kharkoff.

(706) FАхтнам, H. B. (1905). Lankesterella tritonis. Z.A., zxix., p. 257.

(707) Fuv, P. C. (1909). Hämogregarinen im Bluto Surinamiseher Sohlangen. A.P.K., xviii., p. 190.

(708) França, C. (1908). Une Hómogregarine de l'Anguille (H. bettencourli). A.L.C.P., ii., p. 109.

(709) - (1908). H. splendens (Labbé). Ibid., p. 123.

(710) - (1909). Hémogregarines de Lacerts ocellata. Ibid., p. 339.

(711) - (1910). Parasites endooellulaires du Psammodromus algirus. Ibid., iii., p. 1.

(712) - (1910). Hémogregarines de Lacerta muralis. Ibid., p. 21.

(713) Hams, C. W. (1909). H. stepanowi in the Blood of Turtles A.P.K., xvii., p. 307.

(714) KoIDzomi, M. (1910). H. вp. in Clemmys japonicus. A.P.K., xviii., p. 260.

(715) Laveran, A., and PeTtit, A. (1909). Los Hémogregarines de quelques Sauriens d'Afrique. B.S.P.E., ii., p. 506.

(716) - - (1910). Les Formes de Multiplication endogene de $H$. sebai. C.R.A.S., cli., p. 182.

(717) - - (1910). H. agama. C.R.S.B., Ixviii., p. 744.

(718) - - (1910). Le Role d'Hyalomma Algyptium L. dans la Propagation de H. mauritanica. C.-R. Assoc. France (Lille), p. 723.

(719) MrLler, W. W. (1909). Hepatozoon perniciosum and its Sexual Cyclo in the Intermediate Host, a Mite (Lelaps echidninus). Hygienis Laboratory Bulletin, No. 46 (June, 1908).

(720) Neresheimer, E. (1909). Das Eindringen von Lankesterella spec. in dio Froschblutkörperchen. A.P.K., xvi., p. 187.

(721) Patton, W. S. (1906). On a Parasite found in the Blood of Palm Squirrels. S.M.I., 24.

(722) - (1908). The Hæmogregarines of Mammals and Reptiles. Py., i., p. 319.

(723) Porter, A. (1908). Leucocytozoon musculi. P.Z.S., i908, p. 703.

(724) Prow azer, S. v. (1907). Ueber Hämogregarinen. A.K.G.A., xxvi., p. 32.

(725) Robmerson, M. (1910). Life-Cycle of H. nicorice. Q.J.M.S., lv., p. 741.

(726) Sambon, L. W., and Srligmann, C. G. (1907). Hæmogregarines of Snakes. Trans. Pathd. Soc. London, lviii., p. 310.

(727) SerTz (1910). Die Hartmannsche Binukleaten. C.B.B.P.K. (I. Abth. Orig.), lvi., p. 308.

See also No. 528.

(f) Piroplasms.

(728) Bettencourt, A., Franca, C., and Borges, I. (1907). Piroplasmose bacilliforme chez le Daim. A.I.C.P., i., p. 341.

(729) BowHrl, T. (1905). Equine Piroplasmosis, or "Biliary Fever" „J.H., v., p. 7.

(730) Brent, A., and Hindle, E. (1908). Morphology, etc., of Piroplasma canis. A.T.M.P., ii., p. 233.

(731) Bruce, D., Hamerton, A. E., Bateman, H. R., and Mackie, F. P. (1910). Amakebe : a Disease of Calves in Uganda. P.R.S. (B.), lxxxii., p. 256.

*(732) Christophers, S. R. (1907). P. canis and its Life-Cycle in the Tiok. S.M.I., 29.

(733) Dschunkowsky, E., and LuHs, J. (1909). Protozoenkrankhciten des Blutes des Haustierc in 'Transkaukasien. Ber. IX. Int. Tierarztl. Kongr. Haag. 
(734) Dschonkowsky, E., and LuHs, J. (1909). Entwickelungsiormen von Piroplasmen in Zecken. Ibid.

(735) Fantham, H. B. (1907). The Chromatin-Masses of P. bigeminum (Babesia bovis. Q.J.M.S., li., p. 297.

(736) Franoa, C. (1910). La Classification des Piroplasmes et Description de deux Formes. A.I.C.P., iii., p. 11.

(737) GoNDER, R. (1906). Achromaticus vesperuginis. A.K.G.A., xxiv., p. 220.

(738) - (1910). Die Entwicklung von Theileria parva. A.P.K., xxi., p. 143.

(739) - (1911). Th. parva und Babesia mutans Küstenfieberparasit and Psendoküstenfieberparasit. Ibid., p. 222.

(740) - (1911). Die Entwicklung von Th. parra. II. A.P.K., xxii., p. 170.

(741) Krvoshits, K. (1907). Babesia canis. A.P.K., viii., p. 294.

(742) KLEINE, F. K. (1906). Kultivierungsversuch der Hundepiroplasmen. Z.H., liv., p. 10.

(743) KоCH, R. (1906). Entwioklungsgeschichto der Piroplasmen. Ibid., p. 1.

(744) MAYER, M. (1910). Das ostafrikanische Küsten fieber der Rinder. A.S.T.H. xiv., Beiheft 7, p. 307.

(745) Nedmann, R. O. (1910). Die Blutparasiten von Vesperugo. A.P.K., xviii., p. 1.

(746) NicoLLE, C. (1907). Une Piroplasmoso nonvelle d'un Rongeur. C.R.S.E., lriii., p. 213.

(747) Nutrale, G. H. F., and Fastham, H.B. (1910). Theileria parva. Py., iii., p. 117.

*(748) - and Graham-Smith, G. S. (1906, 1907). Canine Piroplasmosis V. and VI. J.H., vi., p. 585 ; vii., p. 232.

(749) - - (1908). Multiplication of Piroplasma bovis, $P$. pitheci in the circu. lating Blood compared with that of $P$. canis. $P y$., i., p. 134.

(750) - - (1908). Development of $P$. canis in Cultures. Ibid., p. 243.

(751) Sмгтн, T., and Kmвовns, F. L. (1893). Southern Cattle Fever. U.S. Dept. of Agriculture, Eighth and Ninth Reports Bureau Animal Industry, 1891, 1892, p. 77.

(752) Then,Er, A. (1910). Texasfieber, Rotwasser und Gallenkrankheit der Rinder. Zeitschr. f. Infektionskrankheiten der Haustiere, viii., p. 39.

(753) Y $\triangle$ RIMOFF, W. L., StounikOFY, W. J., and KOHL-Y $\triangle$ KIMOFT, N. (1911). L. Achromaticus vesperuginus. A.P.K., xxiv., p. 60 .

(g) Incertæ Sedis.

(754) Nicolle, C., and Manceadx, L (1909). Un Protozosire nonvean du Gondi. C.R.A.S., cxlviii., p. 369 .

(755) Patella, V. (1910). Corps de Kurloff-Demel dans quelqnes Mononucléaires du Sang des Cobayes. La Génèse Endothéliale des Leucocytes Mononucléaires du Sang (Siens, Imprimerie St. Bernardin), p. 211.

(756) Skidern, H. (1911). Protozoon-like Bodies in Yellow-Fever Patients. Journ. Pathol. Bacterid., xv., p. 282.

(757) - (1911). Etiology of Yellow Fever. Yellow Fever Bureau Bulletin, i., p. 229.

\section{CHAPTER XVI}

\section{SPOROZOA-NEOSPORIDIA}

\section{A. Cinidosporidia}

\section{(a) General Works.}

*(758) Aderbach, M. (1910). Die Cnidosporidien. Leipzig : Werner Klinkhardt.

(b) Myxosporidia.

(759) AWERINzEW, S. (1909). Die Sporenbildung bei Ceratomyxa drepanopsetta. A.P.K., ziv., p. 72.

(760) - (1911). Sporenbildung bei Myxidium sp. aus der Gallenblase von Cottus scorpius. A.P.K., xxiii., p. 199.

(761) Chatron, E. (1911). Paramyxa paradoxa. C.R A.S., clii., p. 631.

762) Dorlein, F. (1898). Myxosporidien. Zoo. Jahrbücher (Abth. f. Anat. u. Ontog.), ri., p. 281. 
(763) Kryssmurt, G. (1908). Die Entwioklung von Myxobolus pfeifferi, I. and II. A.P.K., zi., p. 252.

(764) Leane, L, and Hrsse, E. (1906). La Paroi sporale des Myrosporidies. C.R.A.S., oxlii., p. 720 .

(765) - - (1907). Coccomyxa morovi. C.R.A.S., oxlv., p. 85.

(765.5) MrkcinR, L. (1910) La sexualité ohez les Myrosporidies et chez les Miorosporidies. Acad. Roy. Belgique, Mím. 8ı. (2), ii., No. 6.

(766) Prxin, M. (1904). Die Drehkrankheit der Salmoniden (Lentospora cerebralis). A.P.K., v., p. 145.

(767) ScHRöDrR, O. (1907). Entwicklungsgesohiohte der Myxosporidien. Spharomyza labrazesi (sabrazesi). A.P.K., ix., p. 359.

(768) - (1910). Die Anlage der Sporocyste (Pansporoblasten) bei Sphceromyxa sabrazesi. A.P.K., xix., p. 1.

\section{(c) Actinomyxidia.}

(769) CАULLaRY, M., and Mrswn, F. (1905). Spharactinomyxon stolci. A.P.K., vi., p. 272.

\section{(d) Microsporidia}

(770) Awrenzzew, S., and Fremor, K. (1911). Die Sporenbildung bei Glugea anomala. A.P.K., xxiii., p. 1.

(771) Chatron, E., and Krmmpr, A. (1911). Les Protistes du genre.Octosporea. B.S.Z.F., xxxvi., p. 178.

(772) Hesse, E. (1904). Lo Développement de Thelohania legeri. C.R.S.B., Ivii., p. 571.

(773) - (1905). Myxocystis mrazeki. C.R.S.B., lviii., p. 12.

(774) Leger, L., and DuboscQ, O. (1909). Perezia lankesterice. A.Z.E. (5), i., Notes et Revue, p. Ixxix.

(775) - and Hesse, E. (1910). Cnidosporidies des Larves d'Ephémerés. C.R.A.S., cl., p. 411.

(776) Merci kr, L. (1908). Neoplasie du Tissu Adipeux chez les Blattes Parasitées par une Microsporidie. A.P.K., xi., p. 372.

(777) Mrazer, A. (1910). Auffassung der Myxooystiden. A.P.K., xviii., p. 245.

(778) Preez, C. (1904). Uno Microsporidic parasite du Carcinus manas. C.R.S.B., Ivii., p. 214.

(779) - (1905). Miorosporidies Parasites des Crabes d'Areachon. Bull. Stat. Bid. Arcachon, viii.

(780) - (1908). Duboscqia legeri. C.R.S.B., lxv., p. 631.

(781) SchrodEr, O. (1909). Thelohania chatogastris. A.P.K., xiv., p. 119.

(782) SchU в rRq, A. (1910). Microsporidien aus dem Hoden der Barbe. A.K.G.A., xxxiii., p. 401.

(783) Shiwago, P. (1909). Vermehrung bei Pleistophora periplaneta. Z.A., xxxiv., p. 647.

(784) StrapmL, W. (1904). Nosema anomalum. A.P.K., iv., p. 1.

(785) - (1909). Nosema bombycis. A.P.K., xvi., p. 281.

(786) - (1910). Morphologie der Miorosporidien. Z.A., xxxv., p. 801.

(787) WrissenbzRo, R. (1911). Einige Mikrosporidien aus Fisohen (Nosema lophii, Glugea anomala, Gl. Hertwigii). S.B.G.B., p. 344.

(787.5) Woodcock, H.M. (1904). On My xosporidia in Flatfish. Trans. Liverpool Bid. Soc., xviii., p. 126.

See also Nos. 18, 25, and 26.

\section{(e) Sarcosporidia.}

(788) BeteoH, L. v. (1909). Entwioklungsgange der Sarcosporidien. C.B.B.P.K. (I Abth. Orig.), lii., p. 566.

(788.5) CRAwley, H. (1911). Sarcocystis rileyis Proc. Acad. Philadelphia, 1911, p. 457.

(789) Daring, S. T. (1910). Experimental Sarcosporidiosis in the Guinea-Pig. J.E.M., xii., p. 19.

(790) Erdmans, R. (1910). Kern und metaobiomatische Korper bei Sarkosporidien. A.P.K., xx., p. 239.

(791) - (1910). Sarcocystis muris in der Muskulatur. S.B.G.B., p. 377.

(792) Frmbiarr, J. (1910). Sarkosporidien. V $r_{s}$.h. Zool.-Bot. Ges. Wien, Ix., p. (73). 
(793) Laveran, A. and Mesnit, F. (1899). La Morphologie des Sarcosporidies. O.R.S.B., li., p. 245.

(794) NĖare, L. (1910). Io Stado intestinal de la Saroosporidie de la Souris. C.R.S.B., lxviii., p. 997.

(795) Neari, A. (1908, 1910). Ueber Sarkosporidien, I. and II. C.B.B.P.K. (I Abth. Orig.), xlvii., pp. 56 and 612 ;IJI., Ibid., lv., F. 373.

(797) Trionmann, E. (1911). Die Teilungen der Keime in der Cyste von Sarcocystis tenella. A.P.K., xxii., p. 239.

(798) Vomlemm, P. (1902). Sarcocystis tenella. C.R.A.S., oxxxiv., p. 1152.

(799) Watson, E. A. (1909). Sarcosporidiosis: Its Association with IncoDisease, eto. Journ. Comp. Pathol. Therapeut., xxii., p. 1.

\section{B. Haplosporidia.}

(800) Beattie, J. M. (1906). Rhinosporidium kinealyi. Journ. Pathol. Bacterid., xi., p. 270.

(801) Cajlekry, M., and Chapphlimer, A. (1906). Anurosporidium pelseneeri. C.R.S.B., lx., p. 325.

(802) - and MrSNII, F. (1905). Les Haplosporidies. A.Z.E. (4), iv., p. 101.

(803) Chatтon, E. (1907). Caullerya mesnili. C.R.S.B., lxii., p. 529.

(804) - (1908). Blastulidium posdophthorum. C.R.S.B., lxiv., p. 34.

(805) Crawley, H. (1905). Coelospordium blatella. Proc. Acad. Philadelphia, lvii., p. 158.

(806) Krwa, H. D. (1907). Eertramia bufonis. Ibid., lix., p. 273.

(807) Laveran, A., and Petrit, A. (1910). Une Epizootie des Truites. C.R.A.S., cli., p. 421 .

(808) MrNchis, E. A., and Fantham, H. b. (1905). Rhinosporidium kinealyi. Q.J.M.S., xlix., p. 521.

(809) RIDEWOOD, W. G., and FANTHaM, H. B. (1907). Neurosporidium cephalodisci. Q.J.M.S., li., p. 81 .

(810) RobertsoN, M. (1908). A Haplosporidian of the Genus Ichthyosporidium. Proc. R. Phys. Soc. Edinburgh, xvii., p. 175. (811) - (1909). An Iohthyosporidiau causing Disease in Sea-Trout. P.Z.S.,
1909, p. 399 .

(812) Stempal, W. (1903). Die Gattung Polycaryum. A.P.K., ii., p. 349.

(813) WARREN, E. (1906). Bertramia kirkmanni. Ann. Natal. Gout. Mus., i., p. 7. (814) WRIGHT, J. (1907). Rhinosporidium kinealyi. New York Med. Journ., December 21 .

\section{Ixometa Seds.}

(815) AWERINzEw, S. (1909). Lymphocystis johmstonei. A.P.K., xiv., p. 335.

(816) - (1911). Die Entwicklungsgesohichte von Lymphocystis jolinstonei. A.P.K., xxii., p. 179.

(817) Chatron, E. (1906). La Biologie, eto., des Amoebidium. A.Z.E. (4), v., Notes et Rovue, p. xvii.

(818) - (1907). Pansporella perplexa. O.R.S.B., 1xii.: p. 42.

(819) - (1910). Gastrocystis gilruthi. A.Z.E. (5), v., Notes et Revue, p. oxiv.

(820) GnruTH, J. A. (1910). Gastrocystis gilruthi. Proc. Roy. Soc. Victoria (n.s.), xxiii., p. 19.

(821) GraxaTA, L. (1908). Capillus intestinalis. Biologica, ii., p. 1.

(822) Krassmstsonm, J. M. (1909). Neue Sporozoen bei Insekten. A.P.K., xiv., p. 1.

(823) LEGKr, L. and Dubosce. 0 (1909). Les Chytridiopsis. A.Z.E. (5), i. Notes ot Revue, p. ix.

(824) WooDOock, H. M. (1904). Lymphocystis johnstonei. Trans. Biol. Soc. Liverpool, xviii p. 143. 


\section{CHAPTER XVII}

\section{INFUSORIA}

\section{(a) General Work.}

(825) Hartog, M. (1910). Infueoria. Encyclop. Brit., eleventh edition, xiv., p. 557.

*(826) Hicksow, S. (1903). The Infusoria. A Treatise on Zoology (Lankester) (London : A. and C. Black), p. 361.

\section{(b) Ciliata.}

See also, Nos. 16, 32, 33, 38.5, 44, 50, 53, 73, 93, 96, 102, 104, 106-109, 111-113. $115,121,122,124.126,136-148,148,149,155,162,165-167,170-173,177,181.183$, 197-199, 201, 205, 206, 208, 209, 211, 214-220, and 346.

(827) Bradchamp, P. DE, and Coumn, B. (1910). Sur Hastatella radians. A.Z.E. (5), v., Notes et Revue, p. xxviii.

(828) BuSCHKIKL, A. L. (1911). Ichthyophthirius multifliis. A.P.K., xxi., p. 61. (829) Cadluery, M., and Mesnic, F. (1903). La Structure nucléaire d'un Infu. soire Parasite des Actinies (Foettingeria actiniarum). C.R.S.B., lv., p. 806.

(830) - - (1907). L'Appsreil nucléaire d'un Infusoire (Rhizocaryum concaerum). C.R.Ass. Franc. Reims.

(831) CXiptDe, C. (1910). Les Infusoires astomes. A.Z.E. (5), iii., p. $34 \mathrm{I}$.

(831.5) Chatron, E. (1911). Perikaryon cesticola and Conchophrys davidoff. A.Z.E. (5), viii., Notes et Revue, p. viii.

(832) Counn, B. (19n9). Deux Formes nonvelles d'Infusoires Discotriches. A.Z.E. (5), ii. Notes et Revue, p. xxi.

(833) DuвeLL, C. C. (1909). Infusoria parasitic in Cophalopoda. Q.J.M.S., liii., p. 183.

(834) Faure-Fremiet, E. (1905). L'Appareil fixateur chez les Vorticellida. A.P.K., vi., p. 207.

(835) - (1907). Mitochondries et Sphéroplastes chez les Infusoires ciliés. C.R.S.B., lxii., p. 523.

(836) - (1908). Tintinnidium inquilinum. A.P.K., xi., p. 225.

(837) - (1908). L'Ancystropodium maupasi. A.P.K., xiii., p. 121.

(838) - (1909). Le Macronucléus des Infusoires ciliés. B.S.Z.F., xxxiv., p. 55.

(839) - (1910). Le Mycterothrix tuamotuensis. A.P.K., хx., p. 223.

(840) Gonder, R. (1905). Kernverhältnisse bei den in Cephalopoden schmarotzenden Infusorien. A.P.K., v., p. 240.

(841) HambURaer, C. (1903). Trachelius ovum. A.P.K., ii., p. 445.

(842) - (1904). Die Konjugation von Paramecium bursaria. A.P.K., iv., p.199.

(843) - and Buddenbrock, v. (1911). Nordische Ciliata mit Ansschluss der Tintinnoidea. Brundt and Apstein, Nordisches Plankton.

(844) JoskPH (1907). Kernverhältnisse von Loxodes rostrum. A.P.K., viii., p. 344.

(845) KASANZErF, W. (1910). Loxodes rostrum. A.P.K., xx., p. 79.

(846) Krernik, E. (1909). Chilodon hexastichus. B.A.S.C., p. 75.

(847) Korord, C. A. (1903). Protophrya oricala. Mark Anniversary Volume, p. 111.

(848) LEGER, L., and Dubosca, O. (1904). Les Astomata representent-ils un Groupe naturel ? A.Z.B. (4), ii., Notes et Revue, p. xcviii.

(849) - - (1904). Les Infusoires endoparasites. A.Z.E. (4), ii., p. 337.

(850) Martini (1910). Über einen bei amöbenruhrähnlichen Dysenterien vorkommenden Ciliaten. Z.H., lxvii., p. 387.

(851) MAsT, S. O. (1909). The Reactions of Didinium nasutum. B.B., xvi., p. 91.

(851.5) MaUPaS, E. (1888). La Multiplioation des Infusoires oiliés. A.Z.E. (2), vi., p. 165.

(852) Metcale, M. M. (1907). Excretory Organs of Opalina. A.P.K., x., pp. 183, 365.

(853) - (1909). Opalina : Its Anatomy, ete. A.P.K., xiii., p. 195.

(854) Meunier, A. (1910). Microplankton des Mers de Barents ct de Kara. Duo d'Orleans, Campagne Arctique de 1907. Brussels. 
(855) Mrtrophanow, P. (1905). La Structure, eto., des Trichooystes des Para. mécies. A.P.K., v., p. 78.

(856) Nereshermer, E. R. (1803). Die Höhe histologischer Differenzierung bei heterotrichen Ciliaten. A.P.K., ii., p. 305.

(857) Nereshermek, E. (1907). Die Fortpflanzung der Opalinen. A.P.K., Suppl. i., p. 1.

(858) - (1908). Fortpflanzung eines parasitischen Infusors (Ichthyophthirius), S.B.G.M.P., xriii.

(859) Prowazer, S. v. (1904). Der Enoystierungvorgang bei Dileptus. A.P.K., iii., p. 64 .

(860) - (1909). Conjugation von Lionotus. Z.A., xxxiv., p. 626.

(861) - (1909). Formdimorphismus bei Ciliaten Infusorien. M.I.O.C., i., p. 105.

(862) Roux, J. (1899). Quelques Infusoires ciliés des Environs de Genève. Rev. Suisse Zool., vi., p. 657.

(863) Schewiakorf, W. (1893). Die geographische Verbreitung der SusswasserProtozoen. Mem. Acrd. Imp. St..Petersbourg (vii.), xli.

(864) ScHRÖDER, O' (1906). Campanella umbellaria. A.P.K., vii., p. 75.

(865) - (1906). Epistylis qlicatilis. Ibid., p. 173.

(866) - (1906). Vorticella monilata. Ibid., p. 395.

(867) - (1906). Stentor coruleus und St. raeselii. A.P.K., viii., p. 1.

(868) Schовотz, H. (1908). Pycnothrix monocystoides. Denkschr. Ges. Jena, xiii., p. 1.

(869) ScHwEYER, A. (1909). Tintinnodeenweichkörper, etc. A.P.K., xviii., p. 134.

(870) SIEDLECKI, M. (1902). L'Herpetophrya astoma. B.A.S.C., p. 356.

(871) Stern, F. v. $(1859,1867)$. Der Organismus der Infusionthiere: I. Hypotricha; II. Heterotricha. Leipzig : W. Engelmann.

(872) Stevens, N. M. (1904). On Licnophora and Boveria. A.P.K., iii., p. 1.

(873) Thon, K. (1905). Bau von Didinium nasutum. A.P.K., v., p. 281.

(874) Walrer, E. L. (1909). Sporulation in the Parasitic Ciliata. A.P.K., xvii., p. 297.

\section{(c) Acinetaria.}

(875) Awerinzew, S. (1904). Astrophrya arenaria. Z.A., xxvii., p. 425.

(876) Chatron, E., and Colur, B. (1910). Un Acinetien commensal d'un Copepode, Rhabdophrya trimorpha. A.Z.E. (5), v., Notes et Revue, p. cxxx viii.

(877) ConLrs, B. (1907). Sur quelques Acinétiens. A.Z.E. (4), vii., Notes et Revue, p. xciii.

(878) - (1908). Sur Tokophrya cyclopum. A.Z.E. (4), viii., Notes et Revue, p. xxxiii.

(879) - (1909). La Conjugaison, gemmiforme chez les Acinétiens. C.R.A.S., cxlviii., p. 1416.

(880) - (1909). Les Formes hypertrophiques et la Croissance dégénérative chez queiques Acinétiens. C.R.A.S., cxlix., p. 742.

(881) - (1909). Sur deux Acinétiens. Ibid., p. 1407.

(882) - (1909). La Symétrie, etc., des Embryons d'Acinétiens. A.Z.E. (5), ii., Notes et Revue, p. xxriv.

(883) FunpJev, J. (1910). Tocophrya quadripartita. A.P.K., xxi., p. 117.

(884) HАRTOG, M. (1902). Notes on Suctoria. A.P.K., i., p. 372.

(885) Hickson, S. J., and WaDsworTH, J. T. (1902). Dendrocometes paradoxus. Q.J.M.S., xlv., p. 325.

(886) - - (1909). Dendrosoma radians. Q.J.M.S., liv., p. 141.

(887) IsHra A, C. (1897). Eine in Misaki vorkommende Art von Ephelota. Journ. Coll. Sci. Tokyo, x., p. 119.

(888) Martin, C. H. (1909). On Acinetaria. Parts I. and II. Q.J.M.S., liii., p. 351. Part III. Ibid., p. 629.

(889) Perez, C. (1903). Lernoophrya capitata. C.R.S.B., lv., p. 98. 


\section{CHAPTER XVIII}

(a) Classiflcation:

(890) Awrrnnzew, S. (1910). Die Stellung im System und die Klassifizierung der Protozoen. B.C., xxx., p. 465.

(891) Dorrenn, F. (1902). Das System der Protozoen. A.P.K., i., p. 169.

(892) Hartmann, M. (1911). Das System der Protozoen. Vide Prowazek (14),. p. 41 ; and No. 675 .

\section{(b) Spirochætes.}

*(893) Bosanedet, W. C. (1911). Spiroohætes. Philadelphia and London: W. B. Saunders Company.

(894) - (1911). Sp. anodonta Keysselitz. Q.J.M.S., Ivi., p. 387.

(895) Do bel, C. C. (1911). On Cristispira veneris and the Classificatiun of Spirochætes Q.J.M.S., lvi., p. 507.

(896) FanthaM. H. B. (1911). Life-Cycle of Spirochætes. A.T.M.P., v., p. 479.

(897) Gross, J. (1910). Cristispira nov, gen. Mitt. zool. Stnt. Neapel, xx., p. 41.

(898) - (1911) Freilebende Spironemaceen. Ibid., p. 188.

(899) - (1911). Nomenclatur der Sp. pallida. A.P.K., xxiv., p. 109.

(900) Hindle, E. (1912). Life-Crcle of $S p$. gallinarum. Py., iv., p. 463.

(001) Krzysztalowicz, F., and Siedleck I, M. (1905). La Structure, etc., de Sp. pallida. B.A.S.C., p. 713.

(902) Leishman, W. B. (1910). Mechanism of Infection in Tick Fever and Hereditary Transmission of $S p$. duttoni in the Tick. Trans. Soc. Trop. Med. Hyg., iii., p. 77.

(903) SchaUdinN, F. (1905). Sp. pallida. Deutsch. Med. Wochenschr., xxxi., p. 1665 .

(904) ZUELZER, M. (1911). Sp. plicatilis. A.P.K., xxiv., p. 1.

\section{(c) Chlamydozoa.}

(906) Acton, H. W., and Harvey, W. F. (1911). Negri Bodies. Py., iv., p. 255.

(907) Awerinzew, S. (1910). Die Krebsgeschwülste. C.B.B.P.K., lvi. (I Abtl. Orig.), p. 506.

(908) Calkins, G. N. (1904). Cytoryctes variola, Guarnieri. Journ. Med. Research (Special Variola Number), xi., p. 136.

(909) HartmanN, M. (1910). Chlamydozoen. C.B.B.P.K. (I Abth. Ref.), zlvii., Beiheft, p. 94.

(010) Neari, A. (1909). Die Morphologie und der Entwicklungszyklus des Parasiten der Tollwut. Z.H., ete., lxiii., p. 421.

(911) Prowazer, S. v. (1907). Chlamydozoa. A.P.K., x., p. 336.

(912) - and AragaO, H. DE B. (1909). Variola-Untersuohungen. M.I.O.C., i., p. 147.

(913) - LiPschUtz, B., and Others (1911). Chlamydozon, etc. Vide Prowazek (14).

(914) Sirgel, J. (1905). Die Atiologie der Pocken und der Maul- und Klauenseuohe : des Soharlachs : der Syphilis. Abhandl. k. preuss Akad. Wiss. (Anhang.) 


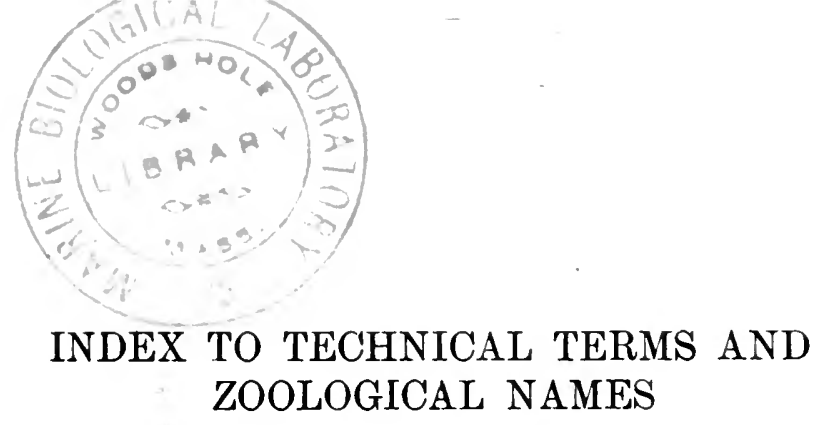

The numerals printed in hosvier black typo rofer to pages on whioh the meaning of the word or the systematio position of a gonus, family, or order are fully explained.

AOANTEARIA, 251, 256

Acanthin, 37, 253

Acanthocystis, 37, 48, 91, 245, 248

- aculeata, 117, 118 (Fig. 61), 123 (Fig. 68)

- chastophora, 37 (Fig. 18)

Acanthometra, 258

- elastica, 250 (Fig. 105)

- pellucida, 255

Acanthometridas, 37

Acophalina, 339

Achromaticus, 364, 382

- vesperuginis, 382

Achromatin, 65

Acineta, 461

- orandis, 11 (Fig. 10)

- papillifera, 16

Aoinotaria, 430, 455

Acinetidas, 461

Aorasiz, 243

Acrasis, 243

Actinobolus radians, 141

Actinocephalidas, 339

Actinocephalus oligacanthus, 327 (Fig. 142)

Actinomma asteracanthion, 254 (Fig. 107)

Aotinomyxidia, 409

Actinophrys, 117, 215, 245, 248

- $80 l, 90$ (Fig. 46), 132 (Fig. 71), 151

Aotinopoda, 218

Actinospharium, 43, 50 (Fig. 22), 68, $74,77,78,80,91,138,144,150,193$, $198,207,209,214,216,245,248$

- eichhorni, 7 (Fig. 3), 81 (Fig. 37), 115 (Fig. 62), 116 (Fig. 63)

Adaptive polymorphism, 164

Adelea, 175, 176, 348, 352, 393

- hartmanni, 344, 347, 348

- ovata, 344, 345 (Fig. 153), 346, 347 (Fig. 154), 352

Adeleida, 352, 354, 355

Adeleidea, 352, 394

Adinida, 278

Adoral epiral, 442

Adult, 212

Athalium, 242

Aflagellar, 287

Agamete8, 180, 181

Agrmogony, 181

Agamont, 181

Agglomerstion, 128, 209, 305

Agglutination, 128

Agglutinin, 128

Aggregata, 23, 168, 325, 348, 353

- facquemeti, 121 (Fis. 67)

Aogregatidos, $\mathbf{8 5 3}$
Alcohol, offects of, 204

Allogromia, 230

- ovoidea, 235

Aiternation of generations, 181

Alveolar layer, 435

Alvooli, 42

Amicronucleate, 211

Amitosis, 105

Amaba, 218

- albida, 221 (Fig. 87)

- binucleata, 78, 95, 214, 223

- diploidea, 222 (Fig. 88)

- diplomitotica, 108, 109 (Fig. 56)

- flava, 221

- limax, 46, 47 (Fig. 20), 206, 217, 219

- minuta, 221, 223

- mucicola, 220

- proleus, 6 (Fig. 2), 47, 191, 205, 209, $215,216,217,219,220,222,230$

- radiosa, 217,219

- terricola, 48, 190 (Fig. 82), 214, 220

- verrucosa, 32, 45, 48, 50,51 (Fig. 23), $198,214,219$

- vespertilio, 217

Amceba, form-ohanges, 216 (Fjg. 85)

Amcbæa, 217

Amobidium, 428

Amcbodiastase, 193

Amceboflagellata, 463

Amœbogenix, 325, 466

Amceboid, $\mathbf{3 0}$

Amœbula, 169

Amphikaryon, 96

Amphileptus, 439

Amphimixis, 150, 154

Amphinnoleus, 96

Amylum, 188

Anserobic, 196

Anaplasma marginale, 383

Ancystropodium, 441

Angeiocystis audouinias, 349

Anisogamy, 126, 132, 175

Anisonema, 274

7 grande, 53 (Fig. 25)

Anisonemina, 274

Anisospore, 215, 254

Annulus, 276

Anophelinæ, 358

Anoplophrya, 171, 439, 443, 449, 452

- branchiarum (roduotion), 145 (Flg. 74)

Anoplophryino, 197, 452

Anurosporidium, 424

Aphrothoraos, 247

Apiosoma, 379

Apodinium, 278 
Aposporogony, $\mathbf{3 6 8}$

Arcella, 64, 65, 72, 78, 126, 148, 173, 199, $201,215,216,229$

- vulgaris, 67 (Fig. 32), 110 (Fig. 57), 177,178 (Fig. 80)

Arohæooyte8, 133

Arohoplasm, 79, 103

Arenacoous, 34, 231

Asporooystea. 388

Aspirigera, 439

Arrhenoplasm, 129

Artificlal classifioation, 463

Assimilatlun, 187

Assoolatlon, 127, 330

Astasia, 274

- tenax, 33 (Fig. 15)

Astasiidas, 274

Astomata, 438, 489, 451

Astrodisculus, 248

Astrophrya, 461

- arenaria, 456

Athene noclua, 390

Attraction-8phere, 103

Attraotion-8plndle, 104

A ulacantha, 256

Autocyst, 417

Autogamy, 138, 306

Automixis, 140

Antophya, 84

Avoiding resction, 202

Axopodium, 48, 53, 60, 87, 199, 465

Axostyle, 36, 259, 289, 311

Azoosporidæ, 218

Babesia, 357, 379, 394

- bovis (bigemina), 379, 384

- mutans, 380, 382

Babesioses, 378

Baoteria, 5, 98

Badhamia, 242

- utricularis, 240 (FIg. 99), 241 (Fig. 100)

Balantidium, 439, 440

- coli, 440

- minutum, 440

Banana-treo, 136

Barroussia, 352

- alpina, 344, 345 (Flg. 153)

- caudata, 348

- ornata, 346, 352

- spiralis, 344, 348

Barotaxis, 202, 207

Basal granulo, 82, 92, 200, 443

- rim, 443

Benedenia, 853

Bertramia, 424

- asperospora, 424

- bufonis, 424

- capitellos, 424

- kirkmanni, 424

Bertramiida, 424

Bilatoral symmotry, 31, 250

Biloculina depressa sholls, 233 (Fig. 94)

Blnary fission, 100

Binuclearity, 96

BInucleats, 85, 280, 388

Bloblast, 40, 41

Bionomice, 15

Black sporos, 364

Biastocoele, 133

Blastodinidas, 278

Blastodinium, 278

Blastogener, 418
Blastomore, 133

Blastulidium pasdophthorum, 424

Blepharoplast, $52,59,82,262,286,288$, 289

Bodo, 270, 281, 319

- edax, 319

- gracilis, 271 (Fig. 115)

- lacertos, 270

- saltans, 271 (Fjg. 115), 319

Bodonida, 268, 270

Body-form, 29

Bud, 122

Buetschlia, 439

Bursaria, 439

Bursarida, 439

Callyntrochlamys phronimos, 327

Calonympha, 276

Calymma, 251, 252

Calyx, 89

Campanella, 440, 446, 447

- umbellaria, 434 (Fig. 183)

Camptonema, 51, 248

- nutans, 91 (Flg. 47)

Cancer, 473

Capilltium, 241

Capillus intestinalis, $\mathbf{4 2 8}$

Capsulogenous cell, 399, 403

Carchesium, 145, 192, 194, 440, 441, 449

Caryorycles, 473

Caryospora, 349, 352

- simplex, 352

Caryotropha, 195, 344, 348, 352

- mesnilii, 349,352

Caryotrophidx, $\mathbf{3 5 2}$

Caullerya, 424.

- mesnili, 424

Coll, 1, 98, 464

Coll-anus, 433

Coll-division, 121

Cell-mombrane, 45

Coll-mouth, 63

Coll-thoory, 133

Central capsulo, 250

- grain, 91

- spindle, 103

Contriale, 73, 80, 97, 262

Controdesmose, $36,58,59,82,103$

Centropyxis, $148,173,229$

- aculeata, 36, 230

Controsomo, 58, 59, 73, 79, 262, 288

Controsphere, 80

Cophalina, 339

Cephaloidophora, 337

Cophalont, 181, 326

Ceratimyxa, 242

Ceratium, 278

Ceratocorys, 278

L horrida, 277 (Fig, 121)

Ceratomyxa, 408

- drepanopsettas, 402 (Flg. 166), 403

- sphírulosa, 409

Ceratomyxidas, 408

Cerabophyllus fasciatus, 291

Cercomonadidos, 268, 270

Cercomonas, 270, 271

- crassicauda, 271 (Flg. 114)

Chagasia hartmannt, 344,347

Chalat othoraca, 248

Chomotaxis, 202

Chiliferidas, 439

Ckilodon, 145, 439, 448 
Chilodon cucullulus, 435 (Fig. 184)

- dentatus, 440

Chilomonas, 208, 274

Chlamydodontidas, 439

Chlamydomonadidos, 275

Chlamydomonas, 275

Chlamydomyxa, 214, 243, 244

Chlamydophora, 248

Chlamydophrys, 237

- schaudinni, 237

- stercorea, 17, 237

Chlamydozoa, 470

Chloromyxida, 407, 409

Chloromyxum, 409

- leydigi, 400 (Fig. 164), 409

Chlorophyll, 13, 63, 188, 261

Choanoflagellata, 261, 271

Choanoflagellida. 271

Chondriosome, 41

Chromatín, 65, 69

Chromatoid grains, 67, 289, 311

Chromatophoro, 13, 63, 188, 261

Chromldia, 6, 65, 97, 150, 215, 328

Chromidial fragnentation, 101

Chromidina, 452

Chromidiogamy, 126, 416

Chromidiosome, 65, 103

Chromomonadina, 274

Chrornophyll, 188

Chromoplast, 13, 63

Chromosomo, 103

Chromulina, 274

- flavicans, 15

Chrysamœba, 274

Chrysomonadina, 14, 274

Chytridiopsis, 428

- socius, 428

Ciliary apparatus, 442, 444 (Fig. 186)

Cillata, 430, 432 (Fig. 181)

Cilioflagollata, 277

Clliophora, 462

Ciliophrys, 248

Ciliospore, 169

Cilium, 12, 53, 92, 199, 200, 442, 454

Circumfluenoo, 189

Circumvallation, 189

Cirrus, 55, 445

Cladomonas, 270

Cladothrix pelomyxo, 227

Classification, 462

Clathrulina, 39, 245, 248

- clegans, 38 (Fig. 19)

Clepsydrina, 335, 339

Cnidosporidla, 399

Coccidla, 341, 389

Coccidiidas, 352

Coccidioides immitis, 17

Cocoidiomorpha, 388, 395

Cocoidiosis, 343

Coccidium, 101, 166, 173, 174, 346, 352

- cuniculi, 341,351

- mitrarium, 344

- oviforme, 341

- rouxi, 349

- schubergi, 102 (Flg. 50), 106 (Figs. 51,52 ), 127 (Fig. 69), 146 (Fig. 75), 204, 342 (Fig. 152), 353, 354

-. stiedos, 341

Coccoid bodies, 468

Coocolith, 274

Coccolithophoridas, 274

Coccomyxa, 408
Cdccomyxa morovi, 409

Cochleuria faurei, 442

Cochliopodium, 229

Codonosiga botrytis, 260 (Fig. 110.

Colosporidiidos, 399, 424

Colosporidium, 424

- blatellos, 424

Colozoic, 324

Coleps, 438, 441

Collar, 57, 89, 261

Collecting-pusule, 277

Collodagia, 255

Collozoum, 256

Colpidium, 208

Colpoda, 439

Conchophrys, 439

Conjugant, 126, 448

Conjugation, 126, 448

Conorhinus megistus, 291, 302

Contact-8timulus, 207

Contractlle vaouolo, 60, 196, 197, 862, 437,447

- system, 445, 446

Contractility, 200, 201

Copromonas, 171, 274

- major, 268

- subtilis, 264 (Fig. 111), 268

Copromyxa, 243

Copularium, 355

Copulation, 126

Corps en barillet, 344

Cortex, 45

Cortical layer, 32

Cortlcate, 45

Costia, 258, 272

- necatrix, 16, 272

Cothurnia, 440

Craspedomonads, 261, 271

Craspedotella, 279

Cristispira, 466, 469

- anodontas, 468

- balbianii, 467

- pectinis, 469 (Fjg. 194)

Crithidia, 281, 282,-287, 308, 312, 320, 321

- campanulata, 313

- gerridis, 313

- melophagia, 290

- minuta, 312 (Fig. 135)

Cryptocystes, 412

Cryplodifflugia, 229, 230

Cryptomonadina, 15, 274

Cryplomonas, 274

- schaudinni, 15

Cryptosporidium, 349, 352

- muris, 344, 352

Crystal-8poro8, 254

Cuirass, 33, 45, 276

Culicinæ, 358

Culturo d'attonte, 304

Cutiole, 45

Cyclasterium, $\mathbf{4 7 0}$

Cyclical transmission, 290

Cyclochata, 440, 441

Cycloposthium, 439

Cyclosis, 192, 194, 437

Cyclospora, 352

- caryolitica, 176, 198, 344, 348, 349, 352

Cyst, 154

Cystal residuum, 349

Cystobia chiridotas, 341 
Cystobia holothuria, 128 (Fig. 70)

- irregularis, 331

- minchinit, 336

Cystoflagellata, 257, 278

Cystotrypanosoma, 304

Cytooyst, 344

Cytomere, 344

Cytomiorosome, 41

Cytopharynx, 63, 261, 433, 442

Cytoplasm, 6, 7, 99

Cytopyge, 433

Cytorhyctes, 471

- aphtharum, 471

- luis, 471

- scarlatinw, 471

- vaccina, 471

Cytoryctes, 470,471

Cytostome, 63, 190, 191, 261, 433, 452

Cy tozoio, 324

Dactylosoma splendens, 378

Dofocation, 233

Degeneration, 208

Dendrocometes, 457, 460, 461

Dendrocometid $\omega, \mathbf{4 6 1}$

Dendrosoma, 456, 458, 461

- radians, 78, 460 (Fig. 193)

Dendrosomid $\omega, 461$

Dendrosomides paguri, 455

Doprossion, 131, 135, 197, 208

Derbesia, 90

Desmothoraca, 248

Deutoblast, 426

Doutomeritc, 327

Deutoplasmic, 41

Dcvescovina, 276

Dexiotricha, 440

Dictyostelium, 243

Didinium, 145, 438, 442, 449

Didymophycs, 330

Difflugia, 34, 35, 50, 65, 66, 78, 126, 140 , $149,199,215,216,229$

- spiralis, 34 (Fig. 16)

- urceolata, 214, 229, 230

Diffuse infiltration, 400

Digestlon, 192

Dileptus, 439

Dimastigamœba, 268

Dimorpha, 249

- nutans, 249 (Fig. 104)

Dincnympha, 276

Diritera, 278

Dinobryon, 274

Dinoflagellata, 257, 276

Dinophysido, 278

Dinophysis, 278

Diphtheria, 470

Diplocystis minor, 128 (Fig. 70)

Diplodina, 174

Diplosome, 78

Diplozos, 273

Direct division, 101

Direot transmission, 290

Discophrya, 439

Discorbina, 232 (Fig. 93, vii)

Disporea, 408

Dizolo, 349

Doliocystidco, 339

Dourine, 26, 285, 289

Drehkrankhoit, 400

Drepanidia, 395

Drepanidium, 372
Duboscqia, 418

- legeri, 418

Earth-amobæ, 220

Echinomera, 333

Echinopyxis, 101

Fotoplasm, 43, 45, 435

Eotosaro, 43

Eotocohize, 839

Eotosporea, 325

Eimeria, 346, 852

- falciformis, 346

- nepos, 346

Eimeridas, 352

Eimeridea, 352, 394

Electrical stimull, effeots of, 208

Elementary corpusoles, 472

Elleipsisoma, 387

- thomsoni, 387

Enchelidos, 439

Enchyloms, 41, 72

Encystment, 164

Endogenous bndding, 124

- cyole, 184

Endoparasita, 962

Endophrys rotatorium, 249

Endoplasm, 43, 62, 437

Endoral mombrane, 145

Endosarc, 43

Endosohiza, 338

Endosomo, 73

Endospore, 835

Endospores, 242, 825

Endotrypanum, 807

- schaudinni, 307 (Fig. 133)

End-pleoe, 443

Enorgid, 121

Entamaba, 220

- africana, 226

- blattas, 47, 220, 223

- buccalis, 220

- coli, 18, 138 (Fig. 73), 139, 223, 224 (Fig. 89), 225

- histolytica, 18, 46, 293, 224, 225 (Fig. 90)

- minuta, 226

- muris, 220

- ramarum, 220

- tetragena, 226

- williamsi, 225

Entodinium, 439, 141

Entozolo, 16

Enzymes. Soo Ferments

Ephelota, 457, 481

- buefschliana, 457

- oemmipara, 460

Eployto, 45, 327

Eplmerite, 45, 826

Epispore, 335

Epiatylis, 470, 441

- plicatilis, 144 (Fig. 186, K), 140

- umbellaria, 447

Epithelioma contagiosum, 470

Epizoio, 16

Equating division, 104

Equatorial plate, 103

Ergastoplasm, 41

Erythropsis, 277

Etheogenesis, 138, 315

Eucocoidia, $\mathbf{8 5 2}$

Eucoccidium, 353

Eucyrtidium, 256 
Eucyritidium cranioides, 256 (Fig. 109)

Euflagellata, 257

Eúglena, 14, 33, 52 (Fig. 24), 107, 202, 274

- gracilis, 188

- spirogyra, 8 (Fjg. 4)

- viridis, 188, 205 (Fig. 84)

Euglenidos, 274

Euglenoid movoment, 33

Euglonoidina, 273

Euglypha, 34, 35, 214, 287

- alveolata, 111, 112 (Fig. 59), 113 (Fig. 60)

Eugregarinæ, 328, 339

Fuplasmodida, 242

Euplotes, 194, 440, 448

- harpa, 433 (F1g. 182)

- patella, 433 (Fig. 182)

Euplotidos, 440

Eutrypanosome, 292

Ex-conjugant, 153

Exoretion, 197

Exoretory canals, 447

Exflagellation, 357, 362, 364, 365, 390

Exogenous oycle, 184

Eye-spot, 205

Faloiform body, 324

Fat, 194

Fatty dogeneration, 210

Feoding oanals, 437

Fomalo 6ox, 158

Formont8, 193, 194

Fortilization-spindlo, 127, 348

Flloplasmodida, 243

Filose, 48

Fission, 100

Fixation, 441

Flagollar, 287

Flagellata, 82, 257

Flagellispore, 168

Flagellosis, 313

Flagollula, 169

Flagellum, 6, 51, 199, 200, 289, 454, 465

Fattingeria, 438

Fottingeriidas, 439

Food-vaonole, 50, 62, 191, 194, 437

Foot-and-mouth disosso, 470

Foraminifera, 217, 231

Form-produotion, 31

Frambœsla, 467

Frondicularia, 232 (Fig. 93, 17.)

Frontonia, 439,442

- leucas, 206, 447 (Fig. 187)

Fulora, 441

Fuligo, 242

- septica, 239 (Flg. 97)

Galvanotaxis, 202, 208

Gamoto, 125, 448

Gametid, 334

Gamotooyte, 126

Gamogony, 181

Gamont, 126, 181

Ganymedes, 330

Gas-vaouolo, 64

Gastrocystis gilruthi, 427, 428 (Flg. 179)

Gemmatlon, 122

Gommula, 459

Gemmule, 471

Generative ohromatin, 71

Geotaxis, 207

Germ, 165
Germ-colls, $13 \mathrm{C}$

Germen, 130

Germinative infection, 24

Glaucoma, 439

- colpidium, 197, 206

- scintillans, 445

Glenodinium, 278

- cinclum, 277 (Fig. 120)

Globidium, 387

- multifidum, 387

Globigerina, 231, 232 (Fig. 93, v1.

Glossina morsilans, 291

- palpalis, 291, 303, 301

Olugea, 412, 417, 418

- anomala, 411, 415, 417 (Fir. 174), 4]8

- stephani, 412

Gonium, 276

- pectorale, 275 (Flg. 119)

Granellø, 238

Granellarium, 238

Gregarina, 174, 335, 339

- blattarum, 339

- munieri, 58 (Fig. 29)

- ovata, 332, 333 (Fig. 146), 335, 339

- polymorpha, 9 (Fig. 7), 339

Gregarines, sporogony, 331 (Fig. 144)

Gregariniform phases, 315

Gregarinoidoa, 326

Grogarinula, 168, 324

Gromia, 231

- oviformis, 49 (Fig. 21)

Guarnieri's bodies, 470

Curleya, 418

Gymnamcbæ, 218

Gymnoarnidos, 278

Gymnodinium, 278

Gymnosporo, 165

Gymnostomata, 439, 142

Gymnozoum, 439, 442

- viviparum, 439

Hæmamœbæ, 357, 389

Hæmatochrome, 188

Homatococcus, 188, 275, 379

- pluvialis, 111 (Fig. 58)

Hamatomonas, 308

Hosmatopinus spinulosus, 291, 301

Hosmocystidium, 358, 364

- diploglossi, 358, 365

- metschnikovi, 358

- simondi, 358, 365

- tropiduri, 365

Hæmoflagellato8, 258, 280

Hamogregarina, 372

- agama, 373

- balfouri, 376

- bicapsulata, 372

- canis, 377

- funambuli, 377

- gerbilli, 376, 377, 390

- jaculi; 876

- muris, 23, 376, 390

- musculi, 352, 377

- nicoria, 373, 375

- peramelis, 376

- sebai, 377

- stepanowi, 107 (Fig. 53), 372, 373, 374 (Fig. 159), 375, 390

Hæmogтegarines, 357, 371, 390

Hasmogregarinidos, 378

Hasmoproteus, 365,391

- columbos, 365, 366 (Fig. 157), 390, 391 
Hosmoproteus danilewskvi, 365

- nocturo, 365, 390

- oryzivoras, 368

Hæmosporidia, 356

Haliphysema, 35, 231

- tumanowiczii, 35 (Fig. 17)

Halteria, 439

Halteridos, 439

Halteridia, 389, 391

Halteridium, 357, 365, 391

Haplosporidia, 398, 423

Haplosporidiidos, 423, 424

Haplosporidium, 424

Hastatella radians, 441

Helcosoma tropicum, 393, 412

Heliozos, 90, 218, 244.

Hemiclepsis marginata, 291, 298, 303

Hemispeira asterio, 441

Henneguya, 409, 426

Hepatozoon, 372

- muris, 376

- perniciosum, 376

Hereditary transmission, 24, 290

Herpetomonas, 281, 282, 292, 313, 319, 320

- muscas-domestica, 137, 138, 282 (Fig. 124), 315

Herpetophrya, 452

Heterokaryote, 449, 453

Hoteromastigoto, 259

Heteroneminos, 274

Heterophrys, 248

- fockei, 248 (Fig. 103)

Hoterotricha, 433

Hexactinomyxon, 409

Hexamitus, 258, 272

Histocyte8, 130, 133

Histoplasma, 319

- capsulatum, 319

Histozoic, 324

Holomastigina, 270

Holomastigote, 259

Holophrya, 439

Holopbytic, 13, 187, 188, 261

Holotricha, 439

Holotrichous larvæ, 459

Holozolo, 8, 13, 187, 261

Homaxon, 39, 250

Hoplitophrya, 452

House, 33, 45

Hyalosphora gregarinicola, 341

Hyalosphenia, 34

- ouneata, 34 (Fig. 16)

Hydrophobla, 470

Hymenostomata, 439, 442

Hypershromasy, 71

Hypnocyst, 166

Hypocoma, 460

- acinetarum, 460

Hypocomidas, 460

Hypothallus, 240

Hypotrioha, 433, 440

Hypotrichous larvæ, 459, 460

Ichthyophthiriasie, 450

Ichthyophthirius, 448, 453

- multifitis, 16, 21, 450, 451

Ichthyosporidium, 424

Idiochromatin, 71

Idiochromidia, 150

Immanoplasma, $\mathbf{3 8 8}$

- scyllii, 388
Imperforate, 231

Import, 189

Incubation-period, 292, 361

Incurvation, $\mathbf{4 6 8}$

Indirect division, 101

- transmission, 280

Infusorie, 2, 12, 152 (Fig. 77), 153, 430

Ingestion, 204

Initial body, 472

Intestinal flagellates, 258

Invagination, 180

Involution stages, 296

Isogamy, 126, 175

Isomastigote, 259

Isospore, 215, 254

Isotricha, 439

Isotrichido, 439

Jania, 276

Kala-azar, 316

Kalpidorhynchres, 332

Karyogamy, 126

Karyokinesis, 101, 119

Karyolysus, 372

- lacertarum, 372, 378.

Karyosome, 76, 288

Kutaphorio aotion, 208

Kentrochona, $\mathbf{4 0}$

Kentrochonopsis, 440

Kinotonucleus, 78, 85, 200, 286, 288, 289 , 392

Klossia, 348, 352

- helicina, 352

Klossiella, 352

- muris, 352

Kurloff-Demel bodies, 388

Labyrinthula, 243, 244

Labyrinthulidea, 243

Lagena, 232 (Fig. 93, 1i.)

Lagenophryincs, 440

Lagenophrys, 440

Lamblia, 272

- intestinalis, 31, 272, 273 (Fig. 117)

- sanouinis, 272

Lankesterella, 189, 372, 378

- ranarum, 372,378

Lankesteria ascidico, 327, 329 (Fig. 143)

- culicis, 327

Latent bodies, 296

Laverania, $\mathbf{3 5 8}$

Legendrea loyezos, 141

Legerella, 348, 349, 352, 355, 388

- nova, 352

Legercllidas, 852

Legeria, $\mathbf{3 5 3}$

Legerina, $\mathbf{3 5 3}$

Leishmania, 258, 281, 816, 320, 321. 393,394

- domovani, 316 (Fig. 138), 317 (Fig. 139), 473

- infantum, 316,317

- tropica, 87, 316, 317, 318 (Mis. 140), 412,473

Lentospora cerebralis, 100

Leptodiscus, 278

Leptomonas, 52, 281, 282, 898, 308, 813, $319,320,321$

- biitschlii, 282

- jaculum, 314 (Fig. 136), 315

Leptotheca, $\mathbf{4 0 8}$ 
Leptotheca agilis, 201, 401 (Fig. 165)

- ranarum, $\mathbf{4 0 8}$

Leptotrypanosome, 298, 314

Lermasophrya, 461

Lothal, 19

Leucocylogregarina, 372

Lgucocytozos, 372

Levcocytozoon, 357, 369, 390, 392

- Lovati, 370

- muris, 378

- piroplasmoides, 319

- ratti, 376

- sabruzesi, 371

- ziemanni, 369 (FLR. 158), 370,371

Leucophrys, 439

- patula, 440

Loucoplests, 188

Leydenia gemmipara, 237

Licnophora, 440, 441, 446, 449

Licnophoridas, 440

Life-cyole, 129, 130

Light-porception, 201

Light-produotion, 201

Linellæ, 238

Linin, 72

Lionotus, 439

Lithocircus, 256

- productus, 252 (Fig. 106)

Lithocystis schneideri, 331

Lobopodia, 47, 199

Lobosa, 217, 219

- testacoa, 229

Lobose, 47

Lophomonadida, 276

Lophomonas, 36, 88, 261, 276

- blattarum, 17, 18, 89 (Fig. 45), 263 , 276

- striata, 276

Lophophora, 278

Lorica, 33, 45, 276, 441

Loxodes, 439, 448

Luminosity, 201

Lymphocystis, 428

- johnstonei, 426, 427 (Fig. 178)

Lymphocytozoon, $\mathbf{3 8 8}$

- cobaya, 388

Ienchia, 365

Macramceba, 148

Macroconjugant, 153, 449

Macrogamete, 126

Macrogamy, 181, 161, 172

Macrogonidie, 267

Macromerozoite, 379

Macront, 426

Macronucleus, 78, 107, 430, 437, 448, 458

Macrosohizogony, 344

Macrosohizont, 344, 873

Macrosporea, 254, 255, 416

Macrostoma, 272

- mesnili, 272

Mal de caderas, 285

Malaria, 358, 359

Male sox, 159

Mallory's bodies, 470

Malpighiella refringens, 228

Mantlo-flbres, 103

Mastigoenceba, 213, 261, 268

Mastigella, 77, 268

- vitrea, 83 (Fig. 40), 265, 266 (Fig. 112)

Mastigina, 265, 267, 268

- setosa, 82 (Figs. 38,39 )
Mastigophora, 12,257

Mastigotricha, 455

Maturation, 142

Maupasia, 454

- paradoxa, 454 (Fig. 189, B)

Messlos, 470

Mechanical stimuli, effeots of, 207

Mechanical transmission, 290

Megaloephærio, 184, 238

Megastoma, 272

- entericum, 272, 273 (Fig. 117)

Melanin, 64, 198, 357

Mombrane (nuolear), 76

Membranellæ, 55, 443, 445

Membranulæ, $4 \mathbf{4 5}$

Merocyatis, 352

- kathos, 352

Merogregarina amaroucii, 336

Merogregarinidas, $\mathbf{3 4 1}$

Meront, 898, 413

Merozoite, 169, 325

Merozoon, 210

Mesomitosis, 111

Motabolio, 38

Metaohromatinio grains, 67, 420, 481

Metacineta, 460

Metacinetida, 460

Metagenesis, 266

Metamera, 932, 339

Motamitosis, 111

Metaplastio, 40, 63

Metazoa, 2

Micramœba, 148

Microconjug ant, 153, 172, 448

Microgamete, 126, 448

Microgamy, 182, 172

Miorogonidia, 267

Microlklosia, 488

Micromerozoite, $\mathbf{3 7 8}$

Mleront, 426

Micronuolens, 78, 113, 114 (Fig. 61), 288, $332,333,430,437,448$

Miorosohizont, $\mathbf{3 4 4}, 878$

Miorosome, 40

Micrbapherio, 184, 233

Microspore, 254, 255, 416

Miorosporidis, 411

Microthoracidas, 499

Microthorax, 439

Miesoher's tubes, 419,422

Minchinia, 852

- caudata, 348

- chitonis, 349, 352

Mitochondria, 41, 448

Mitosis, 101

Mixotrophto, 188

Mollusoum contagiosum, 17, 470

Monad, 466

Munadidos, 270

Monas, 270

Monaxon, 39, 250

Monera, 78

Moniliform, 77

Monocercomonas, 272

Monocystis, 23, 174 (Fig. 79), 328, 336 , 339

- coronata, 328

- pareudrili, 331

- rostrata, 332, 333,335

Monokaryon, 121, 255

Monomastigoto, 259

Monomastix, 455 
Monomastix ciliatus, 454 (Fig. 189, A), 455

Monomorphio kpecieg, 163

Monopylaria, 251, 256

Monospora, 339

Monosporea, 409

Monothalamous, 36, 232

Monozoa, 273

Monozoio, 348

Mother-oyst, 138

Movement, 199

Movements of grogarines, 327

Multicilia, 249, 261, 270, 454

- lacustris, 269 (Fig. 113), 270

- palustris, 269 (Fig. 113), 270

Multiplo flssion, 100, 120

- gemmation, 122

- promitosis, 120

Multiplicative phase, 20, 166

Multipolar mitosis, 120

Murrina, 285

Mycotosporidium, 243

Mycetozos, 218, 239, 268

Mycterothrix, 446

Myocyte, 327

Myonemes, 57, 201, 253, 259, 286, 445

Myophrisk8, 258

Myxamœba, 239

Myxidiidos, 400

Myxidium, 409

- bergense, 407

- lieberkihni, 400, 401, 409

- sp., 406

Myxobolidas, 22, 23, 409

Myxobolus, 409

- cerebralis, 400

- neurobius, 400

- pfeifferi, 405, 406 (Fig. 168)

Myxocystis, 417,418

Myxoflagellate, 239

Myxogastres, 242

Myxomycetes, 239, 242

Myxopodia, 253

Myxosporidia, 399

Myxotheca, 231

\section{Nagana, 19}

Narcotics, effects of, 204

Nasbollaria, 256

Nassula, 439

Natural olassiffcation, 463

Nebenkern, 95

Nebenkörper, 278

Negri's bodies, 470

Nematocyst, 447

Neogamous, 127, 330

Neosporidia, 325, 398, 466

Nephroselmis, 275

Nervous eystem, 146

Neuronemes, 446

Neuroryctes, 470

- hydrophobia, 171

Neurosporidium, 424

- cephalodisct, 424

Nicollia, $\mathbf{8 8 0}$

- quadrigemina, 380, 381

Nina. Boe Plenocephalus

Noctiluca, 201, 213, 279

- miliaris, 119 (Fig. 65), 279

Nodosaria, 232 (Fig. 93, 3)

Nosema, 418

- apts, 412
Nosema bombycis, 24, 411, 413, 114 (Fig. 172)

Nuolear membrane. 76

- sap, 72

Nuclearia, 248

Nuclearia-stage, 177

Nucleo-oytoplasmlo ratío, 70

Nucleolo-centrosome, 95

Nucleolus, 76, 103

Nucleophaga, 473

Nuolens, 6, 7, 65, 96

- 8ooundns, 95

Nuda, 217, 218

Nummulites, 232 (Fig. 93, 11)

Nutation, 51

Nutrition, 187

Nuttallia, 880

- equi, 380

- herpestidis, 380

$N$ yctotherus, $439,440,447$

- condiformis, 10 (Fig. 9), 444 (Fig. $186, \mathrm{~F})$

- faba, 440

Octomitida, 272

Octrmitus, 36, 258, 272

- dufardini, 272 (Flg. 116)

Octosporea, 418

- muscas domesticos, 138

Octozoio, 349

CEcomonas, 270

(Esophagus, 261, 433

Oikomonas, 270

Oligospores, 418

Oligosporulea, 424

Oligotrichs, 439

Oroyst, 348

Ö̈oyte, 143

Ookinete, 305, 362

Opalina, 196, 198, 208, 209, 439, 440. $447,448,452,454$

- caudata, 452

- intestinalis, 152

- ranarum, 447, 452, $\$ 53$

Opalininas, 452

Opalinopsis, 452

Opercularia, 145, 440

- faurei, 442

Operoulum, 441

Ophrydium, 438, 440

Ophryocystida, 341

Ophryocystis, 337, 339

Orcheobius, $\mathbf{3 5 2}$

Ophryodendridas, 461

Ophryodendron, 455,461

Ophryoscolecida, 439

Ophryoscolex, 439, 141

Orcheobius herpobdella, 346, 348, 349, 352

Organella, 1

Oriental soro, 316

Osmotaris, 203

Orum, 125

Oxyrrhis, 52, 278

- marina, 878 (Fig. 123)

Oxytricha, 202, 440

Oxytrichidas, 440

Pansporella perplexa, 427

Pansporoblast, 405, 417, 423

Pantastomina, 268

Parsbasal apparatus, 89

Paracoccidium prevoti, 349 
Paraglycogen, 41, 63, 195, 327

Paramastigote, 259

Paramecidas, 439

Paramecium, 61, 114 (Fig. 61), 171, 191, 192, 194, 196, 197, 198, 203 (Fig. 83), $205,206,208,210,437,439,442,443$

- bursaria, 449

- caudatum, 107 (Fig. 53), 436 (Fig. 185), 444 (Fig. 186, D, E), 447 (Fig. 187)

Paramaba, 228

- eilhardi, 94 (Flg. 49), 95, 228

- hominis, 228

Paramylnm, 63, 188, 195

Paramyxa, 243, 409

- paradoxa, 409

Paramyxidia, 409

Paraplasma flavigenum, 379

Parasito, 8, 14

Parietal cell, 403

Parthenogenesis, 137

Parthenogonidia, 267

Partial karyogamy, 126, 153, 453

Pathogenic, 19

- amœbæ, 226

Paulinella, 214

Pearl-stage, 334

Pébrine, 24, 411

Pectinellæ, 442

Peduncle, 31

Pelliclc, 32, 45, 435

Pelomyxa, 78, 144, 150, 205, 214, 227

- palustris, 227 (Fig. 91)

Pettomyces, 243

Peneroplis, 15, 235

Peranema, 274

- trichophorum, 273

Peranemidas, 274

Perezia, 418

Perforate, 231

Peridinialos, 276

Peridinida, 278

Peridinium, 278

- divergens, 278 (Fig. 122)

Peridium, 241

Perikaryon, 439

Periplast, 45, 259

Peripylaria, 251, 255

Perist ome, 433, 442

Peritricha, 433, 438, 440, 441, 442, 448

Peritrichous larvæ, 459

Peritromido, $\mathbf{4 4 0}$

Peritromus, $\mathbf{4 4 0}$

Pernicjous malaria, 358

Peroral mombrane, 445

Phacus, 274

- triqueter, 274 (Flg. 118).

Phrenocystes, 412

Phacodaria, 256

Pliaedium, 252

P'losphorosconce, 201, 278

Pliot otaxis, 202, 205

Phylogeny, 463

Physurum didermoides, 242

Physodes, 244

Phytoflagellata, 274

Phytomonadina, 274

Phytomyxinas, 243

Piroplasma, 24, 357, 379, 393, 394

- bigeminum (bovis), 379 (Fig. 160), 384,385 (Fig. 162)

- caballi, 379
Piroplasma canis, 382, 383 (Fig. 161), 384, 385 (Fig. 162), 387

- donovani, 393

- hominis, 379

Piroplasmoses, 378

Piroplasm8, 378, 390

Plagiotomidas, 439

Planont, 398, 408, 413, 423

Planorbulina, 232 (Fig. 93, 9)

Plasmodiophora, 243

- brassica, 149 (Fig. 76), 243

Plasmodium, 100, 128, 240, 398, 423

Plasmodium, 357

- brasilianum, 364

- cynomolor, 364

- diploglossi, 358

- falciparum, 358, 359, 360 (Fjg. 156)

- inui, 364

- kochi, 364

- malarias, 358, 359

- pitheci, 359, 364

- procox, 358

- relictum, 358

- virax, 137 (Fig. 72), 358, 359, 360 (Fig. 156)

- vassali, 364

Plasmodi oma, 462

Plasmogamy, 128

Plasmotomy, 100

Plastin, 73, 103

Plastinoid granules, 41, 195, 346

Plastogamy, 128, 209

Plogepoda, 462

Pleistophora, 418

- longifilis, 413 (Fig. 171), 415, 416

- periplanetio, 416

- species, 413

Pleodorina californica, 267

Pleuronema, 55, 439, 442

- chrysalis, 56 (Fig. 27)

Pleuronemida, 439

Podophrya, 461

- $f x a, 456$ (Fig. 190, C), 458

- gemmipara, 108 (Fig. 55)

- mollis, 456 (Fig. 190, A)

Podophryidae, 461

Polar bodies, 143

- capsulo, 399 (Fig. 163)

- conos, 117

- flament, 399

- masscs, 110

- platos, 117

Polycaryum, 424

Polychromophilus, 364

Polycystid, 326

Polycyttaria, 256

Polyonorgid nuclei, 121, 151, 255

Polykaryon, 121, 255

Polymastigidas, 272

Polymastigina, 271

Polymastigoto, 259

Polymastix, 272

l'olymoı phism, 162, 163, 297, 311

I'olyspora, $\mathbf{3 3 9}$

Polysporoa, 409, 418

Polysporuloa, 424

L'olystomella, 210

- crispa, 139, 234 (Fig. 95), 235, 236 (Fig. 96)

Polythalamous, 36, 232

Polytomella agilis, 86 (Fig. 43)

Polytrema, 231 
Polytricha, 439

Polyzoic, 349

Poneramœba, 224

Pontobdella muricata, 291, 303

Pontomyxa thava, 218

Porospora, 337, 340

— gigantea, 74 (Fig. 35), 336,339 (Fig. 150)

- legeri, 336

Porosporidas, 341

Pouchctia, 62

- cornuta, 61 (Fig. 31)

Prchonsile tentacle, 457

Probosoidiform Individuals, 455

Proboscidium, 442

Prococcldia, 352

Protlagellat a, 469

Promitosis, 109

Pronucleus, 127

Propagat iro cell, 405

- phase, 21, 166

Propulsive psoudopodium, 401

Prorocontracce, 276

Prorocentrum, 278

Prorodon, 439

- teres, 32 (Fig. 14), 444 (Fig. 186, B, $C), 446$

Proteomyxa, 217, 268

Proteosoma, 358, 364, 365, 393

Protista, 4, 5

Protoblast, 426

Frotococcacoæ, 15

Protoentospora ptychodera, 229

Protokaryon, 75, 87, 108

Protomorito, 327

Protomonadina, 270

Protophrya, 452

- ovicola, 452

Protophyta, 8

Protopla6m, 29, 40

Frotozos, 2, 10, 464

Prowazck's bodies, 470

Prowazekia, 260, 271, 281, 319, 321,322

- asiatica, 319

- cruzi, 319

- parva, 319, 320 (Fig. 141)

- weinbergi, 319,320 (Fig. 141)

Pseudochlamys-stago, 170,177

Psendoplasmodida, 243

Psoudoplasmodium, 242

Pseudopodiospore, 160

Pseudopodium, 30, 46, 90, 199, 214, 400, 465

Pseudospora, 213, 218, 249

Psorosperm, 165, 323

Pterocephalus, 173, 327, 329, 330, 339

- gracilis, 174 (Fig. 79), 332 (Fig. 145), 334 (Fig. 147)

- nobilis, 339

Pulsellum, 52, 258

Pusulc, 277

Pycnothrix, 452

- monocystoides, 443, 446, 447, 452

Pyramimonas, 275

Pyronoid, 63, 188, 261

Pyrodinium, 201,278

Pyrosoma, 379

Pyxinia, 329, 330

Quartan malaria, 358, 359

Radiolaria, 218, 249

Radium-rays, oflects of, 205
Rainoy's corpuscles, 410

Roactions of protozoa, 201

Reoapitulative forms, 170

Roduoing division, 104

Reduotlon, 142, 145, 335

Reduotion-nuolol, 144

Redwater, 378

Rogoneration, 208, 210

Rejurenoscence, 155

Rolapso (malarial), 363

Reserve-materials, 195, 196

Resorvolr-vacnole, 262

Rospiration, 195

Reticulosa, 217, 218

Rotlculozo, 48

Retioulum (nuclear), 75, 103

- (protoplasmio), 41

Rhabdogonie, 325, 466

Rhabdophrya, $\mathbf{4 6 1}$

- trimorpha, 455

Rhaphidiophry8, 245

Rhootaxis, 207

Rhinosporidium, 424

- kinealyi, 424,425 (Fig. 177)

Rhizomastigine, 265, 268, 465

Rhizoplast, 82

Rhizopoda, 213, 217

Rhyncheta, 457,460

Rhynchoflagellata, 278

Right host8, 291

Rod-apparatus, 433, 439

Röntgen-rays, effects of, 206

Rostrum, $\mathbf{3 2 6}$

Saccamina, 232 (Fig. 93, 1)

Sack-pusule, 277

Sapropelic, 14

Saprophytic, 8, 14, 187, 194, 262

Saprospira, 467, 469

- glandis, 468

Saprozoic, 14

Sarcocystine, 20, 420, 421

Sarcocystis, 20, 419

- bertrami, 420

- muris, 419, 420, 421, 422 (Fig. 176)

- rilevi, 420

- tenclla, 419, 420, 421 (Fig. 175)

Sarcocyte, 327

Sarcode, 40

Sarcodina, 11, 213

Sarcosporidia, 20, 410

Scaiotrioha, 440

Scarlet foror, 470

Schaudinne.'la, 355

Schewiakovella schmeili, 425

Schizocystidoo, 339, 341

Schizocystis, 339

- gregarinoides, 336, 338 (Fig. 149)

Schizogonea, 418

Schizogony, 166, 324, 392

Schizogrogarinæ, 328, 339

Sohizokineto, 373

Sohlzont, 166, 181, 324

Schizontocyte, 344

Schizotrypanum, 285, 307, 392

- cruzi, 28, 295 (Fig. 128), 296, 302 (Flg. 132), 307

Schizozoite, 344, 428

Solorotium, 166, 240

Scopula, 441, 456, 459

Scyph idia, 440, 441

Secondary nucloi, 66 
Secrotion, 197, 198

Selenidiida, 341

Selenidium, 338

- caulleryi, 336, 337 (Fig. 148)

Selenococcidium, 352, 354

- intermedium, 344, 350 (Fig. 155), 351

Sonility, 131, 135, 155

Sensory organs, 201, 446

Soparation-8pindle, 104

Septate, 326

Serumsporidia, 425

Sex, 154

Sexual differontiation, 160, 170, 176

- phases of try panosomes, 305

Shell, 33, 45, 232 (Fig. 93)

Siedleckia, 339, 352

Silicoflagollata, 274

Sloeping sickness, 26

Smithia, 380

- microti, 380

Soma, 130

Somatlo number, 143

Sorophora, 243

Sorosphara, 243

Sorus, 242

Souma, 304

Spesmoneme, 446

Species, 141, 162

Spermatocyte, 143

Spermatozoon, 125

Sphwractinomyxon, 409

- stolci, 409, 410 (Fig. 170)

Sphærollaria, 255

Spharomyxa sabrazesi, 404 (Fig. 167), 405

Spharophrya, 461

Sphærozoa, 256

Sphere, 95

Spheroplast, 41, 448

Spicule, 36

Spindle (nuolear), 103

Spirigera, 442

Epirillacea, 467

Spirillar forms, 319

Spirillum, 467

Spirochosta, 466

-plicatilis, 466

- ziemanni, 371, 468

Spirochøtes, 466

Spirochona, 440

Spirochonida, 440

Spiroloculina, 232 (Fig. 93, 5)

Spironema, 467, 469

Spironemaces, 469

Spiroschaudinnia, 467

- anserina, 167

- duttoni, 467, 468

- gallinarum, 467

- obermeieri, 467

- recurrentis, 467

Spirostomum, 196, 197, 208, 438, 439, 445

- ambiguum, 431 (Fig. 180)

s̀ponyomonas, 270

- splendida, 84 (Fig. 41)

- urella, 85 (Fig. 42)

Sporal rosiduum, 349

Sporangium, 240, 241

Spore, 165, 166, 323

Spore-formation, 166

Sporotia, 150

Sporoblast, 325

Sporocyst. 165
Sporocyst-mothor-coll, 403

Sporoduct, 335

Sporogons, 181, 325

Sporomyx :, 243

Sporont, 166, 181, 325, 326

Sporophoro, 242

Sporoplasm, 405

Sporozoa, 12, 323, 462, 466

Sporozoito, 169, 324

Sporulation, 122, 165, 166

Spumellaria, 255

Stannomida, 238

Starvation, 195, 210

Stemonitis flaccida, 82

- fusca, 240 (Fig. 98)

Stemm-psendopodium, 101

Stempellia, 418

- mutabilis, 418

Stenophora, 329

Stentor, 61, 202, 211, 437, 438, 439, 441, 445,446

- ccruleus, 444 (Fig. 186, $4, I$ )

- niger 444 (Fig. 186, G)

- rocselii, 10 (Fig. 8)

Stentoridos, 439

Stephanosphora, 267, 276

Steroomarium, 238

Stercome, 194, 233

Stigma, 61, 205, 262

Stomatophora coronata, 328

Streaming morements, 199

Strongrloplasmata, 470

Stylonychia, 438, 440

- histrio, 444 (Fig. 186, $H$ )

- mytilus, 211, 459 (Fig. 192)

Stylorhynchus, 173 329, 330, 339

- longicollis, 174 (Fig. 79), 327 (Fig. 142), 339

Suctoria, 455

Suctorial tontacle, 190, 458

Sulous, 276

Surface-tension, 200

Surra, 26

Swarm-spore, 169, 396

Symbiosis, 15

Symbiotic algæ, 197

Synactinom yxon, 409

Syngamy, 126, 438

Synkaryon, 127

Syphilis, 467

Syzy6y, 330

Tachyblaston, 460

Tactio, 202

Tactile bristles, 443

- organs, 201

Taniocystis, 327, 339

Taxis, 202

Technitella thompsoni, 34

Toloblast, 426

Telomyxa, 418

- glugeiformis, 418

Telosporldia, 325, 395, 466

Temporature, effocts of, 206

Tentaoulifera, 455

Tortian malaria, 358,359

Tost, 33

Testacea, 217, 219

Tetramyxa, 243

Tetratrichomonas, 272

Totrazoio, 349

Thalamophora, 219 
Thalassicolla, 255

- pelavica, 30 (Fig. 13)

Thalassophysa, 255

Thocamobæ, 219, 229

Theileria, 379

- pariva, $380,382,386$

Thelohania, 418

- chatogastris, 416 (Flg. 173)

- contejeani, 412

- manadis, 416

Thelyplasm, 129

Thormotaxis, 202, 206

Thigmotaxis, 207

Thyroid oxtract, effocte of, 204

Tinotin-body, 458

Tintinnidas, 439, 441, 443, 447

Tocophrya, $\mathbf{4 6 1}$

- cyclopum, 461

- limbata, 460

- quadripartita, 210, 456 (Fłg. $1 \& 0 . B$ ) 460

Toddia, $\mathbf{3 8 7}$

- bufonis, 387

Tolerant, 21

Tonicity, offects of, 207

Total karyogamy, 126, 453

Toxocystis homari, 426

Toxoplasma, 319, 387

- canis, 387

- cuniculi, 387

- gondii, 387

- talpa, 387

Trachelidas, 439

Trachelius, 439

- ovum, 441, 448

Trachelocerca, 439, 448, 453

- phonicopterus, 120 (Fig. 66), 449, 450 (Fig. 188)

Trachoma, 470

Tractellum, 52, 259

Trailing flagellum, 53, 260

Transmission of trypanosomos, 289

Transmutation of energy, 199

Treponema, 467

- pallidum, 467, 468

- pertenue, 467

Triactinomyxon, 409

Trichia varia, 241 (Fig. 101)

Trlohites, 442

Triohocyst, 46, 435, 447 (Fig. 187)

Trichodina, 440, 441

Trichomastix, 260, 271

Trichomonas, 17, 36, 56, 258, 260, 271

- eberthi, 8 (Fig. 5 ), 36

- hominis, 272

- vaginalis, 272

Trichonympha, 276

- hertwigi, 276

Trichony mphlda, 463

Trichonymphida, 89, 276, 454

Trichophrya, $\mathbf{4 6 1}$

Trichophryida, 461

Trichorhynchus, 446

Trichosphorium, 51, 216, 229

- sieboldi, 73 (Fig. 34), 182 (Fig. 81)

Trimastigamœba, 268

Tripyiaria, 251, 256

Tritoblast, 426

Trizoic, 349

Trophio phase, 324

Trophochromatin, 71

Trophocbromidia, 150
Trophonucleus, $7885,286,288$

Trophozoito, 324

Tropical malaria, 358

Trypanomonad, 282, 298, 299

Trypanomorpha, 308

Trypanophis orobbeni, 309

Trypanoplasma, 56, 78, 87, 260, 271, 281, $287,308,309,321,322$

- abramidis, 310 (Fig. 134)

- borreli, 312

- congeri, 312

- dendrocoli, 309

- gryllotalpas, 309, 310

- gurneyorum, 78 (Fig. 36)

- helicis, 309, 311, 312

- intestinalis, 312

- keysselitzi, 310 (Fig. 134)

- ranas, 319

- vaginalis, 309

Trypanosoma, 270, 280, 283, 308, 320, 321

- balbianii, 467

- blanchardi, 25

- brucii, 19, 25, 26, 27 (Flg. 12), 285 , $291,296,305,306,308$

- cazalboui, 304

- cruzi, 285, 295, 296

- cuniculi, 25, 26 (Fig. 11)

- dimorphon, 304

- drosophilas, 315 (Fig. 137)

- duttoni, 25, 26 (FIg. 11)

- elyomis, 25, 26 (Fig. 11)

- equinum, 285, 305

- equiperdum, 22, 26, 285

- evansi, 26, 27 (Flg. 12), 285

- gambiense, 19, 26, 27 (Fig. 12), 285, $291,296,297,303,304,305,300$

- oranulosum, 288, 297, 298 (Fig. 129)

- grayi, 304

- hippicum, 285

- inopinatum, 290

- lewisi, 19, 25, 26 (Fig. 11), 28, 263,

- 286, 291. 292, 293 (Fig. 127), 297, 299, 300 (Fig. 130), 301 (Fig. 131). $305,306,308$

- longocaudense, 294

- mega, 284 (Fig 125)

- microli, 25, 26 (Fjg. 11)

- nanum, 27 (Fig. 12)

- noctuce, 59 (Flg. 30), 137, 144, 158. $283,297,305,306,308,391$

- pecaudi, 304

- percas (myonomos), 58 (Fig. 28)

- rabinouitschi, 25

- raiळ, 291, 303

- remaki, 9 (Fig. 6 )

- rhodesiense, 26, 286

- rotatorium, 59 (Fig. 30 ), 283, 297, 307

- sanguinis, 283

- vittata, 303

- vivax, 27 (F1g. 12), 287, 291, 204

Trypanosomos, 8yngamy, 136

Trypanosomida, 268, 270

Trypanotoxin, 20

Trypanozoon, 308

Uitramicroseopic stagos, $3 \mathrm{CB}$

Unciform Individuals, 455

Undulating mombrano, 55, 260, 286, 287, 443

Undulina ranarum, 283

Unicoliular, 1, 3 
Urceolarinas, $\mathbf{4 4 0}$

Urhsemoflagellat, 468

Urnula, 457, 460

- cpistylidis, 457 (Eir 191), 460

Urnulidos, 460

Urospora lagidis gametos, 174 (Fig. 79)

Urosporidium, 424

Urosilyla, 440.

Vaccinla, 470

Vacuolo, 43

Vaginicola, 440

Vampyrella, 218

- lateritia, 219 (Fig. 86)

Variola, 470

Vegetative chromatin, 71

Vermiform Individuals, 455

Vestibule, 433

Volutin, 68, 195, 289

Volvocida, 267,275

Volvox, 3, 131, 206, 267, 276

Vorticella, 440, 441, 445, 446
Vorticella microstoma, 172 (Fig. 78)

- monilata, 446

Vorticellida, $\mathbf{4 4 0}$

Vorticellin $\omega, \mathbf{4 4 0}$

Wagnerella, 48, 51, 92, 120, 245, 246, 248

- borealis, 93 (Fig. 48), 247 (Fig. 102)

Wrong hosts 291

Xenophya, 34, 238

Xenophyophora, 218, 237

Yaws, 467

Yollow cells, 252

Zoochlorolla, 15, 252

Zoomyxa, 243

Zoospore, 169, 26.

Zoosporidas, 218

Zoothamnium, 440

Zooxanthellæ, 15, 252

Zygote, 125 
MEMORANDA 
MEMORANDA 
520

MEMORANDA 


\section{Messrs. Edward Arnold \& Co.'s Scientific \& Technical Publications.}

\section{PHYSICS}

GENERAL ASTRONOMY. By H. SPencer Jones, M.A., B.Sc., Senior Assistant at the Royal Observatory, Greenwich. viii +392 pages, with 102 diagrams and 24 plates. SECOND IMPRESSION. Demy 8 vo, 2 Is. net.

ASTRONOMICAL DISCOVERY. By H. H. TURNER, D.Sc., F.R.S., Savilian Professor of Astronomy in the University of Oxford. xii +225 pages, with 15 plates. Demy 8vo, Ios. 6d. net.

ISOTOPES. By F. W. Aston, D.Sc., M.A., F.R.S., Fellow of Trinity College, Cambridge. viii + 152 pages, with diagrams and plates. THIRD IMPression. Demy 8vo, 9s. net.

IONS, ELECTRONS, AND IONIZING RADIATIONS. By J. A. Crowther, M.A., Sc.D., University Lecturer in Physics as applied to Medical Radiology at Cambridge University. THird EDition. 'Thoroughly revised. xii + 292 pages. Demy 8vo, I2s. 6d. net.

THE BECQUEREL RAYS AND THE PROPERTIES OF RADIUM. By Lord RAYLEIGH, F.R.S., formerly Professor of Physics at the Imperial College of Science and Technology. SEcond Edition. vi +215 pages, with illustrations. Demy 8 vo, ros. net.

AN INTRODUCTION TO THE THEORY OF OPTICS. By Sir Arthur Schuster, Sc.D., F.R.S., Honorary Professor of Physics in the Victoria University of Manchester. SECOND Edition. xvi +352 pages, 180 illustrations. Demy 8 vo, I 8 s. net.

THE DYNAMICAL THEORY OF SOUND, By HoRACE LAMB, D.Sc., LL.D., F.R.S., Rayleigh Lecturer in the University of Cambridge and Emeritus Professor of Mathematics in the Victoria University of Manchester.

[New Edition in Preparation.

COLOUR VISION. A Discussion of the Leading Phenomena and their Physical Laws. By W. PEddie, D.Sc., F.R.S.E., Harris Professor of Physics at University College, Dundee. xii +208 pages. Illustrated. Demy 8 vo, I 2 s. $6 \mathrm{~d}$. net.

AERONAUTICS IN THEORY AND EXPERIMENT. By W. L. Cowley, A.R.C.S., D.I.C., and H. LEVy, M.A., B.Sc., F.R.S.E., Assistants at the National Physical Laboratory. With numerous illustrations and diagrams. SEcond Edition. xii +332 pages. Demy 8vo, 25s. net.

NAVIGATION NOTES AND EXAMPLES. By Instructor Captain S. F. CARD, B.A., R.N., late Head of the Navigation Department and Lecturer in Navigation at the Royal Naval College, Greenwich. Third Edırıon. xviii +252 pages. Demy 8vo, r2s. 6d. net.

AIR NAVIGATION NOTES AND EXAMPLES. By Instructor Captain S. F. CARD, B.A., R.N. viii + 140 pages, with 61 figures. Demy 8 vo, Ios. 6 d. net.

A TEXT-BOOK OF PHYSICS. By R. S. Willows, M.A. (Camb.), D.Sc. (London), late Head of the Department of Mathematics and Physics at the Sir John Cass Technical Institute; Member of the Council of the Physical Socicty. Second Edition. Sixth Impression. viii +520 pages, with 320 diagranis. Large crown $8 \mathrm{vo}$, 9s. net.

HEAT. By W. J. R. Calvert, M.A., Harrow School. viii +344 pages, with 138 diagrams. Crown $8 \mathrm{vo}, 6 \mathrm{~s}$.

ELECTRICITY AND MAGNETISM. By C. E. AsHFord, M.A., Headmaster of the Royal Naval College, Dartmouth. With over 200 diagrams. Third Revised Edition. Crown 8vo, 4s. 6d.

LONDON : EDWARD ARNOLD \& CO., 4I \& 43 MADDOX ST., W.I. 
MAGNETISM AND ELECTRICITY. By J. PALEY YoRkE, Head of the Physics and Electrical Engineering Department, L.C.C. School of Marine Engineering, Poplar. Crown 8 vo, $5 \mathrm{~s}$.

AN INTRODUCTION TO PRACTICAL PHYSICS. By E. $\mathrm{H}$. BARTon, D.Sc., F.R.S.E., Professor of Experimental Physics, University College, Nottingham; and the late T. I'. BLACK, M.Sc., Ph.D. 55 Figures. SECond EDITION. Crown 8vo, 5s.

ADVANCED EXAMPLES IN PHYSICS. By A. O. Allen, M.A., B.Sc., A.R.C.S., Lecturer in Optics at Leeds University. Second Edrtion. Crown 8vo, 2s. 6 d. net.

\section{MATHEMATICS}

FIVE-FIGURE TABLES OF MATHEMATICAL FUNCTIONS. Comprising Tables of Logarithms, Powers of Numbers, Trigonometric, Elliptic, and other Transcendental Functions. By J. B. DALE, M.A., Assistant Professor of Mathematics at King's College, London. Demy 8 vo, 4s. 6d. net.

LOGARITHMIC AND TRIGONOMETRIC TABLES (To Five Places of Decimals). By J. B. DALE, M.A. Demy 8vo, 2s. 6d. net.

THE CALCULUS FOR ENGINEERS. By JOHN PERry, M.E., D.Sc., F.R.S., late Emeritus Professor of Mechanics and Mathematics in the Royal College of Science, London. Thirtentil Impression. viii +382 pages. Crown 8 vo, 8s. $6 \mathrm{~d}$.

CALCULUS FOR SCHOOLS. By R. C. FAwDry, M.A., Head of the Military and Engineering Side at Clifton College; and C. V. DURELL, Senior Mathematical Master at Winchester College. Crown 8 vo. With Answers. In one volume, 6s. 6d. Part I, 3s. 6d.; Part II, 4s.

AN INTRODUCTION TO PROJECTIVE GEOMETRY. BY L. N. G. FILON, M.A., D.Sc., F.R.S., Professor of Applied Mechanics, University College, University of London. THiRD EDition, viii +261 pages. Crown $8 \mathrm{vo}, 7 \mathrm{~s} .6 \mathrm{~d}$.

MATHEMATICAL DRAWING. Including the Graphic Solution of Equations. By the late G. M. Mincuin, M.A., F.R.S.; and J. B. DALE, M.A. Third Edition, Second Impression. vi + 455 pages. Demy $8 \mathrm{vo}$, cloth, 8s. 6 d. net. HIGHER ALGEBRA. By W. P. Milne, M.A., D.Sc., Professor of Mathematics in the University of Leeds. xii +586 pages. Crown $8 \mathrm{vo}, 8 \mathrm{~s}$. $6 \mathrm{~d}$.

HOMOGENEOUS CO-ORDINATES. By W. P. Milne, M.A., D.Sc. xii +164 pages. Crown 8 vo, 6s. net.

EXAMPLES IN THE MATHEMATICAL THEORY OF ELECTRICITY AND MAGNETISM. By J. G. Lea THeM, M.A., Fellow of St. John's College, Cambridge. Crown 8vo, cloth, $2 \mathrm{~s}$.

VECTORS AND ROTORS: with applications. By O. HENRICI, Ph.D., LL.D., F.R.S.; and G. C. Turner, B.Sc., Head of the Mathematics and Physics Department, Goldsmiths' College. xvi +204 pages. Crown 8vo, 5s.

GEOMETRICAL CONICS. By G. IV. CAUNT, M.A., Lecturer in Mathematics, Armstrong College, Newcastle-on-Tyne; and C. M. Jessop, M.A., I'rofessor of Mathematics, Arınstrong College, Newcastle-on-Tyne. Crown 8vo, 3s.

\section{ENGINEERING}

STRENGTH AND STRUCTURE OF STEEL AND OTHER METALS. By IV. E. DAlbY, F.R.S., M.A., B.Sc., M.Inst.C.E., M.I.M.E., University Professor of Engineering at the City and Guilds (Engineering) College. Very fully illustrated. $19^{2}$ pages and $3^{8}$ plates. $8 \mathrm{vo}, 18 \mathrm{~s}$. net.

LONDON : EDWARD ARNOLD \& CO., 41 \& 43 MADDOX ST., W.I, 
STEAM POWER. By Professor W. E. DAlby, F.R.S., M.Inst.C.E., M.I.M.E. Second Edition. xvi +760 pages, with 250 diagrams. 8vo, 25s. net. The Total Energy and Temperature Diagram and the Total Energy and Entropy Diagram (Mollier Diagram) are obtainable separately, price 6d. net each (by post in a tube, IId.).

VALVES AND VALVE GEAR MECHANISMS. By Professor W. E. DALBY, F.R.S. xviii + 366 pages, 202 illustrations. SEcond Impression. Royal 8 vo, 24s. net.

THE BALANCING OF ENGINES. By Professor W. E. Dalby, F.R.S. THird Edition. xii +283 pages, I 84 ilhustrations. Demy 8vo, I2s. 6d. net.

PROPERTIES OF STEAM AND THERMODYNAMIC THEORY OF TURBINES. By H. L. Callendar, F.R.S., Professor of Physics in the Imperial College of Science and Technology. 544 pages, numerous diagrams. 8vo, 30s. net.

THE CALLENDAR STEAM TABLES. 3s. 6d. net. ABRIDGED CALLENDAR STEAM TABLES. (Centigrade Units.) 8 vo, is. net.

ABRIDGED CALLENDAR STEAM TABLES. (Fahrenheit Units.) 8 vo, Is. net. These abridged tables contain the necessary data in a convenient form for Engineers and Students engaged in steam work.

THE CALLENDAR STEAM DIAGRAM. (Centigrade Units.) This is supplied with "The Properties of Steam," and also with "The Callendar Steam Tables." It can be obtained separately, price 6d. net (by post in a tube, ird.).

THE CALLENDAR STEAM DIAGRAM. (Fahrenheit Units.) This is not supplied with "The Properties of Steam" or with "The Callendar Steam Tables." 6d. net (by post in a tube, IId.).

HEAT DROP TABLES : ABSOLUTE PRESSURES. Calculated by H. Moss, M.Se., A.R.C.S., from the Formulæ and Steam Tables of Professor H. L. Callendar, F.R.S. 64 pages. Cloth, 5s. net.

HEAT DROP TABLES: H.P. GAUGE PRESSURES, L.P. ABSOLUTE PRESSURES. Calculated by H. Moss, Imperial College of Science, from Professor Callendar's Formulæ and Steam Tables. Cloth, 5s. net.

CORRECTION TABLES FOR THERMODYNAMIC EFFICIENCY. Caleulated by C. H. NAYLOR, Assoc.M.Inst.C.E. Cloth, 5s. net.

STEAM TURBINE DESIGN. With Especial Reference to the Reaction Type, and including Chapters on Condensers and Propeller Design. By JoHs MorRow, M.Sc., D.Eng. (Armstrong College, Newcastle-on-Tyne).

[New Edition in Preparation.

RAILWAY ELECTRIC TRACTION. By F. W. CARTER, M.A., M.I.E.E., British Thomson-Houston Co., Rugby. viii +4 I2 pages, with 204 illustrations and Io folding plates. Demy 8 vo, 25s. net.

HYDRAULICS. For Engineers and Engineering Students. By F. C. LEA, D.Sc., M.Inst.C.E., Professor of Civil Engineering in the University of Birmingham. Fourtil Edition. xii + 594 pages, 400 diagrams. Demy 8vo, i 8s. net.

ELEMENTARY HYDRAULICS. For Technical Students. By F. C. LEA, D.Sc., M.Inst.C.E. viii + 224 pages, with 156 diagrams. Crown 8 vo, 7s. 6 d. net. MODERN METHODS OF WATER PURIFICATION. BY JOHN Don, I.I.C., A.M.I.Mech.E., and Jous Cuisholm, A.M.I.Mech.E. Second Edition. xviii +398 pages, 106 illustrations. Demy 8 vo, r6s. net.

MODERN ROADS. By H. P. Boulwois, M.Inst.C.E., F.R.San.Inst., etc., Chairman of the Council of the Roads Improvement Association, etc. xii +302 pages. Demy 8 vo, I $6 \mathrm{~s}$. net.

REINFORCED CONCRETE DESIGN. VOL. I. : THEORY. BY OSCAR FABER, D.Sc., A.M.Iust.C.E., Chief Engineer to Messrs. Trollope and Colls, Ltd., and P.G. Bowie, A.M.Inst.C.E. $x x+332$ pages, 158 diagrams. SECond Edition. Demy 8vo, i4s. net. Vol. II. : Practice. ISy Oscar Faber, D.Sc., A.M.Inst.C.E. $\mathrm{xii}+246$ pages, 89 diagrams. Demy 8 vo, 18 s. net.

LONDON : EDIVARD ARNOLD \& CO., 41 \& 43 MADDOX ST., W.1. 
THE PRACTICE OF RAILWAY SURVEYING AND PERMANENT WAY WORK. By S. Wright PERrotT, M.A.I., M.Inst.C.E., Professor of Civil Engineering in the University of Liverpool ; and F.E.G. BADGER, A.M.Inst.C.E. viii +304 pages, with 140 diagrams. Demy 8 vo, 3os. net.

SURVEYING. By WV. N. Thomas, M.Sc. (Birmingham), B.Sc. Eng. (London), Assoc.M.Inst.C.E., A.M.I.Mech.E., A.M.Inst. M. and Cy.E., Demonstrator in Engineering at the University of Oxford. viii +536 pages and 298 diagrams. 8 vo, 25s. net.

THE FIELD ENGINEER'S HANDBOOK. By G. C. Wells and A. S. Clay, B.Sc. Second Edition. viii +227 pages, 7 I illustrations. Small 8 vo, 8s. 6d. net.

TRAVERSE TABLES. With an Introductory Chapter on Co-ordinate Surveying. By Henry Lours, M.A., D.Sc., M.I.C.E., Professor of Mining; and G. W. CAUnt, M.A., Lecturer in Mathematics, Armstrong College, Newcastle-on-Tyne. SECOND EDITION. 8vo, 5s. 6d. net.

TACHEOMETER TABLES. By H. Louis, M.A., D.Sc., M.I.C.E. ; and G. W. CAunt, M.A. 8vo, Ios. 6d. net.

A TEXT-BOOK OF ELECTRICAL ENGINEERING. By Dr. A. Tilomälen. Translated by Professor G. W. O. Howe, D.Sc. Fourti Edition. xii +482 pages, 480 diagrams. Demy 8 vo, 28 s. net.

THE PRINCIPLES OF ELECTRICAL ENGINEERING AND THEIR APPLICATION. By Dr. G. KAPP, late Professor of Electrical Engineering in the University of Birmingham. Volume I.: Principles. Fully illustrated. xii +356 pages. Demy 8 vo, i 8s, net. Volume II.: Application. $x+388$ pages. I 8 s. net.

THE FOUNDATIONS OF ALTERNATE CURRENT THEORY. By C. V. DrySDALE, D.Sc. (London), M.I.E.E., F.R.S.E. xii +300 pages, 190 illustrations. Demy 8vo, Ios. 6d. net.

THE THEORY OF MACHINES. By R. F, McKay, M.Sc., A.M.Inst. C.E., A.M.I.Mech.E. Second Edition. viii +440 pages, 407 diagrams. Demy 8 vo, 20s. net.

GRINDING MACHINERY. By J. J. Guest, M.A., M.I.Mech.E., Professor of Mechanical and Electrical Engineering, Artillery College, Woolwich. xii +444 pages, with illustrations. Demy 8 vo, I6s. net.

STEAM BOILERS AND BOILER ACCESSORIES. By the late W. INCIILEY, B.Sc. $x i+4$ I 2 pages, r4I illustrations. Crown 8vo, 8s. $6 \mathrm{~d}$. net.

THE PRACTICAL DESIGN OF MOTOR-CARS. By J. GunN. viii +256 pages, 162 illustrations. Demy 8vo, I 2S. 6d. net.

THE STRENGTH AND ELASTICITY OF STRUCTURAL MEMBERS. By R. J. Woods, M.E., M.Inst.C.E. Second Edition. xii +310 pages, 292 illustrations. Demy 8vo, 145. net.

THE THEORY OF STRUCTURES. By R. J. Woods, M.E., M.Inst. C.E. $x i i+276$ pages, 157 illustrations. Demy 8 vo, 12S. 6 d. net.

THE ITALIAN ORDERS OF ARCHITECTURE. By CHARLES Gourlay, B.Sc., A.R.I.B.A., Professor of Architecture and Building in the Royal Technical College, Glasgow. With 32 full-page plates. Large 4 to (12" $\times 9 \frac{1^{\prime \prime}}{}$ ), 6s. net.

MECHANICAL DRAWING. With Special Reference to the Needs of Mining Students. By Joseph Husband, B.Eng., A.M.I.C.E., Professor of Civil Engineering at Sheffield University. With 40 plates. Quarto, 3s.6d. net.

MACHINE SKETCHES AND DESIGNS. By Professor A. CruickSHANK, M.I.Mech.E., and R. F. McKAY, M.Sc., A.M.Inst.C.E. viii + 40 pages of illustrations, with short Explanatory Notes. SEcond EdrTron. Quarto, 2s. 6d. net.

LONDON : EDWARD ARNOLD \& CO., 4 I \& 43 MADDOX ST., W.I. 
PETROL ENGINE CONSTRUCTION AND DRAWING. BY W. E. DoMsETT, Wh. Ex., A.M.I.A.E. 26 plates, with descriptive letterpress and questions for technical students. Demy 4 to, 3s. 6 d. net.

EXAMPLES IN ELECTRICAL ENGINEERING. By J. F. GiLl, M.Sc., B.Eng., A.M.I.Mech.E. ; and F. J. TEAGo, M.Sc., M.I.E.E., The University, Liverpool. Crown 8vo, 7s. 6d. net.

EXERCISES IN ELECTRICAL ENGINEERING. For the Use of "Second Year" Students in Universities and Technical Colleges. By T. MATIIER, F.R.S., M.I.E.E., Professor of Electrical Engineering, City and Guilds (Engineering) College; and G. W. O. Howe, D.Sc., M.I.E.E., Assistant Professor of Electrical Engineering, City and Guilds (Engineering) College. THIRD EDIIION. Crown 8vo, 2s. net.

\section{GEOLOGY AND MINING}

THE GEOLOGY OF THE BRITISH EMPIRE. BY F. R. C. REED, Sc.D., li.G.S., formerly Assistant to the Professor of Gcology in the University of Cambridge. viii +480 pages, with 25 maps and sections. Demy 8 vo, 30s. net.

COAL IN GREAT BRITAIN. By Walcot Gibson, D.Sc., F.G.S., F.R.S.E., Head of the Geological Survey of Scotland. viii $+3 I_{2}$ pages, with 50 diagrams and 8 plates. Demy $8 \mathrm{vo}, 2$ Is. net.

OIL FINDING : An Introduction to the Geological Study of Petroleum. By E. H. Cunningham Craig, B.A., F.G.S., Second Edition. Second Impression. xii $+32+$ pages, 13 plates and 20 illustrations. Demy 8 vo, cloth, I $6 \mathrm{~s}$. net.

THE DRESSING OF MINERALS. By H. Louis, D.Sc., M.I.M.E., M.I.C.E., Professor of Mining and Lecturer on Metallurgy at Armstrong College, Newcastle-on-Tyne. $\mathrm{x}+544$ pages, $4 \mathrm{I} 6$ illustrations. Super royal $8 \mathrm{vo}$, cloth, $30 \mathrm{~s}$. net.

WINDING ENGINES AND WINDING APPLIANCES : Their Design and Economical Working. By G. MCCULLOCH, A.M.I.M.E., Inspector of Machinery in the Department of Mines, West Australia; and T. C. FUters, M.Inst.M.E. viii +452 pages, I 75 illustrations. Demy 8 vo, 2 Is. net.

A TEXTBOOK OF GEOLOGY. By P. Lake, M.A., F.G.S., Royal Geographical Society Reader in Regional and Physical Geography in the University of Cainbridge; and R. H. RAst ALL, Sc.D., F.G.S., Lecturer in Economic Geology in the University of Cambridge. xiv +508 pages, fully illustrated. THIRD EDITION:
Demy 8vo, 2Is. net.

OUTLINES OF PALAONTOLOGY. By H. H. Swinnerton, D.Sc., F.G.S., Professor of Geology at University College, Nottingham. Fully Illustrated. Demy 8 vo, cloth.

THE GEOLOGY OF ORE DEPOSITS. By H. H. ThOMAS, M.A., B.Sc., and D. A. MAcAlister, Assoc. R.S.M., of the Geological Survey of Great Britain. xii $+4 \mathrm{I} 6$ pages and 65 illustrations. Crown 8 vo, $8 \mathrm{~s}$. $6 \mathrm{~d}$. net.

THE GEOLOGY OF BUILding STONES. By J. Allen Howe, B.Sc., Curator of the Museum of Practical Geology. viii +455 pages, fully illustrated. Crown 8 vo, 8s. 6d. net.

THE GEOLOGY OF SOILS AND SUBSTRATA. By the late H. B. WoODWARD, F.R.S. xvi +366 pages, with illustrations. Crown 8 vo, 8s. 6d. net. GEOLOGICAL AND TOPOGRAPHICAL MAPS : Their Interpretation and Use. By A. R. Diverryhouse, D.Sc., F.G.S. SEcond EdITION. viii + 133 pages, with 90 illustrations. Demy 8 vo, 6s, net.

AN INTRODUCTION TO MINE SURVEYING. BY T. BRYSON, A.R.T.C., M.Inst.M.E., Lecturer in Mining at the Mining and Technical College, Wigan, and G. M. Cha MBers, M.Inst.M.E., Certificated Collicry Manager and Surveyor of Mines. Fully illustrated. viii +288 pages, $6 \mathrm{~s}$. net.

LONDON : EDWARD ARNOLD \& CO., 4 I \& 43 MADDOX ST., IV.I. 


\section{CHEMISTRY AND METALLURGY}

METALS AND METALLIC COMPOUNDS. By. U. R. Evans, M.A., King's College, Cambridge. 4 vols., obtainable separately. Demy 8 vo. Vol. 1., 21s. net. Vol. II., I $8 \mathrm{~s}$. net. Vol. III., I4s. net. Vol. IV., I 8 s. net.

ASSAYING IN THEORY AND PRACTICE. By E. A. WRAigit, A.R.S.M., M.I.M.M., late Senior Demonstrator in the Assaying Laboratory of the Royal School of Mines, London. xii +324 pages, 70 illustrations. Demy 8 vo, Ios. 6 d. net.

SERVICE CHEMISTRY. By the late ViviaN B. LEwes, F.I.C., F.C.S. ; and J. S. S. Brame, F.I.C., F.C.S., Professor of Chemistry, Royal Naval College, Greenwich. liffth Edition. xvi +576 pages. lllustrated. Demy 8vo, 2 is.

FUEL. Solid, Liquid, and Gaseous. By J. S. S. Brame, F.I.C. Second Edition. xiv +372 pages, with 73 illustrations and 55 tables. Demy 8vo, I5s. net.

PETROL AND PETROLEUM SPIRITS. A Description of their Sources, Preparation, Examination, and Uses. By W. E. Gooday, A.R.S.M., D.I.C., A.M.Inst.P.T. With a Preface by Sir Jonn Cadman, K.C.M.G. xii + I35 pages. Illustrated. Demy 8 vo, ros. 6 d. net.

THE ABSORPTION OF NITROUS GASES. By H. W. WebB, M.Sc., F.I.C., Head of Chemistry Department, Cardiff Technical College. Illustrated. Demy 8 vo, 25s. net.

THE CHEMISTRY AND TESTING OF CEMENT. By C. H. Descil, D.Sc., Ph.D., Professor of Metallurgy in the University of Sheffield.

[New Edition in Preparation.

THE CHEMISTRY AND MANUFACTURE OF HYDROGEN. BY P. Litinerland Teed, A.R.S.M., A.I.M.M., Major, R.A.F. Illustrated. Demy 8 vo, cloth, Ios. 6d. net.

THE PRINCIPLES OF APPLIED ELECTRO-CHEMISTRY. By A. J. Allmand, D.Sc., Professor of 'Chemistry, King's College, London. xii +547 pages, with 136 figures. Demy 8 vo, 25 s. net.

FORENSIC CHEMISTRY. By A. LucAs, O.B.E., F.I.C., Director of the Government Analytical Laboratory and Assay Office, Cairo. viii +268 pages. Demy 8vo, I 5s. net.

ORGANIC CHEMISTRY FOR ADVANCED STUDENTS. By Julius B. Colien, Ih.D., B.Sc., F.R.S., Professor of Organic Chemistry in the University of Leeds. Fourth Edition, in Threc Parts. Each Part obtainable scparately. Demy 8 vo, cloth.

THE CHEMICAL SYNTHESIS OF VITAL PRODUCTS AND THE INTER-RELATIONS BETWEEN ORGANIC COMPOUNDS. By the late Professor Raphael Meldola, F.R.S., F.I.C., etc. xvi $+33^{8}$ pages. Super royal 8 vo, 2 Is. net.

BIO-CHEMISTRY. A Study of the Origin, Reactions, and Equilibria of Living Matter. By the late Benjamin Moore, M.A., D.Sc., F.R.S., Whitley Professor of Bio-Chemistry in the University of Oxford. viii +340 pages. Demy 8vo, 2 Is net.

A LABORATORY HANDBOOK OF BIO-CHEMISTRY. BY P. C. Raiment, B.A., M.R.C.S., and G. L. Peskett, B.A., The University, Oxford. Crown 8 vo, 5s. net.

CHEMICAL DISINFECTION AND STERILIZATION. By S. RIDEAL, D.Sc., F.I.C., and E. K. RideAL, M.A., D.Sc., F.I.C. viii + 3 I 3 pages. Demy 8 vo, 2Is. net.

LEAD POISONING AND LEAD ABSORPTION. By T. M. LEgGe, M.D., H.M. Medical Inspector of liactories; and K. W. GOADBY, M.R.C.S., D.P.H. 308 pages. Illustrated. Demy 8vo, I 2s. 6d. net.

INDUSTRIAL POISONING. Translated from Dr. Rambousek's "Gewerbliche Vergiftungen" by Dr. T. M. LEGGE. Demy 8vo, I4s. net.

LONDON : EDWARD ARNOLD \& CO., 4 I \& 43 MADDOX ST., W.I* 
PHYSICAL CHEMISTRY : its Bearing on Biology and Medicine. By J. C. Philıp, D.Sc., F.R.S., Professor of Physical Chemistry in the Imperial College of Science and Technology. Second Edition. Crown 8vo, 8s. 6d. net.

ELEMENTARY PHYSICAL CHEMISTRY. By W. H. BARRETt, M.A., Har row School. viii +247 pages, with 6I diagrams. 6s.

THE CHEMISTRY OF COLLOIDS AND SOME TECHNICAL APPLICATIONS. By W. W. TAYLOR, M.A., D.Sc., Lecturer in Chemical Physiology in the University of Edinburgh. viii +332 pages. SEcond Editron. Crown 8vo, Ios. 6d. net.

PRACTICAL CHEMICAL PHYSIOLOGY. By W. W. TAYlor, M.A., D.Sc. Crown 8vo. Ordinary Edition, 4s. 6d. net ; Isterleaved Edition, 5s. net.

LECTURES ON THEORETICAL AND PHYSICAL CHEMISTRY. By the late Dr. J. H. van'T Hoff. Translated by R. A. LEHFELd, D.Sc. In three vols., demy 8vo, 29s. net, or separately: PART 1. Chemical Dynamics. I2s. net. Part II. Chemical Statics. 8s. 6d. net. Part III. Relations Between ProPERTIES AND COMPOSITION. 8s. 6d. net.

ANALYTICAL MICROSCOPY. By T. E. WALLIS, B.Sc., Lecturer in Botany at the School of Pharmacy of the Pharmaceutical Society of Great Britain. viii + I50 pages. Illustrated. Crown $8 \mathrm{vo}$, cloth, $6 \mathrm{~s}$. net.

AN INORGANIC CHEMISTRY. By H. G. DENhaM, M.A., D.Sc., $\mathrm{Ph} . \mathrm{D} .$, Professor of Chemistry in the University of Cape Town. xii $+68+$ pages, with I44 diagrams. Crown 8vo, I2s. 6d. net.

INORGANIC CHEMISTRY. A Textbook for Schools. By E. J. Holmyard, B.A., Head of the Science Department, Clifton Collegc. viii +564 pages, with IIg diagrams and Io plates. Crown 8vo, 6s. 6d.

A HANDBOOK OF ORGANIC ANALYSIS : QUALITA'TIVE AND QUANTITATIVE. By H. T. ClARKE, B.Sc., A.I.C., with an Introduction by Professor J. Norman Collie, F.R.S. xvi $+26+$ pages. Tinird Edition. Crown 8 vo, 6s. 6d. net.

THE CHEMISTRY OF BREADMAKING. By Janes GRAnt, M.Sc. Techn., F.I.C., F.C.S., Lecturer and Examiner in the Victoria University and School of Technology, Manchester; Head of the School of Bakery and Confectionery. THIRD Edition. viii +230 pages. Illustrated. Crown 8 vo, $6 s$. net.

CONFECTIONERS' RAW MATERIALS. By James Grant, M.Sc. Techn., F.I.C., F.C.S. vili +174 pages. Illustrated. Crown 8vo, 8s. 6d. net.

AN INTRODUCTION TO BACTERIOLOGICAL AND ENZYME CHEMISTRY. By Gilbert J. Fowler, D.Sc., F.I.C., Professor of BioChemistry in the Indian Institute of Scicnce, Bangalore. viii +328 pages. Illustrated. Crown 8vo, 8s. 6d. net.

INORGANIC CHEMISTRY FOR SCHOOLS. By W. M. HootoN, M.A., M.Sc., Chief Chemistry Master at Repton School. 4I 6 pages. Crown 8vo, 5s. ELEMENTS OF INORGANIC CHEMISTRY. By the late W. A. Shenstone, F.R.S. Sixth Edition, revised and partly rewritten by R. G. Durrant, M.A., Assistant Master, Marlborough College. Crown 8vo, 6s. 6d.

OUTLINES OF INORGANIC CHEMISTRY. With Special Reference to its Historical Development. By E. B. Ludlam, I.Sc., F.C.S., late Head of Chemical Department, Clifton Collegc. xvi +368 pages. Illustrated. Crown 8vo, 5s.

MINING PHYSICS AND CHEMISTRY. By J. W. Whitaker, B.Sc., Lecturer in the Mining Department, University College, Nottingham. xii +268 pages. Crown 8 vo, 9s. net.

QUALITATIVE ANALYSIS: IN THEORY AND PRACTICE. By P. W. Robertson, M.A., Ph.D., Professor of Chemistry, Victoria University College, New Zealand; and D. H. BuRLEIGH, M.Sc., D.I.C., Science Master, Christ's Hospital. Demy 8vo, 4s. 6d.

LONDON : EDWARD ARNOLD \& CO., 41 \& 43 MADDOX ST., W.I. 
QUALITATIVE AND VOLUMETRIC ANALYSIS. By W. M.

Hooton, M.A., M.Sc. Demy 8vo, 3s. 6d. net.

EXERCISES IN CHEMICAL CALCULATION. By H. F. Coward, D.Sc., and W. H. Perkins, M.Sc. viii + 152 pages. Crown 8vo, 3s. 6d. net. FIRST AID IN THE LABORATORY AND WORKSHOP. BY A. A. Eldridge, B.Sc., and H. V. A. Briscoe, D.Sc. Cloth, is. 3d. net.

\section{BIOLOGY}

FOUNDERS OF OCEANOGRAPHY AND THEIR WORK. By Sir William HeRdman, C.B.E., D.Sc., F.R.S., Emeritus Professor in the University of Liverpool. Demy 8vo, cloth.

MANUAL OF ENTOMology. By H. Maxwell Lefroy, M.A., Professor of Entomology in the Imperial College of Science and Technology. xvi +552 pages. Fully Illustrated. Demy 8 vo, cloth, 35s. net.

THE MECHANISM OF LIFE. In Relation to Modern Physical Theory. By J. Johnstone, D.Sc., Professor of Oceanography in the University of Liverpool. xii +248 pages, with 53 diagrams. Demy 8vo, 15s. net.

ANIMAL LIFE IN DESERTS. By P. A. Buxton, M.A. xvi+172 pages, with 14 plates. Demy 8 vo, 10s. 6d. net.

AN INTRODUCTION TO THE STUDY OF THE PROTOZOA. With special reference to the Parasitic Forms. By the late E. A. Minchin, M.A., Ph.D., F.R.S. xii + 520 pages, 194 diagrams. Demy 8vo, 25s. net.

HUMAN EMBRYOLOGY AND MORPHOLOGY. By Sir ARTIIUR KEITH, F.R.S., M.D., F.R.C.S., Conservator of the Museum and Hunterian Professor, Royal College of Surgeons, England. Fourth Edition. viii +491 pages. Demy 8 vo, 3os. net.

THE MigRations OF Fish. By Alexander Meek, M.Sc., F.L.S., F.Z.S., Professor of Zoology, Armstrong College, Newcastle-on-Tyne, and Director of the Dove Marine Laboratory. With illustrations and maps. $x x+428$ pages. Demy 8vo, 18s. net.

ARBOREAL MAN. By F. Wood Jones, M.B., D.Sc., Professor of Anatomy in the University of London. With $8 \mathrm{I}$ illustrations. Demy $8 \mathrm{vo}, 8 \mathrm{~s}$. $6 \mathrm{~d}$. net.

THE DEVELOPMENT OF BRITISH FORESTRY. By A. C. FORBES, F.H.A.S. xii +274 pages, 70 illustrations. Demy 8 vo, ros. 6 d. net.

THE BODY AT WORK. A Treatise on the Principles of Physiology. By Alex. Hill, F.R.C.S. xii +448 pages, with 46 illustrations. Demy 8 vo, $16 \mathrm{~s}$. net.

THE Evolution THEORY. By Dr. August Weismann, Professor of Zoology in the University of Freiburg in Breisgau. Translated by Professor J. Arthur Thomson, and Margaret Thomson. Two Vols., xvi + 4 I 6 and viii +396 pages, with over I30 illustrations. Royal 8vo, 32s. net.

MANUAL OF HUMAN PHYSiOLOGY. By Leonard Hill, M.B., F.R.S. xii +484 pages, with 177 illustrations. Crown 8 vo, 6s.

ANIMAL BEHAVIOUR. By C. Lloyd Morgan, LL.D., F.R.S., Professor of Psychology and Ethics in the University of Bristol. SEcond Edition. viii +344 pages, with 26 illustrations. Crown 8 vo, 8s. 6d. net.

A HANDBOOK OF THE CONIFER AND GINGKOACE压. By W. DALL1MORE and A. B. JACKSON. With 32 plates' and 120 diagrams. Medium 8 vo, cloth.

A CLASS-BOOK OF BOTANY. By G. P. Mudge, A.R.C.Sc. (London), F.Z.S., Lecturer on Biology at the London Hospital Medical College; and A. J. MASLEN, F.L.S. xvi +512 pages, with 228 illustrations. Crown 8vo, 8s. 6d. net.

LONDON: EDWARD ARNOLD \& CO., 41 \& 43 MADDOX ST., W.r. 



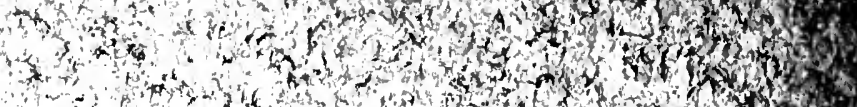
and

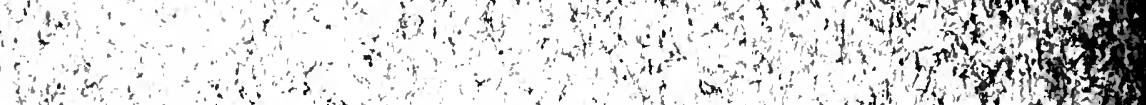
(1)

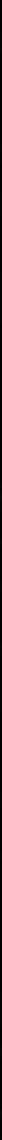




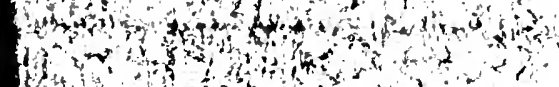
(1) (1) $=4+4$

\section{की}

a

fis

eri, en $3^{4}=2$ and

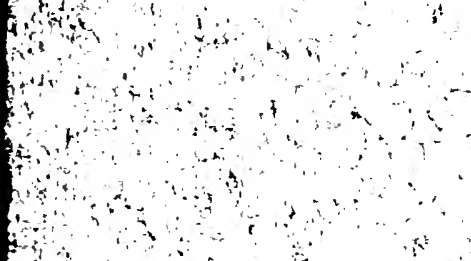




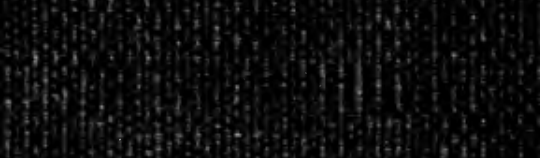

15.

ith

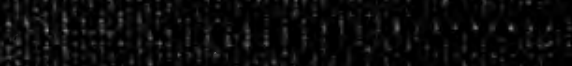

8:

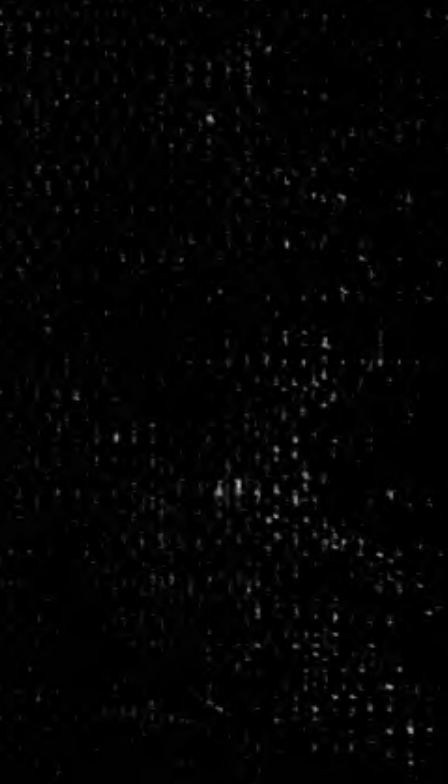

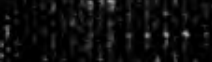

